\title{
Modeling and Testing of Unbalanced Loading and Voltage Regulation
}

Subcontract Report NREL/SR-581-41805 July 2007

\section{Final Report}

M.W. Davis

DTE Energy

Detroit, Michigan

R. Broadwater

Electrical Distribution Design Inc.

Blacksburg, Virginia

J. Hambrick

Virginia Polytechnic Institute and State University Blacksburg, Virginia 


\section{Modeling and Testing of Unbalanced Loading and Voltage Regulation}

\section{Final Report}

M.W. Davis

DTE Energy

Detroit, Michigan

R. Broadwater

Electrical Distribution Design Inc.

Blacksburg, Virginia

J. Hambrick

Virginia Polytechnic Institute and State University Blacksburg, Virginia

NREL Technical Monitor: Thomas Basso

Prepared under Subcontract No. ZAT-5-32616-06

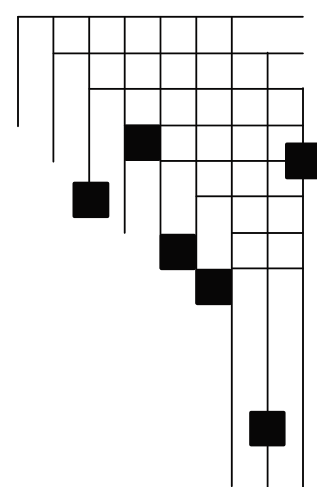


The National Renewable Energy Laboratory is a national laboratory of the U.S. Department of Energy (DOE) managed by Midwest Research Institute for the U.S. Department of Energy under Contract Number DE-AC36-99GO10337. This report was prepared as an account of work sponsored by the California Energy Commission and pursuant to a M\&O Contract with the United States Department of Energy (DOE). Neither Midwest Research Institute, nor the DOE, nor the California Energy Commission, nor any of their employees, contractors, or subcontractors, makes any warranty, express or implied, or assumes any legal liability or responsibility for the accuracy, completeness, or usefulness of any information, apparatus, product, or process disclosed, or represents that its use would not infringe privately owned rights. Reference herein to any specific commercial product, process, or service by trade name, trademark, manufacturer, or otherwise, does not necessarily constitute or imply its endorsement, recommendation, or favoring by Midwest Research Institute, or the DOE, or the California Energy Commission. The views and opinions of authors expressed herein do not necessarily state or reflect those of Midwest Research Institute, the DOE, or the California Energy Commission, or any of their employees, or the United States Government, or any agency thereof, or the State of California. This report has not been approved or disapproved by Midwest Research Institute, the DOE, or the California Energy Commission, nor has Midwest Research Institute, the DOE, or the California Energy Commission passed upon the accuracy or adequacy of the information in this report.

\section{NOTICE}

This report was prepared as an account of work sponsored by an agency of the United States government. Neither the United States government nor any agency thereof, nor any of their employees, makes any warranty, express or implied, or assumes any legal liability or responsibility for the accuracy, completeness, or usefulness of any information, apparatus, product, or process disclosed, or represents that its use would not infringe privately owned rights. Reference herein to any specific commercial product, process, or service by trade name, trademark, manufacturer, or otherwise does not necessarily constitute or imply its endorsement, recommendation, or favoring by the United States government or any agency thereof. The views and opinions of authors expressed herein do not necessarily state or reflect those of the United States government or any agency thereof.

Available electronically at http://www.osti.gov/bridge

Available for a processing fee to U.S. Department of Energy and its contractors, in paper, from:

U.S. Department of Energy

Office of Scientific and Technical Information

P.O. Box 62

Oak Ridge, TN 37831-0062

phone: 865.576 .8401

fax: 865.576 .5728

email: mailto:reports@adonis.osti.gov

Available for sale to the public, in paper, from:

U.S. Department of Commerce

National Technical Information Service

5285 Port Royal Road

Springfield, VA 22161

phone: 800.553 .6847

fax: 703.605.6900

email: orders@ntis.fedworld.gov

online ordering: http://www.ntis.gov/ordering.htm

This publication received minimal editorial review at NREL 


\section{List of Acronyms}

$\begin{array}{ll}\text { ANSI } & \text { American National Standards Institute } \\ \text { BL } & \text { beginning location } \\ \text { CAP } & \text { capacitor } \\ \text { CC } & \text { constant current } \\ \text { CP } & \text { constant power } \\ \text { CT } & \text { current transformer } \\ \text { DG } & \text { distributed generation/distributed generator } \\ \text { DR } & \text { distributed resource(s) } \\ \text { EL } & \text { end location } \\ \text { GMD } & \text { geometric mean distance } \\ \text { HL } & \text { heavy load } \\ \text { HV } & \text { high voltage } \\ \text { LL } & \text { light load } \\ \text { LTC } & \text { load tap changer/load tap-changing } \\ \text { LV } & \text { low voltage } \\ \text { ML } & \text { midlocation } \\ \text { PF } & \text { power factor } \\ \text { VDC } & \text { voltage-dependent current } \\ \text { VR } & \text { voltage regulator } \\ \text { VRR } & \text { voltage regulating relay } \\ \text { VT } & \text { voltage transformer }\end{array}$




\section{Executive Summary}

\section{Introduction}

A distributed generation (DG) penetration limit study (Davis 2003) indicated a range of DG sizes can be interconnected with a distribution circuit. The DG size limit is dependent on system voltage, the location of the DG on the circuit, and system protection, voltage regulation, and other issues related to DG and circuit characteristics. The study showed that the DG could be larger if it was allowed to actively regulate voltage, rather than operate at a fixed unity power factor.

Fixed power factor operation of the DG has a minimum effect on the existing traditional voltage regulation controls of the circuit because, as the system voltage changes, the field current of the DG synchronous generator is adjusted to bring the kilovar output typically back to zero and maintain the desired kilowatt setpoint. Therefore, the only effect on the system voltage is the kilowatt injection at that point on the distribution circuit. However, if the DG synchronous machine is allowed to absorb or export volt-amperes reactive, the voltage can be decreased or increased at that point on the circuit, and much larger kilowatt injections - and thus, larger DG-can be installed on the circuit.

A number of problems are associated with interconnecting DG with a distribution circuit. Some are related to circuit design and operation, and others are related to the analytical tools used to evaluate DG operation. Distribution circuits are primarily designed for radial, oneway power flow, and distribution line voltage regulators are typically designed to regulate voltage based on a unidirectional flow of power. When DG is interconnected with the circuit, two-way flows can result. In addition, most of the load served on a distribution circuit is single-phase, yet most of the analytical tools used to evaluate circuit performance are based on balanced three-phase loads and balanced three-phase line circuit impedances. When balanced three-phase power flow programs are used to calculate the voltage profile on a distribution circuit and determine if voltage limits are being violated by DG, the accuracy of the service voltages at the individual single-phase loads on the single-phase laterals is a concern because only the three-phase portion of the circuit is modeled. American National Standards Institute Standard C84.1 voltage limits may be satisfied based on three-phase balanced load/impedance analysis, but the voltages at single-phase loads may be violated when the DG operates or shuts down.

This is a significant concern for utilities because liability issues arise when customer equipment is damaged because of $\mathrm{HV}$ or LV on a circuit. Therefore, it is critical to evaluate the effects of DG on the distribution circuit voltage profile to ensure customers do not receive service voltages (at the customer billing meter) outside Range A or Range B of American National Standards Institute C84.1. This can be accomplished by using modeling and simulation tools that recognize single-phase loads, unsymmetrical distribution transformer connections, and unbalanced line impedances. 


\section{Purpose}

The purpose of the project is to:

- Explain how voltage regulation reduces voltage spread

- Define the effects of unbalanced loading and voltage on system protection and DG output ratings

- Develop models for an actual distribution circuit, its voltage regulation equipment, and all the DG generator types, recognizing unbalanced loading and unbalanced circuit impedances

- Validate these models by comparing power flow simulations and voltage profiles with actual measured circuit data

- Determine the optimum generator operating conditions (i.e., $\mathrm{P}$ and $\mathrm{Q}$ ) to provide the greatest improvement in released capacity, reduced energy losses, and voltage regulation

- Determine the maximum DG penetration limits with synchronous generator real and reactive power injections.

\section{Project Objectives}

The project objectives are five-fold:

- To develop a load model for an actual $13.2-\mathrm{kV}$ distribution circuit that represents how the real and reactive load on the circuit changes when the voltage is raised or lowered with capacitor switching, distribution line voltage regulator step changes, and load tap changer and DG voltage regulation

- To develop models for distribution overhead and underground circuit line elements, transformers, shunt capacitors, step voltage regulators, and synchronous, induction, and inverter DG generators

- To verify the models by comparing the power flow simulated data with actual circuit measured data

- To determine the maximum DG size that can be interconnected with the circuit

- To determine the optimum generator operating conditions for maximum released capacity savings, reduced energy losses, and voltage regulation improvement.

\section{Project Results}

- The circuit load was modeled using constant current, constant power, and a voltagedependent current. The voltage-dependent current model best represented the load characteristics of the circuit, with a variance of only $2 \%$. The constant current model had a variance of $3.9 \%$, and the constant power model, which is typically used, had a variance of $12.5 \%$. 
The voltage-dependent current model that best represents how the load changes with changes in source voltage is:

$$
\begin{aligned}
& \frac{\% \Delta \mathrm{P}}{\% \Delta \mathrm{V}}=1.26 \\
& \frac{\% \Delta \mathrm{Q}}{\% \Delta \mathrm{V}}=4.66 .
\end{aligned}
$$

- During heavy load conditions when all voltage regulation equipment was turned on, the circuit tag end voltage improvement was $14.87 \mathrm{~V}$ over only the load-tap changing transformer voltage. When the first step regulator was turned on, the tag end voltage improved $4.11 \mathrm{~V}$. With the second, the voltage increased another $11.34 \mathrm{~V}$. With the first capacitor turned on, the voltage increased only $0.97 \mathrm{~V}$. When the second was turned on, the total voltage gain was $1.75 \mathrm{~V}$. When the third was turned on, another $1.66-\mathrm{V}$ rise occurred at the tag end. It may be necessary to operate DG to absorb voltamperes reactive to prevent high voltage during light-load conditions.

- The highest unbalanced voltage of $1.52 \%$ occurred during heavy load conditions with load tap changer regulation and one step regulator turned on. Because most synchronous generators trip when the unbalanced voltage is more than $3 \%$, unbalanced three-phase power flow studies should always be conducted on the circuit to ensure unbalance does not exceed 3\% at the point of interconnection (not the point of common coupling). Adding load tap changer regulation during heavy load conditions lowered the maximum unbalanced voltage to $1.44 \%$; adding step regulators worsened the unbalanced voltage. This was to be expected because an increase in voltage causes an increase in load and unbalance. When all three capacitors were turned on, the maximum unbalanced voltage was reduced to $1.31 \%$. Turning all regulation on lowered the maximum unbalanced voltage to $1.31 \%$. The 13 -utility unbalanced voltage survey showed a maximum measured voltage unbalance of $5.94 \%$. The high imbalances occurred on open delta transformer connections.

- The highest voltage unbalance during light-load conditions was $1.26 \%$.

- At many locations on the circuit, the current imbalance exceeded 20\%. Most synchronous machines trip at 10\%-20\% current imbalance; therefore, studies must determine the current imbalance at the location where the DG is to be sited.

Otherwise, it may never operate without tripping. The load imbalance at the substation reached $4 \%$ at heavy load and $6 \%$ at light load.

- The causes of unbalanced voltage and current and how unbalanced conditions affect protective relaying are described. The neutral relay is set to trip for ground faults, and the trip value may have to be increased to account for increased neutral current because of unbalance.

- Significant unbalanced loading can occur even though voltages are balanced at the source. Reclosers and the substation breaker with ground fault-sensing circuits are affected by load imbalance. Unequal single-phase load connected line-to-line does not produce neutral current in the ground relay. 
- Fuse preload because of unbalanced loading can cause fuses to become unselective with other protective devices such as reclosers and lead to misoperation.

- Reducing unbalanced loading reduces the losses created by the neutral current in the neutral conductor.

- Unbalanced three-phase voltages have a significant effect on the heating of induction and synchronous generators. For example, a 5.5\% voltage unbalance can cause an approximate $25 \%$ increase in temperature. The phase currents with unbalanced voltages are greatly unbalanced, on the order of four to five times the voltage unbalance. If overload relay protection settings are raised because of unbalance, the generator may not be protected against overload and open phases.

- Heating of induction generators because of voltage unbalance is affected by phase rotation because it affects which phase has the highest line currents. This means that negative sequence current protection must be used to protect the induction generator from failure because of voltage unbalance. The negative sequence losses are proportional to the square of the negative sequence voltage. The generator may have to reduce output below nameplate rating to avoid overheating with voltage unbalance. An equation was developed to determine machine rating under unbalanced voltages if one knows the unbalanced voltage and the ratio of the positive-to-negative sequence impedances of the induction generator. A 5\% voltage unbalance causes a $3.2 \%-10.7 \%$ power output derate, depending on the positive-to-negative sequence impedance ratio.

- Models were developed for:

○ Line impedance

- Line voltage drop

- Line loss (which was validated using unbalanced and balanced line configurations and unbalanced and balanced load conditions)

- Transformers for three-phase and single-phase and different loading combinations

- Secondary and service impedances

- Shunt capacitors and step regulators

○ Synchronous, induction, and inverter generation.

The models were validated by measuring power quantities throughout the circuit on the peak day and comparing these data with simulation data. The percent variance between field-measured data and simulation data for phase currents at eight nodes throughout the circuit were within $6 \%$. The highest phase voltage variance was only $1.5 \%$. 
- The voltage spread, measured as the difference between the highest three-phase voltage and the lowest single-phase voltage for the heavy-load condition, was $25.2 \mathrm{~V}$ with no regulation at the substation and on the distribution circuit. When all regulation was implemented, the voltage spread was reduced to only $10.4 \mathrm{~V}$, and there were no voltage criteria violations. For the light-load condition, the voltage spread was $16.65 \mathrm{~V}$ for no regulation. With all regulation operating, the spread was only $2.91 \mathrm{~V}$.

- The load imbalance at the substation was 4\% for heavy load and 5.45\% for light load. Adding step regulators always worsened the voltage unbalance because of the effect of the voltage-dependent current model.

- Three voltage control strategies were tested for the $400-\mathrm{kW}$ induction generator at low and high substation primary voltage, for a total of six simulations. Three voltage control strategies were tested for the $400-\mathrm{kW}$ inverter-based generation at low and high substation primary voltage, for a total of six simulations. Thirteen voltage control strategies were tested for high voltage, and 13 were tested for low voltage, for the synchronous generator at the beginning, mid-, and end locations of the circuit, for a total of 78 simulations. The maximum released capacity of $10.44 \%$ was achieved with the $1,000-\mathrm{kW}$ synchronous generator with $\mathrm{P}=100 \%$ and $\mathrm{Q}=100 \%$. The voltage improvement was $0.82 \%$, and the loss reduction was $0.56 \%$ out of a $5.4 \%$ base.

- The optimum location for the DG with the highest released capacity of $10.44 \%$ is at the source of the circuit because it directly offsets the load current and load losses of the circuit. The optimum DG location for loss reduction is at the end of the circuit. Adding generation here reduces, on a prorata basis, the load and the length of the circuit. To improve voltage regulation, there is little difference between locating the DG at the midpoint or end of the circuit. However, there is a slightly better improvement at the midpoint for circuits on which the conductor size of the entire three-phase backbone is the same.

- The DG penetration study showed synchronous DG had a real power limit of 13,980 $\mathrm{kW}$ at the tag end of the circuit. The optimum location was at the midpoint, with the lowest single-phase voltage improvement of $1.7 \%$ and a real power loss savings of $2.04 \%$. The base case real power losses were $5.4 \%$. The DG penetration study found that the maximum real and reactive power output limit was $14,490 \mathrm{~kW}$ and 2,007 $\mathrm{kVAr}$, which allows a larger DG to be installed than when only real power is injected. Again, the optimum location was at the midpoint of the circuit. However, in this case, the lowest single-phase voltage was improved $4.55 \%$ versus only $1.7 \%$ in the real power limit case. The real power loss savings were marginally better, with a $2.3 \%$ savings versus $2.04 \%$.

\section{Conclusions}

- The voltage-dependent current model best represents how the real and reactive components of the load change with changes in voltage. This model, which had an error of only $2 \%$, should always be used in lieu of the constant power model, which had an error of $12.5 \%$. 
- Heavy-load conditions produce the highest voltage unbalance, and adding a load tap changer and one step voltage regulator caused the maximum percent voltage unbalance to be $1.52 \%$. The typical limit for voltage unbalance is $3 \%$. It is recommended that a three-phase unbalanced power flow study be conducted on a circuit before a synchronous generator is installed because the unit may be installed at a location where the voltage unbalance causes the unit to trip. In this case, it will never operate at that location. Furthermore, the current imbalance cannot exceed $10 \%-20 \%$, or the synchronous DG will trip. There were many locations on the distribution circuit at which the current imbalance exceeded $20 \%$. Inverter-based generation was not sensitive to voltage or current unbalance and operated up to $100 \%$ current imbalance.

- The models developed for the line elements, distribution equipment, and generation and the simulations conducted during peak load conditions compared favorably with the actual phase currents, phase voltages, and power factors measured at eight locations throughout the circuit. However, it is essential to know the phasing of the loads on the circuit to obtain this high degree of accuracy. The variance between simulated and measured data was less than $6 \%$ for phase currents and did not exceed $1.5 \%$ for phase voltages. These low variances indicate the models are accurate enough to represent actual circuit operation under unbalanced load conditions. The only measured circuit data needed to perform accurate simulations are the phase voltages and currents at the source of the circuit and the regulator, capacitor, and DG locations.

- Unbalanced load can cause neutral relaying trip settings to be increased and preloading on fuses, which results in inselectivity with other protective devices.

- Unbalanced voltage can derate the output of synchronous and induction generators up to $10.7 \%$.

- The optimum location of DG on a circuit for the highest released capacity of $10.44 \%$ is at the source of the circuit. The greatest loss reduction of $0.56 \%$ occurs when the DG is located at the tag end of the circuit. There is little difference between locating the DG at the midpoint or the tag end for the greatest improvement in voltage regulation.

- The maximum DG real and reactive output was $14,490 \mathrm{~kW}$ and 2,007 kVAr, and the optimum location was at the midpoint of the circuit. The real power loss savings were $2.3 \%$ versus the base case of $5.4 \%$. The lowest single-phase voltage was improved $4.55 \%$.

\section{Recommendations}

- Always model distribution circuit loads with a voltage-dependent current, and use a validated unbalanced three-phase power flow to determine the percent unbalanced load and voltage during the peak load and at the locations where DG is planned. Using a balanced three-phase power flow or simplified single-phase power flow will not indicate the unbalanced load and voltage at locations where DG is planned. A location may be selected where a synchronous or induction generator may never operate because of circuit unbalance conditions. 
Successful modeling of a distribution circuit using an unbalanced three-phase power flow requires knowledge of which phases the loads are connected to. Otherwise, incorrect unbalanced load and voltage data will result. The use of three-phase metering is not warranted because the highest load imbalance occurs during light load and the highest unbalanced voltage occurs during heavy load. It is not practical to install metering at various locations throughout the circuit to capture light and heavy load unbalanced power quantities over a long period of time (such as a year) when simulations can produce similar information with nearly the same accuracy in considerably less time.

- The optimum voltage regulation method for the least voltage spread used the substation transformer load tap changer, step regulators, capacitors, and DG. The maximum released capacity is achieved when the DG is located at the beginning of the circuit, the maximum loss reduction is achieved at the end, and the best voltage regulation occurs at the middle. It is recommended that the DG be located at the midpoint of the circuit to produce the best overall improvements in voltage regulation, loss reduction, and released capacity.

- The circuit selected had voltage unbalance conditions that closely agreed with the average voltage unbalance measurements taken at 13 major utilities. Therefore, the results should be representative of the average distribution circuit.

It was shown that single-phase DG connected to the appropriate phases can improve the phase unbalanced conditions. Simulation studies can be used to determine to which phases to connect single-phase DG.

- Voltage regulation and system protection issues are the most difficult for interconnecting DG with a distribution circuit. It is recommended that inverter-based generation be considered as the preferred DG type where the level of unbalanced voltage and current may prevent synchronous and induction generators from operating on the circuit.

- It is recommended that future research develop an optimal control algorithm to control the substation transformer load tap changer, the bidirectional step regulators, the switched capacitors, and the DG. This could be accomplished for less than $\$ 500,000$ because the equipment is already installed on the Milford Circuit 8103 .

\section{Benefits to California}

Test data indicate the models developed for this project accurately represent the operation of a distribution circuit. Applying these models results in DG siting that ensures the DG will perform its intended function and produce the highest released capacity savings, lowest energy losses, and improved voltage regulation. 


\section{Table of Contents}

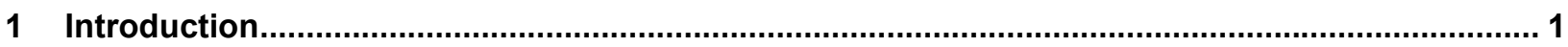

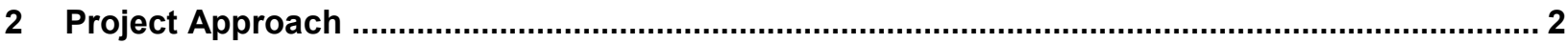

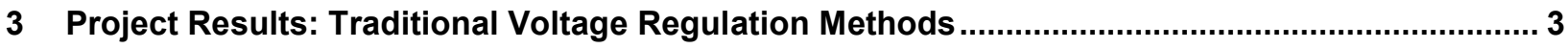

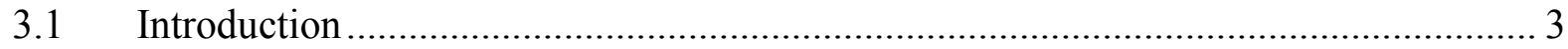

3.2 Distributed Generation Installed at Substation ................................................ 3

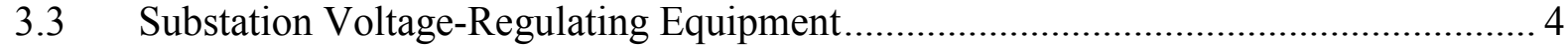

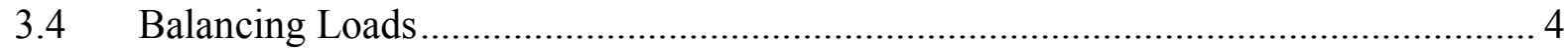

Increased Primary System Voltage ........................................................................ 4

3.6 Increased Conductor Size or Reduced Conductor Spacing..................................... 4

3.7 Conversion of Single-Phase Sections to Three-Phase .............................................. 4

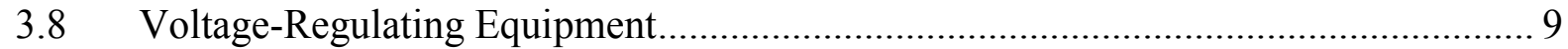

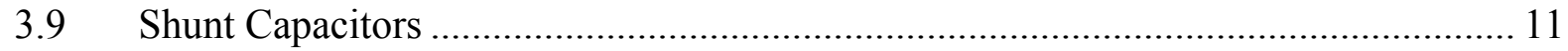

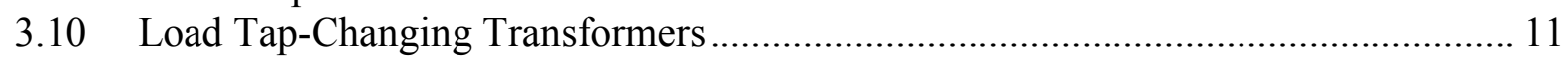

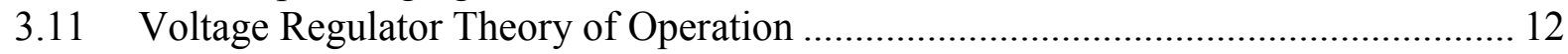

3.12 Bridging Reactor or Preventive Autotransformer ................................................. 16

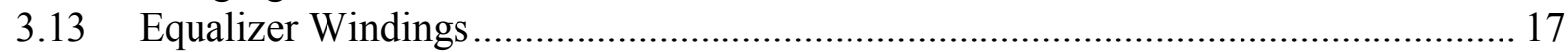

3.14 Types of Step Regulators ............................................................................... 19

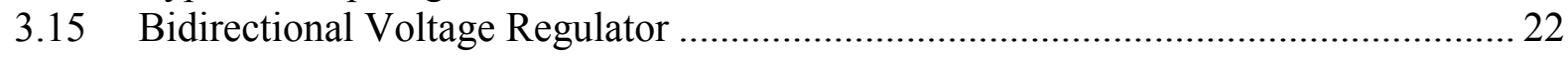

3.16 Step Voltage Regulator Control System ........................................................... 25

3.17 Voltage and Current Equations ...................................................................... 26

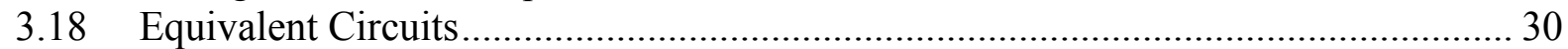

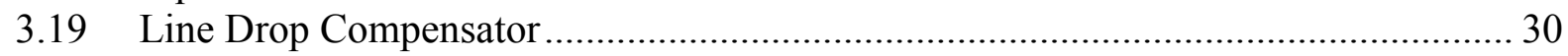

3.20 Regulator Ratings .................................................................................. 32

3.21 Closed Delta - Leading Current and Lagging Current Connections ........................ 37

3.22 Compensator Settings ............................................................................... 41

3.23 Other Compensator Settings and Corrections ....................................................... 44

3.24 Step Regulators in Series ................................................................................ 44

3.25 Fixed Capacitors on Circuits with Regulators ...................................................... 45

3.26 Switched Capacitors on Circuits with Regulators............................................... 46

3.27 Switched Capacitors Downstream from Regulators ............................................ 48

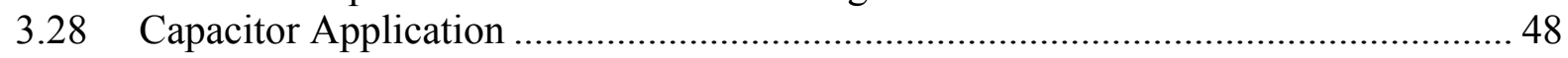

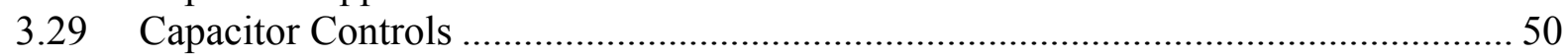

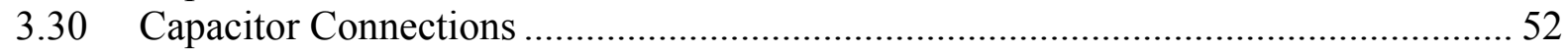

4 Project Results - Effects of Unbalanced Loading and Voltage on System Protection and

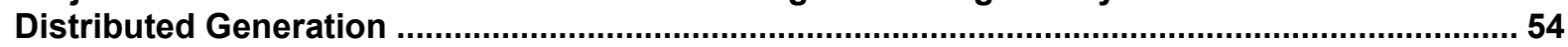

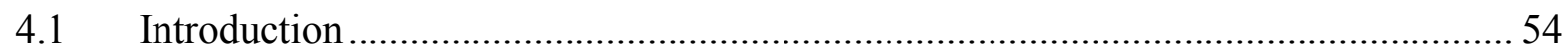

4.2 System Protection Design Philosophy for Grounded and Ungrounded Systems ....... 54 4.2.1 Four-Wire Grounded Wye Systems .......................................................... 58

4.2.2 Three-Wire Ungrounded Delta Systems ..................................................... 58

4.3 Unbalanced Voltage Effects on Protective Relaying ............................................. 59 
4.4 Unbalanced Current Effects on Protective Relaying ………….................................. 60

4.4.1 Single-Phase Operation of Protective Devices .................................................6 60

4.4.2 Operation of Circuit Protective Devices for Ground Faults ............................ 61

4.4.3 Single-Phase Operation of Disconnect and Isolation Devices............................. 62

4.4.4 Unbalanced Load Connected Line-to-Line ...................................................... 62

4.4.5 Fuse Preload Because of Unbalanced Loading or Unbalanced Voltages ....... 64

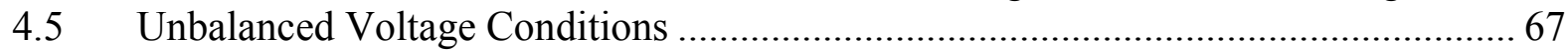

4.5.1 Induction Machines Operating on Unbalanced Voltages....................................68 68

4.5.2 Synchronous Generators Operating on Unbalanced Voltages ......................... 88

$5 \quad$ Project Results - Development of Models............................................................................ 92

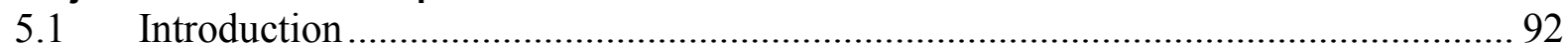

5.2 Three-Phase Substation Transformer Models ............................................................ 92

5.3 Three-Phase and Single-Phase Distribution Transformer Connections for

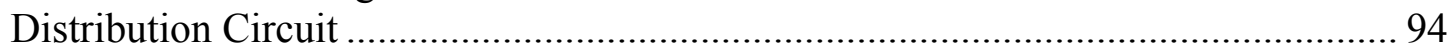

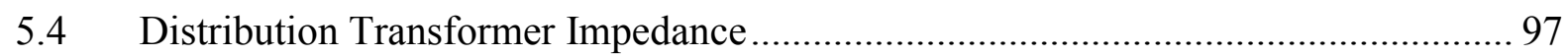

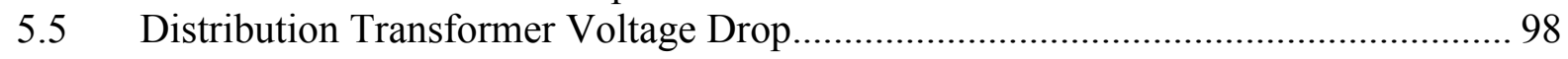

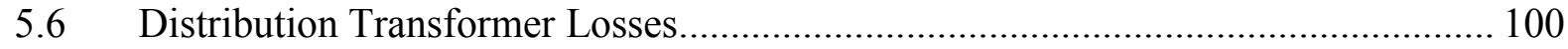

5.6.1 Load Losses......................................................................................... 100

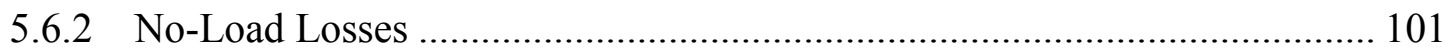

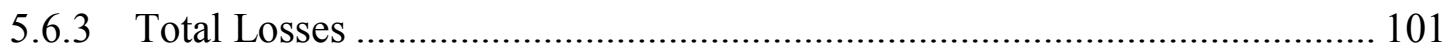

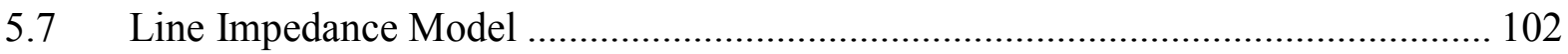

5.8 Line Voltage Drop Model .................................................................................. 105

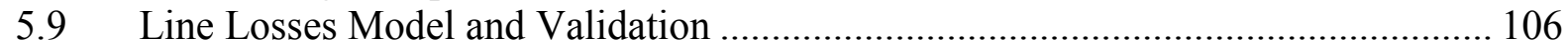

5.10 Secondary and Service Impedances and Voltage Drops.......................................... 124

5.11 Secondary and Service Real Losses ................................................................. 125

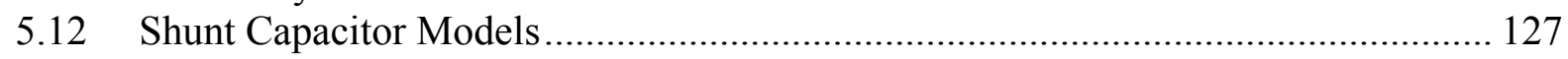

5.13 Step Voltage Regulator Models ..................................................................... 130

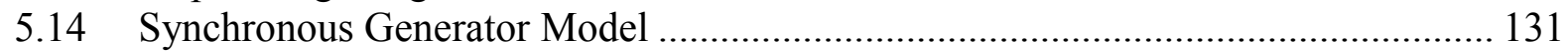

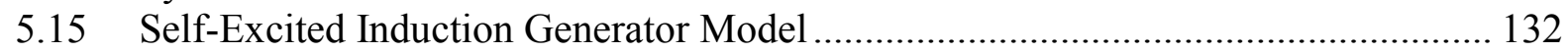

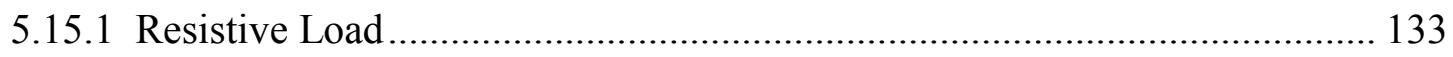

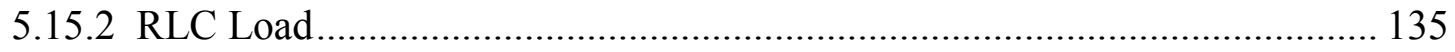

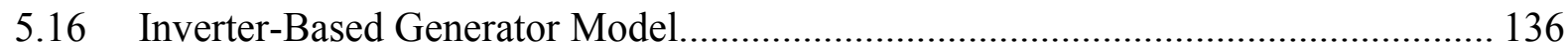

6 Project Results - Circuit Voltage Profiles Under Different Loading Conditions ....................... 139

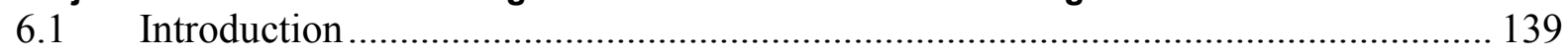

6.2 Circuit Simulations and Voltage Profiles................................................................... 144

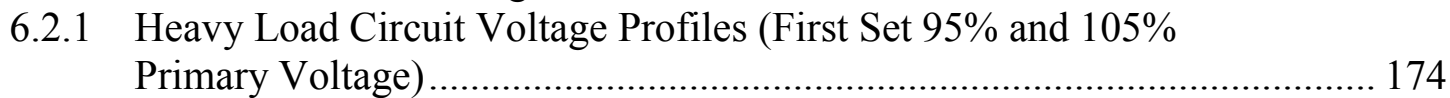

6.2.2 Heavy Load Comparison Circuit Voltage Profiles (Second Set 95\%

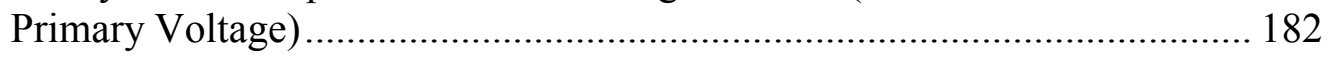

6.2.3 Voltage Profile Data as a Function of Distance from Substation to Tag End.....

6.2.4 Light Load Circuit Voltage Profiles (Third Set 95\% and 105\% Primary Voltage).

6.2.5 Light Load Comparison Circuit Voltage Profiles (Fourth Set 95\% Primary Voltage) 


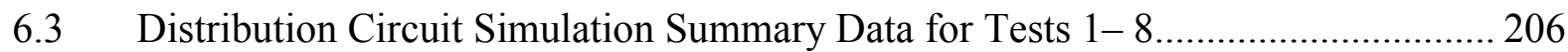

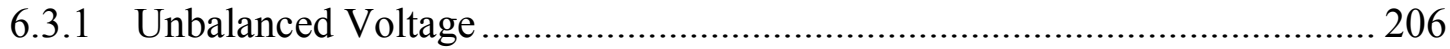

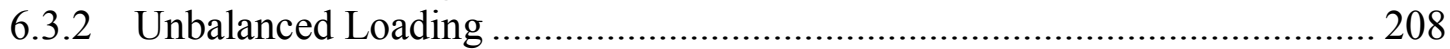

6.4 Significant Results and Conclusions............................................................. 208

7 Project Results - Design of Field Voltage Regulation and Metering Equipment ...................... 211

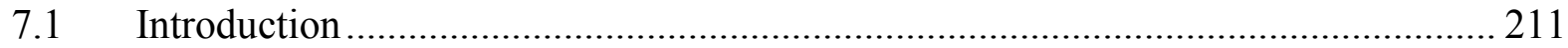

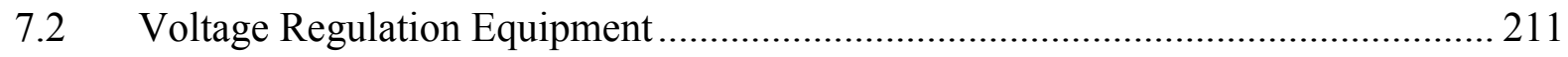

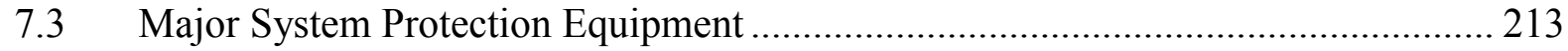

7.4 Metering Equipment and Accuracy of Measurements.......................................... 214

7.4.1 Substation Metering ............................................................................. 214

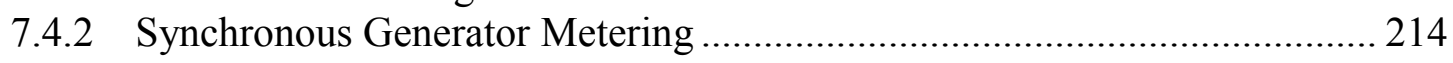

7.4.3 Capacitor Location Metering .............................................................. 215

7.4.4 Voltage Regulator Locations............................................................ 215

7.4.5 Single-Phase Voltage Customer Metering ............................................ 215

8 Project Results - Distributed Generation Control Strategies for Field Verification.................. 216

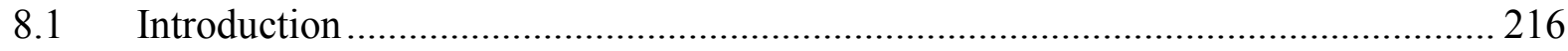

$8.2 \quad 1,000-\mathrm{kW}$ Synchronous Generator................................................................ 216

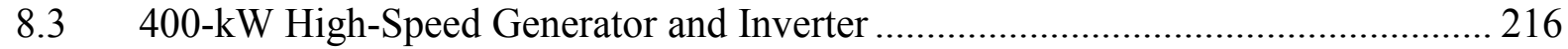

$8.4 \quad 400-\mathrm{kW}$ Self-Excited Induction Generator ....................................................... 216

8.5 Voltage Regulation Simulations and Field Verification Strategy 17 ..................... 216

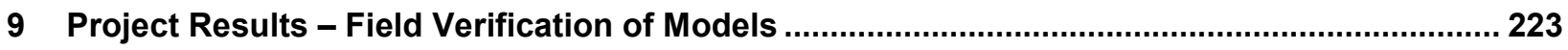

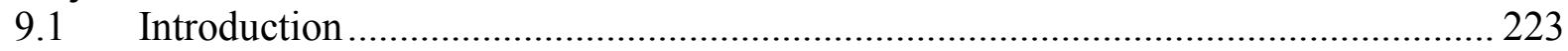

9.2 Circuit and Generation Measured Data............................................................ 223

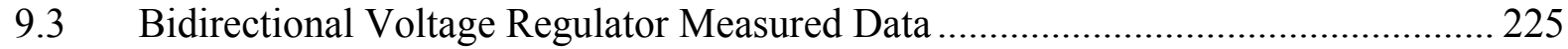

9.4 Circuit Equipment (Capacitors) and Customer-Measured Data ............................. 225

9.5 Percent Variance Between Simulated and Field-Measured Data .......................... 225

9.6 Summary of Variance Results.......................................................................... 231

10 Project Results - Distributed Generation Penetration Limits Using Maximum Real Power and Maximum Real and Reactive Power Methods of Voltage Control ..................................... 232

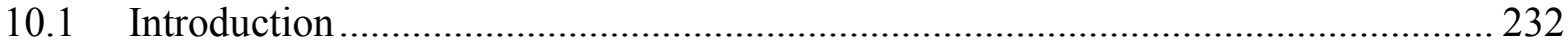

10.2 Real Power Distributed Generation Size Limitation.............................................. 232

10.3 Real and Reactive Distributed Generation Size Limitation ................................... 234

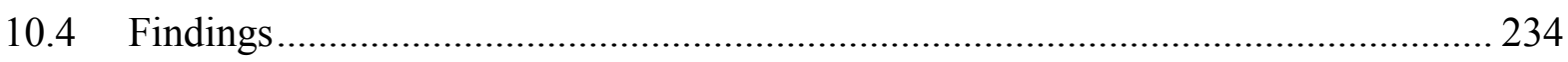

11 Project Results - Distributed Generation Voltage Regulation and Optimum Generator Conditions for Maximum Improvement of Voltage Regulation, Loss Reduction, and

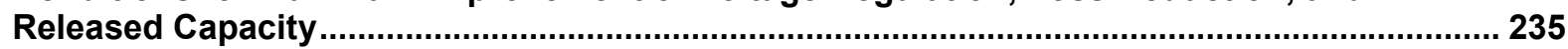

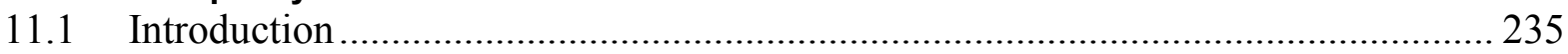

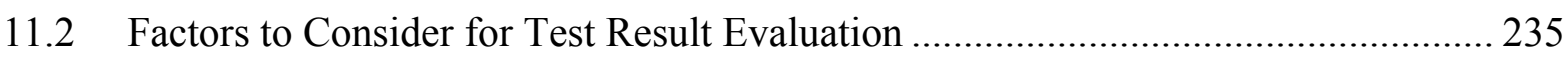

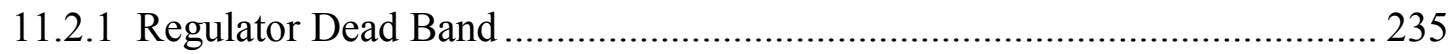

11.2.2 Voltage-Dependent Current Source ..................................................... 236 
11.3 Interpreting the Distributed Generation Voltage Regulation Application Results .. 236

11.3.1 Induction Generator Voltage Regulation Application (High Voltage) Heavy Load, Table 44 ............................................................................. 236

11.3.2 Inverter Generator Voltage Regulation Application (High Voltage) Heavy Load, Table 45 .............................................................................. 238

11.3.3 Synchronous Generator Voltage Regulation Application (Beginning Location, High Voltage) - Heavy Load, Table 46. 239

11.3.4 Synchronous Generator Voltage Regulation Application (End Location, High Voltage) - Heavy Load, Table 47 .

11.3.5 Synchronous Generator Voltage Regulation Application (Midlocation, High Voltage) - Heavy Load, Table 48 240

11.3.6 Synchronous Generator Voltage Regulation Application (Beginning Location, Low Voltage) Heavy Load, Table 49

11.3.7 Synchronous Generator Voltage Regulation Application (End Location, Low Voltage) - Heavy Load, Table 50 ...

11.3.8 Synchronous Generator Voltage Regulation Application (Midlocation, Low Voltage) - Heavy Load, Table 51 ...................................................... 240

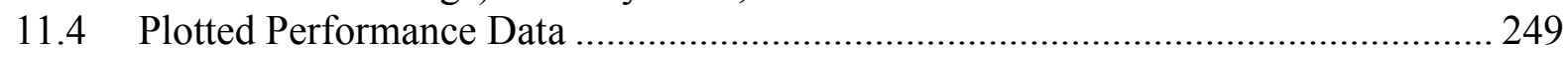

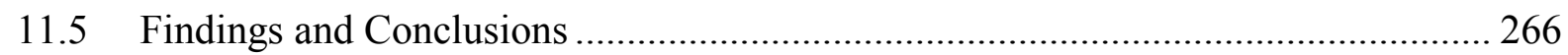

11.5.1 Synchronous Generator (Low Voltage on Primary of Substation) - Heavy Load Condition ...................................................................................... 266

11.5.2 Synchronous Generator (High Voltage on Primary of Generator Substation) - Heavy Load Condition............................................................ 267

11.5.3 Induction Generator for Heavy Load Condition ........................................... 268

11.5.4 Inverter Generator for Heavy Load Condition............................................... 269

11.6 Optimum Generator Conditions for Maximum Improvements - Heavy

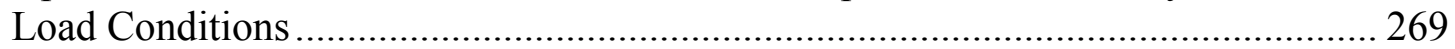

11.7 Optimum Generator Conditions for Maximum Improvements - Light

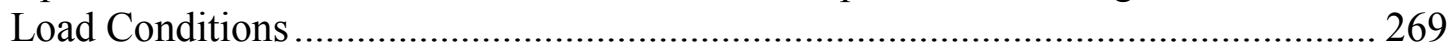

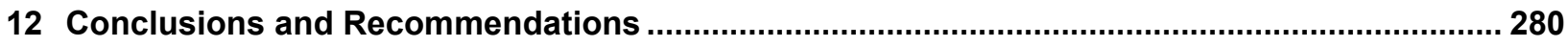

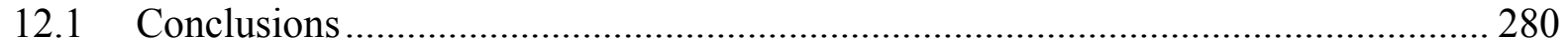

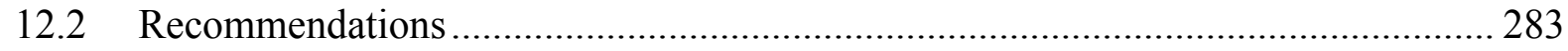

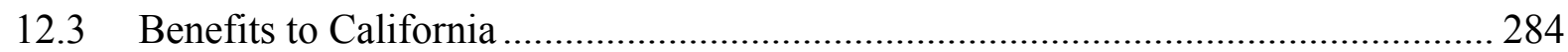

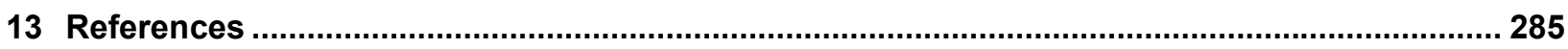

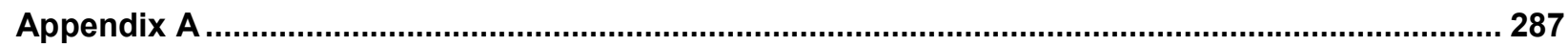

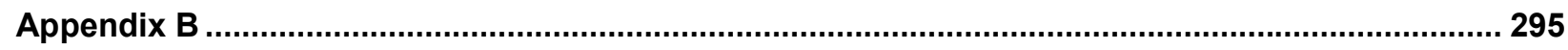




\section{List of Figures}

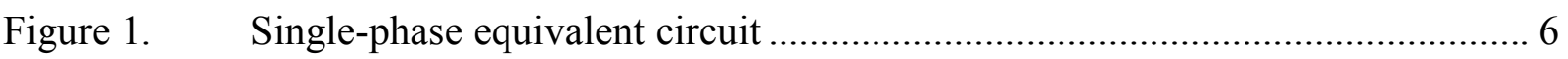

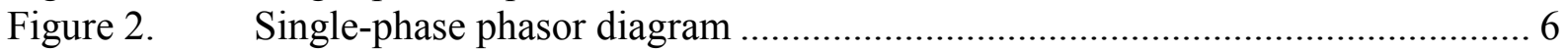

Figure 3. Two-phase lateral - voltage drop for load connected from A and B to neutral. 7

Figure 4. Two-phase lateral - voltage drop for load connected from A and C to neutral. 8

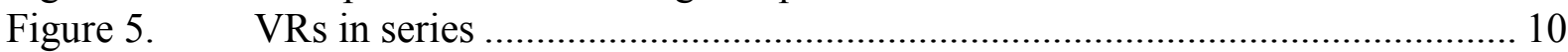

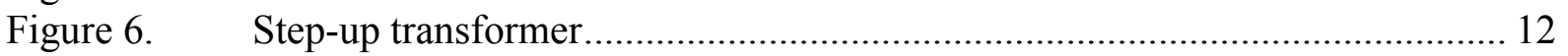

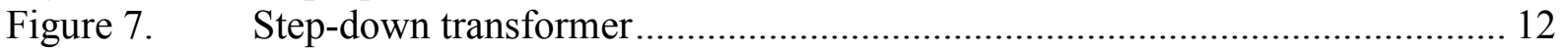

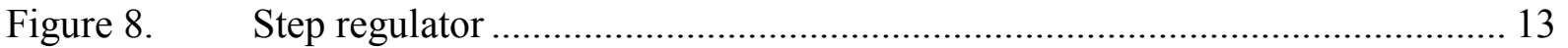

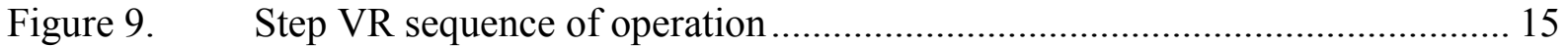

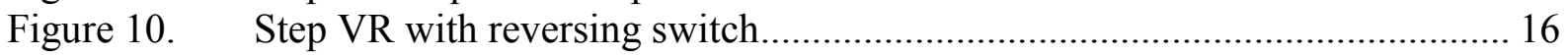

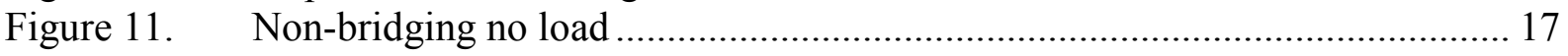

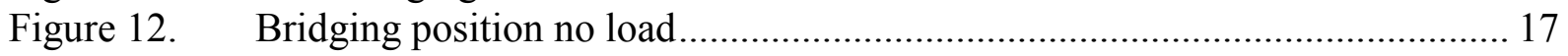

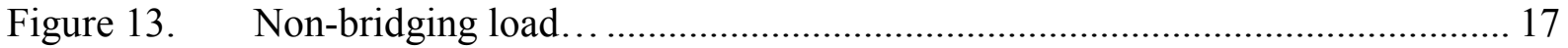

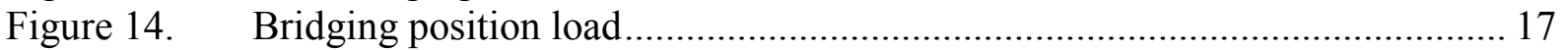

Figure 15. Application of equalizer windings ........................................................... 18

Figure 16. Case A - without equalizer windings.............................................................. 18

Figure 17. Case B - without equalizer windings........................................................... 18

Figure 18. Case $\mathrm{C}$ - with equalizer windings bridging position …….............................. 18

Figure 19. Case D - with equalizer windings non-bridging position............................... 19

Figure 20. ANSI Type A - series winding located on load side ..................................... 19

Figure 21. ANSI Type B - series winding located on source side .................................. 20

Figure 22. DR sources create bidirectional flows through a step VR .............................. 21

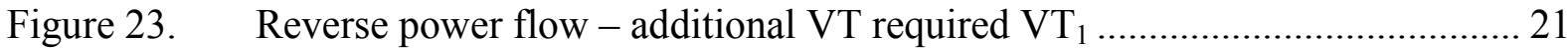

Figure 24. Reverse power flow - differential transformer ................................................. 21

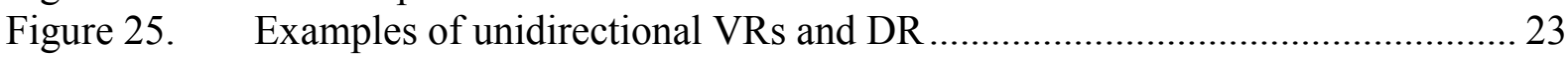

Figure 26. Examples of bidirectional VRs and DR ..................................................... 24

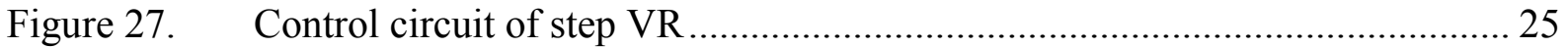

Figure 28. Type A step regulator - raise position ......................................................... 26

Figure 29. Type A step regulator - lower position.......................................................... 26

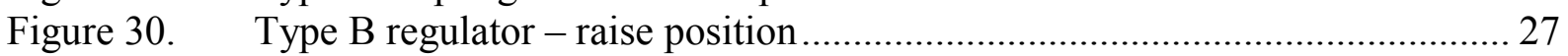

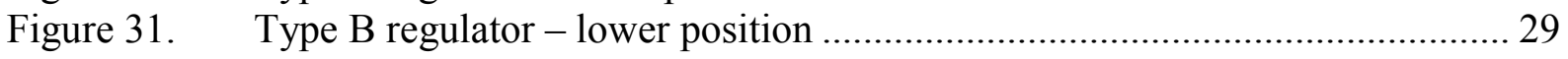

Figure 32. Control system and line drop compensator..................................................... 31

Figure 33. Phasor diagram for line drop compensator ...................................................... 31

Figure 34. Three single-phase VRs connected wye on a four-wire, three-phase circuit... 33

Figure 35. Voltage phasor diagram of wye-connected, three single-phase VRs............... 34

Figure 36. Two single-phase VRs connected open delta on a three-wire,

Figure 37. Voltage phasor diagram of open delta-connected two single-phase VRs....... 36

Figure 38. Voltage diagram of closed delta-connected three single-phase VRs ............... 38

Figure 39. Closed delta-connected three single-phase VRs - leading connection ............. 39

Figure 40. Closed delta-connected three single-phase VRs - lagging connection............. 40

Figure 41. Current at the regulator is not equal to current in the last section before the

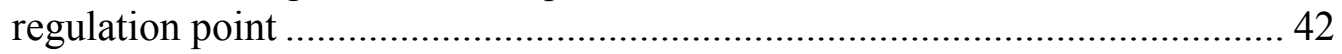


Figure 42. Phasor diagram of the effect of capacitors on the line drop compensator and regulator output voltages before and after relay settings are revised....... 46

Figure 43. Control system and line drop compensator with use of a shunt capacitor at the location of the regulator

Figure 44. Voltage across impedance $\mathrm{Z}$ in relay circuit is equal to voltage rise

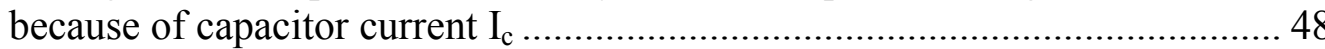

Figure 45. Application of shunt capacitors............................................................ 49

Figure 46. Phasor diagram of the reduction in the sending-end voltage with a shunt capacitor .............................................................................. 51

Figure 47. Voltage and current control of capacitor switching .................................. 51

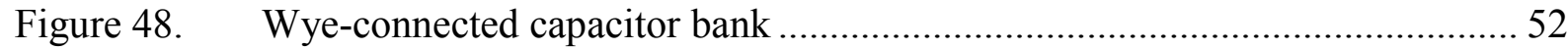

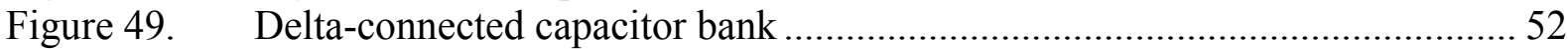

Figure 50. Series resonance condition with open phases ........................................... 53

Figure 51. Radial distribution circuit with typical system protection devices ................. 54

Figure 52. Radial distribution circuit showing load ............................................ 55

Figure 53. Radial distribution circuit showing protection zones................................ 55

Figure 54. Relative tripping times for protective devices ...................................... 56

Figure 55. Relative time-current curves for protective devices................................... 57

Figure 56. Three-phase diagram of a four-wire grounded system …............................ 58

Figure 57. Three-phase diagram of a three-wire ungrounded system ............................ 59

Figure 58. Single-phase operation of protective devices on a four-wire wye circuit of

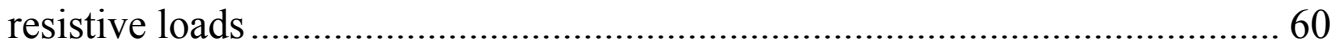

Figure 59. Diagram of substation breaker and relays with ground-sensing circuit.......... 61

Figure 60. Current flow in phase-to-phase-connected load........................................ 63

Figure 61. Reduced melt time because of preloading of universal K link fuses .............. 64

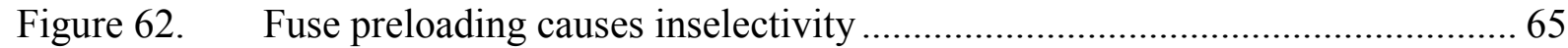

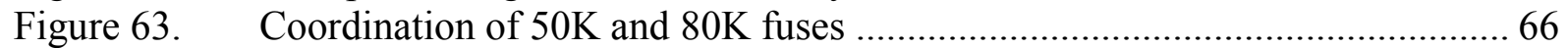

Figure 64. Inselectivity of $80 \mathrm{~K}$ and $50 \mathrm{~K}$ fuses for unbalanced loading of $20 \% \ldots \ldots \ldots \ldots . . .67$

Figure 65. Positive and negative sequence system of voltages .................................. 71

Figure 66. Positive sequence equivalent circuit for induction motor/generator............... 73

Figure 67. Positive sequence induction motor/generator equivalent circuit for copper losses and shaft power............................................................. 73

Figure 68. Simplified positive sequence induction motor/generator equivalent circuit.... 73

Figure 69. Slip of rotor with respect to negative sequence field and positive

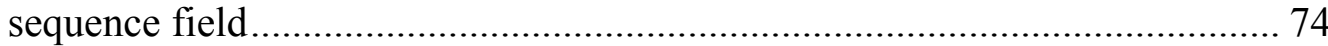

Figure 70. Negative sequence equivalent circuit for induction motor/generator ............. 76

Figure 71. Negative sequence induction motor/generator equivalent circuit for copper losses and shaft power................................................................... 76

Figure 72. Simplified negative sequence induction motor/generator equivalent circuit operating at small values of slip ................................................................. 76

Figure 73. Phasor diagram for minimum heating in Phase $A, \theta=180^{\circ} \ldots \ldots \ldots \ldots \ldots \ldots \ldots . . . . . . . . . . . .87$

Figure 74. Phasor diagram for maximum heating in Phase $\mathrm{A}, \theta=0^{\circ}$......................... 87

Figure 75. Round rotor synchronous machine showing negative sequence current paths

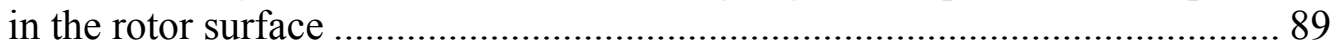

Figure 76. Typical time-current curves for the Type SGC relay................................. 91

Figure 77. Delta-wye-grounded three-phase transformer............................................. 92 
Figure 78. Four-wire, wye-grounded, overhead distribution line with multi-grounded

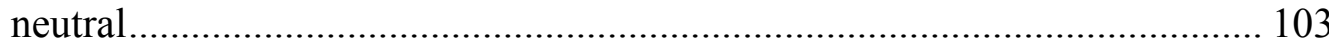

Figure 79. Flat line spacing configuration.......................................................... 104

Figure 80. Configuration, Phase, and Neutral Spacings; Phase and Neutral Sizes; and

Conductor Types ....................................................................... 107

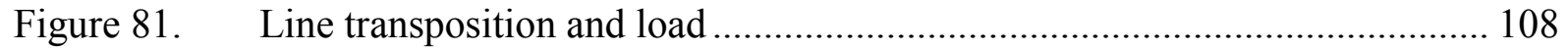

Figure 82. Total line losses versus load imbalance for each line configuration............. 118

Figure 83. Percent losses versus load imbalance for each line configuration ................ 118

Figure 84. Voltage imbalance versus load imbalance for each line configuration.......... 119

Figure 85. Sequence currents versus load imbalance for each line configuration .......... 119

Figure 86. Voltage imbalance versus current imbalance equilateral spacing line........... 120

Figure 87. Voltage imbalance versus current imbalance non-transposed line ............... 120

Figure 88. Voltage imbalance versus current imbalance transposed line....................... 121

Figure 89. Sequence currents versus current imbalance equilateral-spaced line ............ 121

Figure 90. Sequence currents versus current imbalance for non-transposed line .......... 122

Figure 91. Sequence currents versus current substance for transposed line.................. 122

Figure 92. Distribution transformer servicing secondaries and services...................... 124

Figure 93. Distribution service drop .................................................................... 124

Figure 94. Steady-state synchronous machine model .............................................. 131

Figure 95. Two-phase primitive machine to be interconnected with an RLC load......... 132

Figure 96. Stator direct axis with an $\mathrm{R}$ load added................................................... 133

Figure 97. RLC load $\mathrm{R}_{\mathrm{L}}, \mathrm{L}_{\mathrm{L}}$, and $\mathrm{C}_{\mathrm{L}}$ connected to the self-excited capacitor $\mathrm{C}_{\mathrm{S}} \ldots \ldots \ldots \ldots 135$

Figure 98. One-line diagram of 400-kW inverter-based generator and prime mover..... 136

Figure 99. Voltage pullback curves ................................................................. 137

Figure 100. Inverse time-current characteristic .................................................... 138

Figure 101. Milford Substation one-line diagram ................................................ 140

Figure 102. Milford Circuit DC 8103 ....................................................................... 142

Figure 103. HL circuit voltage profiles for all tests

(first set $-95 \%$ and 105\% substation primary voltage) ........................... 174

Figure 104. Test $1 \mathrm{HL}$ circuit voltage profiles for $95 \%$ and $105 \%$ substation primary voltage (no LTC) ................................................................................. 175

Figure 105. Test 2 HL circuit voltage profiles for $95 \%$ and $105 \%$ substation primary voltage (LTC) .................................................................................... 176

Figure 106. Test 3 HL circuit voltage profiles for $95 \%$ and $105 \%$ substation primary voltage (LTC and VR 1)

Figure 107. Test 4 HL circuit voltage profiles for $95 \%$ and $105 \%$ substation primary voltage (LTC, VR 1, and VR 2) .......................................................... 178

Figure 108. Test 5 HL circuit voltage profiles for $95 \%$ and $105 \%$ substation primary voltage (LTC and CAP 1)

Figure 109. Test 6 HL circuit voltage profiles for $95 \%$ and $105 \%$ substation primary voltage (LTC, CAP 1, and CAP 2) 180

Figure 110. Test 7 HL circuit voltage profiles for $95 \%$ and $105 \%$ substation primary voltage (LTC, CAP 1, CAP 2, and CAP 3)

Figure 111. Test $8 \mathrm{HL}$ circuit voltage profiles for $95 \%$ and $105 \%$ substation primary voltage (LTC, VR 1, VR 2, CAP 1, CAP 2, and CAP 3)... 182 
Figure 112. Comparison A: Test 2 and Test 3 HL circuit voltage profiles for $95 \%$ substation primary voltage (LTC versus LTC and VR 1). 183

Figure 113. Comparison B: Test 3 and Test 4 HL circuit voltage profiles for $95 \%$ substation primary voltage (LTC and VR 1 versus LTC, VR 1, and VR 2).. 184

Figure 114. Comparison C: Test 2 and Test 5 HL circuit voltage profiles for $95 \%$ substation primary voltage (LTC versus LTC and CAP 1).... 185

Figure 115. Comparison D: Test 5 and Test 6 HL circuit voltage profiles for $95 \%$ substation primary voltage (LTC and CAP 1 versus LTC, CAP 1, and CAP 2).

Figure 116. Comparison E: Test 6 and Test 7 HL circuit voltage profiles for $95 \%$ substation primary voltage (LTC, CAP 1, and CAP 2 versus LTC, CAP 1, CAP 2, and CAP 3).

Figure 117. Comparison F: Test 2 and Test 8 HL circuit voltage profiles for $95 \%$ substation primary voltage (LTC vs. LTC, VR 1, VR 2, CAP 1, CAP 2, and CAP 3). 188

Figure 118. Comparison G: Test 7 and Test 8 HL circuit voltage profiles for $95 \%$ substation primary voltage (LTC, CAP 1, CAP 2, and CAP 3 versus LTC, VR 1, VR 2, CAP 1, CAP 2, and CAP 3) 189

Figure 119. LL circuit voltage profiles for all tests (third set $-95 \%$ and $105 \%$ substation primary voltage)

Figure 120. Test 1 LL circuit voltage profiles for $95 \%$ and $105 \%$ substation primary voltage (no LTC).

Figure 121. Test 2 LL circuit voltage profiles for $95 \%$ and $105 \%$ substation primary voltage (LTC).

Figure 122. Test 3 LL circuit voltage profiles for $95 \%$ and $105 \%$ substation primary voltage (LTC and VR 1)

Figure 123. Test 4 LL circuit voltage profiles for $95 \%$ and $105 \%$ substation primary voltage (LTC, VR 1, and VR 2)....

Figure 124. Test 5 LL circuit voltage profiles for $95 \%$ and $105 \%$ substation primary voltage (LTC and CAP 1)

Figure 125. Test 6 LL circuit voltage profiles for $95 \%$ and $105 \%$ substation primary voltage (LTC, CAP 1, and CAP 2)

Figure 126. Test 7 LL circuit voltage profiles for $95 \%$ and $105 \%$ substation primary voltage (LTC, CAP 1, CAP 2, and CAP 3)

Figure 127. Test 8 LL circuit voltage profiles for $95 \%$ and $105 \%$ substation primary voltage (LTC, VR 1, VR 2, CAP 1, CAP 2, and CAP 3).

Figure 128. Comparison A: Test 2 and Test 3 LL circuit voltage profiles for $95 \%$ substation primary voltage (LTC versus LTC and VR 1). 200

Figure 129. Comparison B: Test 3 and Test 4 LL circuit voltage profiles for $95 \%$ substation primary voltage (LTC versus LTC, VR 1, and VR 2) 201

Figure 130. Comparison C: Test 2 and Test 5 LL circuit voltage profiles for $95 \%$ substation primary voltage (LTC versus LTC and CAP 1).... 202

Figure 131. Comparison D: Test 5 and Test 6 LL circuit voltage profiles for 95\% substation primary voltage (LTC and CAP 1 versus LTC, CAP 1, and CAP 2). 
Figure 132. Comparison E: Test 6 and Test 7 LL circuit voltage profiles for 95\% substation primary voltage (LTC, CAP 1, and CAP 2 versus LTC, CAP 1, CAP 2, and CAP 3)

Figure 133. Comparison F: Test 2 and Test 8 LL circuit voltage profiles for $95 \%$ substation primary voltage (LTC versus LTC, VR 1, VR 2, CAP 1, CAP 2, and CAP 3).

Figure 134. Comparison G: Test 7 and Test 8 LL circuit voltage profiles for $95 \%$ substation primary voltage (LTC, CAP 1, CAP 2, and CAP 3 versus LTC, VR 1, VR 2, CAP 1, CAP 2, and CAP 3) .............................................. 206

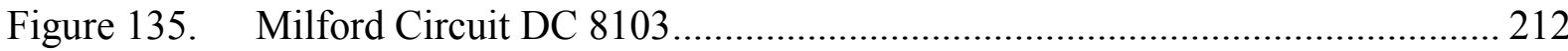

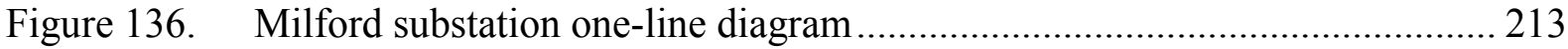

Figure 137. DR control strategies for a 1,000-kW synchronous machine ...................... 217

Figure 138. DR control strategies for a 400-kW high-speed generator and inverter current mode.......................................................................................... 218

Figure 139. DR control strategies for a 400-kW self-excited induction generator ........... 219

Figure 140. Daily circuit load profile and time stamps ............................................ 224

Figure 141. HL condition - voltage rise or drop (in volts on a $120-\mathrm{V}$ base) as a function of induction generator $+/$ - reactive power with a constant real power output of $400 \mathrm{~kW}$ 254

Figure 142. HL condition - reduction or increase in voltage (in percent on a $120-\mathrm{V}$ base) as a function of induction generator $+/$ - reactive power with a constant real power output of $400 \mathrm{~kW}$

Figure 143. HL condition - reduction or increase of real power (in percent of total circuit kilowatts) as a function of induction generator $+/$ - reactive power with a constant real power output of $400 \mathrm{~kW}$

Figure 144. HL load condition - reduction or increase of capacity (in percent of total circuit kilovolt-amperes) as a function of induction generator $+/$ - reactive power with a CP output of $400 \mathrm{~kW}$.

Figure 145. HL condition - voltage rise or drop (in volts on a $120-\mathrm{V}$ base) as a function of inverter generator $+/$ - reactive power with a constant real power output of $400 \mathrm{~kW}$....

Figure 146. HL condition - reduction or increase in voltage (in percent on a $120-\mathrm{V}$ base) as a function of inverter generator $+/$ - reactive power with a constant real power output of $320 \mathrm{~kW}$ or $400 \mathrm{~kW}$

Figure 147. HL condition - reduction or increase of real power (in percent of total circuit kilowatts) as a function of induction generator $+/$ - reactive power with a constant real power output of $320 \mathrm{~kW}$ or $400 \mathrm{~kW}$

Figure 148. HL condition - reduction or increase of capacity (in percent of total circuit kilovolt-amperes) as a function of induction generator $+/$ - reactive power with a CP output of $320 \mathrm{~kW}$ or $400 \mathrm{~kW}$

Figure 149. High substation primary voltage, HL condition - voltage rise or drop (in volts on a $120-\mathrm{V}$ base) as a function of location and synchronous generator +/- reactive power with a constant real power output of $984 \mathrm{~kW} .258$

Figure 150. Low substation primary voltage, HL condition - voltage rise or drop (in volts on a $120-\mathrm{V}$ base) as a function of location and synchronous generator $+/$ - reactive power with a constant real power output of $984 \mathrm{~kW} . .258$ 
Figure 151. High substation primary voltage, HL condition - voltage rise or drop (in volts on a $120-\mathrm{V}$ base) as a function of location and synchronous generator $+/$ - reactive power with a constant real power output of $246 \mathrm{~kW}$.. 259

Figure 152. Low substation primary voltage, HL condition - voltage rise or drop (in volts on a $120-\mathrm{V}$ base) as a function of location and synchronous generator $+/$ - reactive power with a constant real power output of $246 \mathrm{~kW}$.. 259

Figure 153. High substation primary voltage, HL condition - reduction or increase in voltage (in volts on a $120-\mathrm{V}$ base) as a function of location and synchronous generator $+/$ - reactive power with a constant real power output of $984 \mathrm{~kW}$

Figure 154. Low substation primary voltage, HL condition - voltage rise or drop (in volts on a $120-\mathrm{V}$ base) as a function of location and synchronous generator $+/$ - reactive power with a constant real power output of $984 \mathrm{~kW}$.. 260

Figure 155. High substation primary voltage, HL condition - reduction or increase in voltage (in volts on $120-\mathrm{V}$ base) as a function of location and synchronous generator $+/$ - reactive power with a constant real power output of $246 \mathrm{~kW}$.. 261

Figure 156. Low substation primary voltage, HL condition - reduction or increase in voltage (in volts on a $120-\mathrm{V}$ base) as a function of location and synchronous generator $+/$ - reactive power with a constant real power output of $246 \mathrm{~kW}$ 261

Figure 157. High substation primary voltage, HL condition - reduction or increase of real power loss (in percent total circuit kilowatts) as a function of location and synchronous generator $+/$ - reactive power with a constant real power output of $984 \mathrm{~kW}$

Figure 158. Low substation primary voltage, HL condition - reduction or increase of real power loss (in percent total circuit kilowatts) as a function of location and synchronous generator $+/$ - reactive power with a constant real power output of $984 \mathrm{~kW}$

Figure 159. High substation primary voltage, HL condition - reduction or increase of real power loss (in percent total circuit kilowatts) as a function of location and synchronous generator $+/$ - reactive power with a constant real power output of $246 \mathrm{~kW}$

Figure 160. Low substation primary voltage, HLcondition - reduction or increase of real power loss (in percent total circuit kilowatts) as a function of location and synchronous generator $+/$ - reactive power with a constant real power output of $246 \mathrm{~kW}$

Figure 161. High substation primary voltage, HL condition - reduction or increase of capacity (in percent total circuit kilowatts) as a function of location and synchronous generator $+/$ - reactive power with a constant real power output of $984 \mathrm{~kW}$

Figure 162. Low substation primary voltage, HL condition - reduction or increase of capacity (in percent total circuit kilowatts) as a function of location and synchronous generator $+/$ - reactive power with a constant real power output of $984 \mathrm{~kW}$ 
Figure 163. High substation primary voltage, HL condition - reduction or increase of capacity (in percent total circuit kilowatts) as a function of location and synchronous generator $+/$ - reactive power with a constant real power output of $246 \mathrm{~kW}$

Figure 164. Low substation primary voltage, HL condition - reduction or increase of capacity (in percent total circuit kilowatts) as a function of location and synchronous generator $+/$ - reactive power with a constant real power output of $246 \mathrm{~kW}$

Figure A-1. Normal leakage currents to ground because of distributed capacitance ........ 287

Figure A-2. Normal leakage currents to ground because of distributed capacitance phasor diagram ..................................................................................... 288

Figure A-3. Increased leakage current on the unfaulted phases and resultant ground current $\mathrm{I}_{\mathrm{G}}$ and resultant zero sequence voltage $3 \mathrm{~V}_{\mathrm{O}}$ 288

Figure A-4. Increased leakage current on the unfaulted phases and resultant ground current $\mathrm{I}_{\mathrm{G}}$ and resultant zero sequence voltage $3 \mathrm{~V}_{\mathrm{O}}$ - phasor diagram

Figure B-1. Percent change in kilovolt-amperes or current from percent change in $\mathrm{P}$ and $\mathrm{Q}$ 296

Figure B-2. Percent change in kilovolt-amperes or current. 296

Figure B-3. Zero sequence current on load side becomes negative sequence current to the generator... 299

Figure B-4. Determining $\mathrm{I}_{\mathrm{AG}}$ 300

Figure B-5.

Determining $\mathrm{I}_{\mathrm{AG} 2}$ 301

Figure B-6.

Determining I AGO 301 


\section{List of Tables}

Table 1. Comparison of National Electrical Manufacturers Association Standard Method with Correct Voltage Unbalance Calculations Using

Symmetrical Components ........................................................................... 70

Table 2. Per-Unit Line Currents for Percent Negative Sequence Voltage

Single-Cage Induction Motor....................................................................... 79

Table 3. Per-Unit Line Currents for Percent Negative Sequence Voltage

Double-Cage Induction Motor ..................................................................... 79

Table 4. Double- and Single-Cage Characteristics for 10-hp, Four-Pole, 220-V,

Three-Phase, Wye-Connected Ungrounded Motors ..................................... 80

Table 5. Single-Cage Induction Motor - 10 hp, 220 V, Four Pole,

Wye-Connected Ungrounded ............................................................... 81

Table 6. Double-Cage Induction Motor - 10 hp, 220 V, Four Pole,

Wye-Connected Ungrounded ................................................................... 81

Table 7. Maximum Allowable Power Output in Percent of Rated While Operating at Unbalanced Voltages.............................................................................. 86

Table 8. Comparison of Gafford, Duesterohoeft, and Mosher (1959) and Tracey

(1954) Motor Derate Data Operating at Unbalance Voltage ......................... 86

Table 9. Unbalance Voltage Survey Results of Closed and Open Delta

Transformer Connections........................................................................... 88

Table 10. Distribution Transformer No Load (Core Losses) and Load

(Copper Losses) ................................................................................. 99

Table 11. Equilateral Spacing Kilowatt Loss Evaluation ........................................ 112

Table 12. Non-Transposed Kilowatt Loss Evaluation ............................................. 114

Table 13. Transposed Kilowatt Loss Evaluation .......................................................... 116

Table 14. The Effects of Circuit Spacing and Unbalanced Load on Percent

Kilowatt Losses ..................................................................................... 123

Table 15. Voltage Drop Summary - Transformer, Secondary, and Service Drop ........ 125

Table 16. Peak Day Real Losses Comparison.......................................................... 127

Table 17. Circuit Customer Load Characteristics ....................................................... 140

Table 18. Measurement Locations and Data Collection ............................................. 144

Table 19. Matrix of Voltage Regulation Simulations ................................................ 145

Table 20. Voltage Profile Data for HL Tests 1 Through 8 at 95\% and 105\%

Substation Primary Voltage ....................................................................... 149

Table 21. Voltage Profile Data for Light Load Tests 1 Through 8 at 95\% and 105\%

Substation Primary Voltage ..................................................................... 158

Table 22. Simulation Summary Data for Tests 1 Through 8 ..................................... 167

Table 23. Simulation Summary Data for Unbalanced Loading (Tests 1 Through 8) .... 172

Table 24. HL Circuit Voltage Profiles (First Set) ..................................................... 175

Table 25. Voltage Profile Comparison for HL and 95\% Primary Voltage ................... 183

Table 26. LL Circuit Voltage Profiles (Third Set) ................................................... 190

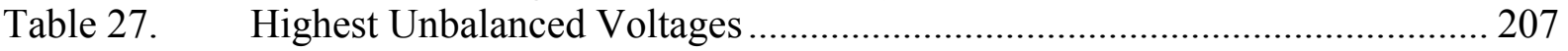

Table 28. Voltage Regulation Equipment ............................................................. 211 


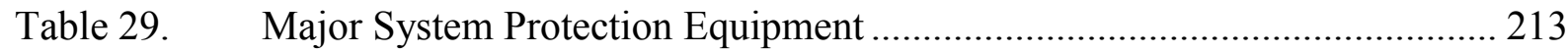

Table 30. Measurement Locations and Data Collection .............................................. 214

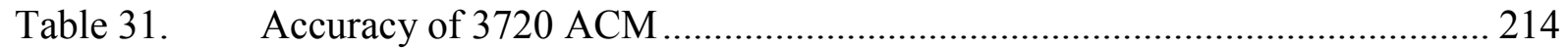

Table 32. Matrix of Voltage Regulation Simulations and Control Strategy 17

for Field Verification..................................................................................... 220

Table 33. Test Dates and Data Collection Periods ..................................................... 223

Table 34. Circuit and Generation Measured Data........................................................ 226

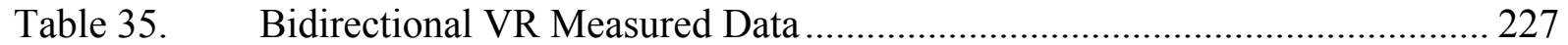

Table 36. Circuit Equipment and Customer-Measured Data .......................................... 228

Table 37. Field Verification Data - July 17, 2006 - DR Generation On........................ 229

Table 38. Field Verification Data - July 29, 2006 - DR Generation On........................ 229

Table 39. Field Verification Data - July 31, 2006 - DR Generation Off ....................... 230

Table 40. Percent Variance Between Actual and Simulated Currents, Voltages,

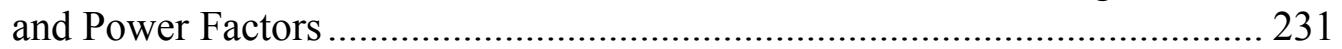

Table 41. Maximum Limit for Real Power Injection.................................................... 233

Table 42. Maximum Limit for Real and Reactive Power Injection ................................ 233

Table 43. Explaining the Meaning of Positive and Negative Improvements for DG Applications .................................................................................. 238

Table 44. Induction Generator Voltage Regulation Application (LV, HV) - HL ......... 241

Table 45. Inverter Generator Voltage Regulation Application (LV, HV) - HL ............ 242

Table 46. Synchronous Generator Voltage Regulation Application (BL, HV) - HL .... 243

Table 47. Synchronous Generator Voltage Regulation Application (EL, HV) - HL .... 244

Table 48. Synchronous Generator Voltage Regulation Application (ML, HV) - HL ... 245

Table 49. Synchronous Generator Voltage Regulation Application (BL, LV) - HL .... 246

Table 50. Synchronous Generator Voltage Regulation Application (EL, LV) - HL..... 247

Table 51. Synchronous Generator Voltage Regulation Application (ML, LV) - HL ... 248

Table 52. Distributed Generator Voltage Regulation Application Summary - HL....... 250

Table 53. Distributed Generator Voltage Regulation Application Summary - LL ....... 252

Table 54. Optimum Generator Conditions for Maximum Improvements - HL ............. 270

Table 55. Optimum Generator Conditions for Maximum Improvement - LL ............... 271

Table 56. Induction Generator Voltage Regulation Application (LV, HV) - LL.......... 272

Table 57. Inverter Generator Voltage Regulation Application (LV, HV) - LL ............ 273

Table 58. Synchronous Generator Voltage Regulation Application (BL, HV) - LL .... 274

Table 59. Synchronous Generator Voltage Regulation Application (EL, HV) - LL..... 275

Table 60. Synchronous Generator Voltage Regulation Application (ML, HV) - LL ... 276

Table 61. Synchronous Generator Voltage Regulation Application (BL, LV) - LL ..... 277

Table 62. Synchronous Generator Voltage Regulation Application (EL, LV) - LL ..... 278

Table 63. Synchronous Generator Voltage Regulation Application (ML, LV) - LL.... 279

Table B-1. Simulation Summary Data for Tests 7 and 8 at 95\% Voltage and HL Conditions and a Comparison of Modeling Results for CC, CP, and VDC .. 303

Table B-2. Comparison of Line Currents for Simulated and Actual Measurements for Each Load Model and Each Test 7 and 8 …………................................ 304

Table B-3. Sequence Currents for Each Test ………………………………….......... 305

Table B-4. Simulation Data by Node for Test 8 at 95\% HL with LTC, VR 1, VR 2, and Capacitors 1, 2, and 3 Regulation Methods Implemented (CC Model).. 306 
xxiv 


\section{Introduction}

An earlier study (Davis 2003) reported that the maximum-sized distributed generation (DG) that can be installed on a distribution circuit depends on:

- Circuit system voltage

- The location of DG on the circuit

- Circuit configuration and characteristics

- System protection issues

- Voltage regulation issues

- DG characteristics.

The study also found that larger DG units can be installed on higher-system-voltage circuits and at the substation with fewer effects on system protection and voltage regulation. If the DG actively regulates voltage, larger units can be interconnected because high-voltage (HV) conditions during light-load (LL) conditions can be curtailed if the DG absorbs voltamperes reactive.

These findings were the results of tests that used (3Ф) balanced power flows on distribution circuits with balanced loads. However, most distribution circuits have unbalanced loading conditions, unbalanced line impedances, and unbalanced voltage conditions. Therefore, there is no assurance that these results represent what happens on the single-phase (S $\Phi)$ laterals of a three-phase circuit.

The single-phase portion of the circuit may serve more than $90 \%$ of the load. Just because the voltage limits are satisfied on the three-phase portion of the circuit does not mean there are no voltage limit violations on the single-phase loads. Installing DG on the circuit adds another level of complexity to the analysis of the distribution system, but it can also contribute added benefits of released capacity, lower energy losses, and improved voltage regulation.

The objectives of this project are to:

1. Select a distribution circuit and install DG to reduce an overload and improve voltage regulation

2. Develop models and run simulations to calculate voltage profiles on the distribution circuit with the load tap changer (LTC), step regulators, capacitors, and DG regulating the voltage

3. Install metering on the circuit and DG installations and compare actual measured data with simulations to verify the models

4. Determine the maximum-sized DG and the optimum DG operating conditions that will provide the greatest released capacity, lowest energy losses, and most improved voltage regulation. 


\section{Project Approach}

The approach of the project is to:

1. Develop a load model of a distribution circuit to represent how circuit load changes with changes in voltage

2. Develop circuit line elements and circuit equipment models

3. Develop DG models for synchronous, induction, and inverter generation

4. Model an entire 13.2-kV distribution circuit with all three-phase and single-phase loads connected to the correct phases of the distribution circuit

5. Install a 1,000-kW synchronous generator on the circuit and install the appropriate metering equipment to measure the power quantities of the circuit, including single-phase loads

6. Validate the models by conducting a multitude of power flow simulations and comparing the simulations with circuit-measured data on the circuit peak day (with DG on and off)

7. Report the variance between simulated and measured power quantities

8. Determine the largest DG that can be installed on the circuit without violating voltage, thermal, and reverse power criteria

9. Determine the optimum DG operating conditions to give maximum released capacity, reduced energy losses, and improved voltage regulation. 


\section{Project Results: Traditional Voltage Regulation Methods}

\subsection{Introduction}

A number of methods can improve voltage regulation. Some use voltage regulation equipment to raise or lower voltage at the substation or on the distribution circuit to reduce the voltage difference between LL and heavy-load conditions. Others reduce the impedance of the circuit to reduce the voltage difference or spread, and still others reduce the load current (i.e., improve the power factor) to reduce the voltage drop and, thus, the voltage spread.

Methods that can be applied at the substation include:

- Use DG voltage regulators (VRs).

- Apply capacitors at the distribution substation.

- Apply voltage-regulating equipment, such as LTC transformers and bus or circuit voltage-regulating equipment, at the substation.

- Balance the loads on the circuits.

- Transfer loads to other substations.

- Install new substations and circuits.

- Install substation transformers with reduced reactance.

Methods that can be used on the distribution circuit include:

- Increase the primary system voltage of the circuit.

- Increase the conductor size of the circuit or reduce conductor spacing and, consequently, reduce reactance.

- Change circuit sections from single-phase to three-phase.

- Transfer loads to other circuits.

- Apply voltage-regulating equipment on the circuit.

- Install DG on the circuit.

- Apply shunt (or series) capacitors on the circuit.

- Balance loads.

- Increase the distribution transformer size.

- Increase the conductor size of secondaries and services.

\subsection{Distributed Generation Installed at Substation}

The generator bus voltage can be regulated to maintain a fixed voltage for changes in load and reactive requirements. The generator field current can be varied to match the changes in load current. The voltage can be increased or decreased as the load increases or decreases. 


\subsection{Substation Voltage-Regulating Equipment}

One of the most common types of substation voltage-regulating equipment is the load tap changing (LTC) transformer. Step- or induction-type VRs may be installed between the secondary of the transformer and the secondary bus or on the secondary bus. Also, step- or induction-type regulators may be installed on individual circuits. Because circuit voltage is a function of voltage spread at the secondary of the substation bus, voltage-regulating equipment allows for a greater voltage drop in the circuit.

\subsection{Balancing Loads}

If a circuit has poor voltage regulation, it could be due to a significant difference in phase loading. A $20 \%$ or more load imbalance on the primary of a circuit, especially during HL conditions, is not uncommon. In addition, a high load imbalance can cause substation transformers and VRs to overload, based on the highest phase load. Balanced loading should be achieved throughout the circuit, not just at the substation.

\subsection{Increased Primary System Voltage}

Increasing the system voltage reduces the load by the inverse ratio of the voltage change, but the voltage regulation changes as the square of the voltage change. Changing from a threewire ungrounded delta to a four-wire grounded wye increases the voltage by the $\sqrt{3}$ but reduces the voltage drop to $1 / 3$ of the drop in the three-wire delta. However, it is common to increase the allowable load when this conversion is made to a higher system voltage; therefore, the voltage drop improvement is less than $2 / 3$. This method is more expensive than using supplemental voltage regulation because it involves re-insulating the line and changing out electric transformation (i.e., transformers) and other equipment.

\subsection{Increased Conductor Size or Reduced Conductor Spacing}

Increasing conductor size is another expensive method to achieve improved voltage regulation. Increasing the size of the conductor reduces the resistance and, thus, the voltage drop and real losses. However, it may necessitate a rebuilding of the line because of the larger and heavier conductor. An alternative is to reduce the spacing between the phase conductors, which reduces the reactance and lowers the voltage drop.

\subsection{Conversion of Single-Phase Sections to Three-Phase}

Most general-purpose circuits are single-phase. For single-phase circuits, voltage drop occurs in both the phase conductor and the neutral for the wye systems and in both the phase conductors for the delta systems. Adding two conductors to a single-phase wye system, and assuming the existing load is evenly distributed among the three phases, results in $1 / 6$ of the voltage drop that occurs on the single-phase wye lateral. This is demonstrated below.

$$
\begin{array}{ll}
\Delta \mathrm{V}_{\mathrm{S} \Phi}=2 \mathrm{I}_{\mathrm{S} \Phi} \mathrm{Z} \text { and } & \text { Equation } 3.1 \\
\% \Delta \mathrm{Vs} \Phi=2 \mathrm{I}_{\mathrm{S} \Phi} \mathrm{Z} / \mathrm{VLN} \times 100 & \text { Equation } 3.2
\end{array}
$$


where

$$
\begin{aligned}
& \Delta \mathrm{V}_{\mathrm{S} \Phi}=\text { Voltage drop per unit length } \\
& \mathrm{I}_{\mathrm{S} \Phi}=\text { Line current } \\
& \mathrm{Z}=\text { Impedance per unit length } \\
& \mathrm{V}_{\mathrm{LN}}=\text { Line-to-neutral voltage. }
\end{aligned}
$$

For the three-phase case,

$$
\begin{aligned}
& \Delta \mathrm{V}_{3 \Phi}=\mathrm{I}_{3 \Phi} \mathrm{Z} \\
& \% \Delta \mathrm{V}_{3 \Phi}=\left(\mathrm{I}_{3 \Phi} \mathrm{Z}\right) / \mathrm{VLN} \times 100
\end{aligned}
$$

Equation 3.3

Equation 3.4

where

$$
\mathrm{I}_{3 \Phi}=\mathrm{I}_{\Phi \Phi} / 3 .
$$

Using equations $3.2,3.4$, and 3.5 , the ratio of percent voltage drops is thus:

$$
\begin{aligned}
\% \Delta \mathrm{V}_{\mathrm{S} \Phi} / \% \Delta \mathrm{V}_{3 \Phi} & =\left[\left(2 \mathrm{I}_{\mathrm{S} \Phi} \mathrm{Z}\right) / \mathrm{VLN}\right] /\left[\left(\mathrm{I}_{3 \Phi} \mathrm{Z}\right) / \mathrm{VLN}\right] \\
& =\left(2 \mathrm{I}_{\mathrm{S} \Phi}\right) / \mathrm{I}_{3 \Phi} \\
& =\left[(2)(3)\left(\mathrm{I}_{3 \Phi}\right)\right] / \mathrm{I}_{3 \Phi}=6
\end{aligned}
$$

Equation 3.6

Therefore, the voltage drop is six times less for the three-phase circuit than for the singlephase circuit for the same load. Of course, undoubtedly, the load will increase over time after the single-phase lateral is converted to three-phase. However, this does illustrate the concerns when single-phase DGs and three-phase DGs are added to the circuit.

If only one phase conductor, rather than two, is added to a single-phase wye system, the problem becomes more complicated because the voltage drop depends on the two phases selected, the $\mathrm{R} / \mathrm{X}$ ratio of the conductor, and the load power factor $(\mathrm{PF})$.

To describe the differences between the single-phase case and the two two-phase cases, circuit diagrams and phasor diagrams are used. Figure 1 and Figure 2 show the single-phase case, in which $\mathrm{I}_{\mathrm{A}}$ is the phase current that is equal to the neutral current $\mathrm{I}_{\mathrm{N}}$. 


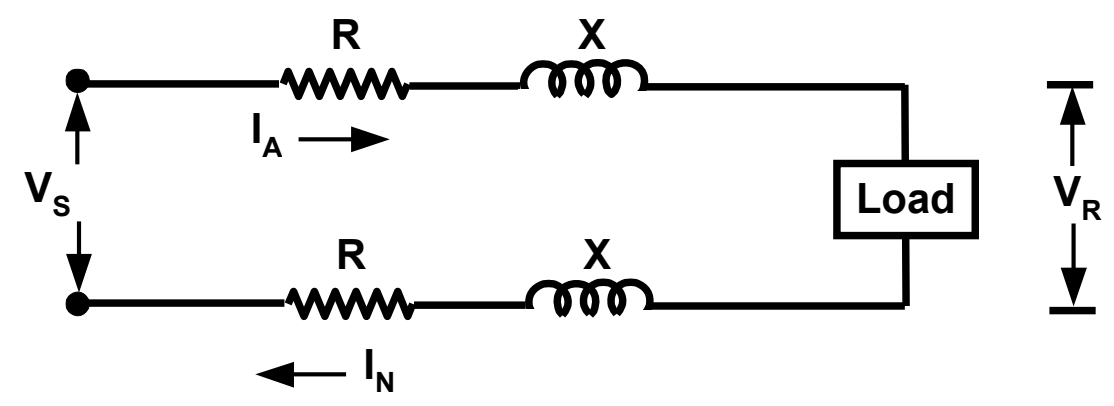

Figure 1. Single-phase equivalent circuit

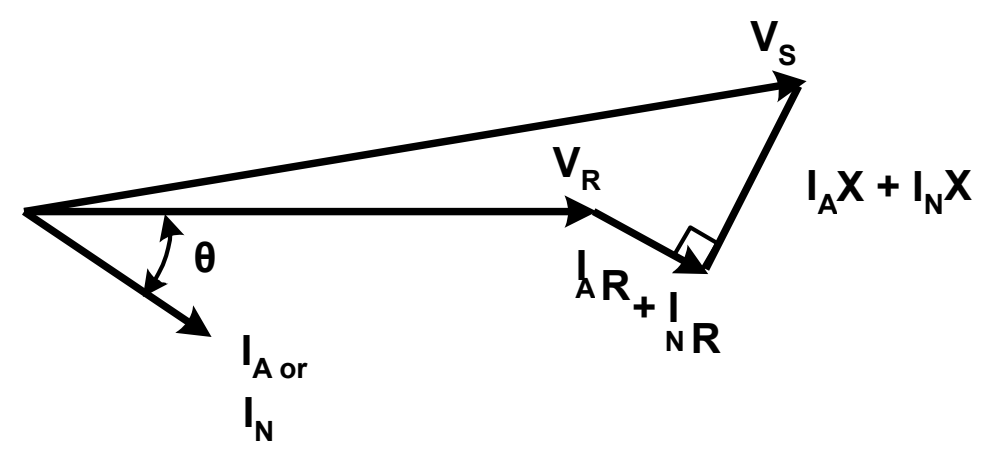

Figure 2. Single-phase phasor diagram

The approximate voltage drop consists of four components:

$$
\Delta \mathrm{V}=\left|\mathrm{I}_{\mathrm{A}}\right| \mathrm{R} \operatorname{Cos} \theta+\left|\mathrm{I}_{\mathrm{N}}\right| \mathrm{R} \operatorname{Cos} \theta+\left|\mathrm{I}_{\mathrm{A}}\right| \mathrm{X} \operatorname{Sin} \theta+\left|\mathrm{I}_{\mathrm{N}}\right| \mathrm{X} \operatorname{Sin} \theta . \quad \text { Equation } 3.7
$$

In each of the two-phase cases, the single-phase load is divided equally among the two phases; therefore, $\mathrm{I}_{\mathrm{A}}$ of the single-phase case is twice the magnitude of $\mathrm{I}_{\mathrm{A}}$ in the two-phase cases.

In Figure 3 and Figure 4 , it should be noted that the sending-end voltage $V_{S A}$ for Phase $A$ with loads connected to phases $\mathrm{A}$ and $\mathrm{C}$ to neutral, as in Figure 4, is less than the sending-end voltage VSA for Phase A with loads connected to phases A and B to neutral, as shown in Figure 3. The primary reason is because the $\mathrm{I}_{N} \mathrm{X}$ term is in phase with $\mathrm{V}_{\mathrm{RA}}$ in Figure 3 , whereas it is rotated counterclockwise in Figure 4. Also, the load power factors and the $R / X$ ratios must be taken into account when calculating the voltage drop. 

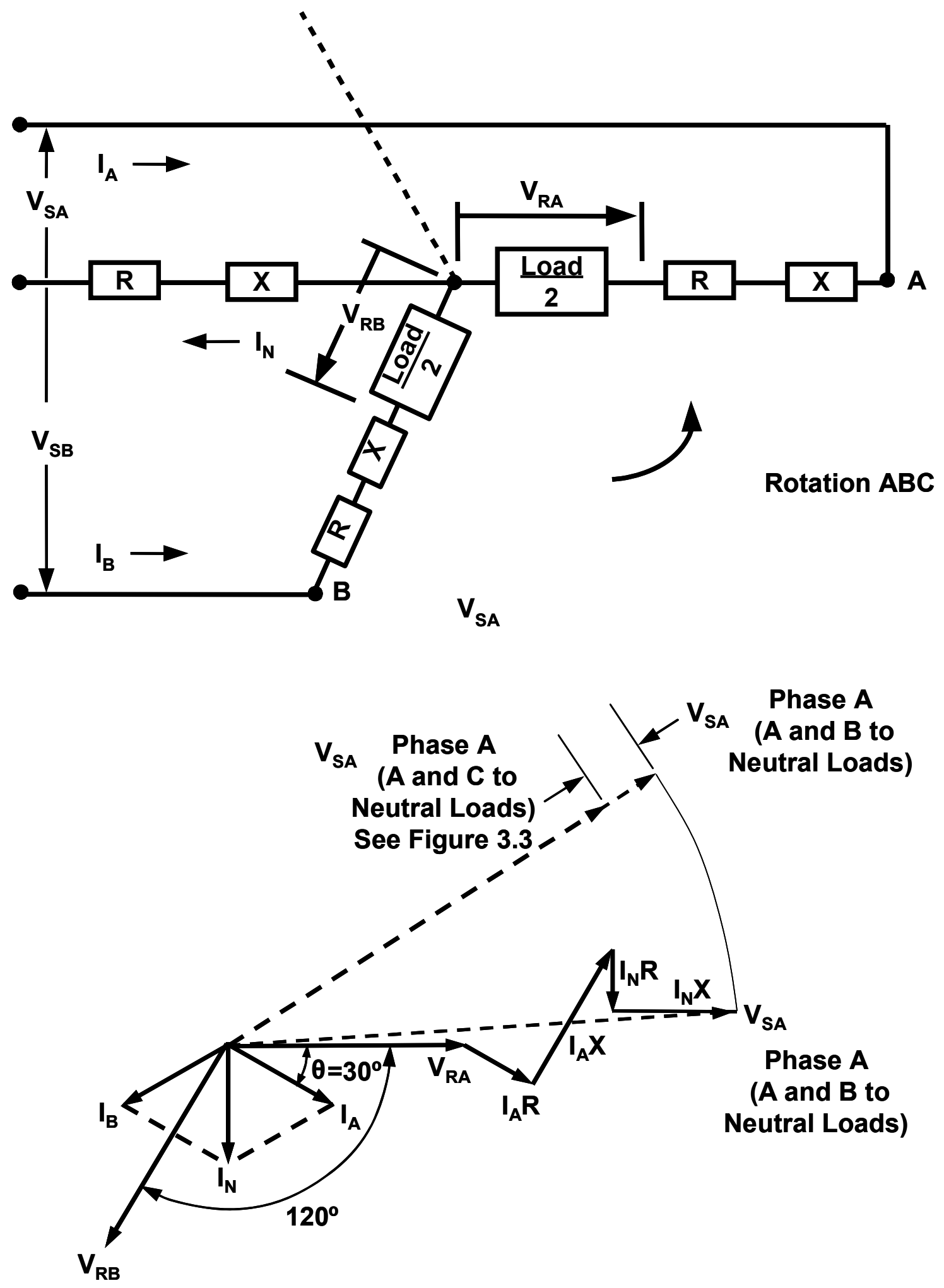

Figure 3. Two-phase lateral - voltage drop for load connected from A and B to neutral 


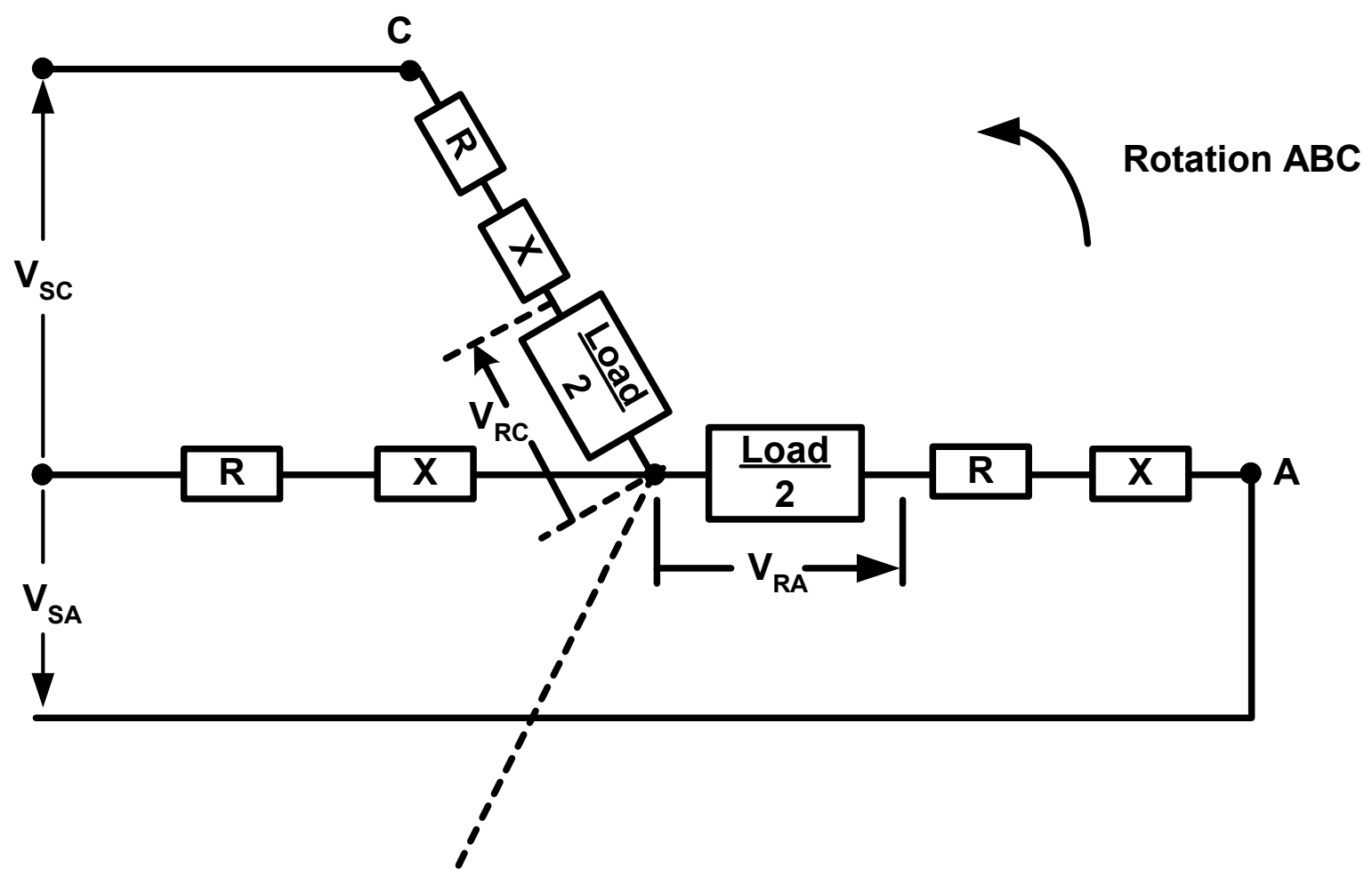

Phase A

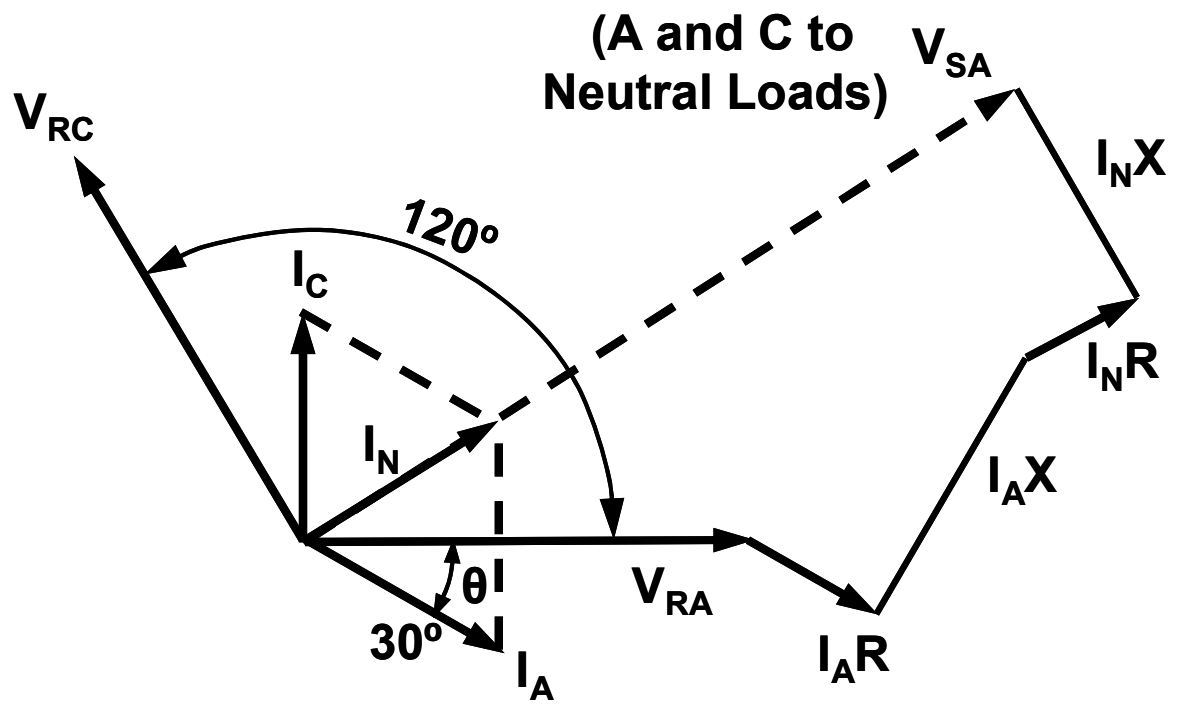

Figure 4. Two-phase lateral - voltage drop for load connected from $A$ and $C$ to neutral 


\subsection{Voltage-Regulating Equipment}

VRs may be installed at the substation secondary voltage bus, at the circuit position of the substation, or out on the circuit. Their primary purpose is to reduce voltage spread so voltage is maintained within American National Standards Institute (ANSI) C84.1 Range A for normal conditions and Range B for infrequent operating conditions. Regulators may be used to "boost" the voltage for heavily loaded circuits or "buck" the voltage for lightly loaded circuits or circuits that have fixed shunt capacitors. For this reason, it may be necessary to use switched shunt capacitors to prevent (LV, switched on) during heavily loaded conditions and HV (switched off) during lightly loaded conditions.

VRs may be applied in series, as shown in Figure 5. Where two or more are needed, fixed boost regulators may solve the LV problem if $\mathrm{HV}$ is not experienced during LL conditions. Fixed boost can be achieved by adding taps on the distribution transformers. In the upper portion of Figure 5, the voltage profile is given with the LTC VR installed at the substation. The voltage profile drops below the minimum voltage at the second and third loads. When the first (1) bus VR is added, the voltage at load (2) is within limits, but the voltage at load (3) is still below the minimum level. Adding the second (2) line regulator puts the voltage spread within limits for all three loads. 


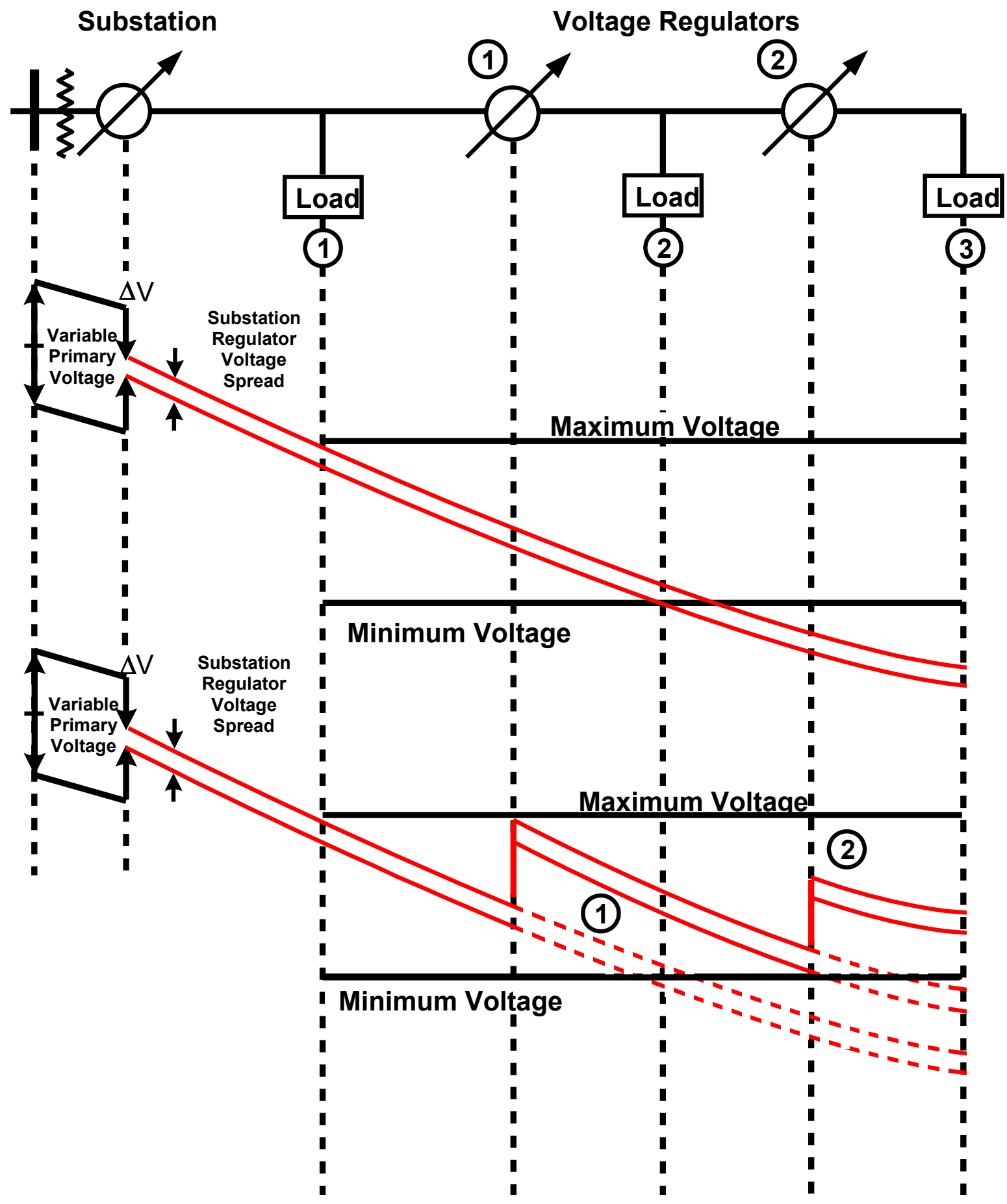

Figure 5. VRs in series 


\subsection{Shunt Capacitors}

Installing shunt capacitors causes a voltage rise or a reduced voltage drop. The capacitor current is leading and, when multiplied by the series circuit (inductive) reactance, causes a voltage rise. This rise is not dependent on load current and is highest at the capacitor location. The percentage of voltage rise is calculated as

$$
\% \Delta \mathrm{V} \text { rise }=[(3 \Phi \mathrm{kVAr})(\mathrm{l})(\mathrm{X})] /\left[\mathrm{kV}^{2}(10)\right] \quad \text { Equation } 3.8
$$

where

$3 \Phi \mathrm{kVAr}=$ The three-phase capacitor $\mathrm{kVAr}$ or $\mathrm{kVA}$ because, for the capacitor, $\mathrm{kVAr}$ $=\mathrm{kVA}$

$\mathrm{X}=$ Inductive line reactance in $\Omega$ per unit length

$\mathrm{kV}=$ Line-to-line voltage

$1=$ Distance in unit length from the substation source to the capacitor.

When the capacitor installed is single-phase, $\mathrm{S} \Phi \mathrm{kVAr}$ is used in Equation 3.8. However, the $\mathrm{X}$ is multiplied by two because there are two conductors, and $\mathrm{kV}$ is the line-to-line voltage between these two conductors. As an example, if a $600-\mathrm{kVAr}$, three-phase capacitor is installed on a $13.2-\mathrm{kV}$ wye system at 3 miles and the line reactance of a $636-\mathrm{k} \mathrm{cmil,} \mathrm{all-}$ aluminum conductor is $0.536 \Omega /$ mile/conductor, then

$$
\% \Delta \mathrm{V} \text { rise }=[(600)(3)(0.536)] /\left[(13.2)^{2}(10)\right]=0.554 \% . \quad \text { Equation } 3.9
$$

For single-phase,

$$
\% \Delta \mathrm{V} \text { rise }=[(200)(3)(0.536)(2)] /\left[(7.62)^{2}(10)\right]=1.108 \%, \quad \text { Equation } 3.10
$$

or twice the percent voltage rise. As noted earlier, the voltage rise will be the same for LL and HL conditions. Therefore, it may be necessary to install capacitor switches. The amount of capacitors needed to correct an LV problem is dependent on the conductor size, load power factor, and how the load is distributed on the circuit.

\subsection{Load Tap-Changing Transformers}

LTC transformers are often referred to as tap-changing under load. Load tap changers are applied to power transformers at the substation. They are used to control the voltage on the LV or secondary side to a fixed value with a variable primary voltage input, as shown in Figure 5. Also, LTCs may be used to control reactive power flow by shifting the phase angle of the transformer secondary voltages. The regulation range is typically 8,16 , and 32 steps with $\pm 10 \%$ of rated voltage. The 32 -step LTC is the most common for LTC transformers in substations. The 32 steps are divided into 16 steps raise and 16 steps lower, or a $5 / 8 \%$ change per step based on $\pm 10 \%$ voltage range. The change in steps is made without interrupting the circuit by using a mid-tapped autotransformer, called a preventive autotransformer. The steptype VR theory of operation is similar to the LTC transformer. 


\subsection{Voltage Regulator Theory of Operation}

In general, the VR is a transformer. If two windings are wound on a common magnetic core and the numbers of turns is different, then the voltages across these windings will be different. The alternating voltage applied to the first coil will induce a voltage in the second coil, and the magnitude of voltage induced will be dependent on the turn ratio between these two windings. In the case of a regulator, the first coil is referred to as the primary, or exciting, winding and the second as the regulating, or series, winding.

The step regulator is an autotransformer, the windings of which are connected in series and wound on the same magnetic core. There are two types of autotransformers: step-up and stepdown. Figure 6 shows a step-up transformer with a turn ratio of 10. For example, if $\mathrm{N}_{1}=100$ and $\mathrm{N}_{2}=10$, then $\mathrm{a}=\mathrm{N}_{1} / \mathrm{N}_{2}=10$. If the series winding is connected in series with the exciting or shunt winding that has the polarity, as shown in Figure 6, then $120 \mathrm{~V}$ applied to the exciting winding will result in $12 \mathrm{~V}$ on the series winding, which, when added to the primary voltage, results in a $132-\mathrm{V}$ output. When the polarity of the series winding is reversed, as shown in Figure 7, then the $12 \mathrm{~V}$ on the series winding is subtracted from the $120 \mathrm{~V}$ primary voltage, and the output is $108 \mathrm{~V}$. This is called a step-down transformer. To obtain a smaller change in voltage, the series winding is divided into eight equal parts, called taps, as shown in Figure 8.

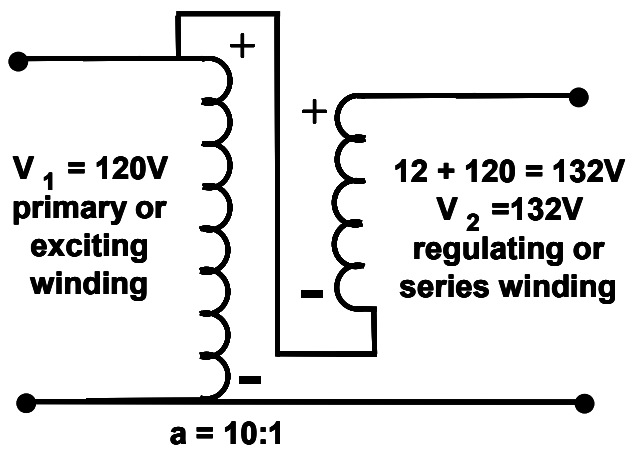

Figure 6. Step-up transformer

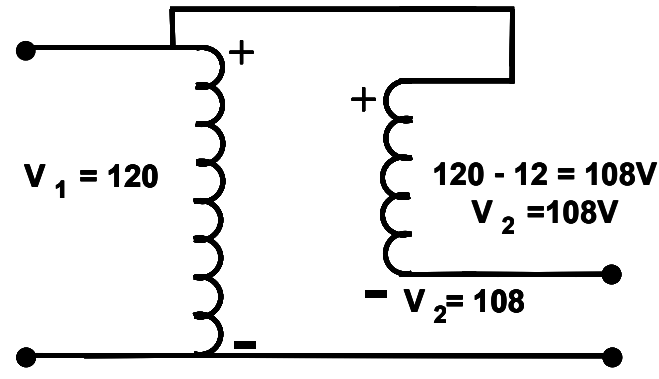

Figure 7. Step-down transformer 


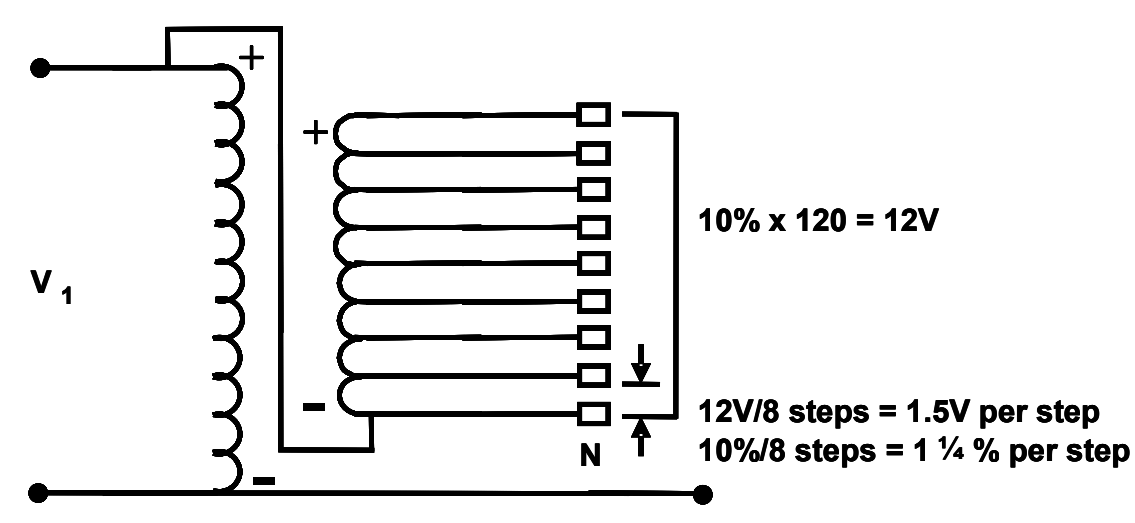

Figure 8. Step regulator

The output voltage can be varied from $120 \mathrm{~V}$ if tapped at the neutral position, $\mathrm{N}$, up to $132 \mathrm{~V}$, with steps of $12 \mathrm{~V}$ divided by 8 or $1.5 \mathrm{~V}$ per step, or $11 / 4 \%$ changes in voltage per tap setting. The problem with this arrangement is the interruption of the circuit each time the tap changes. To avoid this, the moving tap can be broken into two fingers such that, as the moving tap changes from one tap position to the next, one finger is always in contact with a tap. This is shown in Figure 9. To prevent shorting out the turns of the series winding, as shown in Figure $9 \mathrm{~b}$, when one finger is on one tap and the other finger is on the next tap, a preventive autotransformer is midtapped and connected to each finger. The center tap of this autotransformer is connected to the load bushing (L). In Figure 9a, when both fingers are on the neutral tap " 0 ," they are at the same voltage, and the center tap of the preventive auto is at the same voltage. When $120 \mathrm{~V}$ is applied to the primary, the voltage from the preventive auto, sometimes referred to as a bridging reactor, is at $120 \mathrm{~V}$. When the moving fingers move toward Tap 1, the top finger is on Tap 1, while the bottom finger is on Tap 0. As shown in Figure 9b, there is a $11 / 4 \%$ voltage difference between Tap 0 and Tap 1 and the two fingers. But when the bridging reactor is connected between these two fingers and its center tap is connected to the load, the load will see one half of the $11 / 4 \%$, or $5 / 8 \%$, as shown in (2) of Figure $9 \mathrm{~b}$. This is called the bridging position $5 / 8 \%$. When the top finger moves farther up and is resting on Tap 1 , and the bottom finger is resting on Tap 1, then the center tap is at the same voltage as the two fingers and Tap 1. Now the voltage at the load is up 1 1/4\% voltage (see Figure 9c). For higher-voltage outputs, this process repeats where the fingers are in non-bridging and bridging positions until the top tap is reached and a full $10 \%$ voltage increase is attained. 


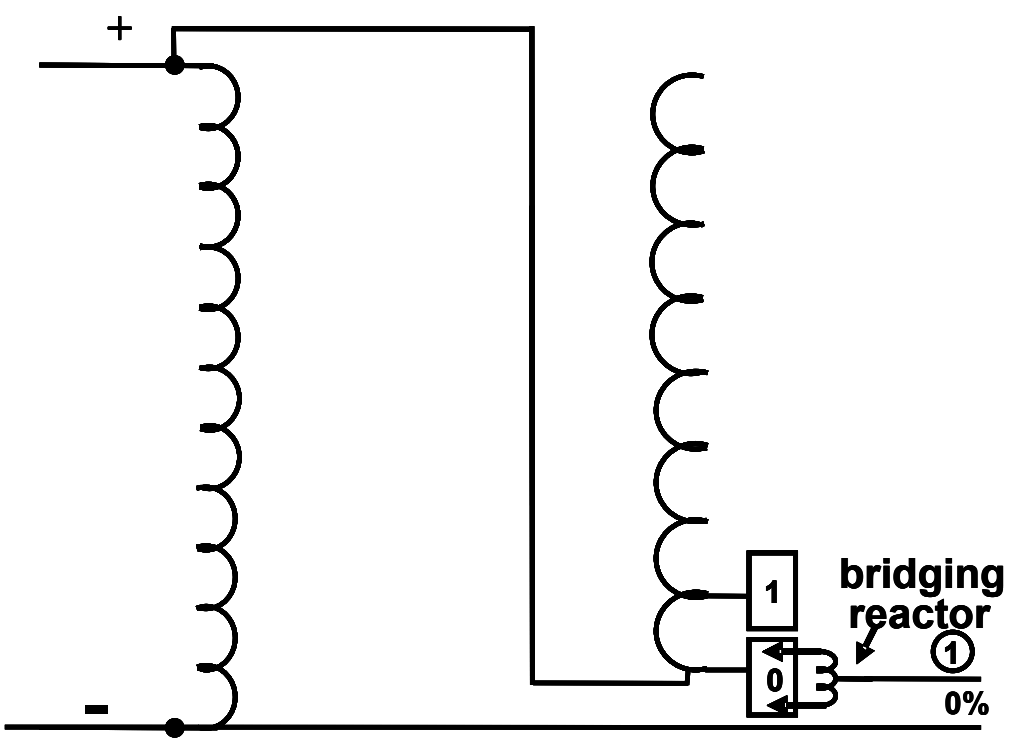

a. Non-bridging position $0 \%$

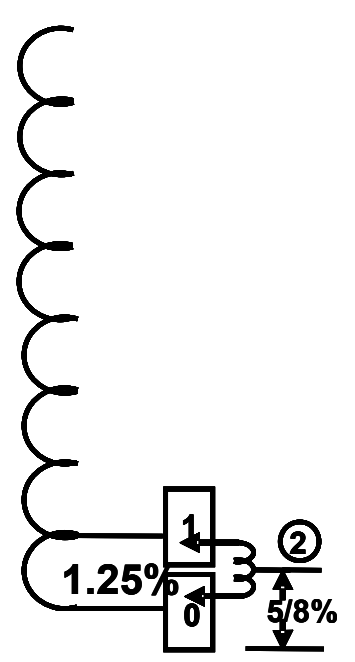

b. Bridging position $\mathbf{5 / 8} \%$ 


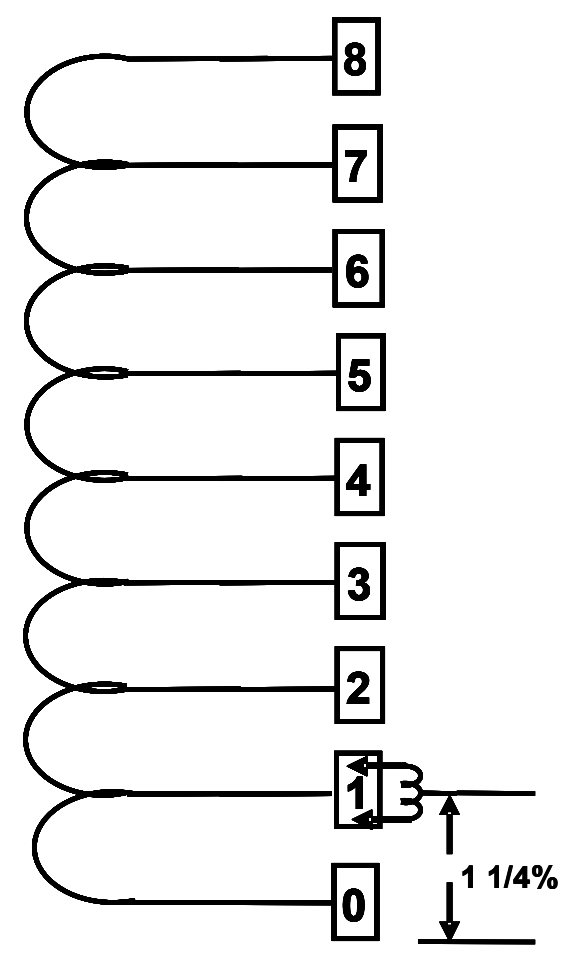

c. Non-bridging position $11 / 4 \%$

Figure 9. Step VR sequence of operation

For decreases in voltage, the process is reversed until both fingers are resting on Tap 0 . This process has addressed only increases in voltage above the primary voltage. To lower the voltage below the primary voltage, it is necessary to reverse the polarity, which is similar to the step-down transformer of Figure 7. This is accomplished by adding the reversing switch of Figure 10, which changes the polarity of the series winding. Now, the regulator can raise or lower the voltage by $\pm 10 \%$. 


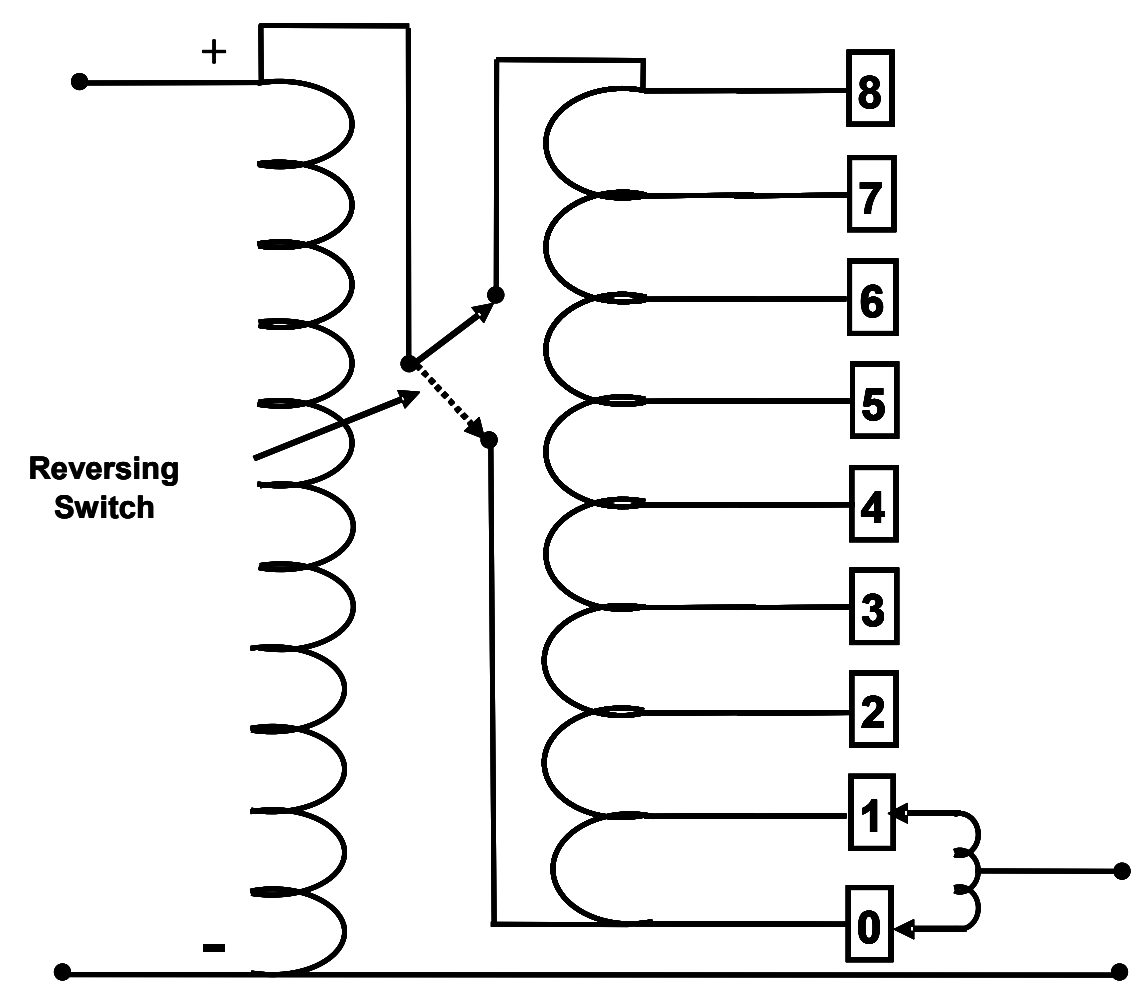

Figure 10. Step VR with reversing switch

\subsection{Bridging Reactor or Preventive Autotransformer}

Before, the bridging reactor was introduced to achieve one-half of a voltage step between taps and eliminate shorted turns when the moving fingers were on different taps. The current in these fingers is not the same when moving from non-bridging and bridging positions.

Four conditions are examined: (1) non-bridging no-load, (2) bridging no-load, (3) non-bridging load, and (4) bridging load. Figure 11 illustrates the non-bridging no-load condition. When both fingers are on the same tap (1) and no load, there is no voltage difference across the bridging reactor and the load current $\mathrm{I}_{\mathrm{L}}=0$. When the top finger moves to Tap 2, and the bottom finger remains on Tap 1 (bridging position), a voltage appears across the reactor. A circulating current (IC) flows from the higher voltage of Tap 2 to the lower voltage of Tap 1. See Figure 12. The current through the top finger is the same as the bottom-finger current because there is no load. With no load, there is a current in the reactor for every bridging position. This explains why current is flowing even though there is no load current on the circuit.

When load ( $\left.\mathrm{I}_{\mathrm{L}}\right)$ is applied and the top and bottom fingers are on the same tap (Figure 13), the current is equal in the two fingers because since the ampere turns in the reactor are balanced and $I_{a}=1 / 2 I_{L}$ and $I_{b}=1 / 2 I_{L}$. When the fingers are on different taps, such as the bridging position of Figure 14, a circulatory current flows. Now, the top-finger current $I_{a}=1 / 2 I_{L}+I_{C}$, and the bottom-finger current $\mathrm{I}_{\mathrm{b}}=1 / 2 \mathrm{I}_{\mathrm{L}}-\mathrm{I}_{\mathrm{C}}$. The circulatory current is approaching $50 \%$ of the value of the load current and flows from the higher voltage on Tap 2 to the lower voltage on Tap 1. 
The current in the top finger is higher than the current in the bottom finger because of this circulating current. This difference can be reduced with equalizer windings.

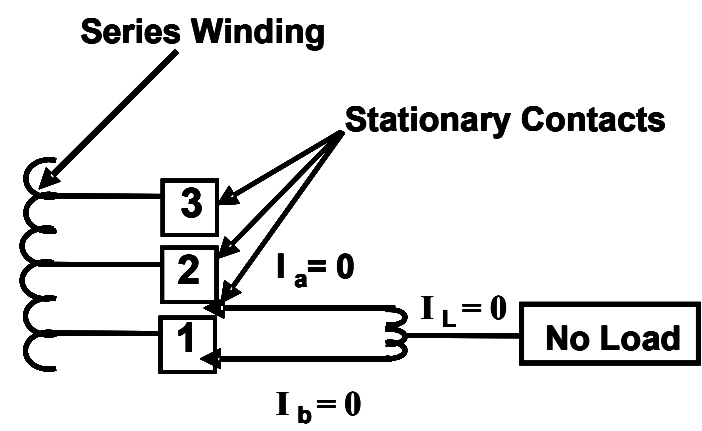

Figure 11. Non-bridging no load

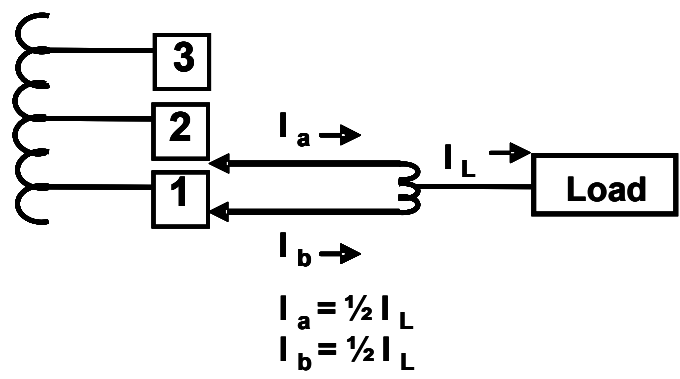

Figure 13. Non-bridging load

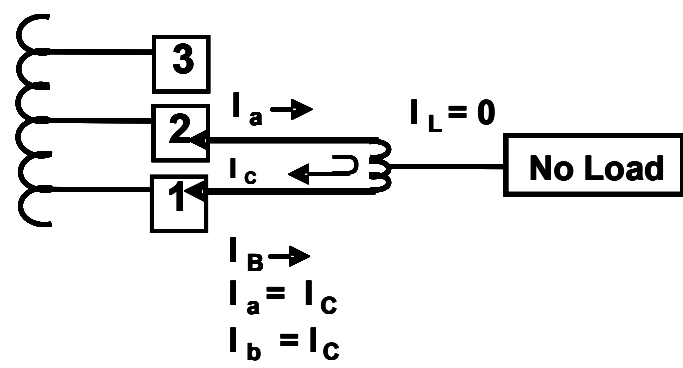

Figure 12. Bridging position no load

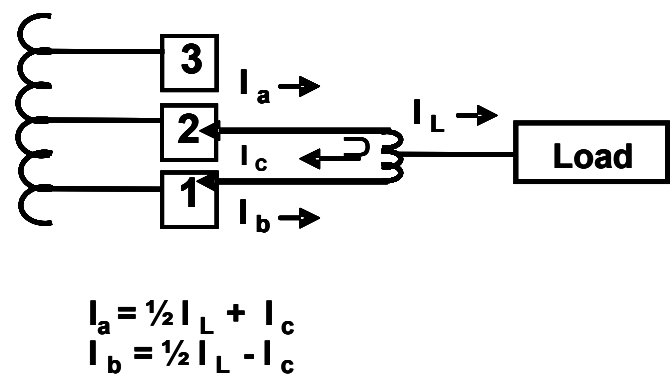

Figure 14. Bridging position load

\subsection{Equalizer Windings}

Equalizer windings are wound on the same central core as the primary and series windings and equal about $1 / 2$ the number of turns between each tap on the series winding. These windings reduce the voltage during switching and thus prolong the life of the tap changer and equalize the current on the fingers because the net circulating current reverses for odd- and even-numbered taps. Figure 15 shows the application of the equalizer windings.

Four cases are studied: one set without equalizer windings and one set with equalizer windings. Case A, Figure 16, shows what happens when the lower finger moves from Tap 2 to Tap 1. When the lower finger, $b$, moves off Tap 2, the voltage between $b$ and 2 is $V$, which causes an arc on $b$ and contact erosion. But when the lower finger, $b$, moves from Tap 2 of Case B to Tap 3 (Figure 17), there is no arc because there is no voltage difference.

When equalizer windings are present in Case $C$ (Figure 18) and finger b moves to Tap 1, the voltage difference between $b$ and 2 is $1 / 2 \mathrm{~V}$. Therefore, the contact erosion is reduced. Case $\mathrm{D}$ (Figure 19) shows the non-bridging case, in which finger b moves from 2 to 3 . Here, when $b$ moves off of 2 , a voltage difference of $1 / 2 \mathrm{~V}$ appears between 2 and $b$, so that the arcing is the same as in Case $\mathrm{C}$. The contact wear is thus equalized between bridging and non-bridging positions. 


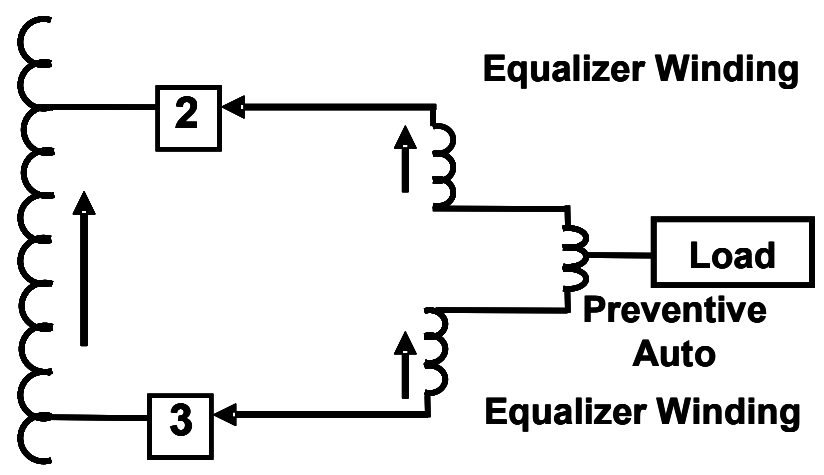

Figure 15. Application of equalizer windings

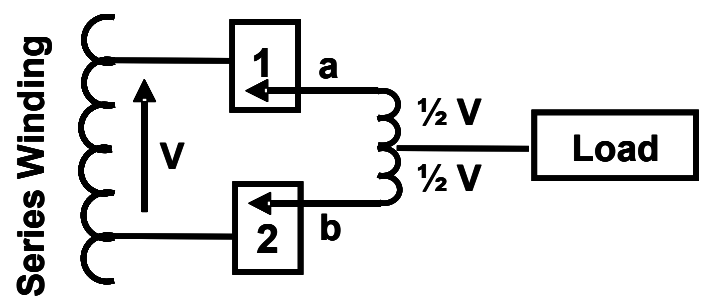

Figure 16. Case A - without equalizer windings

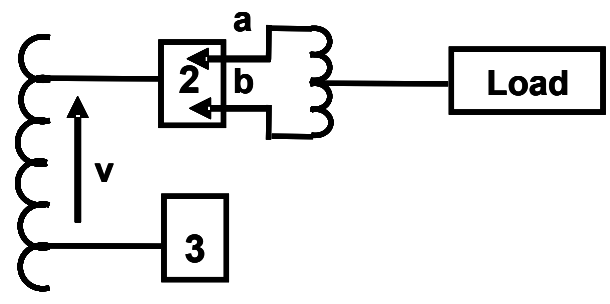

Figure 17. Case B - without equalizer windings

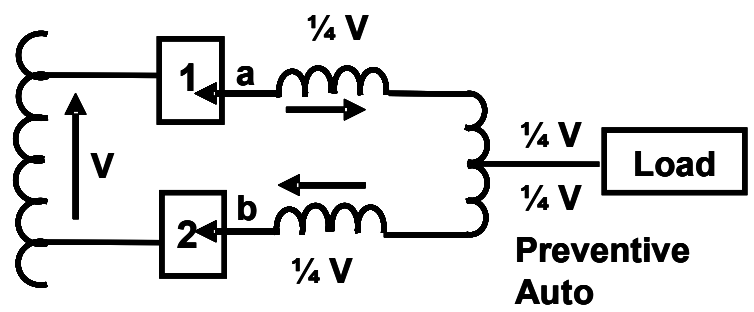

Figure 18. Case $\mathrm{C}$ - with equalizer windings bridging position 


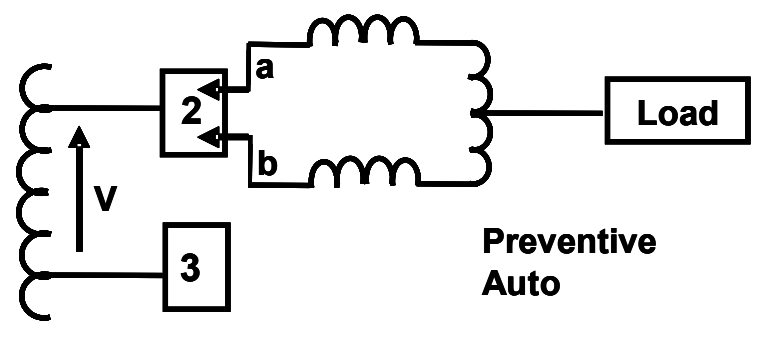

Figure 19. Case $D$ - with equalizer windings non-bridging position

\subsection{Types of Step Regulators}

There are three types of VRs: Type A, Type B, and bi-directional (or cogeneration). For Type A, the primary is connected to the exciting winding or the shunt winding. See Figure 20. The series winding is connected to the shunt winding via the reversing switch, as shown in Figure 10. Therefore, the series winding is connected to the load side. Type B, shown in Figure 21, is more common. Its series winding is connected to the source side.

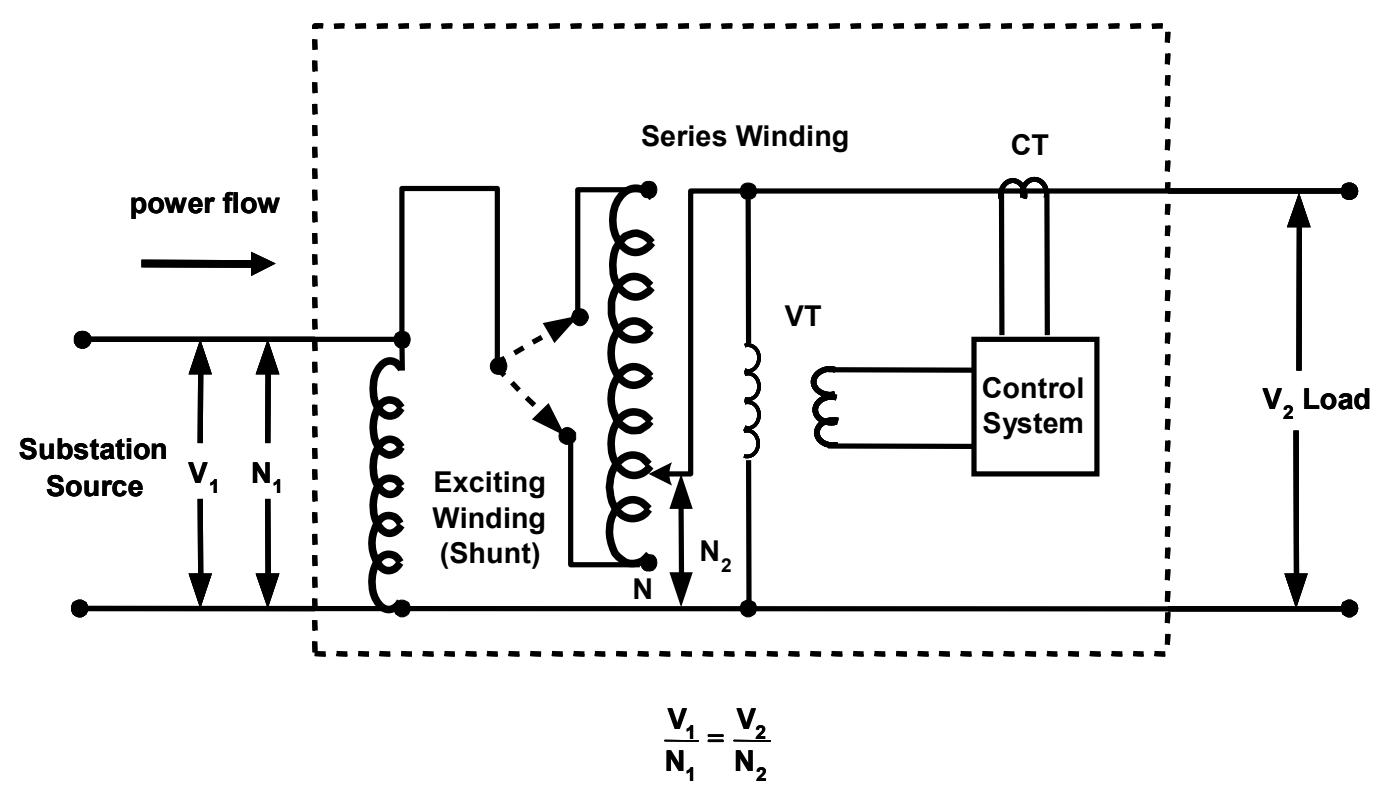

Figure 20. ANSI Type A - series winding located on load side 


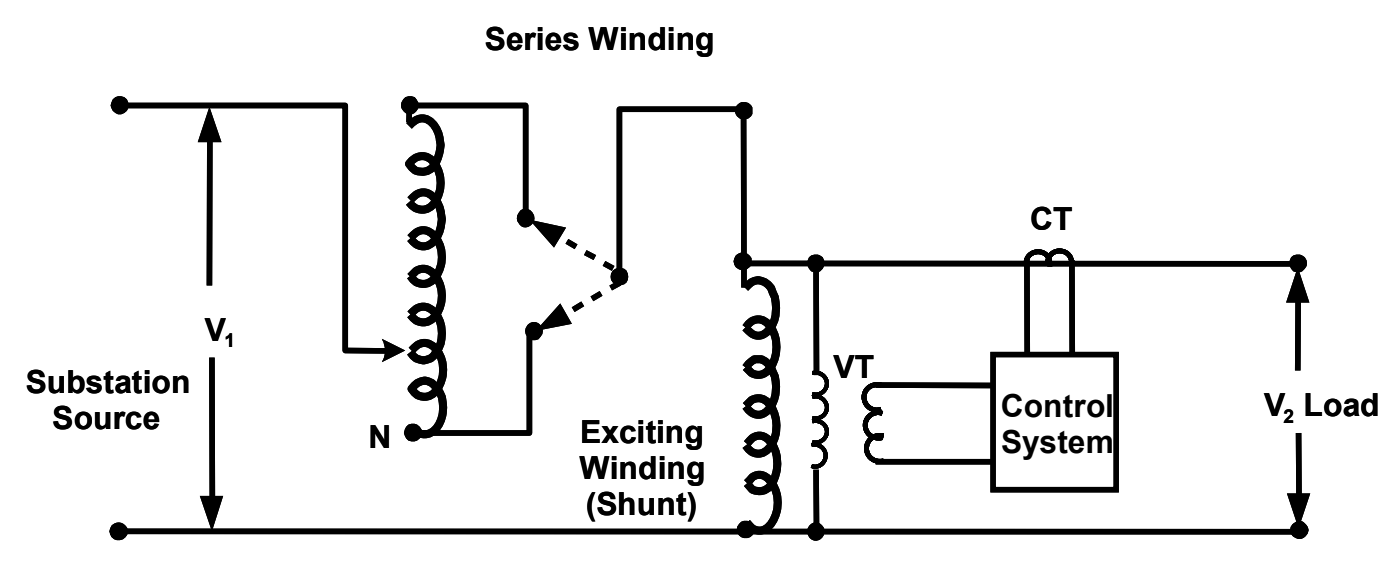

Figure 21. ANSI Type B - series winding located on source side

When applying Type A and B regulators, there is only one source of power (e.g., the substation source). However, there are applications in which power may flow from either direction through the regulator, as shown in Figure 22. In this figure, the regulator is located between two power sources: the substation and a distributed resource (DR). In Figure 23, assume the power flows from left to right. The voltage transformer (VT) $\mathrm{VT}_{2}$ is connected across the load side. When the $\mathrm{VT}_{2}$ senses $\mathrm{LV}$ and the regulator voltage is adjusted up, the voltage at the output is increased. Now, suppose the power flows in the reverse direction because of the operation of the DR in Figure 22. Because $\mathrm{VT}_{2}$ is now connected across the source side, it will measure the $V_{2}$ voltage. When the $V_{2}$ voltage is low, the control system will cause the voltage to rise and thus insert more series winding, $\mathrm{N}_{2}$. The voltage turns ratio equation,

$$
\frac{\mathrm{V}_{1}}{\mathrm{~N}_{1}}=\frac{\mathrm{V}_{2}}{\mathrm{~N}_{2}},
$$

shows that increasing $\mathrm{N}_{2}$ will cause $\mathrm{V}_{1} / \mathrm{N}_{1}$ to change. Because $\mathrm{N}_{1}$ is the turns on the exciting winding, which is a constant, $\mathrm{V}_{1}$ voltage will drop even further, thus lowering voltage $\mathrm{V}_{1}$ rather than raising the voltage. This will cause the regulator to go to the maximum raised position. If the voltage $\mathrm{V}_{2}$ changes, then the regulator will chase up and down continuously between all raise and all lower.

Changes must correct this problem. First, the regulator must recognize the power flow has reversed. This can be accomplished using the current transformer (CT) input to the control system. Second, the VT must sense the voltage on the $\mathrm{V}_{1}$ side or the load side for the reversepower use. Third, there must be a change in the line drop compensator settings because the regulation point has now changed to a different location with different circuit $\mathrm{R}$ and $\mathrm{X}$ values and voltage settings. Another solution is shown in Figure 24, in which the change in power flow is sensed, but a differential transformer reads the difference between $\mathrm{V}_{1}$ and $\mathrm{V}_{2}$. Here, the control system will compensate for the voltage difference between $V_{1}$ and $V_{2}$ and correct for the LV or HV problem, which is independent of the power flow. This is called a bidirectional VR. 


\section{Substation}

Source

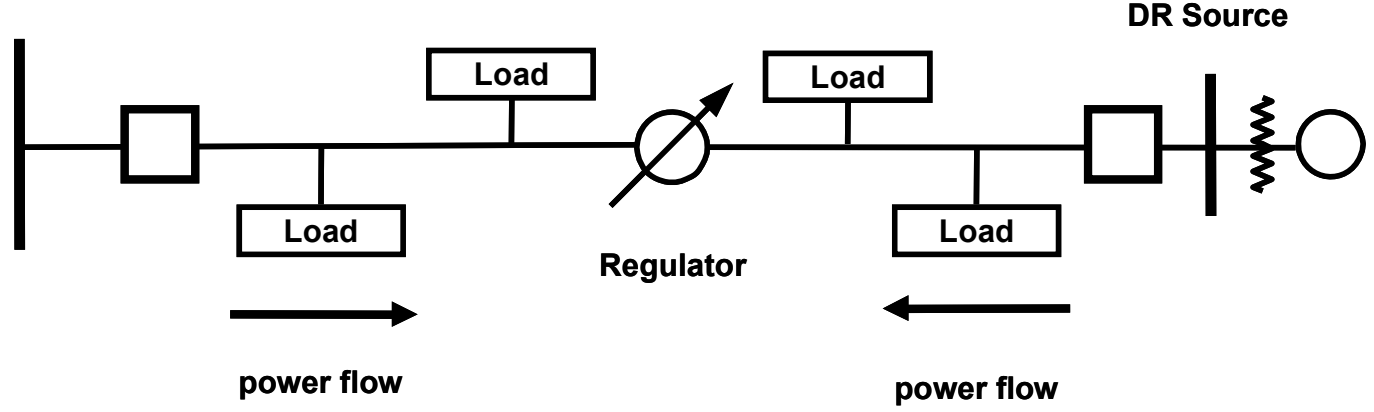

Figure 22. DR sources create bidirectional flows through a step VR

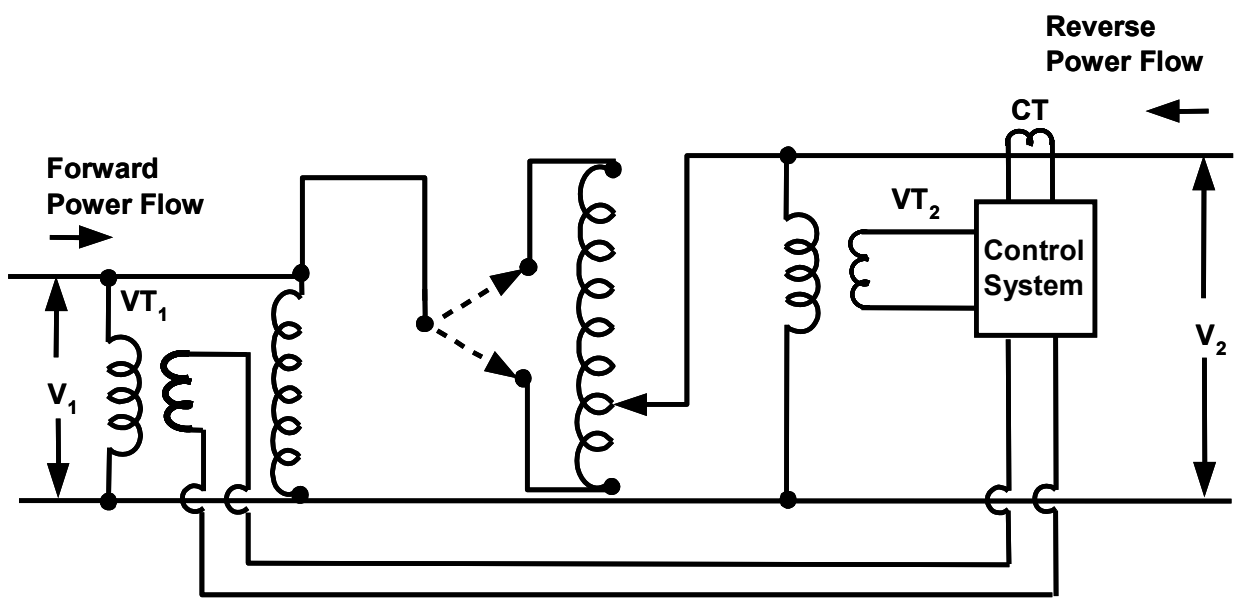

Notes: (1) Reverse flow - use $\mathrm{VT}_{1}$ voltage

(2) Logic controls motor rotation direction

Figure 23. Reverse power flow - additional VT required $\mathrm{VT}_{1}$

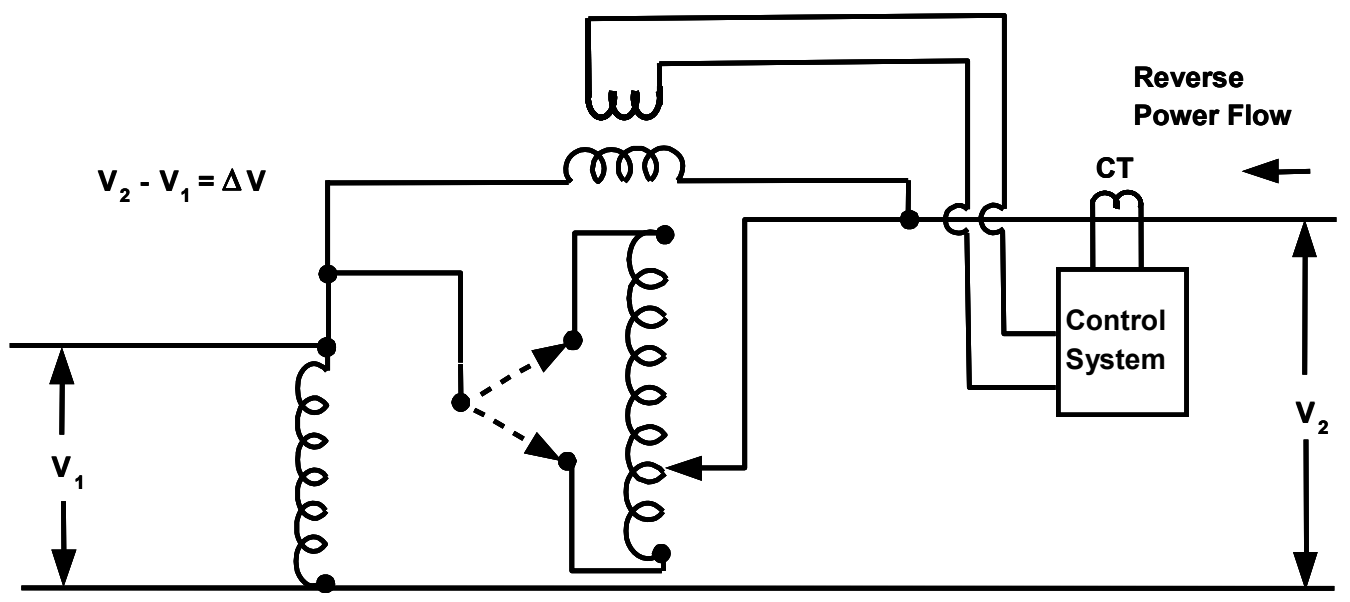

Figure 24. Reverse power flow - differential transformer 


\subsection{Bidirectional Voltage Regulator}

The bidirectional VR may use either of the methods outlined in Figure 23 or Figure 24, but a VT must be added for the method shown in Figure 23. Therefore, it is common to have the logic, based on the direction of current flow, calculate the voltage on either the source or load side based on one VT voltage measurement. Also, the logic in the control system knows the position of the reversing switch and the tap position number by counting the number of tap positions from neutral, either raise or lower. This results in a lower-cost regulator for DR applications. In addition, the $\mathrm{R}_{\mathrm{L}}$ and $\mathrm{X}_{\mathrm{L}}$ settings are usually different when the current reverses direction; therefore, this regulator must have a set of forward settings and a set of reverse settings.

Figure 25 shows four application cases - a, b, c, and d-for the unidirectional step VR. The first case (a) is a simple application of an LTC transformer (1) at the substation. The voltage drop in the feeder to the feed point is compensated using the $\mathrm{R}_{1}$ and $\mathrm{X}_{1}$ values of the circuit to provide a fixed voltage $\mathrm{VR}_{1}$ at the regulation point as the load current and load power factor change and as the primary voltage on the LTC transformer changes. The second case (b) shows the LTC transformer regulation device (1) regulating to $\mathrm{VR}_{1}$ and a DR (2) regulating voltage at $\mathrm{VR}_{2}$. The third case (c) uses an $\mathrm{LTC}$ transformer (1) regulating to $\mathrm{VR}_{1}$ and a unidirectional step regulator (2) regulating to voltage $\mathrm{VR}_{2}$. It is not necessary to know the $R_{2}$ and $X_{2}$ values for this case, but they must be known for cases e, $f$, and $g$, which follow in Figure 26. For consistency, these values are shown in all the latter application cases. The fourth case (d) has three methods of voltage regulation: the LTC (1), the unidirectional regulator (2), and a capacitor (3). All of these cases are used in the modeling and circuit simulation studies to determine resultant voltage profiles.

Figure 26 consists of three additional cases - e, f, and g-in which bidirectional VRs and DR are applied to regulate the voltage on the circuit. The fifth case, e, shows LTC regulation (1), bidirectional voltage regulation (2), DR regulation (3), and a reactive compensation capacitor (4). If the DR is an induction generator, a capacitor is often used to provide the voltage if it becomes isolated from the circuit because an induction generator does not create voltage. But, the kilowatt injection at 3 will improve the voltage because less load current flows through the circuit elements. The sixth case, $\mathrm{f}$, adds an additional bidirectional regulator (3). The final case, $g$, involves the addition of a second DR, which does not regulate voltage. However, the DR at 4 does provide Var production and Var absorption to regulate voltage. It should be remembered the loads in Figure 25 and Figure 26 are shown as lumped loads. In actual applications, the loads are distributed throughout the circuit. All these cases will be analyzed in detail to show the effects of DR generation, DR voltage regulation on a normal voltage- regulated circuit that contains an LTC, step regulators, and capacitors. 


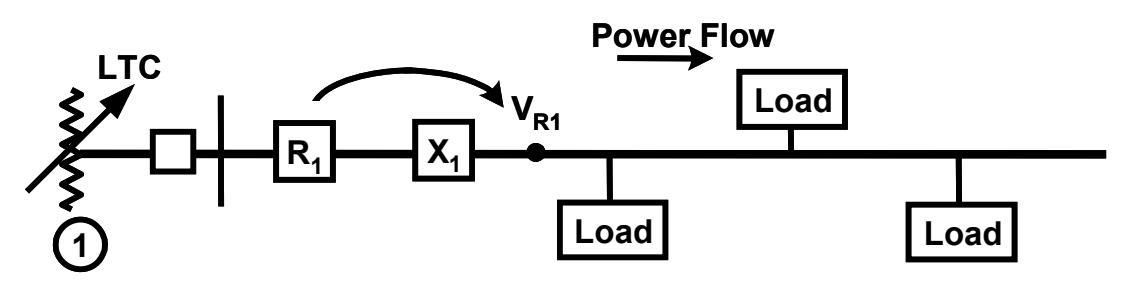

(a)

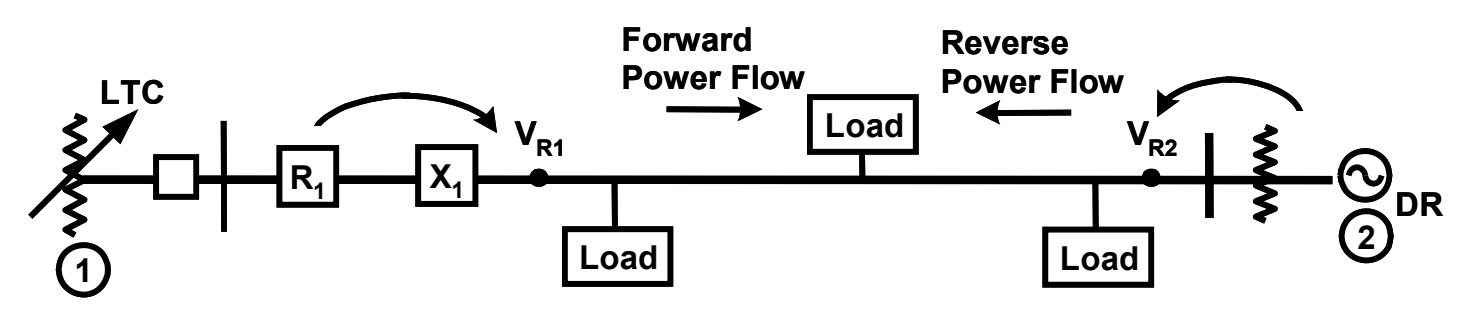

(b)

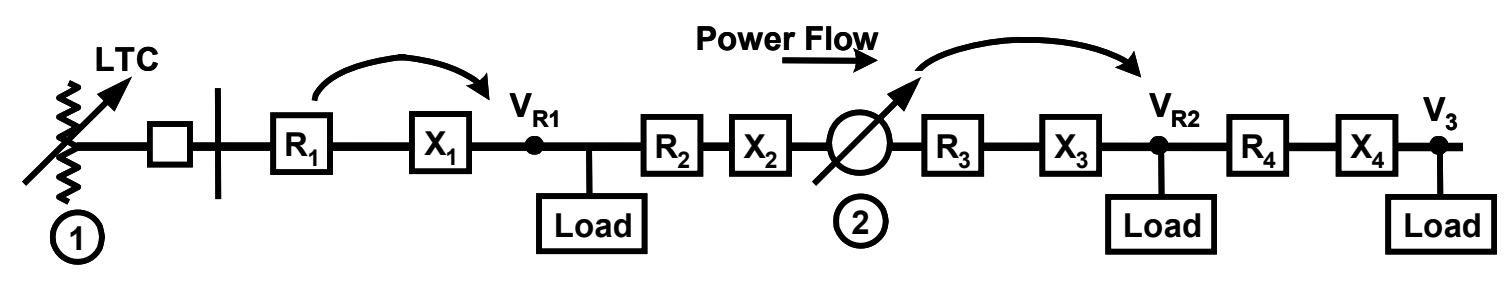

(c)

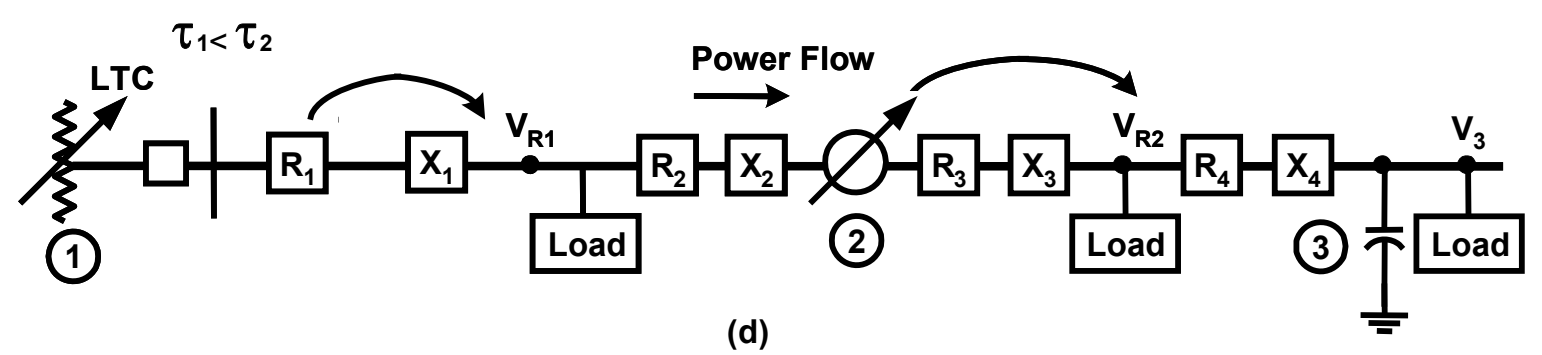

Figure 25. Examples of unidirectional VRs and DR 


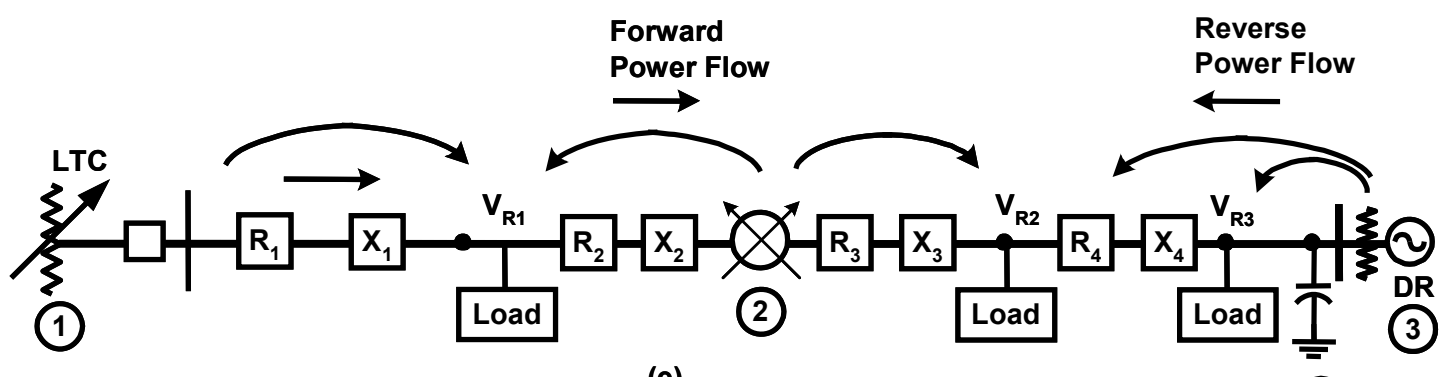

(e)
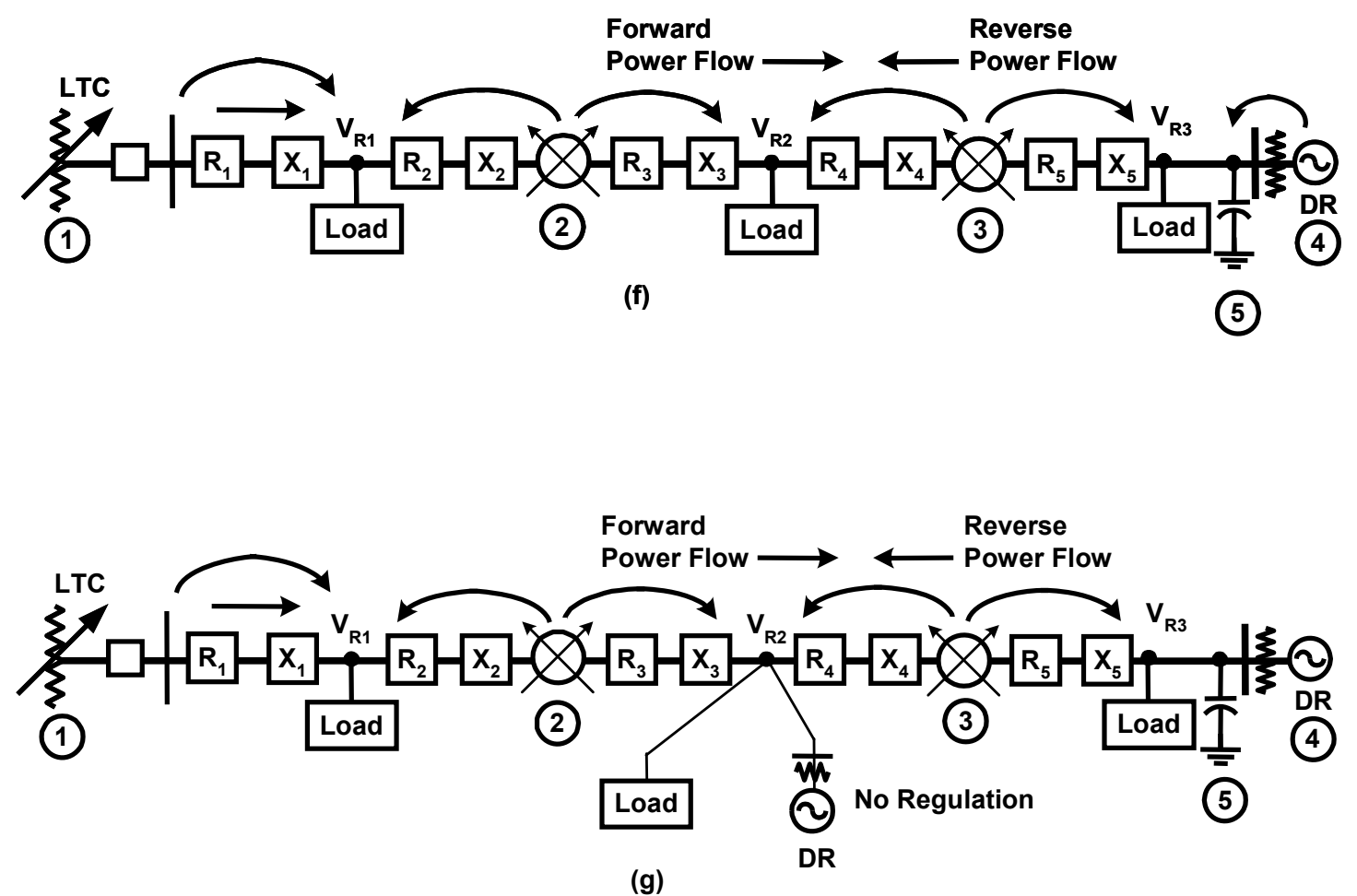

Figure 26. Examples of bidirectional VRs and DR 


\subsection{Step Voltage Regulator Control System}

Four settings are required to control voltage:

- Set point voltage - The voltage required at the load on a $120-\mathrm{V}$ base

- Voltage bandwidth - The voltage variation from the set point voltage at the load. If the set point voltage is $125 \mathrm{~V}$ and the bandwidth is $2 \mathrm{~V}$, the regulator will control the voltage within $126 \mathrm{~V}$ and $124 \mathrm{~V}$

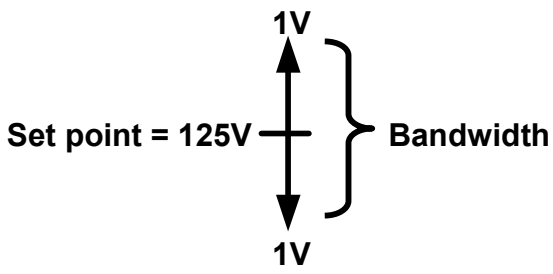

- Time delay - The time between when a change in voltage is sensed and when the change in voltage occurs. The time delay allows for the short-term surge currents, such as motor-starting currents, to occur so that tap changing does not occur during these temporary periods of voltage drop

- Line drop compensator - The compensator for the voltage drop in the circuit between the regulator and the load. The $\mathrm{R}$ and $\mathrm{X}$ values of the line are set to determine the voltage drop in the line.

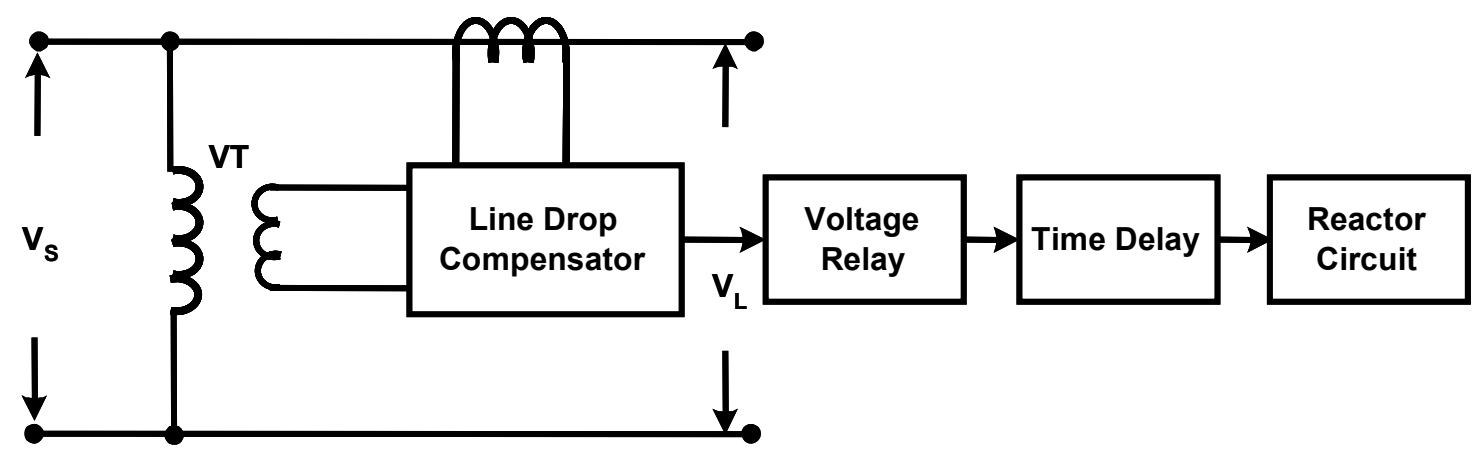

Figure 27. Control circuit of step VR

The components of the control circuit are illustrated in Figure 27. The kilovolt-ampere rating of the regulator is determined using the same approach as that used for the autotransformer because since the shunt and series windings form an autotransformer. The kilovolt-ampere rating is usually $10 \%$ of the rated current flowing through the series winding. 


\subsection{Voltage and Current Equations}

As previously noted, there are three types of VRs: Type A, Type B, and bidirectional. The most common is Type B. Figure 28 shows Type A. Here, the preventive autotransformer winding is connected to the load terminal $(\mathrm{L})$. The regulator is in the raise, $r$, position; the $\mathrm{I}_{2}$ current is "up"; and the excitation current of the shunt winding, $\mathrm{I}_{1}$, is "down." The exciting current varies because it is connected to the source, $\mathrm{S}$. When the reversing switch is connected to the lower terminal (1), the $I_{2}$ current is reversed ("down"), as in Figure 29, and the $\mathrm{I}_{1}$ current is "up." This is because the polarity of the series winding has changed because of the reversing switch changing its polarity.

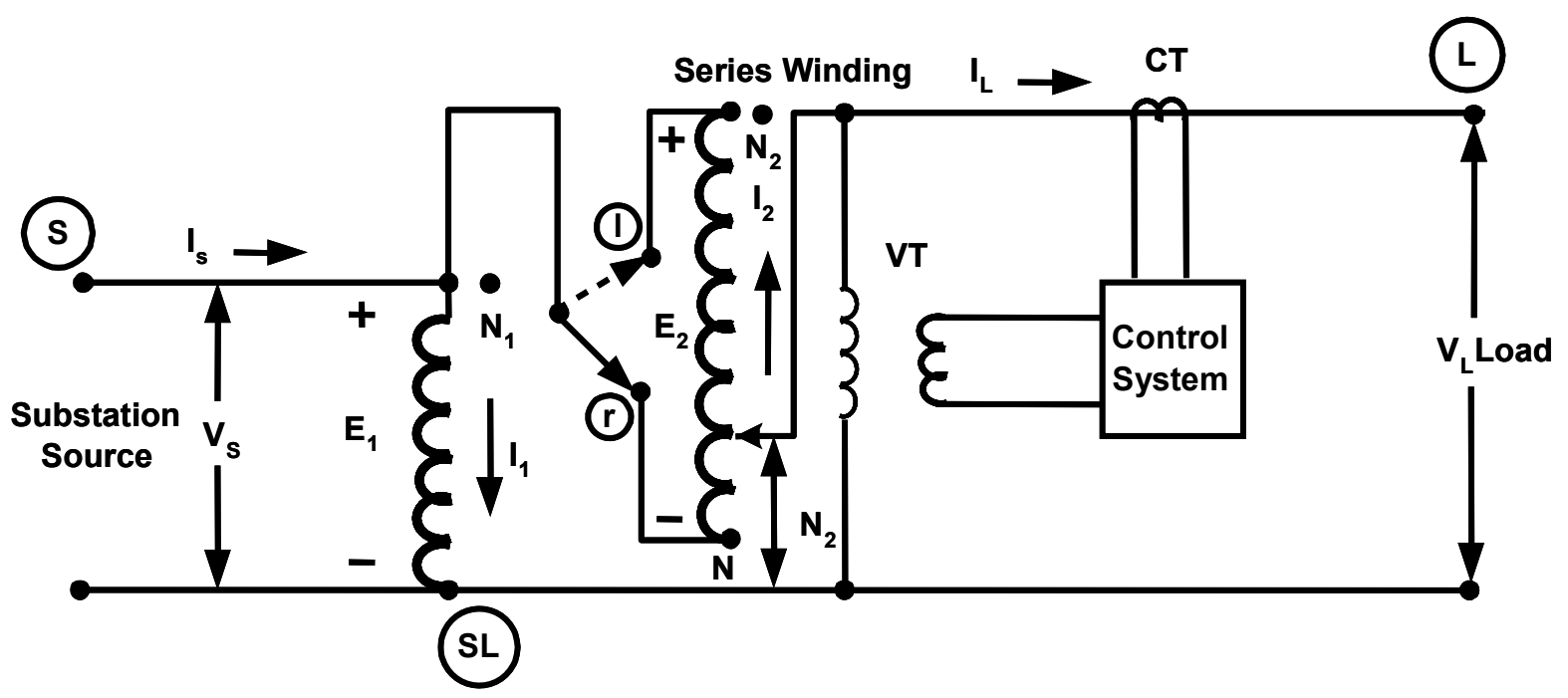

Figure 28. Type A step regulator - raise position

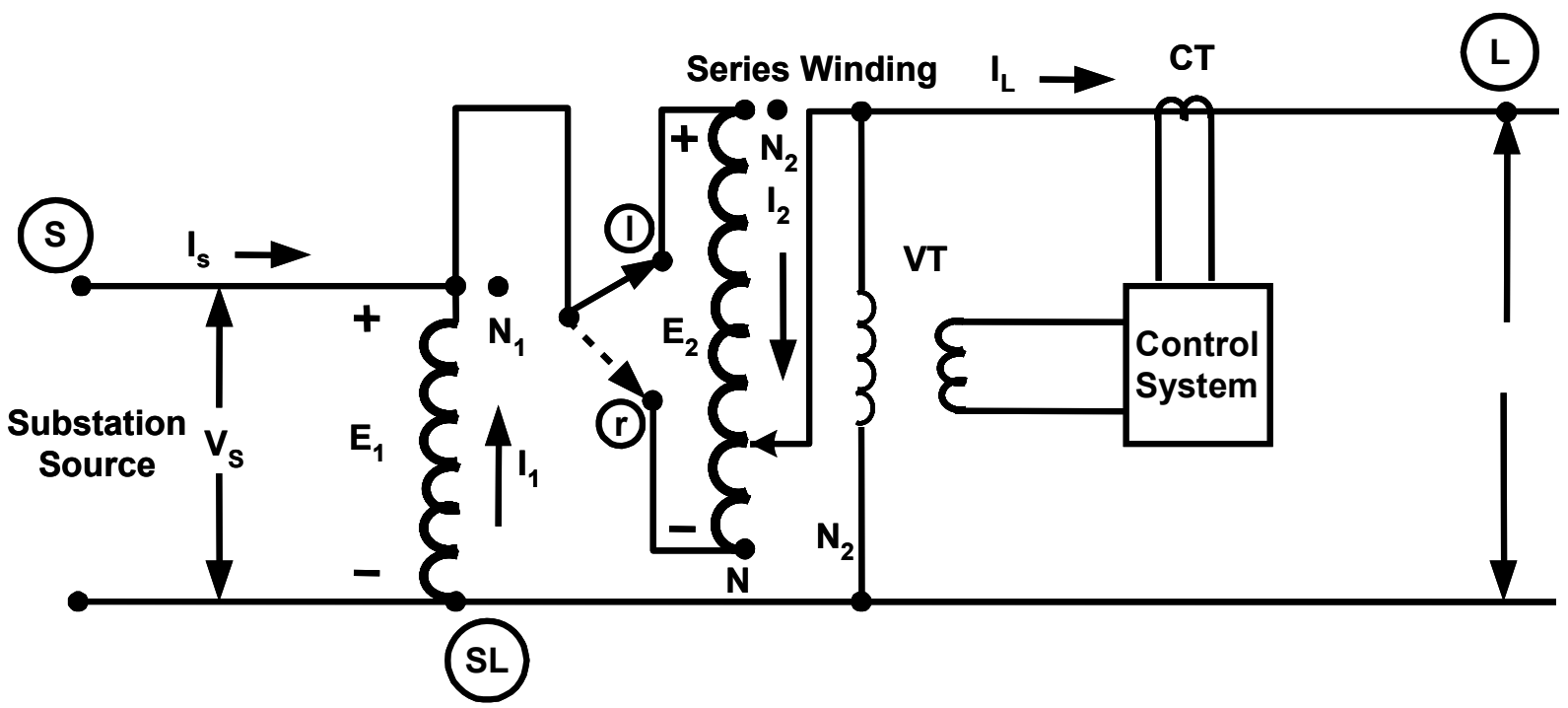

Figure 29. Type A step regulator - lower position

For the Type B regulator, the preventive auto is connected to the source terminal (S). Figure 30 shows the Type $B$ regulator in the raise position (r). 


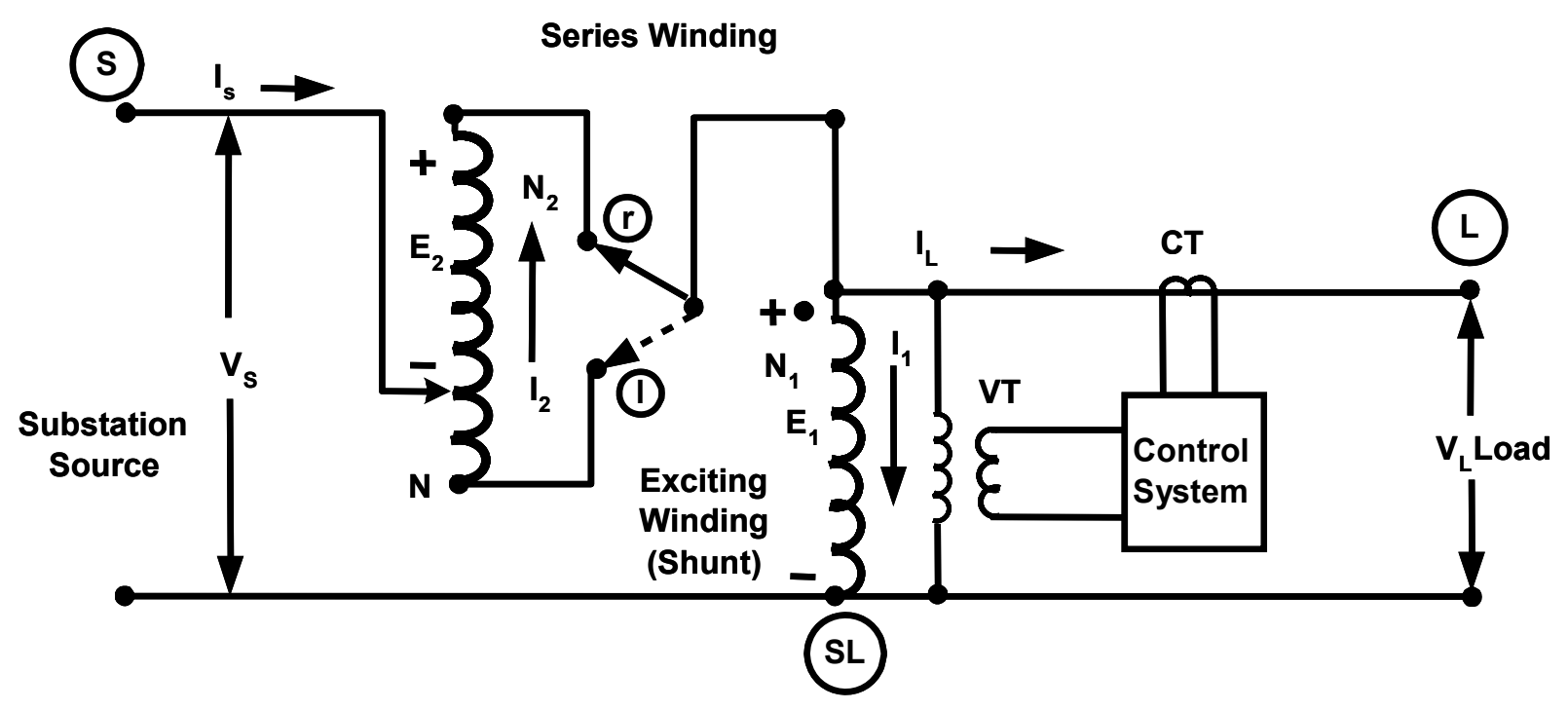

Figure 30. Type $B$ regulator - raise position

Here, the excitation current is constant because the shunt winding is connected to the load side $(\mathrm{L})$ or the regulated circuit side.

For the Type B regulator in the raise position of Figure 30, the voltage and current equations may be written as:

\section{Raise Position Equations}

$$
\begin{aligned}
& \mathrm{N}_{1} \mathrm{I}_{1}=\mathrm{N}_{2} \mathrm{I}_{2} \\
& \frac{\mathrm{E}_{1}}{\mathrm{~N}_{1}}=\frac{\mathrm{E}_{2}}{\mathrm{~N}_{2}} \\
& \mathrm{I}_{\mathrm{L}}=\mathrm{I}_{\mathrm{S}}-\mathrm{I}_{1} \\
& \mathrm{~V}_{\mathrm{S}}=\mathrm{E}_{1}-\mathrm{E}_{2} \\
& \mathrm{I}_{\mathrm{L}}=\mathrm{I}_{\mathrm{S}}-\mathrm{I}_{1} \\
& \mathrm{E}_{1}=\mathrm{V}_{\mathrm{L}} \\
& \mathrm{I}_{\mathrm{S}}=\mathrm{I}_{2}
\end{aligned}
$$

Equation 3.12

Equation 3.13

Equation 3.14

Equation 3.15

Equation 3.16

Equation 3.17

Equation 3.18

From equations 3.13 and 3.17 ,

$$
\mathrm{E}_{2}=\left(\mathrm{N}_{2} / \mathrm{N}_{1}\right) \mathrm{E}_{1}=\left(\mathrm{N}_{2} / \mathrm{N}_{1}\right) \mathrm{V}_{\mathrm{L}} .
$$


From Equations 3.12 and 3.18,

$$
I_{1}=\left(N_{2} / N_{1}\right) I_{2}=\left(N_{2} / N_{1}\right) I_{S} \text {. }
$$

Equation 3.20

From Equations 3.15, 3.17, and 3.19,

$$
\begin{aligned}
& \mathrm{V}_{\mathrm{S}}=\mathrm{E}_{1}-\mathrm{E}_{2}=\mathrm{E}_{1}-\left(\mathrm{N}_{2} / \mathrm{N}_{1}\right) \mathrm{V}_{\mathrm{L}}=\mathrm{V}_{\mathrm{L}}-\left(\mathrm{N}_{2} / \mathrm{N}_{1}\right) \mathrm{V}_{\mathrm{L}} \\
& \mathrm{V}_{\mathrm{S}}=\left(1-\left(\mathrm{N}_{2} / \mathrm{N}_{1}\right) \mathrm{V}_{\mathrm{L}} .\right.
\end{aligned}
$$

From Equations 3.16 and 3.20,

$$
\begin{aligned}
& \mathrm{I}_{\mathrm{L}}=\mathrm{I}_{\mathrm{S}}-\mathrm{I}_{1}=\mathrm{I}_{\mathrm{S}}-\left(\mathrm{N}_{2} / \mathrm{N}_{1}\right) \mathrm{I}_{\mathrm{S}} \\
& \mathrm{I}_{\mathrm{L}}=\left(1-\mathrm{N}_{2} / \mathrm{N}_{1}\right) \mathrm{I}_{\mathrm{S}} .
\end{aligned}
$$

Defining the raise turns ratio term as

$$
a_{r}=\left(1-N_{2} / N_{1}\right)
$$

and substituting into equations 3.21 and 3.22,

$$
\begin{aligned}
& \mathrm{V}_{\mathrm{S}}=\mathrm{a}_{\mathrm{r}} \mathrm{V} \\
& \mathrm{I}_{\mathrm{L}}=\mathrm{a}_{\mathrm{r}} \mathrm{I}_{\mathrm{S}} .
\end{aligned}
$$

Equation 3.25

Using Figure 31, the voltage and current equations for the lower regulator position may be defined as:

\section{Lower Position Equations}

$$
\begin{array}{lc}
\mathrm{N}_{1} \mathrm{I}_{1}=\mathrm{N}_{2} \mathrm{I}_{2} & \text { Equation } 3.26 \\
\frac{\mathrm{E}_{1}}{\mathrm{~N}_{1}}=\frac{\mathrm{E}_{2}}{\mathrm{~N}_{2}} & \text { Equation } 3.27 \\
\mathrm{I}_{\mathrm{L}}=\mathrm{I}_{\mathrm{S}}+\mathrm{I}_{1} & \\
\mathrm{~V}_{\mathrm{S}}=\mathrm{E}_{1}+\mathrm{E}_{2} & \text { Equation } 3.28 \\
\mathrm{E}_{1}=\mathrm{V}_{\mathrm{L}} & \text { Equation } 3.29 \\
\mathrm{I}_{\mathrm{S}}=\mathrm{I}_{2} . & \text { Equation } 3.30 \\
&
\end{array}
$$




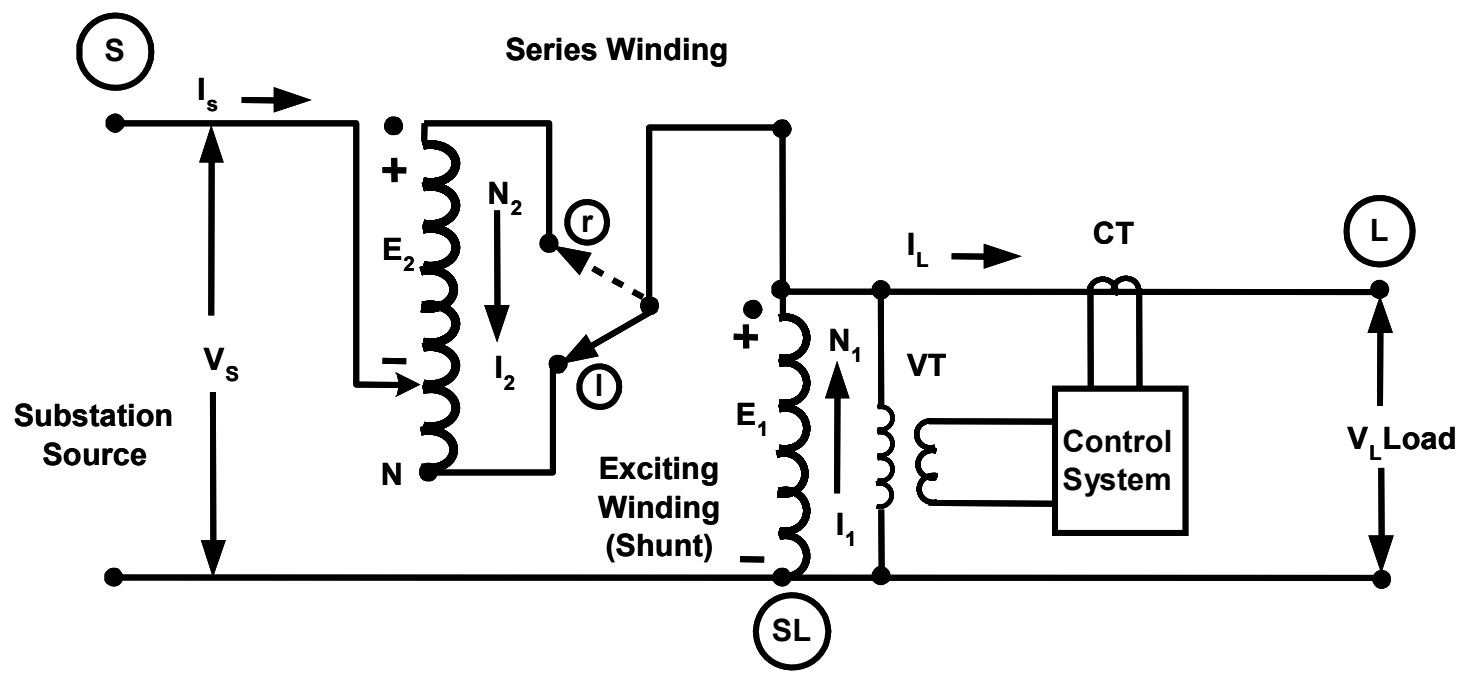

Figure 31. Type B regulator - lower position

From equations 3.27 and 3.30,

$$
\mathrm{E}_{2}=\left(\mathrm{N}_{2} / \mathrm{N}_{1}\right) \mathrm{E}_{1}=\left(\mathrm{N}_{2} / \mathrm{N}_{1}\right) \mathrm{V}_{\mathrm{L}}
$$

Equation 3.32

From equations 3.26 and 3.31 ,

$$
I_{1}=\left(N_{2} / N_{1}\right) I_{2}=\left(N_{2} / N_{1}\right) I_{S} .
$$

From equations 3.29, 3.30, and 3.32,

$$
\begin{aligned}
& \mathrm{V}_{\mathrm{S}}=\mathrm{E}_{1}+\left(\mathrm{N}_{2} / \mathrm{N}_{1}\right) \mathrm{E}_{1}=\mathrm{V}_{\mathrm{L}}+\left(\mathrm{N}_{2} / \mathrm{N}_{1}\right) \mathrm{V}_{\mathrm{L}} \\
& \mathrm{V}_{\mathrm{S}}=\left(1+\mathrm{N}_{2} / \mathrm{N}_{1}\right) \mathrm{V}_{\mathrm{L}} .
\end{aligned}
$$

From equations 3.28 and 3.33 ,

$$
\begin{aligned}
& \mathrm{I}_{\mathrm{L}}=\mathrm{I}_{\mathrm{S}}+\mathrm{I}_{1}=\mathrm{I}_{\mathrm{S}}+\left(\mathrm{N}_{2} / \mathrm{N}_{1}\right) \mathrm{I}_{2} \\
& \mathrm{I}_{\mathrm{L}}=\mathrm{I}_{\mathrm{S}}\left(1+\mathrm{N}_{2} / \mathrm{N}_{1}\right) .
\end{aligned}
$$

Defining the lower position turns ratio term as

$$
\begin{aligned}
& \mathrm{a}_{1}=\left(1+\mathrm{N}_{2} / \mathrm{N}_{1}\right) \text {, then } \\
& \mathrm{V}_{\mathrm{S}}=\mathrm{a}_{1} \mathrm{~V}_{\mathrm{L}} \\
& \mathrm{I}_{\mathrm{L}}=\mathrm{a}_{1} \mathrm{I}_{\mathrm{S}} .
\end{aligned}
$$

Equation 3.37

Equation 3.38 
Notice that the only difference between voltage and current raise equations 3.21 and 3.22 and voltage and current lower equations 3.34 and 3.35 is the sign of the turns ratio $\mathrm{N}_{2} / \mathrm{N}_{1}$. The sign of the raise position is negative, and the sign of lower position is positive. It is not necessary to know the turns ratio of equations $3.21,3.22,3.34$, and 3.35 because each tap represents a voltage change of $5 / 8 \%$ or 0.00625 p.u. Therefore, $a_{\mathrm{r}}$ and $\mathrm{a}_{1}$ can be described as

$$
\mathrm{a}_{\mathrm{r}}, \mathrm{a}_{1}=1-,+(0.00625) \text { tap position for a Type B regulator. }
$$

Equation 3.39

For the Type A regulator, the $\mathrm{a}_{\mathrm{r}}$ and $\mathrm{a}_{\mathrm{l}}$ are

$$
\mathrm{a}_{\mathrm{r}}, \mathrm{a}_{1}=1+,-(0.00625) \text { tap position. }
$$

Equation 3.40

\subsection{Equivalent Circuits}

Because the series impedance and shunt admittance of a step VR are negligible, their effects can be ignored. But if desired, this effect can be included in the same manner as the equivalent circuit for an autotransformer.

\subsection{Line Drop Compensator}

For VRs to operate properly, when applied at a substation or on a circuit, and maintain voltage at the regulation point, line drop compensators are required. The voltage at the regulation point is to be constant even through the load power factor and the load change. This is achieved by setting the resistance and reactance controls on the control panel of the regulator.

The voltage-regulating relay of Figure 32 causes the regulator to return to the preset voltage, VR, when a change in voltage occurs. For the VR to compensate for the voltage drop in the circuit to the regulation point, an additional voltage must be added between the VT output and the voltage-regulating relay (VRR) so that the VRR sees a reduced voltage proportional to the load current and load power factor. The current from the VT is almost in phase with the voltage because the resistance of its secondary circuit is high compared with the reactance of this circuit. The current from the CT adds current through the $\mathrm{R}_{\mathrm{L}}$ and $\mathrm{X}_{\mathrm{L}}$ that has the same phase angle as the load current and is proportional to the load current. The VRR is adjusted so that, with no load current, the regulator output is equal to the set point voltage at the regulation point. The compensator $\mathrm{R}_{\mathrm{L}}$ and $\mathrm{X}_{\mathrm{L}}$ elements are adjusted so they are proportional to the $R_{L}$ and $X_{L}$ of the circuit between the regulator and regulation point. The phasor diagram of Figure 33 shows how the regulation point voltage, $V_{R}$, or the voltage across the VRR is determined from the output voltage of the regulator, $\mathrm{V}_{\mathrm{O}}$ (from the VT), and the $\mathrm{R}_{\mathrm{L}}$ and $\mathrm{X}_{\mathrm{L}}$ values of the circuit. $\mathrm{I}_{\mathrm{L}}$ is the load current, and $\theta$ is the power factor angle of the load current from the CT. The $I_{L} R_{L}$ voltage drop is the voltage drop across the $R_{L}$ of the compensator, and $\mathrm{I}_{\mathrm{L}} \mathrm{X}_{\mathrm{L}}$ is the voltage drop across the $\mathrm{X}_{\mathrm{L}}$ of the compensator. 


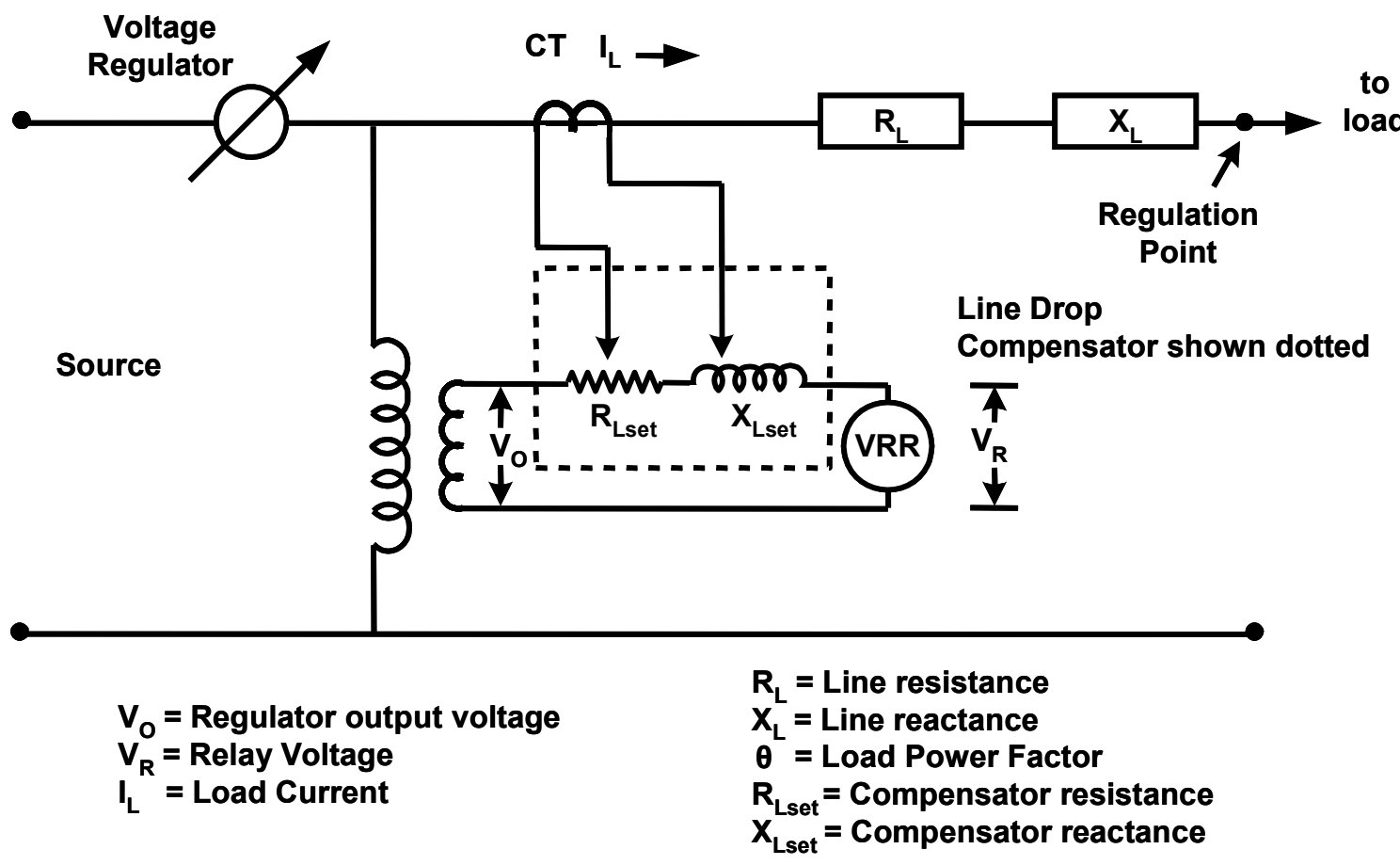

Figure 32. Control system and line drop compensator

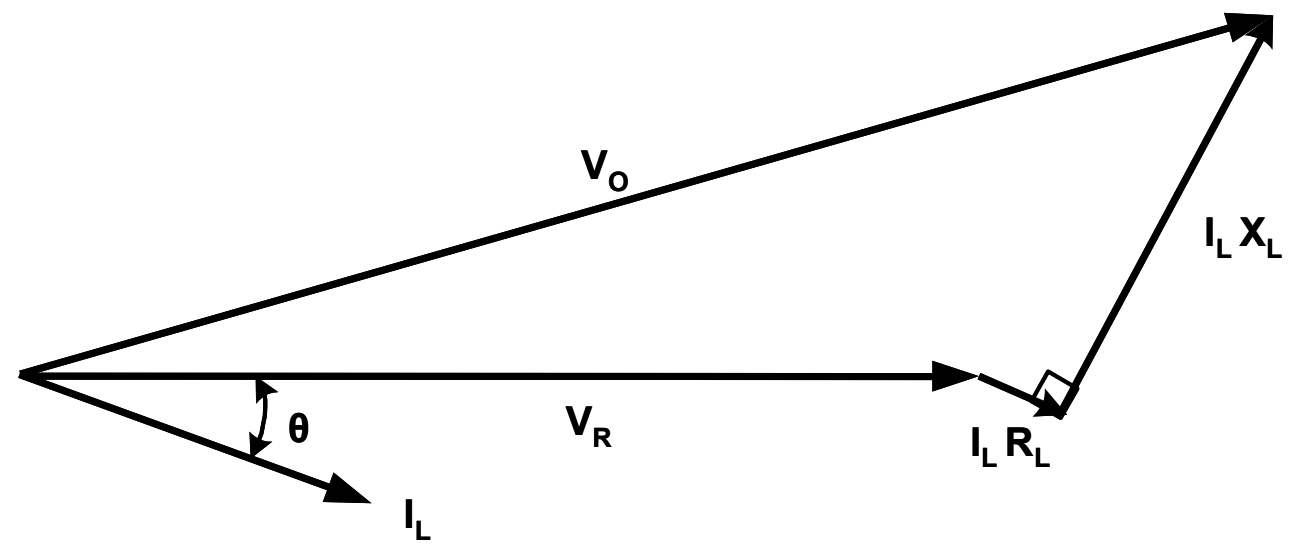

Figure 33. Phasor diagram for line drop compensator

The values $\mathrm{R}_{\mathrm{L}}$ and $\mathrm{X}_{\mathrm{L}}$ are calibrated in volts on the dials of the compensator panel. When the dial is set at a voltage value, this is the voltage compensation at rated current in the secondary of the $\mathrm{CT}$. The regulator rating must be determined before the $\mathrm{R}_{\mathrm{L}}$ and $\mathrm{X}_{\mathrm{L}}$ settings can be defined. 


\subsection{Regulator Ratings}

If $\mathrm{V}_{\mathrm{O}}$ is the output voltage and $\mathrm{V}_{\mathrm{i}}$ is the input voltage for a single-phase regulator, then the percent regulation range is

$$
\% \mathrm{R}=\left[\left(\left|\mathrm{V}_{\mathrm{o}}\right|-\left|\mathrm{V}_{\mathrm{i}}\right|\right) / \mathrm{V}_{\mathrm{i}} \mid\right] \times 100
$$

in either the buck or boost direction. The regulation kilovolt-amperes is defined as

$$
\mathrm{kVA}_{\text {Regulation }}=\left[\left(\left|\mathrm{V}_{\mathrm{o}}\right|-\left|\mathrm{V}_{\mathrm{i}}\right|\right) / \mathrm{V}_{\mathrm{o}} \mid\right] \mathrm{kVA}_{\mathrm{S} \Phi} \text { circuit, }
$$

and the regulator kilovolt-amperes rating is

$$
\begin{gathered}
\left\{\left[\left(\left|\mathrm{V}_{\mathrm{o}}\right|-\left|\mathrm{V}_{\mathrm{i}}\right|\right) /\left|\mathrm{V}_{\mathrm{i}}\right|\right][100 \times \mathrm{kVA} \mathrm{S} \text { circuit }]\right\} / 100 \\
\left.\mathrm{kVA}_{\mathrm{S} \Phi} \text { Rating }=\left[\% \mathrm{R}_{(\mathrm{kVA}} \mathrm{S} \text { circuit }\right)\right] / 100=\left(\% \mathrm{R}\left|\mathrm{kV}_{\mathrm{LL}}\right|\left|\mathrm{I}_{\mathrm{L}}\right|\right) / 100,
\end{gathered}
$$

Equation 3.43

Equation 3.44

where $\mathrm{kV}_{\mathrm{LL}}$ is line-to-line voltage, and $\mathrm{I}_{\mathrm{L}}$ is the load current. For the three-phase, four-wire wye system of Figure 34, Equation 3.44 is used, but the kilovolt-amperes of the circuit are now three-phase.

$$
\begin{aligned}
& \operatorname{kVA}_{3 \Phi \text { Rating }}=\left[\% \mathrm{R}\left(\mathrm{kVA}_{3 \Phi} \text { circuit }\right)\right] / 100 \text { or } \\
& \mathrm{kVA}_{3 \Phi \text { Rating }}=\left[\% \mathrm{R} \sqrt{3}\left|\mathrm{kV}_{\mathrm{LL}}\right|\left|\mathrm{I}_{\mathrm{L}}\right|\right] / 100 .
\end{aligned}
$$

Equation 3.45

Figure 35 shows the phasor diagram for the wye connection. Notice that the regulated voltages are in phase with the input voltages. For a $13.2-\mathrm{kV}$ three-phase, four-wire wye system and an $8,000-\mathrm{kVA}$ circuit load, $\mathrm{I}_{\mathrm{L}}=350 \mathrm{~A}$. With $\pm 10 \%$ regulation, the rating is

$$
\mathrm{kVA}_{3 \Phi \text { Rating }}=[(10)(\sqrt{ } 3)(13.2 \mathrm{kV})(350 \mathrm{amps})] / 100=800 \mathrm{kVA} .
$$

Equation 3.46 


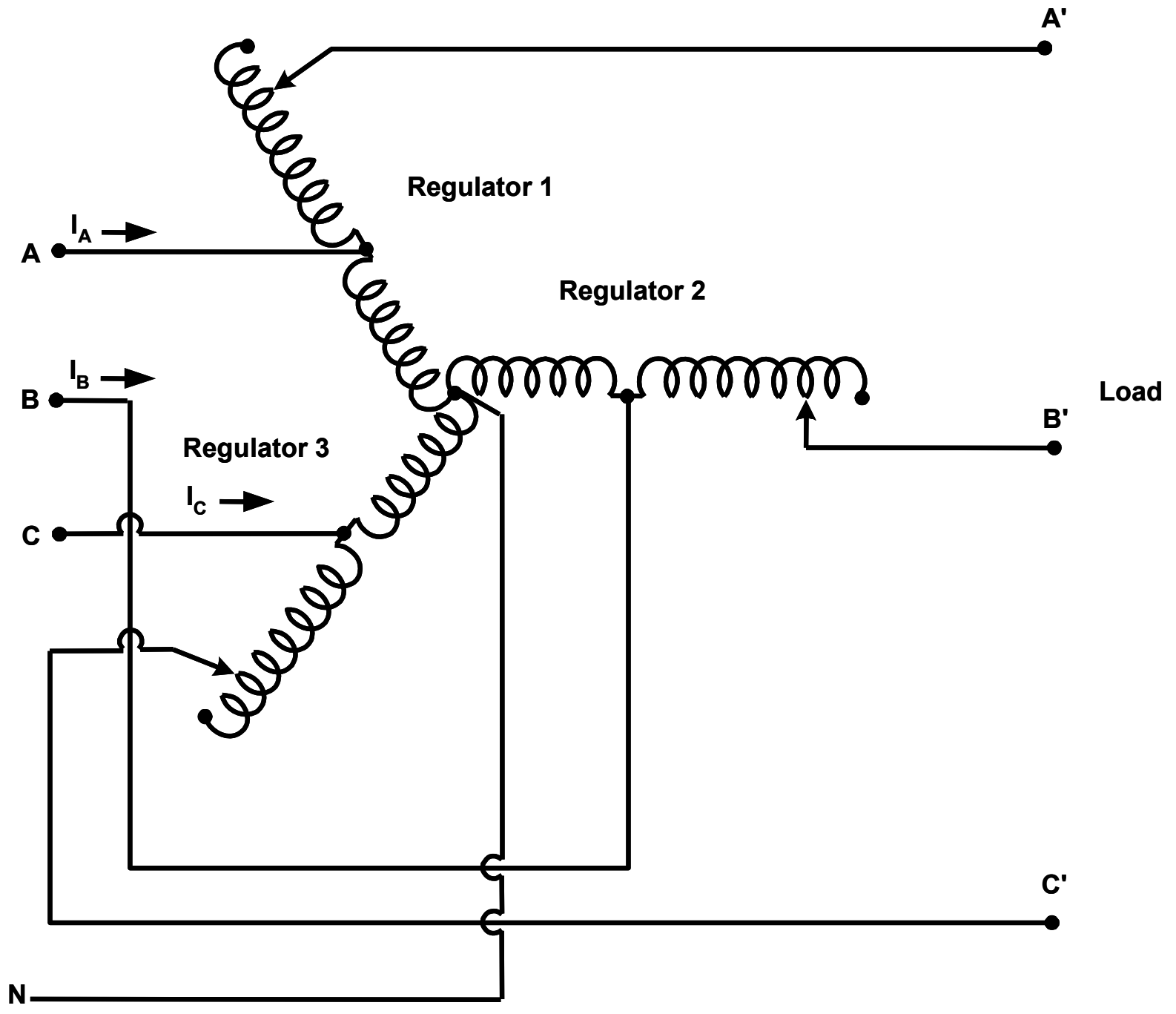

Figure 34. Three single-phase VRs connected wye on a four-wire, three-phase circuit 


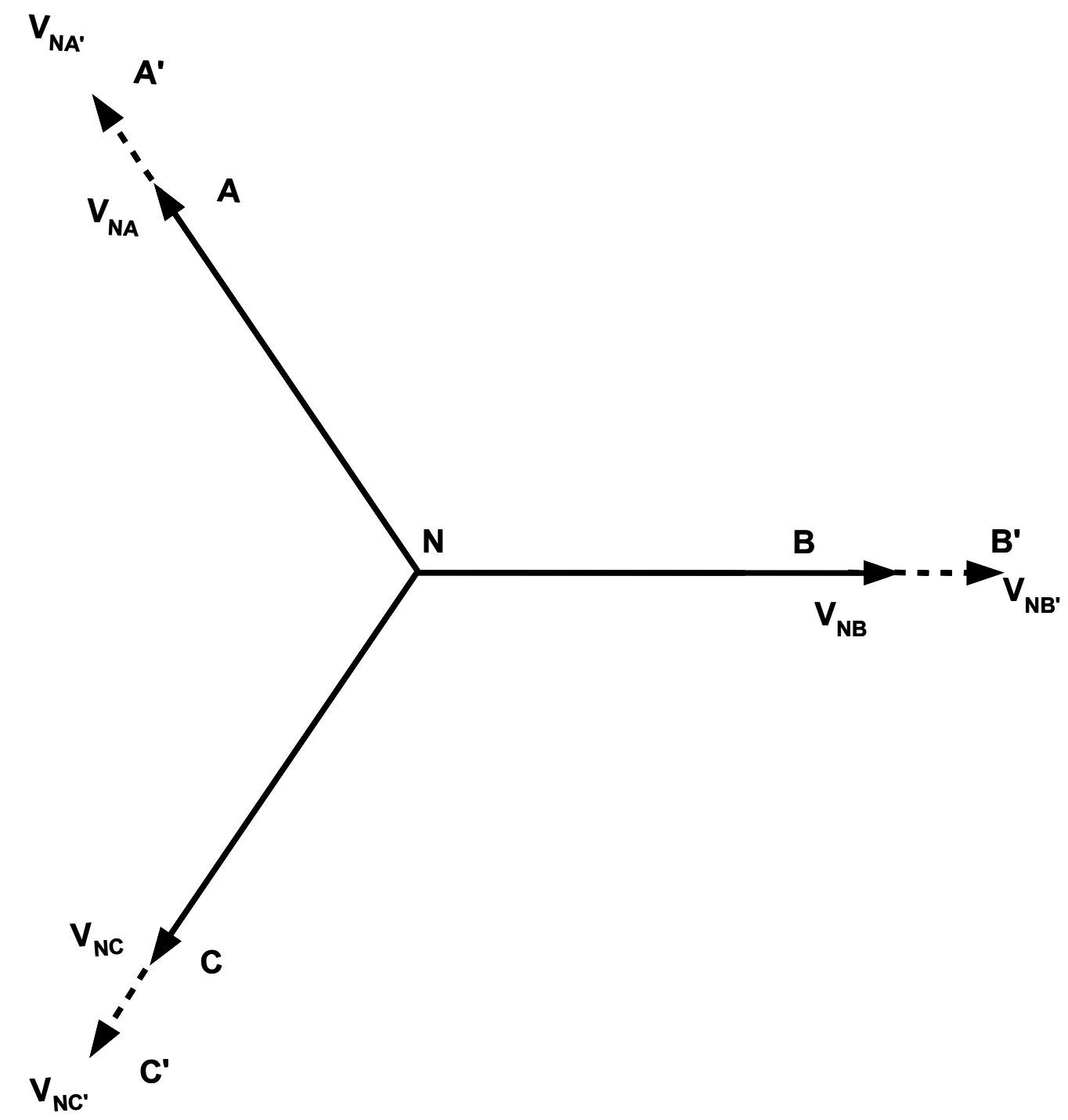

Figure 35. Voltage phasor diagram of wye-connected, three single-phase VRs

Notice, from Equation 3.46, that the kilovolt-ampere three-phase rating of the $\pm 10 \%$ regulator is just $10 \%$ times the three-phase circuit load. For a three-phase, three-wire circuit, Equation 3.46 applies because the regulator is connected in wye, but there is no connection to the neutral of the regulator.

Sometimes, two single-phase regulators are connected open delta or "vee" to obtain voltage regulation on a delta three-wire system. This connection is shown in Figure 36. For its phasor diagram, see Figure 37. The same percentage increase in voltage on $V_{C B}$ to $V_{C}{ }^{\prime}$ and $V_{B A}$ to

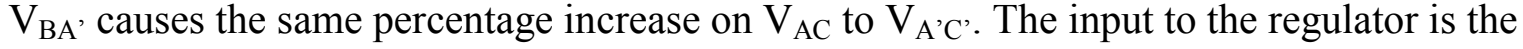
line voltage, not the line-to-neutral voltage, as in Figure 34. From Equation 3.44, the singlephase rating can be used because the voltage is line-to-line. The-three phase circuit $\mathrm{kVA}=\sqrt{ }$ $3 \mathrm{kV}_{\mathrm{LL}} \mathrm{I}_{\mathrm{L}}$, and each single-phase regulator kilovolt-ampere rating is

$\mathrm{kVA}_{\mathrm{S} \Phi \text { Rating }}=\left[\left(\% \mathrm{R} \sqrt{ } 3\left|\mathrm{kV}_{\mathrm{LL}}\right|\left|\mathrm{I}_{\mathrm{L}}\right|\right)\right] /[(100)(\sqrt{ } 3)] . \quad$ Equation 3.47 


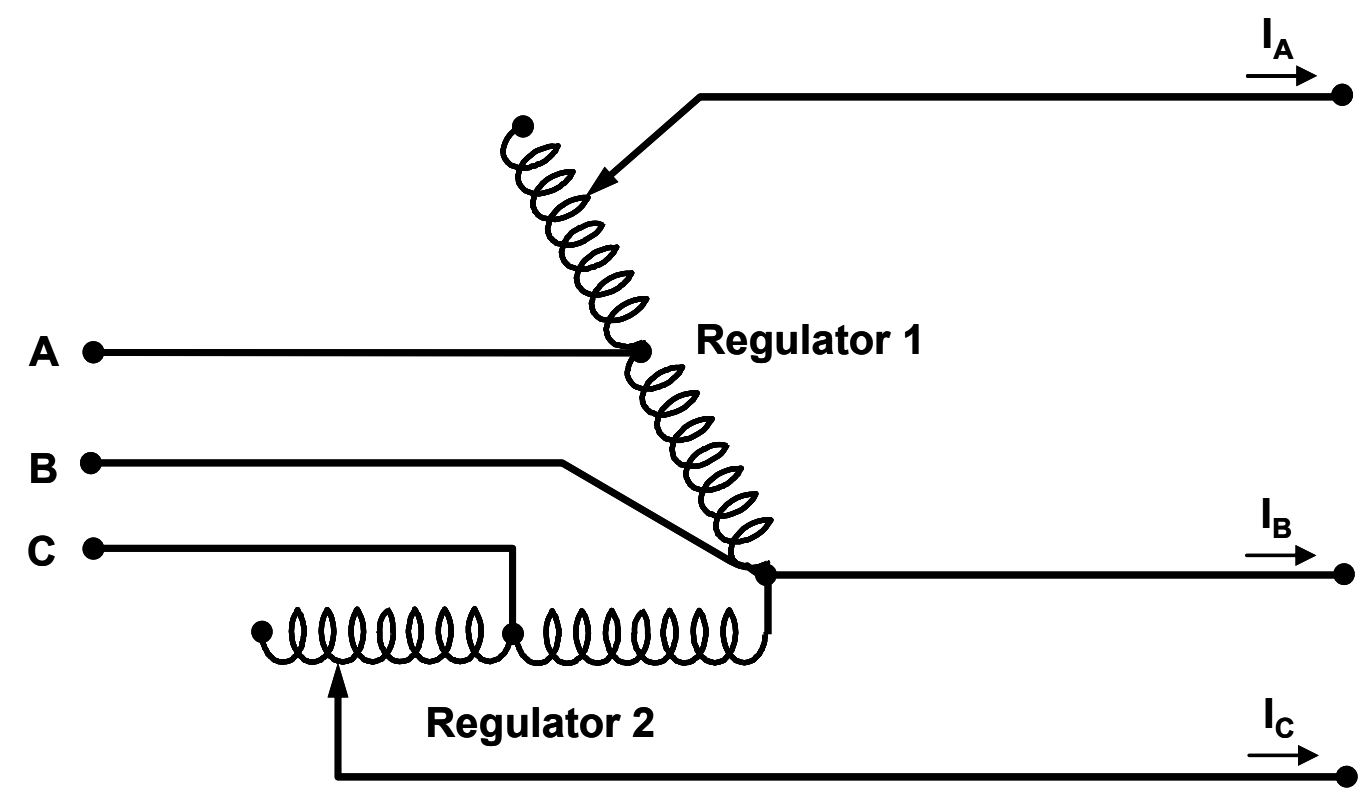

Figure 36. Two single-phase VRs connected open delta on a three-wire, three-phase circuit 


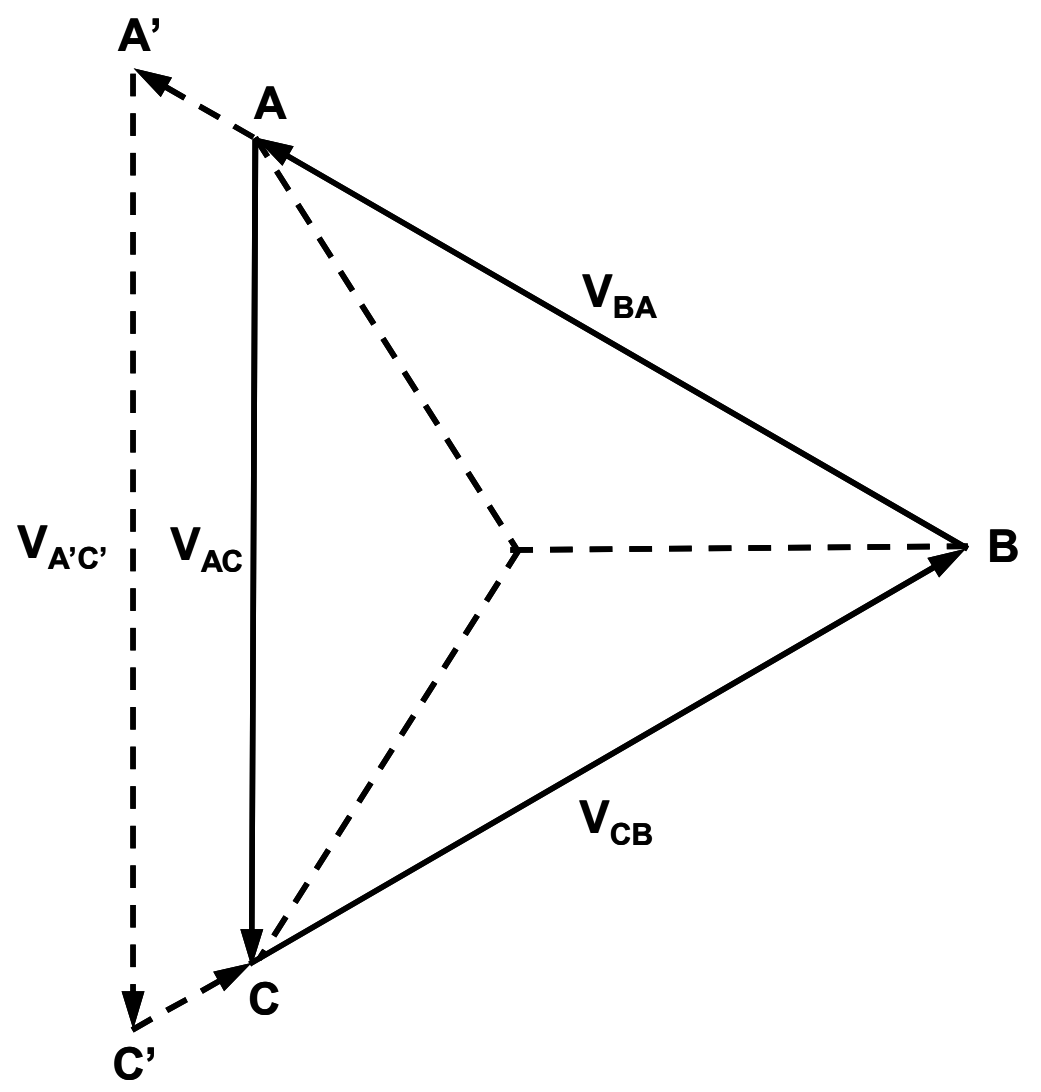

\section{Regulator Input Voltages $\mathrm{V}_{\mathrm{BA}} \mathrm{V}_{\mathrm{CB}} \mathrm{V}_{\mathrm{AC}}$ Regulator Output Voltages $V_{B^{\prime}}, V_{C^{\prime} B} V_{A^{\prime} C^{\prime}}$}

Figure 37. Voltage phasor diagram of open delta-connected two single-phase VRs

The $\sqrt{ } 3$ is needed in the denominator to obtain the phase current. When a $\pm 10 \%$ regulation is substituted for $\% \mathrm{R}$ in Equation 3.47., the kilovolt-ampere rating is

$$
\begin{aligned}
\mathrm{kVA}_{\mathrm{S} \Phi \text { Rating }} & =\left[(10)\left(\mathrm{kVA}_{3 \Phi} \text { circuit }\right)\right] /[(100 \sqrt{ } 3)] \\
& =\left(\mathrm{kVA}_{3 \Phi} \text { circuit }\right) /(17.32) .
\end{aligned}
$$

Equation 3.48

For a $4.8-\mathrm{kV}$, three-phase, three-wire delta system and a 2,000-kVA, three-phase circuit load, the load current is $241 \mathrm{~A}$. Applying Equation 3.48 and using $\pm 10 \%$ regulation, each singlephase unit connected in open delta is

$$
\mathrm{kVA}_{\mathrm{S} \Phi \text { Rating }}=[(10)(2,000)] /[(100)(17.32)]=115 \mathrm{kVA} . \quad \text { Equation } 3.49
$$

In this case, the rating is $6 \%$ of the three-phase circuit load, not the $10 \%$ calculated for the four-wire wye system above. 
Three-phase regulation can be achieved for a closed delta. Closing the delta with the third regulator does not allow more circuit load, but it does increase the percentage regulation from $\pm 10 \%$ to $\pm 15 \%$. This is shown in the phasor diagram of Figure 38. Each regulator of the closed delta carries the phase load current, as was the case for the open delta, and the regulator rating is determined from equations 3.47 and 3.48 , which is identical to the regulator rating obtained in the open delta configuration. There are two possible connections for the closed delta: the load current leading the line-to-line voltage across the shunt winding by $30^{\circ}$ and current lagging the line-to-line voltage by $30^{\circ}$. Each of these will be explained.

\subsection{Closed Delta - Leading Current and Lagging Current Connections}

The leading current connection and its phasor diagram are shown in Figure 39, and the lagging current connection and its phasor diagram are shown in Figure 40. A phase shift results between the input and output voltages, as shown in Figure 38, and different phase shifts occur depending on the tap and boost or buck positions. The smaller phase shifts occur for the lower tap positions. Although there are no advantages to either $30^{\circ}$ leading or lagging connections, it may be necessary to go beyond the range of the compensator settings for one versus the other connection. Therefore, one of these connections may be chosen to solve this problem. It is generally not recommended to use wye-connected, single-phase regulators for the delta system because the neutral will shift for unbalanced loads. Because there are separate controls for each regulator, this may cause a different response and result in a neutral shift. 


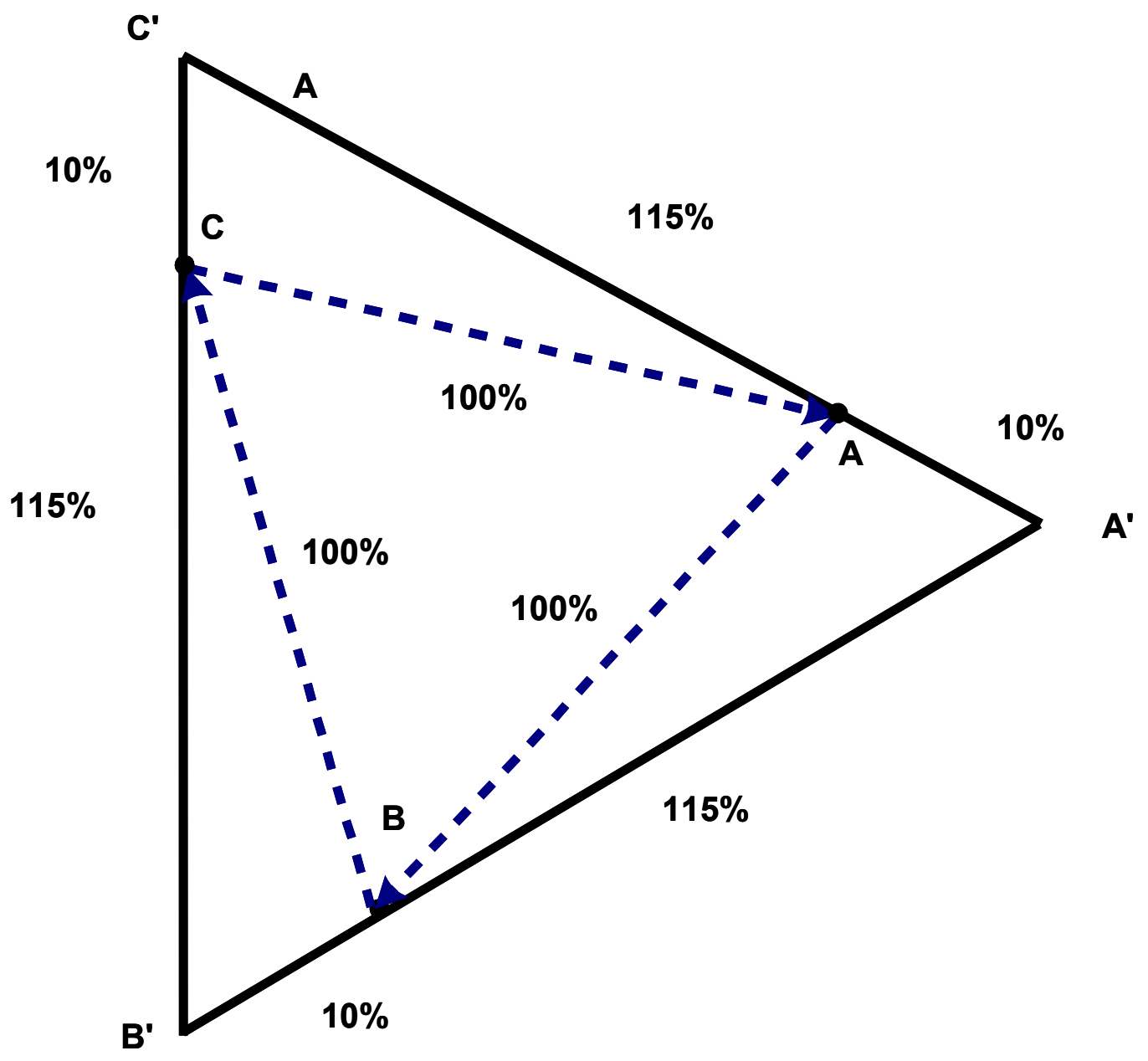

Figure 38. Voltage diagram of closed delta-connected three single-phase VRs 


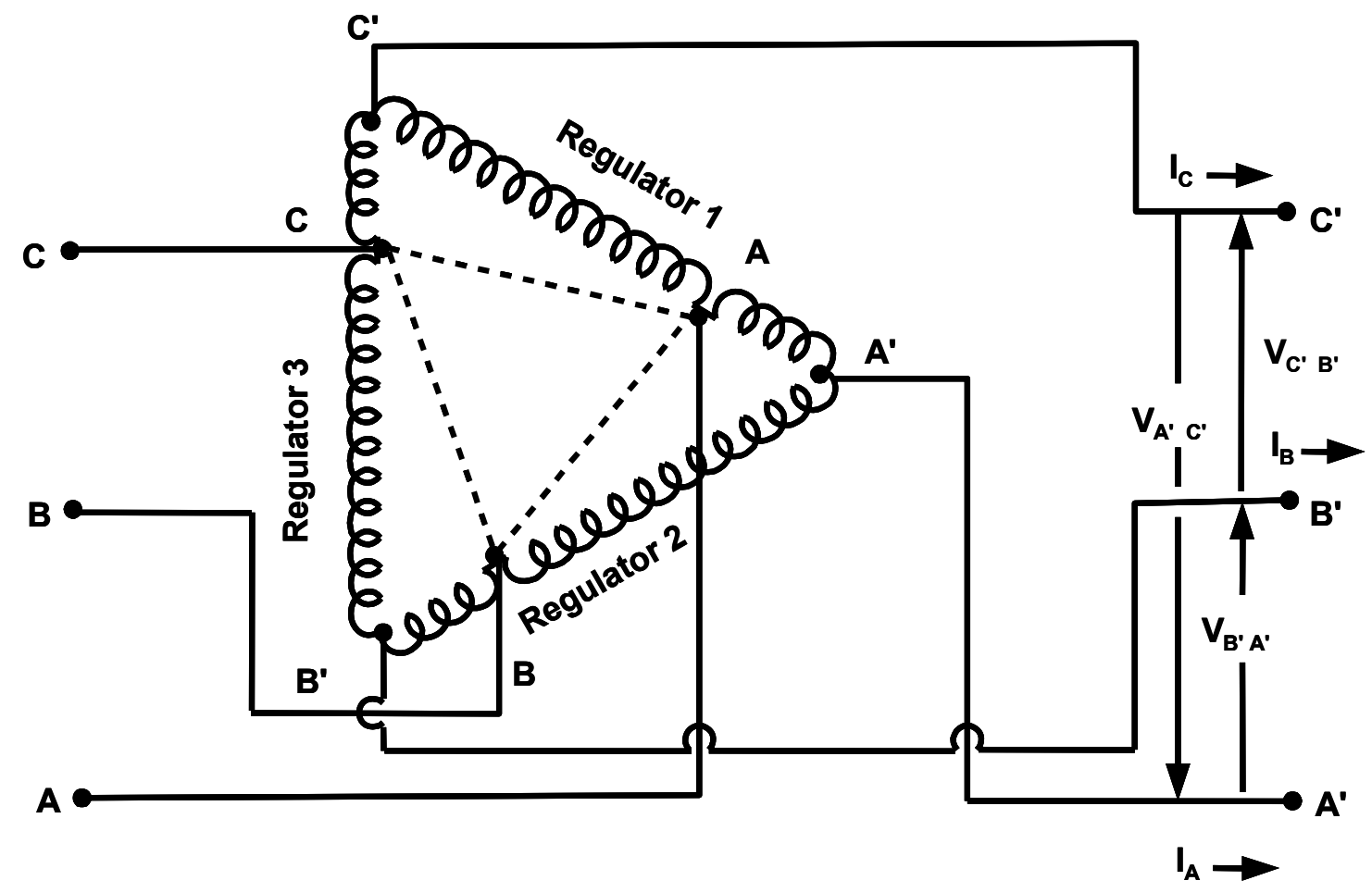

Regulator 3

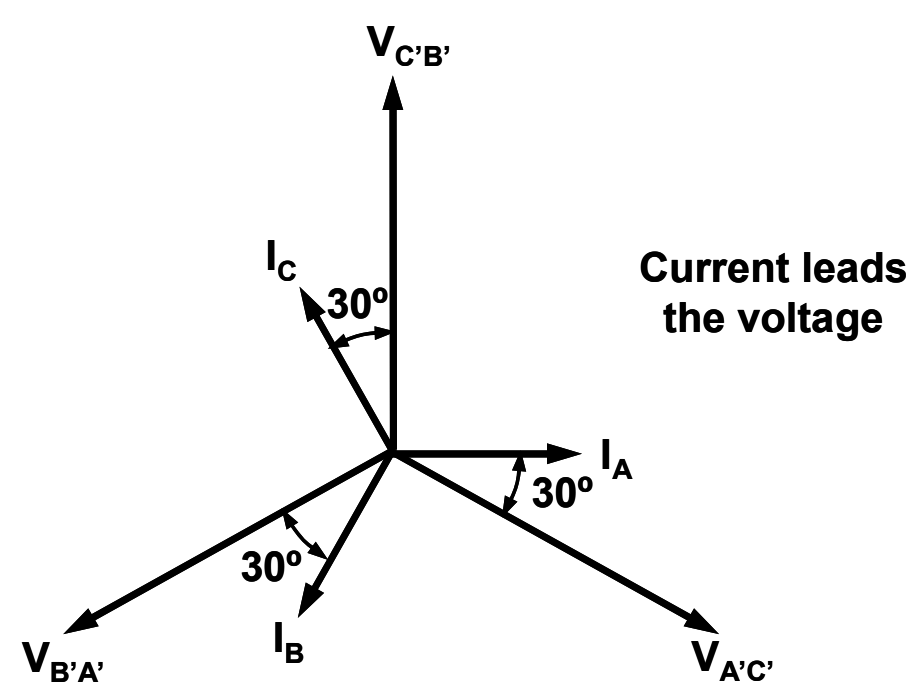

Regulator 2

Regulator 1

Figure 39. Closed delta-connected three single-phase VRs - leading connection 

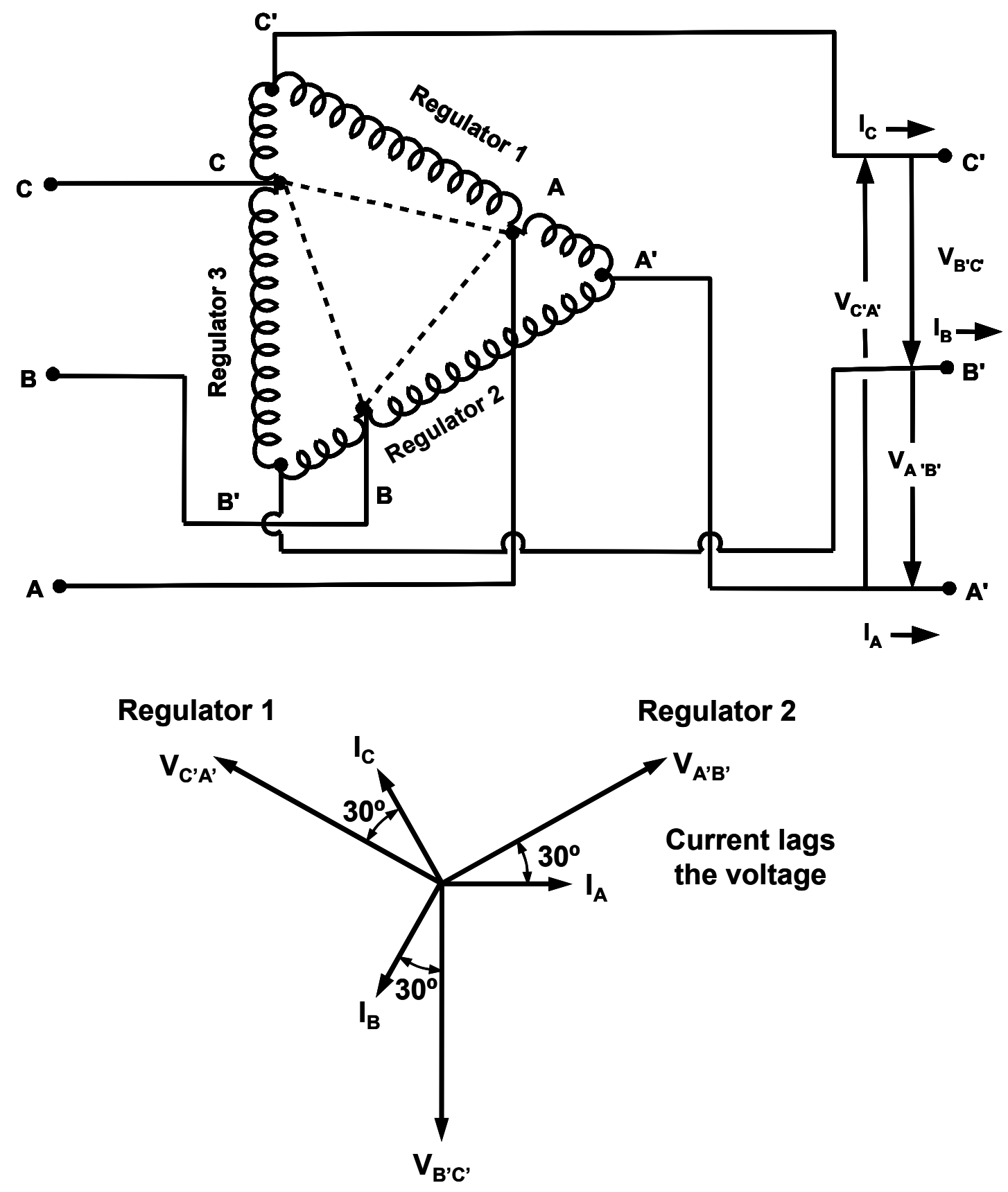

Regulator 3

Figure 40. Closed delta-connected three single-phase VRs - lagging connection 


\subsection{Compensator Settings}

If no load is connected between the regulator and the regulation point, it is easy to determine the settings of $R_{L}$ and $X_{L}$. This is the case for a typical substation step regulator, as in Figure 32 and Figure 33. The $\mathrm{R}_{\mathrm{L}}$ setting and $\mathrm{X}_{\mathrm{L}}$ settings are determined from equations 3.50 and 3.51 as

$$
\begin{aligned}
& \mathrm{R}_{\mathrm{Lset}}=\left[\left(\mathrm{CT}_{\mathrm{P}} / \mathrm{N}_{\mathrm{VT}}\right)\right]\left(\mathrm{R}_{\mathrm{L}}\right)(1) \\
& \mathrm{X}_{\mathrm{Lset}}=\left[\left(\mathrm{CT}_{\mathrm{P}} / \mathrm{N}_{\mathrm{VT}}\right)\right]\left(\mathrm{X}_{\mathrm{L}}\right)(1)
\end{aligned}
$$

Equation 3.51

where

$\mathrm{R}_{\mathrm{Lset}}=$ Dial setting for the resistance in the feeder portion of the circuit given in volts

$\mathrm{X}_{\mathrm{Lset}}=$ Dial setting for the reactance in the feeder portion of the circuit given in volts

$\mathrm{N}_{\mathrm{VT}}=\mathrm{VT}$ ratio of primary voltage/secondary voltage

$\mathrm{CT}_{\mathrm{P}}=$ Primary rating of the $\mathrm{CT}$ given in amperes

$\mathrm{R}_{\mathrm{L}}=$ Resistance per conductor in unit length from the LTC to regulation point, normally given in ohms per mile

$\mathrm{X}_{\mathrm{L}}=$ Reactance per conductor in unit length from the LTC to regulation point, normally given in ohms per mile

$1=$ Unit length in miles to the regulation point.

The settings for $\mathrm{R}_{\mathrm{Lset}}$ and $\mathrm{X}_{\mathrm{Lset}}$ can now be determined. But first, the regulator rating must be calculated.

From Equation 3.46, the rated amperes for three single-phase regulators connected wye and rated $13.8 \mathrm{kV}, 1,000 \mathrm{kVA}$, and \pm 10 regulation is $418 \mathrm{~A}$. The $\mathrm{CT}$ ratio is $500: 5$, and the VT ratio is $13.8 \mathrm{kV} / \sqrt{ } 3=7,960: 120$. The regulator is located 3 miles from the regulation point, and the conductor size is $636 \mathrm{kcmil}$ all aluminum. From the resistance and reactance values of

$\mathrm{X}=0.536 \Omega /$ mile/conductor (see Equation 3.9)

$\mathrm{R}=0.164 \Omega /$ mile/conductor,

and using equations 3.50 and 3.51 ,

$$
\begin{array}{ll}
\mathrm{X}_{\mathrm{Lset}}=[(500) /(7960 / 120)][(0.536)(3)]=12.12 \mathrm{~V} & \text { Equation 3.50 } \\
\mathrm{R}_{\mathrm{Lset}}=[(500) /(66.3)][(0.164)(3)]=3.71 \mathrm{~V} . & \text { Equation } 3.51
\end{array}
$$


The control panel dial settings are based on the rated current of 500 A for the CT primary, not the load current or the rated current for the regulator. For step regulators installed on the circuit, the primary rating of the $\mathrm{CT}$ is the same as the regulator current rating. Hence, the regulator current rating can be substituted for the $\mathrm{CT}$ primary current rating in equations 3.50 and 3.51. Load may be connected between the regulator and the voltage regulation point; therefore, the magnitude of the current flowing through the $\mathrm{CT}$ of the regulator does not equal the magnitude of the current flowing through the balance of the $\mathrm{R}_{\mathrm{L}}$ and $\mathrm{X}_{\mathrm{L}}$ of the circuit to the regulation point. This is especially true for regulators installed beyond the feed point voltage $V_{R}$. Equations 3.50 and 3.51 can be corrected with the aid of Figure 41.

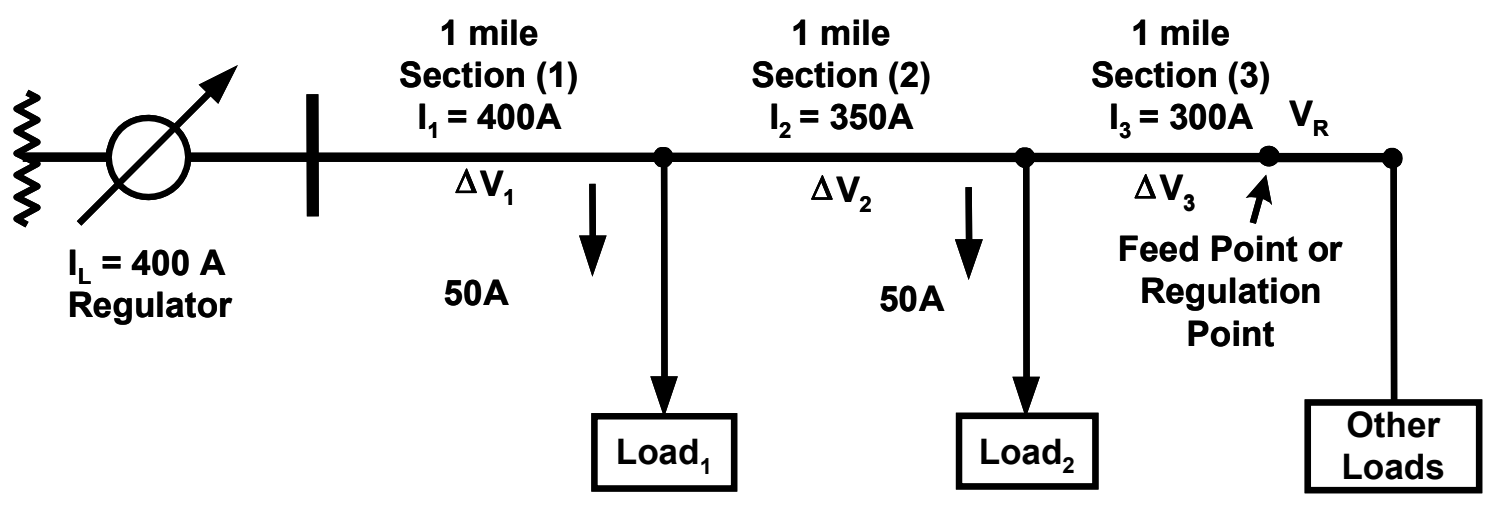

Figure 41. Current at the regulator is not equal to current in the last section before the regulation point

The $R_{L}$ set and $X_{L}$ set can be changed to consider the effect of load being served before the regulation point.

$$
\mathrm{R}_{\mathrm{Lset}}=\left(\mathrm{C} \mathrm{T}_{\mathrm{P}} / \mathrm{N}_{\mathrm{VT}}\right) /\left(\mathrm{R}_{\mathrm{LE}}\right) \text {, }
$$

Equation 3.52

where the effective resistance $\mathrm{R}_{\mathrm{LE}}$ when multiplied by $\mathrm{I}_{\mathrm{L}}$ gives the same voltage drop as the sum of the drops through the various sections of line (e.g., Section 1, Section 2, and Section 3 in Figure 41). Thus, $\mathrm{R}_{\mathrm{LE}}$ can be written as

$$
\begin{aligned}
& \mathrm{n} \\
& \mathrm{R}_{\mathrm{LE}}=\left(\sum_{\mathrm{i}=1}\left|\Delta \mathrm{V}_{\mathrm{iR}}\right|\right) / \mathrm{I}_{\mathrm{L}} \\
& \mathrm{n} \\
& \sum\left|\Delta V_{\mathrm{iR}}\right|=\left|\mathrm{I}_{1}\right| \mathrm{R}_{\mathrm{L} 1} 1_{1}+\left|\mathrm{I}_{2}\right| \mathrm{R}_{\mathrm{L} 2} \mathrm{l}_{2}+\left|\mathrm{I}_{3}\right| \mathrm{R}_{\mathrm{L} 3} \mathrm{l}_{3} \ldots\left|\mathrm{I}_{\mathrm{n}}\right| \mathrm{R}_{\mathrm{Ln}} 1_{\mathrm{n}} . \\
& \mathrm{i}=1 \\
& \text { Equation } 3.53 \\
& \text { Equation } 3.54 \\
& \mathrm{n} \\
& \sum\left|\Delta \mathrm{V}_{\mathrm{iR}}\right|=\text { The total voltage drop from regulator to the regulation point. } \\
& \mathrm{i}=1
\end{aligned}
$$


$\mathrm{R}_{\mathrm{LE}}=$ Effective resistance

$\left|\mathrm{I}_{\mathrm{L}}\right|=$ Load current flowing through the regulator

$\left|I_{1}\right|,\left|I_{2}\right|,\left|I_{3}\right|, \ldots\left|I_{n}\right|=$ The load current in each line section to the regulation point

$\mathrm{R}_{\mathrm{L} 1}, \mathrm{R}_{\mathrm{L} 2}, \mathrm{R}_{\mathrm{L} 3} \ldots \mathrm{R}_{\mathrm{Ln}}=$ The resistance in ohms per mile of each section from regulator to the regulation point

$11,12,13, \ldots 1_{\mathrm{n}}=$ The length of conductor in miles for each section from regulator to the regulation point

$\mathrm{n}=$ number of line sections.

The

$$
\mathrm{X}_{\mathrm{Lset}}=\mathrm{CT}_{\mathrm{p}} / \mathrm{N}_{\mathrm{VT}}\left(\mathrm{X}_{\mathrm{LE}}\right) \text {, }
$$

Equation 3.55

where $\mathrm{X}_{\mathrm{LE}}$ takes on the same form as equations 3.53 and 3.54 for $\mathrm{R}_{\mathrm{LE}}$. Based on Figure 41, and Equation 3.54, the $\mathrm{R}_{\mathrm{LE}}$ is found as follows:

$$
\begin{aligned}
\sum_{i=1}^{3}\left|\Delta \mathrm{V}_{\mathrm{iR}}\right| & =(400)(0.164)(1)+(350)(0.164)(1)+(300)(0.164)(1) \\
& =65.6+57.4+49.2=172.2 \mathrm{~V} .
\end{aligned}
$$

Equation 3.56

From Equation 3.53,

$$
\begin{aligned}
& \mathrm{R}_{\mathrm{LE}}=172.2 / 400=0.4305 \Omega \\
& \mathrm{R}_{\mathrm{Lset}}=(500 / 66.3) 0.4305=3.25 \mathrm{~V} .
\end{aligned}
$$

In reference to the $\mathrm{R}_{\mathrm{Lset}}$ values of equations 3.53 and 3.58, the value of Equation 3.58 is lower because the load current has stepped down after having served loads 1 and 2 . Thus, the total voltage drop is less and the compensation is less.

Equation 3.54 has considered the power factor angle to be the same for each section. However, in fact, this angle may not be the same for each section. A more practical approach is to measure the load current at the regulator, the load power factor, and the voltages at the regulation point and the regulator output at the same time. From these data and the $\mathrm{X} / \mathrm{R}$ ratio out to the regulation point, and by applying the voltage drop equation, the effective values of $\mathrm{R}_{\mathrm{LE}}$ and $\mathrm{X}_{\mathrm{LE}}$ can now be calculated.

$$
\Delta \mathrm{V}=\mathrm{I}_{\mathrm{L}} \mathrm{R}_{\mathrm{LE}} \operatorname{Cos} \theta+\mathrm{I}_{\mathrm{L}} \mathrm{X}_{\mathrm{LE}} \operatorname{Sin} \theta
$$

Equation 3.59 


\subsection{Other Compensator Settings and Corrections}

Settings for three-phase regulators connected wye and three single-phase regulators connected wye are determined using the same approach as that outlined above. However, when single-phase regulators are connected in open delta, the control voltage and current in the compensator do have the same phase relationship as the wye-connected regulators. For example, the open delta connection described in Figure 36 and Figure 37 show the control voltage and current as $\mathrm{V}_{\mathrm{BA}}$ and $\mathrm{I}_{\mathrm{A}}$ for Regulator 1 and as $\mathrm{V}_{\mathrm{CB}}$ and $\mathrm{I}_{\mathrm{C}}$ for Regulator 2, which are $30^{\circ}$ out of phase with the wye connection shown dashed in Figure 37. The current of one regulator lags the voltage by $30^{\circ}$ plus the power factor angle, and the current of the other regulator leads the voltage by $30^{\circ}$ minus the power factor angle. Therefore, phase-shifting networks are used, or corrections are made to the settings. On the control panel of substation regulators, a $30^{\circ}$ shift forward or $30^{\circ}$ shift back is selected to correct this problem.

For single-phase connected open delta regulators applied to the circuit, corrections are made to the settings by using a $30^{\circ}$ phase shift in the voltage on the compensator circuit. For the lead unit regulator in an open delta connection, the corrected settings are determined by multiplying the original settings obtained earlier by the quantity $\cos 30^{\circ}-\mathrm{j} \sin 30^{\circ}$, or

$$
\mathrm{R}_{\mathrm{LC}}+\mathrm{j} \mathrm{X}_{\mathrm{LC}}=(0.866-\mathrm{j} 0.5)\left(\mathrm{R}_{\mathrm{L}}+\mathrm{j} \mathrm{X}_{\mathrm{L}}\right), \quad \text { Equation } 3.60
$$

where $\mathrm{R}_{\mathrm{LC}}+\mathrm{j} \mathrm{X}_{\mathrm{LC}}$ are the corrected values.

For the lag unit regulator in an open delta connection, the corrected settings are found by multiplying the original settings by the quantity $\cos 30^{\circ}+\mathrm{j} \sin 30^{\circ}$, or

$$
R_{L C}+j X_{L C}=(0.866-j 0.5)\left(R_{L}+j X_{L}\right) .
$$

Equation 3.61

Because these new, corrected settings require the phase angle to be moved back $30^{\circ}$ or forward $30^{\circ}$, negative RL values result when increasing the phase angle, and negative $\mathrm{X}_{\mathrm{L}}$ values result when decreasing the phase angle.

\subsection{Step Regulators in Series}

Often, to obtain the needed voltage profile on a circuit and not subject customers to LV or $\mathrm{HV}$, two or more regulators are installed. This was illustrated in Figure 5. The solutions to the problems associated with these installations are dependent on the time delay settings, the bandwidth settings, and the voltage magnitude of the tap position steps. Other factors include changes in voltage at the primary side of the substation transformer, size of loads, location of loads, and the rate of change of load current and the resultant voltage.

Generally, the best solution is a short first regulator time delay, so the voltage is changed before the second regulator begins to make a change. The second regulator then completes its change before the third regulator begins to make a change, and so on. However, the time delay on the last regulator should not be long enough to subject customers to a long period of LV or HV. 
However, there are exceptions to this rule if large loads are installed on the tag end of a circuit. In this case, the preferred solution is to have the last regulator respond first to the voltage correction and then readjust after the regulators upstream have operated. The optimum solution is the least number of regulator voltage adjustments for all the regulators on the circuit. The application of capacitors complicates the problem because, when capacitors are switched "on," the input voltage to the nearby regulators goes up, and the regulators may operate to control the voltage within limits.

\subsection{Fixed Capacitors on Circuits with Regulators}

When a fixed capacitor is installed at the regulation point or downstream on the circuit, no changes to the compensator settings of equations 3.50 and 3.51 are needed. This is because the capacitor current $\mathrm{I}_{\mathrm{C}}$ is included with the $\mathrm{I}_{\mathrm{L}}$ current, and the current in the $\mathrm{CT}$ of the compensator includes both $\mathrm{I}_{\mathrm{L}}$ and $\mathrm{I}_{\mathrm{C}}$. This assumes no load is connected between the regulator at the substation and the regulation point or between the regulator on the circuit and the regulation point. When load is connected between the regulator and the regulation point, then equations 3.52 and 3.55 for the $R_{L s e t}$ and $X_{L s e t}$ are not correct. This is because the $I_{C} R_{E}$ and $I_{C} X_{E}$ are not equal to the $I_{C} R_{a}$ and $I_{C} X_{a}$, where the actual line resistance is $R_{a}$ and the actual line reactance is $X_{a}$. This is shown in Figure 42. This phasor diagram shows the received voltage difference before and after the fixed capacitor is installed is

$$
\left|V^{\prime}{ }_{R C}\right|-\left|V_{R C}\right|=\left|I_{C}\right|\left(X_{a}-X_{E}\right) .
$$

Equation 3.62

This equation applies when the capacitor is located at the regulation point or downstream. This effect can be taken into account by changing the compensator settings or the VRR setting. The easiest and most common solution is to change the VRR setting. However, if the compensator settings are to be revised, then equations $3.53,3.54$, and 3.55 must be modified to include $\mathrm{I}_{\mathrm{C}}$, or

$$
\begin{aligned}
& \mathrm{R}_{\mathrm{LE}}=\left[\sum_{\mathrm{i}=1}^{\mathrm{n}}\left|\Delta \mathrm{V}_{\text {ir }}\right|\right] /\left(\left|\mathrm{I}_{\mathrm{L}}+\mathrm{I}_{\mathrm{C}}\right|\right) \\
& \mathrm{n}
\end{aligned}
$$

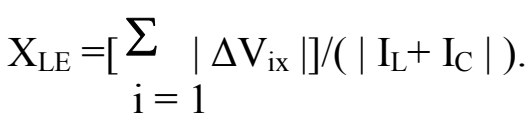




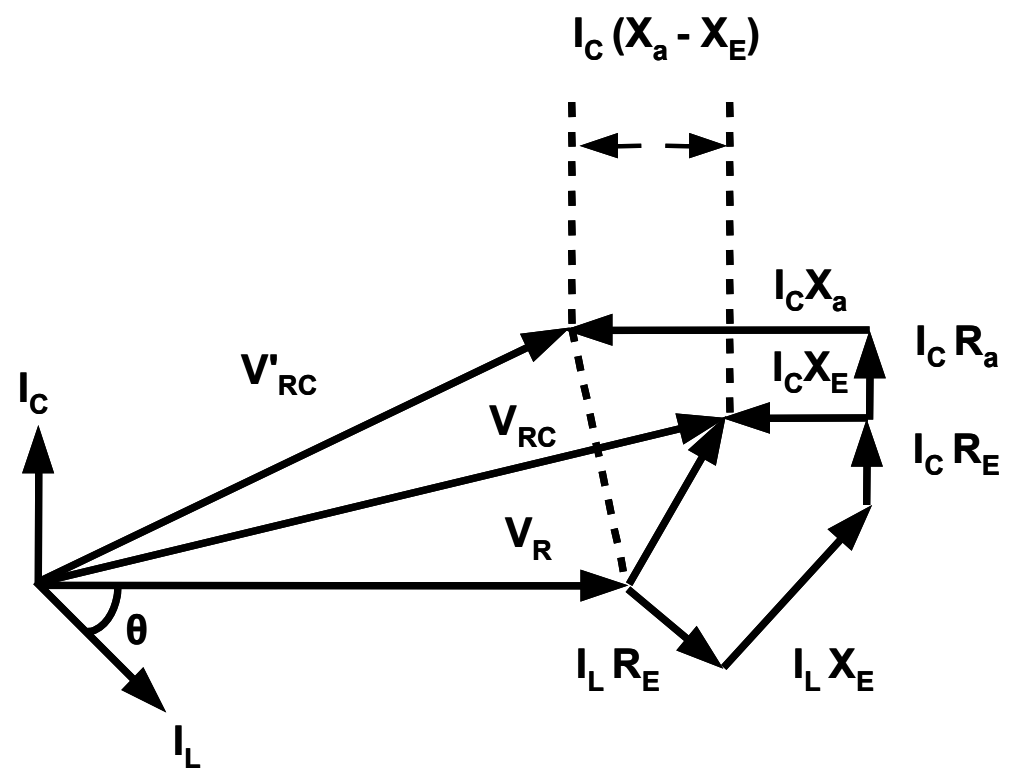

Figure 42. Phasor diagram of the effect of capacitors on the line drop compensator and regulator output voltages before and after relay settings are revised

$\mathrm{V}_{\mathrm{R}}=$ Relay setting and regulation point voltage without capacitors

$\mathrm{R}_{\mathrm{a}}=$ Actual resistance from regulator to regulation point

$\mathrm{R}_{\mathrm{E}}=$ Effective line resistance from the regulator to regulation point or the resistive voltage drop to regulation point.

$\mathrm{I}_{\mathrm{L}}=$ Load current at regulator

$\mathrm{X}_{\mathrm{a}}=$ Actual reactance from regulator to regulation point

$\mathrm{X}_{\mathrm{E}}=$ Effective line reactance from the regulator to the regulation point

$\mathrm{V}_{\mathrm{RC}}=$ Regulator output voltage (relay $\mathrm{R}$ and $\mathrm{X}$ settings) after capacitors are "on"

$\mathrm{V}_{\mathrm{R}^{\prime} \mathrm{C}}=$ Regulator output voltage to have VR at regulation point after capacitors are "on" or $\mathrm{V}_{\mathrm{R}}-\mathrm{I}_{\mathrm{C}}\left(\mathrm{X}_{\mathrm{L}}-\mathrm{X}_{\mathrm{E}}\right)$

$\mathrm{V}=$ Voltage at regulation point with adjusted relay settings because of capacitors

If a fixed capacitor is installed between the regulator and the regulation point, the $\mathrm{I}_{\mathrm{C}}$ current flows only to the capacitor and is not in the $\mathrm{I}_{\mathrm{L}}$ current at the regulation point. Again, the best method to correct this problem is to change the VRR setting.

\subsection{Switched Capacitors on Circuits with Regulators}

When a switched capacitor is switched "on," the effect on the regulator settings is the same as that of a fixed capacitor. If a switched capacitor is installed at the same location as the regulator, then the regulator can be operated properly when the capacitor is turned "on" or "off." If the switched capacitor is installed on the source side of the regulator, regulator operation is not affected by the capacitor because the $\mathrm{I}_{\mathrm{C}}$ current does not flow through the regulator. However, it is common practice to locate the capacitor on the load side because the capacitive current will lower the $\mathrm{I}_{\mathrm{L}}$ current through the regulator. In this case, regulator operation is affected by the capacitor current, and the compensating circuit must be modified. This is explained in Figure 43. If the switched capacitor is voltage-controlled and the 
capacitor is located on the load side of the regulator, the VT of the capacitor control should be located on the source side because the regulator operation will not affect the capacitor operation. Notice in Figure 43, with a CT in the capacitor circuit connected to the CT of the regulator control, that there is no $\mathrm{I}_{\mathrm{C}}$ current in the compensator $\mathrm{R}_{\mathrm{L}}$ and $\mathrm{X}_{\mathrm{L}}$.

Another method of control is shown in Figure 44, in which an impedance has been added in series with the $\mathrm{R}_{\mathrm{L}}$ and $\mathrm{X}_{\mathrm{L}}$ of the compensator circuit that has a voltage drop equal to the voltage rise because of the capacitor. When the capacitor is switched "on," the auxiliary contact is open, which adds a voltage drop in the compensator circuit. When the capacitor is switched "off," the contact shorts out the impedance Z, and the regulator operation reverts back to normal, as though the capacitor were not there.

A third method of control is to change the VRR setting or the compensator settings as described earlier. The VRR setting is determined with the capacitor on, but a full setting increase should not be used because too low of a voltage will now occur at LL when the capacitor is off.

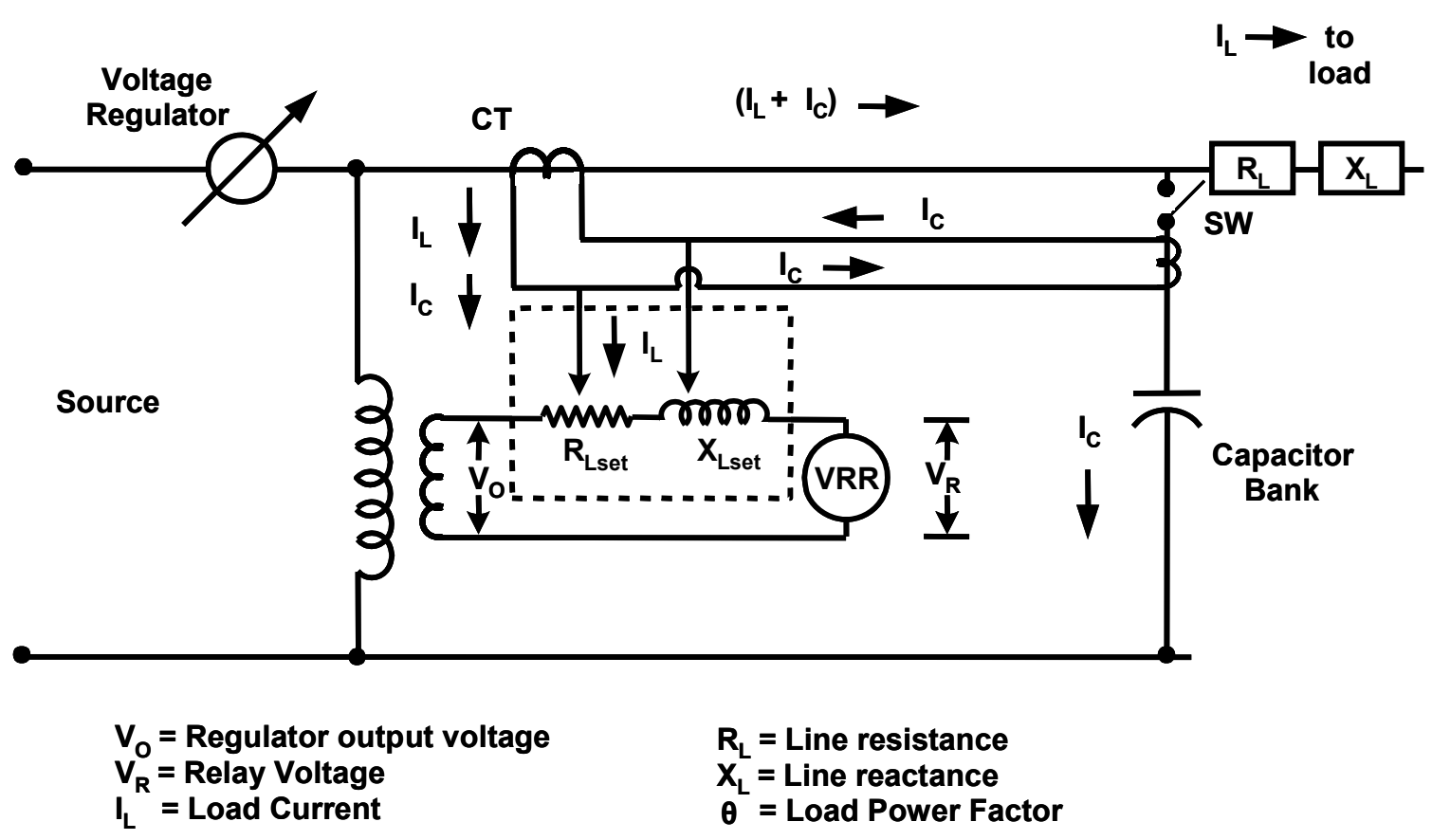

Figure 43. Control system and line drop compensator with use of a shunt capacitor at the location of regulator 


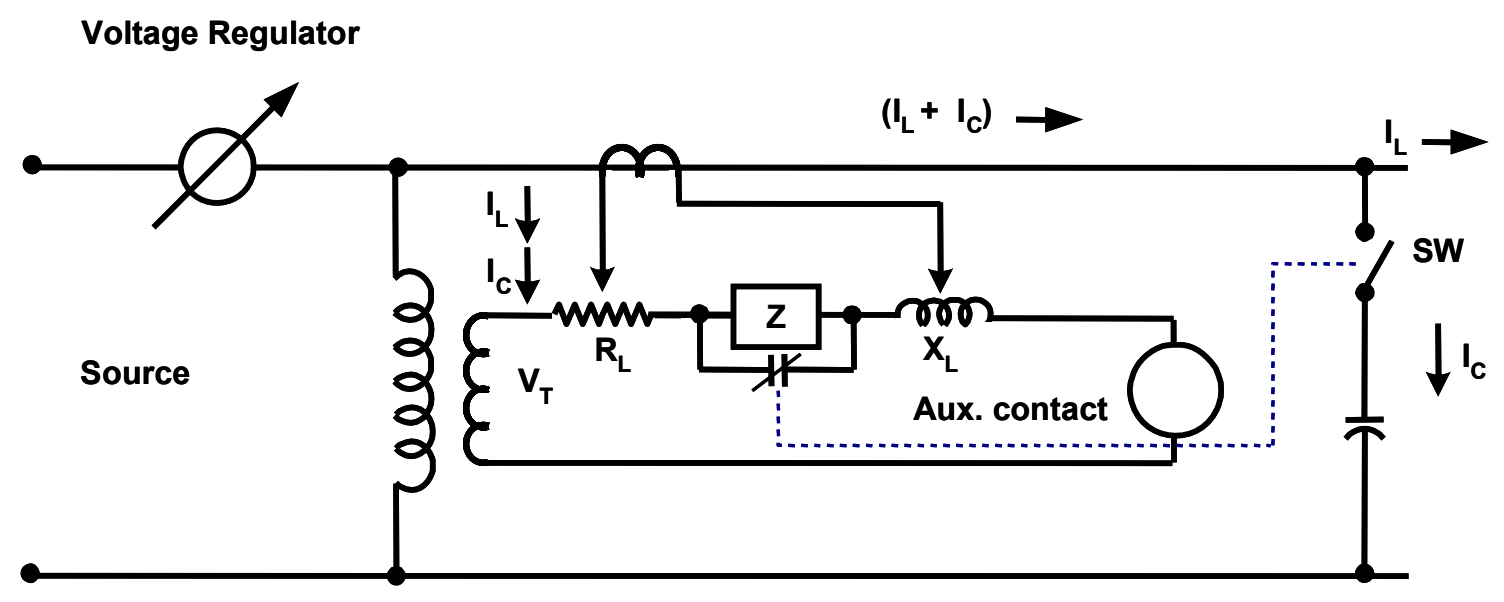

Figure 44. Voltage across impedance $Z$ in relay circuit is equal to voltage rise because of capacitor current $I_{c}$

\subsection{Switched Capacitors Downstream from Regulators}

When switched capacitors are installed downstream from the regulators, the compensator settings or the voltage setting on the VRR can be changed. The easiest approach is to change the VRR setting. Coordination of regulators and switched capacitors on the same circuit is generally not a problem because the bandwidth on the capacitor voltage control relay is greater than the regulator bandwidth and the time delay for the capacitor control is more than the regulator time delay (which is similar to regulators installed in series, in which the downstream regulators have a greater time delay than the upstream regulators). Also, the voltage change at the capacitor when it switches on is high compared with what the upstream regulators see.

\subsection{Capacitor Application}

As noted earlier, capacitors can be added to distribution circuits to improve voltage regulation. Equation 3.8 showed that voltage rise (a reduction in load current $\mathrm{I}_{\mathrm{L}}$ ) can be calculated if one knows the single-phase kilovolt-amperes reactive or three-phase kilovoltamperes reactive, the distance from the source to the capacitor, the inductive line reactance, and the line-to-line voltage. The voltage rise is independent of the load magnitude. Therefore, it is common to install switches to turn them on and off to not subject customers to HV during LL conditions, when the voltage drop in the circuit is low.

Capacitors are rated in kilovars, the value of which is derived from the capacitance $\mathrm{C}$ in microfarads, the rms voltage $\mathrm{V}$, and the frequency, or

$$
\mathrm{kVAr}=\left(\mathrm{V}^{2} 2 \pi f \mathrm{C} \times 10^{-6}\right) / 1,000 \text {. }
$$

Equation 3.65

Notice the kilovar value is proportional to the square of the voltage. As voltage drops on a circuit, the kilovar value decreases, and the voltage rise decreases. 
There are two types of capacitors: series and shunt. Series capacitors are not discussed here because their application can cause a sub-synchronous resonance condition. Often, additional resistance must be added to correct this problem. Shunt capacitors supply a source of kilovars similar to an overexcited synchronous generator. They are often applied to induction generators to supply a source of voltage. Shunt capacitors lower or counteract the lagging component of circuit current and, thus, increase the voltage at their location. This improves the voltage regulation. Because the lagging component of current is reduced, capacitors reduce the $\mathrm{I}_{2} \mathrm{R}_{\mathrm{L}}$ real losses and the $\mathrm{I}_{2} \mathrm{X}_{\mathrm{L}}$ reactive losses. They are a low-cost solution to improving the power factor of load because synchronous generator volt-amperes reactive are about four times the cost of static capacitors. This reduces the kilovolt-ampere loading on synchronous generators, which in turn, allows more kilowatt generation (if this is not limited by the prime mover kilowatt output). When capacitors are added to a circuit, they reduce the load current, release capacity to serve future load, and reduce the cost to serve kilowatts. Correction to unity power factor where no reactive current exists in the load current is generally not economical. Power factors ranging from about 0.975 up to 0.980 are normally the most economic, as seen by the generation source. The diminishing return effect is illustrated in Figure 45.

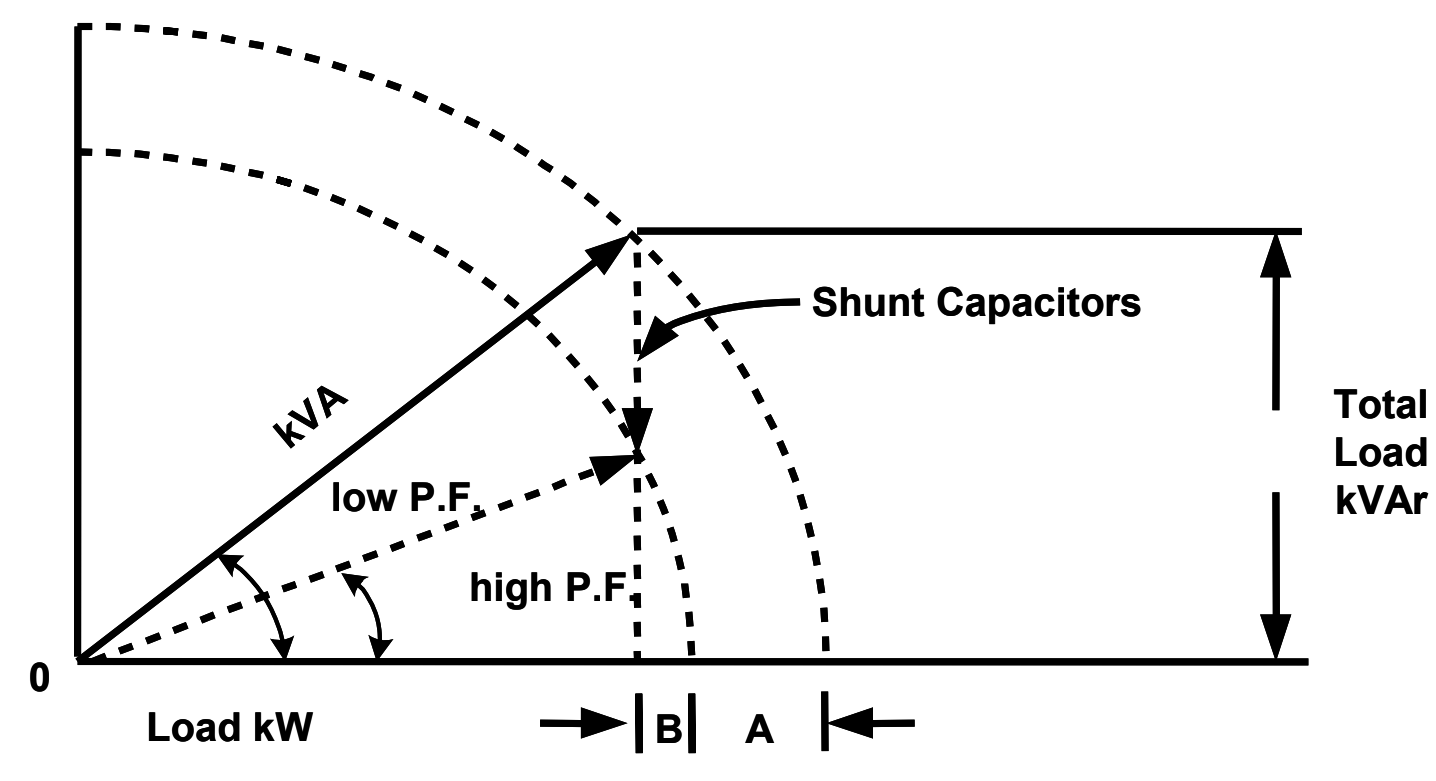

Change in kVA

Figure 45. Application of shunt capacitors

Applying enough kilovar shunt capacitance to offset just half the load kilovars results in a large kilovolt-ampere reduction of "A." If an additional equal amount of kilovars is added, it results in only a small kilovolt-ampere reduction of "B." Thus, the lower the power factor, the more benefit that is derived from installing shunt capacitors. 
The voltage drop $\Delta \mathrm{V}$ in a circuit can be approximated by

$$
\begin{aligned}
& \Delta V=I_{r} R+I_{x} X, \text { or } \\
& \Delta V=I(R \operatorname{Cos} \theta+X \operatorname{Sin} \theta),
\end{aligned}
$$

where:

$$
\begin{aligned}
& I=\text { Load current } \\
& I_{r}=\text { Real component of current } \\
& I_{x}=\text { Reactive component of current } \\
& \theta=\text { Load power factor angle. }
\end{aligned}
$$

When a capacitor is added, Equation 3.66 becomes

$$
\Delta \mathrm{V}=\mathrm{I}_{\mathrm{r}} \mathrm{R}+\mathrm{I}_{\mathrm{X}} \mathrm{X}-\mathrm{I}_{\mathrm{c}} \mathrm{X}
$$

where:

$\mathrm{I}_{\mathrm{c}}=$ Capacitive reactive components of current.

If too many volt-amperes reactive are added to the circuit during LL conditions, the circuit can become overcompensated because $\mathrm{I}_{\mathrm{C}}$ is proportional to $\mathrm{V}$, not the load.

Figure 46 shows the phasor diagram when a shunt capacitor is added at the load. The sending-end voltage with the capacitor applied $\left(\mathrm{V}_{\mathrm{SC}}\right)$ is less than the sending-end voltage without the capacitor $\left(\mathrm{V}_{\mathrm{S}}\right)$.

\subsection{Capacitor Controls}

Capacitor control can be accomplished through the:

- Time clock

- Voltage

- Current

- Temperature

- Time clock with voltage override

- Time clock with different VRR settings

- Current with voltage override

- Radio or other communication medium

- Power factor.

The most common and simplest control is time clock control because the load profile on most circuits is predictable. As such, the time clock turns the capacitor on at a set time in the morning and off at a set time at night.

Figure 47 shows a voltage-current control in which the voltage can be measured at the capacitor or another location on the circuit and the VRR signals the capacitor switch to close. 


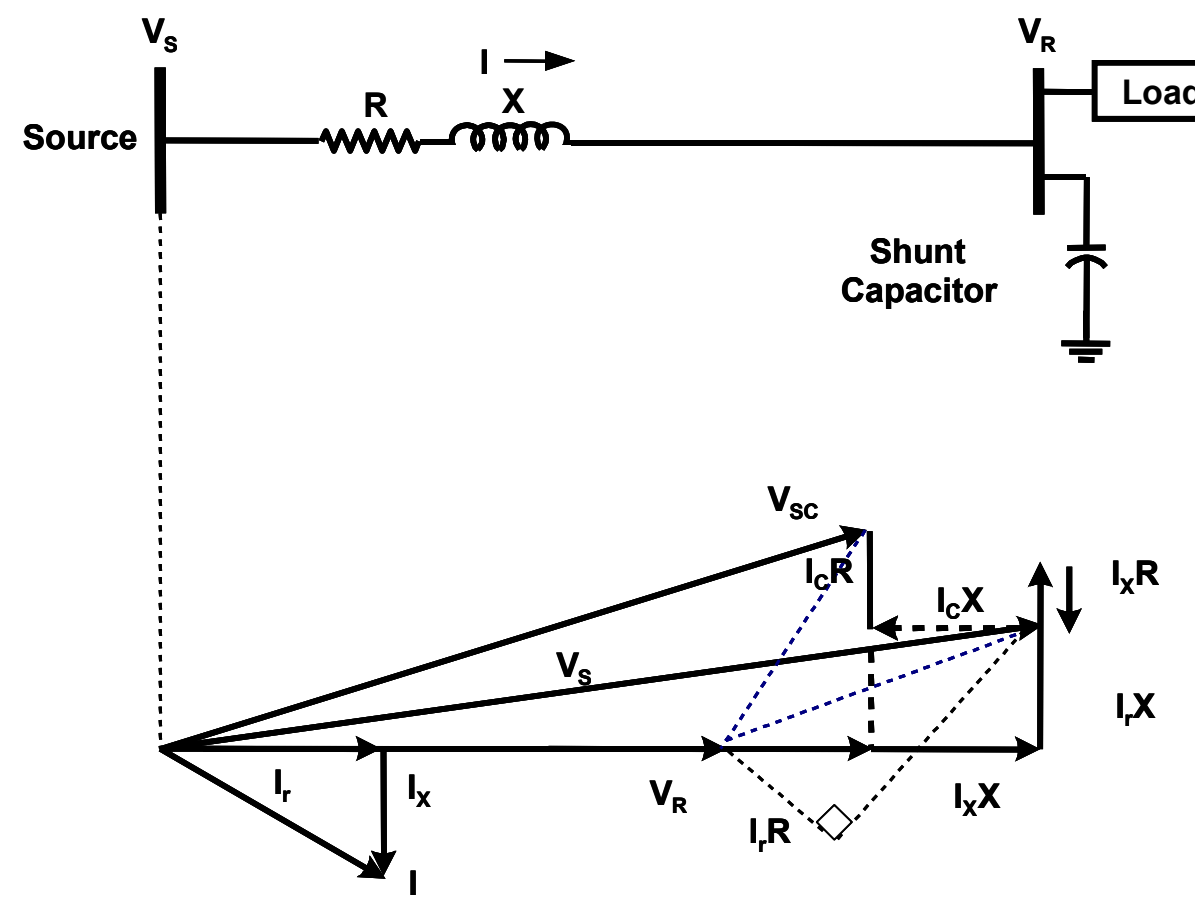

The $l_{x} X$ Component is parallel to and opposite to the $I_{c} X$ component.

$V_{R}=$ voltage at the load or receiving end voltage.

$V_{S}=$ sending end voltage

$V_{s c}=$ sending end voltage with capacitor

Figure 46. Phasor diagram of the reduction in the sending-end voltage with a shunt capacitor

The resistor $\mathrm{R}$ is chosen so that its voltage drop represents the drop in voltage to the regulation point. If the power factor varies, it may be necessary to add a reactance with the resistance.

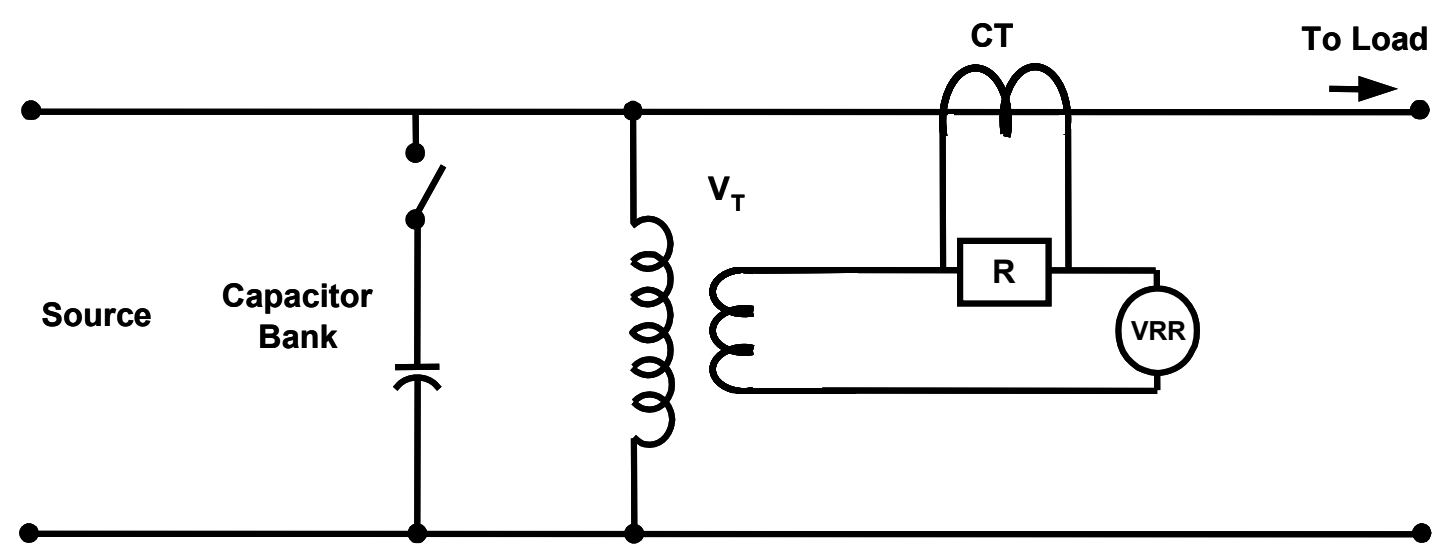

Figure 47. Voltage and current control of capacitor switching 


\subsection{Capacitor Connections}

Three-phase capacitor banks are normally connected delta or wye with the neutral grounded as in Figure 48 or wye with the neutral ungrounded as in Figure 49. Delta-connected capacitors or ungrounded wye-connected capacitors may result in a resonant condition when one or more of the phase conductors on the source is open, as shown in Figure 50. The capacitor bank can provide voltage to the loads when an open phase (or phases) occurs.

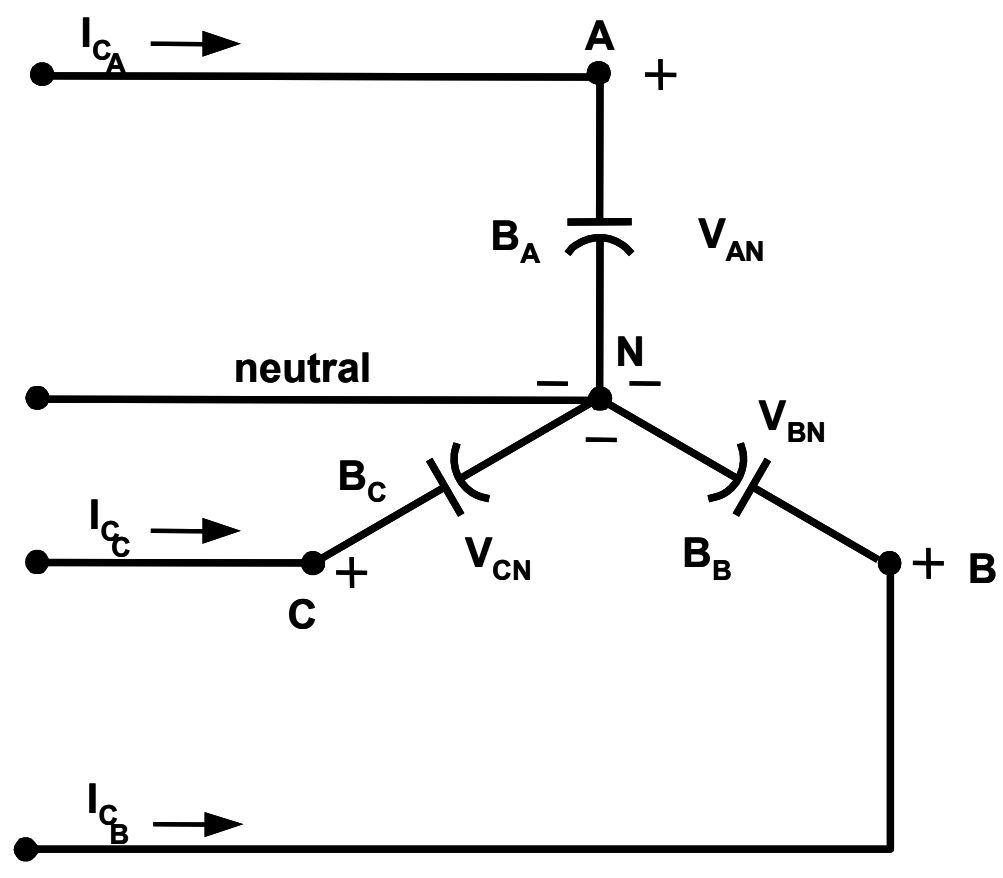

Figure 48. Wye-connected capacitor bank

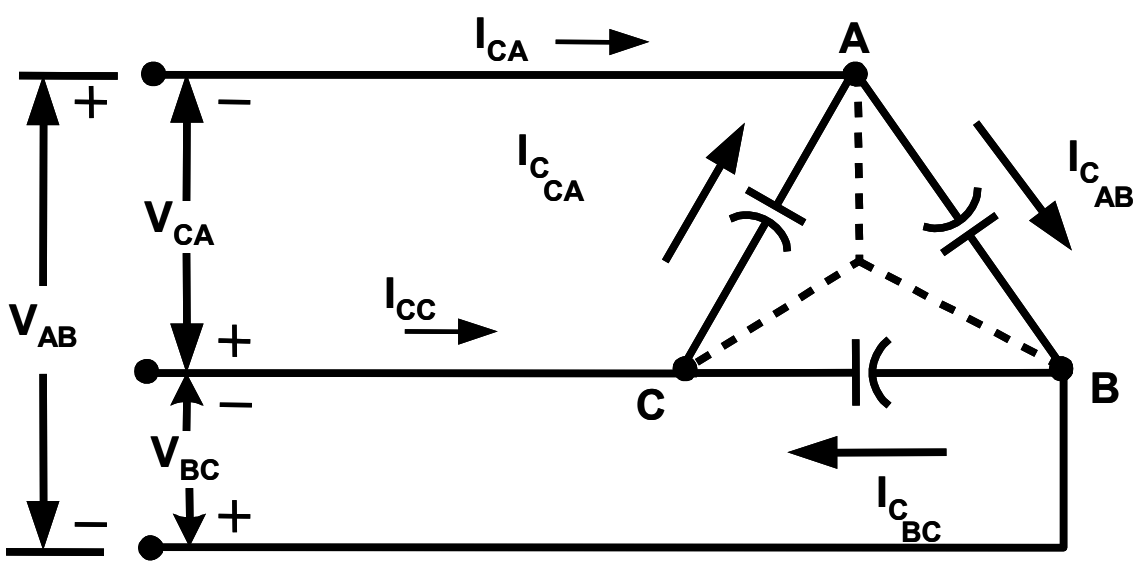

Figure 49. Delta-connected capacitor bank 


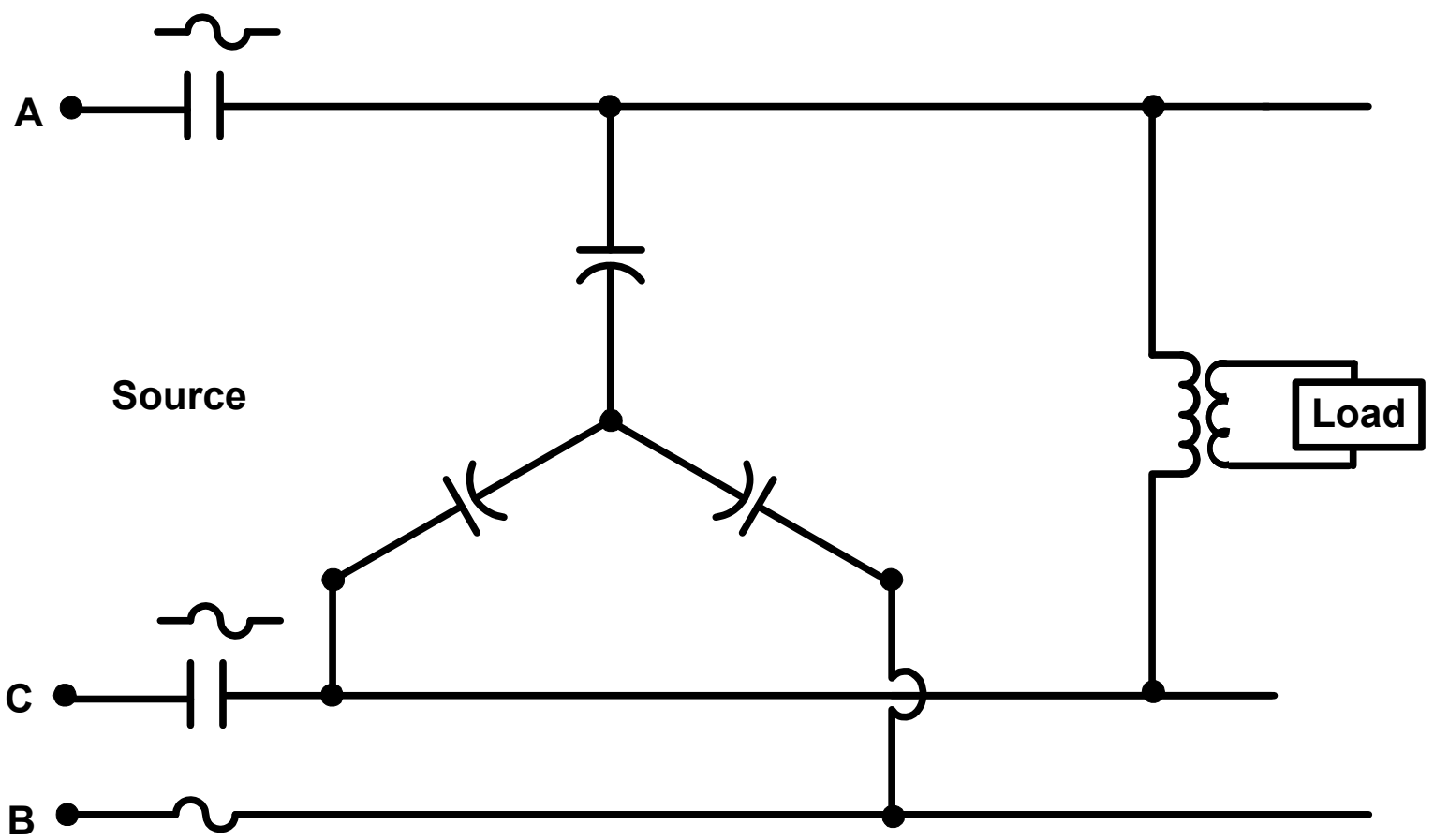

Figure 50. Series resonance condition with open phases

Putting capacitors in series with transformers feeding load may create a series resonant circuit. For this reason, it is not recommended that capacitor banks be put on the load side of single-phase fuses, sectionalizers, or reclosers. Grounded wye capacitors are used on fourwire wye systems. Grounded wye capacitors applied to ungrounded wye or ungrounded delta systems cause ground currents and overvoltages during ground faults. Therefore, grounded wye banks are not used on delta systems or ungrounded wye systems. Delta-connected banks are used on delta systems and ungrounded wye systems. 


\section{Project Results - Effects of Unbalanced Loading and Voltage on System Protection and Distributed Generation}

\subsection{Introduction}

This section describes the effects of unbalanced voltage and load on system protection. The effects of unbalanced voltage and load on sensitive ground relaying were studied for a selected circuit, and adequate protective relaying limits were determined for different levels of unbalance and different energy conversion devices.

This section includes an overview of system protection philosophy, focused on the issues associated with unbalanced loading. Descriptions of the operation of protective devices under unbalanced conditions are also provided. For a detailed discussion of protective relay theory and operation, consult Elmore (1994), Electrical Distribution (2005), and Mason.

\subsection{System Protection Design Philosophy for Grounded and Ungrounded Systems}

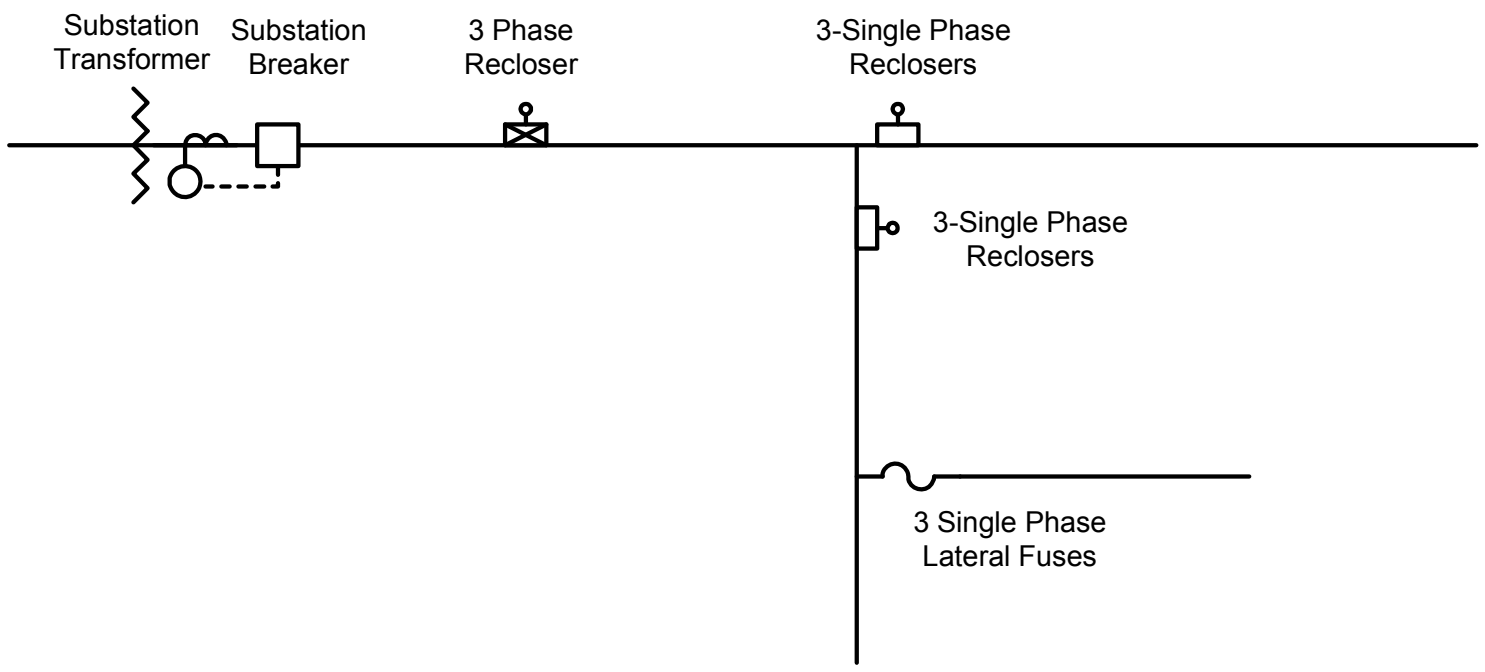

Figure 51. Radial distribution circuit with typical system protection devices

Figure 51 shows overcurrent relaying application for the entire circuit. The protective devices must be capable of carrying the intended load (loadability), sensing and clearing faults (sensitivity), and selectively de-energizing the minimum amount of load to isolate faults (selectivity).

Typical loads, as seen by major protective devices, are shown in Figure 52. Devices at the substation must carry the load of the entire circuit; devices downstream carry less load. The amount of unbalance in the phase currents may or may not be greatest at the substation breaker. 


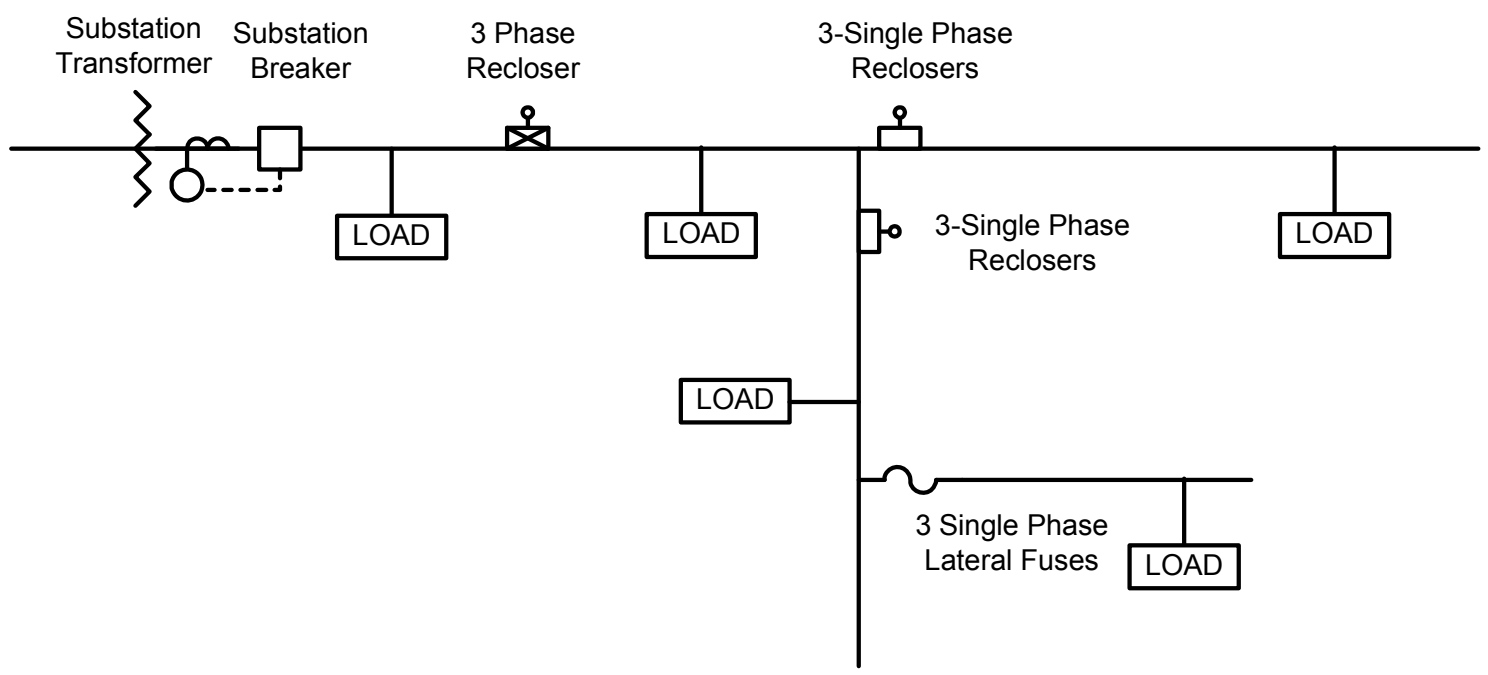

Figure 52. Radial distribution circuit showing load

Figure 53 shows the portions of the circuit where the protective device must sense faults. Note that, for full circuit protection, the zone of protection for the upstream device must extend beyond (overlap) the beginning of the next downstream device.

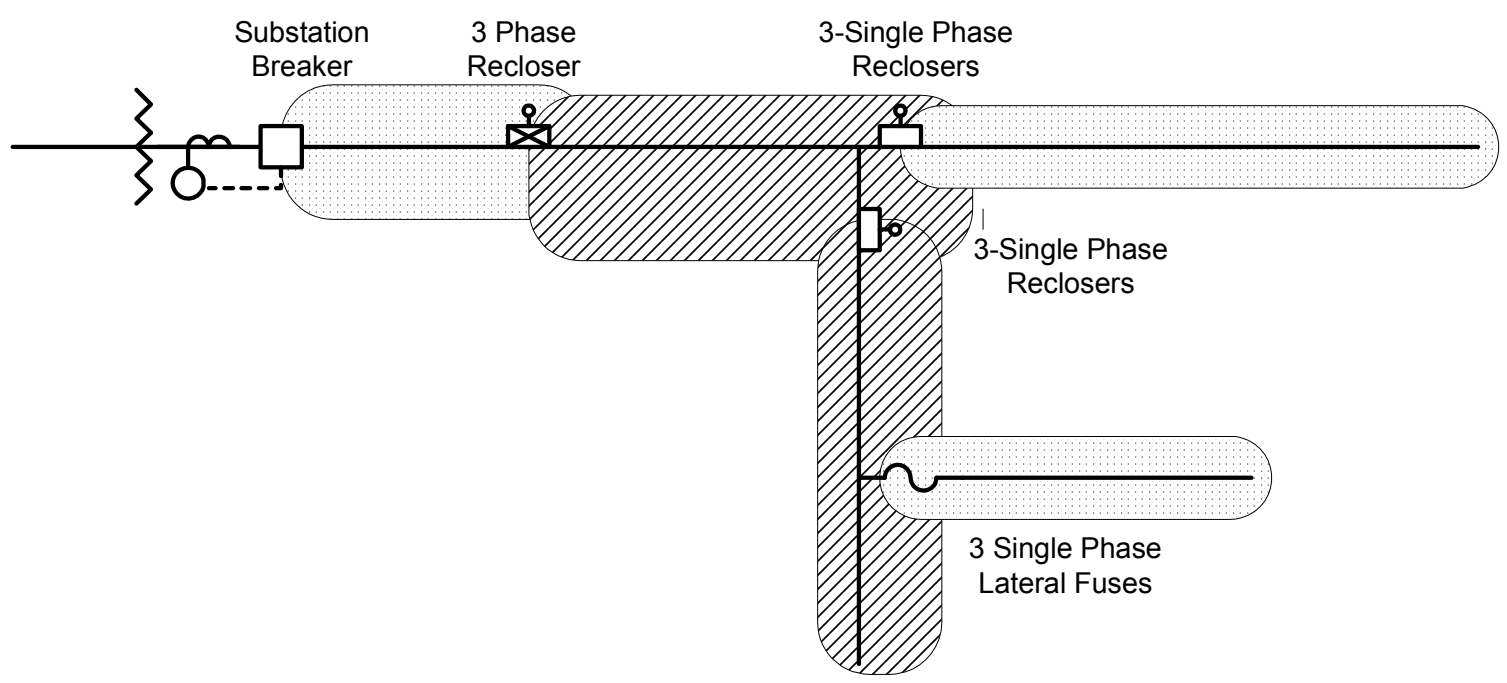

Figure 53. Radial distribution circuit showing protection zones

Figure 54 shows the relative tripping times for the substation breaker, the three-phase recloser, and the single-phase recloser. As the distance between a protective device and a fault increases, tripping time increases. For a fault downstream at point A, the single-phase recloser will trip in about 0.5 seconds. The three-phase recloser will trip in about 0.8 seconds. If the single-phase recloser operates correctly, the three-phase recloser should not operate, and only load beyond B will be interrupted. 


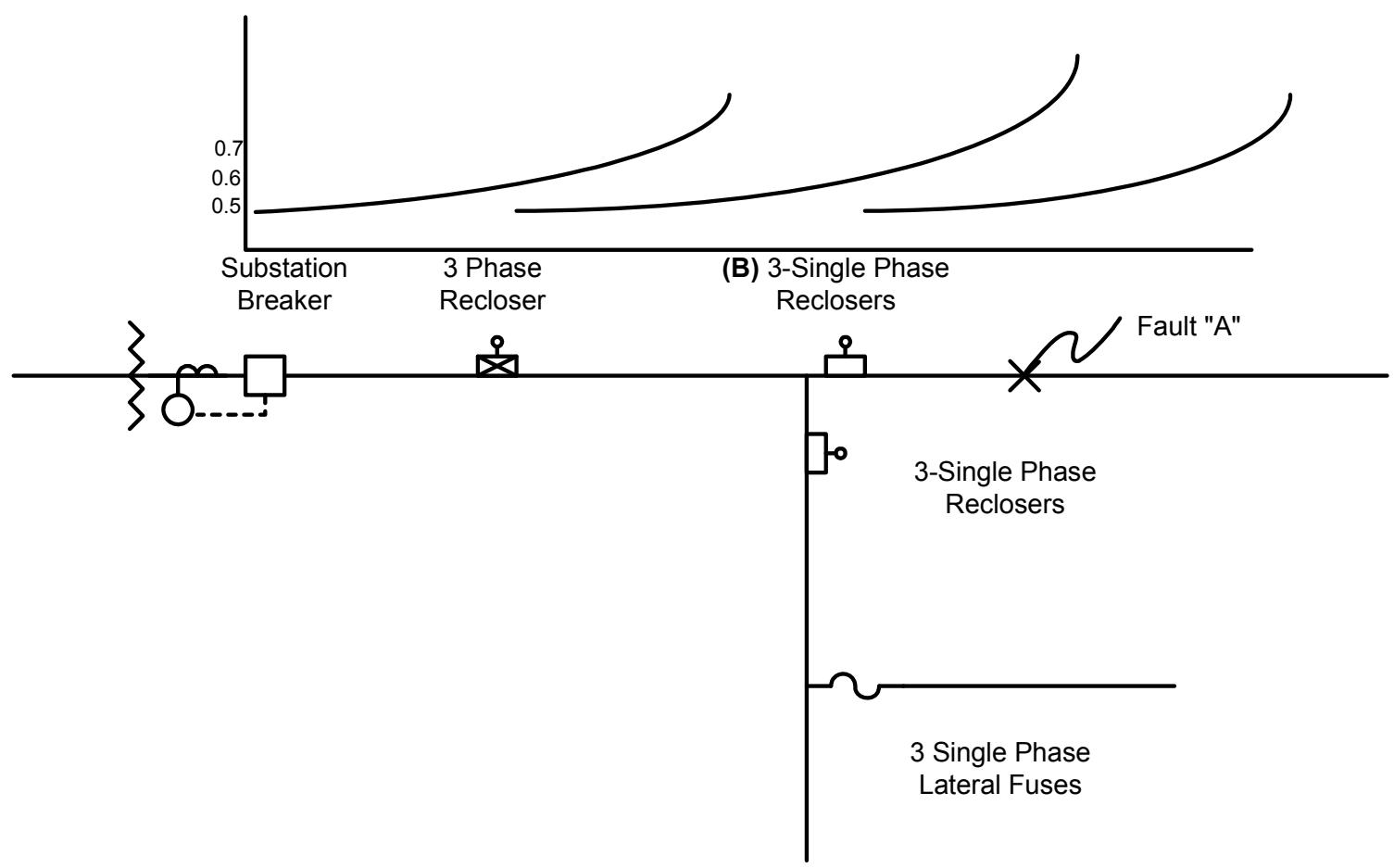

Figure 54. Relative tripping times for protective devices

Figure 55 shows the time-current characteristics for a typical breaker and reclosers. For any specific value of current, the device closest to the substation operates last, and the device furthest from the substation operates first. 


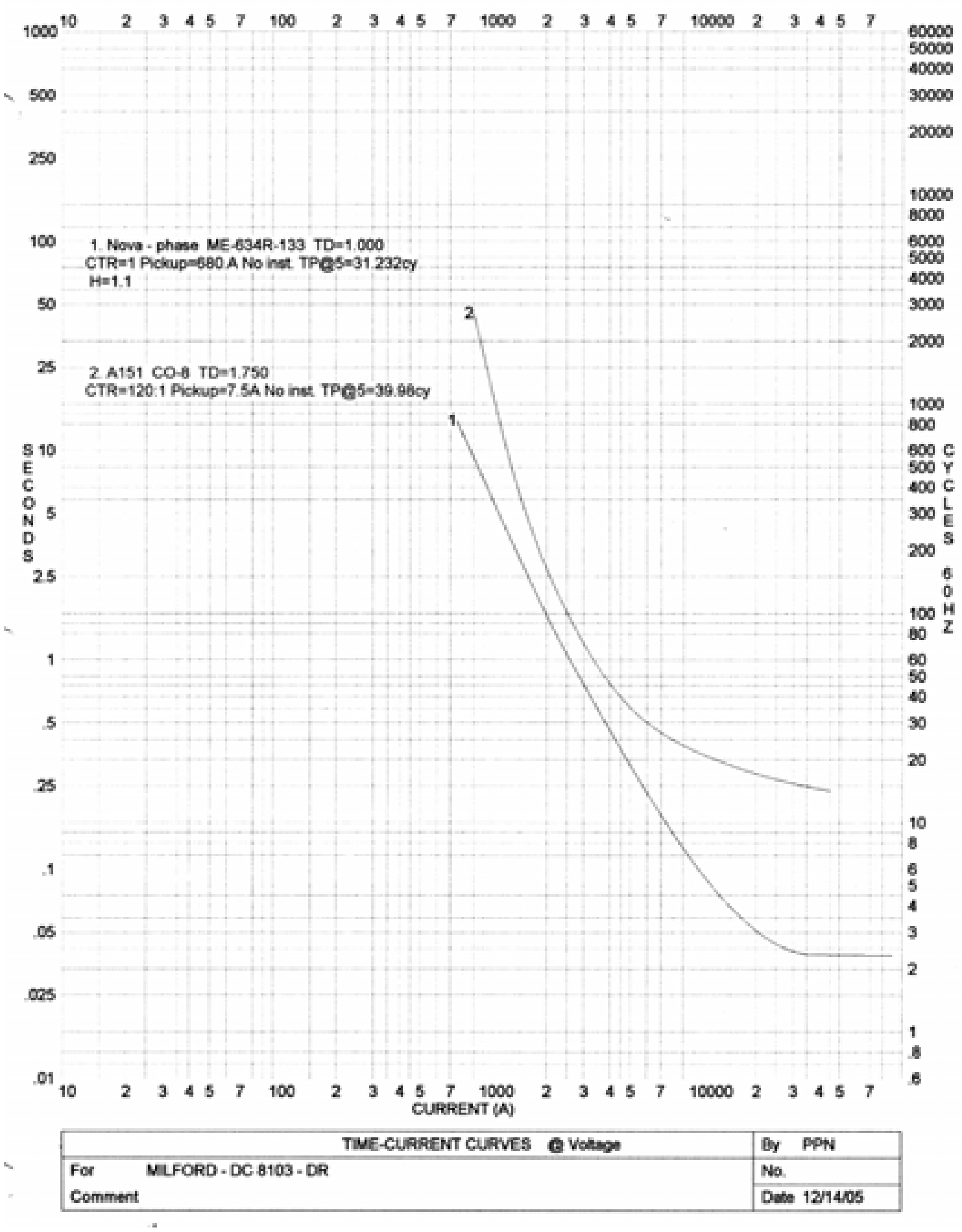

Figure 55. Relative time-current curves for protective devices 


\subsubsection{Four-Wire Grounded Wye Systems}

Four-wire grounded wye systems have a substation transformer with a grounded wye winding sourcing the circuit bus. The primary of the transformer is typically connected in a delta. The neutral point of the transformer secondary is grounded and taken out on the circuit as the neutral with the three-phase conductors. The three-phase diagram in Figure 56 shows details of the connections at the substation and load on the circuit. A single-phase load connected line-to-ground will cause current to flow in the neutral of the system unless it is balanced by equal amounts of other single-phase loads connected to the alternate phases.

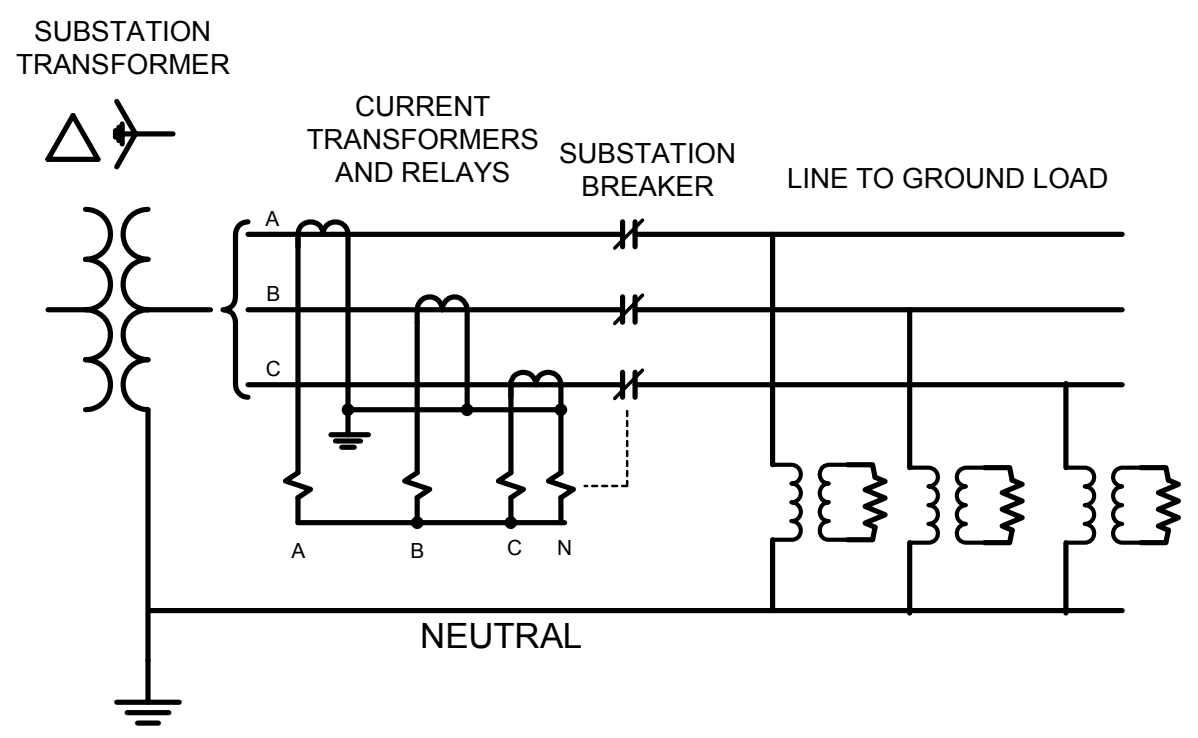

Figure 56. Three-phase diagram of a four-wire grounded system

\subsubsection{Three-Wire Ungrounded Delta Systems}

Three-wire ungrounded delta systems have a substation transformer with an ungrounded delta winding sourcing the circuit bus. The primary of the transformer may be configured in an ungrounded wye, grounded wye, or delta. Figure 57 shows details of the connections at the substation and load connected on the circuit. The ungrounded delta system differs from the grounded wye system because the load is always connected phase-to-phase. Typically, there is no neutral or ground relay connected to detect ground faults, as is the case for the four-wire grounded wye system. A small amount of current will flow if a phase conductor makes solid contact with ground. Some systems may use a ground current relay to detect this. A ground detector system is typically installed to alarm for unintentional grounds caused by downed conductors or foreign objects making contact with a phase conductor. Ground detector systems and why ground currents will flow on ungrounded systems are explained in Appendix A. 


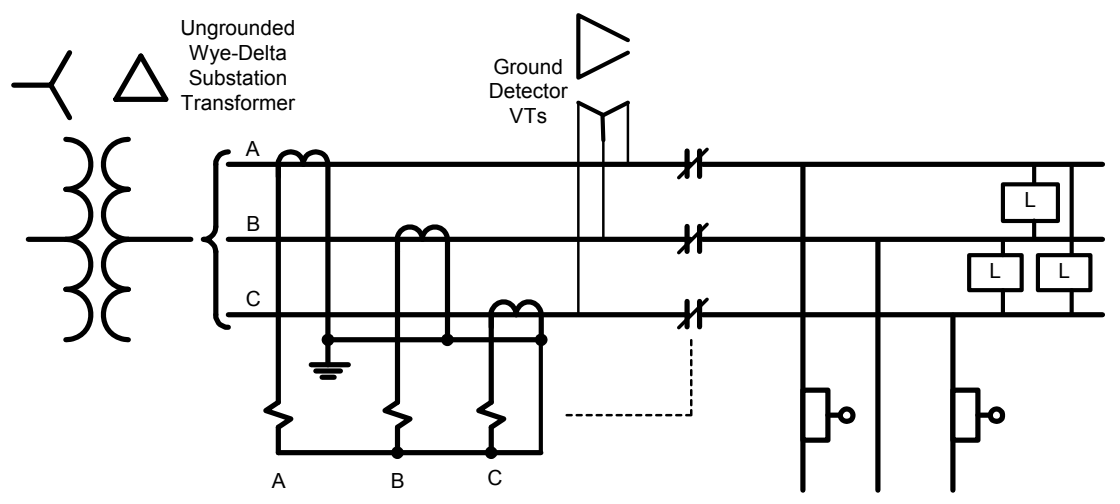

Figure 57. Three-phase diagram of a three-wire ungrounded system

\subsection{Unbalanced Voltage Effects on Protective Relaying}

Unbalanced voltages can cause an addition to current already in the neutral conductor and relay because of unbalanced load. For the case in which balanced loads are served from a distribution circuit with balanced voltages, no current is in the neutrals of a four-wire system. For the case in which the voltage is unbalanced and the load is primarily resistive and connected line-to-ground, the load will decrease on the lowest voltage phase and increase on the highest voltage phase. The current imbalance will result in a current flow in the neutral conductor and ground (earth). For the case in which the motor load consists primarily of single-phase loads connected line-to-ground, lowering the voltage on one phase will cause the current to increase on that phase. This is due to the essentially constant power (CP) characteristic of the motor. This current imbalance will cause additional neutral current.

Additional unbalance can result from failed capacitor cans (units) within a capacitor bank. The volt-amperes reactive from the capacitor bank are directly proportional to the voltage squared. (See Equation 3.65 in Section 3.) Unbalancing the voltage causes an unequal supply of volt-amperes reactive and an unbalanced loading effect with increased neutral current.

The ultimate effect of unbalanced voltage on the neutral current of the distribution circuit can depend on a number of factors, including:

- The mix of single-phase resistances and single-phase motor load

- Capacitor size, location, and unbalance because of unit failures

- Current unbalance and significant increased losses from unbalanced voltages applied to three-phase induction and synchronous machines

- Unsymmetrical transformer connections (e.g., open-delta, open-wye, and unequal impedances)

- Untransposed lines

- Open phases because of single-phase fuse blowing and single-phase protective devices

- Malfunctioning single-phase VR within a three-phase or open-delta or open-wye configuration 
- Single-phase VRs connected in a bank and operating to correct a voltage unbalance condition

- Single-phase VRs operating on a long single-phase portion of a three-phase circuit.

Additional neutral current caused by unbalanced voltage on the circuit will flow in the neutral relay $(51 \mathrm{~N})$ at the substation if neutral current flows through any three-phase protective device downstream from the substation. However, circuit unbalance can occur on a portion of the circuit but be balanced with other connected load such that no neutral current reaches the neutral relay element of that protective device. This relay must be set to trip for ground faults. The setting may have to be increased to take into account the increased neutral current because of the unbalanced condition.

\subsection{Unbalanced Current Effects on Protective Relaying}

Unbalanced loading can occur even though voltages are balanced at the source. The factors that affect unbalanced loading are similar to those factors that affect unbalanced voltage.

\subsubsection{Single-Phase Operation of Protective Devices}

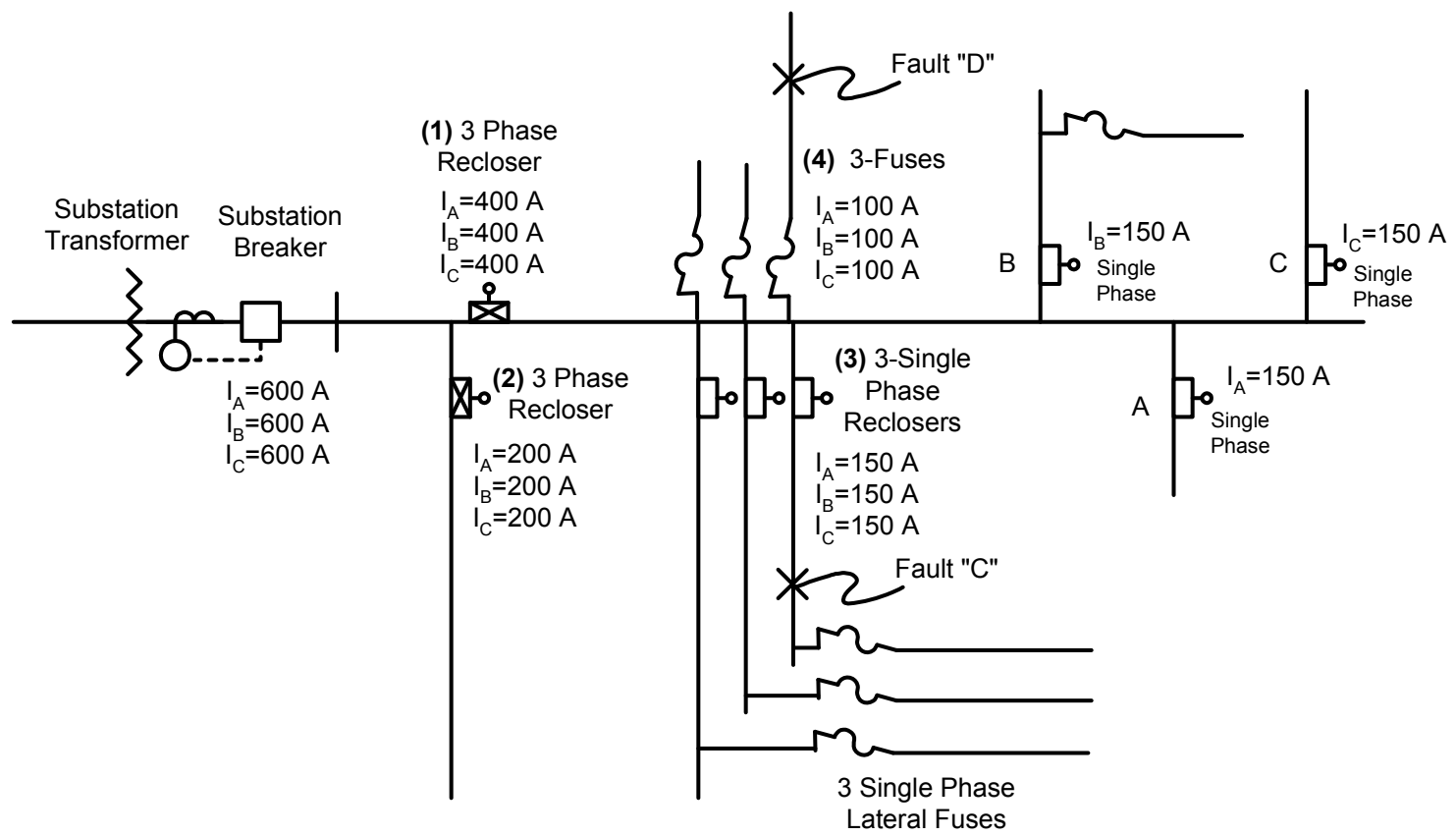

Figure 58. Single-phase operation of protective devices on a four-wire wye circuit of resistive loads

Figure 58 shows that protective devices may be three-phase devices, a set of three singlephase devices, or single-phase devices that protect single-phase laterals. Operation of one of these single-phase devices on a previously balanced loaded circuit will cause unbalanced loading and neutral current. When the circuit is in a normal state with the loads given in Figure 58, the three-phase recloser (1) has no current in the neutral relay element. When the single-phase recloser (3) on Phase $\mathrm{C}$ opens because of the fault at $\mathrm{C}$, load of $150 \mathrm{~A}$ is lost on Phase $\mathrm{C}$ downstream of the recloser. The phase currents on the substation breaker change to: 


$$
\mathbf{I}_{\mathbf{A}}=600 \mathrm{~A} \angle 0^{\circ}, \mathbf{I}_{\mathbf{B}}=600 \mathrm{~A} \angle-120^{\circ}, \mathbf{I}_{\mathbf{C}}=600 \mathrm{~A}-150 \mathrm{~A} \angle 120^{\circ}=450 \mathrm{~A} \angle 120^{\circ} .
$$

Current in the neutral element is determined by vectorally adding the three-phase currents.

$$
\begin{aligned}
\mathbf{I}_{\mathbf{N}} & =\mathrm{I}_{\mathrm{A}} \angle 0^{\circ}+\mathrm{I}_{\mathrm{B}} \angle-120^{\circ}+\mathrm{I}_{\mathrm{C}} \angle 120^{\circ} \\
\mathbf{I}_{\mathbf{N}} & =600 \mathrm{~A} \angle 0^{\circ}+600 \mathrm{~A} \angle-120^{\circ}+450 \mathrm{~A} \angle 120^{\circ} \\
& =150 \mathrm{~A} \angle-60^{\circ}
\end{aligned}
$$

\subsubsection{Operation of Circuit Protective Devices for Ground Faults}

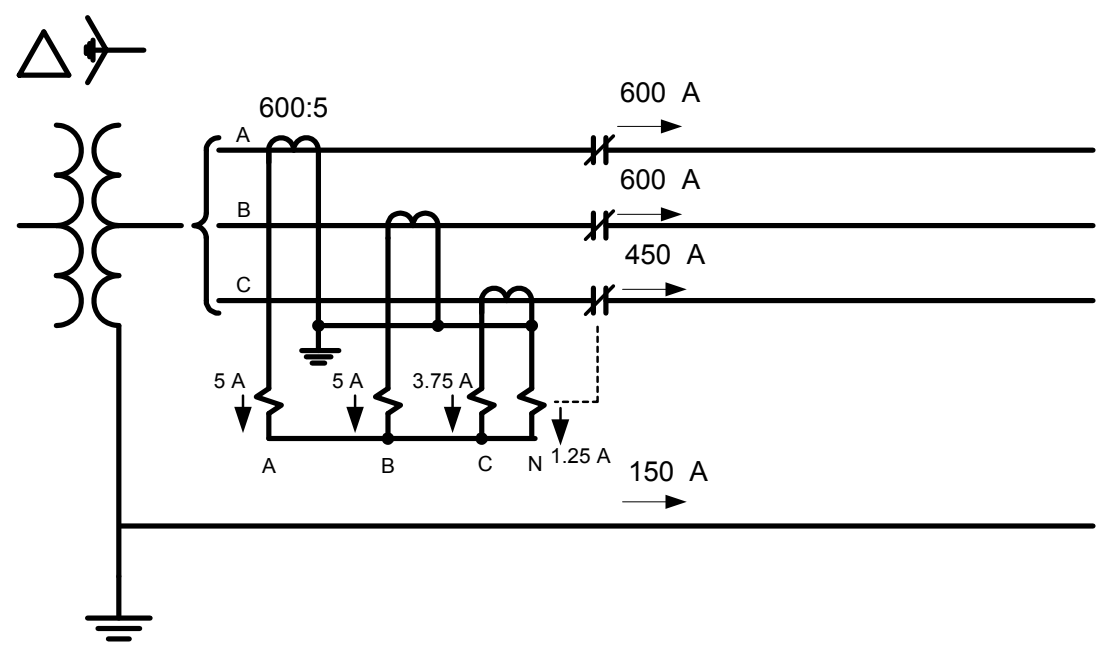

Figure 59. Diagram of substation breaker and relays with ground-sensing circuit

The equipment arrangement shown in Figure 59 is typical for a four-wire grounded system. The substation transformer, CTs, protective relay current coils, and circuit breaker are represented. For simplicity, it is assumed that all load is resistive and connected line-toground and that the phase currents are in phase with their respective voltages. Unbalanced currents resulting from phase-to-phase-connected load may not show up in the ground or neutral relay sensing circuits.

Unbalanced current flowing to the load on the circuit will result in current in the neutral relay coil $(\mathrm{N})$. The example shows loads of $600 \mathrm{~A}, 600 \mathrm{~A}$, and $450 \mathrm{~A}$, respectively, on A, B, and C phases. Taking into account the $\mathrm{CT}$ ratio of 120 , the neutral current in the relay element is $150 / 120=1.25 \mathrm{~A}$. The setting is based on two times the minimum load, which is 1.8 MVA or $157 \mathrm{~A}$.

$$
2 \mathrm{I}_{\min }=2 \frac{1,800 \mathrm{kVA}}{\sqrt{3} \times 13.2 \mathrm{kV}}=2 \times 78.73=157 \text { amperes } \quad \text { Equation } 4.2
$$

Because the setting is $157 / 120=1.31 \mathrm{~A}$ and neutral current is $1.25 \mathrm{~A}$, the breaker will not open for the unbalance caused by clearing Fault C, but the single-phase recloser (3) will open and lock out for the permanent fault. (See Figure 58.) 
Reclosers with ground fault-sensing circuits will be affected similarly by load unbalance. The same equations can be used for the recloser and the circuit breaker.

Another case to consider is the opening of a fuse (4) in Figure 58 for a fault at D. The unbalance current is $100 \mathrm{~A}$, which results in $\mathrm{I}_{\mathrm{N}}=100 \mathrm{~A} \angle-60^{\circ}$ and a relay element current of $0.833 \mathrm{~A}$. Because this is below the setting of $1.31 \mathrm{~A}$, the breaker will not trip, and the fuse (4) will isolate the fault.

The fault current that occurred at D and caused the 150-A fuse (4) to blow was significantly higher than the resultant unbalanced current (neutral current) after the fault cleared. For example, the fault current magnitude could range from 1,500 to 7,000 A. In this case, the neutral relay would see from 12.5 A to 58.3 A for approximately $0.1-0.06$ seconds (fuseclearing time). See Figure 63.

\subsubsection{Single-Phase Operation of Disconnect and Isolation Devices}

Opening the fused disconnect (5) of Figure 58 has the same effect as the cleared fault D on the neutral current. Disconnects are often opened to allow maintenance and repair of downstream equipment.

\subsubsection{Unbalanced Load Connected Line-to-Line}

Load that is connected line-to-line will not produce current in the neutral relay element. Even unequal single-phase load connected line-to-line will not produce neutral current in the ground relay. For example, unequal load connected line-to-line will result in negative sequence current but no zero-sequence neutral current because

$$
\mathrm{I}_{\mathrm{A}}+\mathrm{I}_{\mathrm{B}}+\mathrm{I}_{\mathrm{C}}=0 \text {. }
$$

Equation 4.3

The equations for the substation breaker also apply in this case. However, caution must be exercised to use the correct phase angle of the current in each phase. Figure 60 shows resistive load connected between $\mathrm{B}$ and $\mathrm{C}$ phases. Current flowing in the load equals the voltage across the load divided by the resistance. 


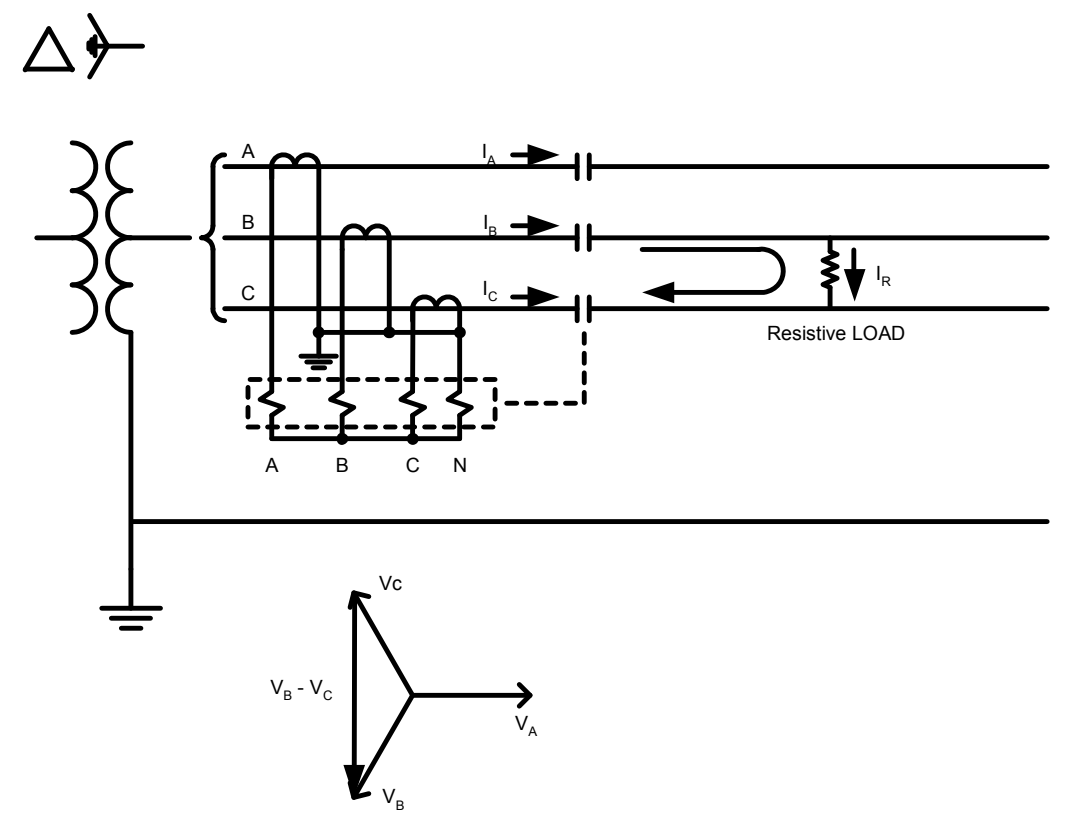

Figure 60. Current flow in phase-to-phase-connected load

For this circuit, assume:

$$
\begin{aligned}
& \mathbf{V}_{\mathbf{A}}=7,620 \square \boldsymbol{Q}^{\prime} \\
& \mathbf{V}_{\mathbf{B}}=7,620 \square \text { Úl } 20^{\circ} \\
& \mathbf{V}_{\mathbf{C}}=7,620 \square \mathbf{i} 20^{\circ} \\
& \square \ddot{\mathrm{E}}_{\mathrm{BC}}=\mathrm{V}_{\mathrm{B}}-\mathrm{V}_{\mathrm{C}}=13,200 \square \dot{\mathrm{U}} 90 \\
& \mathrm{P}_{\mathrm{L}}=100 \mathrm{~kW} \square \ddot{\mathbb{E}}_{\mathrm{L}}=100,000 \text { Watts } \div 13,200 \text { volts }=7.56 \text { amperes }
\end{aligned}
$$

For this circuit, current in Phase B returns on Phase C. Or $\mathrm{I}_{\mathrm{B}}=-\mathrm{I}_{\mathrm{C}}$.

Voltage across the load equals the difference between the voltages $V_{B}$ and $V_{C}$ or $V_{B}-V_{C}$. Current in the load equals the voltage across the load divided by the resistance or $\left(\mathrm{V}_{\mathrm{B}^{-}}\right.$ $\left.V_{C}\right) / R_{L}$. If no other load is on the circuit, then $I_{B}=I_{R}=-I_{C}$ and $I_{A}=0$.

Using the equations for the substation breaker:

$$
\begin{aligned}
& \mathbf{I}_{\mathrm{N}}=\mathbf{I}_{\mathrm{A}}+\mathbf{I}_{\mathrm{B}}+\mathbf{I}_{\mathbf{C}} \\
& \mathbf{I}_{\mathrm{A}}=0 \square \boldsymbol{U}^{\prime} \quad \mathbf{I}_{\mathrm{B}}=7.56 \square \mathrm{U}^{\prime} 90^{\circ} \quad \mathbf{I}_{\mathbf{C}}=7.56 \square \mathrm{U}^{\prime} 90^{\circ}
\end{aligned}
$$

(Current in amperes)

$$
\mathbf{I}_{\mathbf{N}}=0 \square \mathbf{0}^{\rho}+7.56 \square \mathrm{U}^{\prime} 90^{\circ} \quad 7.56 \square \mathrm{U}^{\prime} 90^{\circ}=0
$$

Equation 4.6 
Even though the load is unbalanced, it is important to consider how the load is connected, which has an effect on the phase angle of the currents flowing, as seen at the substation breaker and the respective relays.

\subsubsection{Fuse Preload Because of Unbalanced Loading or Unbalanced Voltages}

Load unbalance can cause fuses to become unselective with other protective devices (such as reclosers). This inselectivity is due to load-unbalanced currents heating the fuse element and causing a shift in the time-current characteristic. In Figure 61, the melting time as a percentage of the published time-current characteristic for a fuse is shown as a function of the load current in percentage of the fuse ampere rating. Notice that the melt time is reduced to $52 \%$ when the load current on one of the phases of the three-phase circuit is $150 \%$ of the fuse ampere rating.

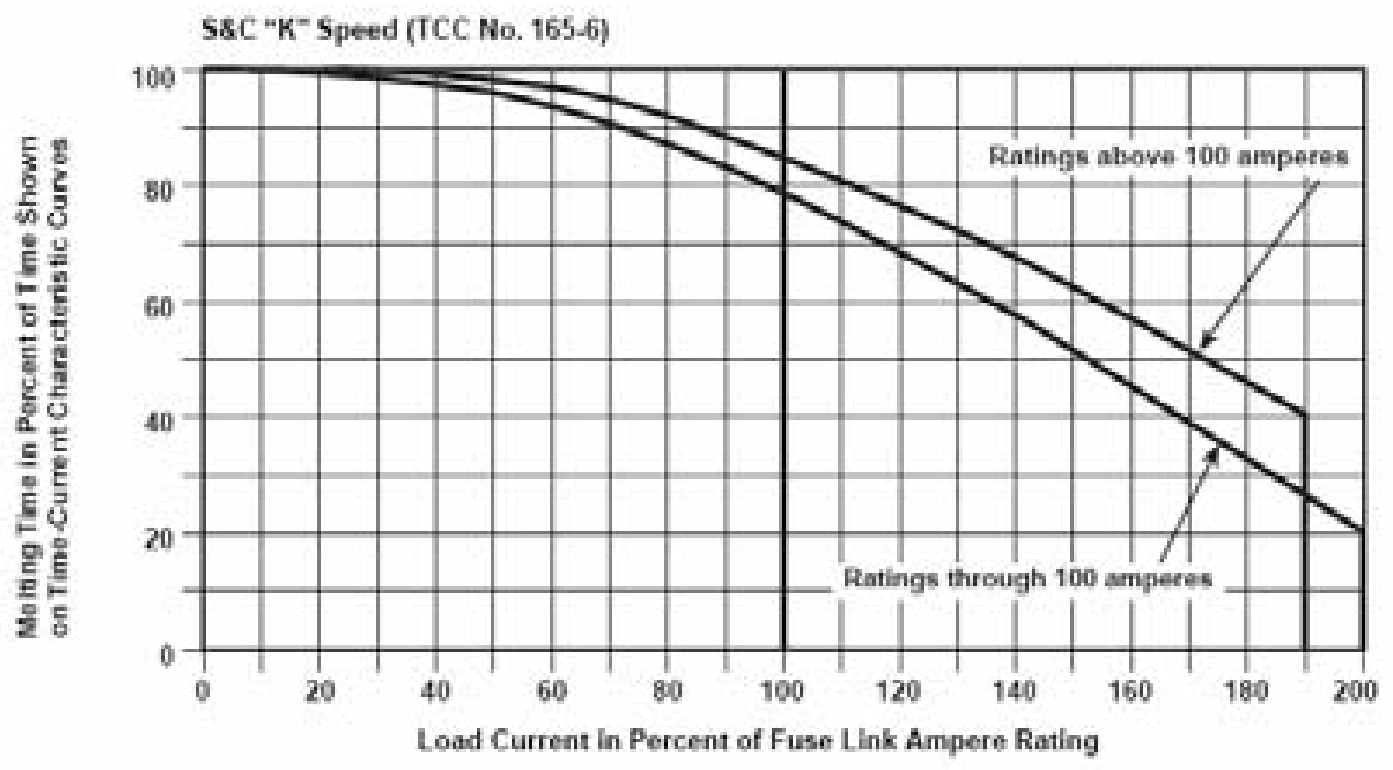

Figure 61. Reduced melt time because of preloading of universal $\mathrm{K}$ link fuses (S\&C Electric Co. 2000)

To illustrate this coordination issue, Figure 62 shows a distribution circuit with an unbalanced three-phase lateral that serves a number of single-phase loads. For simplicity, each single-phase load is fused with a $50 \mathrm{~K}$ fuse. The three-phase load, as seen by the $80 \mathrm{~K}$ fuses, is unbalanced by $20 \%$. 


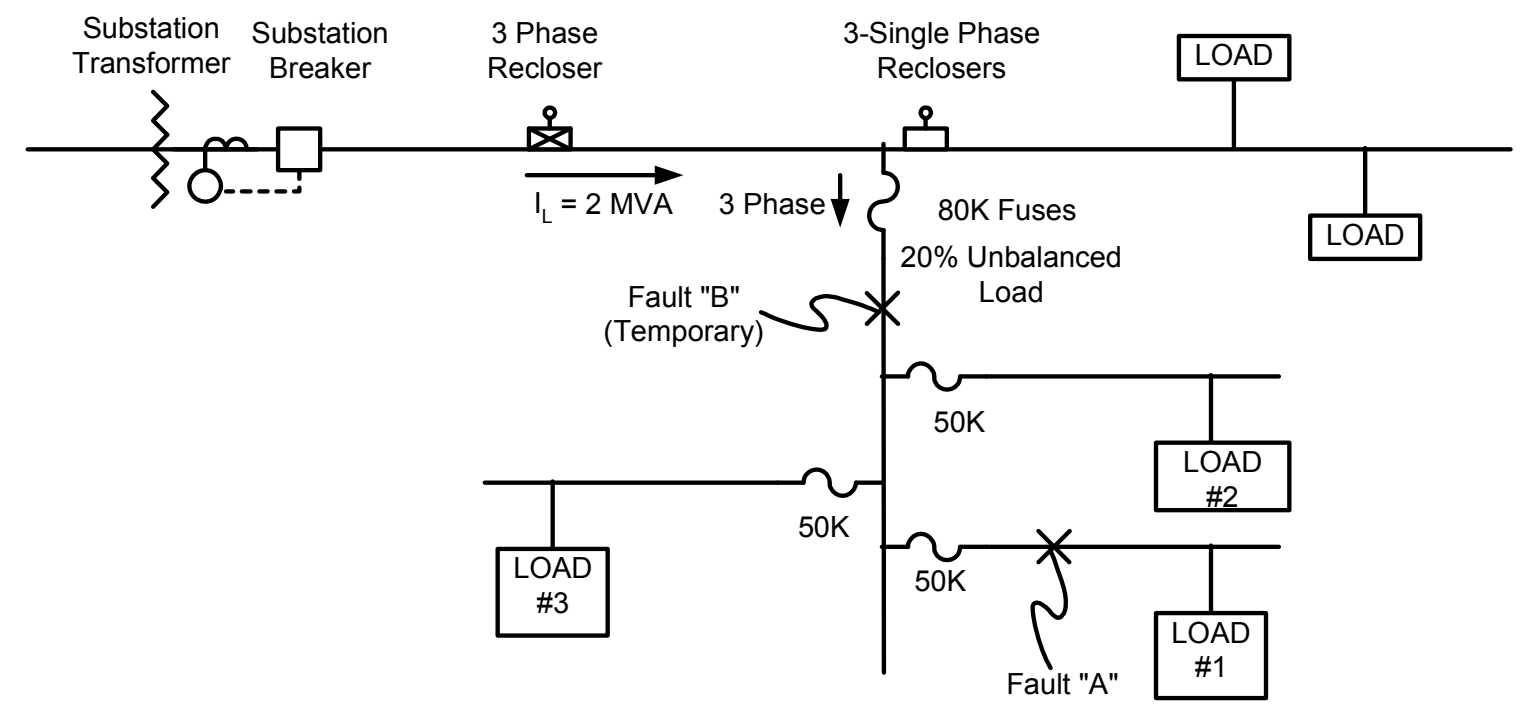

Notes:

Loading of 13.2-kV three-phase lateral:

$\mathrm{I}_{\text {Lavg }}=87.50 \mathrm{~A}$

$\mathrm{I}_{\text {Lhigh phase }}=105 \mathrm{~A}$

Figure 62. Fuse preloading causes inselectivity

The average current is $87.5 \mathrm{~A}$, and the high-load phase current is $120 \%$ of average, or $105 \mathrm{~A}$. Figure 63 shows the fuse time-current for the $80 \mathrm{~K}$ fuse (Curve 1) and the $50 \mathrm{~K}$ fuses (Curve 2) with no preloading on any phase. Notice that these curves do not intersect until a fault greater than 2,000 A occurs on this lateral. If a fault, A, of $600 \mathrm{~A}$ or greater occurs on this lateral with a $150 \%$ preload, then the $80 \mathrm{~K}$ fuse and the $50 \mathrm{~K}$ fuses would blow near simultaneously, thus causing the unnecessary loss of loads 1,2 , and 3, rather than only Load 1. 


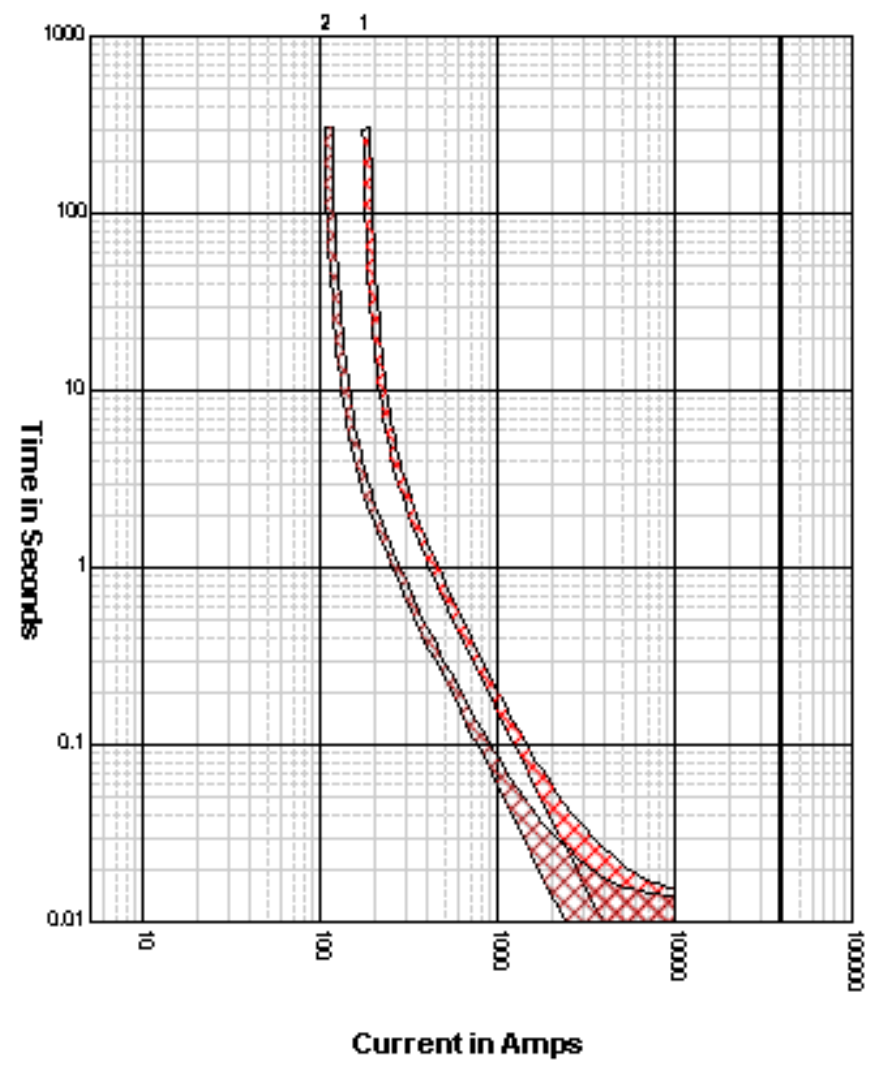

Figure 63. Coordination of $50 \mathrm{~K}$ and $80 \mathrm{~K}$ fuses (S\&C Electric Co.) 


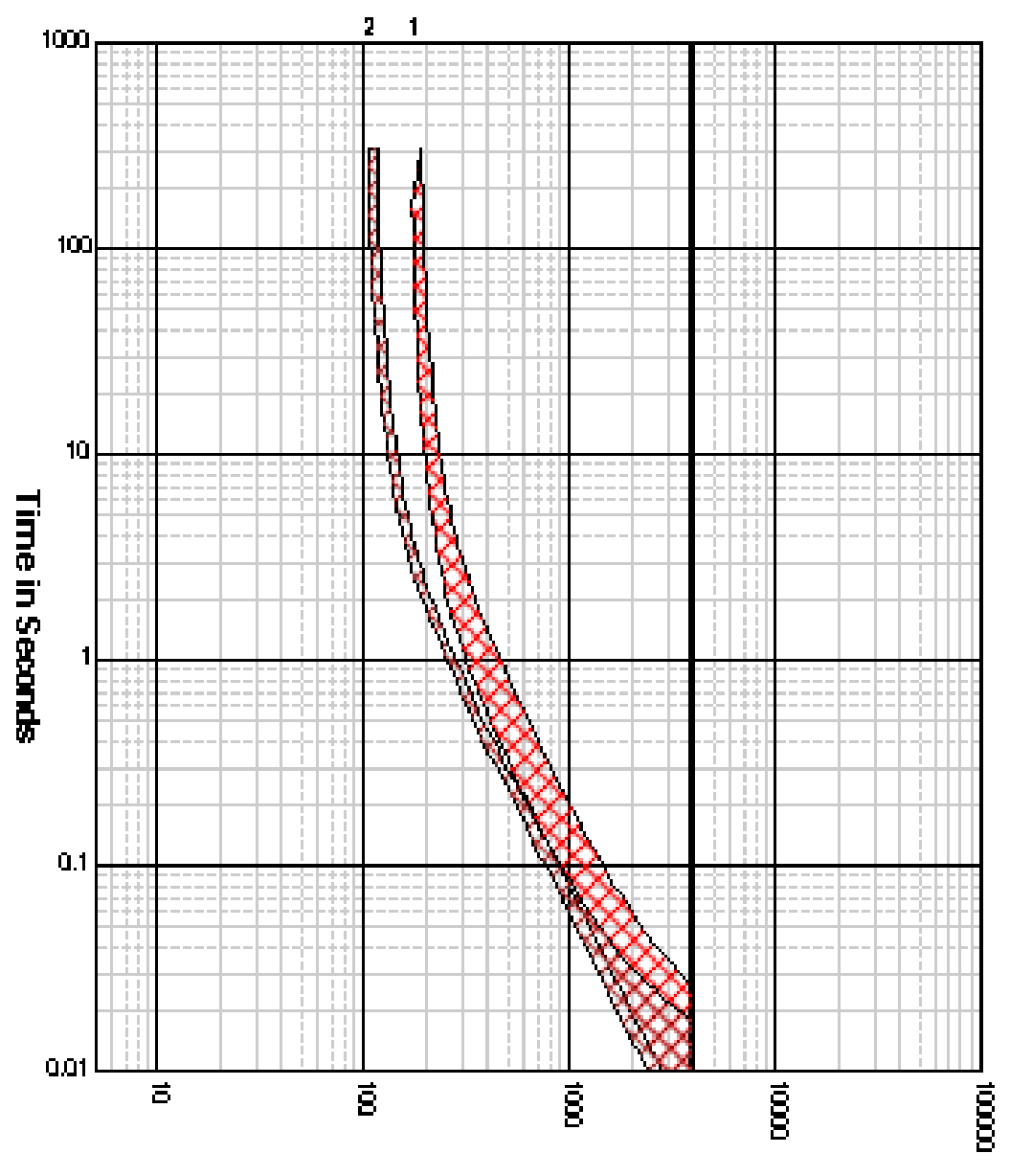

Current in Amps

Figure 64 . Inselectivity of $80 \mathrm{~K}$ and $50 \mathrm{~K}$ fuses for unbalanced loading of $20 \%$ (S\&C Electric Co.)

Another example of this inselectivity may occur when the $80 \mathrm{~K}$ fuse is preloaded because of unbalance and a temporary fault is placed at B of Figure 62 . The fault would cause the recloser to open on the fast curve, the $80 \mathrm{k}$ fuse would blow because of preload, and all of the load on the lateral would be lost before the recloser restores power to the then-unfaulted lateral. Without the preloading because of the unbalance, the $80 \mathrm{~K}$ fuse would not have blown, and the recloser would have restored power after the temporary fault cleared.

Because unbalanced voltages create unbalanced currents, similar effects of inselectivity can occur between protective devices.

\subsection{Unbalanced Voltage Conditions}

Unbalanced voltages may exist on distribution primaries and secondaries because: 
- The loads on a distribution circuit are not all three-phase but predominately single-phase

- The distribution phases are not fully transposed to balance the line impedances

- The phase conductors are different and thus have different impedances

- The transformer banks serving the three-phase loads are not identical single-phase units of the same size and impedance

- The transformer connections are either open delta or open wye

- One of the phase conductors is open upstream from the load because of a blown single-phase fuse or other open single-phase protective device

- The phase conductor is broken

- Individual units of capacitors within a capacitor bank fail and cause unbalanced conditions.

It is important to keep the three-phase circuits as closely balanced as possible to prevent overloading the equipment on the highest loaded phase. Also, it is recommended that the unbalanced loading be kept to no more than $10 \%-20 \%$ because it permits more sensitivity in setting the ground fault relay.

Reducing the unbalanced loading reduces the losses created by the neutral current in the neutral conductor. Unbalance tends to be greater during off-peak conditions and at points at which the single-phase laterals are tapped to the three-phase primary. Unbalanced threephase voltages have a significant effect on the design and operation of transformers and induction and synchronous machines. Unbalance causes a voltage with a rotation that is opposite to the voltage of a balanced supply. These opposite rotating voltages, called "negative sequence voltages," produce a flux in the air gap of these machines that counters the rotor rotation and causes high line currents in the phases that feed the machines. These higher line currents cause additional losses in the motors, generators, and distribution system.

\subsubsection{Induction Machines Operating on Unbalanced Voltages}

The National Electrical Manufacturers Association Standard MG1-14.34 (National Electrical Manufacturers Association 1978) specifies the voltage unbalance at the terminals of the polyphase induction motor is not to exceed $1 \%$. The voltage unbalance, in percentage, is defined as

Percent voltage unbalance $=100 \times \frac{\text { maximum voltage deviation from avg. voltage }}{\text { average voltage }}$

Equation 4.7

The standard goes on to say that: 
"A relatively small unbalance in voltage will cause a considerable increase in temperature rise. In the phase with the highest current, the percentage increase in temperature rise will be approximately two times the square of the percent voltage unbalance. The increase in losses and consequently the increase in average heating of the whole winding will be slightly lower than the winding with the highest current.

"To illustrate the severity of this condition, an approximate 3.5 percent voltage unbalance will cause an approximate 25 percent increase in temperature rise."

The breakdown torque and locked rotor torque are decreased for unbalanced voltages. The phase currents with unbalanced voltages are greatly unbalanced, on the order of four to five times the voltage unbalance. This creates a problem of choosing the correct overload protection because the proper setting for one unbalanced condition may not be adequate for another unbalanced condition. If the setting is raised, the motor may not be protected against overload and open phases.

The load or generation may have to be reduced below the nameplate output rating to avoid overheating. For example a $5 \%$ voltage unbalance might result in a $25 \%$ reduction in the nameplate rating. If lowering the load is not possible, the size of the motor would have to be increased if the unbalanced abnormal condition prevails and cannot be corrected.

Equation 4.7 is usually accurate enough to predict the percent voltage unbalance for unbalanced conditions less than $3 \%$, but at $5 \%$ or greater, the method of symmetrical components should be used. The example below (Table 1) compares the percent voltage imbalance determined from Equation 4.7 with the correct percent voltage unbalance calculated using symmetrical components. The correct voltage unbalance is calculated by first determining the positive sequence voltage $V_{1}$ from the measured line-to-line voltages, $\mathrm{V}_{\mathrm{A}}, \mathrm{V}_{\mathrm{B}}$, and $\mathrm{V}_{\mathrm{C}}$. The Law of Cosines is used to calculate the angles once the magnitudes are measured. The positive sequence voltage $\mathrm{V}_{1}$ is

$$
V_{1}=1 / 3\left(V_{A}+a V_{B}+a^{2} V_{C}\right) .
$$

Equation 4.8

The negative sequence voltage, $V_{2}$,

$$
\mathrm{V}_{2}=1 / 3\left(\mathrm{~V}_{\mathrm{A}}+\mathrm{a}^{2} \mathrm{~V}_{\mathrm{B}}+\mathrm{a} \mathrm{V}_{\mathrm{C}}\right),
$$

Equation 4.9

is then determined. Next the negative sequence voltage is divided by the positive sequence voltage. The percent voltage unbalance $\% \mathrm{~V}_{\mathrm{u}}$ is found from

$$
\% \mathrm{~V}_{\mathrm{u}}=\frac{\mathrm{V}_{2}}{\mathrm{~V}_{1}} \times 100 .
$$

Equation 4.10

The zero sequence voltage is

$$
\mathrm{V}_{0}=1 / 3\left(\mathrm{~V}_{\mathrm{A}}+\mathrm{V}_{\mathrm{B}}+\mathrm{V}_{\mathrm{C}}\right) \text {, }
$$


which is zero for $\mathrm{V}_{\mathrm{A}}+\mathrm{V}_{\mathrm{B}}+\mathrm{V}_{\mathrm{C}}=0$. All bolded symbols are vector quantities.

Table 1. Comparison of National Electrical Manufacturers Association Standard Method with Correct Voltage Unbalance Calculations Using Symmetrical Components

\begin{tabular}{|c|c|c|c|c|c|c|c|c|}
\hline \multirow[b]{2}{*}{$\mathrm{V}_{\mathrm{A}}$} & \multicolumn{2}{|c|}{ Case I } & \multicolumn{2}{|c|}{$\begin{array}{c}\text { Case II } \\
\text { Rev. Rotation }\end{array}$} & \multicolumn{2}{|c|}{ Case III } & \multicolumn{2}{|c|}{$\begin{array}{c}\text { Case IV } \\
\text { Rev. Rotation }\end{array}$} \\
\hline & 232 & & 225 & & 233 & & 226 & \\
\hline$V_{B}$ & 230 & & 230 & & 228 & & 228 & \\
\hline $\mathrm{V}_{\mathrm{C}}$ & 225 & & 232 & & 226 & & 233 & \\
\hline $\mathrm{V}_{\text {avg }}$ & 229 & & 229 & & 229 & & 229 & \\
\hline $\mathrm{V}_{\mathrm{A} 1}$ & 229.0 & $59.7^{\circ}$ & 228.9 & $60.7^{\circ}$ & 228.9 & $59.3^{\circ}$ & 229.0 & $60.3^{\circ}$ \\
\hline $\mathrm{V}_{\mathrm{A} 2}$ & 3.56 & $105.2^{\circ}$ & 4.13 & $-103.0^{\circ}$ & 4.30 & $76.5^{\circ}$ & 4.00 & $-76.5^{\circ}$ \\
\hline Equation 4.7 & $1.75 \%$ & & $1.75 \%$ & & $1.75 \%$ & & $1.75 \%$ & \\
\hline Equation 4.10 & $1.55 \%$ & & $1.80 \%$ & & $1.88 \%$ & & $1.75 \%$ & \\
\hline
\end{tabular}

In the above comparison, little difference exists between the percent unbalance using Equation 4.7 and Equation 4.10. Equation 4.10 will be used for all calculations of percent unbalanced voltage beginning at $3 \%$.

The effects of unbalanced voltages are greater unbalanced line currents, significantly increased losses, decreased torques, and increased slip.

\subsubsection{Positive and Negative Sequence Equivalent Circuits}

The positive and negative sequence voltages (Wagner and Evans 1933) are defined in equations 4.8 and 4.9. They each form symmetrical three-phase systems, as shown in Figure 65.

The positive sequence system consists of three equal voltages with an angle of $120^{\circ}$ between them. When applied to the stator winding, the rotating field revolves in the positive direction. When the negative sequence system is applied to the stator winding, the field rotates in the opposite direction of the field produced by the positive sequence voltages. The results can be superimposed because they act independently. 


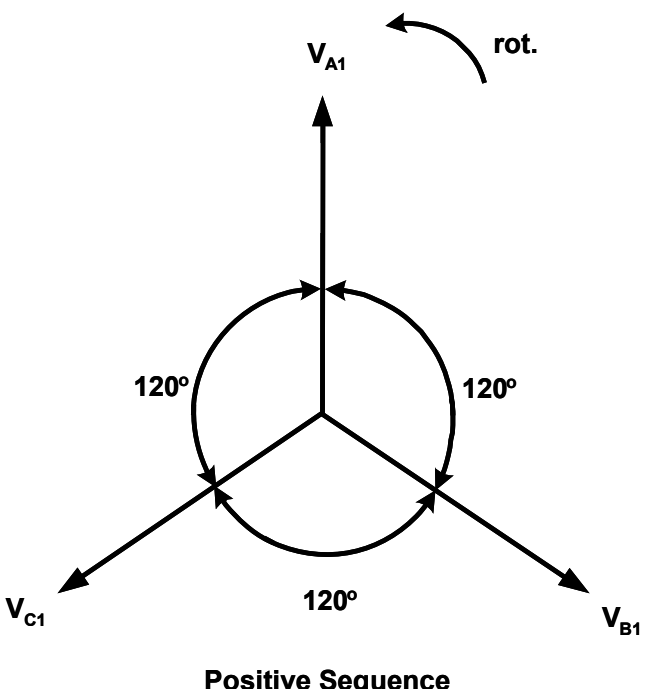

Positive Sequence
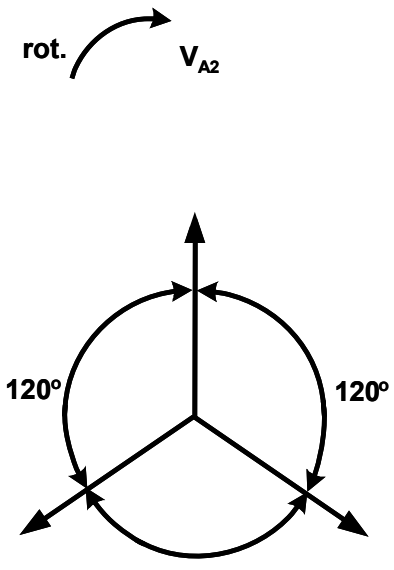

$120^{\circ}$

$\mathbf{V}_{\mathbf{c 2}}$

Negative Sequence

Note: Notice the negative sequence system of vectors rotates in the opposite direction to positive sequence. But when vectorally adding positive and negative sequence vectors, both systems must rotate in the counterclockwise direction with $\mathrm{A}_{\mathrm{V}} \mathrm{V}_{\mathrm{B} 1}$, and $\mathrm{V}_{\mathrm{C} 1}$ for positive and $\mathrm{V}_{\mathrm{A} 2}, \mathrm{~V}_{\mathrm{C} 2}$, and $\mathrm{V}_{\mathrm{B} 2}$ for negative.

Figure 65. Positive and negative sequence system of voltages

\subsection{Positive Sequence Equivalent Circuit}

The equivalent circuit is shown in Figure 66 for the positive sequence system. The symbols are:

$\mathrm{R}_{\mathrm{S}}=$ Resistance per phase for the stator

$\mathrm{R}_{\mathrm{r} 1}=$ Resistance per phase for the rotor referred to the stator

$\mathrm{X}_{\mathrm{S}}=$ Leakage reactance per phase for the stator

$\mathrm{X}_{\mathrm{r} 1}=$ Leakage reactance per phase for the rotor referred to the stator

$\mathrm{X}_{\mathrm{m}}=$ magnetizing reactance

$\mathrm{s}=$ Slip

$\mathrm{I}_{\mathrm{s} 1}=$ Stator current per phase for the positive sequence

$\mathrm{I}_{\mathrm{r} 1}=$ Rotor current per phase referred to the stator for the positive sequence.

$$
\frac{\mathrm{R}_{\mathrm{r}_{1}}}{\mathrm{~S}}=\mathrm{R}_{\mathrm{r}_{1}}+\mathrm{R}_{\mathrm{r}_{1}} \frac{(1-\mathrm{s})}{\mathrm{s}}
$$

Multiplying by $\mathrm{I}_{\mathrm{r} 1}^{2}$ results in

$$
I_{r_{1}}{ }^{2} \frac{R_{r_{1}}}{s}=I_{r_{1}}^{2} R_{r_{1}}+I_{r_{1}}^{2} R_{r_{1}} \frac{(1-s)}{s} .
$$


Now,

$\mathrm{I}_{\mathrm{r}_{1}}{ }^{2} \frac{\mathrm{R}_{\mathrm{r}_{1}}}{\mathrm{~S}}=$ Power input to the rotor per phase,

Equation 4.14

$\mathrm{I}_{\mathrm{r}_{1}}^{2} \mathrm{R}_{\mathrm{r}_{1}}=$ Copper losses in the rotor per phase,

Equation 4.15

$\mathrm{I}_{\mathrm{r}_{1}}^{2} \mathrm{R}_{\mathrm{r}_{1}} \frac{(1-\mathrm{s})}{\mathrm{S}}=$ Loss in a fictitious resistance that is equal to the gross mechanical power developed by the rotor per phase without including the loss for friction and windage,

Equation 4.16

and

$$
\mathrm{R}_{\mathrm{r}_{1}} \frac{(1-\mathrm{s})}{\mathrm{S}}=\text { Fictitious resistance. }
$$

The equivalent circuit of Figure 66 can now be changed to that of Figure 67 using equations 4.15 and 4.16. The gross mechanical power output for three phases without the friction and windage losses subtracted is from Equation 4.16.

$$
\mathrm{P}_{1(3 \text { Ф output })}=3 \mathrm{I}_{\mathrm{r}_{1}}^{2} \mathrm{R}_{\mathrm{r}_{1}} \frac{(1-\mathrm{s})}{\mathrm{s}}, \text { watts }
$$

The gross mechanical power may be represented in terms of torque, $\mathrm{T}$, or,

$$
\mathrm{P}_{1(3 \text { Ф output })}=\frac{(\mathrm{T})(2 \pi \mathrm{rpm})(\text { h.p. })}{33,000}
$$

Because the actual revolutions per minute of the rotor are equal to the synchronous revolutions per minute times (1-s) and $\mathrm{T}$ is the torque in pound-feet, then

$$
\frac{\mathrm{T} 2 \pi \mathrm{rpm} \text { sync. }(1-\mathrm{s})}{33,000}=\frac{3 \mathrm{I}_{\mathrm{r}_{1}}{ }^{2} \mathrm{R}_{\mathrm{r}_{1}}}{746} \frac{(1-\mathrm{s})}{\mathrm{s}} .
$$




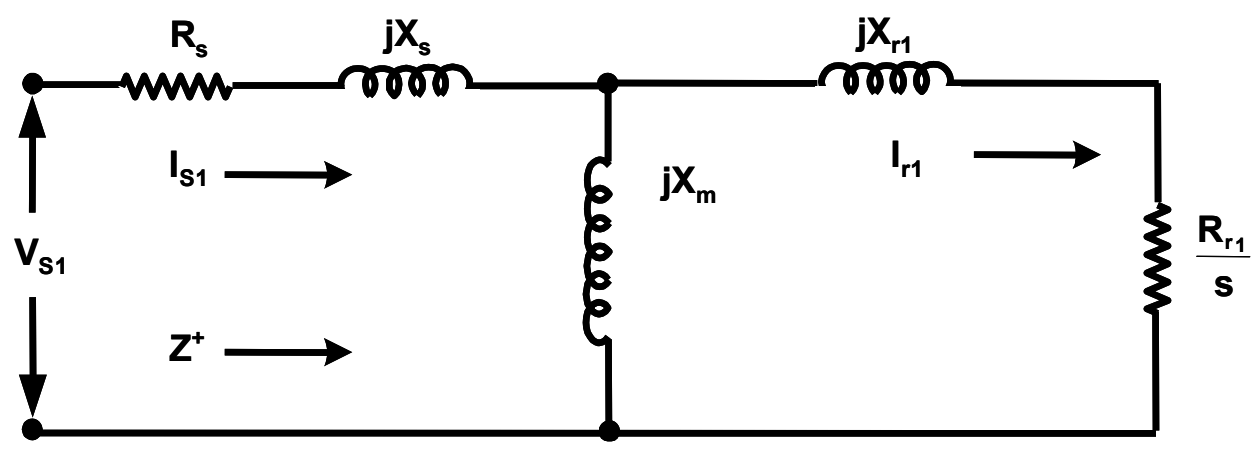

Figure 66. Positive sequence equivalent circuit for induction motor/generator

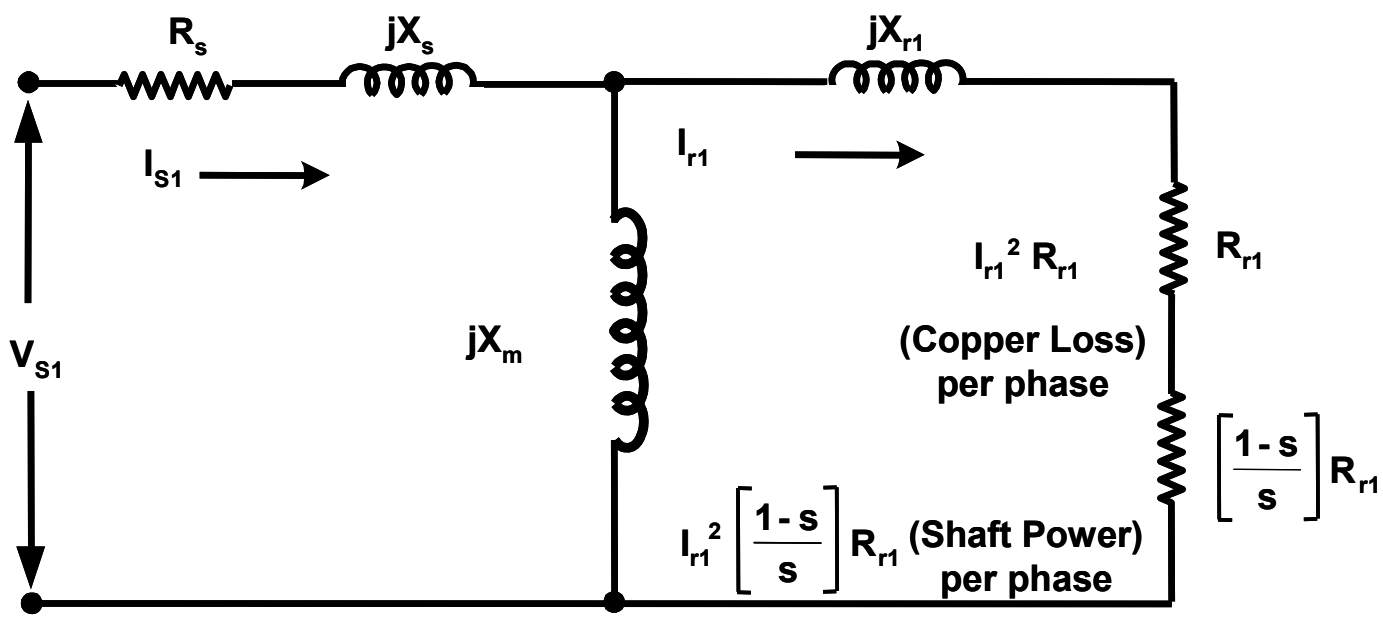

Figure 67. Positive sequence induction motor/generator equivalent circuit for copper losses and shaft power

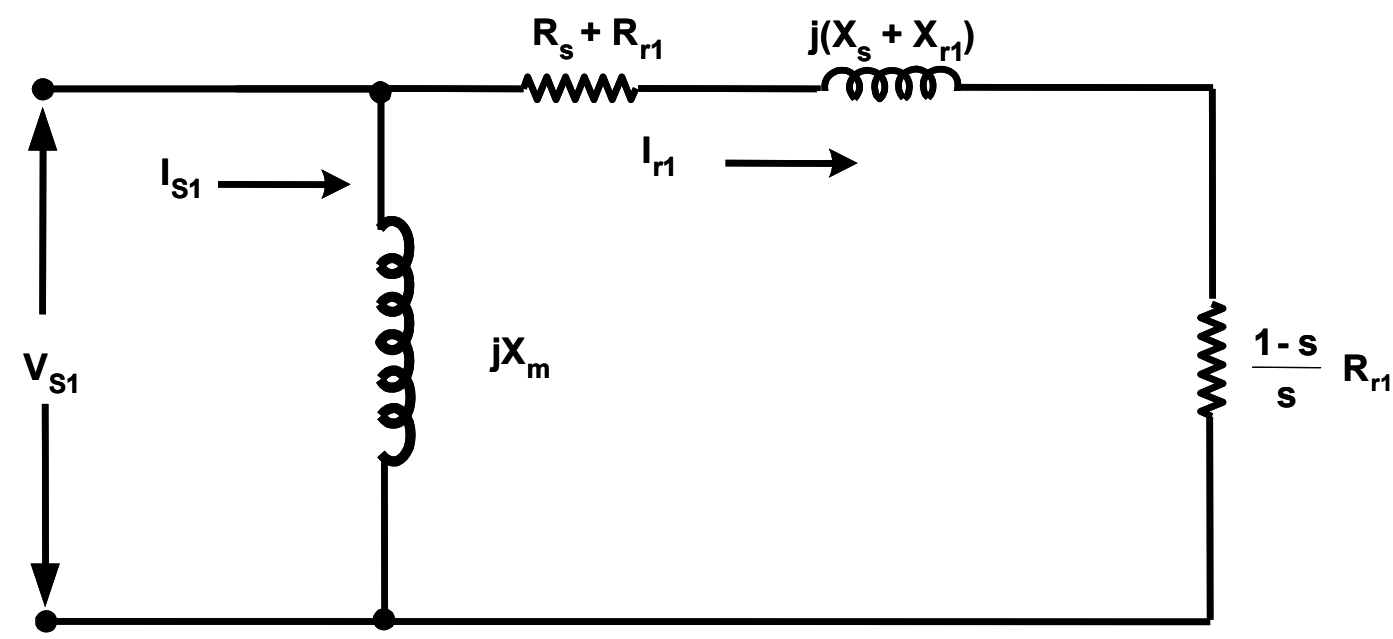

Figure 68. Simplified positive sequence induction motor/generator equivalent circuit 
The

$$
\text { rpm sync. }=\frac{120 f}{\mathrm{p}}=\frac{120 \mathrm{x} \text { frequency }}{\text { no. of poles }}
$$

and

$$
\begin{aligned}
& \mathrm{T} \mathrm{lb}-\mathrm{ft}=\frac{33,000}{2 \pi \times 746} \quad \frac{\mathrm{p}}{120 f} \frac{3 \mathrm{I}_{\mathrm{r}_{1}}{ }^{2} \mathrm{R}_{\mathrm{r}_{1}}}{\mathrm{~s}}, \\
& \mathrm{~T} \mathrm{lb} \mathrm{ft}=0.1765 \frac{\mathrm{p}}{f} \frac{\mathrm{I}_{\mathrm{r}_{1}}{ }^{2} \mathrm{R}_{\mathrm{r}_{1}}}{\mathrm{~s}} .
\end{aligned}
$$

The equivalent circuit of Figure 68 is an approximation of that in Figure 67, with the $\mathrm{j} \mathrm{X}_{\mathrm{m}}$ term moved out to the terminals. Because the magnitude of $X_{m}$ is large compared with those of the other terms in the equivalent circuit, this approximation is acceptable.

\subsection{Negative Sequence Equivalent Circuit}

The rotor is rotating in the direction of the positive sequence field with slip (s). The negative sequence field is rotating in the opposite direction at synchronous speed.

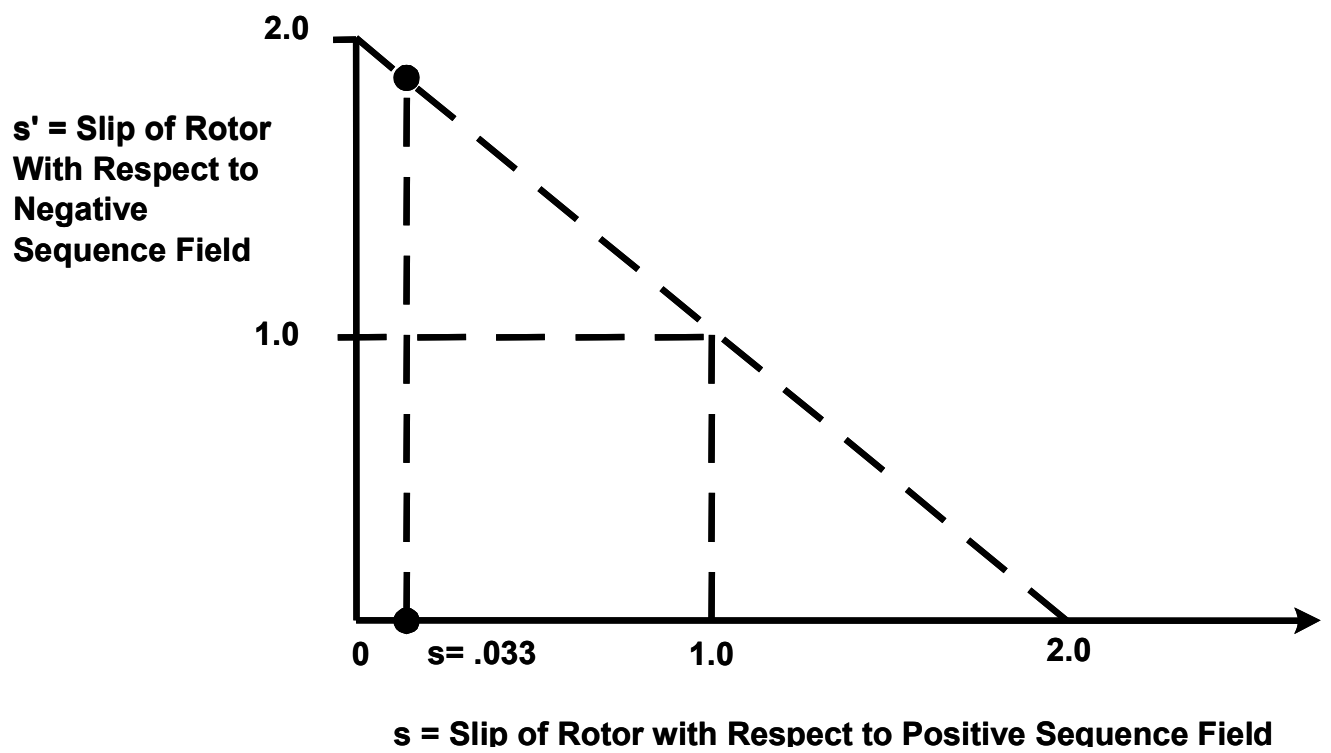

Figure 69. Slip of rotor with respect to negative sequence field and positive sequence field

If the speed of the field in the negative direction is $\omega$ and the speed of the rotor in the positive direction is $\omega(1-s)$, then the slip, with respect to the negative sequence field, is

$$
\mathrm{s}^{\prime}=\frac{\omega+\omega(1-\mathrm{s})}{\omega}=2-\mathrm{s} .
$$


Also, this could be obtained from Figure 69. When $s=0$, then $s^{\prime}=2$. When the rotor is at standstill or $s=1$, then $s^{\prime}=1$. When $s=2$, then $s^{\prime}=0$. The negative sequence equivalent circuit of Figure 71 will be similar to the positive sequence of Figure 67, but the term $\frac{(1-s)}{s} R_{r 1}$ of the positive sequence will be replaced with $\frac{\left(1-s^{\prime}\right)}{s^{\prime}} R_{r 2}$.

Because $\mathrm{s}^{\prime}=2-\mathrm{s}$, from Equation 4.24, then

$$
\begin{aligned}
\frac{\left(1-s^{\prime}\right)}{s^{\prime}} R_{r 2}= & \frac{1-(2-s)}{2-s} R_{r 2} \\
& =-\frac{(1-s)}{2-s} R_{r 2} .
\end{aligned}
$$

Equation 4.25

Because the slip (s) is small, typically $2 \%-3 \%$,

$$
-\frac{(1-\mathrm{s})}{2-\mathrm{s}} \mathrm{R}_{\mathrm{r} 2} \cong-\frac{\mathrm{R}_{\mathrm{r} 2}}{2}
$$

which is shown in the simplified equivalent circuit of Figure 72 . The negative sequence mechanical power $\mathrm{P}_{2}$ for all three phases is from Equation 4.25 and equal to

$$
\mathrm{P}_{2(3 \phi \text { output })}=-3\left(\frac{1-\mathrm{s}}{2-\mathrm{s}}\right) \mathrm{R}_{\mathrm{r}_{2}} \mathrm{I}_{\mathrm{r}_{2}}{ }^{2} \text {, watts }
$$

where $\mathrm{I}_{\mathrm{r} 2}{ }^{2}$ is the negative sequence rotor current referred to the stator. The gross mechanical power output because of both positive and negative sequence voltages is

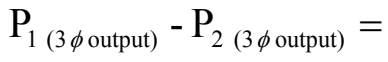

$$
\begin{aligned}
& 3 \mathrm{I}_{\mathrm{r} 1}{ }^{2} \mathrm{R}_{\mathrm{r} 1}\left(\frac{1-\mathrm{s}}{\mathrm{s}}\right)-3 \frac{1-\mathrm{s}}{2-\mathrm{s}} \mathrm{R}_{\mathrm{r} 2} \mathrm{I}_{\mathrm{r}_{2}}{ }^{2} \text {, watts . }
\end{aligned}
$$

Equation 4.28

The gross torque $\mathrm{T}$ is

$$
\mathrm{T}=0.1765 \frac{\mathrm{p}}{f} \mathrm{R}_{\mathrm{r} 2}\left[\left(\frac{\mathrm{I}_{\mathrm{r} 1}^{2}}{\mathrm{~s}}\right)-\left(\frac{\mathrm{I}_{\mathrm{r} 2}^{2}}{2-\mathrm{s}}\right)\right] \mathrm{lb}-\mathrm{ft} .
$$




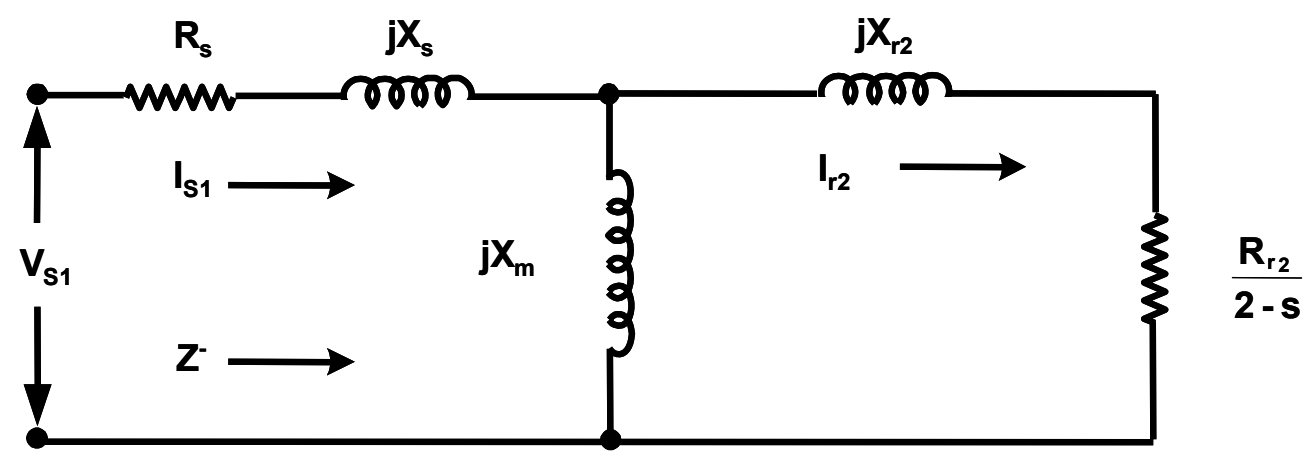

Figure 70. Negative sequence equivalent circuit for induction motor/generator

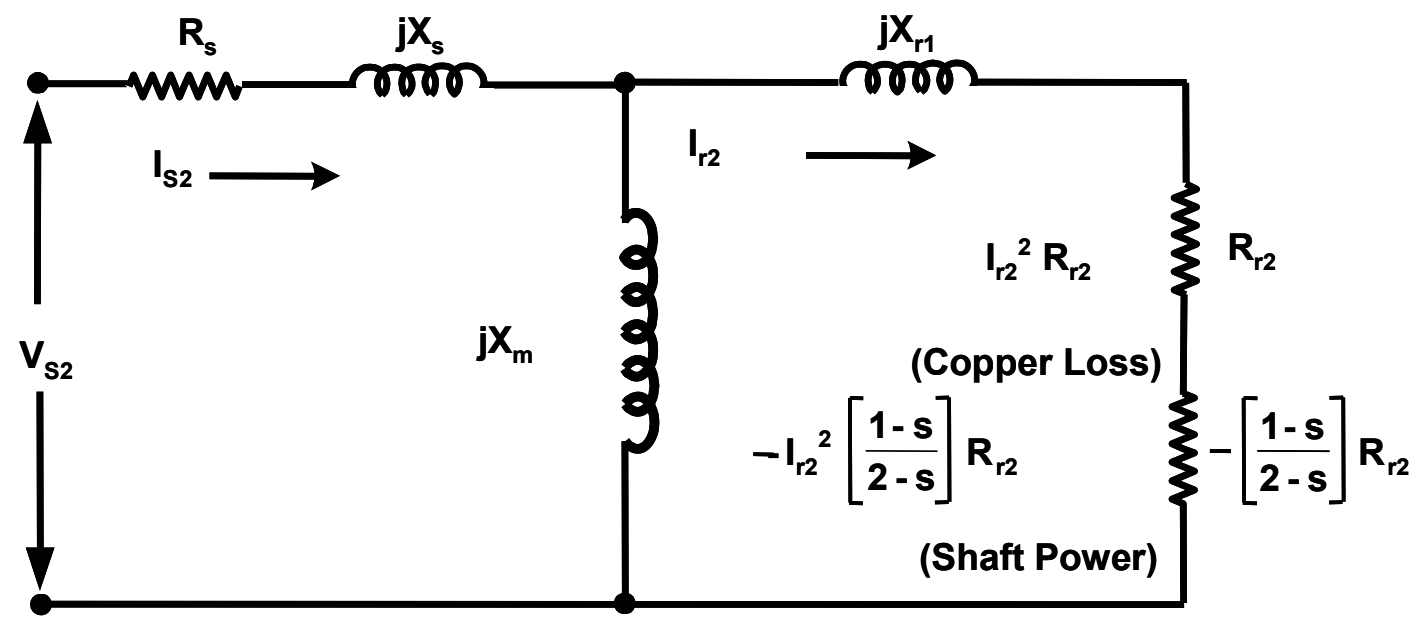

Figure 71. Negative sequence induction motor/generator equivalent circuit for copper losses and shaft power

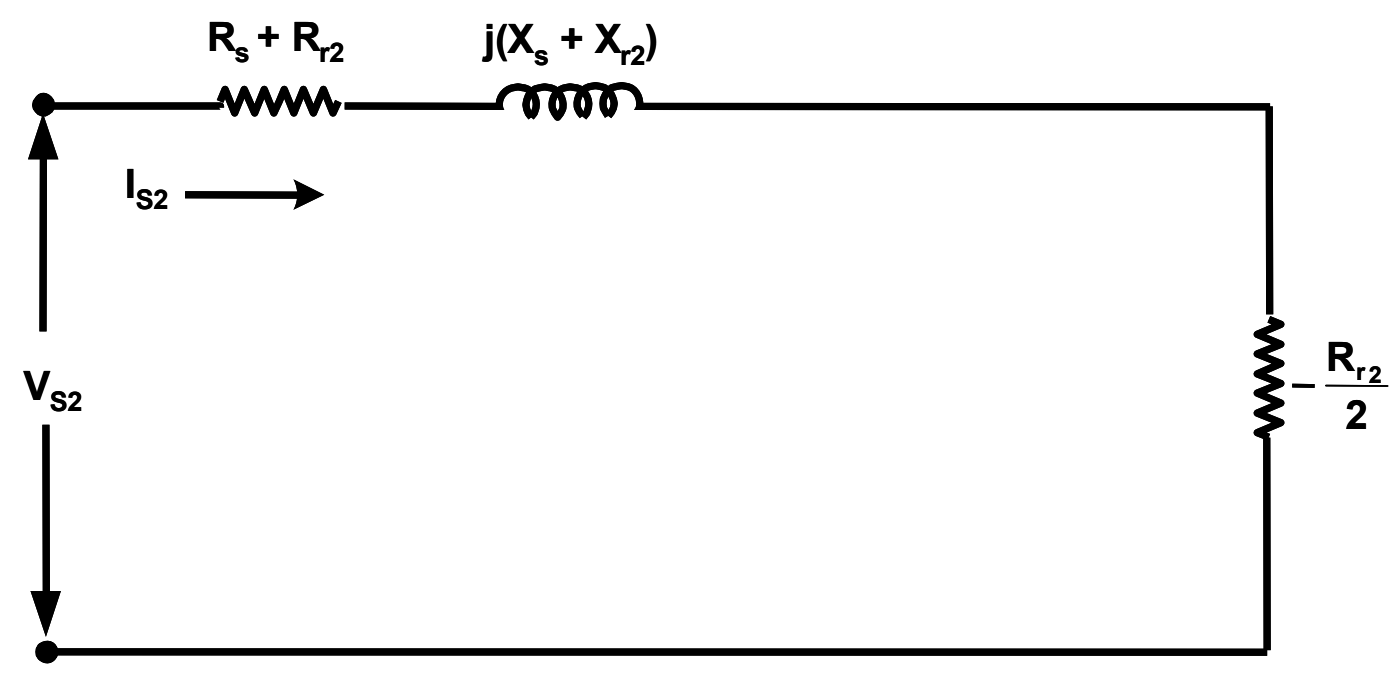

Figure 72. Simplified negative sequence induction motor/generator equivalent circuit operating at small values of slip 
Because the negative sequence power output is negative (see Equation 4.27), the power output is reduced with negative sequence voltages, which reduces the nameplate capability of the machine. An example demonstrates the significance of this.

\subsection{Negative Sequence Voltages Increase Losses}

The example uses test data from Williams (1954). Table 2 and Table 3 show the line currents for various percent unbalanced voltages, as measured for single-cage and double-cage induction motors with similar performance characteristics. These motors are 10-hp, fourpole, $220-\mathrm{V}$, three-phase, and wye-connected ungrounded. The process of determining the effects of unbalanced voltages is detailed below.

1. From the percent unbalanced voltages or the negative sequence divided by the positive sequence voltage, measure the line currents $\mathrm{I}_{\mathrm{A}}, \mathrm{I}_{\mathrm{B}}$, and $\mathrm{I}_{\mathrm{C}}$ for both $\mathrm{ABC}$ and $\mathrm{ACB}$ rotation.

2. Calculate the negative sequence currents for $3 \%, 5 \%, 8 \%, 10 \%$, and $11 \%$ negative sequence voltages. Repeat this step for the single-cage and double-cage induction machines for both ABC and ACB rotation. See Table 2 and Table 3. Two major conclusions should be noted from the test data of these tables:

A. Phase rotation affects which of the phases has the highest line current. This presents a real problem for protecting the motor/generator from overcurrent failure. At a 3\% negative sequence voltage, for the single cage-motor, Phase $\mathrm{C}$ has the highest phase current $\mathrm{I}_{\mathrm{C}}$ of $1.12 \mathrm{p}$.u. for $\mathrm{ABC}$ rotation. The Phase $\mathrm{A}$ motor has the highest phase current $\mathrm{I}_{\mathrm{A}}$ of $1.145 \mathrm{p}$.u. for $\mathrm{ACB}$ rotation. When $\mathrm{V}_{2} / \mathrm{V}_{1} \%$ is increased to $5 \%, \mathrm{I}_{\mathrm{C}}$ increases to $1.19 \mathrm{p}$.u. for $\mathrm{ABC}$ rotation, and $\mathrm{I}_{\mathrm{A}}$ is 1.245 p.u. for ACB rotation.

Overcurrent motor/generator protection is typically installed on two of the three phases. If the protection is installed on phases $\mathrm{A}$ and $\mathrm{B}$ for the singlecage motor with $\mathrm{ABC}$ rotation and set to trip at $115 \%$, then the motor protection will not trip to protect the motor because $\mathrm{I}_{\mathrm{A}}$ is 1.09 p.u. and $\mathrm{I}_{\mathrm{B}}$ is only 0.760 p.u. However, phase $\mathrm{I}_{\mathrm{C}}$ current is 1.19 p.u. Similarly, if this same motor is operated at $5 \%$ negative sequence voltage with $\mathrm{ACB}$ rotation and the protection is installed on phases $\mathrm{B}$ and $\mathrm{C}$, then the $\mathrm{I}_{\mathrm{A}}$ current is 1.245 p.u., and the $\mathrm{I}_{\mathrm{B}}$ and $\mathrm{I}_{\mathrm{C}}$ currents are only 0.980 p.u. and 0.825 p.u., respectively. Again, the protection will not trip to protect the motor because the currents $\mathrm{I}_{\mathrm{B}}$ and $\mathrm{I}_{\mathrm{C}}$ are below 0.980 p.u., yet $\mathrm{I}_{\mathrm{A}}$ current is at 1.245 p.u., or $24.5 \%$ over rated current. Following this same case, even with $\mathrm{V}_{2} / \mathrm{V}_{1} \%=11 \%$, the $\mathrm{I}_{\mathrm{B}}$ and $\mathrm{I}_{\mathrm{C}}$ currents are only 1.040 p.u. and 0.695 p.u., respectively, yet the $\mathrm{I}_{\mathrm{A}}$ current is 1.535 p.u., or $53.5 \%$ over rated current. The overcurrent protection still has not operated to trip and protect the motor. This conclusion indicates that negative sequence current protection must be used to protect the motor or generator to prevent failure because of unbalanced voltages. This condition is not the same for $A B C$ rotation. In fact, at $V_{2} / V_{1} \%=8 \%, I_{A}=1.165$ p.u., $I_{B}=$ 0.620 p.u., and $\mathrm{I}_{\mathrm{C}}=1.31$ p.u. Therefore, the overcurrent motor protection under worst-case conditions would trip the motor at $\mathrm{I}_{\mathrm{C}}=1.31 \mathrm{p}$.u. if the protection was installed on phases A and B. 
Although the problem is less severe for the double-cage motor, the phase current $\mathrm{I}_{\mathrm{A}}$ for rotation $\mathrm{ACB}$ reaches 1.48 p.u., or $48 \%$ over rated current at $\mathrm{V}_{2} / \mathrm{V}_{1} \%=11 \%$. Yet overcurrent tripping would occur for $\mathrm{ABC}$ rotation at $\mathrm{V}_{2} / \mathrm{V}_{1} \%=8 \%$.

B. The percent negative sequence current is independent of phase rotation. For example, referring to the single-cage motor at $\mathrm{V}_{2} / \mathrm{V}_{1} \%=5 \%$, the $\mathrm{I}_{2} / \mathrm{I}_{1} \%=$ $25.08 \%$ for $\mathrm{ABC}$ rotation and $\mathrm{I}_{2} / \mathrm{I}_{1} \%=25.68 \%$ for $\mathrm{ACB}$ rotation. The same is true for the double-cage motor. The phase rotation does not affect the result, but the percent negative sequence current is somewhat less at $21.6 \%$. This confirms the need for negative sequence current protection because a safe level of overcurrent can be selected to protect the motor/generator that is independent of phases selected for protection and the phase rotation applied to the terminals of the motor or generator.

3. From the motor characteristics of Table 4, determine the positive and negative sequence equivalent circuits and the positive $\mathrm{Z}^{+}$and negative sequence $\mathrm{Z}^{-}$ impedances. The positive sequence impedance $Z^{+}$is shown in Figure 66 and is given as Equation 4.30; the negative sequence impedance $Z^{-}$is shown in Figure 70 and is given as Equation 4.31 .

$\mathrm{Z}^{+}=\left[\mathrm{R}_{\mathrm{s}}+\frac{\left(\frac{\mathrm{R}_{\mathrm{r} 1}}{\mathrm{~s}}\right) \mathrm{X}_{\mathrm{m}}{ }^{2}}{\left(\frac{\mathrm{R}_{\mathrm{r} 1}}{\mathrm{~s}}\right)^{2}+\left(\mathrm{X}_{\mathrm{r} 1}+\mathrm{X}_{\mathrm{m}}\right)^{2}}\right]+\mathrm{j}\left[\mathrm{X}_{\mathrm{s}}+\frac{\left(\frac{\mathrm{R}_{\mathrm{r} 1}}{\mathrm{~s}}\right)^{2} \mathrm{X}_{\mathrm{m}}+\mathrm{X}_{\mathrm{r} 1} \mathrm{X}_{\mathrm{m}}\left(\mathrm{X}_{\mathrm{r} 1}+\mathrm{X}_{\mathrm{m}}\right)}{\left(\frac{\mathrm{R}_{\mathrm{r} 1}}{\mathrm{~s}}\right)^{2}+\left(\mathrm{X}_{\mathrm{r} 1}+\mathrm{X}_{\mathrm{m}}\right)^{2}}\right]$,

Equation 4.30

$Z^{-}=\left[R_{s}+\frac{\left(\frac{R_{r 2}}{2-s}\right) X_{m}{ }^{2}}{\left(\frac{R_{r 2}}{2-s}\right)^{2}+\left(X_{r 2}+X_{m}\right)^{2}}\right]+j\left[X_{s}+\frac{\left(\frac{R_{r 2}}{2-s}\right)^{2} X_{m}+X_{r 2} X_{m}\left(X_{r 2}+X_{m}\right)}{\left(\frac{R_{r 2}}{2-s}\right)^{2}+\left(X_{r 2}+X_{m}\right)^{2}}\right]$.

Equation 4.31 
Table 2. Per-Unit Line Currents for Percent Negative Sequence Voltage Single-Cage Induction Motor

\begin{tabular}{|c|c|c|c|c|c|c|c|c|c|}
\hline $\mathrm{V}_{2} / \mathrm{V}_{1} \%$ & $\mathrm{I}_{\mathrm{A}}$ & $\begin{array}{l}\text { Ref } \\
\text { ngle }= \\
0\end{array}$ & $\mathrm{I}_{\mathrm{B}}$ & angle & $\mathrm{I}_{\mathrm{C}}$ & angle & $\mathrm{I}_{1}$ & $\mathrm{I}_{2}$ & $\mathrm{I}_{2} / \mathrm{I}_{1} \%$ \\
\hline \multicolumn{10}{|c|}{ Single Cage ABC Rotation } \\
\hline 3.000 & 1.045 & 0.000 & 0.855 & -108.556 & 1.120 & 133.638 & 1.001 & 0.155 & 15.437 \\
\hline 5.000 & 1.090 & 0.000 & 0.760 & -102.181 & 1.190 & 141.371 & 0.999 & 0.251 & 25.086 \\
\hline 8.000 & 1.165 & 0.000 & 0.620 & -91.012 & 1.310 & 151.757 & 0.997 & 0.399 & 40.052 \\
\hline 10.000 & 1.230 & 0.000 & 0.520 & -80.787 & 1.410 & 158.652 & 0.997 & 0.514 & 51.565 \\
\hline 11.000 & 1.262 & 0.000 & 0.486 & -78.353 & 1.441 & 160.712 & 0.999 & 0.551 & 55.206 \\
\hline & \multicolumn{9}{|c|}{ Single Cage ACB Rotation } \\
\hline 3.000 & 1.145 & 0.000 & 0.975 & 131.161 & 0.890 & -124.435 & 0.154 & 0.997 & 647.554 \\
\hline 5.000 & 1.245 & 0.000 & 0.980 & 138.578 & 0.825 & -128.196 & 0.257 & 0.999 & 389.448 \\
\hline 8.000 & 1.385 & 0.000 & 1.000 & 148.378 & 0.748 & -135.496 & 0.400 & 0.999 & 249.577 \\
\hline 10.000 & 1.485 & 0.000 & 1.030 & 154.850 & 0.705 & -141.618 & 0.502 & 1.001 & 199.494 \\
\hline 11.000 & 1.535 & 0.000 & 1.040 & 157.737 & 0.695 & -145.465 & 0.552 & 1.001 & 181.522 \\
\hline
\end{tabular}

Table 3. Per-Unit Line Currents for Percent Negative Sequence Voltage Double-Cage Induction Motor

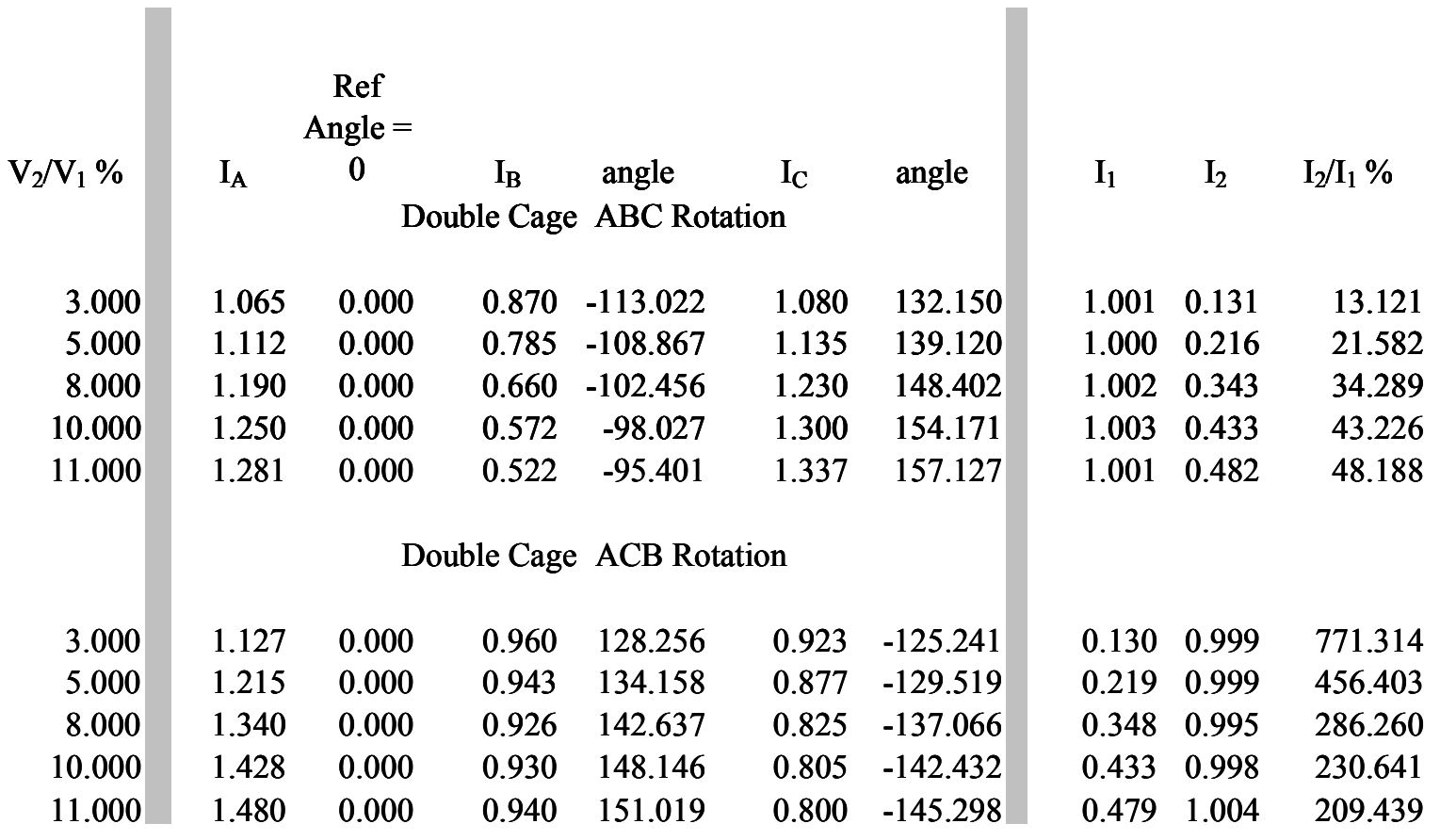


4. From the negative sequence voltage $V_{2}$ and the negative sequence impedance $Z^{-}$, the negative sequence amperes are calculated and displayed in Table 5. Calculate the negative sequence stator losses from $3 \mathrm{I}_{\mathrm{s} 2}{ }^{2} \mathrm{R}_{1}$, where $\mathrm{I}_{\mathrm{s} 2}$ is the negative sequence stator current of Table 5 and Rs is the stator resistance of $0.153 \Omega / \Phi$, shown in Table 4 .

5. The negative sequence rotor losses are calculated from $(2-s) 3 \mathrm{I}_{\mathrm{r} 2}^{2}\left(\mathrm{R}^{-}-\mathrm{R}_{\mathrm{s}}\right)$, where the negative sequence rotor current $\mathrm{I}_{\mathrm{r} 2}$ can be approximated with the negative sequence stator current $\mathrm{I}_{\mathrm{s} 2}{ }^{[4]}, \mathrm{R}^{-}$is the resistance portion of the negative sequence impedance $Z^{-}$of Table 4 , and $R_{s}$ is the stator resistance. The total negative sequence losses are found by adding the negative sequence stator and rotor losses.

Table 4. Double- and Single-Cage Characteristics for 10-hp, Four-Pole, 220-V, Three-Phase, Wye-Connected Ungrounded Motors

\begin{tabular}{|c|c|}
\hline Double-Cage Motor & Single-Cage Motor \\
\hline $\mathrm{R}_{\mathrm{s}}=0.153 \Omega / \Phi$ & $\mathrm{R}_{\mathrm{s}}=0.153 \Omega / \Phi$ \\
\hline $\begin{array}{l}X_{s}=0.500 \Omega / \Phi \\
X_{m}=14.3 \Omega / \Phi\end{array}$ & $\begin{array}{l}X_{s}=0.500 \Omega / \Phi \\
X_{m}=14.3 \Omega / \Phi\end{array}$ \\
\hline $\begin{aligned} \mathrm{R}_{\mathrm{r} 1} & =0.188 \Omega / \Phi @ \\
\mathrm{~s} & =0.033(2 \mathrm{~Hz})\end{aligned}$ & $\begin{aligned} \operatorname{Rr} 1 & =0.188 \Omega / \Phi @ \\
\mathrm{~s} & =0.033(2 \mathrm{~Hz})\end{aligned}$ \\
\hline $\begin{aligned} \operatorname{Rr} 2 & =0.507 \Omega / \Phi @ \\
\mathrm{~s} & =1.967(118 \mathrm{~Hz})\end{aligned}$ & $\begin{aligned} \mathrm{R}_{\mathrm{r} 2} & =0.205 \Omega / \Phi @ \\
\mathrm{~s} & =1.967(118 \mathrm{~Hz})\end{aligned}$ \\
\hline $\begin{aligned} \mathrm{X}_{\mathrm{r} 1} & =0.760 \Omega / \Phi @ \\
\mathrm{~s} & =0.033(2 \mathrm{~Hz})\end{aligned}$ & $\begin{aligned} \mathrm{X}_{\mathrm{r} 1} & =0.420 \Omega / \Phi @ \\
\mathrm{~s} & =0.033(2 \mathrm{~Hz})\end{aligned}$ \\
\hline $\begin{aligned} \mathrm{X}_{\mathrm{r} 2} & =0.482 \Omega / \Phi @ \\
\mathrm{~s} & =1.967(118 \mathrm{~Hz})\end{aligned}$ & $\begin{aligned} \mathrm{X}_{\mathrm{r} 2} & =0.415 \Omega / \Phi @ \\
\mathrm{~s} & =1.967(118 \mathrm{~Hz})\end{aligned}$ \\
\hline$Z^{+}=4.01+j 2.43=4.703^{\prime} 1.2^{\circ}$ & $Z^{+}=4.02+\mathrm{j} 2.13=4.55 \bigsqcup_{28.0^{\circ}}$ \\
\hline$Z^{-}=0.395+\mathrm{j} 0.970=1.05 \square 7.8^{\circ}$ & $Z^{-}=0.252+\mathrm{j} 0.903=.938 \backslash 74.4^{\circ}$ \\
\hline$@ 10 \% \frac{\mathrm{V}_{2}}{\mathrm{~V}_{1}}$ & $@ 10 \% \frac{\mathrm{V}_{2}}{\mathrm{~V}_{1}}$ \\
\hline$v_{2}=\frac{220}{\sqrt{3}} \times 0.10=12.7$ volts & $V_{2}=\frac{220}{\sqrt{3}} \times 0.10=12.7$ volts \\
\hline $\mathrm{I}_{2}=\frac{\mathrm{V}^{2}}{\mathrm{Z}^{-}}=\frac{12.7}{1.05}=12.1$ amperes & $\mathrm{I}_{2}=\frac{\mathrm{V}^{2}}{\mathrm{Z}^{-}}=\frac{12.7}{.938}=13.54$ amperes \\
\hline$\frac{\mathrm{Z}^{+}}{\mathrm{Z}^{-}}=\frac{4.70}{1.05}=4.48$ & $\frac{Z^{+}}{Z^{-}}=\frac{4.55}{0.938}=4.85$ \\
\hline
\end{tabular}


Table 5. Single-Cage Induction Motor - 10 hp, 220 V, Four Pole, Wye-Connected Ungrounded

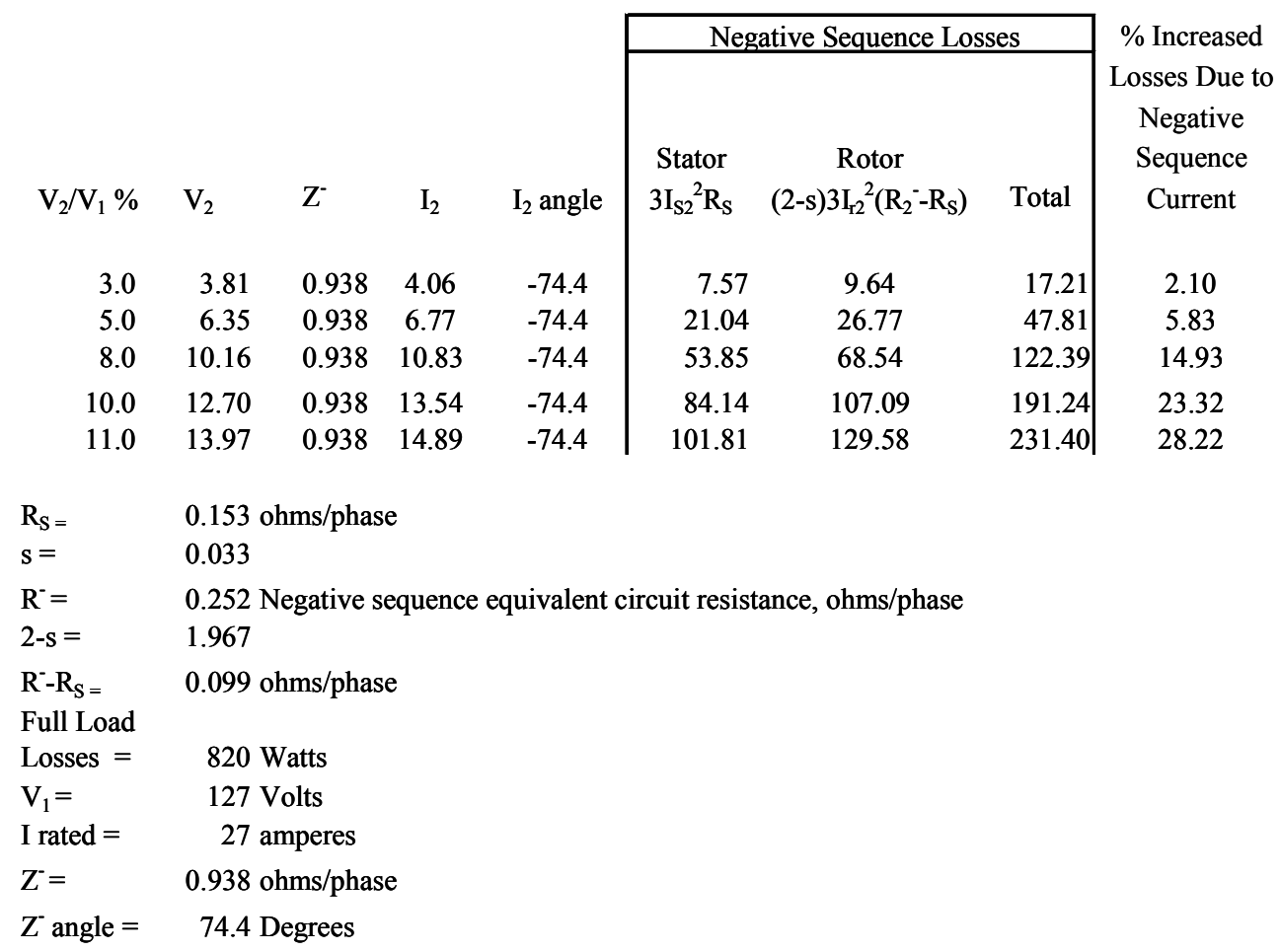

Table 6. Double-Cage Induction Motor - 10 hp, 220 V, Four Pole, Wye-Connected Ungrounded

\begin{tabular}{|c|c|c|c|c|c|c|c|c|}
\hline & & & & & & & & \\
\hline & & & & & $\mathrm{Neg}$ & ative Sequence Loss & & $\%$ Increased \\
\hline $\mathrm{V}_{2} / \mathrm{V}_{1} \%$ & $\mathrm{~V}_{2}$ & $Z^{-}$ & $\mathrm{I}_{2}$ & $\mathrm{I}_{2}$ angle & $\begin{array}{l}\text { Stator } \\
3 \mathrm{I}_{\mathrm{S} 2}{ }^{2} \mathrm{R}_{\mathrm{S}}\end{array}$ & $\begin{array}{c}\text { Rotor } \\
(2-\mathrm{s}) 3 \mathrm{I}_{\mathrm{r} 2}^{2}\left(\mathrm{R}_{2}^{-}-\mathrm{R}_{\mathrm{S}}\right)\end{array}$ & Total & $\begin{array}{c}\text { Sequence } \\
\text { Current }\end{array}$ \\
\hline 3.000 & 3.81 & 1.05 & 3.63 & -67.8 & 6.043438 & 18.80 & 24.85 & 3.03 \\
\hline 5.000 & 6.35 & 1.05 & 6.05 & -67.8 & 16.78733 & 52.23 & 69.02 & 8.42 \\
\hline 8.000 & 10.16 & 1.05 & 9.68 & -67.8 & 42.97556 & 133.71 & 176.68 & 21.55 \\
\hline 10.000 & 12.70 & 1.05 & 12.10 & -67.8 & 67.14931 & 208.92 & 276.06 & 33.67 \\
\hline 11.000 & 13.97 & 1.05 & 13.30 & -67.8 & 81.25066 & 252.79 & 334.04 & 40.74 \\
\hline $\mathrm{R}_{\mathrm{S}=}$ & $0.153 \mathrm{c}$ & $\mathrm{ms} / \mathrm{pha}$ & & & & & & \\
\hline$s=$ & 0.033 & & & & & & & \\
\hline $\mathrm{R}^{-}=$ & $0.395 \mathrm{~N}$ & egative & equence & quivalent & ircuit resist & ance, ohms/phase & & \\
\hline $2-\mathrm{s}=$ & 1.967 & & & & & & & \\
\hline $\mathrm{R}^{-}-\mathrm{R}_{\mathrm{S}}=$ & $0.242 \mathrm{c}$ & $\mathrm{ms} / \mathrm{pha}$ & & & & & & \\
\hline Full Load & & & & & & & & \\
\hline Losses $=$ & $820 \mathrm{~V}$ & atts & & & & & & \\
\hline$V_{1}=$ & $127 \mathrm{Y}$ & & & & & & & \\
\hline I rated $=$ & $27 a$ & nperes & & & & & & \\
\hline$Z^{-}=$ & $1.05 \mathrm{c}$ & $\mathrm{ms} / \mathrm{pha}$ & & & & & & \\
\hline $\mathrm{Z}^{-}$angle $=$ & $67.8 \mathrm{I}$ & egrees & & & & & & \\
\hline
\end{tabular}


6. The percent increased losses because of the negative sequence currents are determined by dividing the total negative sequence losses of Step 5 by the full load losses.

An example using the double-cage induction motor applied to a $10 \%$ negative sequence supply voltage illustrates steps $3-5$. From Table 6:

The negative sequence stator losses are

$$
3 \mathrm{I}_{\mathrm{s} 2}^{2} \mathrm{R}_{\mathrm{s}}=(3)(12.10)^{2}(0.153)=67.2 \mathrm{~W} .
$$

The negative sequence rotor losses are

$$
\begin{array}{r}
(2-\mathrm{s}) 3 \mathrm{I}_{\mathrm{r} 2}^{2}\left(\mathrm{R}^{-}-\mathrm{R}_{\mathrm{s}}\right)=(2-0.033)(3)(12.10)_{2}(0.395-0.153)=209.1 \mathrm{~W} . \\
\text { Equation } 4.33
\end{array}
$$

The total negative sequence losses $=276.3 \mathrm{~W}$.

Equation 4.34

The full load losses are equal to $820 \mathrm{~W}$. Therefore, the percent increased losses because of a $10 \%$ negative sequence voltage is

$$
\% \Delta \text { losses }\left(@ \mathrm{~V}_{2} / \mathrm{V}_{1}=10 \%\right)=276.3 / 820 \times 100=33.7 \% \text {. Equation } 4.35
$$

This value is shown in Table 6 for $\mathrm{V}_{2} / \mathrm{V}_{1} \%=10 \%$. The negative sequence losses are proportional to the square of the negative sequence voltage. Or, at $11 \%$ negative sequence voltage, the increased losses are, from Equation 4.35, equal to

$$
\left(\frac{11 \%}{10 \%}\right)^{2} 33.7 \%=40.7 \% \text {. }
$$

Equation 4.36

Notice that the percent increased losses are much higher for the double-cage motor/generator than for the single-cage motor/generator at the same percent negative sequence voltage. This is primarily because the negative sequence resistance of the rotor $(0.507 \Omega / \Phi)$ for the double-cage motor is much higher than the positive sequence resistance of the rotor $(0.188 \Omega / \Phi)$. By comparison, the negative sequence resistance of the rotor $(0.205 \Omega / \Phi)$ for the single-cage motor is only slightly higher than the positive sequence resistance $(0.188 \Omega / \Phi)$. For example, at $\mathrm{V}_{2} / \mathrm{V}_{1} \%=10 \%$, the increased losses are $23.3 \%$ for the single cage but $33.7 \%$ for the double cage. This is a significant problem because the double-cage machines are typically the larger motor and generator units. 


\subsection{Negative Sequence Voltage Affects Shaft Output Power and Reduces Capacity} The next series of questions involves how negative sequence voltage affects the shaft output power and increased losses reduce the nameplate capacity. The gross mechanical power of the machine because of the positive and negative sequence voltage will be calculated. Again, using the test data, two examples - one for the single-cage motor and one for the double-cage motor-are considered.

For a negative sequence voltage of $10 \%$, applied to a single-cage motor,

$$
\begin{aligned}
& I_{1}=\frac{V_{1}}{Z^{+}}=\frac{127}{4.55}=27.9 \text { amperes, and } \\
& I_{2}=\frac{V_{2}}{Z^{-}}=\frac{12.7}{.938}=13.54 \text { amperes. }
\end{aligned}
$$

Because $\mathrm{R}_{\mathrm{r} 1}=0.188 \Omega / \Phi$ and $\mathrm{R}_{\mathrm{r} 2}=0.205 \Omega / \Phi$, applying equations 4.23 and 4.27 results in

$$
\begin{aligned}
& \mathrm{P}_{1(3 \Phi \text { output })}-\mathrm{P} 2_{(3 \Phi \text { output })}=3 \mathrm{I}_{\mathrm{r} 1}^{2} \mathrm{R}_{\mathrm{r} 1} \frac{1-\mathrm{s}}{\mathrm{S}}-3 \mathrm{I}_{\mathrm{r} 2}^{2} \mathrm{R}_{\mathrm{r} 2} \frac{1-\mathrm{s}}{2-\mathrm{S}} \\
& \mathrm{P}_{3 \Phi \text { output }}=(3)(27.9) 2(0.188) \frac{(1-.033)}{.033}-(3)(13.54) 2(0.205) \frac{(1-.033)}{2-.033} \\
& =(439.02)(29.30)-(112.75)(0.4916) \\
& \mathrm{P}_{3 \text { Ф output }}=12,863.3 \text { watts }-55.43 \text { watts. } \\
& \text { Positive Sequence Negative Sequence } \\
& \text { Power Output Power Output }
\end{aligned}
$$

Notice that, at $10 \%$ negative sequence voltage, the power because of the negative sequence voltage is only $0.43 \%$ of the positive sequence power, or

$$
\text { Decreased power }=\frac{55.43}{12,863.3}=-0.43 \% \text {. }
$$

Therefore, voltage imbalance does not materially affect the power output of the rotor, but the rotor and stator copper losses increased $23.3 \%$ because of the negative sequence currents, as shown in Table 4. It is the increased losses, not the reduction in power because of the negative sequence voltage, that derate the motor. 
When a $10 \%$ negative sequence voltage is applied to a double-cage motor, the negative sequence reverse power as a percentage of positive sequence power is $0.91 \%$, or more than twice that for the single-cage motor. The percentage increase in rotor and stator copper losses is $33.7 \%$, for $44.6 \%$ higher losses than for the single-cage motor.

The additional copper losses cause an increase in temperature above ambient temperature and reduce the power output capability of the motor or generator. The effect of this increase in temperature or the derating of the induction machine is illustrated inTable 7 Table 7 . The higher the ratio of the positive-to-negative sequence impedances, the greater the capacity reduction. The negative sequence rotor values $\left(\mathrm{R}_{\mathrm{r} 2}, \mathrm{X}_{\mathrm{r} 2}\right)$ are not readily known, but Tracey (1954) found the ratio $\mathrm{Z}^{+} / \mathrm{Z}^{-}$can be approximated by dividing the locked rotor current by the rated full load current.

If one knows the ratio of $\mathrm{Z}^{+} / \mathrm{Z}^{-}$and the unbalanced voltage applied, an equation can be developed for derating the motor capacity. The equation is based on the assumptions that the total copper loss for the stator and rotor that corresponds to any stator current is proportional to that current squared and the change in rotor resistance with frequency is negligible.

Because the positive sequence current will result in the same total copper losses as balanced conditions and rated load,

$$
\mathrm{I}_{\mathrm{s}}^{2} \text { rated }=\mathrm{I}_{\mathrm{s} 1}^{2}+\mathrm{I}_{\mathrm{s} 2}^{2}
$$

Equation 4.39

where $_{\text {Is rated }}$ is the rated stator current, and $\mathrm{I}_{\mathrm{s} 1}$ and $\mathrm{I}_{\mathrm{s} 2}$ are the positive and negative sequence currents.

From Equation 4.39,

$$
\mathrm{I}_{\mathrm{s} 1}=\sqrt{\mathrm{I}_{\mathrm{s} \text { rated }}^{2}-\mathrm{I}_{\mathrm{s} 2}^{2}}
$$

Defining

$$
\begin{aligned}
& I_{\mathrm{s} 2}=\frac{V_{2}}{Z^{-}} \text {, and } I_{s \text { rated }}=\frac{V_{1}}{Z^{+}} \text {, then } \\
& I_{s 2}=\left(\frac{V_{2}}{V_{1}} \frac{Z^{+}}{Z^{-}}\right) I_{\text {s rated. }}
\end{aligned}
$$

Substituting Equation 4.41 into Equation 4.40,

$$
I_{s 1}=I_{s \text { rated }} \sqrt{1-\left(\frac{V_{2}}{V_{1}} \frac{Z^{+}}{Z^{-}}\right)} .
$$

To prevent the motor from overheating, the positive sequence stator current cannot be greater than the value given in Equation 4.42. Remember that the negative sequence torque is very small. Therefore, the rating under unbalance conditions is 


$$
\left[\begin{array}{l}
\text { Machine Rating } \\
\text { under unbalanced voltages }
\end{array}\right]=\sqrt{1-\left(\frac{\mathrm{V}_{2}}{\mathrm{~V}_{1}} \frac{\mathrm{Z}^{+}}{\mathrm{Z}^{-}}\right)^{2}} \times\left[\begin{array}{l}
\text { Machine } \\
\text { N.P. Rating }
\end{array}\right] \text {. Equation } 4.43
$$

(Note: $\frac{\mathrm{V}_{2}}{\mathrm{~V}_{1}}$ is given in p.u. in Equation 4.43, not in percent.)

It will be shown (see Equation 4.75) that when a phase conductor is opened on a three-phase supply to the induction machine, at standstill, the motor will not start. For example, when $\mathrm{Z}^{+} / \mathrm{Z}^{-}=5$ and $\mathrm{V}_{2} / \mathrm{V}_{1} \%=20 \%$, from Equation 4.43 , the machine rating is zero! When Equation 4.43 is applied to a ratio of $Z^{+} / Z^{-}=7$, the zero rating occurs at $V_{2} / V_{1} \%=15 \%$; when it is applied to a ratio of 9 , the zero rating occurs at $\mathrm{V}_{2} / \mathrm{V}_{1}=11 \%$. These zero rating values correspond with the value of the $\mathrm{V}_{2} / \mathrm{V}_{1} \%$ unbalance voltage, which occurs when an open phase conductor condition is applied to each of these machines. This result also applies to other $\mathrm{Z}^{+} / \mathrm{Z}^{-}$ratio machines.

From Table 4 , the $\mathrm{Z}^{+} / \mathrm{Z}^{-}$ratio for the single-cage motor is 4.85 , and the ratio for the doublecage is 4.48. Using the approximate ratio value of 5 and applying the results of Table 7, for a $\mathrm{V}_{2} / \mathrm{V}_{1} \%=5 \%$, the motor must be derated to $97 \%$ of rated power. Obviously, this is a generalized result, and temperature rise and service factor data must be used from the actual design to obtain realistic and specific results. However, the test results of Gafford, Duesterohoeft, and Mosher (1959) and Tracey (1954), shown in Table 8, have comparable deratings for the same unbalanced voltages.

No single-phase current or percent negative sequence current is indicative of the actual temperature rise. The losses in the stator are unevenly distributed because the line currents are not equal, and this causes hot spots, unbalanced temperatures, and resistances. Doublecage motors have to dissipate more losses than single-cage motors for the same negative sequence voltage. An unbalanced voltage condition not to exceed 3\% to 5\% is the recommended limit based on the line current not to exceed $120 \%$ (i.e., $\mathrm{I}_{\mathrm{C}}=119 \%$ ) to $125 \%$ (i.e., $\mathrm{I}_{\mathrm{A}}=124.5 \%$ ) of rated current, as shown in Table 2 . This results in percent negative currents of about $25 \%-22 \%$ and increased losses of $5.85 \%-8.45 \%$ for single- and doublecage machines, respectively. 
Table 7. Maximum Allowable Power Output in Percent of Rated While Operating at Unbalanced Voltages

\begin{tabular}{cccc}
\hline $\mathrm{V}_{2}$ & $\frac{\mathrm{Z}^{+}}{\mathrm{V}_{1}}=\mathbf{Z ^ { - }}$ & $\frac{\mathrm{Z}^{+}}{\mathrm{Z}^{-}}=\mathbf{7}$ & $\frac{\mathrm{Z}^{+}}{\mathrm{Z}^{-}}=\mathbf{9}$ \\
\cline { 2 - 4 } $3 \%$ & 99.8 & 97.8 & 96.3 \\
$5 \%$ & 96.8 & 93.7 & 89.3 \\
$8 \%$ & 91.7 & 82.8 & 69.4 \\
$10 \%$ & 86.6 & 71.4 & 43.6 \\
$11 \%$ & 83.5 & 63.8 & 0 \\
$13 \%$ & 76.0 & 41.5 & 0 \\
$14 \%$ & 71.4 & 19.9 & 0 \\
$15 \%$ & 66.1 & 0 & 0 \\
$20 \%$ & 0 & 0 & 0 \\
\hline
\end{tabular}

Notes:

From Tracey (1954)

(1) $Z^{+}=$Motor impedance to $V_{1}$ (positive sequence voltages) at rated

(2) $Z^{-}=$Motor impedance to $V_{2}$ (negative sequence voltages) at rated load

(3) $Z^{+} / Z^{-} \approx$ Locked rotor current/rated current

Table 8. Comparison of Gafford, Duesterohoeft, and Mosher (1959) and Tracey (1954) Motor Derate Data Operating at Unbalance Voltage

\begin{tabular}{|c|c|c|c|c|}
\hline \multirow[b]{2}{*}{$\frac{V_{2}}{V_{1}} \%$} & \multirow[b]{2}{*}{$\begin{array}{l}\text { P.u. Negative } \\
\text { (Gafford, } \\
\text { Duesterohoeft, } \\
\text { and Mosher) } \\
\text { Sequence } \mathrm{I}_{2}\end{array}$} & \multicolumn{3}{|c|}{ \% Power Derate } \\
\hline & & $\begin{array}{l}\text { P Output } \\
\text { (Gafford, } \\
\text { Duesterohoeft, } \\
\text { and Mosher) } \\
\text { p.u. @ Rated } \\
\text { Temp. Rise } \\
\end{array}$ & $\begin{array}{l}\Delta \text { P\% } \\
\text { (Gafford, } \\
\text { Duesterohoeft, } \\
\text { and Mosher) }\end{array}$ & $\begin{array}{l}\frac{Z^{+}}{Z^{-}}=5 \\
\Delta P \% \\
\text { (Tracey) }\end{array}$ \\
\hline 0 & 0 & 1.22 & 0 & 0 \\
\hline 3 & 0.160 & 1.20 & 1.64 & 1.0 \\
\hline 5 & 0.265 & 1.17 & 4.10 & 3.0 \\
\hline 8 & 0.420 & 1.10 & 9.84 & 8.0 \\
\hline 10 & 0.515 & 1.03 & 15.6 & 13.0 \\
\hline 11 & 0.570 & 0.98 & 19.7 & 16.5 \\
\hline 13 & 0.660 & 0.85 & 30.3 & 23.5 \\
\hline 15 & 0.750 & 0.66 & 45.9 & 34.0 \\
\hline
\end{tabular}


Note: The phasor diagram of Figure 73 was used as the voltage source by Gafford, Duesterohoeft, and Mosher (1959) to determine the heating condition of phases A, B, and C.

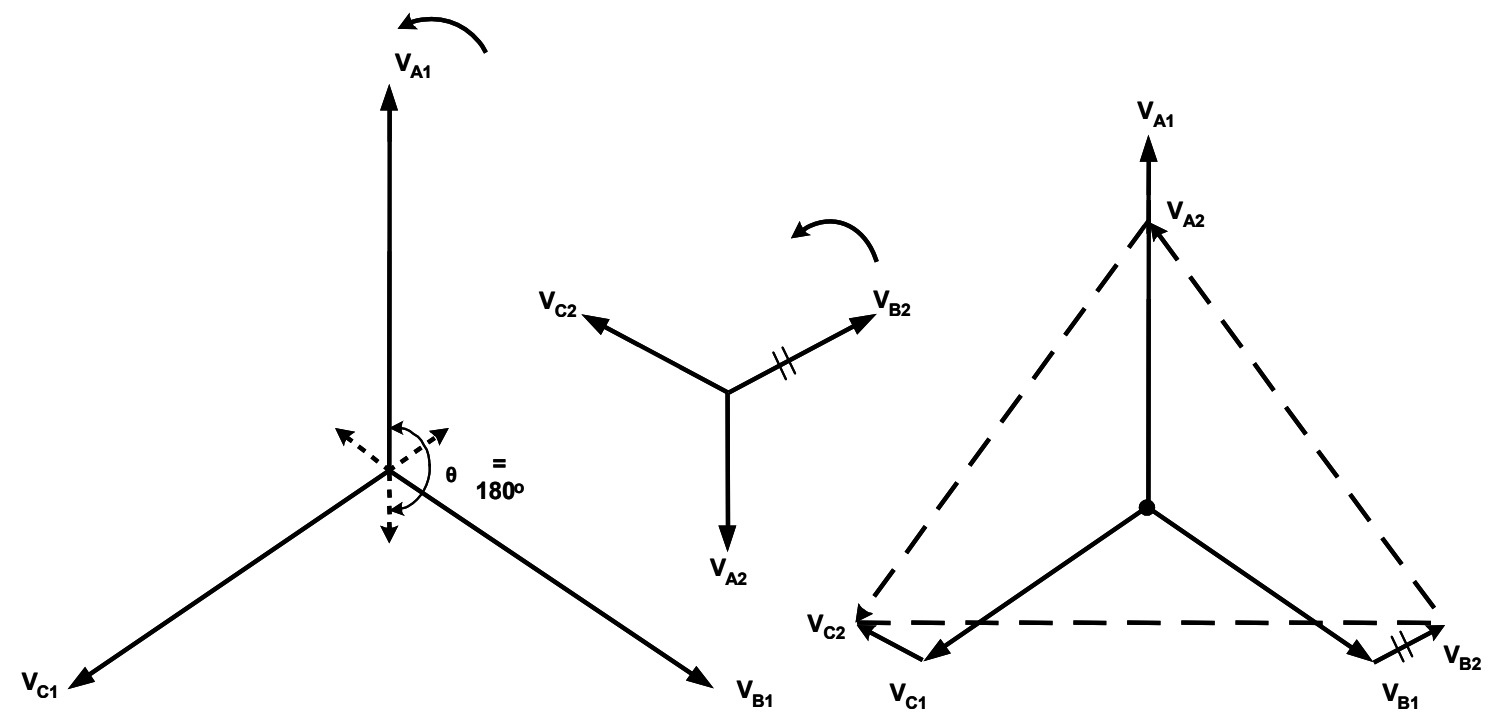

Figure 73. Phasor diagram for minimum heating in Phase $A, \theta=180^{\circ}$

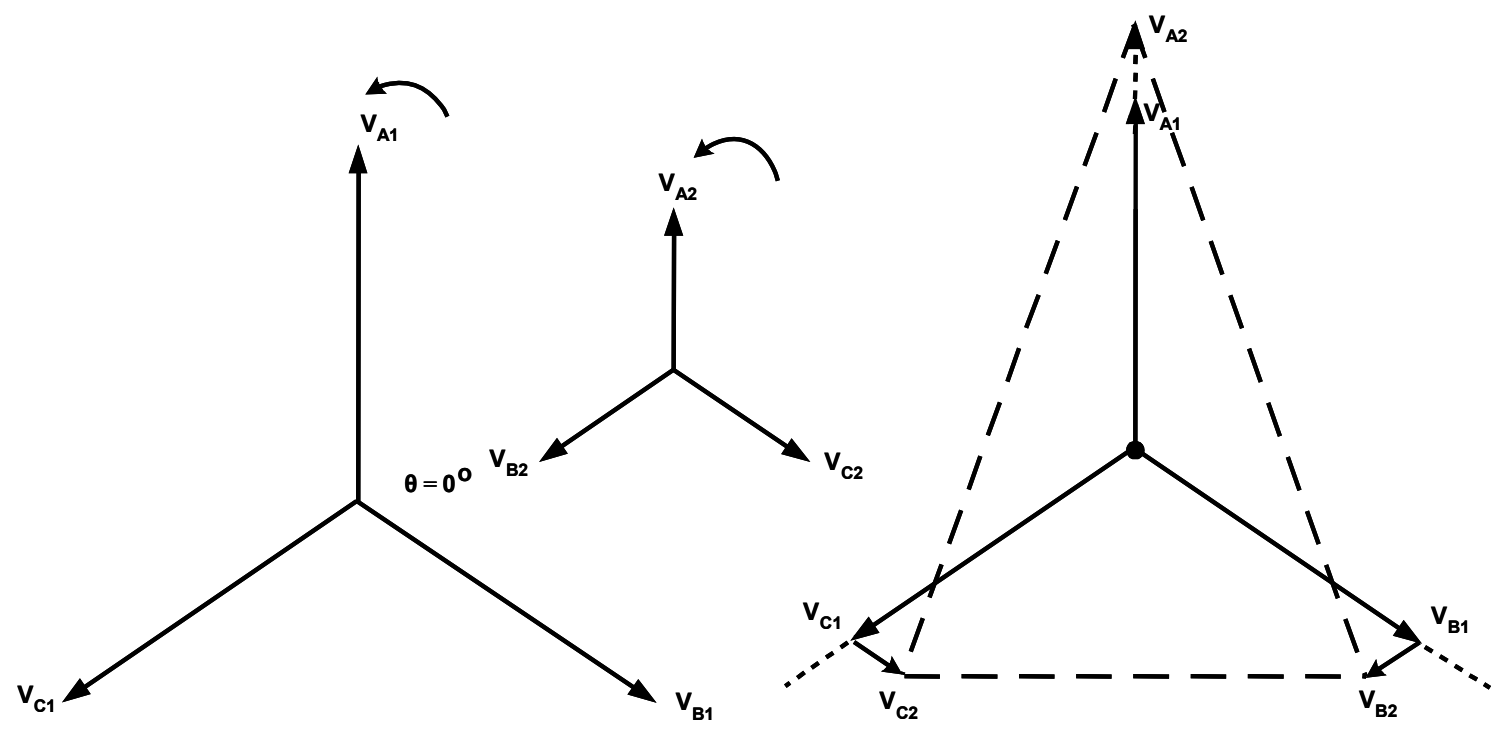

Figure 74. Phasor diagram for maximum heating in Phase $A, \theta=0^{\circ}$

\subsubsection{Unbalance Voltage Survey}

The survey results of 13 utility systems are shown in Table 9. The data show the majority of unbalanced conditions occur with open delta transformer connections. But even then, the average unbalance is only $1.98 \%$, with the highest being $10 \%$. 
Table 9. Unbalance Voltage Survey Results of Closed and Open Delta Transformer Connections

\begin{tabular}{|c|c|c|c|}
\hline & $\begin{array}{c}\text { Wye and } \\
\text { Closed Delta }\end{array}$ & Open Delta & Total \\
\hline Total $\mathrm{N}$ of Tests & 919 & 290 & 1209 \\
\hline Max. \% Unbal. & 5.94 & 10 & 10 \\
\hline Min. \% Unbal. & 0.0 & 0.0 & 0.0 \\
\hline Avg. \% Unbal. & 0.83 & 1.98 & 1.10 \\
\hline \multirow[t]{2}{*}{ N of tests @0\% Unbal. } & 142 & 9 & 151 \\
\hline & \multicolumn{3}{|c|}{$\%$ of Tests } \\
\hline \multicolumn{4}{|l|}{$\frac{\mathrm{V}_{2}}{\mathrm{~V}_{1}} \%$ NEMA } \\
\hline $0-0.5$ & 25.5 & 7.9 & 21.2 \\
\hline $0.51-1$ & 47.2 & 15.5 & 39.6 \\
\hline $1.01-1.5$ & 14.7 & 21.7 & 16.4 \\
\hline $1.51-2$ & 5.3 & 12.8 & 7.1 \\
\hline $2.01-2.5$ & 2.7 & 10.3 & 4.6 \\
\hline $2.51-3$ & 2.2 & 13.8 & 5.0 \\
\hline $3.01-3.5$ & 0.9 & 5.9 & 2.1 \\
\hline $3.51-4$ & 0.8 & 4.8 & 1.7 \\
\hline $4.01-4.5$ & 0.1 & 2.8 & 0.7 \\
\hline $4.51-5$ & 0.3 & 1.4 & 0.6 \\
\hline$>5$ & 0.3 & 3.1 & 1.0 \\
\hline
\end{tabular}

Notes:

(1) 1209 test (13-utility survey)

(2) Average unbalanced $=1.1 \%$

(3) $12.5 \%$ of tests $=0 \%$ unbalance $60 \%$ of tests $<1 \%$ unbalance $85 \%$ of tests $<2 \%$ unbalance

(4) Average unbalance $=0.83 \%$ for closed banks

(5) Average unbalance $=1.98 \%$ for open delta

\subsubsection{Synchronous Generators Operating on Unbalanced Voltages}

Unbalanced three-phase stator currents applied to synchronous generators cause negative sequence phase currents that, in turn, cause a double frequency current in the surface of the rotor. These currents flow through the retaining rings, slot wedges, and field winding and cause high temperatures and possibly failure. 


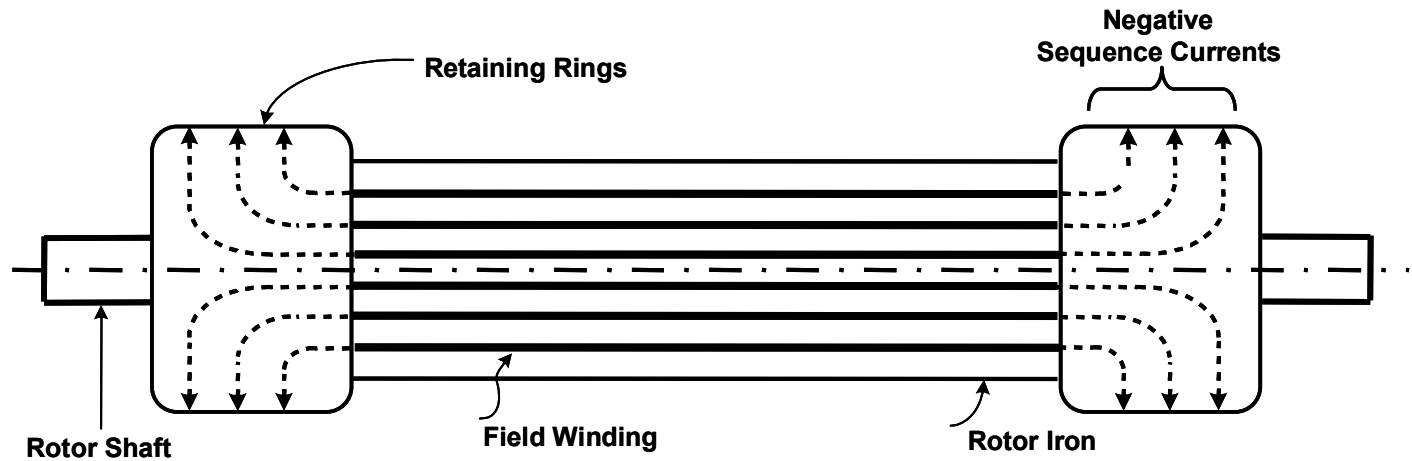

Figure 75. Round rotor synchronous machine showing negative sequence current paths in the rotor surface

Typically, the unbalance comes from single-phase main unit transformers with different impedances, unbalanced loads, open phases, and faults. Phase-to-phase faults create the highest negative sequence currents. A line-to-ground fault on the wye side of a delta-wye step-up transformer creates a line-to-line fault, as seen by the generator. The fault current from a line-to-ground fault on the generator is less than the line-to-line fault, and the openphase condition produces less negative sequence current than the phase-to-phase or phase-toground fault.

Because the negative sequence current components rotate in the opposite direction of the rotation of the rotor, the flux produced by these currents has a frequency twice the synchronous speed. The skin effect causes these double frequency currents to be concentrated on the surface of the poles and the teeth of the rotor. These currents flow along the axial length of the rotor until they contact the retaining rings, which are shrunk onto the ends of the rotor iron, as in Figure 75. A small portion of these negative sequence currents flows in the field winding. The negative sequence current beating of the rotor and retaining rings causes these rings to expand and become loose on the rotor iron.

\subsubsection{Negative Sequence Heating}

As was the case with induction machines, most of the increased losses for the synchronous generator because of negative sequence currents occur in the rotor. The temperature rise over time is proportional to the $\mathrm{I}_{\mathrm{s} 2}{ }^{2} \mathrm{t}$, where the $\mathrm{I}_{\mathrm{s} 2}$ is the stator negative sequence current, and $\mathrm{t}$ is in seconds. The safe limit is established based on

$$
\mathrm{K}=\mathrm{I}_{\mathrm{s} 2}{ }^{2} \mathrm{t},
$$

where

$\mathrm{K}=$ Constant based or a specific design and size of generator

$\mathrm{I}_{\mathrm{s} 2}=$ The negative sequence stator current $(\mathrm{rms})$

$\mathrm{t}=$ Time in seconds. 
The safe limit value of $\mathrm{K}$ is determined by measuring the temperature of the rotor with negative sequence current from the stator according to ANSI C50.13 and establishing the safe operating continuous unbalanced current capability of the generator. The safe limit is based on rated kilovolt-amperes and maximum current not to exceed $105 \%$ of rated current in any phase. Typically, the maximum permissible stator negative sequence $\mathrm{I}_{\mathrm{S} 2}$ current is $10 \%$ of the rated stator current, but the specific value recommended by the manufacturer should be used to set the value for the negative sequence relay.

\subsubsection{Negative Sequence Relay}

The negative sequence relays may be of definite-time-delay type or inverse-time type. The device 46 function is an inverse time overcurrent. Figure 76 shows typical time-negative sequence current curves for the Type SGC relay. Values for K can be calculated (G.E. Multilin 1998) using the p.u. locked rotor current ILR for a motor. If the p.u. ILR $=5$,

$$
\mathrm{K}=\frac{175}{\mathrm{I}_{\mathrm{LR}}^{2}}=7 .
$$

Values ranging up 40 have been used. Also, synchronous generator values for $\mathrm{K}$ vary from about 4 to 8 . A negative sequence pickup current setting of about $15 \%$ of full load current results in about 300 seconds. As shown in Table 2, a 15.44\% negative sequence current results in a $3 \%$ voltage imbalance for the single-cage induction motor. 


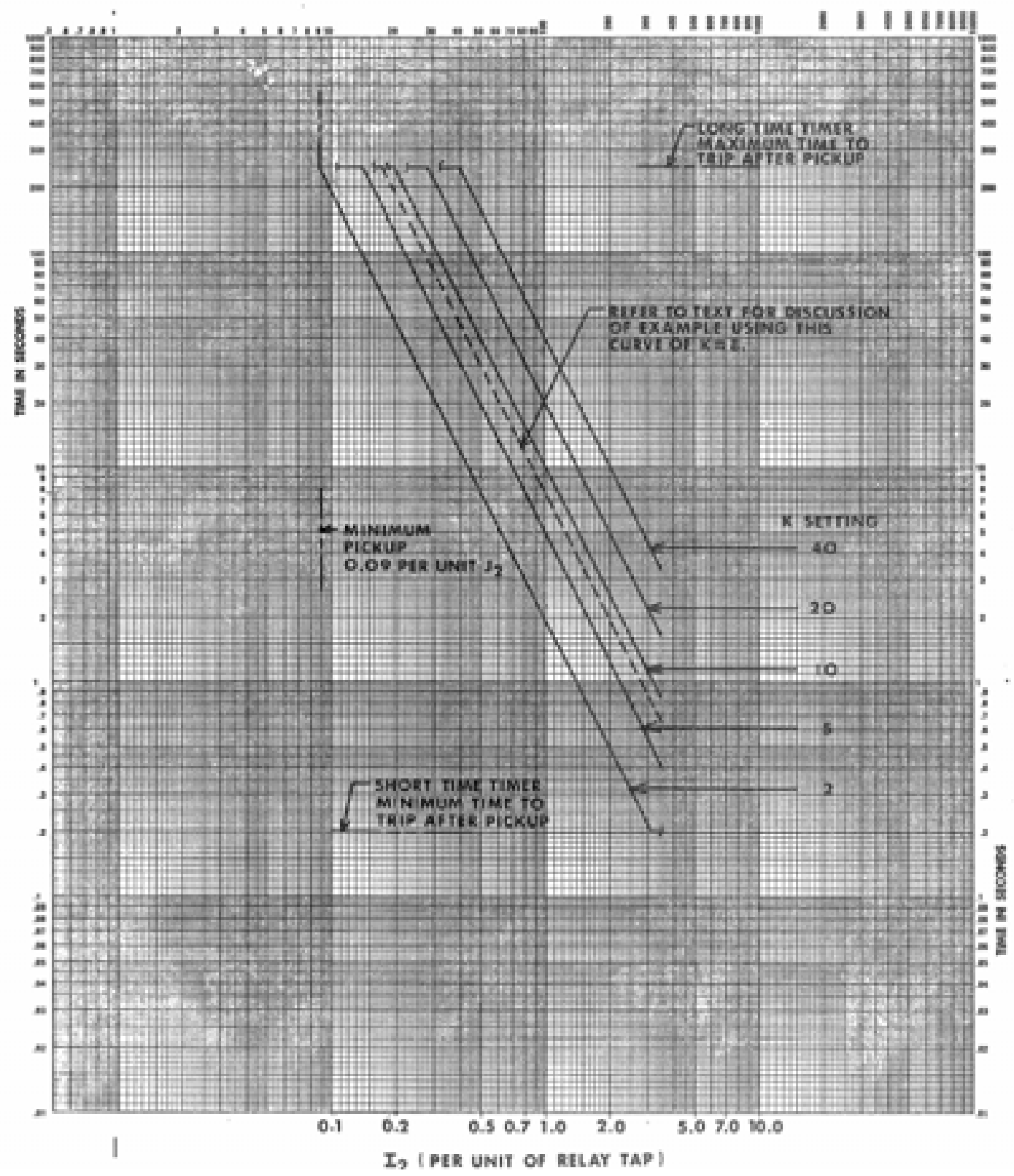

Figure 76. Typical time-current curves for the Type SGC relay 


\section{Project Results - Development of Models}

\subsection{Introduction}

This section focuses on the development of models for DG and the distribution circuit voltage regulation equipment for unbalanced power systems. The voltage regulation models were developed for the substation LTC transformer, bidirectional step regulator, capacitors, and each of the distribution circuit transformer connections. In addition, line impedance, voltage drop, and losses were modeled, as were secondary and service drop impedance, voltage drop, and losses. Finally, a shunt capacitor model for a wye-grounded connection and models for synchronous, induction, and inverter-based generators were created.

\subsection{Three-Phase Substation Transformer Models}

Three-phase transformers are used at the distribution substation to transform voltage from the sub-transmission or, in some cases, transmission system down to distribution circuit levels. These three-phase transformers may have HV fixed taps or LV fixed taps or can be LTC or under LTC transformers. The typical distribution circuit is a four-wire wye multi-grounded system fed from a delta-wye-grounded, three-phase substation transformer. However, there are three-wire, ungrounded delta distribution circuits, and they are normally fed from deltadelta three-phase substation transformers. Three-phase and single-phase transformers on the circuit feed three-phase customer load or combinations of single-phase and three-phase loads. Also, single-phase transformers on the circuit feed single-phase loads.

The delta-wye grounded transformer connection is shown in Figure 77.
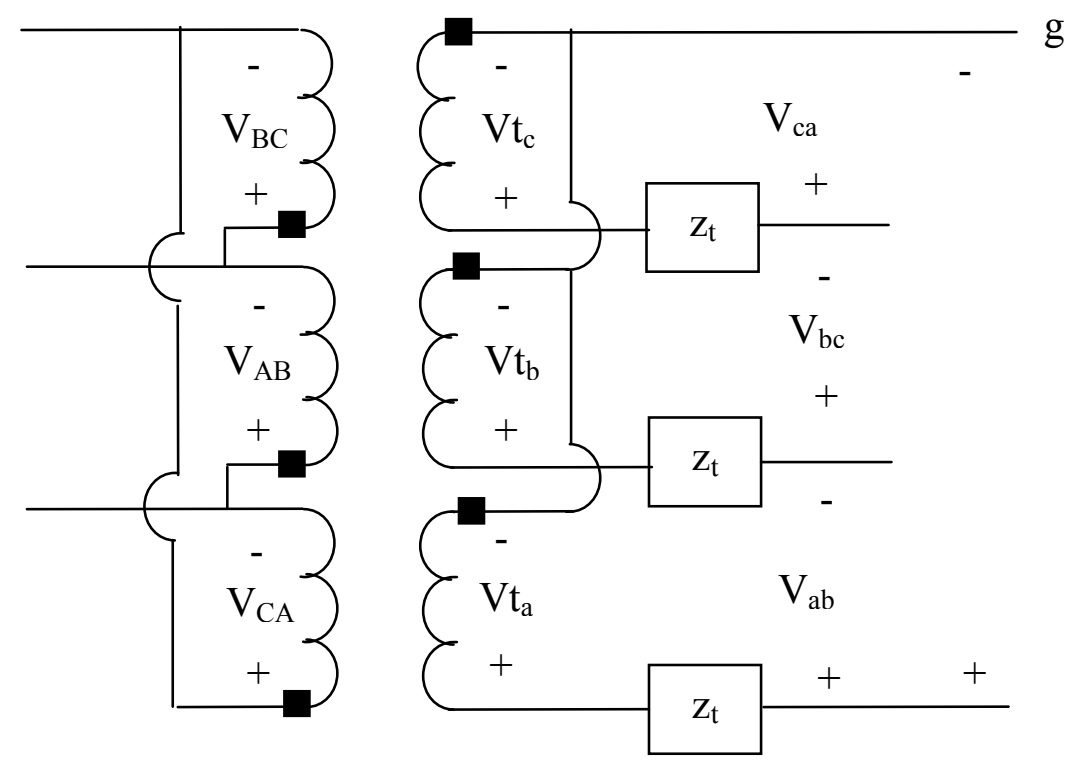

Figure 77. Delta-wye grounded three-phase transformer

From this figure, the primary-side line-to-line voltages can be written as a function of the ideal secondary-side voltages. 


$$
\left[\begin{array}{c}
\mathrm{V}_{\mathrm{AB}} \\
\mathrm{V}_{\mathrm{BC}} \\
\mathrm{V}_{\mathrm{CA}}
\end{array}\right]=\left[\begin{array}{ccc}
0 & -\mathrm{n}_{\mathrm{t}} & 0 \\
0 & 0 & -\mathrm{n}_{\mathrm{t}} \\
-\mathrm{n}_{\mathrm{t}} & 0 & 0
\end{array}\right] \cdot\left[\begin{array}{c}
\mathrm{V}_{\mathrm{ta}} \\
\mathrm{V}_{\mathrm{tb}} \\
\mathrm{V}_{\mathrm{tc}}
\end{array}\right]
$$

where the turns ratio $n_{t}$ is defined as

$$
\mathrm{n}_{\mathrm{t}}=\frac{\mathrm{V}_{\mathrm{LLHS}}}{\mathrm{V}_{\mathrm{LNLS}}}
$$

Equation 5.2

The ideal secondary voltage can be written as a function of the secondary-side line-to-neutral voltage as:

$$
\mathrm{V}_{\mathrm{tabc}}=\mathrm{V}_{\mathrm{LGabc}}+\mathrm{Z}_{\mathrm{tabc}} \mathrm{I}_{\mathrm{abc}}
$$

Equation 5.3

where

$$
Z_{t}=\left[\begin{array}{ccc}
Z_{t} & 0 & 0 \\
0 & Z_{t} & 0 \\
0 & 0 & Z_{t}
\end{array}\right]
$$

The primary line-to-line voltage as a function of the secondary line-to-ground voltage and the secondary current $I_{a b c}$ is

$$
\left[\begin{array}{l}
V_{A B} \\
V_{B C} \\
V_{C A}
\end{array}\right]=\left[\begin{array}{ccc}
0 & -n_{t} & 0 \\
0 & 0 & -n_{t} \\
-n_{t} & 0 & 0
\end{array}\right] \cdot\left(\left[\begin{array}{l}
V_{a} \\
V_{b} \\
V_{c}
\end{array}\right]+\left[\begin{array}{ccc}
z_{t} & 0 & 0 \\
0 & z_{t} & 0 \\
0 & 0 & z_{t}
\end{array}\right] \cdot\left[\begin{array}{c}
I_{a} \\
I_{b} \\
I_{c}
\end{array}\right]\right) .
$$

To make the transformer model consistent with other system models and therefore easier to calculate, the secondary-side line-to-ground voltage should be expressed as a function of the primary-side line-to-neutral voltage. This can be done using the theory of symmetrical components and algebraic manipulation. The resulting expression is

$$
\begin{aligned}
& {\left[\begin{array}{l}
V_{A B} \\
V_{B C} \\
V_{C A}
\end{array}\right]=\left[\begin{array}{ccc}
1 & -1 & 0 \\
0 & 1 & -1 \\
-1 & 0 & 1
\end{array}\right] \cdot\left[\begin{array}{l}
V_{A} \\
V_{B} \\
V_{C}
\end{array}\right]} \\
& {\left[\begin{array}{ccc}
1 & -1 & 0 \\
0 & 1 & -1 \\
-1 & 0 & 1
\end{array}\right] \cdot\left[\begin{array}{l}
V_{A} \\
V_{B} \\
V_{C}
\end{array}\right]=\left[\begin{array}{ccc}
0 & -n_{t} & 0 \\
0 & 0 & -n_{t} \\
-n_{t} & 0 & 0
\end{array}\right] \cdot\left(\left[\begin{array}{c}
V_{a} \\
V_{b} \\
V_{c}
\end{array}\right]+\left[\begin{array}{ccc}
z_{t} & 0 & 0 \\
0 & z_{t} & 0 \\
0 & 0 & z_{t}
\end{array}\right] \cdot\left[\begin{array}{c}
I_{a} \\
I_{b} \\
I_{c}
\end{array}\right]\right)}
\end{aligned}
$$




$$
\begin{aligned}
& \frac{1}{n_{t}}\left[\begin{array}{ccc}
0 & 0 & -1 \\
-1 & 0 & 0 \\
0 & -1 & 0
\end{array}\right] \cdot\left[\begin{array}{ccc}
1 & -1 & 0 \\
0 & 1 & -1 \\
-1 & 0 & 1
\end{array}\right] \cdot\left[\begin{array}{l}
V_{A} \\
V_{B} \\
V_{C}
\end{array}\right]=\left[\begin{array}{l}
V_{a} \\
V_{b} \\
V_{c}
\end{array}\right]+\left[\begin{array}{ccc}
z_{t} & 0 & 0 \\
0 & z_{t} & 0 \\
0 & 0 & z_{t}
\end{array}\right] \cdot\left[\begin{array}{c}
I_{a} \\
I_{b} \\
I_{c}
\end{array}\right] \\
& {\left[\begin{array}{l}
V_{a} \\
V_{b} \\
V_{c}
\end{array}\right]=\frac{1}{n_{t}}\left[\begin{array}{ccc}
1 & 0 & -1 \\
-1 & 1 & 0 \\
0 & -1 & 1
\end{array}\right] \cdot\left[\begin{array}{l}
V_{A} \\
V_{B} \\
V_{C}
\end{array}\right]-\left[\begin{array}{ccc}
z_{t} & 0 & 0 \\
0 & z_{t} & 0 \\
0 & 0 & z_{t}
\end{array}\right] \cdot\left[\begin{array}{l}
I_{a} \\
I_{b} \\
I_{c}
\end{array}\right] .}
\end{aligned}
$$

Equation 5.6

\subsection{Three-Phase and Single-Phase Distribution Transformer Connections for Distribution Circuit}

The following equations determine the voltage drop on the wye-grounded primary system feeding different transformer connections and three-phase and single-phase loading. The factor $0.9 \mathrm{I}_{\mathrm{L}}$ in some of the equations represents the portion of the neutral current in the neutral conductor, the remainder of which is current in the earth.

\section{Wye-Grounded Primary System Transformer Connections and Voltage Regulation}

\section{Case I.}
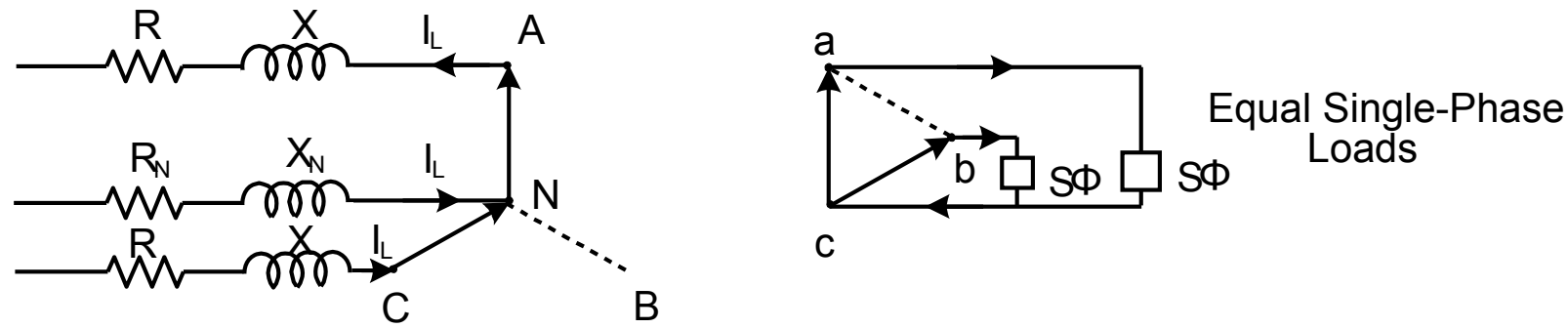

$\mathrm{V}_{\mathrm{R}_{\mathrm{NA}}}=\mathrm{I}_{\mathrm{L}}[\mathrm{R} \operatorname{Cos} \theta+\mathrm{X} \operatorname{Sin} \theta]+0.9 \mathrm{I}_{\mathrm{L}}\left[\mathrm{R}_{\mathrm{N}} \operatorname{Cos}\left(\theta-60^{\circ}\right)+\mathrm{X}_{\mathrm{N}} \operatorname{Sin}\left(\theta-60^{\circ}\right)\right]$

Equation 5.7

$\mathrm{V}_{\mathrm{R}_{\mathrm{CN}}}=\mathrm{I}_{\mathrm{L}}[\mathrm{R} \operatorname{Cos} \theta+\mathrm{X} \operatorname{Sin} \theta]+0.9 \mathrm{I}_{\mathrm{L}}\left[\mathrm{R}_{\mathrm{N}} \operatorname{Cos}\left(\theta+60^{\circ}\right)+\mathrm{X}_{\mathrm{N}}\left(\theta+60^{\circ}\right)\right]$

Equation 5.8

\section{Case II.}
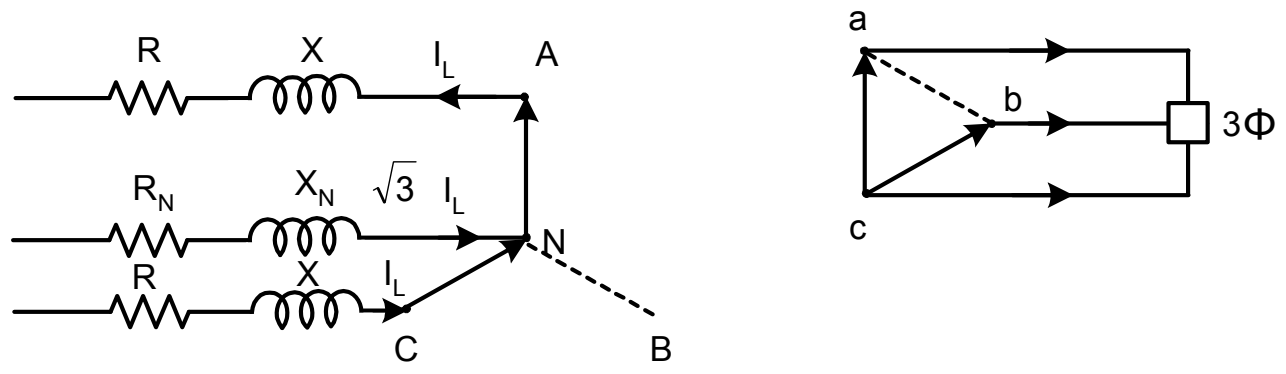
$\mathrm{V}_{\mathrm{R}_{\mathrm{NA}}}=\mathrm{I}_{\mathrm{L}}\left[\mathrm{R} \operatorname{Cos}\left(\theta-30^{\circ}\right)+\mathrm{X} \operatorname{Sin}\left(\theta-30^{\circ}\right)\right]+0.9 \sqrt{3} \mathrm{I}_{\mathrm{L}}\left[\mathrm{R}_{\mathrm{N}} \operatorname{Cos}\left(\theta-60^{\circ}\right)+\mathrm{X}_{\mathrm{N}} \operatorname{Sin}(\theta-\right.$ $\left.\left.60^{\circ}\right)\right]$

Equation 5.9

$\mathrm{V}_{\mathrm{R}_{\mathrm{CN}}}=\mathrm{I}_{\mathrm{L}}\left[\mathrm{R} \operatorname{Cos}\left(\theta+30^{\circ}\right)+\mathrm{X} \operatorname{Sin}\left(\theta+30^{\circ}\right)\right]+0.9 \sqrt{3} \mathrm{IL}\left[\mathrm{R}_{\mathrm{N}} \operatorname{Cos}\left(\theta+60^{\circ}\right)+\mathrm{X}_{\mathrm{N}} \operatorname{Sin}(\theta+\right.$ $\left.60^{\circ}\right)$ ]

Equation 5.10

\section{Case III.}

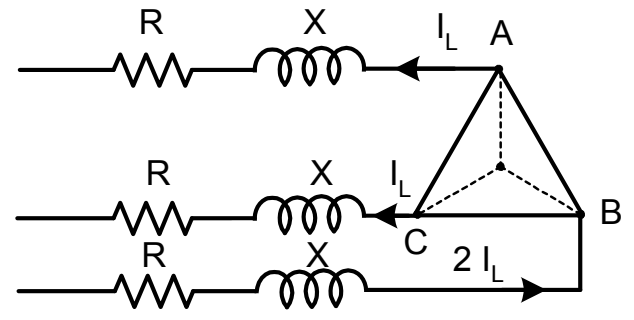

$\left.\mathrm{V}_{\mathrm{R}} \mathrm{NA}_{\mathrm{L}}\left[\mathrm{R} \operatorname{Cos}\left(\theta-60^{\circ}\right)+\mathrm{X} \operatorname{Sin} \theta-60^{\circ}\right)\right]$

$\mathrm{V}_{\mathrm{R}_{\mathrm{BN}}}=2 \mathrm{I}_{\mathrm{L}}(\mathrm{R} \operatorname{Cos} \theta+\mathrm{X} \operatorname{Sin} \theta)$

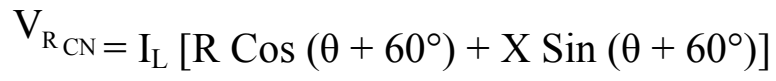

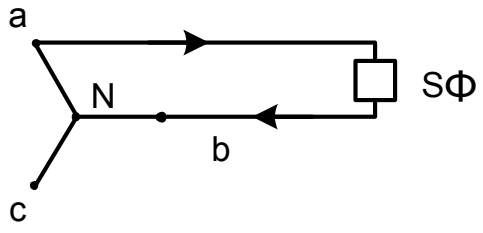

Equation 5.11

Equation 5.12

Equation 5.13

\section{Case IV.}

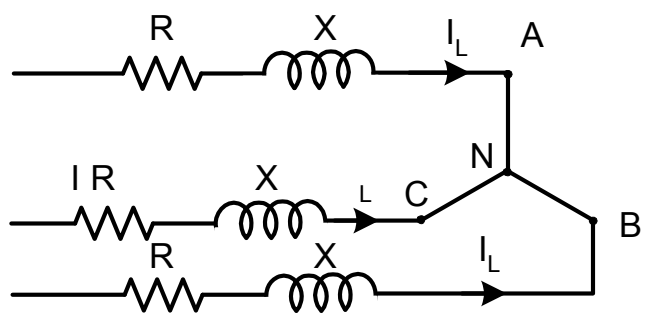

$\mathrm{V}_{\mathrm{R}_{\mathrm{AN}}}=\mathrm{I}_{\mathrm{L}}(\mathrm{R} \operatorname{Cos} \theta+\mathrm{X} \operatorname{Sin} \theta)$ on L-N Base Voltage

$\mathrm{V}_{\mathrm{R}_{\mathrm{BN}}}=\mathrm{I}_{\mathrm{L}}(\mathrm{R} \operatorname{Cos} \theta+\mathrm{X} \operatorname{Sin} \theta)$ on L-N Base Voltage

$\mathrm{V}_{\mathrm{R}_{\mathrm{CN}}}=\mathrm{I}_{\mathrm{L}}(\mathrm{R} \operatorname{Cos} \theta+\mathrm{X} \operatorname{Sin} \theta)$ on L-N Base Voltage

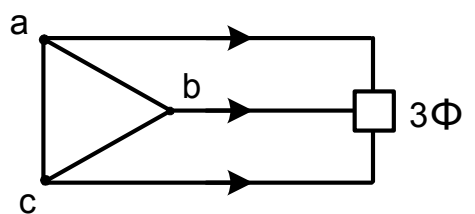

Equation 5.14

Equation 5.15

Equation 5.16 


\section{Case V.}

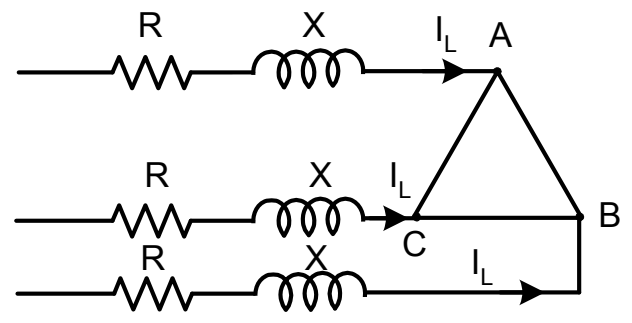

$\mathrm{V}_{\mathrm{R}_{\mathrm{NA}}}=\mathrm{I}_{\mathrm{L}}(\mathrm{R} \operatorname{Cos} \theta+\mathrm{X} \operatorname{Sin} \theta)$

$\mathrm{V}_{\mathrm{R}_{\mathrm{NB}}}=\mathrm{I}_{\mathrm{L}}(\mathrm{R} \operatorname{Cos} \theta+\mathrm{X} \operatorname{Sin} \theta)$

$\mathrm{V}_{\mathrm{R}_{\mathrm{NC}}}=\mathrm{I}_{\mathrm{L}}(\mathrm{R} \operatorname{Cos} \theta+\mathrm{X} \operatorname{Sin} \theta)$

\section{Case VI.}

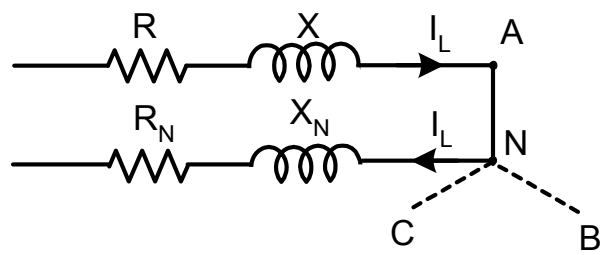

$\left.\mathrm{V}_{\mathrm{R}_{\mathrm{NA}}}=\mathrm{I}_{\mathrm{L}}\left[\mathrm{R}+\mathrm{R}_{\mathrm{N}}\right) \operatorname{Cos} \theta+(\mathrm{X}+\mathrm{XN}) \operatorname{Sin} \theta\right]$

Case VII.

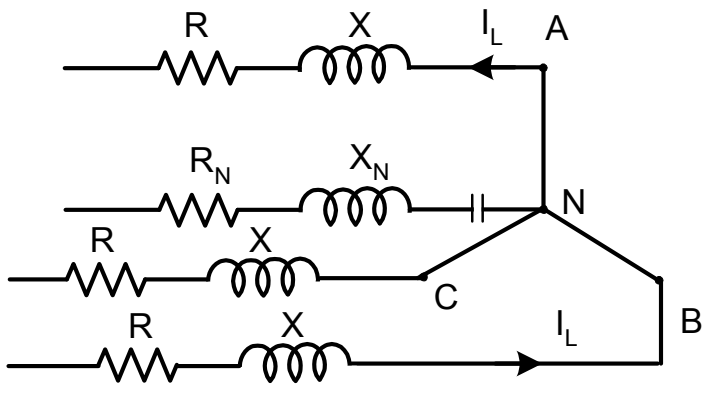

$\mathrm{V}_{\mathrm{R}_{\mathrm{BA}}}=2 \mathrm{I}_{\mathrm{L}}(\mathrm{R} \operatorname{Cos} \theta+\mathrm{X} \operatorname{Sin} \theta)$

$\mathrm{V}_{\mathrm{R}_{\mathrm{NA}}}=\mathrm{I}_{\mathrm{L}}\left[\mathrm{R} \operatorname{Cos}\left(\theta-30^{\circ}\right)+\mathrm{X} \operatorname{Sin}\left(\theta-30^{\circ}\right)\right]$

$\mathrm{V}_{\mathrm{R}_{\mathrm{BN}}}=\mathrm{I}_{\mathrm{L}}\left[\mathrm{R} \operatorname{Cos}\left(\theta+30^{\circ}\right)+\mathrm{X} \operatorname{Sin}\left(\theta+30^{\circ}\right)\right]$

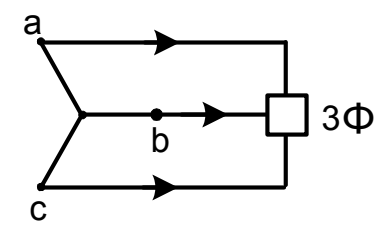

Equation 5.17

Equation 5.18

Equation 5.19

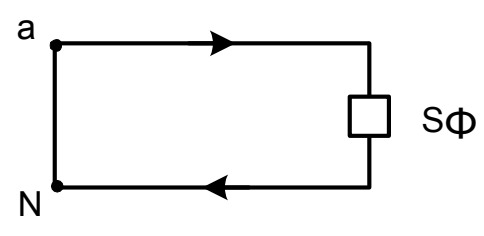

Equation 5.20

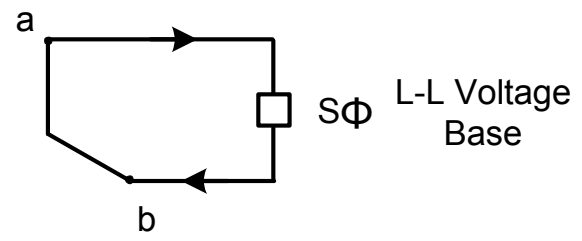

Equation 5.21

Equation 5.22

Equation 5.23 


\section{Case VIII.}
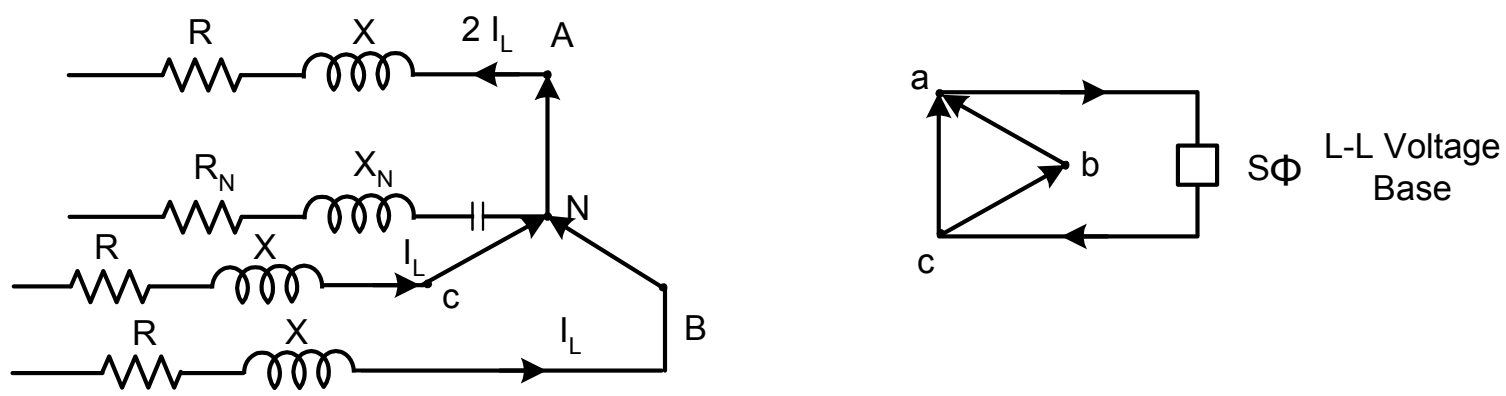

$\mathrm{V}_{\mathrm{R}_{\mathrm{NA}}}=2 \mathrm{I}_{\mathrm{L}}(\mathrm{R} \operatorname{Cos} \theta+\mathrm{X} \operatorname{Sin} \theta)$

Equation 5.24

$\mathrm{V}_{\mathrm{R}_{\mathrm{BN}}}=\mathrm{I}_{\mathrm{L}}\left[\mathrm{R} \operatorname{Cos}\left(\theta+60^{\circ}\right)+\mathrm{X} \operatorname{Sin}\left(\theta+60^{\circ}\right)\right]$

Equation 5.25

$\mathrm{V}_{\mathrm{R}_{\mathrm{CN}}}=\mathrm{I}_{\mathrm{L}}\left[\mathrm{R} \operatorname{Cos}\left(\theta-60^{\circ}\right)+\mathrm{X} \operatorname{Sin}\left(\theta-60^{\circ}\right)\right]$

Equation 5.26

\section{Case IX.}
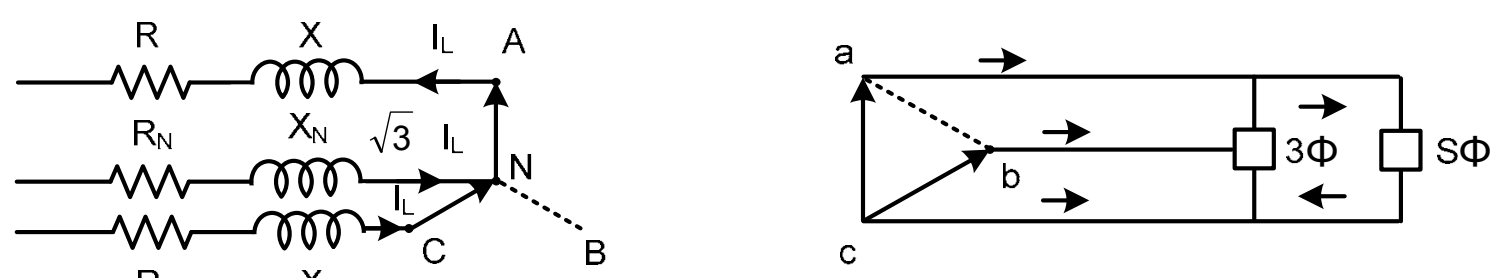

$\mathrm{V}_{\mathrm{R}_{\mathrm{NA} 3 \Phi}+\mathrm{NAS} \Phi}=\mathrm{I}_{\mathrm{L} 3 \Phi}\left[\mathrm{R} \operatorname{Cos}\left(\theta_{3 \Phi}-30^{\circ}\right)+\mathrm{X} \operatorname{Sin}\left(\theta_{3 \Phi}-30^{\circ}\right)\right]+0.9 \sqrt{3} \mathrm{I}_{\mathrm{L} 3 \Phi}\left[\mathrm{R}_{\mathrm{N}} \operatorname{Cos}\left(\theta_{3 \Phi}-\right.\right.$

$\left.\left.60^{\circ}\right)+\mathrm{X}_{\mathrm{N}} \operatorname{Sin}\left(\theta_{3 \Phi}-60^{\circ}\right)\right]+\mathrm{I}_{\mathrm{LS} \Phi}\left[\mathrm{R} \operatorname{Cos} \theta_{\mathrm{S} \Phi}+\mathrm{X} \operatorname{Sin} \theta_{\mathrm{S} \Phi}\right]+0.9 \mathrm{I}_{\mathrm{L} \mathrm{S} \Phi}\left[\mathrm{RN} \operatorname{Cos}\left(\theta_{\mathrm{S} \Phi}-60^{\circ}\right)+\right.$ $\left.\mathrm{X}_{\mathrm{N}} \operatorname{Sin}\left(\theta_{\mathrm{S} \Phi}-60^{\circ}\right)\right]$

Equation 5.27

$\mathrm{V}_{\mathrm{R}_{\mathrm{CN} 3 \Phi}}=\mathrm{I}_{\mathrm{L} 3 \Phi}\left[\mathrm{R} \operatorname{Cos}\left(\theta_{3 \Phi}+30^{\circ}\right)+\mathrm{X} \operatorname{Sin}\left(\theta_{3 \Phi}+30^{\circ}\right)\right]+0.9 \sqrt{3} \mathrm{I}_{\mathrm{L} 3 \Phi}\left[\mathrm{R}_{\mathrm{N}} \operatorname{Cos}\left(\theta_{3 \Phi}+60^{\circ}\right)\right.$

$\left.+X_{N} \operatorname{Sin}\left(\theta_{3 \Phi}+60^{\circ}\right)\right]$

Equation 5.28

$\mathrm{V}_{\mathrm{R}_{\mathrm{CA} 3 \Phi}}+\mathrm{C}_{\mathrm{AS} \Phi}=\mathrm{I}_{\mathrm{L} 3 \Phi}\left[\mathrm{R} \operatorname{Cos}\left(\theta+60^{\circ}\right)+\mathrm{X} \operatorname{Sin}\left(\theta+60^{\circ}\right)+\mathrm{I}_{\mathrm{L} 3 \Phi}\left[\mathrm{R} \operatorname{Cos}\left(\theta-60^{\circ}\right)+\mathrm{X} \operatorname{Sin}\right.\right.$ $\left.\left(\theta-60^{\circ}\right)\right]+\mathrm{I}_{\mathrm{LS} \Phi}\left[\mathrm{R} \operatorname{Cos}\left(\theta-30^{\circ}\right)+\mathrm{X} \operatorname{Sin}\left(\theta-30^{\circ}\right)\right]$

Equation 5.29

\subsection{Distribution Transformer Impedance}

The percent resistance and percent reactance of transformers can be determined from load losses and percent impedance. 
The percent $\mathrm{Z}$ is defined as

$$
\% Z=\sqrt{\% R^{2}+\% X^{2}}
$$

and percent $\mathrm{R}$ is determined from

$$
\% \mathrm{R}=\frac{\text { Load Losses (watts) }}{10 \mathrm{kVA}}
$$

Equation 5.31

Using the 25-kVA transformer load losses from Table 10, then

$$
\% \mathrm{R}=\frac{397 \text { watts }}{(10)(25)}=1.59
$$

From Equation 5.30, the $\% \mathrm{R}$ of Equation 5.31, and the $\% \mathrm{Z}$ of Table $10, \% \mathrm{X}$ is determined as

$$
\begin{aligned}
& \% X=\sqrt{\% Z^{2}-\% R^{2}}, \\
& \% X=\sqrt{(2.58)^{2}-(1.59)^{2}}=2.03 .
\end{aligned}
$$

\subsection{Distribution Transformer Voltage Drop}

In one knows the $\mathrm{R}$ and $\mathrm{X}$ values, the load current, and the power factor, the voltage drop can be determined. The actual $\mathrm{R}$ and $\mathrm{X}$ values in ohms are found from the $\% \mathrm{R}$ and $\% \mathrm{X}$ values as follows:

\section{Voltage Drop for 25-kVA Transformer}

Base voltage $=240 \mathrm{~V}$

Base $\mathrm{kVA}=25 \mathrm{kVA}$

Base current $=\frac{25,000}{240}=104.17$ amperes

Base $Z=\frac{240}{104.17}=2.30 \Omega$ 
Table 10. Distribution Transformer No Load (Core Losses) and Load (Copper Losses)

\begin{tabular}{|c|c|c|c|c|c|c|c|c|c|}
\hline Type & kVA & Phase & Sec. Volt & Pri. Volt & $\begin{array}{c}\text { No- } \\
\text { Load } \\
\text { Losses } \\
\text { Watts }\end{array}$ & $\begin{array}{l}\text { Load } \\
\text { Losses } \\
\text { Watts }\end{array}$ & $\begin{array}{c}\text { Tot } \\
\text { Losses } \\
\text { Watts }\end{array}$ & $\% Z$ & $\% \mathrm{I}_{\mathrm{e}}$ \\
\hline $\mathrm{OH}$ & 15 & $S$ & $120 / 240$ & $4800 / 7620$ & 34 & 280 & 314 & 2.58 & 0.51 \\
\hline $\mathrm{OH}$ & 25 & $S$ & $120 / 240$ & $4800 / 7620$ & 43 & 397 & 440 & 2.58 & 0.27 \\
\hline $\mathrm{OH}$ & 50 & $S$ & $120 / 240$ & $4800 / 7620$ & 103 & 564 & 667 & 1.97 & 0.76 \\
\hline $\mathrm{OH}$ & 100 & S & $120 / 240$ & $4800 / 7620$ & 165 & 1150 & 1315 & 2.10 & 0.21 \\
\hline $\mathrm{OH}$ & 167 & S & $120 / 240$ & $4800 / 7620$ & 267 & 1749 & 2016 & 2.37 & 0.33 \\
\hline PAD & 75 & 3 & $120 / 208$ & $4800 \times 13200 / 7620$ & 283 & 836 & 1119 & 2.43 & 0.78 \\
\hline PAD & 150 & 3 & $120 / 208$ & $13200 / 7620$ & 328 & 2026 & 2354 & 2.37 & 0.34 \\
\hline PAD & 300 & 3 & $120 / 208$ & $13200 / 7620$ & 639 & 3198 & 3837 & 2.50 & 0.55 \\
\hline PAD & 500 & 3 & $120 / 208$ & $4800 \times 13200 / 7620$ & 1140 & 4085 & 5225 & 3.45 & 0.46 \\
\hline PAD & 1000 & 3 & $480 Y / 277$ & 4160 & 1160 & 7601 & 8761 & 5.51 & 0.23 \\
\hline PAD & 1500 & 3 & $480 Y / 277$ & $4800 \times 13200$ & 1516 & 10294 & 11810 & 6.04 & 0.17 \\
\hline PAD & 2000 & 3 & $480 Y / 277$ & $4800 \times 13200$ & 1894 & 12933 & 14827 & 5.75 & 0.15 \\
\hline ISO & 333 & $S$ & $4800 / 8320$ & $7620 / 13200$ & 416 & 2937 & 3353 & 3.43 & 0.35 \\
\hline PAD & 50 & $\mathrm{~S}$ & $120 / 240$ & 7620 & 107 & 675 & 782 & 1.92 & 0.30 \\
\hline PAD & 100 & $\mathrm{~S}$ & $120 / 240$ & 7620 & 173 & 1074 & 1247 & 2.34 & 0.26 \\
\hline PAD & 167 & $\mathrm{~S}$ & $120 / 240$ & $4800 \times 13200 / 7620$ & 231 & 1466 & 1697 & 2.72 & 0.20 \\
\hline
\end{tabular}

Actual R ohms $=1.59 \% \times 2.30 \Omega$

$$
=0.0366 \Omega
$$

Actual X ohms $=2.0 \% \times 2.30 \Omega$

$$
=0.0460 \Omega
$$

Actual $\mathrm{Z}$ ohms $=2.58 \% \times 2.30 \Omega$

$$
=0.0593 \Omega
$$

Assuming a power factor of $0.90\left(25.84^{\circ}\right)$, a load current of 1.40 times rated kilovoltamperes, and a voltage of $105 \%$ of rated voltage, the current would be

$$
I=\frac{(104.17 \text { amperes })}{1.05} \times 1.40=138.9 \text { amperes }
$$

The approximate voltage drop is then

$$
\begin{aligned}
\Delta \mathrm{V} & =\mathrm{IR} \operatorname{Cos} \theta+\mathrm{I} X \operatorname{Sin} \theta, \\
& =(138.9)[(0.0366)(0.90)+(0.0460)(0.4358)] \\
& =(138.9)[0.03294+0.0200]
\end{aligned}
$$


$\Delta \mathrm{V}=7.36 \mathrm{~V} @ 240 \mathrm{~V}$

$\Delta \mathrm{V} @ 120 \mathrm{~V}=3.68 \mathrm{~V}$.

Equation 5.33

The voltage drop can be reduced if a 50-kVA transformer is substituted for the $25-\mathrm{kVA}$ transformer.

Voltage Drop for 50-kVA Transformer

Base current $=208.34 \mathrm{~A}$

Base $Z=\frac{240}{208.34}=1.15 \Omega$

Actual R ohms $=1.13 \% \times 1.15 \Omega$

$=0.0130 \Omega$

Actual X ohms $=1.62 \% \times 1.15 \Omega$

$=0.0187 \Omega$

Actual $\mathrm{Z}$ ohms $=1.97 \% \times 1.15 \Omega$

$=0.0227 \Omega$

Using a 0.90 power factor, 1.4 times rated current, and $105 \%$ voltage, the current is

$$
I=\frac{208.34}{1.05} \times 1.40=277.8 \text { amperes }
$$

and

$$
\begin{aligned}
& \Delta \mathrm{V}=\mathrm{IR} \operatorname{Cos} \theta+\mathrm{I} X \operatorname{Sin} \theta \\
& =(277.8)[(0.0130)(0.90)+(0.0187)(0.4358)] \\
& =(277.8)[0.0117+0.00815] \\
& \Delta \mathrm{V}=5.51 \mathrm{~V} @ 240 \mathrm{~V} \\
& \Delta \mathrm{V} @ 120 \mathrm{~V}=2.75 \mathrm{~V} .
\end{aligned}
$$

Equation 5.34

\subsection{Distribution Transformer Losses}

Distribution transformer losses consist of load losses and no-load losses.

\subsubsection{Load Losses}

The load losses are proportional to the square of the load current. The test data in Table 10 are given at rated load current. The load loss at loads other than rated load is 


$$
\begin{aligned}
\text { Load loss } & =\left(\frac{I_{l_{\text {oad }}}}{I_{\text {rated }}}\right)^{2} \text { (load loss at rated load), } & \text { Equation 5.35 } \\
& =\left(\frac{\mathrm{kV}_{\text {rated }}}{\mathrm{kV}_{\text {actual }}} \frac{\mathrm{kVA}_{\text {actual }}}{\mathrm{kVA}_{\text {rated }}}\right)^{2} \text { (load loss at rated load). } & \text { Equation 5.36 }
\end{aligned}
$$

For the $25-\mathrm{kVA}$ transformer, the load loss at rated load is $397 \mathrm{~W}$. But at peak load, the load may be as high as $140 \%-200 \%$ of rated load. The load losses at $105 \%$ of rated voltage and $140 \%$ of rated load are from Equation 5.36.

$$
\begin{aligned}
\text { Load losses } & =\left(\frac{7620}{(7620)(1.05)} \times \frac{(25)(1.40)}{25}\right)^{2} \\
& =(1.78)(397) \\
& =706 \mathrm{~W}
\end{aligned}
$$

$$
\text { Percent load loss }=\frac{\text { Load Loss }(\text { watts })}{(1.4)\left(\mathrm{VA}_{\text {rated }}\right)(\operatorname{Cos} \theta)} \times 100=\frac{\text { Load Loss }(\text { watts })}{(1.4)\left(10 \mathrm{kVA}_{\text {rated }}\right)(\operatorname{Cos} \theta)}
$$

Equation 5.37

For the 25-kVA transformer, the percent load loss is

$$
\text { Percent load loss }=\frac{706}{(1.4)(10)(25)(.90)}=2.24 \% .
$$

\subsubsection{No-Load Losses}

The no-load losses are the core losses, dielectric losses, and copper losses caused by the excitation current. However, the core losses because of the hysteresis and eddy current losses are the most significant. From Table 10, the no-load losses for the 25-kVA transformer are $43 \mathrm{~W}$. For operation above rated voltage, the no-load losses must be corrected. The correction factors are 1.15 at $105 \%$ of rated voltage and 1.30 at $110 \%$ of rated voltage. At $105 \%$ voltage,

$$
\begin{aligned}
& \text { No-load losses }=(\text { rated no-load losses })(\text { voltage correction }) \quad \text { Equation } 5.38 \\
& \text { No-load losses }=(43 \mathrm{~W})(1.15)=49.5 \mathrm{~W} .
\end{aligned}
$$

\subsubsection{Total Losses}

Adding the no-load losses corrected for voltage and load losses corrected for voltage and current gives $2.4 \%$ total losses for the $25-\mathrm{kVA}$ transformer. 
Total percent losses $=\frac{706+49.5}{(1.4)(10)(25)(0.90)}=2.40 \% \quad$ Equation 5.39

For the 50-kVA transformer, the percent load losses are

$$
\begin{aligned}
\text { Load losses } & =\left(\frac{7620}{(7620)(1.05)} \times \frac{(50)(1.40)}{50}\right)^{2}(564) \\
& =1,003 \mathrm{~W}
\end{aligned}
$$$$
\text { Percent load losses }=\frac{1003}{(1.4)(10)(50)(0.90)}=1.59 \%
$$

The percent no-load losses are

$$
\text { No-load losses }=(103)(1.15)=118.5 \mathrm{~W} \text {, }
$$

and the total percent losses are

$$
\text { Total percent losses }=\frac{1003+118.5}{(1.4)(10)(50)(0.90)}=1.78 \% . \quad \text { Equation } 5.40
$$

\subsection{Line Impedance Model}

A four-wire, wye-grounded, overhead line distribution circuit that consists of three-phase conductors and one neutral can be represented as a four-by-four matrix. With the aid of Figure 78, the resultant matrix, using Kirckhoff's voltage law, is shown below.

$$
\left[\begin{array}{l}
\mathrm{v}_{\mathrm{A}_{\mathrm{S}}} \\
\mathrm{v}_{\mathrm{B}} \\
\mathrm{v}_{\mathrm{C}_{\mathrm{S}}} \\
\mathrm{v}_{\mathrm{N}_{\mathrm{S}}}
\end{array}\right]=\left[\begin{array}{l}
\mathrm{v}_{\mathrm{A}_{\mathrm{L}}} \\
\mathrm{v}_{\mathrm{B}_{\mathrm{L}}} \\
\mathrm{v}_{\mathrm{C}_{\mathrm{L}}} \\
\mathrm{v}_{\mathrm{N}_{\mathrm{L}}}
\end{array}\right]+\left[\begin{array}{llll}
\mathrm{z}_{\mathrm{A}} & \mathrm{z}_{\mathrm{AB}} & \mathrm{z}_{\mathrm{AC}} & \mathrm{z}_{\mathrm{AN}} \\
\mathrm{z}_{\mathrm{BA}} & \mathrm{z}_{\mathrm{B}} & \mathrm{z}_{\mathrm{BC}} & \mathrm{z}_{\mathrm{BN}} \\
\mathrm{z}_{\mathrm{CA}} & \mathrm{z}_{\mathrm{CB}} & \mathrm{z}_{\mathrm{C}} & \mathrm{z}_{\mathrm{CN}} \\
\mathrm{z}_{\mathrm{NA}} & \mathrm{z}_{\mathrm{NB}} & \mathrm{z}_{\mathrm{NC}} & \mathrm{z}_{\mathrm{N}}
\end{array}\right] \cdot\left[\begin{array}{l}
\mathrm{I}_{\mathrm{A}} \\
\mathrm{I}_{\mathrm{B}} \\
\mathrm{I}_{\mathrm{C}} \\
\mathrm{I}_{\mathrm{N}}
\end{array}\right]
$$




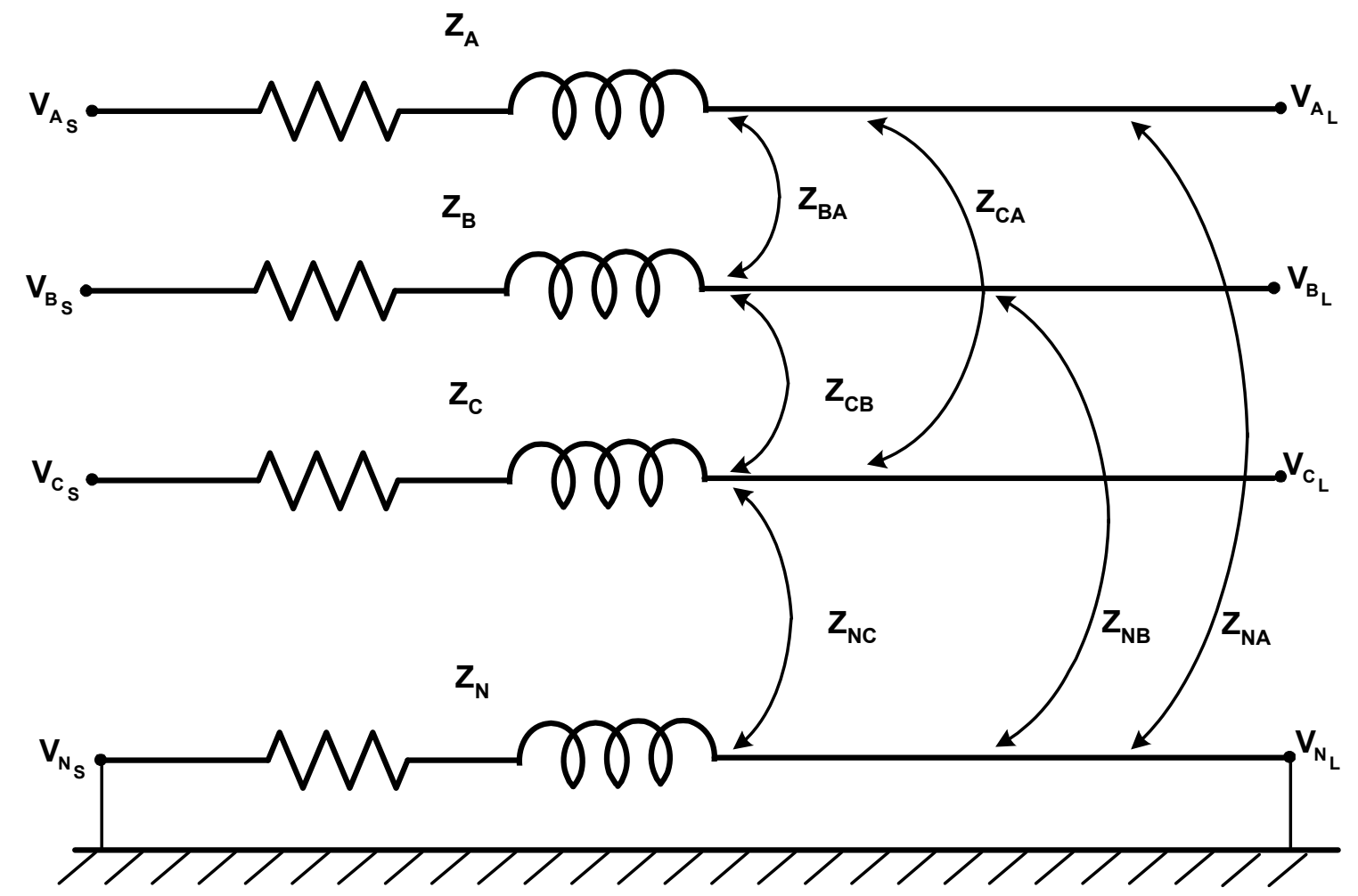

Figure 78. Four-wire, wye-grounded, overhead distribution line with multi-grounded neutral

For a three-conductor cable with one neutral, the impedance matrix is:

$$
Z=\left[\begin{array}{cccc}
Z_{\mathrm{A}} & \mathrm{Z}_{\mathrm{AB}} & \mathrm{Z}_{\mathrm{AC}} & \mathrm{Z}_{\mathrm{AN}} \\
\mathrm{Z}_{\mathrm{BA}} & \mathrm{Z}_{\mathrm{B}} & \mathrm{Z}_{\mathrm{BC}} & \mathrm{Z}_{\mathrm{BN}} \\
\mathrm{Z}_{\mathrm{CA}} & \mathrm{Z}_{\mathrm{CB}} & \mathrm{Z}_{\mathrm{C}} & \mathrm{Z}_{\mathrm{CN}} \\
\mathrm{Z}_{\mathrm{NA}} & \mathrm{Z}_{\mathrm{NB}} & \mathrm{Z}_{\mathrm{NC}} & \mathrm{Z}_{\mathrm{N}}
\end{array}\right] .
$$


For the flat-line configuration used in the Milford circuit, the line spacing is:

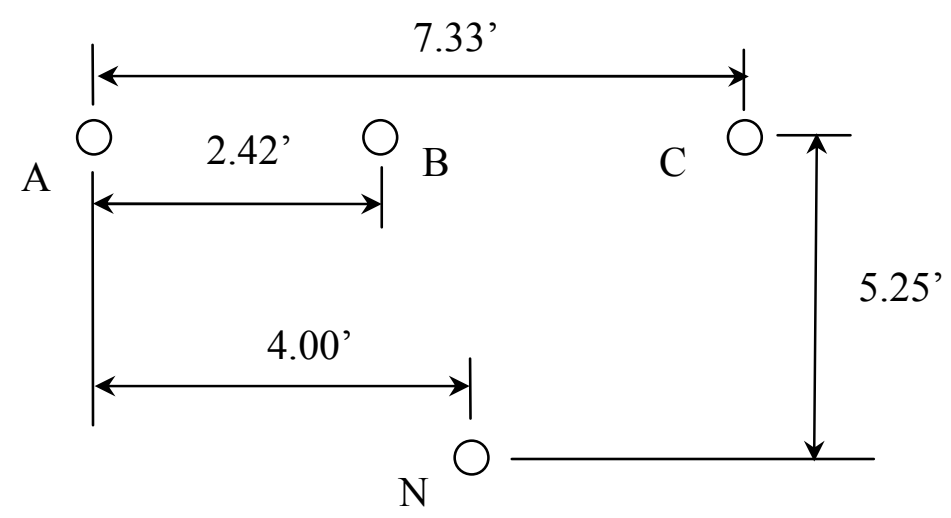

Figure 79. Flat line spacing configuration

The characteristics of the phase and neutral conductors are shown below.

\begin{tabular}{lcccc}
\hline & $\mathbf{A}$ & $\mathbf{B}$ & $\mathbf{C}$ & $\mathbf{N}$ \\
\cline { 2 - 5 } Conductor & & & & \\
GMR (ft) & $036 \mathrm{Al}$ & $636 \mathrm{Al}$ & $636 \mathrm{Al}$ & $1 / 0 \mathrm{ACSR}$ \\
Resistance $(\Omega / \mathrm{mi})$ & 0.146 & 0.0294 & 0.0294 & 0.006 \\
& & & 0.146 & 1.153 \\
\hline
\end{tabular}

The diagonal and off-diagonal elements of the impedance matrix (Kersting 2004) are calculated using:

$$
\begin{array}{ll}
\mathrm{z}_{\mathrm{ii}}=\mathrm{r}_{\mathrm{i}}+0.09530+\mathrm{j} 0.12134\left[\ln \left(1 / \mathrm{GMR}_{\mathrm{i}}\right)+7.93402\right] \Omega / \mathrm{mi} & \text { Equation } 5.42 \\
\mathrm{z}_{\mathrm{ij}}=0.09530+\mathrm{j} 0.12134\left[\ln \left(1 / \mathrm{D}_{\mathrm{ij}}\right)+7.93402\right] \Omega / \mathrm{mi}, & \text { Equation } 5.43
\end{array}
$$

where

$r_{i}=$ Resistance of conductor in $\Omega / \mathrm{mi}$

$\mathrm{D}_{\mathrm{ij}}=$ Distance in feet from conductor $\mathrm{i}$ to conductor $\mathrm{j}$

$\mathrm{GMR}_{\mathrm{i}}=$ Geometric mean radius of the conductor in feet

$0.09530=$ Equivalent resistance of earth $(100 \Omega-\mathrm{m})$

$7.93402=$ Equivalent mutual inductive reactance between a conductor and earth @ $60 \mathrm{~Hz}$.

It should be noted that the diagonal terms account for the self-inductance and resistance of the line and also contain expressions that take into account the effects of the earth return path. The off-diagonal elements account for the mutual inductance of the lines and again take into account the effects of the earth return path.

For the flat-line model of Figure 79, the impedance matrix is: 


$$
\mathrm{Z}=\left[\begin{array}{cccc}
.2413+\mathrm{j} 1.3907 & .0953+\mathrm{j} .85548 & .0953+\mathrm{j} .72101 & .0953+\mathrm{j} .70949 \\
.0953+\mathrm{j} .8554 & .2413+\mathrm{j} 1.3907 & .0953+\mathrm{j} .76963 & .0953+\mathrm{j} .78573 \\
.0953+\mathrm{j} .72101 & .0953+\mathrm{j} .76963 & .2413+\mathrm{j} 1.3907 & .0953+\mathrm{j} .76267 \\
.0953+\mathrm{j} .70949 & .0953+\mathrm{j} .78573 & .0953+\mathrm{j} .76267 & 1.2483+\mathrm{j} 1.3907
\end{array}\right]
$$

Equation 5.44

The Kron reduction technique allows this four-by-four matrix to be reduced to a moreapplicable three-by-three matrix $\mathrm{Z}_{\mathrm{ABC}}$.

$$
Z_{\mathrm{ij}}=\left[\begin{array}{ccc}
Z_{\mathrm{A}} & \mathrm{Z}_{\mathrm{AB}} & \mathrm{Z}_{\mathrm{AC}} \\
Z_{\mathrm{BA}} & \mathrm{Z}_{\mathrm{B}} & \mathrm{Z}_{\mathrm{BC}} \\
Z_{\mathrm{CA}} & Z_{\mathrm{CB}} & Z_{\mathrm{C}}
\end{array}\right]=\left[\begin{array}{ccc}
.2413+\mathrm{j} 1.3907 & .0953+\mathrm{j} .85548 & .0953+\mathrm{j} .72101 \\
.0953+\mathrm{j} .8554 & .2413+\mathrm{j} 1.3907 & .0953+\mathrm{j} .76963 \\
.0953+\mathrm{j} .72101 & .0953+\mathrm{j} .76963 & .2413+\mathrm{j} 1.3907
\end{array}\right]
$$

Equation 5.45

$$
\begin{aligned}
& Z_{\text {in }}=\left[\begin{array}{l}
\mathrm{Z}_{\mathrm{AN}} \\
\mathrm{Z}_{\mathrm{BN}} \\
\mathrm{Z}_{\mathrm{CN}}
\end{array}\right]=\left[\begin{array}{l}
.0953+\mathrm{j} .70949 \\
.0953+\mathrm{j} .78573 \\
.0953+\mathrm{j} .76267
\end{array}\right] \\
& \mathrm{Z}_{\mathrm{nj}}=\left[\begin{array}{llll}
Z_{\mathrm{NA}} & \mathrm{Z}_{\mathrm{NB}} & \mathrm{Z}_{\mathrm{NC}}
\end{array}\right]=\left[\begin{array}{lll}
.0953+\mathrm{j} .70949 & .0953+\mathrm{j} .78573 & .0953+\mathrm{j} .76267
\end{array}\right]
\end{aligned}
$$

Equation 5.47

$$
\begin{aligned}
& \mathrm{Z}_{\mathrm{N}}=\mathrm{ZN}_{\mathrm{N}}=1.2483+\mathrm{j} 1.5835 \\
& \mathrm{Z}_{\mathrm{ABC}}=\mathrm{Z}_{\mathrm{ij}}-\mathrm{Z}_{\mathrm{in}} \mathrm{Z}_{\mathrm{N}}^{-1} \mathrm{Z}_{\mathrm{nj}}=\left[\begin{array}{ccc}
.3404+\mathrm{j} 1.1566 & .0953+\mathrm{j} .85548 & .0953+\mathrm{j} .7210 \\
.0953+\mathrm{j} .85548 & .2413+\mathrm{j} 1.3907 & .0953+\mathrm{j} .76963 \\
.0953+\mathrm{j} .7210 & .0953+\mathrm{j} .76963 & .2413+\mathrm{j} 1.3907
\end{array}\right]
\end{aligned}
$$

Equation 5.48

Equation 5.49

For a non-transposed line of $6.25 \mathrm{mi}(33,000 \mathrm{ft})$, the impedance matrix becomes

$$
\mathrm{Z}_{\mathrm{ABC}}=\left[\begin{array}{lll}
2.1275+\mathrm{j} 7.2289 & 1.3100+\mathrm{j} 3.7384 & 1.2750+\mathrm{j} 2.9420 \\
1.3100+\mathrm{j} 3.7384 & 2.3108+\mathrm{j} 6.9235 & 1.3689+\mathrm{j} 3.0904 \\
1.2750+\mathrm{j} 2.9420 & 1.3689+\mathrm{j} 3.0904 & 2.2530+\mathrm{j} 7.0188
\end{array}\right] . \quad \text { Equation } 5.50
$$

\subsection{Line Voltage Drop Model}

The voltage drop for a given four-wire line segment is calculated using the impedance and matrix for that line segment.

$$
\begin{array}{lr}
\mathrm{V}_{\mathrm{S}}-\mathrm{V}_{\mathrm{L}}=\mathrm{Z}_{\mathrm{ABC}} \mathrm{I}_{\mathrm{ABC}} & \text { Equation } 5.51 \\
\Delta \mathrm{V}=\mathrm{Z}_{\mathrm{ABC}} \mathrm{I}_{\mathrm{ABC}} & \text { Equation } 5.52
\end{array}
$$




$$
\left[\begin{array}{c}
\Delta \mathrm{V}_{\mathrm{A}} \\
\Delta \mathrm{V}_{\mathrm{B}} \\
\Delta \mathrm{V}_{\mathrm{C}}
\end{array}\right]=\left[\begin{array}{ccc}
\mathrm{z}_{\mathrm{A}} & \mathrm{z}_{\mathrm{AB}} & \mathrm{z}_{\mathrm{AC}} \\
\mathrm{z}_{\mathrm{BA}} & \mathrm{z}_{\mathrm{B}} & \mathrm{z}_{\mathrm{BC}} \\
\mathrm{z}_{\mathrm{CA}} & \mathrm{z}_{\mathrm{CB}} & \mathrm{z}_{\mathrm{C}}
\end{array}\right] \cdot\left[\begin{array}{c}
\mathrm{I}_{\mathrm{A}} \\
\mathrm{I}_{\mathrm{B}} \\
\mathrm{I}_{\mathrm{C}}
\end{array}\right]
$$

Equation 5.53

The effects of the admittance matrix have been neglected because of the relatively short line length $(6.25 \mathrm{mi})$.

\subsection{Line Losses Model and Validation}

To validate the line loss model, three line configurations and balanced and unbalanced load conditions are considered. These line configurations are shown in Figure 80. Figure 80a is a balanced impedance triangular or equilateral configuration with a high $\mathrm{B}$ phase. The geometric mean distance (GMD) of the phase spacings shown in Figure 80a is 53 in. (4.42 $\mathrm{ft}$ ), which is the same as the GMD of the flat configuration in Figure 79. Figure $80 \mathrm{~b}$ is the flat non-transposed line, and Figure 80c is the flat transposed line. The dimensions between phases, neutral, and ground are identical in Figure 80b and Figure 80c, but the flat transposed line of Figure 80c has two transpositions.

Figure 81 shows the flat transposed line, which consists of three line segments of $11,000 \mathrm{ft}$ each, for a total length of 33,000 ft. The source is at Node 0 , and the two transpositions are shown at Node 1 and Node 2. The lump load of 9,000 kVA at unity power factor is located at the end of the line at Node 3. 


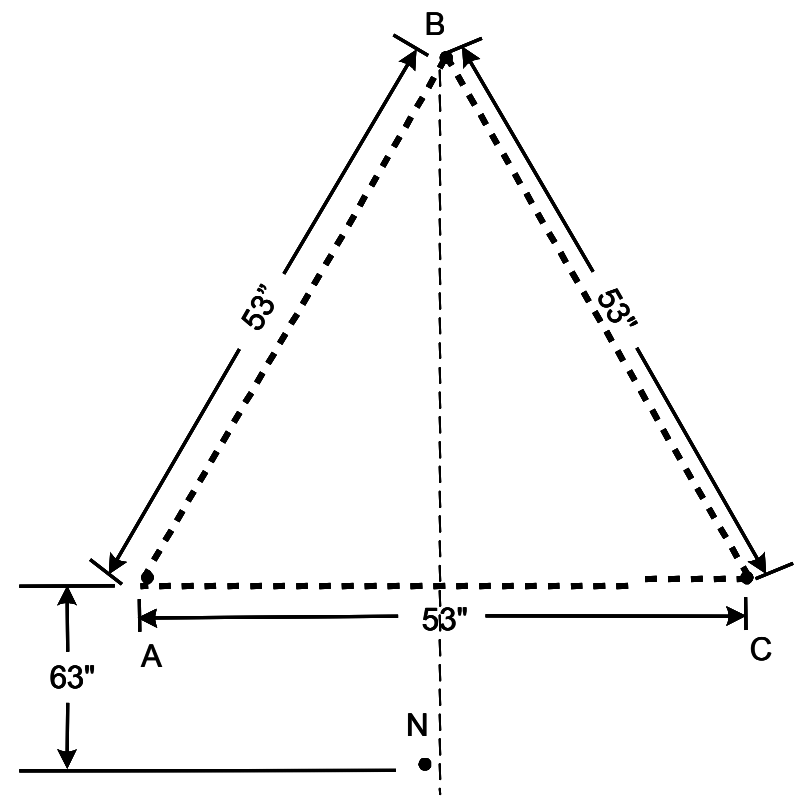

$$
\begin{aligned}
N=1 / 0 \text { ACSR } \\
\Phi=636 \mathrm{Al} \\
\mathrm{GMD}=53^{\prime \prime}
\end{aligned}
$$

(a) Equilateral Spacing

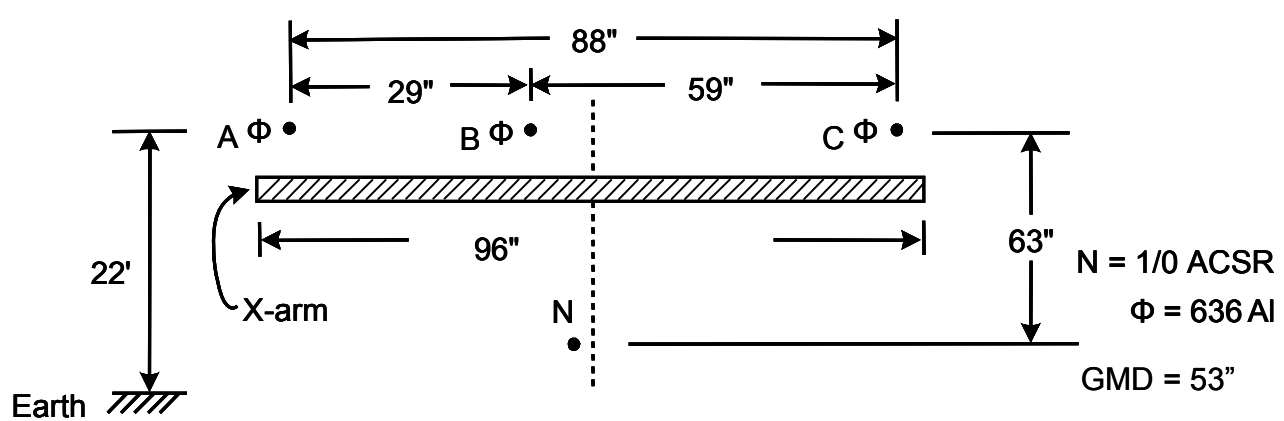

(b) Flat Non-transposed

Note: Neutral to ground distance $=22^{\prime}-63^{\prime \prime}=201^{\prime \prime}\left(16.75^{\prime}\right)$

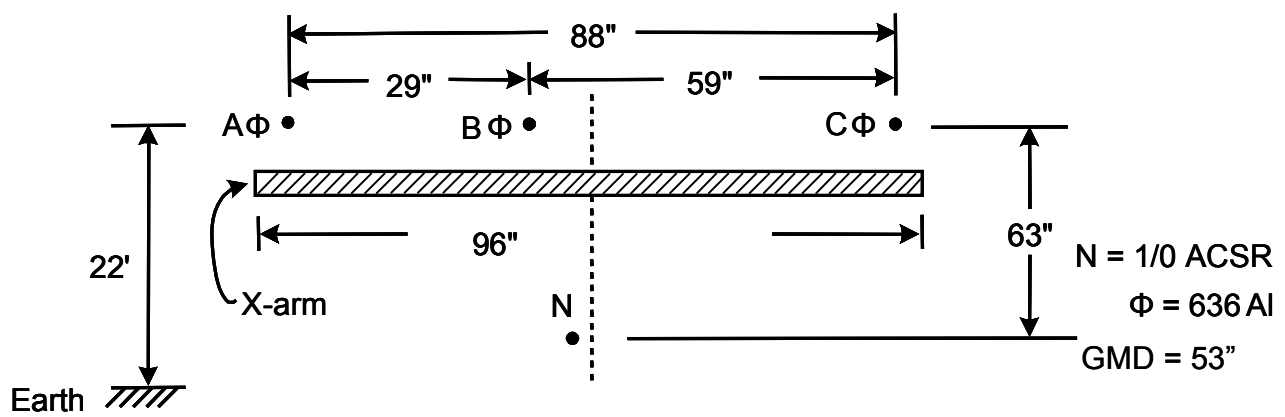

(c) Flat Transposed

Note: Neutral to ground distance $=22^{\prime}-63^{\prime \prime}=201 "$ " $\left(16.75^{\prime}\right)$

Figure 80. Configuration, phase, and neutral spacings; phase and neutral sizes; and conductor types 


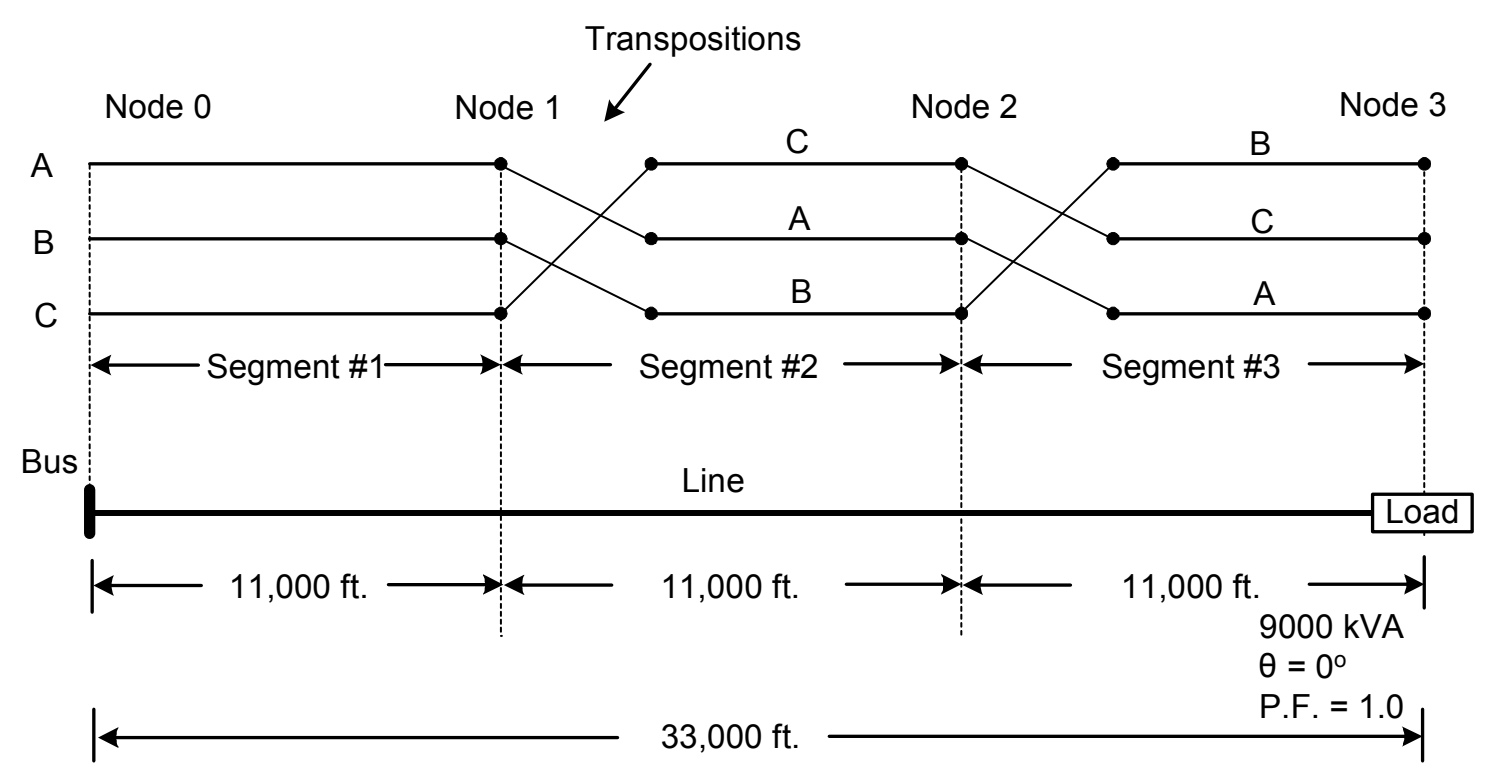

Flat Transposed Line and Load Characteristics

Figure 81. Line transposition and load

The purpose of this validation is to show that with balanced line impedance and balanced load the kilowatt losses are equal in each phase and total kilowatt losses are lowest. In addition, even though the kilowatt losses per phase may not be correct because of the Kron reduction process (which reduces a four-by-four matrix to a three-by-three matrix) the total losses are, in fact, correct. Table 11, Table 12, and Table 13 and the associated graphs provide a summary of balanced and unbalanced loading for the equilateral line spacing, nontransposed flat line spacing, and transposed flat line spacing configurations.

A balanced load of 3,000 kW per phase, modeled using constant impedance and computed using a nominal voltage of $7.62 \mathrm{kV}$, is attached at the end of the non-transposed line at Node 3. The load impedance matrix is

$$
Z_{\text {load }}=\left[\begin{array}{ccc}
19.35 & 0 & 0 \\
0 & 19.35 & 0 \\
0 & 0 & 19.35
\end{array}\right]
$$

where

$$
\mathrm{Z}_{\text {loadii }}=\frac{(7.62 \mathrm{kV})^{2}}{3 \mathrm{MVA}}=19.35
$$

Attaching the load to the end of the above non-transposed line yields 


$$
\mathrm{V}_{\mathrm{S}}=\left(\mathrm{Z}_{\mathrm{load}}+\mathrm{Z}_{\mathrm{ABC}}\right) \mathrm{I}_{\mathrm{ABC}}
$$

Equation 5.56

For a $13.2-\mathrm{kV}$ line, the above load, and the 6.25 -mi non-transposed line, solving for $\mathrm{I}_{\mathrm{ABC}}$ gives

$$
\begin{aligned}
I_{A B C} & =\left(Z_{\text {load }}+Z_{A B C}\right)^{-1} V_{S} \\
I_{A B C} & =\left[\begin{array}{c}
358.4 \angle-10.2 \\
377.8 \angle-130.2 \\
371.3 \angle 108.4
\end{array}\right], A .
\end{aligned}
$$

Equation 5.58

Notice from the non-transposed spreadsheet of Table 12, at Node 3, the magnitudes of phase currents are $\mathrm{I}_{\mathrm{A}}=356.2, \mathrm{I}_{\mathrm{B}}=375.9$, and $\mathrm{I}_{\mathrm{C}}=372.0$. These data are based on the voltage-dependent current (VDC) source model rather than the constant-impedance model data in the matrix above.

The load end voltage $\mathrm{V}$ load is therefore

$$
\begin{aligned}
\mathrm{V}_{\text {load }} & =\mathrm{V}_{\mathrm{S}}-\mathrm{Z}_{\mathrm{ABC}} \mathrm{I}_{\mathrm{ABC}} \\
\mathrm{V}_{\text {load }} & =\left[\begin{array}{c}
6.94 \angle-10.2 \\
7.31 \angle-130.2 \\
7.19 \angle 108.4
\end{array}\right], \mathrm{kV} .
\end{aligned}
$$

The magnitudes of the phase voltages in kilovolts from Table 12 at Node 3 are $V_{A}=6.96, V_{B}$ $=7.31$, and $\mathrm{V}_{\mathrm{C}}=7.24$. The power loss in the line is

$$
\mathrm{P}_{\text {loss }}=\operatorname{Re}\left[\mathrm{S}_{\text {in }}\right]-\operatorname{Re}\left[\mathrm{S}_{\text {out }}\right]=\left[\begin{array}{c}
200.5 \\
71.6 \\
102.8
\end{array}\right], \mathrm{kW} . \quad \text { Equation } 5.60
$$

The total loss for the line is $374.9 \mathrm{~kW}$. This compares to $\Phi \mathrm{A}=194.35 \mathrm{~kW}, \Phi B=72.47 \mathrm{~kW}$, and $\Phi C=84.29 \mathrm{~kW}$, for a total of $351.11 \mathrm{~kW}$ for the VDC model. If the balanced load impedance matrix is replaced with an unbalanced load impedance matrix, the loss results may appear incorrect.

$$
Z_{\text {load }}=\left[\begin{array}{ccc}
12.90 & 0 & 0 \\
0 & 25.81 & 0 \\
0 & 0 & 25.81
\end{array}\right]
$$


The above impedance matrix corresponds to a heavily loaded A phase $(4,500 \mathrm{~kW})$ relative to the $\mathrm{B}$ and $\mathrm{C}$ phases $(2,250 \mathrm{~kW})$. The last line of Table 12 at Node 0 shows the voltage magnitudes in kilovolts of $\mathrm{V}_{\mathrm{A}}=7.620, \mathrm{~V}_{\mathrm{B}}=7.620$, and $\mathrm{V}_{\mathrm{C}}=7.620$. Applying these balanced voltages and unbalanced loads for each phase,

$$
\begin{aligned}
& Z_{A}=\frac{(7620)^{2}}{2250 \times 10^{3}}=12.90, \\
& Z_{B}=\frac{(7620)^{2}}{4500 \times 10^{3}}=25.81, \text { and } \\
& Z_{C}=\frac{(7620)^{2}}{2250 \times 10^{3}}=25.81 .
\end{aligned}
$$

Repeating the above procedure to find the line losses yields

$$
\mathrm{P}_{\text {loss }}=\operatorname{Re}\left[\mathrm{S}_{\text {in }}\right]-\operatorname{Re}\left[\mathrm{S}_{\text {out }}\right]=\left[\begin{array}{c}
516.0 \\
-164.4 \\
57.0
\end{array}\right], \mathrm{kW} \text {. }
$$

Equation 5.62

The total loss for the line is $408.6 \mathrm{~kW}$. The phase losses from the spreadsheet for the nontransposed case are $131.8,390.5$, and -117.4 , corresponding to a heavily loaded B phase $(4,500 \mathrm{~kW})$ in the simulation case, or a total of $404.96 \mathrm{~kW}$. It is obvious in this unbalanced case that the phase loss calculations do not represent the actual losses in each phase; however, the total line loss is correct. The inclusion of the effects of the earth and neutral in the three-by-three impedance matrix is the reason for the dubious phase loss numbers. To obtain correct losses for the individual phases, the $\mathrm{I}^{2} \mathrm{R}$ losses should be calculated. First, the earth and neutral currents should be found.

$$
\mathrm{I}_{\mathrm{N}}=\mathrm{Z}_{\mathrm{Nt}} \mathrm{I}_{\mathrm{ABC}}=60.6 \angle 157.5, \mathrm{~A}
$$

Equation 5.63

where

$$
\mathrm{Z}_{\mathrm{Nt}} \mathrm{I}_{\mathrm{ABC}}=-\mathrm{Z}_{\mathrm{N}}^{-1} \mathrm{Z}_{\mathrm{nj}}
$$

Equation 5.64

The earth current is

$$
\mathrm{I}_{\mathrm{E}}=-\left(\mathrm{I}_{\mathrm{A}}+\mathrm{I}_{\mathrm{B}}+\mathrm{I}_{\mathrm{C}}+\mathrm{IN}_{\mathrm{N}}\right)=127.9 \angle 126.4, \mathrm{~A} .
$$

Equation 5.65

Next, the $I^{2} R$ losses for each phase, earth, and neutral are calculated.

$$
\begin{array}{ll}
\mathrm{P}_{\text {lossA }}=\left|\mathrm{IA}_{\mathrm{A}}\right|^{2} \mathrm{R}_{\mathrm{A}}=209.1, \mathrm{~kW} & \text { Equation } 5.66 \\
\mathrm{P}_{\text {lossB }}=\left|\mathrm{I}_{\mathrm{B}}\right|^{2} \mathrm{R}_{\mathrm{B}}=88.4, \mathrm{~kW} & \text { Equation } 5.67 \\
\mathrm{P}_{\text {lossc }}=|\mathrm{IC}|^{2} \mathrm{R}_{\mathrm{C}}=75.0, \mathrm{~kW} & \text { Equation } 5.68
\end{array}
$$




$$
\begin{aligned}
& P_{\text {losse }}=\left|I_{E}\right|^{2} R_{E}=9.7, k W \\
& P_{\text {loss }}=\left|I_{N}\right|^{2} R_{N}=26.4, k W
\end{aligned}
$$$$
\text { Equation } 5.69
$$

When the losses are summed from the $\mathrm{I}^{2} \mathrm{R}$ calculations, the result is 408.6 . This exactly matches the previously calculated total line loss. The reason for the slight difference between the total losses from the Table 12 spreadsheet data of $404.96 \mathrm{~kW}$ and the total losses of 408.6 $\mathrm{kW}$ above is the spreadsheet data are for a VDC model. The total losses above are based on a constant-impedance model.

Note: The PF in Table 11, Table 12, and Table 13 is the percent power flow imbalance (in kilovolt-amperes). 
Table 11. Equilateral Spacing Kilowatt Loss Evaluation

\begin{tabular}{|c|c|c|c|c|c|c|c|c|c|c|c|c|c|c|}
\hline \multicolumn{3}{|c|}{ Spot Load (kW) } & \multicolumn{3}{|c|}{ kW Losses } & \multirow{2}{*}{$\begin{array}{l}\text { Total kW } \\
\text { Losses }\end{array}$} & \multicolumn{3}{|c|}{ kVar Losses } & \multirow{2}{*}{$\begin{array}{c}\text { Total kVar } \\
\text { Losses }\end{array}$} & \multicolumn{3}{|c|}{ kW Flow } & \multirow[b]{2}{*}{ Total kW Flow } \\
\hline$\Phi A$ & $\Phi \mathrm{B}$ & $\Phi C$ & ФA & \begin{tabular}{|l|}
$\mathrm{B}$ \\
\end{tabular} & $\Phi C$ & & $\Phi A$ & \begin{tabular}{l|l}
$\Phi \mathrm{B}$ \\
\end{tabular} & $\Phi C$ & & ФA & \begin{tabular}{|c|}
$\mathrm{B}$ \\
\end{tabular} & $\Phi C$ & \\
\hline 3000.00 & 3000.00 & 3000.00 & 117.17 & 117.02 & 116.89 & 351.08 & 515.76 & 515.74 & 515.68 & 1547.18 & 2758.79 & 2759.06 & 2759.28 & 8277.13 \\
\hline 3150.00 & 2925.00 & 2925.00 & 143.85 & 94.42 & 113.39 & 351.66 & 568.41 & 507.63 & 472.37 & 1548.41 & 2856.88 & 2713.97 & 2703.31 & 8274.16 \\
\hline 3300.00 & 2850.00 & 2850.00 & 171.35 & 72.44 & 109.62 & 353.41 & 622.80 & 498.63 & 430.76 & 1552.19 & 2951.53 & 2666.94 & 2646.96 & 8265.43 \\
\hline 3450.00 & 2775.00 & 2775.00 & 199.93 & 51.43 & 104.92 & 356.28 & 677.99 & 489.86 & 390.41 & 1558.26 & 3041.81 & 2619.49 & 2589.48 & 8250.78 \\
\hline 3600.00 & 2700.00 & 2700.00 & 228.92 & 31.27 & 100.01 & 360.20 & 734.23 & 480.24 & 351.94 & 1566.41 & 3128.22 & 2570.23 & 2531.83 & 8230.28 \\
\hline 3750.00 & 2625.00 & 2625.00 & 258.85 & 11.66 & 94.75 & 365.26 & 792.19 & 470.22 & 314.54 & 1576.95 & 3211.81 & 2519.64 & 2472.90 & 8204.35 \\
\hline 3900.00 & 2550.00 & 2550.00 & 289.23 & -6.96 & 89.03 & 371.30 & 850.71 & 459.93 & 278.75 & 1589.39 & 3291.28 & 2468.02 & 2413.32 & 8172.62 \\
\hline 4050.00 & 2475.00 & 2475.00 & 320.05 & -24.56 & 82.79 & 378.28 & 909.51 & 449.56 & 244.49 & 1603.56 & 3366.43 & 2415.58 & 2352.90 & 8134.91 \\
\hline 4200.00 & 2400.00 & 2400.00 & 351.17 & -41.40 & 76.48 & 386.25 & 969.41 & 438.57 & 211.70 & 1619.68 & 3438.52 & 2361.61 & 2291.72 & 8091.85 \\
\hline 4350.00 & 2325.00 & 2325.00 & 382.50 & -57.46 & 70.17 & 395.21 & 1030.43 & 426.96 & 180.38 & 1637.77 & 3507.79 & 2306.09 & 2229.77 & 8043.65 \\
\hline 4500.00 & 2250.00 & 2250.00 & 414.10 & -72.43 & 63.39 & 405.06 & 1091.39 & 415.48 & 150.55 & 1657.42 & 3572.73 & 2249.97 & 2166.85 & 7989.55 \\
\hline
\end{tabular}

\begin{tabular}{|c|c|c|c|c|c|c|c|c|c|c|c|c|c|c|}
\hline \multicolumn{15}{|c|}{ Node 0} \\
\hline \multicolumn{3}{|c|}{ Voltage } & \multicolumn{3}{|c|}{ Sequence Voltages (V) } & \multirow[b]{2}{*}{$\mathrm{V} 2 / \mathrm{V} 1$} & \multicolumn{3}{|c|}{ Current } & \multicolumn{3}{|c|}{ Sequence Currents } & \multicolumn{2}{|c|}{ Imbalance } \\
\hline$\Phi A$ & ФВ & $\Phi C$ & V0 & V1 & V2 & & $\Phi A$ & ФB & $\Phi C$ & 10 & 11 & 12 & \begin{tabular}{|l|l|} 
& \\
\end{tabular} & PF \\
\hline 7.62 & 7.62 & 7.62 & 0 & 7620.91 & 0.11 & $0.0 \%$ & 368.22 & 368.25 & 368.28 & 0.02 & 368.24 & 0.01 & $0.0 \%$ & $0.0 \%$ \\
\hline 7.62 & 7.62 & 7.62 & 0 & 7620.91 & 0.11 & $0.0 \%$ & 382.16 & 362.23 & 360.04 & 6.44 & 368.12 & 8.39 & $2.3 \%$ & $4.0 \%$ \\
\hline 7.62 & 7.62 & 7.62 & 0 & 7620.91 & 0.11 & $0.0 \%$ & 395.74 & 355.95 & 351.85 & 12.85 & 367.78 & 16.63 & $4.5 \%$ & $8.0 \%$ \\
\hline 7.62 & 7.62 & 7.62 & 0 & 7620.91 & 0.11 & $0.0 \%$ & 408.87 & 349.61 & 343.58 & 19.24 & 367.2 & 24.7 & $6.7 \%$ & $11.0 \%$ \\
\hline 7.62 & 7.62 & 7.62 & 0 & 7620.91 & 0.11 & $0.0 \%$ & 421.56 & 343.04 & 335.36 & 25.53 & 366.38 & 32.61 & $8.9 \%$ & $15.0 \%$ \\
\hline 7.62 & 7.62 & 7.62 & 0 & 7620.91 & 0.11 & $0.0 \%$ & 433.99 & 336.27 & 327.06 & 31.87 & 365.35 & 40.37 & $11.0 \%$ & $19.0 \%$ \\
\hline 7.62 & 7.62 & 7.62 & 0 & 7620.91 & 0.11 & $0.0 \%$ & 445.97 & 329.36 & 318.74 & 38.14 & 364.09 & 47.97 & $13.2 \%$ & $22.0 \%$ \\
\hline 7.62 & 7.62 & 7.62 & 0 & 7620.91 & 0.11 & $0.0 \%$ & 457.5 & 322.35 & 310.36 & 44.35 & 362.59 & 55.41 & $15.3 \%$ & $26.0 \%$ \\
\hline 7.62 & 7.62 & 7.62 & 0 & 7620.91 & 0.11 & $0.0 \%$ & 468.72 & 315.12 & 301.96 & 50.54 & 360.88 & 62.69 & $17.4 \%$ & $30.0 \%$ \\
\hline 7.62 & 7.62 & 7.62 & 0 & 7620.91 & 0.11 & $0.0 \%$ & 479.63 & 307.68 & 293.51 & 56.72 & 358.97 & 69.83 & $19.5 \%$ & 0.33 \\
\hline 7.62 & 7.62 & 7.62 & 0 & 7620.91 & 0.11 & $0.0 \%$ & 490.12 & 300.16 & 284.99 & 62.84 & 356.83 & 76.81 & $21.5 \%$ & 0.37 \\
\hline
\end{tabular}

\begin{tabular}{|c|c|c|c|c|c|c|c|c|c|c|c|c|c|c|}
\hline \multicolumn{15}{|c|}{ Node 1} \\
\hline \multicolumn{3}{|c|}{ Voltage } & \multicolumn{3}{|c|}{ Sequence Voltages (V) } & \multirow[b]{2}{*}{$\mathrm{V} 2 / \mathrm{V} 1$} & \multicolumn{3}{|c|}{ Current } & \multicolumn{3}{|c|}{ Sequence Currents } & \multicolumn{2}{|c|}{ Imbalance } \\
\hline ФA & $\Phi \mathrm{B}$ & $\Phi C$ & V0 & V1 & V2 & & ФA & ФB & $\Phi C$ & \begin{tabular}{|l|}
10 \\
\end{tabular} & \begin{tabular}{|l|}
11 \\
\end{tabular} & 12 & \begin{tabular}{l|l} 
I2/I1 \\
\end{tabular} & $\mathrm{PF}$ \\
\hline 7.44 & 7.44 & 7.44 & 0.1 & 7444.34 & 0.08 & $0.0 \%$ & 368.22 & 368.25 & 368.28 & 0.02 & 368.24 & 0.01 & $0.0 \%$ & $0.0 \%$ \\
\hline 7.42 & 7.46 & 7.45 & 27.28 & 7444.34 & 10.95 & $0.1 \%$ & 382.16 & 362.23 & 360.04 & 6.44 & 368.12 & 8.39 & $2.3 \%$ & $4.0 \%$ \\
\hline 7.39 & 7.48 & 7.46 & 54.31 & 7444.22 & 21.65 & $0.3 \%$ & 395.74 & 355.95 & 351.85 & 12.85 & 367.78 & 16.63 & $4.5 \%$ & $8.0 \%$ \\
\hline 7.36 & 7.5 & 7.47 & 81.21 & 7444.01 & 32.15 & $0.4 \%$ & 408.87 & 349.61 & 343.58 & 19.24 & 367.2 & 24.7 & $6.7 \%$ & $11.0 \%$ \\
\hline 7.33 & 7.52 & 7.48 & 107.71 & 7443.74 & 42.43 & $0.6 \%$ & 421.56 & 343.04 & 335.36 & 25.53 & 366.38 & 32.61 & $8.9 \%$ & $15.0 \%$ \\
\hline 7.3 & 7.54 & 7.49 & 134.43 & 7443.41 & 52.52 & $0.7 \%$ & 433.99 & 336.27 & 327.06 & 31.87 & 365.35 & 40.37 & $11.0 \%$ & $19.0 \%$ \\
\hline 7.27 & 7.56 & 7.5 & 160.86 & 7443.01 & 62.4 & $0.8 \%$ & 445.97 & 329.36 & 318.74 & 38.14 & 364.09 & 47.97 & $13.2 \%$ & $22.0 \%$ \\
\hline 7.25 & 7.58 & 7.51 & 187.04 & 7442.55 & 72.06 & $1.0 \%$ & 457.5 & 322.35 & 310.36 & 44.35 & 362.59 & 55.41 & $15.3 \%$ & $26.0 \%$ \\
\hline 7.22 & 7.59 & 7.52 & 213.13 & 7442.05 & 81.53 & $1.1 \%$ & 468.72 & 315.12 & 301.96 & 50.54 & 360.88 & 62.69 & $17.4 \%$ & $30.0 \%$ \\
\hline 7.19 & 7.61 & 7.53 & 239.13 & 7441.52 & 90.81 & $1.2 \%$ & 479.63 & 307.68 & 293.51 & 56.72 & 358.97 & 69.83 & $19.5 \%$ & 0.33 \\
\hline 7.16 & 7.63 & 7.54 & 264.96 & 7440.9 & 99.88 & $1.3 \%$ & 490.12 & 300.16 & 284.99 & 62.84 & 356.83 & 76.81 & $21.5 \%$ & 0.37 \\
\hline
\end{tabular}

\begin{tabular}{|c|c|c|c|c|c|c|c|c|c|c|c|c|c|c|}
\hline \multicolumn{15}{|c|}{ Node 2} \\
\hline \multicolumn{3}{|c|}{ Voltage } & \multicolumn{3}{|c|}{ Sequence Voltages (V) } & \multirow[b]{2}{*}{$\mathrm{V} 2 / \mathrm{V} 1$} & \multicolumn{3}{|c|}{ Current } & \multicolumn{3}{|c|}{ Sequence Currents } & \multicolumn{2}{|c|}{ Imbalance } \\
\hline$\Phi A$ & ФВ & $\Phi C$ & V0 & \begin{tabular}{l|l}
$\mathrm{V} 1$ & \\
\end{tabular} & $\mathrm{~V} 2$ & & $\Phi A$ & ФB & $\Phi C$ & \begin{tabular}{l|l}
10 \\
\end{tabular} & \begin{tabular}{l|l}
11 \\
\end{tabular} & 12 & \begin{tabular}{|l|l|}
$\mid 2 / I 1$ \\
\end{tabular} & $\mathrm{PF}$ \\
\hline 7.29 & 7.29 & 7.3 & 0.19 & 7294.81 & 0.15 & $0.0 \%$ & 368.23 & 368.27 & 368.29 & 0.02 & 368.26 & 0.01 & $0.0 \%$ & $0.0 \%$ \\
\hline 7.24 & 7.33 & 7.31 & 54.55 & 7294.79 & 21.95 & $0.3 \%$ & 382.18 & 362.24 & 360.05 & 6.44 & 368.13 & 8.39 & $2.3 \%$ & $3.0 \%$ \\
\hline 7.19 & 7.37 & 7.33 & 108.61 & 7294.48 & 43.37 & $0.6 \%$ & 395.76 & 355.97 & 351.86 & 12.85 & 367.79 & 16.63 & $4.5 \%$ & $7.0 \%$ \\
\hline 7.13 & 7.41 & 7.34 & 162.42 & 7293.96 & 64.36 & $0.9 \%$ & 408.89 & 349.63 & 343.59 & 19.24 & 367.21 & 24.7 & $6.7 \%$ & $10.0 \%$ \\
\hline 7.08 & 7.45 & 7.36 & 215.42 & 7293.28 & 84.91 & $1.2 \%$ & 421.58 & 343.05 & 335.37 & 25.52 & 366.39 & 32.61 & $8.9 \%$ & $13.0 \%$ \\
\hline 7.03 & 7.48 & 7.37 & 268.87 & 7292.42 & 105.1 & $1.4 \%$ & 434.01 & 336.28 & 327.07 & 31.87 & 365.37 & 40.37 & $11.0 \%$ & $17.0 \%$ \\
\hline 6.98 & 7.52 & 7.39 & 321.72 & 7291.4 & 124.86 & $1.7 \%$ & 445.99 & 329.38 & 318.74 & 38.14 & 364.11 & 47.97 & $13.2 \%$ & $20.0 \%$ \\
\hline 6.92 & 7.56 & 7.41 & 374.07 & 7290.21 & 144.19 & $2.0 \%$ & 457.52 & 322.36 & 310.37 & 44.35 & 362.61 & 55.41 & $15.3 \%$ & $23.0 \%$ \\
\hline 6.87 & 7.6 & 7.42 & 426.25 & 7288.9 & 163.12 & $2.2 \%$ & 468.74 & 315.14 & 301.96 & 50.54 & 360.9 & 62.69 & $17.4 \%$ & $26.0 \%$ \\
\hline 6.82 & 7.63 & 7.44 & 478.26 & 7287.49 & 181.69 & $2.5 \%$ & 479.65 & 307.7 & 293.52 & 56.71 & 358.99 & 69.83 & $19.5 \%$ & 0.29 \\
\hline 6.77 & 7.67 & 7.46 & 529.91 & 7285.88 & 199.83 & $2.7 \%$ & 490.14 & 300.18 & 284.99 & 62.84 & 356.84 & 76.81 & $21.5 \%$ & 0.32 \\
\hline
\end{tabular}

\begin{tabular}{|c|c|c|c|c|c|c|c|c|c|c|c|c|c|c|}
\hline \multicolumn{15}{|c|}{ Node 3} \\
\hline \multicolumn{3}{|c|}{ Voltage } & \multicolumn{3}{|c|}{ Sequence Voltages (V) } & \multirow[b]{2}{*}{$\mathrm{V} 2 / \mathrm{V} 1$} & \multicolumn{3}{|c|}{ Current } & \multicolumn{3}{|c|}{ Sequence Currents } & \multicolumn{2}{|c|}{ Imbalance } \\
\hline$\Phi \mathrm{A}$ & बB & $\Phi C$ & V0 & $\mathrm{V} 1$ & $\mathrm{~V} 2$ & & $\Phi A$ & $\Phi \mathrm{B}$ & $\Phi C$ & 10 & 11 & 12 & $|2 /| 1$ & $\mathrm{PF}$ \\
\hline 7.17 & 7.17 & 7.17 & 0.29 & 7174.03 & 0.24 & $0.0 \%$ & 368.24 & 368.27 & 368.3 & 0.02 & 368.26 & 0.01 & $0.0 \%$ & $0.0 \%$ \\
\hline 7.1 & 7.23 & 7.19 & 81.83 & 7173.95 & 32.96 & $0.5 \%$ & 382.19 & 362.25 & 360.06 & 6.44 & 368.14 & 8.39 & $2.3 \%$ & $3.0 \%$ \\
\hline 7.02 & 7.29 & 7.21 & 162.92 & 7173.39 & 65.08 & $0.9 \%$ & 395.77 & 355.97 & 351.86 & 12.85 & 367.8 & 16.63 & $4.5 \%$ & $6.0 \%$ \\
\hline 6.95 & 7.35 & 7.23 & 243.63 & 7172.45 & 96.57 & $1.3 \%$ & 408.9 & 349.63 & 343.59 & 19.24 & 367.22 & 24.7 & $6.7 \%$ & $9.0 \%$ \\
\hline 6.88 & 7.4 & 7.25 & 323.12 & 7171.2 & 127.4 & $1.8 \%$ & 421.59 & 343.06 & 335.38 & 25.52 & 366.4 & 32.61 & $8.9 \%$ & $12.0 \%$ \\
\hline 6.8 & 7.46 & 7.27 & 403.29 & 7169.63 & 157.69 & $2.2 \%$ & 434.02 & 336.29 & 327.07 & 31.87 & 365.37 & 40.37 & $11.0 \%$ & $15.0 \%$ \\
\hline 6.73 & 7.51 & 7.29 & 482.57 & 7167.75 & 187.32 & $2.6 \%$ & 446 & 329.38 & 318.75 & 38.14 & 364.11 & 47.97 & $13.2 \%$ & $17.0 \%$ \\
\hline 6.66 & 7.57 & 7.31 & 561.09 & 7165.55 & 216.31 & $3.0 \%$ & 457.53 & 322.37 & 310.37 & 44.35 & 362.62 & 55.4 & $15.3 \%$ & $20.0 \%$ \\
\hline 6.59 & 7.63 & 7.34 & 639.36 & 7163.11 & 244.72 & $3.4 \%$ & 468.75 & 315.14 & 301.96 & 50.54 & 360.91 & 62.69 & $17.4 \%$ & $23.0 \%$ \\
\hline 6.52 & 7.68 & 7.36 & 717.38 & 7160.47 & 272.57 & $3.8 \%$ & 479.67 & 307.7 & 293.52 & 56.71 & 358.99 & 69.83 & $19.5 \%$ & 0.26 \\
\hline 6.44 & 7.74 & 7.38 & 794.85 & 7157.47 & 299.78 & $4.2 \%$ & 490.16 & 300.18 & 284.99 & 62.84 & 356.85 & 76.81 & $21.5 \%$ & 0.28 \\
\hline
\end{tabular}




\begin{tabular}{|c|c|c|c|c|c|c|c|c|c|c|c|}
\hline \multicolumn{3}{|c|}{ kVar Flow } & $\begin{array}{c}\text { Total kVar } \\
\text { Flow }\end{array}$ & \multicolumn{3}{|c|}{ kW Native Load } & $\begin{array}{c}\text { Total kW } \\
\text { Load }\end{array}$ & \multicolumn{3}{|c|}{ kVar Native Load } & $\begin{array}{c}\text { Total kVar } \\
\text { Load }\end{array}$ \\
\hline 513.61 & 513.62 & 513.49 & 1540.72 & 2641.63 & 2642.04 & 2642.39 & 7926.06 & -2.15 & -2.12 & -2.20 & -6.47 \\
\hline 620.16 & 496.25 & 428.61 & 1545.02 & 2780.18 & 2594.50 & 2537.33 & 7912.01 & -2.64 & -2.38 & -2.15 & -7.17 \\
\hline 675.99 & 487.17 & 388.05 & 1551.21 & 2841.88 & 2568.05 & 2484.56 & 7894.49 & -2.00 & -2.70 & -2.36 & -7.06 \\
\hline 732.12 & 478.01 & 349.25 & 1559.38 & 2899.30 & 2538.95 & 2431.82 & 7870.07 & -2.11 & -2.23 & -2.69 & -7.03 \\
\hline 907.62 & 447.15 & 241.84 & 1596.61 & 3046.38 & 2440.15 & 2270.11 & 7756.64 & -1.89 & -2.41 & -2.65 & -6.95 \\
\hline 967.68 & 436.16 & 208.98 & 1612.82 & 3087.35 & 2403.01 & 2215.24 & 7705.60 & -1.73 & -2.42 & -2.72 & -6.87 \\
\hline 1027.84 & 424.65 & 177.97 & 1630.46 & 3125.30 & 2363.54 & 2159.60 & 7648.44 & -2.58 & -2.31 & -2.41 & -7.30 \\
\hline 1089.67 & 412.92 & 147.93 & 1650.52 & 3158.63 & 2322.40 & 2103.46 & 7584.49 & -1.73 & -2.56 & -2.61 & -6.90 \\
\hline
\end{tabular}

Plot Information:

\begin{tabular}{rcr} 
Node 0 & Total kW & \multicolumn{1}{c}{ kW } \\
I2/l1 & Losses / \\
$0.0 \%$ & 351.08 & kW Flow \\
$2.3 \%$ & 351.66 & $4.2 \%$ \\
$4.5 \%$ & 353.41 & $4.3 \%$ \\
$6.7 \%$ & 356.28 & $4.3 \%$ \\
$8.9 \%$ & 360.20 & $4.4 \%$ \\
$11.0 \%$ & 365.26 & $4.5 \%$ \\
$13.2 \%$ & 371.30 & $4.5 \%$ \\
$15.3 \%$ & 378.28 & $4.7 \%$ \\
$17.4 \%$ & 386.25 & $4.8 \%$ \\
$19.5 \%$ & 395.21 & $4.9 \%$ \\
$21.5 \%$ & 405.06 & $5.1 \%$
\end{tabular}

\begin{tabular}{rrr} 
Node 1 & Node 2 & \multicolumn{1}{c}{ Node 3 } \\
V1/V2 & V1/N2 & V1/N2 \\
$0.0 \%$ & $0.0 \%$ & $0.0 \%$ \\
$0.1 \%$ & $0.3 \%$ & $0.5 \%$ \\
$0.3 \%$ & $0.6 \%$ & $0.9 \%$ \\
$0.4 \%$ & $0.9 \%$ & $1.3 \%$ \\
$0.6 \%$ & $1.2 \%$ & $1.8 \%$ \\
$0.7 \%$ & $1.4 \%$ & $2.2 \%$ \\
$0.8 \%$ & $1.7 \%$ & $2.6 \%$ \\
$1.0 \%$ & $2.0 \%$ & $3.0 \%$ \\
$1.1 \%$ & $2.2 \%$ & $3.4 \%$ \\
$1.2 \%$ & $2.5 \%$ & $3.8 \%$ \\
$1.3 \%$ & $2.7 \%$ & $4.2 \%$
\end{tabular}

\begin{tabular}{rlr}
\multicolumn{1}{l}{ I0 } & \multicolumn{1}{l}{ I } & \multicolumn{1}{l}{ I } \\
0.02 & 368.24 & 0.01 \\
6.44 & 368.12 & 8.39 \\
12.85 & 367.78 & 16.63 \\
19.24 & 367.20 & 24.70 \\
25.53 & 366.38 & 32.61 \\
31.87 & 365.35 & 40.37 \\
38.14 & 364.09 & 47.97 \\
44.35 & 362.59 & 55.41 \\
50.54 & 360.88 & 62.69 \\
56.72 & 358.97 & 69.83 \\
62.84 & 356.83 & 76.81
\end{tabular}


Table 12. Non-Transposed Kilowatt Loss Evaluation

\begin{tabular}{|c|c|c|c|c|c|c|c|c|c|c|c|c|c|c|}
\hline \multicolumn{3}{|c|}{ Spot Load (kW) } & \multicolumn{3}{|c|}{ kW Losses } & \multirow{2}{*}{$\begin{array}{c}\text { Total kW } \\
\text { Losses }\end{array}$} & \multicolumn{3}{|c|}{ kVar Losses } & \multirow{2}{*}{$\begin{array}{c}\text { Total kVar } \\
\text { Losses }\end{array}$} & \multicolumn{3}{|c|}{ kW Flow } & \multirow[b]{2}{*}{ Total kW Flow } \\
\hline ФА & ФB & $\Phi C$ & ФА & ФB & $\Phi C$ & & ФА & \begin{tabular}{|l|l} 
ФB & \\
\end{tabular} & $\Phi C$ & & $\Phi A$ & ФB & $\Phi C$ & \\
\hline 3000.00 & 3000.00 & 3000.00 & 194.35 & 72.47 & 84.29 & 351.11 & 468.69 & 504.08 & 571.12 & 1543.89 & 2673.63 & 2820.78 & 2777.24 & 8271.65 \\
\hline 2925.00 & 3150.00 & 2925.00 & 191.64 & 101.18 & 59.82 & 352.64 & 421.45 & 558.63 & 560.65 & 1540.73 & 2617.99 & 2918.11 & 2734.33 & 8270.43 \\
\hline 2850.00 & 3300.00 & 2850.00 & 187.85 & 130.92 & 36.35 & 355.12 & 376.15 & 614.45 & 549.88 & 1540.48 & 2561.96 & 3010.91 & 2690.10 & 8262.97 \\
\hline 2775.00 & 3450.00 & 2775.00 & 183.15 & 161.60 & 13.77 & 358.52 & 332.63 & 671.65 & 538.71 & 1542.99 & 2505.32 & 3099.54 & 2644.44 & 8249.30 \\
\hline 2700.00 & 3600.00 & 2700.00 & 177.65 & 193.08 & -7.92 & 362.81 & 290.90 & 730.13 & 527.13 & 1548.16 & 2448.06 & 3184.09 & 2597.33 & 8229.48 \\
\hline 2625.00 & 3750.00 & 2625.00 & 171.40 & 225.19 & -28.66 & 367.93 & 250.97 & 789.76 & 515.15 & 1555.88 & 2390.15 & 3264.58 & 2548.82 & 8203.55 \\
\hline 2550.00 & 3900.00 & 2550.00 & 164.51 & 257.80 & -48.44 & 373.87 & 212.83 & 850.44 & 502.77 & 1566.04 & 2331.59 & 3341.09 & 2498.88 & 8171.56 \\
\hline 2475.00 & 4050.00 & 2475.00 & 157.03 & 290.77 & -67.24 & 380.56 & 176.49 & 912.05 & 490.01 & 1578.55 & 2272.37 & 3413.67 & 2447.55 & 8133.59 \\
\hline 2400.00 & 4200.00 & 2400.00 & 149.06 & 323.97 & -85.02 & 388.01 & 141.95 & 974.51 & 476.88 & 1593.34 & 2212.46 & 3482.4 & 2394.83 & 8089.69 \\
\hline 2325.00 & 4350.00 & 2325.00 & 140.67 & 357.27 & -101.76 & 396.18 & 109.20 & 1037.72 & 463.38 & 1610.30 & 2151.87 & 3547.33 & 2340.72 & 8039.92 \\
\hline 2250.00 & 4500.00 & 2250.00 & 131.84 & 390.48 & -117.36 & 404.96 & 78.33 & 1101.20 & 449.63 & 1629.16 & 2090.66 & 3608.08 & 2285.35 & 7984.09 \\
\hline \multicolumn{15}{|c|}{ Node 0} \\
\hline \multicolumn{3}{|c|}{ Voltage } & \multicolumn{3}{|c|}{ Sequence Voltages (V) } & & \multicolumn{3}{|c|}{ Current } & \multicolumn{3}{|c|}{ Sequence Currents } & \multicolumn{2}{|c|}{ Imbalance } \\
\hline ФA & $\Phi \mathrm{B}$ & $\Phi C$ & \begin{tabular}{l|l}
$\mathrm{V} 0$ & \\
\end{tabular} & \begin{tabular}{l|l}
$\mathrm{V} 1$ & \\
\end{tabular} & V2 & $\mathrm{V} 2 / \mathrm{V} 1$ & $\Phi \mathrm{A}$ & $\Phi \mathrm{B}$ & $\Phi C$ & \begin{tabular}{l|l}
10 & \\
\end{tabular} & \begin{tabular}{l|l}
11 \\
\end{tabular} & 12 & \begin{tabular}{|l|l|}
$\mid 2 / I 1$ & \\
\end{tabular} & PF \\
\hline 7.6200 & 7.6200 & 7.6200 & 0 & 7620.91 & 0.11 & $0.0 \%$ & 356.11 & 375.94 & 371.99 & 3.24 & 367.98 & 9.3 & $2.5 \%$ & 0.03 \\
\hline 7.62 & 7.62 & 7.62 & 0 & 7620.91 & 0.11 & $0.0 \%$ & 347.9 & 389.79 & 366.19 & 9.06 & 367.9 & 15.93 & $4.3 \%$ & 0.06 \\
\hline 7.62 & 7.62 & 7.6200 & 0 & 7620.91 & 0.11 & $0.0 \%$ & 339.73 & 403.15 & 360.22 & 15.38 & 367.57 & 23.48 & $6.4 \%$ & 0.1 \\
\hline 7.62 & 7.62 & 7.6200 & 0 & 7620.91 & 0.11 & $0.0 \%$ & 331.58 & 416.08 & 354.06 & 21.72 & 367.01 & 31.17 & $8.5 \%$ & 0.13 \\
\hline 7.62 & 7.62 & 7.6200 & 0 & 7620.91 & 0.11 & $0.0 \%$ & 323.45 & 428.57 & 347.7 & 28.05 & 366.2 & 38.8 & $10.6 \%$ & 0.17 \\
\hline 7.62 & 7.62 & 7.6200 & 0 & 7620.91 & 0.11 & $0.0 \%$ & 315.32 & 440.64 & 341.15 & 34.34 & 365.14 & 46.31 & $12.7 \%$ & 0.2 \\
\hline 7.62 & 7.62 & 7.6200 & 0 & 7620.91 & 0.11 & $0.0 \%$ & 307.19 & 452.3 & 334.41 & 40.59 & 363.85 & 53.68 & $14.8 \%$ & 0.24 \\
\hline 7.62 & 7.62 & 7.6200 & 0 & 7620.91 & 0.11 & $0.0 \%$ & 299.04 & 463.56 & 327.47 & 46.8 & 362.32 & 60.9 & $16.8 \%$ & 0.28 \\
\hline 7.62 & 7.62 & 7.62 & 0 & 7620.91 & 0.11 & $0.0 \%$ & 290.89 & 474.41 & 320.35 & 52.97 & 360.56 & 67.97 & $18.9 \%$ & 0.31 \\
\hline 7.62 & 7.62 & 7.62 & 0 & 7620.91 & 0.11 & $0.0 \%$ & 282.71 & 484.88 & 313.04 & 59.1 & 358.57 & 74.88 & $20.9 \%$ & 0.35 \\
\hline 7.6200 & 7.62 & 7.62 & 0 & 7620.91 & 0.11 & $0.0 \%$ & 274.51 & 494.92 & 305.56 & 65.15 & 356.34 & 81.62 & $22.9 \%$ & 0.38 \\
\hline
\end{tabular}

\begin{tabular}{|c|c|c|c|c|c|c|c|c|c|c|c|c|c|c|}
\hline \multicolumn{15}{|c|}{$\begin{array}{l}\text { Node } 1 \\
\end{array}$} \\
\hline \multicolumn{3}{|c|}{ Voltage } & \multicolumn{3}{|c|}{ Sequence Voltages $(\mathrm{V})$} & \multirow[b]{2}{*}{$\mathrm{V} 2 \mathrm{~N} 1$} & \multicolumn{3}{|c|}{ Current } & \multicolumn{3}{|c|}{ Sequence Currents } & \multicolumn{2}{|c|}{ Imbalance } \\
\hline ФA & $\Phi \mathrm{B}$ & $\Phi C$ & \begin{tabular}{l|l} 
V0 & \\
\end{tabular} & $\mathrm{V} 1$ & V2 & & \begin{tabular}{|l|} 
ФA \\
\end{tabular} & $\Phi \mathrm{B}$ & कC & 10 & 11 & 12 & \begin{tabular}{l|l}
$\mid 2 / I 1$ \\
\end{tabular} & PF \\
\hline 7.38 & 7.49 & 7.46 & 17.54 & 7442.93 & 56.3 & $0.8 \%$ & 356.11 & 375.94 & 371.99 & 3.24 & 367.98 & 9.3 & $2.5 \%$ & 0.03 \\
\hline 7.39 & 7.46 & 7.48 & 42.54 & 7442.11 & 46.62 & $0.6 \%$ & 347.9 & 389.79 & 366.19 & 9.06 & 367.9 & 15.93 & $4.3 \%$ & 0.06 \\
\hline 7.39 & 7.43 & 7.5 & 71.6 & 7441.23 & 37.83 & $0.5 \%$ & 339.73 & 403.15 & 360.22 & 15.38 & 367.57 & 23.48 & $6.4 \%$ & 0.1 \\
\hline 7.4 & 7.4 & 7.52 & 101.14 & 7440.28 & 30.53 & $0.4 \%$ & 331.58 & 416.08 & 354.06 & 21.72 & 367.01 & 31.17 & $8.5 \%$ & 0.13 \\
\hline 7.41 & 7.37 & 7.54 & 130.7 & 7439.27 & 25.77 & $0.3 \%$ & 323.45 & 428.57 & 347.7 & 28.05 & 366.2 & 38.8 & $10.6 \%$ & 0.17 \\
\hline 7.42 & 7.34 & 7.57 & 160.16 & 7438.22 & 24.81 & $0.3 \%$ & 315.32 & 440.64 & 341.15 & 34.34 & 365.14 & 46.31 & $12.7 \%$ & 0.2 \\
\hline 7.43 & 7.3 & 7.59 & 189.47 & 7437.11 & 27.84 & $0.4 \%$ & 307.19 & 452.3 & 334.41 & 40.59 & 363.85 & 53.68 & $14.8 \%$ & 0.24 \\
\hline 7.43 & 7.27 & 7.61 & 218.59 & 7435.97 & 33.64 & $0.5 \%$ & 299.04 & 463.56 & 327.47 & 46.8 & 362.32 & 60.9 & $16.8 \%$ & 0.28 \\
\hline 7.44 & 7.24 & 7.63 & 247.53 & 7434.78 & 40.92 & $0.6 \%$ & 290.89 & 474.41 & 320.35 & 52.97 & 360.56 & 67.97 & $18.9 \%$ & 0.31 \\
\hline 7.45 & 7.21 & 7.65 & 276.27 & 7433.56 & 48.93 & $0.7 \%$ & 282.71 & 484.88 & 313.04 & 59.1 & 358.57 & 74.88 & $20.9 \%$ & 0.35 \\
\hline 7.46 & 7.18 & 7.67 & 304.7 & 7432.31 & 57.25 & $0.8 \%$ & 274.51 & 494.92 & 305.56 & 65.15 & 356.34 & 81.62 & $22.9 \%$ & 0.38 \\
\hline
\end{tabular}

\begin{tabular}{|c|c|c|c|c|c|c|c|c|c|c|c|c|c|c|}
\hline \multicolumn{15}{|c|}{ Node 2} \\
\hline \multicolumn{3}{|c|}{ Voltage } & \multicolumn{3}{|c|}{ Sequence Voltages $(\mathrm{V})$} & \multirow[b]{2}{*}{ V2N1 } & \multicolumn{3}{|c|}{ Current } & \multicolumn{3}{|c|}{ Sequence Currents } & \multicolumn{2}{|c|}{ Imbalance } \\
\hline$\Phi A$ & $\Phi \mathrm{B}$ & $\Phi C$ & V0 & V1 & $\mathrm{V} 2$ & & \begin{tabular}{|c|}
$\mathrm{A}$ \\
\end{tabular} & कB & $\Phi C$ & 10 & $\mid 1$ & 12 & \begin{tabular}{l|l}
$\mid 2 / I 1$ &
\end{tabular} & PF \\
\hline 7.16 & 7.39 & 7.33 & 35.08 & 7291.9 & 112.7 & $1.5 \%$ & 356.14 & 375.94 & 372 & 3.24 & 367.99 & 9.29 & $2.5 \%$ & 0.04 \\
\hline 7.17 & 7.33 & 7.38 & 85.08 & 7290.1 & 93.32 & $1.3 \%$ & 347.93 & 389.8 & 366.2 & 9.06 & 367.91 & 15.91 & $4.3 \%$ & 0.06 \\
\hline 7.18 & 7.27 & 7.42 & 143.2 & 7288.13 & 75.74 & $1.0 \%$ & 339.76 & 403.16 & 360.23 & 15.37 & 367.59 & 23.47 & $6.4 \%$ & 0.09 \\
\hline 7.19 & 7.21 & 7.46 & 202.27 & 7285.99 & 61.12 & $0.8 \%$ & 331.61 & 416.08 & 354.07 & 21.72 & 367.02 & 31.16 & $8.5 \%$ & 0.13 \\
\hline 7.21 & 7.15 & 7.5 & 261.4 & 7283.7 & 51.56 & $0.7 \%$ & 323.47 & 428.58 & 347.71 & 28.04 & 366.21 & 38.79 & $10.6 \%$ & 0.16 \\
\hline 7.22 & 7.09 & 7.54 & 320.31 & 7281.26 & 49.59 & $0.7 \%$ & 315.34 & 440.65 & 341.16 & 34.34 & 365.16 & 46.3 & $12.7 \%$ & 0.19 \\
\hline 7.24 & 7.03 & 7.59 & 378.92 & 7278.69 & 55.61 & $0.8 \%$ & 307.21 & 452.31 & 334.41 & 40.59 & 363.87 & 53.67 & $14.7 \%$ & 0.22 \\
\hline 7.25 & 6.98 & 7.63 & 437.17 & 7276 & 67.19 & $0.9 \%$ & 299.07 & 463.57 & 327.48 & 46.8 & 362.34 & 60.89 & $16.8 \%$ & 0.25 \\
\hline 7.27 & 6.92 & 7.67 & 495.05 & 7273.19 & 81.75 & $1.1 \%$ & 290.91 & 474.43 & 320.36 & 52.97 & 360.58 & 67.96 & $18.8 \%$ & 0.28 \\
\hline 7.28 & 6.86 & 7.71 & 552.53 & 7270.27 & 97.75 & $1.3 \%$ & 282.73 & 484.9 & 313.05 & 59.09 & 358.59 & 74.87 & $20.9 \%$ & 0.31 \\
\hline 7.3 & 6.8 & 7.75 & 609.38 & 7267.27 & 114.39 & $1.6 \%$ & 274.52 & 494.94 & 305.57 & 65.15 & 356.36 & 81.61 & $22.9 \%$ & 0.34 \\
\hline
\end{tabular}

\begin{tabular}{|c|c|c|c|c|c|c|c|c|c|c|c|c|c|c|}
\hline \multicolumn{15}{|c|}{ Node 3} \\
\hline \multicolumn{3}{|c|}{ Voltage } & \multicolumn{3}{|c|}{ Sequence Voltages (V) } & \multirow[b]{2}{*}{$\mathrm{V} 2 / \mathrm{N} 1$} & \multicolumn{3}{|c|}{ Current } & \multicolumn{3}{|c|}{ Sequence Currents } & \multicolumn{2}{|c|}{ Imbalance } \\
\hline$\Phi A$ & ФВ & $\Phi C$ & V0 & V1 & V2 & & $\Phi A$ & ФВ & $\Phi C$ & 10 & 11 & 12 & $\mathrm{I} 2 / \mathrm{I}$ & PF \\
\hline 6.96 & 7.31 & 7.24 & 52.62 & 7169.53 & 169.1 & $2.4 \%$ & 356.17 & 375.94 & 372 & 3.24 & 368 & 9.27 & $2.5 \%$ & 0.05 \\
\hline 6.97 & 7.23 & 7.3 & 127.61 & 7166.6 & 140.03 & $2.0 \%$ & 347.95 & 389.79 & 366.2 & 9.05 & 367.92 & 15.9 & $4.3 \%$ & 0.07 \\
\hline 6.99 & 7.14 & 7.37 & 214.79 & 7163.34 & 113.65 & $1.6 \%$ & 339.78 & 403.16 & 360.23 & 15.37 & 367.6 & 23.47 & $6.4 \%$ & 0.09 \\
\hline 7 & 7.06 & 7.43 & 303.4 & 7159.76 & 91.71 & $1.3 \%$ & 331.63 & 416.08 & 354.07 & 21.71 & 367.03 & 31.15 & $8.5 \%$ & 0.12 \\
\hline 7.02 & 6.98 & 7.49 & 392.08 & 7155.89 & 77.36 & $1.1 \%$ & 323.49 & 428.58 & 347.71 & 28.04 & 366.22 & 38.78 & $10.6 \%$ & 0.15 \\
\hline 7.04 & 6.9 & 7.55 & 480.44 & 7151.74 & 74.38 & $1.0 \%$ & 315.36 & 440.65 & 341.16 & 34.33 & 365.17 & 46.29 & $12.7 \%$ & 0.18 \\
\hline 7.05 & 6.82 & 7.62 & 568.36 & 7147.34 & 83.38 & $1.2 \%$ & 307.23 & 452.32 & 334.41 & 40.58 & 363.88 & 53.66 & $14.7 \%$ & 0.2 \\
\hline 7.07 & 6.74 & 7.68 & 655.73 & 7142.68 & 100.74 & $1.4 \%$ & 299.08 & 463.57 & 327.48 & 46.8 & 362.35 & 60.89 & $16.8 \%$ & 0.23 \\
\hline 7.09 & 6.66 & 7.74 & 742.55 & 7137.8 & 122.56 & $1.7 \%$ & 290.92 & 474.43 & 320.36 & 52.96 & 360.59 & 67.95 & $18.8 \%$ & 0.25 \\
\hline 7.11 & 6.58 & 7.8 & 828.77 & 7132.71 & 146.57 & $2.1 \%$ & 282.74 & 484.9 & 313.05 & 59.09 & 358.6 & 74.86 & $20.9 \%$ & 0.28 \\
\hline 7.14 & 6.5 & 7.86 & 914.04 & 7127.42 & 171.52 & $2.4 \%$ & 274.54 & 494.94 & 305.57 & 65.14 & 356.37 & 81.6 & $22.9 \%$ & 0.31 \\
\hline
\end{tabular}




\begin{tabular}{|c|c|c|c|c|c|c|c|c|c|c|c|}
\hline \multicolumn{3}{|c|}{ kVar Flow } & $\begin{array}{c}\text { Total kVar } \\
\text { Flow }\end{array}$ & \multicolumn{3}{|c|}{ kW Native Load } & $\begin{array}{c}\text { Total kW } \\
\text { Load }\end{array}$ & \multicolumn{3}{|c|}{ kVar Native Load } & $\begin{array}{c}\text { Total kVar } \\
\text { Load }\end{array}$ \\
\hline 465.92 & 501.64 & 569.08 & 1536.64 & 2479.28 & 2748.31 & 2692.95 & 7920.54 & -2.78 & -2.43 & -2.04 & -7.25 \\
\hline 373.75 & 611.81 & 547.65 & 1533.21 & 2374.11 & 2879.99 & 2653.75 & 7907.85 & -2.40 & -2.63 & -2.23 & -7.26 \\
\hline 330.22 & 669.05 & 536.47 & 1535.74 & 2322.17 & 2937.94 & 2630.67 & 7890.78 & -2.41 & -2.60 & -2.24 & -7.25 \\
\hline 288.48 & 727.55 & 524.87 & 1540.90 & 2270.41 & 2991.02 & 2605.25 & 7866.68 & -2.42 & -2.58 & -2.26 & -7.26 \\
\hline 248.53 & 787.22 & 512.87 & 1548.62 & 2218.75 & 3039.39 & 2577.47 & 7835.61 & -2.44 & -2.54 & -2.28 & -7.26 \\
\hline 139.48 & 972.05 & 474.55 & 1586.08 & 2063.40 & 3158.43 & 2479.85 & 7701.68 & -2.47 & -2.47 & -2.33 & -7.27 \\
\hline 106.72 & 1035.28 & 461.04 & 1603.04 & 2011.20 & 3190.07 & 2442.48 & 7643.75 & -2.48 & -2.44 & -2.35 & -7.27 \\
\hline 75.60 & 1099.26 & 447.30 & 1622.16 & 1958.82 & 3217.60 & 2402.71 & 7579.13 & -2.73 & -1.94 & -2.32 & -6.99 \\
\hline
\end{tabular}

Plot Information:

\begin{tabular}{|c|c|c|c|c|c|c|c|c|}
\hline Node 0 & Total kW & $\begin{array}{c}\text { kW } \\
\text { Losses / }\end{array}$ & Node 1 & Node 2 & Node 3 & & & \\
\hline |2/I1 & Losses & kW Flow & V1/V2 & V1/V2 & V2/V1 & 10 & 11 & 12 \\
\hline $2.5 \%$ & 351.11 & $4.2 \%$ & $0.8 \%$ & $1.5 \%$ & $2.4 \%$ & 3.24 & 367.98 & 9.30 \\
\hline $4.3 \%$ & 352.64 & $4.3 \%$ & $0.6 \%$ & $1.3 \%$ & $2.0 \%$ & 9.06 & 367.90 & 15.93 \\
\hline $6.4 \%$ & 355.12 & $4.3 \%$ & $0.5 \%$ & $1.0 \%$ & $1.6 \%$ & 15.38 & 367.57 & 23.48 \\
\hline $8.5 \%$ & 358.52 & $4.3 \%$ & $0.4 \%$ & $0.8 \%$ & $1.3 \%$ & 21.72 & 367.01 & 31.17 \\
\hline $10.6 \%$ & 362.81 & $4.4 \%$ & $0.3 \%$ & $0.7 \%$ & $1.1 \%$ & 28.05 & 366.20 & 38.80 \\
\hline $12.7 \%$ & 367.93 & $4.5 \%$ & $0.3 \%$ & $0.7 \%$ & $1.0 \%$ & 34.34 & 365.14 & 46.31 \\
\hline $14.8 \%$ & 373.87 & $4.6 \%$ & $0.4 \%$ & $0.8 \%$ & $1.2 \%$ & 40.59 & 363.85 & 53.68 \\
\hline $16.8 \%$ & 380.56 & $4.7 \%$ & $0.5 \%$ & $0.9 \%$ & $1.4 \%$ & 46.80 & 362.32 & 60.90 \\
\hline $18.9 \%$ & 388.01 & $4.8 \%$ & $0.6 \%$ & $1.1 \%$ & $1.7 \%$ & 52.97 & 360.56 & 67.97 \\
\hline $20.9 \%$ & 396.18 & $4.9 \%$ & $0.7 \%$ & $1.3 \%$ & $2.1 \%$ & 59.10 & 358.57 & 74.88 \\
\hline $22.9 \%$ & 404.96 & $5.1 \%$ & $0.8 \%$ & $1.6 \%$ & $2.4 \%$ & 65.15 & 356.34 & 81.62 \\
\hline
\end{tabular}


Table 13. Transposed Kilowatt Loss Evaluation

\begin{tabular}{|c|c|c|c|c|c|c|c|c|c|c|c|c|c|c|}
\hline \multicolumn{3}{|c|}{ Spot Load (MW) } & \multicolumn{3}{|c|}{ kW Losses } & \multirow{2}{*}{$\begin{array}{c}\text { Total kW } \\
\text { Losses }\end{array}$} & \multicolumn{3}{|c|}{ kVar Losses } & \multirow{2}{*}{$\begin{array}{c}\text { Total kVar } \\
\text { Losses }\end{array}$} & \multicolumn{3}{|c|}{ kW Flow } & \multirow[b]{2}{*}{ Total kW Flow } \\
\hline$\Phi A$ & $\Phi \mathrm{B}$ & $\Phi C$ & ФA & $\Phi \mathrm{B}$ & $\Phi C$ & & $\Phi A$ & बB & $\Phi C$ & & $\Phi A$ & $\Phi \mathrm{B}$ & $\Phi C$ & \\
\hline 3000.00 & 3000.00 & 3000.00 & 117.05 & 117.04 & 117.06 & 351.15 & 515.80 & 515.77 & 515.79 & 1547.36 & 2758.93 & 2758.99 & 2759.00 & 8276.92 \\
\hline 3150.00 & 2925.00 & 2925.00 & 144.46 & 91.77 & 115.41 & 351.64 & 570.85 & 508.10 & 469.82 & 1548.77 & 2854.89 & 2716.82 & 2702.11 & 8273.82 \\
\hline 3300.00 & 2850.00 & 2850.00 & 172.80 & 67.30 & 112.99 & 353.09 & 627.40 & 500.00 & 425.46 & 1552.86 & 2946.78 & 2673.22 & 2644.53 & 8264.53 \\
\hline 3450.00 & 2775.00 & 2775.00 & 201.93 & 43.65 & 109.86 & 355.44 & 685.35 & 491.46 & 382.72 & 1559.53 & 3034.65 & 2628.21 & 2586.23 & 8249.09 \\
\hline 3600.00 & 2700.00 & 2700.00 & 231.72 & 20.86 & 106.09 & 358.67 & 744.55 & 482.47 & 341.61 & 1568.63 & 3118.54 & 2581.78 & 2527.21 & 8227.53 \\
\hline 3750.00 & 2625.00 & 2625.00 & 262.04 & -1.04 & 101.75 & 362.75 & 804.88 & 473.04 & 302.13 & 1580.05 & 3198.50 & 2533.96 & 2467.46 & 8199.92 \\
\hline 3900.00 & 2550.00 & 2550.00 & 292.76 & -22.01 & 96.92 & 367.67 & 866.23 & 463.17 & 264.30 & 1593.70 & 3274.59 & 2484.74 & 2406.97 & 8166.30 \\
\hline 4050.00 & 2475.00 & 2475.00 & 323.76 & -42.03 & 91.64 & 373.37 & 928.50 & 452.87 & 228.11 & 1609.48 & 3346.87 & 2434.14 & 2345.73 & 8126.74 \\
\hline 4200.00 & 2400.00 & 2400.00 & 354.91 & -61.08 & 85.99 & 379.82 & 991.58 & 442.13 & 193.57 & 1627.28 & 3415.40 & 2382.16 & 2283.73 & 8081.29 \\
\hline 4350.00 & 2325.00 & 2325.00 & 386.12 & -79.12 & 80.03 & 387.03 & 1055.39 & 430.97 & 160.70 & 1647.06 & 3480.26 & 2328.81 & 2220.98 & 8030.05 \\
\hline 4500.00 & 2250.00 & 2250.00 & 417.26 & -96.12 & 73.83 & 394.97 & 1119.83 & 419.41 & 129.49 & 1668.73 & 3541.49 & 2274.10 & 2157.48 & 7973.07 \\
\hline & & & & & & & Node 0 & & & & & & & \\
\hline & Voltage & & Seque & ce Voltage & & & & urrent & & Seq & ence Curre & & $\operatorname{Imb}$ & alance \\
\hline ФA & $\Phi \mathrm{B}$ & $\Phi C$ & \begin{tabular}{l|l} 
V0 \\
\end{tabular} & \begin{tabular}{l|l}
$\mathrm{V} 1$ \\
\end{tabular} & V2 & $\mathrm{V} 2 / \mathrm{V} 1$ & ФA & ФB & $\Phi C$ & \begin{tabular}{|l|l|}
10 \\
\end{tabular} & \begin{tabular}{l|}
11 \\
\end{tabular} & 12 & \begin{tabular}{|l|}
$\mid 2 / I 1$ \\
\end{tabular} & $\mathrm{PF}$ \\
\hline 7.62 & 7.62 & 7.62 & 0 & 7620.91 & 0.11 & $0.0 \%$ & 368.23 & 368.24 & 368.24 & 0 & 368.23 & 0 & $0.0 \%$ & 0 \\
\hline 7.62 & 7.62 & 7.62 & 0 & 7620.91 & 0.11 & $0.0 \%$ & 381.96 & 362.61 & 359.83 & 6.43 & 368.11 & 8.38 & $2.3 \%$ & 0.04 \\
\hline 7.62 & 7.62 & 7.62 & 0 & 7620.91 & 0.11 & $0.0 \%$ & 395.27 & 356.79 & 351.42 & 12.82 & 367.74 & 16.59 & $4.5 \%$ & 0.07 \\
\hline 7.62 & 7.62 & 7.62 & 0 & 7620.91 & 0.11 & $0.0 \%$ & 408.16 & 350.78 & 343 & 19.18 & 367.13 & 24.63 & $6.7 \%$ & 0.11 \\
\hline 7.62 & 7.62 & 7.62 & 0 & 7620.91 & 0.11 & $0.0 \%$ & 420.63 & 344.57 & 334.58 & 25.5 & 366.28 & 32.5 & $8.9 \%$ & 0.15 \\
\hline 7.62 & 7.62 & 7.62 & 0 & 7620.91 & 0.11 & $0.0 \%$ & 432.71 & 338.18 & 326.15 & 31.78 & 365.19 & 40.21 & $11.0 \%$ & 0.18 \\
\hline 7.62 & 7.62 & 7.62 & 0 & 7620.91 & 0.11 & $0.0 \%$ & 444.38 & 331.59 & 317.7 & 38.03 & 363.86 & 47.74 & $13.1 \%$ & 0.22 \\
\hline 7.62 & 7.62 & 7.62 & 0 & 7620.91 & 0.11 & $0.0 \%$ & 455.67 & 324.82 & 309.22 & 44.25 & 362.29 & 55.12 & $15.2 \%$ & 0.25 \\
\hline 7.62 & 7.62 & 7.62 & 0 & 7620.91 & 0.11 & $0.0 \%$ & 466.58 & 317.85 & 300.71 & 50.42 & 360.49 & 62.33 & $17.3 \%$ & 0.29 \\
\hline 7.62 & 7.62 & 7.62 & 0 & 7620.91 & 0.11 & $0.0 \%$ & 477.12 & 310.7 & 292.17 & 56.55 & 358.47 & 69.38 & $19.4 \%$ & 0.33 \\
\hline 7.62 & 7.62 & 7.62 & 0 & 7620.91 & 0.11 & $0.0 \%$ & 487.29 & 303.37 & 283.59 & 62.65 & 356.22 & 76.27 & $21.4 \%$ & 0.36 \\
\hline & & & & & & & Node 1 & & & & & & & \\
\hline & Voltage & & Seque & ce Voltag $€$ & & & & urrent & & Seq & ence Curre & & Imbc & alance \\
\hline$\Phi A$ & $\Phi B$ & $\Phi C$ & V0 & $\mathrm{V} 1$ & V2 & V2/V1 & $\Phi A$ & ФB & $\Phi C$ & 10 & 11 & 12 & $\mid 2 / / 1$ & PF \\
\hline 7.36 & 7.5 & 7.47 & 25.23 & 7444.34 & 60.26 & $0.8 \%$ & 368.23 & 368.24 & 368.24 & 0 & 368.23 & 0 & $0.0 \%$ & 0 \\
\hline 7.33 & 7.53 & 7.48 & 53.13 & 7445.92 & 64.24 & $0.9 \%$ & 381.96 & 362.61 & 359.83 & 6.43 & 368.11 & 8.38 & $2.3 \%$ & 0.04 \\
\hline 7.31 & 7.55 & 7.49 & 81.93 & 7447.38 & 69.72 & $0.9 \%$ & 395.27 & 356.79 & 351.42 & 12.82 & 367.74 & 16.59 & $4.5 \%$ & 0.07 \\
\hline 7.28 & 7.57 & 7.5 & 110.8 & 7448.72 & 76.26 & $1.0 \%$ & 408.16 & 350.78 & 343 & 19.18 & 367.13 & 24.63 & $6.7 \%$ & 0.11 \\
\hline 7.25 & 7.6 & 7.5 & 139.58 & 7449.95 & 83.52 & $1.1 \%$ & 420.63 & 344.57 & 334.58 & 25.5 & 366.28 & 32.5 & $8.9 \%$ & 0.15 \\
\hline 7.22 & 7.62 & 7.51 & 168.23 & 7451.06 & 91.24 & $1.2 \%$ & 432.71 & 338.18 & 326.15 & 31.78 & 365.19 & 40.21 & $11.0 \%$ & 0.18 \\
\hline 7.19 & 7.64 & 7.52 & 196.72 & 7452.07 & 99.22 & $1.3 \%$ & 444.38 & 331.59 & 317.7 & 38.03 & 363.86 & 47.74 & $13.1 \%$ & 0.22 \\
\hline 7.17 & 7.67 & 7.53 & 225.04 & 7452.99 & 107.34 & $1.4 \%$ & 455.67 & 324.82 & 309.22 & 44.25 & 362.29 & 55.12 & $15.2 \%$ & 0.25 \\
\hline 7.14 & 7.69 & 7.54 & 253.18 & 7453.81 & 115.52 & $1.5 \%$ & 466.58 & 317.85 & 300.71 & 50.42 & 360.49 & 62.33 & $17.3 \%$ & 0.29 \\
\hline 7.11 & 7.71 & 7.55 & 281.14 & 7454.54 & 123.67 & $1.7 \%$ & 477.12 & 310.7 & 292.17 & 56.55 & 358.47 & 69.38 & $19.4 \%$ & 0.33 \\
\hline 7.08 & 7.73 & 7.56 & 308.91 & 7455.2 & 131.77 & $1.8 \%$ & 487.29 & 303.37 & 283.59 & 62.65 & 356.22 & 76.27 & $21.4 \%$ & 0.36 \\
\hline & & & & & & & Node 2 & & & & & & & \\
\hline & Voltage & & Seque & ce Voltage & & & & urrent & & Seq & ence Curre & & $\operatorname{lmb}$ & alance \\
\hline ФA & $\Phi \mathrm{B}$ & $\Phi C$ & \begin{tabular}{l|l} 
V0 \\
\end{tabular} & V1 & V2 & V2/V1 & $\Phi A$ & बB & $\Phi C$ & 10 & 11 & 12 & $|2 /| 11$ & $\mathrm{PF}$ \\
\hline 7.27 & 7.38 & 7.24 & 25.24 & 7294.81 & 60.31 & $0.8 \%$ & 368.26 & 368.24 & 368.25 & 0 & 368.24 & 0.02 & $0.0 \%$ & 0.01 \\
\hline 7.21 & 7.42 & 7.25 & 81.75 & 7295.27 & 47.15 & $0.6 \%$ & 381.99 & 362.62 & 359.83 & 6.43 & 368.12 & 8.39 & $2.3 \%$ & 0.02 \\
\hline 7.16 & 7.47 & 7.26 & 140.47 & 7295.49 & 42.66 & $0.6 \%$ & 395.3 & 356.8 & 351.42 & 12.82 & 367.76 & 16.6 & $4.5 \%$ & 0.05 \\
\hline 7.1 & 7.51 & 7.28 & 199.18 & 7295.5 & 48.7 & $0.7 \%$ & 408.19 & 350.79 & 343.01 & 19.18 & 367.15 & 24.64 & $6.7 \%$ & 0.09 \\
\hline 7.05 & 7.55 & 7.29 & 257.64 & 7295.3 & 61.82 & $0.8 \%$ & 420.67 & 344.58 & 334.59 & 25.5 & 366.29 & 32.52 & $8.9 \%$ & 0.12 \\
\hline 6.99 & 7.6 & 7.31 & 315.82 & 7294.91 & 78.2 & $1.1 \%$ & 432.74 & 338.18 & 326.15 & 31.79 & 365.2 & 40.22 & $11.0 \%$ & 0.15 \\
\hline 6.93 & 7.64 & 7.32 & 373.65 & 7294.34 & 95.91 & $1.3 \%$ & 444.42 & 331.6 & 317.7 & 38.03 & 363.87 & 47.76 & $13.1 \%$ & 0.18 \\
\hline 6.88 & 7.68 & 7.34 & 431.15 & 7293.6 & 114.1 & $1.6 \%$ & 455.71 & 324.82 & 309.22 & 44.25 & 362.31 & 55.13 & $15.2 \%$ & 0.21 \\
\hline 6.82 & 7.73 & 7.35 & 488.3 & 7292.7 & 132.4 & $1.8 \%$ & 466.62 & 317.86 & 300.71 & 50.42 & 360.51 & 62.34 & $17.3 \%$ & 0.24 \\
\hline 6.77 & 7.77 & 7.37 & 545.08 & 7291.65 & 150.59 & $2.1 \%$ & 477.16 & 310.71 & 292.16 & 56.55 & 358.48 & 69.39 & $19.4 \%$ & 0.27 \\
\hline 6.72 & 7.81 & 7.38 & 601.49 & 7290.47 & 168.59 & $2.3 \%$ & 487.34 & 303.37 & 283.58 & 62.65 & 356.24 & 76.29 & $21.4 \%$ & 0.3 \\
\hline & & & & & & & Node 3 & & & & & & & \\
\hline & Voltage & & Seque & ce Voltage & & & & urrent & & Seq & ence Curre & & Imbs & alance \\
\hline$\Phi A$ & $\Phi \mathrm{B}$ & कC & \begin{tabular}{l|l} 
V0 \\
\end{tabular} & V1 & V2 & V2/V1 & ФA & ФВ & $\Phi C$ & 10 & \begin{tabular}{|l|}
$I 1$ \\
\end{tabular} & 12 & $12 / 11$ & $\mathrm{PF}$ \\
\hline 7.17 & 7.17 & 7.17 & 0.02 & 7174.03 & 0.06 & $0.0 \%$ & 368.26 & 368.24 & 368.27 & 0 & 368.25 & 0.02 & $0.0 \%$ & 0.01 \\
\hline 7.1 & 7.24 & 7.19 & 88.72 & 7173.81 & 32.68 & $0.5 \%$ & 381.99 & 362.62 & 359.85 & 6.42 & 368.13 & 8.39 & $2.3 \%$ & 0.03 \\
\hline 7.02 & 7.3 & 7.2 & 176.95 & 7173.18 & 64.7 & $0.9 \%$ & 395.3 & 356.8 & 351.44 & 12.81 & 367.76 & 16.6 & $4.5 \%$ & 0.05 \\
\hline 6.94 & 7.37 & 7.22 & 264.74 & 7172.14 & 96.06 & $1.3 \%$ & 408.19 & 350.79 & 343.03 & 19.17 & 367.15 & 24.64 & $6.7 \%$ & 0.08 \\
\hline 6.86 & 7.43 & 7.24 & 352.03 & 7170.74 & 126.76 & $1.8 \%$ & 420.67 & 344.58 & 334.61 & 25.49 & 366.3 & 32.51 & $8.9 \%$ & 0.11 \\
\hline 6.79 & 7.5 & 7.25 & 438.83 & 7168.98 & 156.81 & $2.2 \%$ & 432.75 & 338.18 & 326.17 & 31.78 & 365.21 & 40.21 & $11.0 \%$ & 0.14 \\
\hline 6.71 & 7.56 & 7.27 & 525.1 & 7166.88 & 186.21 & $2.6 \%$ & 444.42 & 331.6 & 317.71 & 38.03 & 363.88 & 47.75 & $13.1 \%$ & 0.16 \\
\hline 6.63 & 7.62 & 7.29 & 610.86 & 7164.47 & 214.97 & $3.0 \%$ & 455.72 & 324.82 & 309.23 & 44.24 & 362.31 & 55.13 & $15.2 \%$ & 0.19 \\
\hline 6.56 & 7.69 & 7.31 & 696.09 & 7161.77 & 243.1 & $3.4 \%$ & 466.63 & 317.86 & 300.72 & 50.41 & 360.52 & 62.34 & $17.3 \%$ & 0.22 \\
\hline 6.48 & 7.75 & 7.33 & 780.78 & 7158.78 & 270.59 & $3.8 \%$ & 477.17 & 310.71 & 292.18 & 56.55 & 358.49 & 69.39 & $19.4 \%$ & 0.24 \\
\hline 6.41 & 7.81 & 7.35 & 864.92 & 7155.53 & 297.47 & $4.2 \%$ & 487.34 & 303.37 & 283.59 & 62.64 & 356.24 & 76.28 & $21.4 \%$ & 0.27 \\
\hline
\end{tabular}




\begin{tabular}{|c|c|c|c|c|c|c|c|c|c|c|c|}
\hline \multicolumn{3}{|c|}{ kVar Flow } & \multirow{2}{*}{$\begin{array}{c}\text { Total kVar } \\
\text { Flow }\end{array}$} & \multicolumn{3}{|c|}{ kW Native Load } & \multirow{2}{*}{$\begin{array}{c}\text { Total kW } \\
\text { Load }\end{array}$} & \multicolumn{3}{|c|}{ kVar Native Load } & \multirow{2}{*}{$\begin{array}{c}\text { Total kVar } \\
\text { Load }\end{array}$} \\
\hline$\Phi A$ & $\Phi \mathrm{B}$ & $\Phi C$ & & \begin{tabular}{|l|}
$\Phi A$ \\
\end{tabular} & ФB & $\Phi C$ & & ФA & कВ & $\Phi C$ & \\
\hline 513.41 & 513.32 & 513.39 & 1540.12 & 2641.88 & 2641.94 & 2641.95 & 7925.77 & -2.40 & -2.45 & -2.40 & -7.25 \\
\hline 568.47 & 505.63 & 467.41 & 1541.51 & 2710.43 & 2625.05 & 2586.70 & 7922.18 & -2.37 & -2.47 & -2.41 & -7.25 \\
\hline 625.05 & 497.51 & 423.04 & 1545.60 & 2773.98 & 2605.92 & 2531.54 & 7911.44 & -2.35 & -2.48 & -2.43 & -7.26 \\
\hline 683.03 & 488.95 & 380.29 & 1552.27 & 2832.72 & 2584.56 & 2476.37 & 7893.65 & -2.32 & -2.51 & -2.43 & -7.26 \\
\hline 742.25 & 479.94 & 339.17 & 1561.36 & 2886.82 & 2560.92 & 2421.12 & 7868.86 & -2.30 & -2.52 & -2.44 & -7.26 \\
\hline 802.62 & 470.50 & 299.68 & 1572.80 & 2936.46 & 2534.99 & 2365.71 & 7837.16 & -2.27 & -2.55 & -2.45 & -7.27 \\
\hline 863.99 & 460.61 & 261.83 & 1586.43 & 2981.83 & 2506.75 & 2310.05 & 7798.63 & -2.24 & -2.56 & -2.47 & -7.27 \\
\hline 926.29 & 450.29 & 225.63 & 1602.21 & 3023.12 & 2476.17 & 2254.09 & 7753.38 & -2.22 & -2.58 & -2.47 & -7.27 \\
\hline 989.39 & 439.53 & 191.09 & 1620.01 & 3060.49 & 2443.24 & 2197.74 & 7701.47 & -2.19 & -2.60 & -2.49 & -7.28 \\
\hline 1053.22 & 428.35 & 158.21 & 1639.78 & 3094.14 & 2407.93 & 2140.95 & 7643.02 & -2.17 & -2.62 & -2.49 & -7.28 \\
\hline 1117.68 & 416.77 & 126.99 & 1661.44 & 3124.24 & 2370.22 & 2083.65 & 7578.11 & -2.15 & -2.64 & -2.50 & -7.29 \\
\hline
\end{tabular}

Plot Information:

\begin{tabular}{rcr} 
Node 0 & Total kW & \multicolumn{1}{c}{ kW } \\
L $2 / 11$ & Losses / & kW Flow \\
$0.0 \%$ & 351.15 & $4.2 \%$ \\
$2.3 \%$ & 351.64 & $4.3 \%$ \\
$4.5 \%$ & 353.09 & $4.3 \%$ \\
$6.7 \%$ & 355.44 & $4.3 \%$ \\
$8.9 \%$ & 358.67 & $4.4 \%$ \\
$11.0 \%$ & 362.75 & $4.4 \%$ \\
$13.1 \%$ & 367.67 & $4.5 \%$ \\
$15.2 \%$ & 373.37 & $4.6 \%$ \\
$17.3 \%$ & 379.82 & $4.7 \%$ \\
$19.4 \%$ & 387.03 & $4.8 \%$ \\
$21.4 \%$ & 394.97 & $5.0 \%$
\end{tabular}

\begin{tabular}{rrr} 
Node 1 & Node 2 & \multicolumn{1}{c}{ Node 3 } \\
V1/V2 & V1/N2 & \multicolumn{1}{c}{ V1/V2 } \\
$0.8 \%$ & $0.8 \%$ & $0.0 \%$ \\
$0.9 \%$ & $0.6 \%$ & $0.5 \%$ \\
$0.9 \%$ & $0.6 \%$ & $0.9 \%$ \\
$1.0 \%$ & $0.7 \%$ & $1.3 \%$ \\
$1.1 \%$ & $0.8 \%$ & $1.8 \%$ \\
$1.2 \%$ & $1.1 \%$ & $2.2 \%$ \\
$1.3 \%$ & $1.3 \%$ & $2.6 \%$ \\
$1.4 \%$ & $1.6 \%$ & $3.0 \%$ \\
$1.5 \%$ & $1.8 \%$ & $3.4 \%$ \\
$1.7 \%$ & $2.1 \%$ & $3.8 \%$ \\
$1.8 \%$ & $2.3 \%$ & $4.2 \%$
\end{tabular}

\begin{tabular}{rlr}
10 & 11 & \multicolumn{1}{l}{12} \\
0.00 & 368.23 & 0.00 \\
6.43 & 368.11 & 8.38 \\
12.82 & 367.74 & 16.59 \\
19.18 & 367.13 & 24.63 \\
25.50 & 366.28 & 32.50 \\
31.78 & 365.19 & 40.21 \\
38.03 & 363.86 & 47.74 \\
44.25 & 362.29 & 55.12 \\
50.42 & 360.49 & 62.33 \\
56.55 & 358.47 & 69.38 \\
62.65 & 356.22 & 76.27
\end{tabular}


Total kW Loss

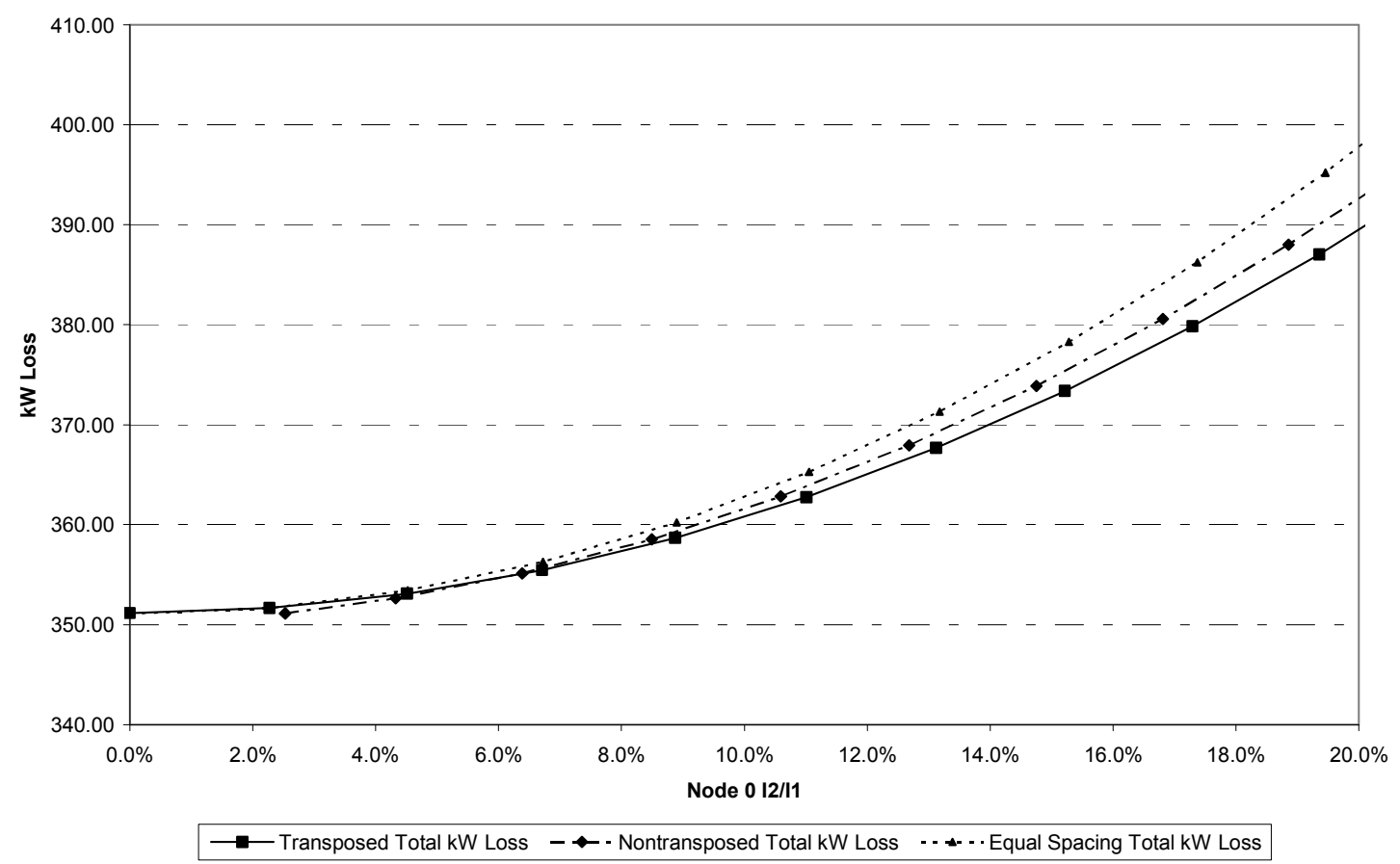

Figure 82. Total line losses versus load imbalance for each line configuration

Total kW Loss / kW Flow

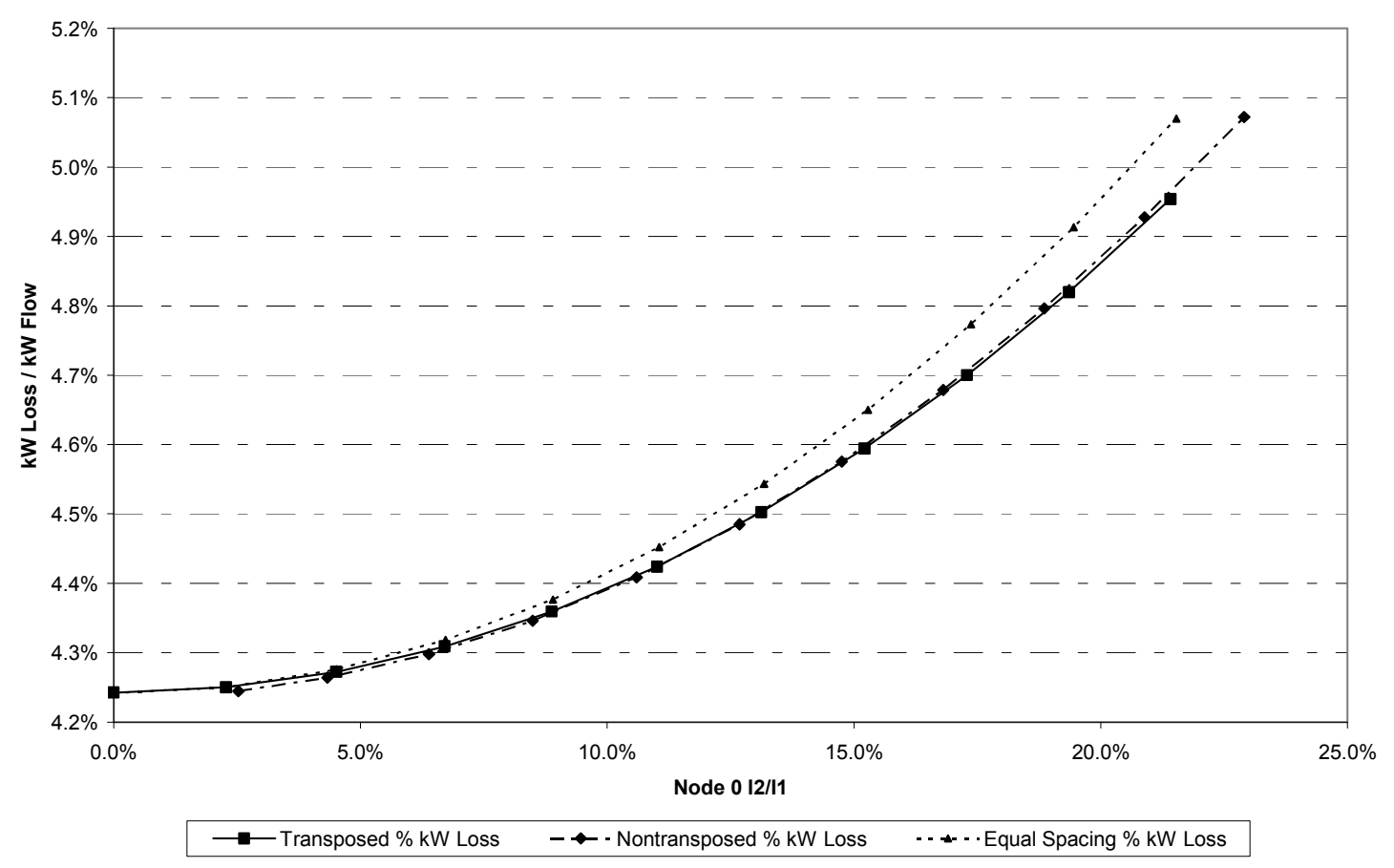

Figure 83. Percent losses versus load imbalance for each line configuration 


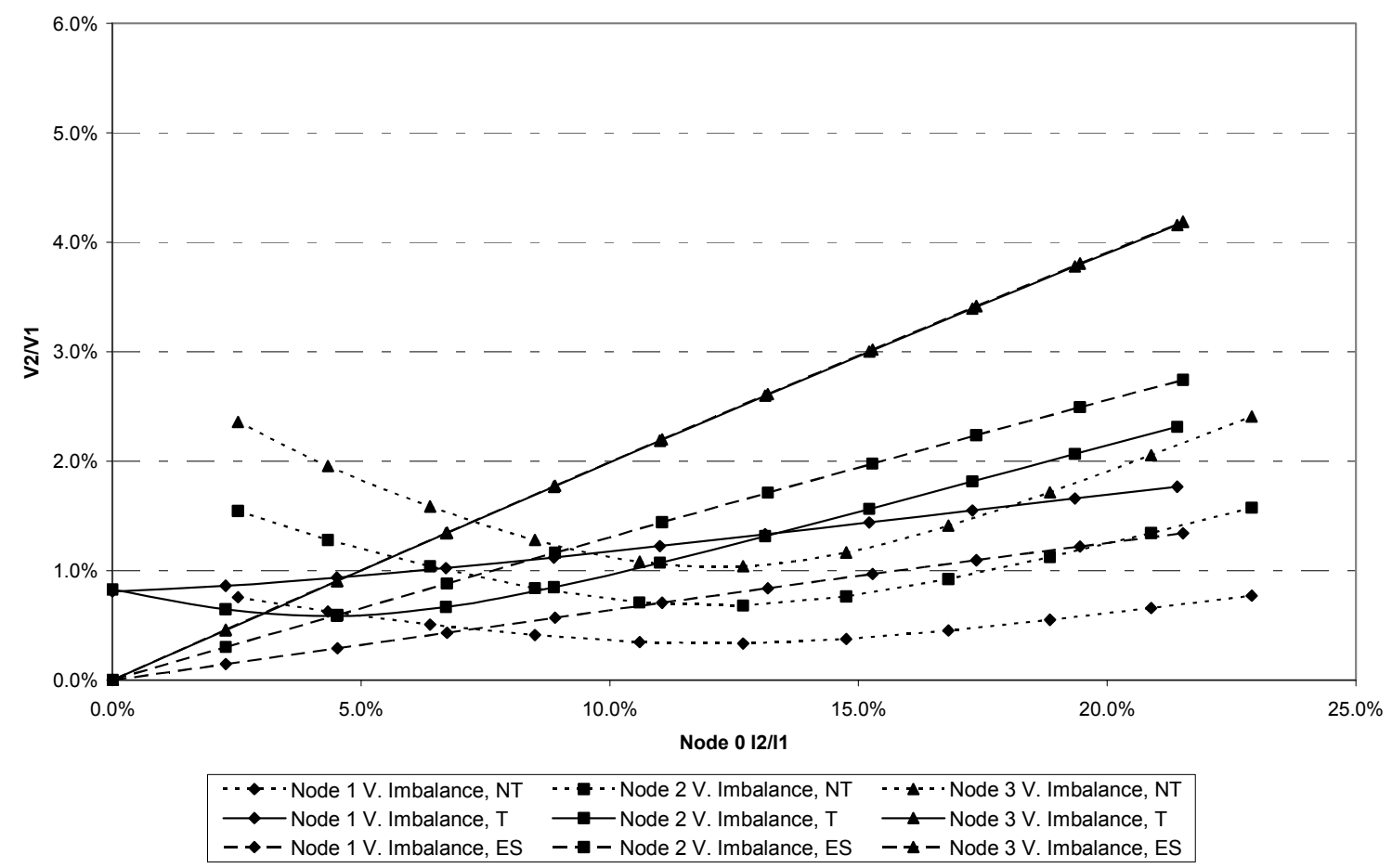

Figure 84. Voltage imbalance versus load imbalance for each line configuration

Sequence Currents vs Current Imbalance

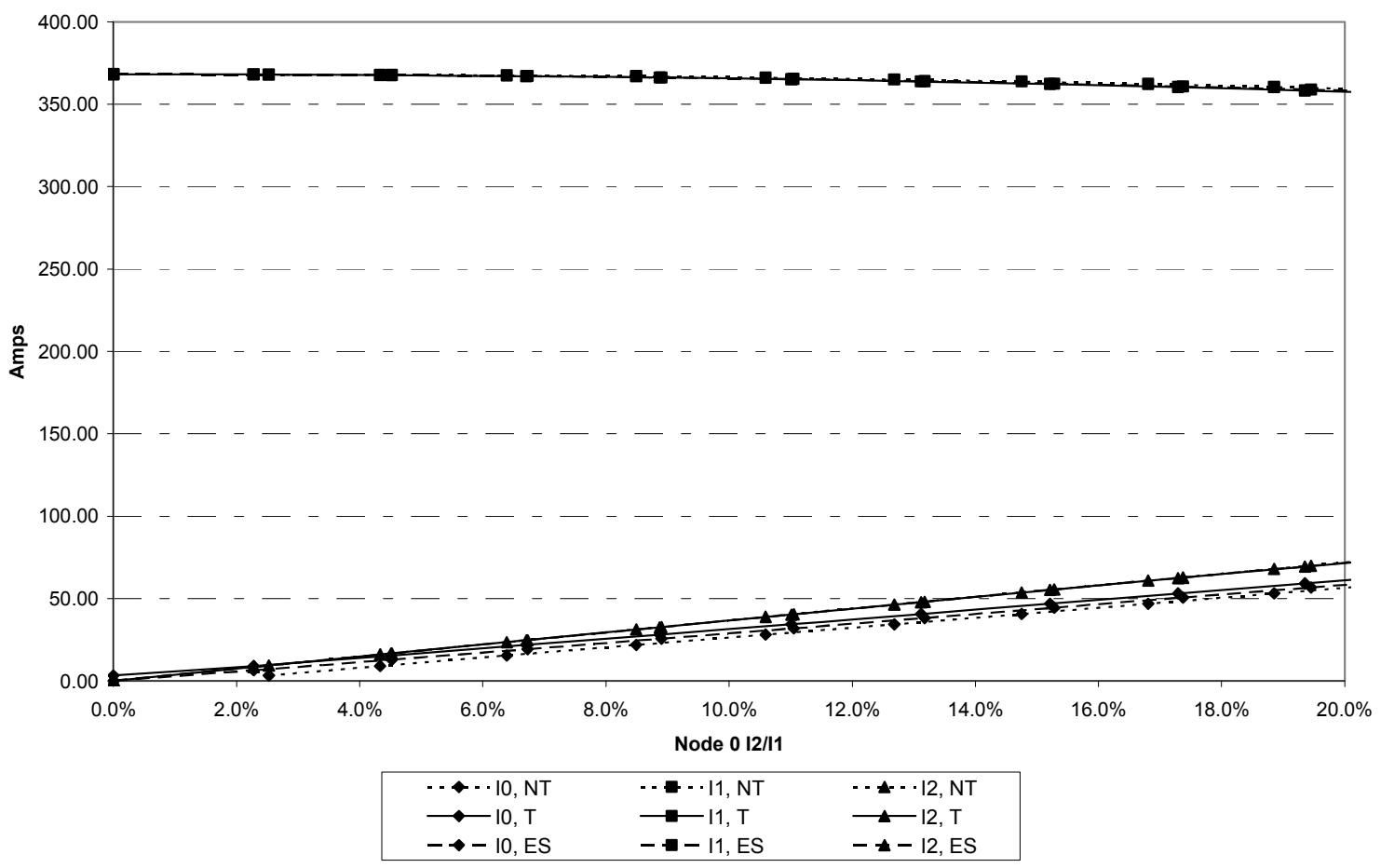

Figure 85. Sequence currents versus load imbalance for each line configuration 


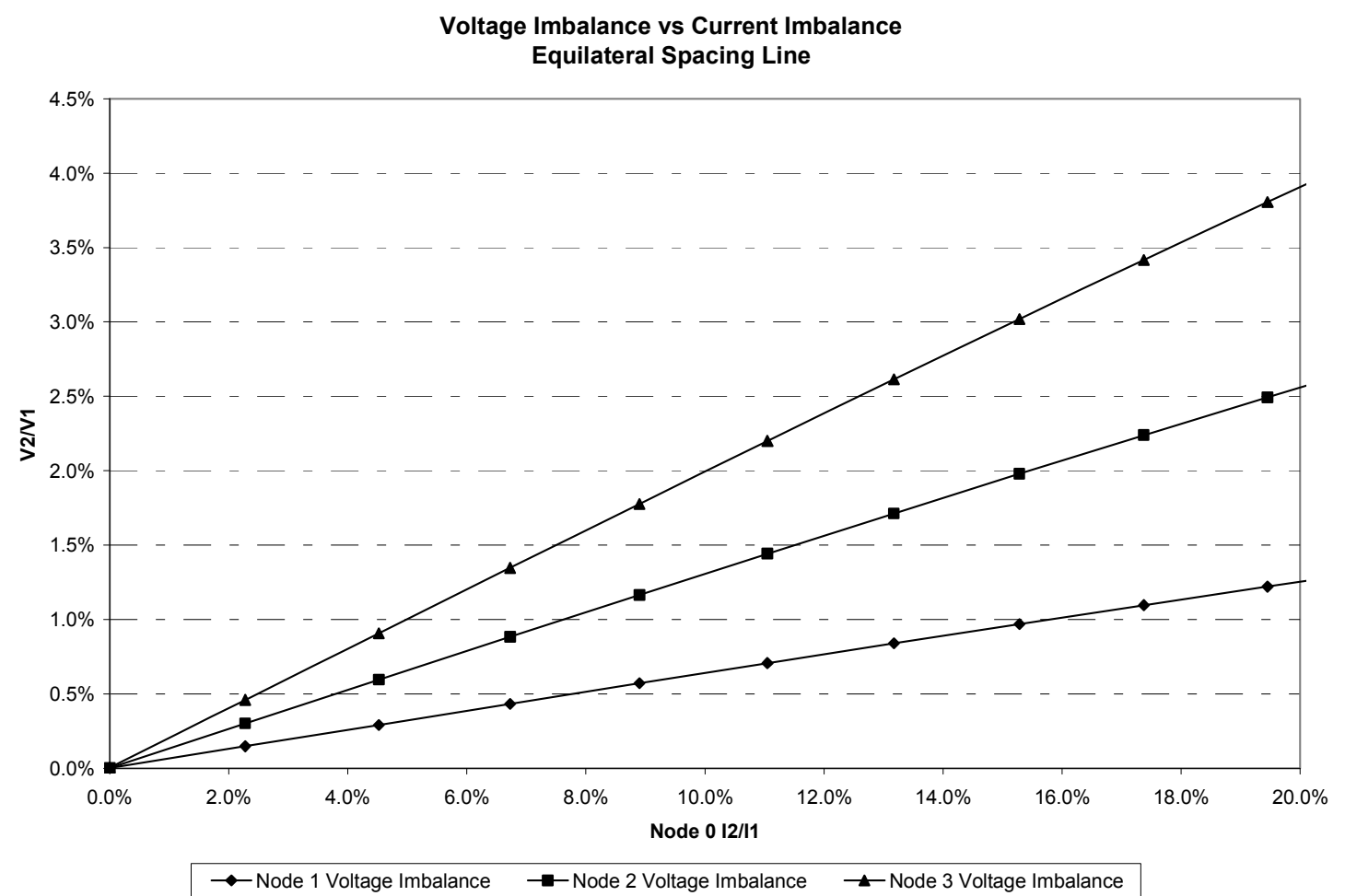

Figure 86. Voltage imbalance versus current imbalance equilateral spacing line

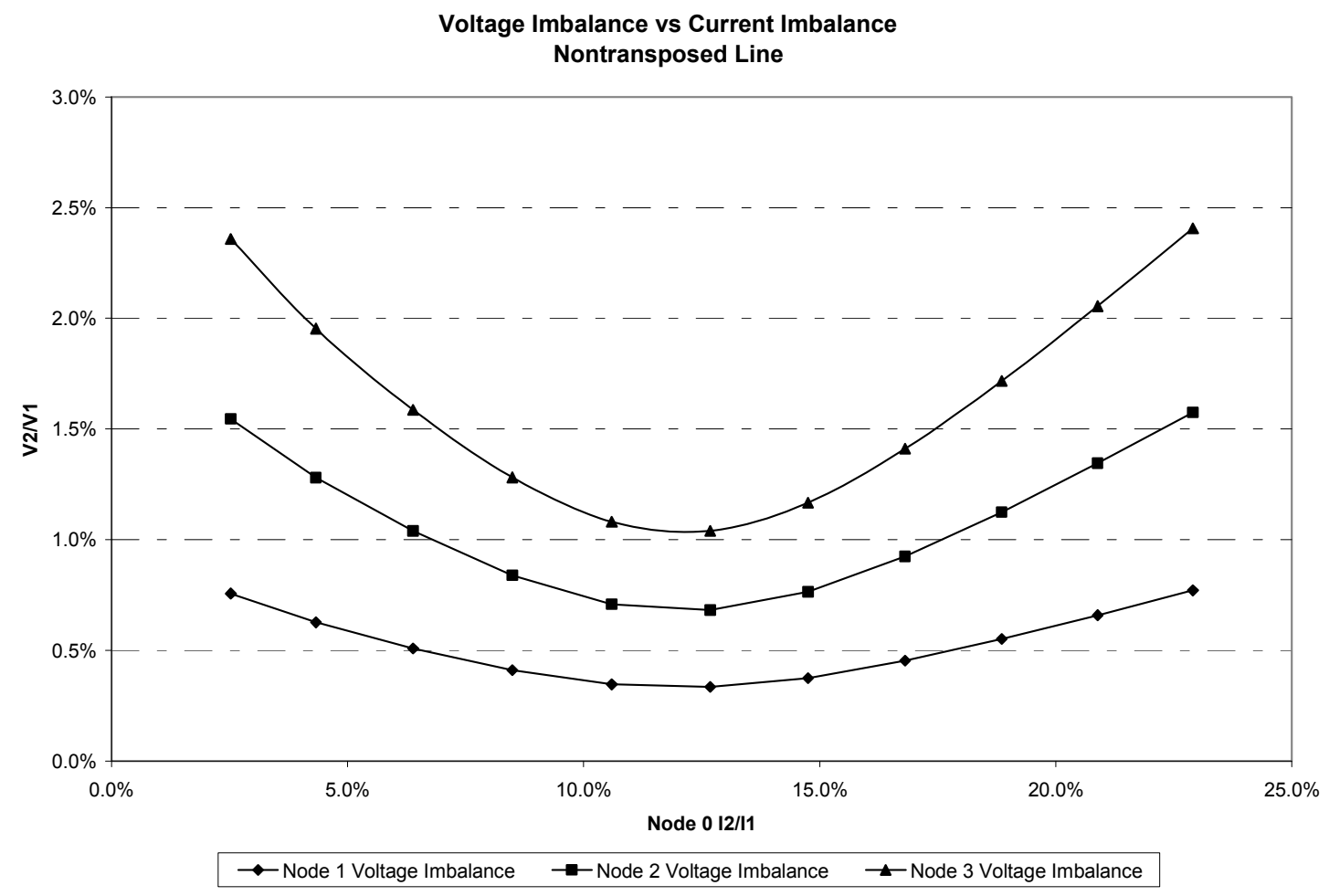

Figure 87. Voltage imbalance versus current imbalance non-transposed line 
Sequence Currents vs Current Imbalance

Transposed Line

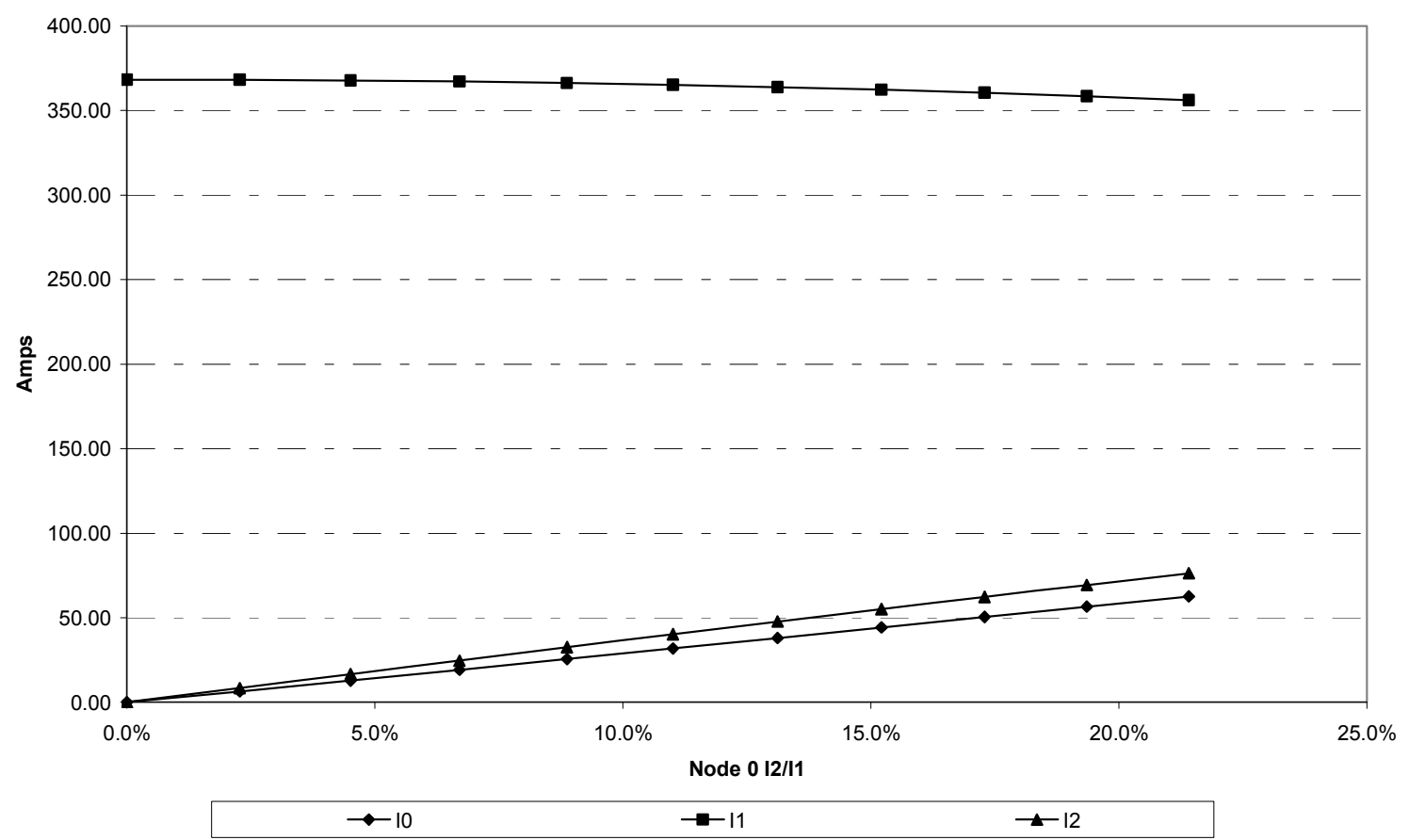

Figure 88. Voltage imbalance versus current imbalance transposed line

Sequence Currents vs Current Imbalance

Equilateral Spaced Line

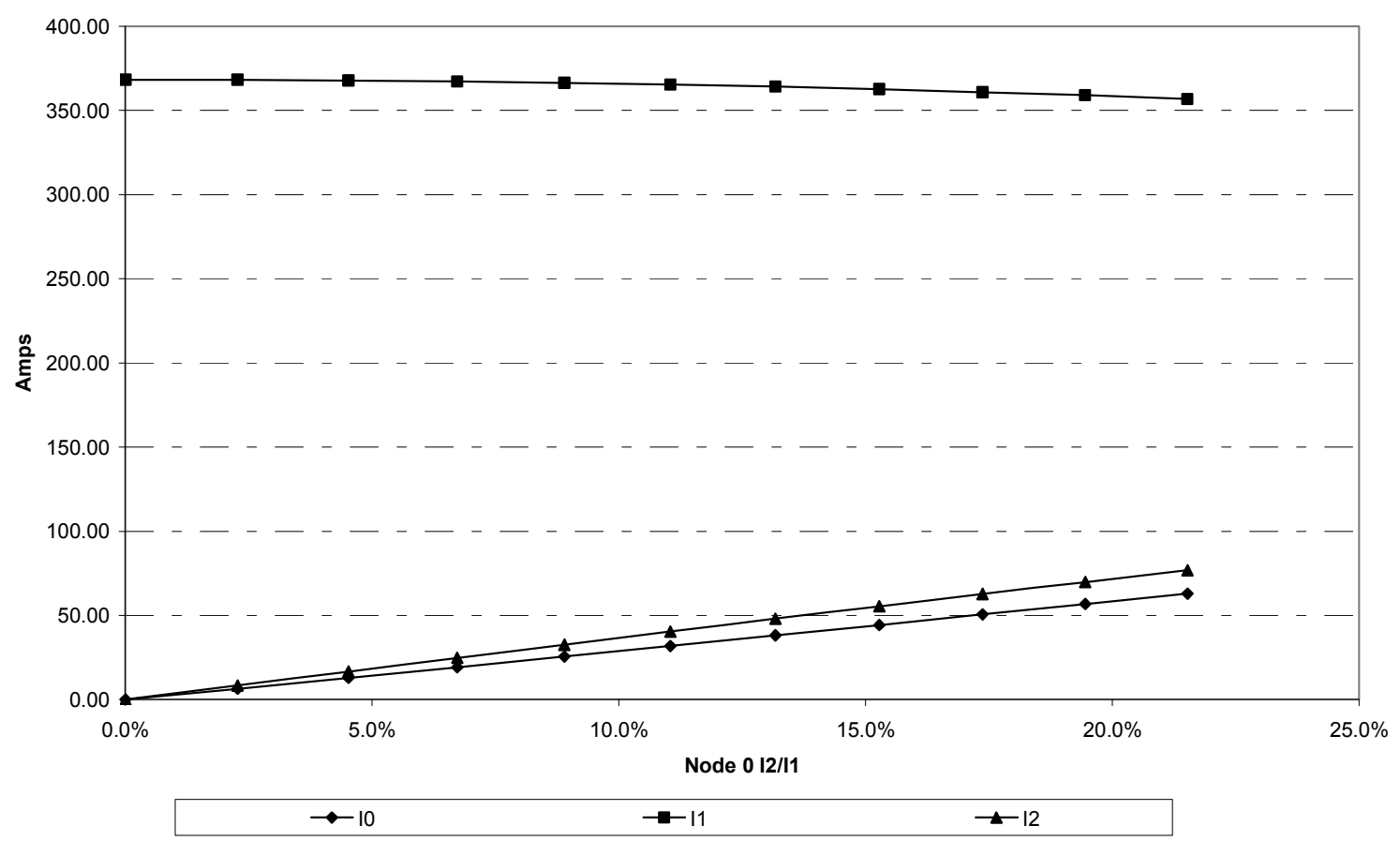

Figure 89. Sequence currents versus current imbalance equilateral-spaced line 
Sequence Currents vs Current Imbalance

Nontransposed Line

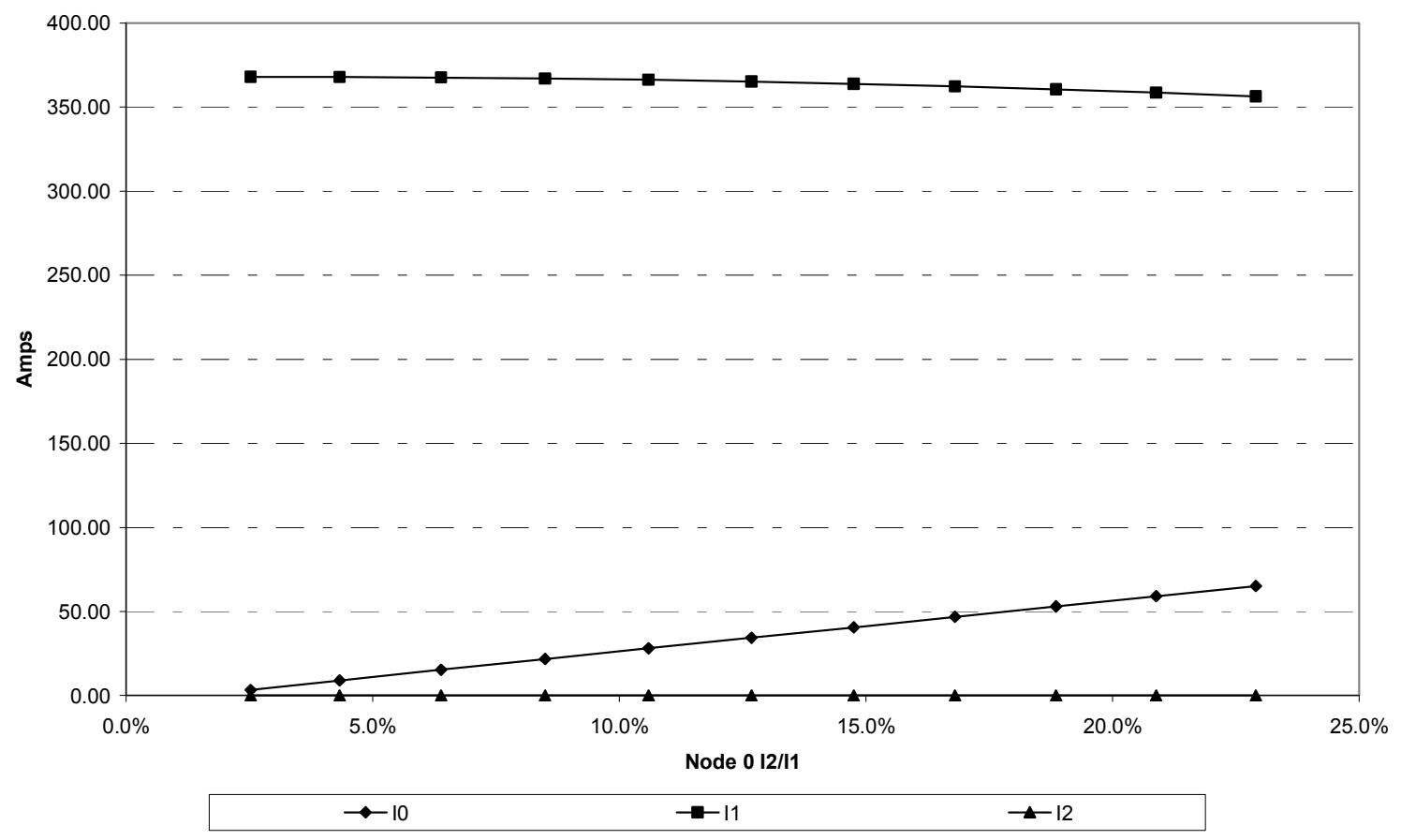

Figure 90. Sequence currents versus current imbalance for non-transposed line

Sequence Currents vs Current Imbalance

Transposed Line

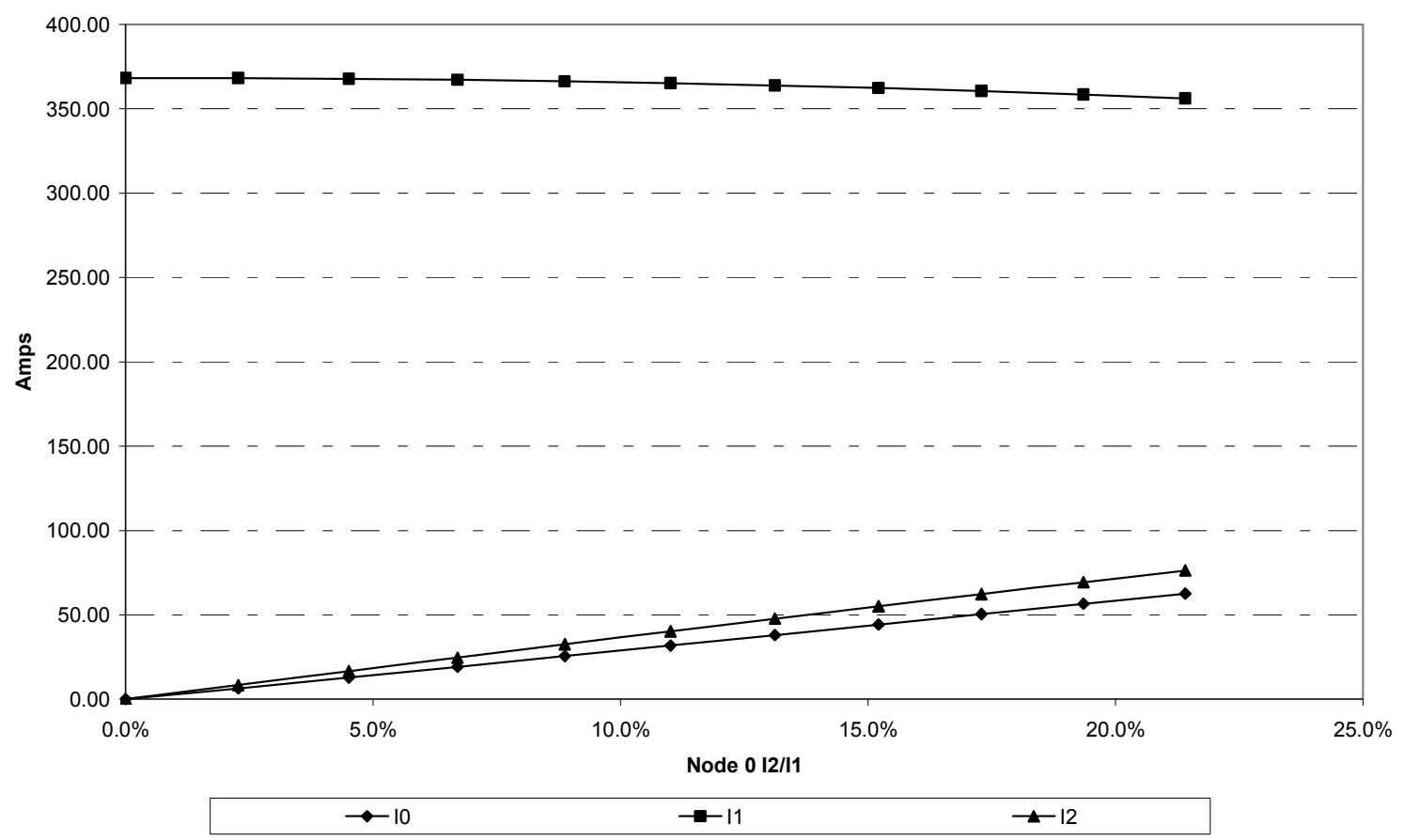

Figure 91. Sequence currents versus current substance for transposed line 
Table 14. The Effects of Circuit Spacing and Unbalanced Load on Percent Kilowatt Losses

\begin{tabular}{|c|c|c|c|c|c|c|c|c|}
\hline \multicolumn{9}{|c|}{ Equilateral Spacing } \\
\hline & \multicolumn{3}{|c|}{ Balanced Load } & \multirow[t]{4}{*}{ Total } & \multirow{2}{*}{\multicolumn{3}{|c|}{$\begin{array}{c}\text { Unbalanced Load } \\
\mathrm{I}_{2} / \mathrm{I}_{1}=21.5 \%\end{array}$}} & Total \\
\hline & \multicolumn{3}{|c|}{$\mathrm{I}_{2} / \mathrm{I}_{1}=0 \%$} & & & & & \\
\hline & $\Phi A$ & ФВ & $\Phi C$ & & $\Phi A$ & ФB & $\Phi C$ & \\
\hline \$l's & 368.22 & 368.25 & 368.28 & & 490.12 & 300.16 & 284.99 & \\
\hline $\mathrm{kW}$ & & & & & & & & \\
\hline Losses & 117.17 & 117.02 & 116.89 & 351.08 & 414.10 & -72.43 & 63.39 & 405.06 \\
\hline$\%$ Losses & & & & $4.2 \%$ & & & & $5.10 \%$ \\
\hline $\begin{array}{c}\text { Native } \\
\text { Load kW }\end{array}$ & & & & 7926 & & & & 7584 \\
\hline \multicolumn{9}{|c|}{ Flat Non-Transposed } \\
\hline & \multicolumn{3}{|c|}{ Balanced Load } & & \multicolumn{3}{|c|}{ Unbalanced Load } & \\
\hline & \multicolumn{3}{|c|}{$\mathrm{I}_{2} / \mathrm{I}_{1}=2.5 \%$} & & \multicolumn{3}{|c|}{$\mathrm{I}_{2} / \mathrm{I}_{1}=22.9 \%$} & \\
\hline & & & & & & & & \\
\hline Фl's & 356.11 & 375.94 & 371.99 & & 274.51 & 494.92 & 305.56 & \\
\hline $\begin{array}{c}\text { kW } \\
\text { Losses }\end{array}$ & 194.35 & 72.47 & 84.29 & 351.11 & 131.84 & 390.48 & -117.36 & 404.96 \\
\hline$\%$ Losses & & & & $4.2 \%$ & & & & $5.10 \%$ \\
\hline $\begin{array}{c}\text { Native } \\
\text { Load kW }\end{array}$ & & & & 7920 & & & & 7579 \\
\hline \multicolumn{9}{|c|}{ Flat Transposed } \\
\hline & \multicolumn{3}{|c|}{ Balanced Load } & & \multicolumn{3}{|c|}{ Unbalanced Load } & \\
\hline & \multicolumn{3}{|c|}{$\mathrm{I}_{2} / \mathrm{I}_{1}=0 \%$} & & \multicolumn{3}{|c|}{$\mathrm{I}_{2} / \mathrm{I}_{1}=21.4 \%$} & \\
\hline Фl's & 36020 & מתחת & 36021 & & 10720 & 30237 & 8005 & \\
\hline $\mathrm{kW}$ & & & & & & & & \\
\hline Losses & 117.05 & 117.04 & 117.06 & 351.15 & 417.26 & -96.12 & 73.83 & 394.97 \\
\hline$\%$ Losses & & & & $4.2 \%$ & & & & $5.0 \%$ \\
\hline $\begin{array}{c}\text { Native } \\
\text { Load kW }\end{array}$ & & & & 7925 & & & & 7578 \\
\hline
\end{tabular}

Notes:

A. Balanced loads result in less loss.

B. Kilowatt losses are greater for the non-transposed line than for the transposed line because $\% \mathrm{l}_{2} / \mathrm{l}_{1}$ is more for the non-transposed line.

C. Unbalanced load is $4,500 \mathrm{~kW}$ on Phase A, $2,250 \mathrm{~kW}$ on Phase B, and 2,250 kW on Phase C. Balanced load is $3,000 \mathrm{~kW}$ on each of the three phases.

Table 14 shows a summary of the effects of circuit spacing and unbalanced load on percent losses. For the balanced load of $3,000 \mathrm{~kW}$ on each phase (total load of $9,000 \mathrm{~kW}$ ), the $4.2 \%$ real losses are the same for the equilateral spacing, flat non-transposed, and flat transposed. For the unbalanced load of 4,500 kW on Phase A, 2,250 kW on Phase B, and 2,250 kW on Phase $\mathrm{C}$, the percent losses are $5.1 \%$ for the equilateral and flat non-transposed configurations and $5 \%$ for the flat transposed configuration. 


\subsection{Secondary and Service Impedances and Voltage Drops}

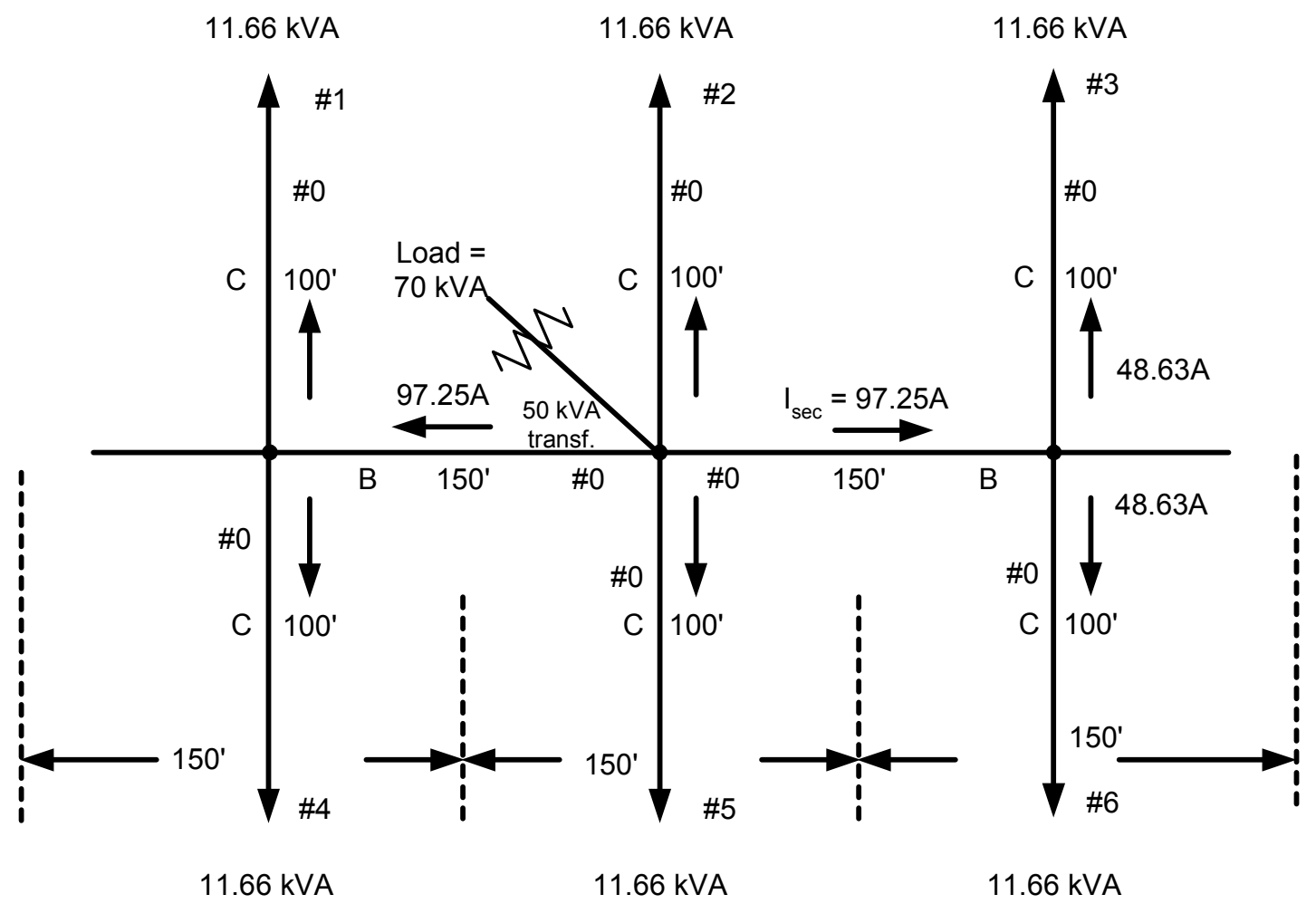

Figure 92. Distribution transformer servicing secondaries and services

Transformer size $=50 \mathrm{kVA}$

$\mathrm{D}_{\mathrm{m}}=$ Maximum coincident total demand on the transformer

$\mathrm{D}_{\mathrm{m}}=70 \mathrm{kVA}$ of load and losses

$\operatorname{Cos} \theta=0.90$

$$
\begin{aligned}
& \mathrm{I}_{\text {sec. }}=1 / 2\left[\frac{70 \mathrm{kVA}-2(11.66 \mathrm{kVA})}{240 \mathrm{~V}}\right]=1 / 2\left[\frac{46.68 \mathrm{kVA}}{240 \mathrm{~V}}\right]=97.25 \mathrm{~A} \\
& \mathrm{I}_{\text {service }}=1 / 2(97.25 \mathrm{~A})=48.63 \mathrm{~A}
\end{aligned}
$$

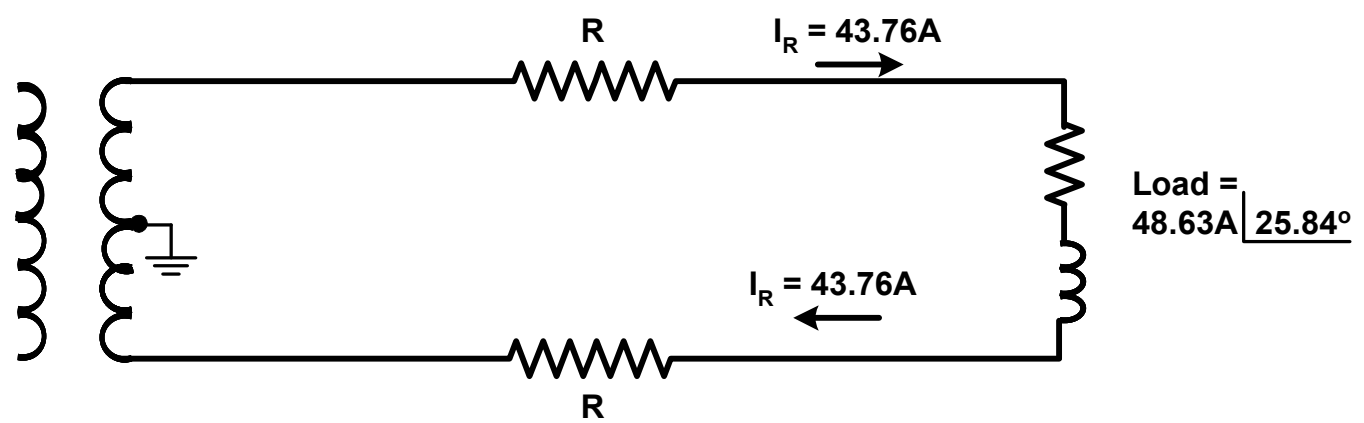

Figure 93. Distribution service drop 
The $\mathrm{R}$ and $\mathrm{X}$ values for the $\# 0$ triplex secondary and service are

$$
\begin{aligned}
& \mathrm{R}=0.211 \Omega / 1000^{\prime} \\
& \mathrm{X}=0.031 \Omega / 1000^{\prime} .
\end{aligned}
$$

The voltage drop in secondaries B is

$$
\begin{aligned}
& \Delta \mathrm{V}=2(\mathrm{I} \mathrm{R} \operatorname{Cos} \theta+\mathrm{I} X \operatorname{Sin} \theta) \\
& =2\left[97.25\left(0.211 \Omega / 1000^{\prime} \times 150^{\prime}\right) 0.90+97.25\left(0.031 \Omega / 1000^{\prime} \times 150^{\prime}\right) .436\right] \\
& =2(2.770+0.197)
\end{aligned}
$$$$
\Delta \mathrm{V}=5.93 \text { volts @ } 240 \mathrm{~V} \text { or } 2.97 \text { volts @ 120V base } \quad \text { Equation } 5.72
$$

\begin{tabular}{|c|c|c|c|}
\hline & \multirow{2}{*}{$\begin{array}{c}25 \mathrm{kVA} \\
\mathrm{N}=3 \\
\text { No Secondary }\end{array}$} & \multicolumn{2}{|c|}{$50 \mathrm{kVA}$} \\
\hline & & $\begin{array}{c}N=2 \\
\text { No Secondary }\end{array}$ & $\begin{array}{c}N=4 \\
\text { Secondary }\end{array}$ \\
\hline Transformer & 3.68 & 2.75 & 2.75 \\
\hline Secondary & -- & -- & 2.97 \\
\hline Service & 0.99 & 0.99 & 0.99 \\
\hline Total & 4.67 & 3.74 & 6.71 \\
\hline
\end{tabular}

The drop in the services $\mathrm{C}$ is

$$
\begin{aligned}
\Delta \mathrm{V} & =2\left[48.63\left(0.211 \Omega / 1000^{\prime} \times 100^{\prime}\right) .90+48.63\left(0.031 \Omega / 1000^{\prime} \times 100^{\prime}\right) .436\right] \\
& =2(0.920+0.067) \\
\Delta \mathrm{V} & =1.97 \text { volts @ } 240 \mathrm{~V} \text { or } 0.99 \text { volts @ } 120 \mathrm{~V} \text { base } \quad \text { Equation } 5.73
\end{aligned}
$$

The voltage drop for customers 2 and 5 is only $0.99 \mathrm{~V}$, but for customers $1,3,4$, and 6 , it is $3.96 \mathrm{~V}(2.97+0.99)$.

Table 15. Voltage Drop Summary - Transformer, Secondary, and Service Drop

\subsection{Secondary and Service Real Losses}

\section{Service Drop Real Losses:}

The resistance of \#0 aluminum service drops for $100^{\prime}$ is

$$
\mathrm{R}_{\text {service }}=2\left(0.211 \Omega / 1000^{\prime} \times 100^{\prime}\right)=0.0422 \Omega . \quad \text { Equation } 5.74
$$


The resistance is doubled because of the forward and return path of the current.

$$
\mathrm{I}_{\text {service }}^{2} \mathrm{R}_{\text {service }}=(48.63)^{2}(0.0422)=99.80 \text { watts per service drop }
$$

Equation 5.75

Service drop total real losses $=(6)(99.80$ watts $)=598.8$ watts $\quad$ Equation 5.76

\section{Secondary Real Losses:}

$$
\begin{aligned}
& \mathrm{R}_{\text {secondary }}=2\left(0.211 \Omega / 1000^{\prime} \times 150^{\prime}\right)=0.0633 \Omega \\
& \mathrm{I}_{\text {sec. }}^{2} \mathrm{R}_{\text {secondary }}=(97.25)^{2}(0.0633)=598.7 \text { watts per secondary }
\end{aligned}
$$

Equation 5.78

Secondary total real losses $=2(598.7$ watts $)=1197.3$ watts Equation 5.79

Total service and secondary real losses $=598.8+1197.3 \quad$ Equation 5.80

$$
=1796.1 \text { watts }
$$

$$
\text { Percent real losses }=\frac{1.796 \mathrm{~kW}}{(1.4)(50 \mathrm{kVA})(0.90)} \times 100=2.9 \% \quad \text { Equation } 5.81
$$

Note: Add kilowatt losses for each circuit element and divide by the total kilowatt flow to obtain total circuit percent losses. Percent losses cannot be added for each element.

A summary of the peak day (July 17, 2006) real power losses for the Milford substation transformer, the DC8103 primary, overhead and padmount transformers, secondary, and services is given in Table 16.

The percent real losses shown in the first column are for the 4.8-kV Hickory Distribution Circuit, the reference of which is given in Note A. These are actual measured losses that total $5.2 \%$, excluding the substation transformer. The next two columns represent the percent real losses for the Milford 8103 circuit. The second column depicts the calculated losses based on the average peak loading of the transformers and services and a lumped load representing the peak circuit load placed at the midpoint to calculate the primary losses. The third column shows the calculated losses determined from the simulation of the circuit for the peak load hour on July 17, 2006. 
Table 16. Peak Day Real Losses Comparison

\begin{tabular}{|c|c|c|c|}
\hline \multirow{4}{*}{$\begin{array}{l}\text { Transmission } \\
\text { Sub-transmission }\end{array}$} & \multicolumn{3}{|c|}{ Percent Losses } \\
\hline & -- & 3.5 & -- \\
\hline & -- & 4.5 & -- \\
\hline & Hickory & Milford 8103 & Milford 8103 \\
\hline Substation transformer & 0.70 & 0.729 & 0.702 \\
\hline Primary & $3.1^{\mathrm{A}}$ & $3.82^{B}$ & $3.59^{\mathrm{C}}$ \\
\hline Overhead and underground transformers & $1.3^{\mathrm{A}}$ & $1.22^{\mathrm{B}}$ & $1.50^{\mathrm{C}}$ \\
\hline Secondary and services & $0.8^{\mathrm{A}}$ & $0.95^{\mathrm{D}}$ & $\begin{array}{l}0.89^{\mathrm{D}} \\
0.31^{\mathrm{E}}\end{array}$ \\
\hline Total circuit losses & 5.2 & 5.99 & $5.98^{\mathrm{D}} 5.4^{\mathrm{E}}$ \\
\hline Total system losses & 13.9 & 14.72 & $14.68 \quad 14.10$ \\
\hline
\end{tabular}
Notes:
A. Data from Davis, Krupa, and Diedzic (1983)
B. Approximate based on average loading of transformers and services and lump load placed at midpoint for the primary losses
C. Distribution Engineering Workstation
D. \#4 MXAT Services used
E. \#O MXAT Services used

\subsection{Shunt Capacitor Models}

When capacitors are connected in parallel, $\mathrm{kVAr}=\mathrm{kVAr}{ }_{1}+\mathrm{kVAr}_{2}+\mathrm{kVAr}_{3}+\ldots$, and when connected in series,

$$
\mathrm{kVAr}=\frac{1}{\frac{1}{\mathrm{kVAr}_{1}}+\frac{1}{\mathrm{kVAr}_{2}}+\frac{1}{\mathrm{kVAr}_{3}}+\ldots}
$$

The capacitive reactance in ohms is

$$
\mathrm{X}_{\mathrm{C}}=\frac{1}{2 \pi f \mathrm{C} \times 10^{-6}},
$$

where $1 \mu \mathrm{f}$ is $2,653 \Omega$ at $60 \mathrm{~Hz}$, or

$$
\mathrm{X}_{\mathrm{C}}=\frac{2653}{\mathrm{C}} \text {, where } \mathrm{C} \text { is } 1 \mu \mathrm{f} \text {. }
$$

From Equation 5.82, $\mathrm{C}$ is then

$$
\mathrm{C}=\frac{10^{6}}{2 \pi f \mathrm{X}_{\mathrm{C}}} \text {. }
$$


Now, $\mathrm{X}_{\mathrm{C}}$ can be defined in terms of voltage and vars.

$$
\mathrm{kVAr}=\mathrm{kV} \mathrm{I}_{\mathrm{X}} \text {, where } \mathrm{I}_{\mathrm{X}}=\frac{\mathrm{V}}{\mathrm{X}_{\mathrm{C}}} .
$$

Because $\mathrm{kV}=\frac{\mathrm{V}}{1000}$, then

$$
\begin{aligned}
& \mathrm{kVAr}=\frac{\mathrm{kV}^{2} 1000}{\mathrm{X}_{\mathrm{C}}} \text {, and } \\
& \mathrm{X}_{\mathrm{C}}=\frac{\mathrm{kV}^{2} 1000}{\mathrm{kVAr}} .
\end{aligned}
$$

The capacitance $\mathrm{C}$ in microfarads can be determined in terms of voltage and Vars by substituting Equation 5.82 into Equation 5.84 and solving for C.

$$
\mathrm{C}=\frac{1000 \mathrm{kVAr}}{\mathrm{kV}^{2} 2 \pi f}
$$

Equation 5.85

If the capacitance $\mathrm{C}$ and voltage are known, then $\mathrm{KVAr}$ can be found from Equation 5.85.

$$
\mathrm{kVAr}=\frac{\mathrm{kV}^{2} 2 \pi f C}{1000}
$$

Equations 5.84, 5.85, and 5.86 are the most commonly used. Two examples follow.

The ohms per phase for a $300-\mathrm{kVAr}$, three-phase, $4.8-\mathrm{kV}$, delta-connected capacitor bank is

$$
\mathrm{kVAr} / \Phi=\frac{300 \mathrm{kVAr}}{3}=100 \mathrm{kVAr} .
$$

The line-to-line voltage applied across each capacitor is $4.8 \mathrm{kV}$. Applying Equation 5.84,

$$
\mathrm{X}_{\mathrm{C}}=\frac{4.8^{2}(1000)}{100}=230.4 \Omega .
$$

The capacitance from Equation 5.85 is

$$
\mathrm{C}=\frac{(1000)(100)}{(4.8)^{2}(377)}=11.51 \mu f .
$$


The ohms per phase for a $600-\mathrm{kVAr}$, three-phase, $13.2-\mathrm{kV}$, wye-connected capacitor bank is

$$
\mathrm{kVAr} / \Phi=\frac{600}{3}=200 \mathrm{kVAr}
$$

The voltage across each capacitor is $\frac{13.2 \mathrm{kV}}{\sqrt{3}},$. Therefore, using Equation 5.84 results in

$$
\mathrm{X}_{\mathrm{C}}=\frac{(7.620)^{2} 1000}{200}=290.3 \Omega
$$

and the capacitance is

$$
C=\frac{(1000)(200)}{(7.620)^{2}(377)}=9.14 \mu f .
$$

Capacitor models can be developed using Equation 5.84, which is repeated below.

$$
\mathrm{X}_{\mathrm{C}}=\frac{\mathrm{kV}^{2} 1000}{\mathrm{kVAr}}
$$

The susceptance $\mathrm{B}_{\mathrm{C}}$ is the reciprocal of $\mathrm{X}_{\mathrm{C}}$; therefore, $\mathrm{B}_{\mathrm{C}}$ from Equation 5.84 becomes

$$
\mathrm{B}_{\mathrm{C}}=\frac{1}{\mathrm{X}_{\mathrm{C}}}=\frac{\mathrm{kVAr}}{\mathrm{kV}^{2} 1000},
$$

where $\mathrm{kVAr}$ is the kilovars per phase and $\mathrm{kV}$ is the applied voltage across the capacitor. For a wye-connected capacitor bank

$$
\mathrm{B}_{\mathrm{C}}=\frac{1}{\mathrm{X}_{\mathrm{C}}}=\frac{\mathrm{kVAr}}{\mathrm{kV}_{\mathrm{LN}}{ }^{2} 1000} \text {. }
$$

For a delta-connected capacitor bank

$$
\mathrm{B}_{\mathrm{C}}=\frac{1}{\mathrm{X}_{\mathrm{C}}}=\frac{\mathrm{kVAr}}{\mathrm{kV}_{\mathrm{LL}}{ }^{2} 1000}
$$

The line currents for the wye-connected bank are written from Equation 5.88.

$$
\begin{aligned}
& \mathrm{V}_{\mathrm{AN}}=\mathrm{I}_{\mathrm{C}_{\mathrm{A}}} \mathrm{j} \mathrm{X}_{\mathrm{A}} \\
& \mathrm{I}_{\mathrm{C}_{\mathrm{A}}}=\frac{\mathrm{V}_{\mathrm{AN}}}{\mathrm{j} \mathrm{X}_{\mathrm{A}}}=\mathrm{VAN} \mathrm{jBA},
\end{aligned}
$$




$$
\begin{aligned}
& \mathrm{I}_{\mathrm{C}_{\mathrm{B}}}=\mathrm{VBNj \textrm {BB }} \\
& \mathrm{I}_{\mathrm{C}_{\mathrm{C}}}=\mathrm{VCNj \textrm {BC }}
\end{aligned}
$$$$
\text { Equation } 5.91
$$

For the delta-connected bank, the phase currents are:

$$
\begin{aligned}
& \mathrm{I}_{\mathrm{C}_{\mathrm{AB}}}=\mathrm{VAB} \mathrm{j} \mathrm{BAB} \\
& \mathrm{I}_{\mathrm{C}_{\mathrm{BC}}}=\mathrm{VBC} \mathrm{j} \mathrm{BBC} \\
& \mathrm{I}_{\mathrm{C}_{\mathrm{CA}}}=\mathrm{VCA} \mathrm{j} \mathrm{BCA} .
\end{aligned}
$$

The line currents are:

$$
\begin{aligned}
& \mathrm{I}_{\mathrm{C}_{\mathrm{A}}}=\mathrm{I}_{\mathrm{C}_{\mathrm{AB}}}-\mathrm{I}_{\mathrm{C}_{\mathrm{CA}}} \\
& \mathrm{I}_{\mathrm{C}_{\mathrm{B}}}=\mathrm{I}_{\mathrm{C}_{\mathrm{BC}}}-\mathrm{I}_{\mathrm{C}_{\mathrm{AB}}} \\
& \mathrm{I}_{\mathrm{C}_{\mathrm{C}}}=\mathrm{I}_{\mathrm{C}_{\mathrm{CA}}}-\mathrm{I}_{\mathrm{C}_{\mathrm{BC}} .}
\end{aligned}
$$

Equation 5.98

In matrix form, equations $5.96,5.97$, and 5.98 can be written as:

$$
\left[\begin{array}{c}
\mathrm{I}_{\mathrm{C}_{\mathrm{A}}} \\
\mathrm{I}_{\mathrm{C}_{\mathrm{B}}} \\
\mathrm{I}_{\mathrm{C}_{\mathrm{C}}}
\end{array}\right]=\left[\begin{array}{ccc}
1 & 0 & -1 \\
-1 & 1 & 0 \\
0 & -1 & 1
\end{array}\right] \bullet\left[\begin{array}{c}
\mathrm{I}_{\mathrm{C}_{\mathrm{AB}}} \\
\mathrm{I}_{\mathrm{C}_{\mathrm{BC}}} \\
\mathrm{I}_{\mathrm{C}_{\mathrm{CA}}}
\end{array}\right] .
$$

\subsection{Step Voltage Regulator Models}

Both Type A and Type B step VRs are modeled. The relationship between the source voltage $V_{S}$ and load voltage $V_{L}$ for the single-phase step VR is:

$$
\mathrm{V}_{\mathrm{S}}=\mathrm{a}_{\mathrm{r}} \mathrm{V}_{\mathrm{L}},
$$

where $a_{r}$ is defined as

$$
\mathrm{a}_{\mathrm{r}}=1 \mp \frac{\text { Total } \% \text { Range }}{\# \text { of Steps }} \times \text { Tap }
$$


with the following sign convention.

\begin{tabular}{lcc}
\hline & Type A & Type B \\
\cline { 2 - 3 } Raise & + & - \\
Lower & - & + \\
\hline
\end{tabular}

The relationship between source current and load current for the single-phase step VR is

$$
\mathrm{I}_{\mathrm{S}}=\frac{1}{\mathrm{a}_{\mathrm{r}}} \mathrm{I}_{\mathrm{L}} .
$$

Equation 5.102

\subsection{Synchronous Generator Model}

The Distribution Engineering Workstation uses two models to represent the synchronous machine: one for power flow and one for transient analysis. Because this study does not involve transient analysis, only the power flow model is considered. Figure 94 is a graphical representation of the power flow synchronous machine model.

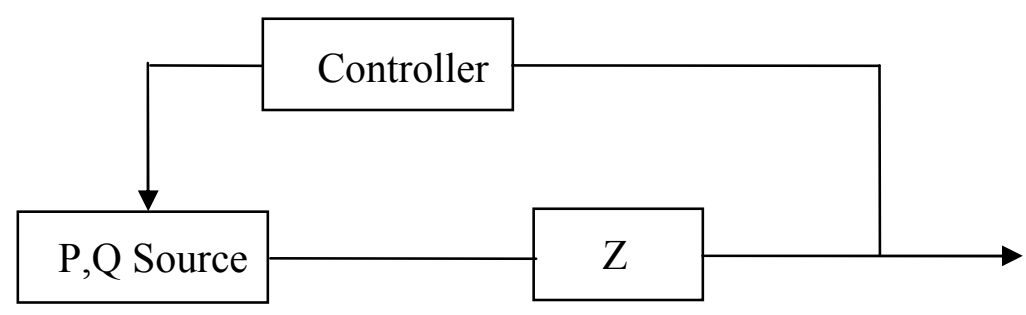

Figure 94. Steady-state synchronous machine model

The synchronous machine model used for power flow consists of a real and reactive power source, along with the impedance $\mathrm{Z}$ (in this case, the synchronous impedance). The controller takes measurements (specifically, voltage magnitude and power factor) from machine terminals and allows the machine to operate in two modes: constant $\mathrm{P}$ with power factor control or constant $\mathrm{P}$ and constant $\mathrm{Q}$.

The model takes into account the minimum and maximum generation limits of the machine, and the controller adjusts the P, Q Source based on the control mode. For example, if the machine is in the constant $\mathrm{P}$ with power factor control mode, the controller will maintain real rated power while varying the reactive power within the machine limits in an attempt to hold the power factor at the point of measurement constant. 


\subsection{Self-Excited Induction Generator Model}

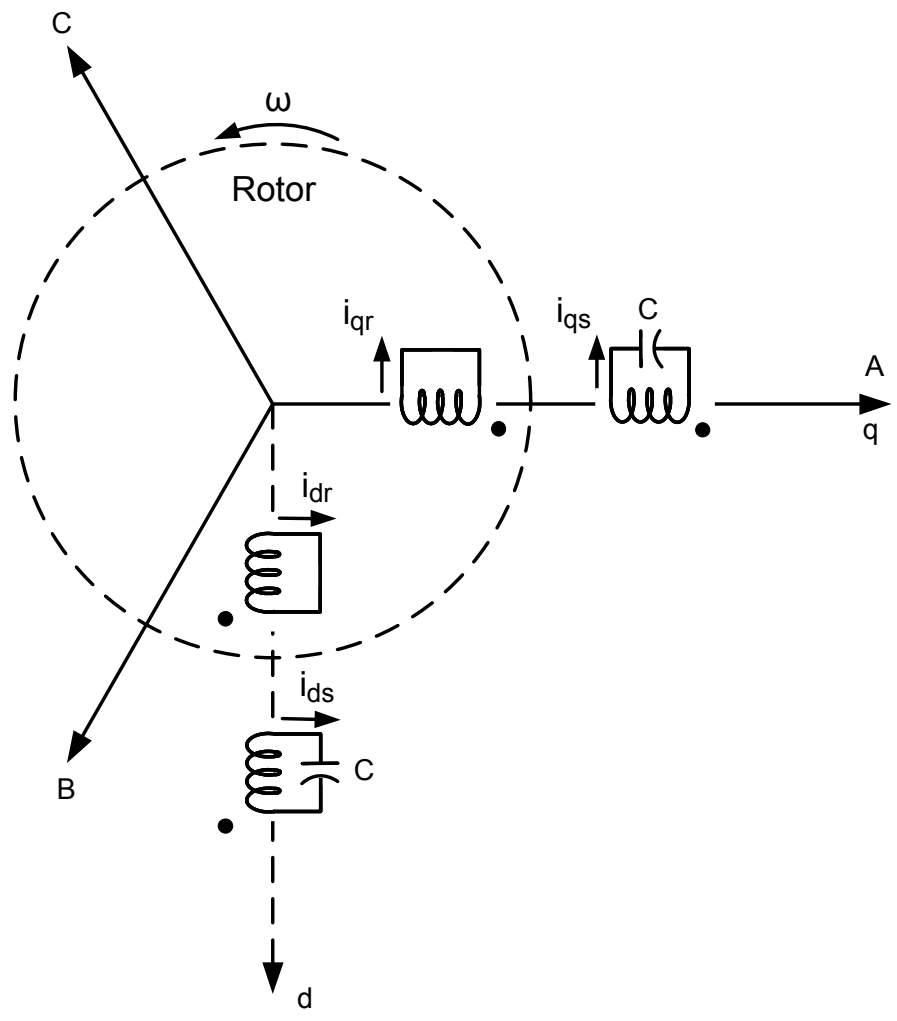

Figure 95. Two-phase primitive machine to be interconnected with an RLC load

A self-excited induction generator can be represented as a two-phase primitive machine with stator windings fixed and rotor windings rotating. The currents $i_{\mathrm{ds}}$ and $\mathrm{i}_{\mathrm{qs}}$ are the stator currents, and $i_{d r}$ and $i_{\text {qr }}$ are the rotor currents in the direct and quadrature axes, respectively. Figure 95 shows the mechanical angular speed of the rotor as $\omega=\mathrm{d} \theta / \mathrm{dt}$. A self-excited induction generator with capacitor added is represented in Equation 5.103. The voltage drop across the capacitor for the direct axis is $\mathrm{v}_{\mathrm{Cd}}$ and for the quadrature axis is $\mathrm{v}_{\mathrm{Cq}}$. The term $1 / \mathrm{pC}$ represents this voltage drop because

$$
\begin{aligned}
& \mathrm{v}=\frac{1}{\mathrm{C}} \square \text { ḑdt, } \\
& \mathrm{p}=\mathrm{d} / \mathrm{dt} \text { and } \\
& \frac{1}{\mathrm{p}}=\square \mathrm{c} d t
\end{aligned}
$$




$\left[\begin{array}{c}\mathrm{v}_{\mathrm{ds}} \\ \mathrm{v}_{\mathrm{qs}} \\ \mathrm{v}_{\mathrm{dr}} \\ \mathrm{v}_{\mathrm{qr}}\end{array}\right]=\left[\begin{array}{cccc}\mathrm{R}_{1}+\mathrm{L}_{1} \mathrm{p}+\frac{1}{\mathrm{pC}} & 0 & \mathrm{Mp} & 0 \\ 0 & \mathrm{R}_{1}+\mathrm{L}_{1} \mathrm{p}+\frac{1}{\mathrm{pC}} & 0 & \mathrm{Mp} \\ \mathrm{Mp} & \omega \mathrm{M} & \mathrm{R}_{2}+\mathrm{L}_{2} \mathrm{p} & { }^{\omega} \mathrm{L}_{2} \\ -\omega \mathrm{M} & \mathrm{Mp} & -\omega \mathrm{L}_{2} & \mathrm{R}_{2}+\mathrm{L}_{2} \mathrm{p}\end{array}\right] \bullet\left[\begin{array}{l}\mathrm{i}_{\mathrm{ds}} \\ \mathrm{i}_{\mathrm{qs}} \\ \mathrm{i}_{\mathrm{dr}} \\ \mathrm{i}_{\mathrm{qr}}\end{array}\right]$

Equation 5.103

This equation represents the no-load condition, and the turns ratio is assumed to be unity. Therefore, if needed, the ratio must be included when referring the rotor parameters to the stator.

The mutual inductance $M$ varies with the relative position between the stator and rotor. $\mathrm{R}_{1}$ and $\mathrm{R}_{2}$ are the stator and rotor resistances, and $\mathrm{L}_{1}$ and $\mathrm{L}_{2}$ are their inductances. The additional voltage drop because of the mutual flux is

$$
\begin{aligned}
& \varphi_{\mathrm{m}}=\mathrm{Mi} \text {, and } \\
& \begin{aligned}
\mathrm{v}=\frac{\mathrm{d} \varphi_{\mathrm{m}}}{\mathrm{dt}} & =\mathrm{M} \frac{\mathrm{di}}{\mathrm{dt}}+\frac{\mathrm{idM}}{\mathrm{dt}} . \\
& =\mathrm{Mpi}+\mathrm{ipM}
\end{aligned}
\end{aligned}
$$

Equation 5.104

There is a nonlinear relationship between the magnetizing reactance and the magnetizing current. Therefore, the mutual inductance $\mathrm{M}$ varies continuously. The term Mpi represents the current variation because of the stator, and the term ipM is because of the rotation of the rotor.

\subsubsection{Resistive Load}

When a resistive load $\mathrm{R}$ is added in parallel with the self-excitation capacitor $\mathrm{C}$, the voltage across this $\mathrm{R}$ load is the same as the voltage across the capacitor. In the direct axis stator of Figure 95, the addition of the load resistor changes the equivalent circuit to Figure 96.

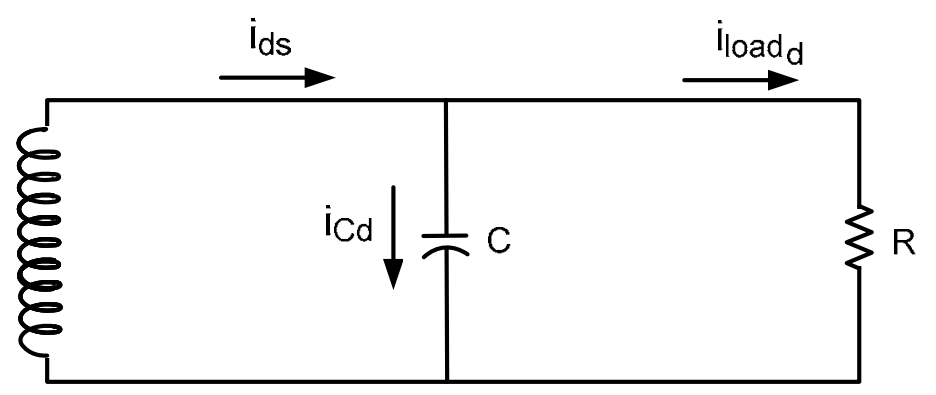

Figure 96. Stator direct axis with an $\mathrm{R}$ load added 
Because $\mathrm{v}_{\text {load } \mathrm{d}}=\mathrm{v}_{\mathrm{Cd}}=\mathrm{R} \mathrm{i}_{\text {load } \mathrm{d}}$

Equation 5.105

$$
\begin{aligned}
& \text { and } i_{C d}=C \frac{d v_{C d}}{d t}=C p v_{C d} \text {, then } \\
& i_{C d}=C \text { p Ri load d. }
\end{aligned}
$$

Because

$$
\begin{aligned}
& \mathrm{i}_{\mathrm{ds}}=\mathrm{i}_{\mathrm{Cd}}+\mathrm{i}_{\text {load d }}, \\
& \mathrm{i}_{\mathrm{ds}}=\mathrm{R} \mathrm{Cp} \mathrm{i}_{\text {load d }}+\mathrm{i}_{\text {load d }} \text { and } \\
& =\mathrm{i}_{\text {load d }}(\mathrm{R} \mathrm{Cpi}+1) .
\end{aligned}
$$

Equation 5.106

From Equation 5.105,

$$
\mathrm{i}_{\text {load d }}=\frac{\mathrm{i}_{\mathrm{ds}}}{\mathrm{R} \mathrm{C} \mathrm{p}+1} \text {. }
$$

Equation 5.107

Substituting Equation 5.107 into 5.105, results in

$$
\mathrm{v} \text { load } \mathrm{d}=\frac{\mathrm{Ri}_{\mathrm{ds}}}{\mathrm{RCp}+1} .
$$

The quadrature load voltage is obtained in a similar manner.

$$
\mathrm{V}_{\text {load } q}=\frac{\mathrm{Ri}_{\mathrm{qs}}}{\mathrm{R} \mathrm{C} \mathrm{p}+1}
$$

Now Equation 5.103 can be rewritten with the addition of the resistive load $\mathrm{R}$ from equations 5.108 and 5.109 .

$\left[\begin{array}{c}\mathrm{v}_{\mathrm{ds}} \\ \mathrm{v}_{\mathrm{qs}} \\ \mathrm{v}_{\mathrm{dr}} \\ \mathrm{v}_{\mathrm{qr}}\end{array}\right]=\left[\begin{array}{cccc}\mathrm{R}_{1}+\mathrm{L}_{1} \mathrm{p}+\frac{\mathrm{R}}{\mathrm{R} \mathrm{Cp}+1} & 0 & \mathrm{Mp} & 0 \\ 0 & \mathrm{R}_{1}+\mathrm{L}_{1} \mathrm{p}+\frac{\mathrm{R}}{\mathrm{R} \mathrm{Cp}+1} & 0 & \mathrm{Mp} \\ \mathrm{Mp} & \omega \mathrm{M} & \mathrm{R}_{2}+\mathrm{L}_{2} \mathrm{p} & { }^{2} \mathrm{~L}_{2} \\ -\omega \mathrm{M} & \mathrm{Mp} & -\omega_{\mathrm{L}_{2}} & \mathrm{R}_{2}+\mathrm{L}_{2} \mathrm{p}\end{array}\right] \bullet\left[\begin{array}{c}\mathrm{i}_{\mathrm{ds}} \\ \mathrm{i}_{\mathrm{qs}} \\ \mathrm{i}_{\mathrm{dr}} \\ \mathrm{i}_{\mathrm{qr}}\end{array}\right]$

Equation 5.110 


\subsubsection{RLC Load}

When an RL load $\mathrm{R}_{\mathrm{L}}$ and $\mathrm{L}_{\mathrm{L}}$ is added in parallel with the self-excitation capacitor Cs, or a combination of this excitation capacitor and other capacitors $\mathrm{C}_{\mathrm{L}}$ on the circuit in parallel, $\mathrm{C}$ in the following equations considers this combination of capacitors. See Figure 97.

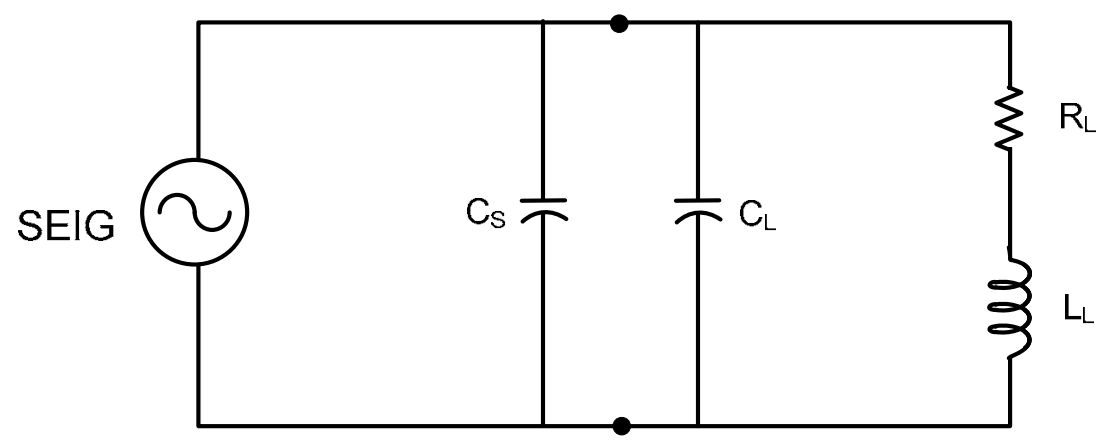

Figure 97. $R L C$ load $R_{L}, L_{L}$, and $C_{L}$ connected to the self-excited capacitor $C_{S}$

Because $\mathrm{v}_{\text {load } \mathrm{d}}=\mathrm{R} \mathrm{i}_{\text {load d }}$ from Equation 5.105, $\mathrm{v}_{\text {load d }}$ with load inductance added is

$$
\mathrm{V}_{\text {load d }}=\mathrm{Ri}_{\text {load d }}+\mathrm{L} \mathrm{pi}_{\text {load d }}
$$

and

$$
\mathrm{i}_{\mathrm{Cd}}=\mathrm{Cpv}_{\text {load d }}=\left(\mathrm{R} \mathrm{C} \mathrm{p}+\mathrm{L} \mathrm{C} \mathrm{p} \mathrm{p}^{2}\right) \mathrm{i}_{\text {load d }} .
$$

Now from Equation 5.112,

$$
\mathrm{i}_{\mathrm{d}_{s}}=\mathrm{i}_{\mathrm{Cd}_{\mathrm{d}}}+\mathrm{i}_{\text {load d }}=\left(\mathrm{R} \mathrm{C} \mathrm{p}+\mathrm{LC} \mathrm{p}^{2}\right) \mathrm{i}_{\text {load d }}+\mathrm{i}_{\text {load d }}
$$

and

$$
i_{\text {load d }}=\frac{i_{d s}}{R C p+L C p^{2}+1} .
$$

From Equation 5.111,

$$
\begin{aligned}
& \mathrm{v}_{\text {load d }}=\mathrm{R} \mathrm{i}_{\text {load d }}+\mathrm{L}_{\mathrm{pi}} \mathrm{i}_{\text {load d }} \text { and } \\
& \mathrm{v}_{\text {load d }}=(\mathrm{R}+\mathrm{L} \mathrm{p}) \mathrm{i}_{\text {load d }} .
\end{aligned}
$$

Substituting $i_{\text {load d }}$ from Equation 5.114 into Equation 5.115 results in

$$
\mathrm{v}_{\text {load d }}=\frac{\mathrm{R}+\mathrm{Lp}}{\mathrm{RCp}+\mathrm{LC} \mathrm{p}^{2}+1} \mathrm{i}_{\mathrm{ds}} .
$$


The quadrature load voltage becomes

$$
\mathrm{V}_{\text {load } q}=\frac{\mathrm{R}+\mathrm{Lp}}{\mathrm{R} \mathrm{C} \mathrm{p}+\mathrm{LC} \mathrm{p}^{2}+1} \mathrm{i}_{\mathrm{qs}}
$$

Equation 5.103 can be rewritten with the addition of resistance load R, inductive load L, and capacitive load $\mathrm{C}$ using Equation 5.117.

Equation 5.118 is the impedance matrix for a single self-excited induction generator serving an RLC load. It must be remembered that the variation in the magnetizing reactance because of the magnetizing current must be included and corrected as in the development of Equation 5.104.

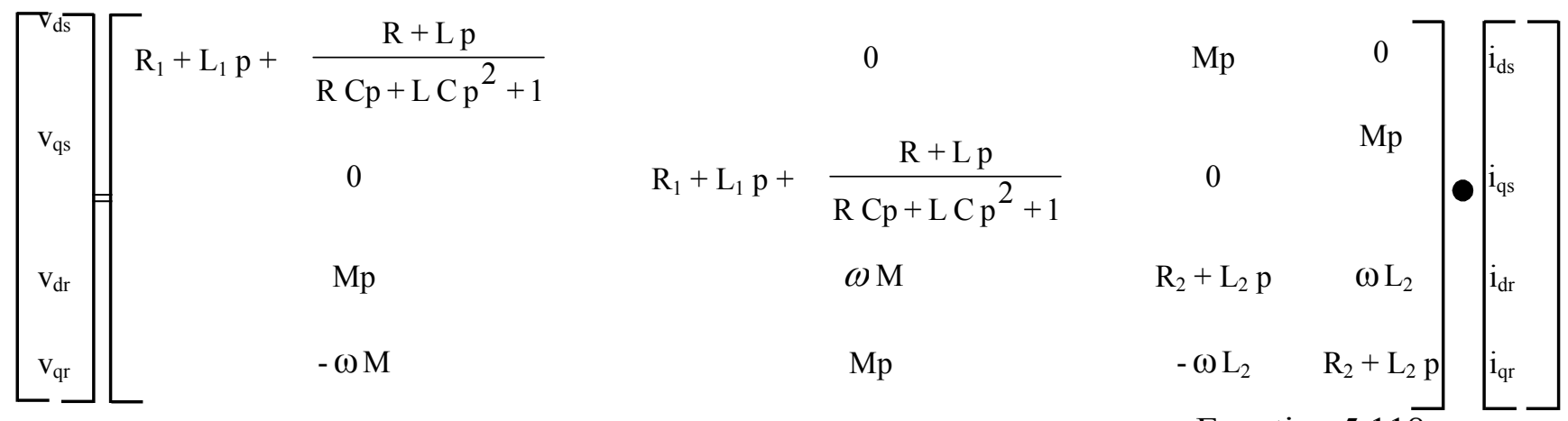

Equation $5.11 \overline{8}$

\subsection{Inverter-Based Generator Model}

The 400-kW, 500-kVA inverter-based generator consists of a high-speed AC generator driven by a 440-kW twin-spool gas turbine engine. Figure 98 is a simplified one-line diagram of the $400-\mathrm{kW}, 60-\mathrm{Hz}$ generator and inverter.

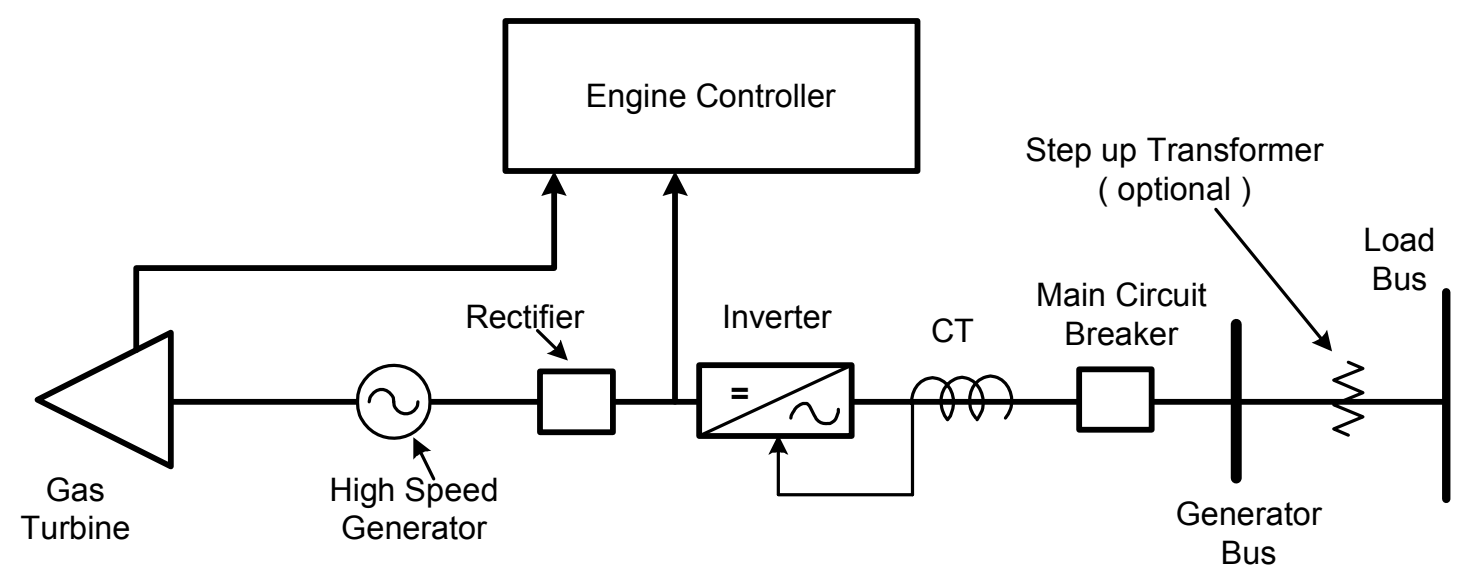

Figure 98. One-line diagram of 400-kW inverter-based generator and prime mover 
The unit has seamless transfer capability so it can operate in a current-source mode parallel to the electric power system with only $10 \%$ load and switch without outage to deliver power in the voltage-source mode to an islanded load. This generating unit has a continuous overload capability of $110 \%$ of rated load at any power factor from -0.8 to 0.8 , and it can follow load steps of $25 \%$ up or down while maintaining frequency to less than $+/-0.1 \mathrm{~Hz}$. The voltage distortion is less than $2 \%$, and it can handle $100 \%$ load imbalance. During short circuits, it can deliver $200 \%$ of rated current for about 8 seconds.

The inverter model is represented by the voltage pullback curves in Figure 99. The unit can deliver 1.10 p.u. current $(602 \mathrm{~A} \mathrm{rms})$ at 1.0 p.u. rated voltage $(277 \mathrm{~V} \mathrm{rms})$ and 2.0 p.u. current at 0.56 p.u. voltage. The inverse time-current characteristic is given in Figure 100.

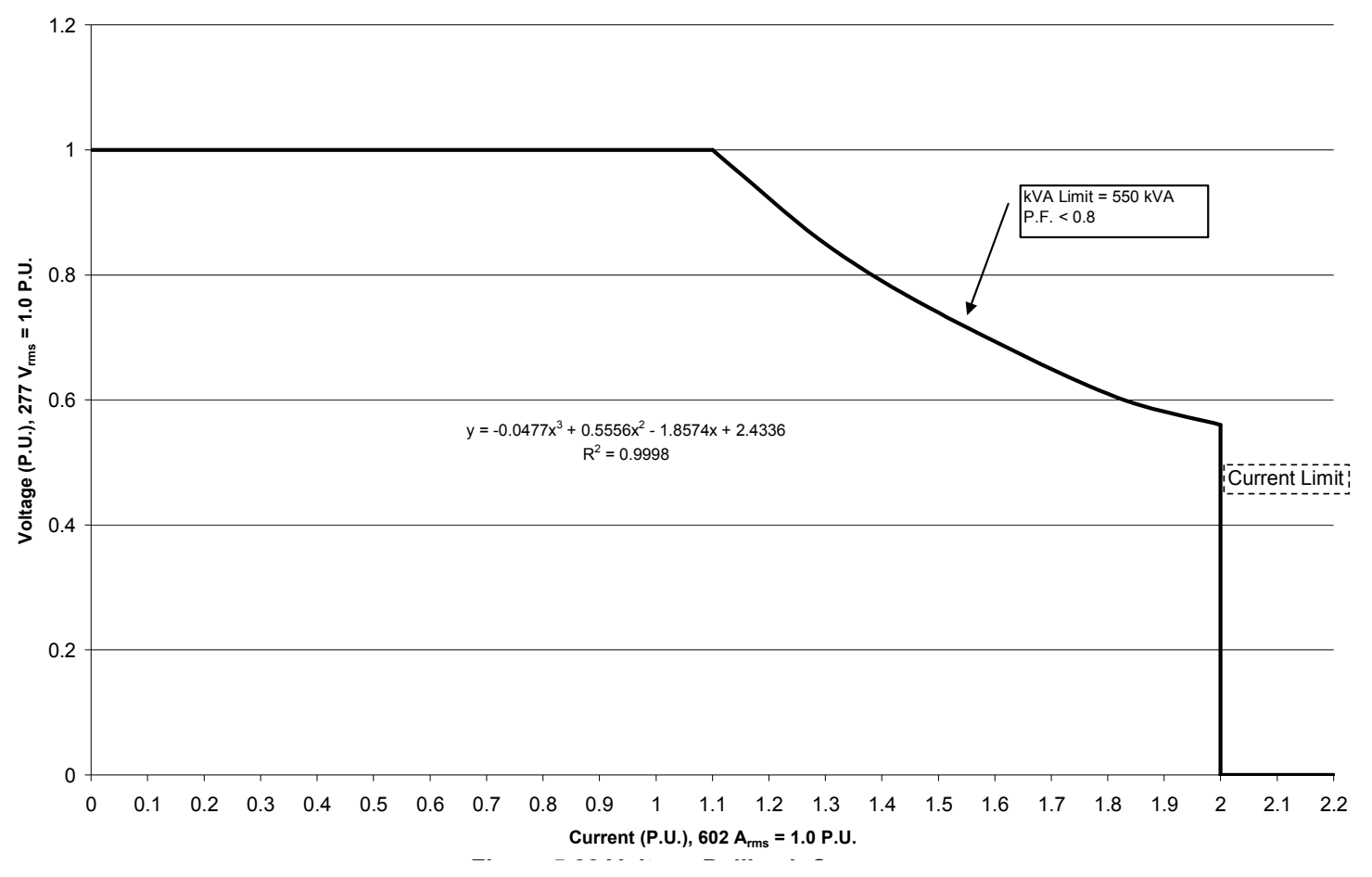

Figure 99. Voltage pullback curves 


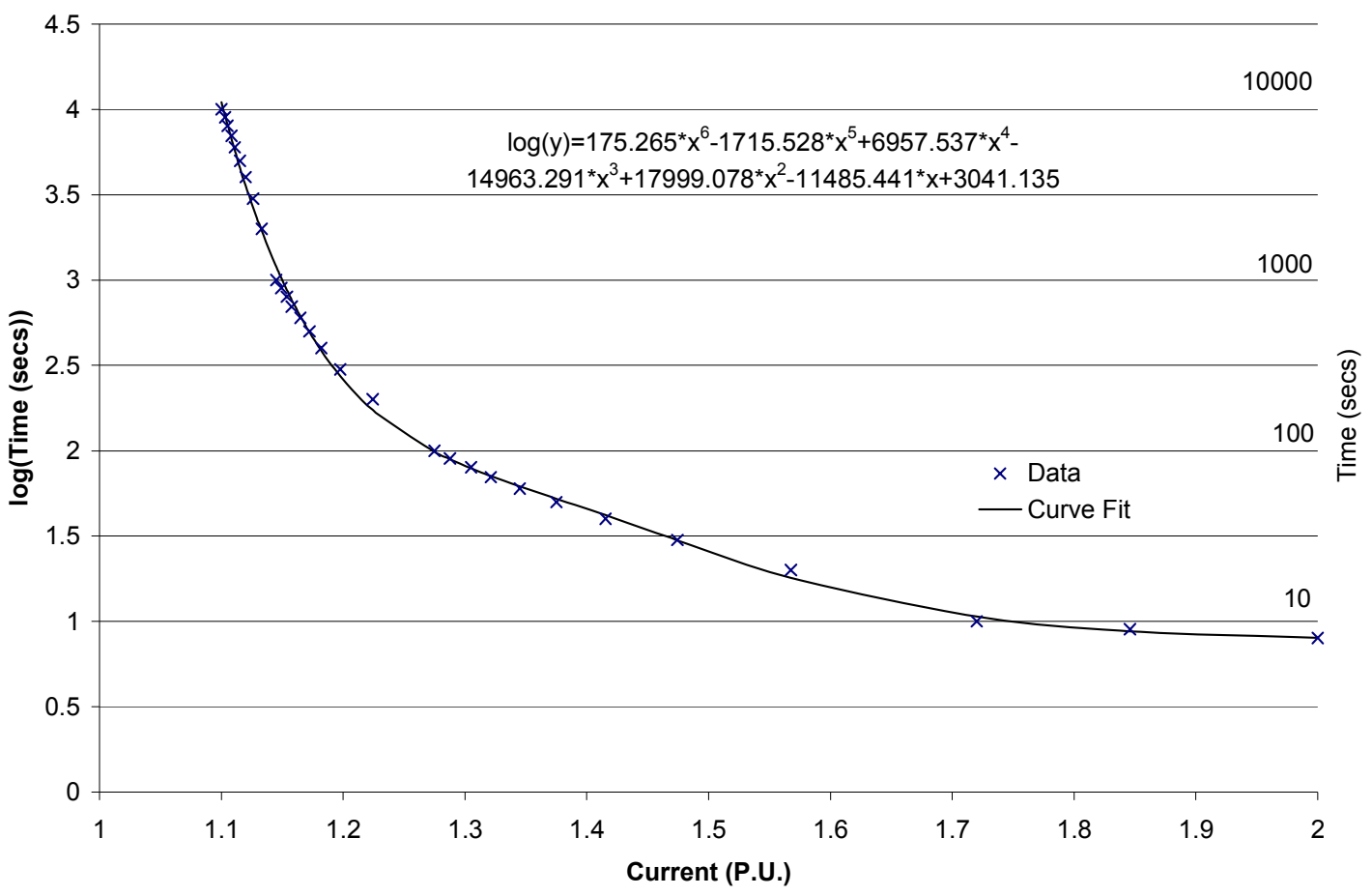

Figure 100. Inverse time-current characteristic 


\section{Project Results - Circuit Voltage Profiles Under Different Loading Conditions}

\subsection{Introduction}

This section summarizes work to perform simulations to determine the circuit voltage profiles of Milford Circuit DC 8103. The two-transformer Milford substation, shown in Figure 101, is fed from two 41.57-kV tie lines and one 41.57-kV trunk line. The Milford DC 8103 circuit is fed by a delta-wye-connected 10-MVA LTC transformer with $\pm 10 \%$ tap positions and 0 to $\pm 5 \%$ fixed taps. The primary voltage is $41.57 \mathrm{kV}$, and the secondary voltage is $13.8 \mathrm{kV}$. The circuit is a $13.8-\mathrm{kV}$, multi-grounded, three-phase wye system, and it serves approximately $76.2 \%$ residential, $4 \%$ commercial, and $19.8 \%$ light industrial loads. See Table 17. The circuit summer peak maximum load is $15.3 \mathrm{MVA}$, and the summer peak minimum load is 5.91 MVA. The annual load factor for the circuit is 0.42 .

The circuit is about $31,000 \mathrm{ft}$, or $5.9 \mathrm{mi}$. The voltage profiles are determined for the peak load day, referred to as the heavy load (HL) condition, and the minimum load day, referred to as the LL condition, using eight traditional methods of voltage regulation. The voltage profiles are shown for the following voltage regulation cases:

- No circuit voltage regulation, with variable transformer primary voltages $(41.57 \mathrm{kV})$ ranging from $87 \%, 95 \%, 100 \%$, and $105 \%$ voltage levels on a $120-\mathrm{V}$ base (The $87 \%$ voltage level represents the lowest voltage for emergency operation, and the $100 \%$ voltage level represents the base case for determining the release capacity. The balance of the simulations represents normal operating conditions for HL and LL conditions with $95 \%$ and $105 \%$ primary substation voltages.)

- The addition of LTC transformer regulation at the substation

- The addition of step VR 1

- The addition of step VR 1 and VR 2

- The addition of capacitor CAP 1

- The addition of capacitors CAP 1 and CAP 2

- $\quad$ The addition of capacitors CAP 1, CAP 2, and CAP 3

- The addition of all traditional voltage regulation methods (i.e., LTC, VR 1, VR 2, CAP 1, CAP 2, and CAP 3). 
Table 17. Circuit Customer Load Characteristics

\begin{tabular}{|c|c|c|c|c|c|c|c|c|}
\hline $\begin{array}{c}\text { Customer } \\
\text { Type }\end{array}$ & $\begin{array}{l}\text { Number of } \\
\text { Customers }\end{array}$ & $\begin{array}{c}\text { Annual } \\
\text { MWH }\end{array}$ & $\begin{array}{l}\text { MW } \\
\text { Avg. }\end{array}$ & $\begin{array}{c}\text { Annual } \\
\text { Load } \\
\text { Factor } \\
\text { Typ. }\end{array}$ & $\begin{array}{l}\text { MW } \\
\text { Peak }\end{array}$ & P.F. & $\begin{array}{l}\text { MVA } \\
\text { Peak }\end{array}$ & $\begin{array}{c}\% \text { on an } \\
\text { MVA } \\
\text { Basis }\end{array}$ \\
\hline Residential & 3178 & 32,610 & 3.72 & 0.35 & 10.6 & 0.91 & 11.7 & 76.3 \\
\hline Commercial & 120 & 2,667 & 0.304 & 0.57 & 0.54 & 0.89 & 0.60 & 3.9 \\
\hline Industrial & 3 & 15,529 & 1.77 & 0.66 & 2.67 & 0.88 & 3.04 & 19.8 \\
\hline Totals & 3301 & 50,806 & 5.79 & 0.42 & 13.8 & .90 & 15.3 & 100.0 \\
\hline
\end{tabular}

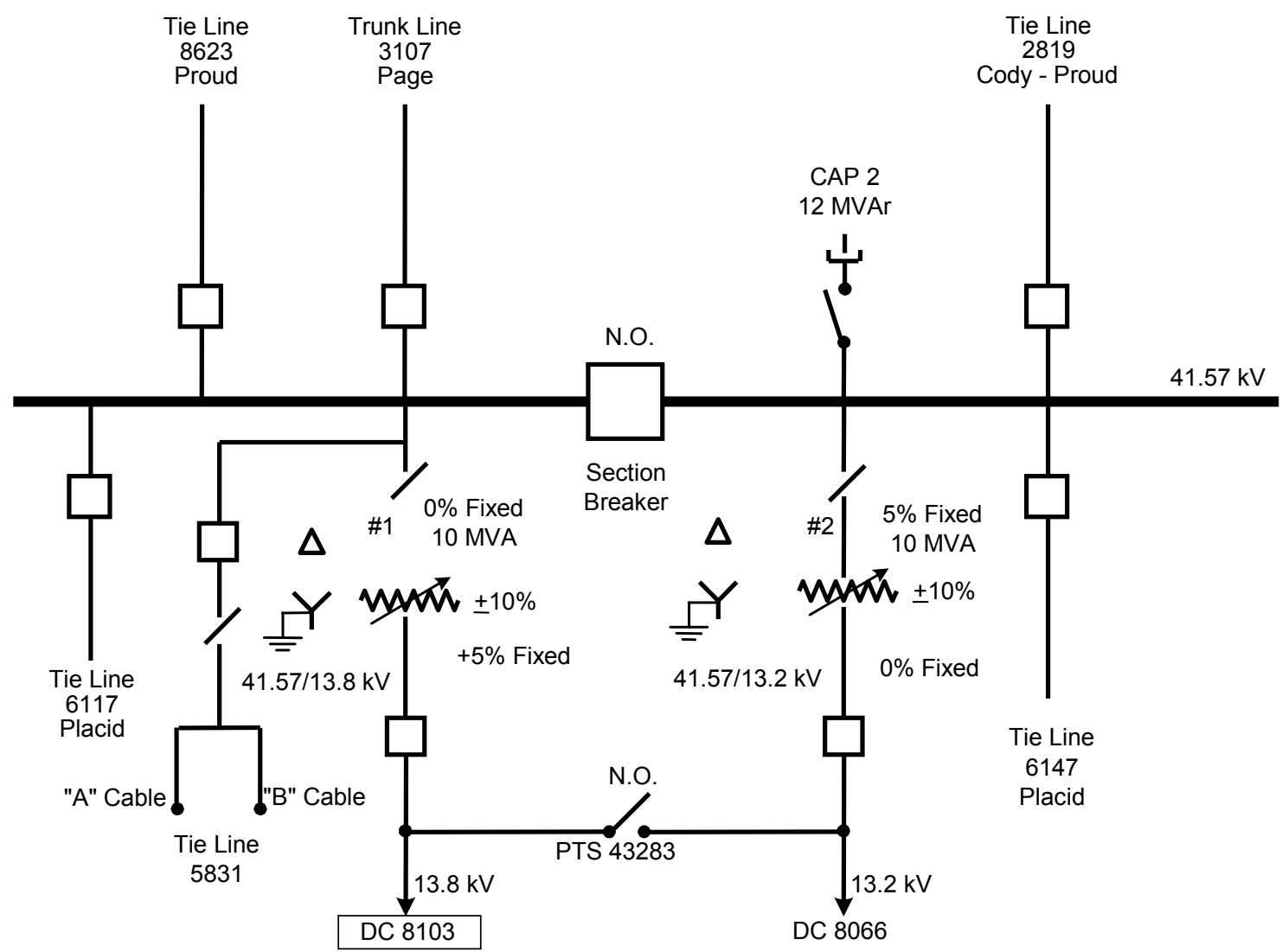

Figure 101. Milford Substation one-line diagram 
The Milford Circuit DC 8103 and substation are shown in Figure 102. It consists of a substation, shown as a cross-hatched square and denoted as Node 0; three-phase sections, represented as heavy-weight solid lines; and single-phase sections, represented as lightweight solid lines. The dotted lines represent two phases. The 10-MVA LTC transformer at the substation has an impedance of $7.02 \%$, with $0, \pm 2.5 \%$, and $\pm 5 \%$ fixed taps with highside and low-side settings of 0 and $\pm 5 \%$. The LTC transformer is $\pm 10 \%$ with \pm 16 steps. The voltage was regulated at $126.0 \mathrm{~V}$ on the secondary bus of the transformer. The circuit has three single-phase step bidirectional VRs (1) and three single-phase step bidirectional VRs (2). These single-phase regulators are each rated $167 \mathrm{kVA}$ and connected wye. The second VR (2) is for study purposes. There are three capacitor banks. CAP 1 is a threephase, $900-\mathrm{kVAr}$ capacity; CAP 2 is a $900-\mathrm{kVAr}$ capacity; and CAP 3 is three-phase, 1200-kVAr capacity. All capacitors are pole-mounted, switched capacitors with wyegrounded connections. 


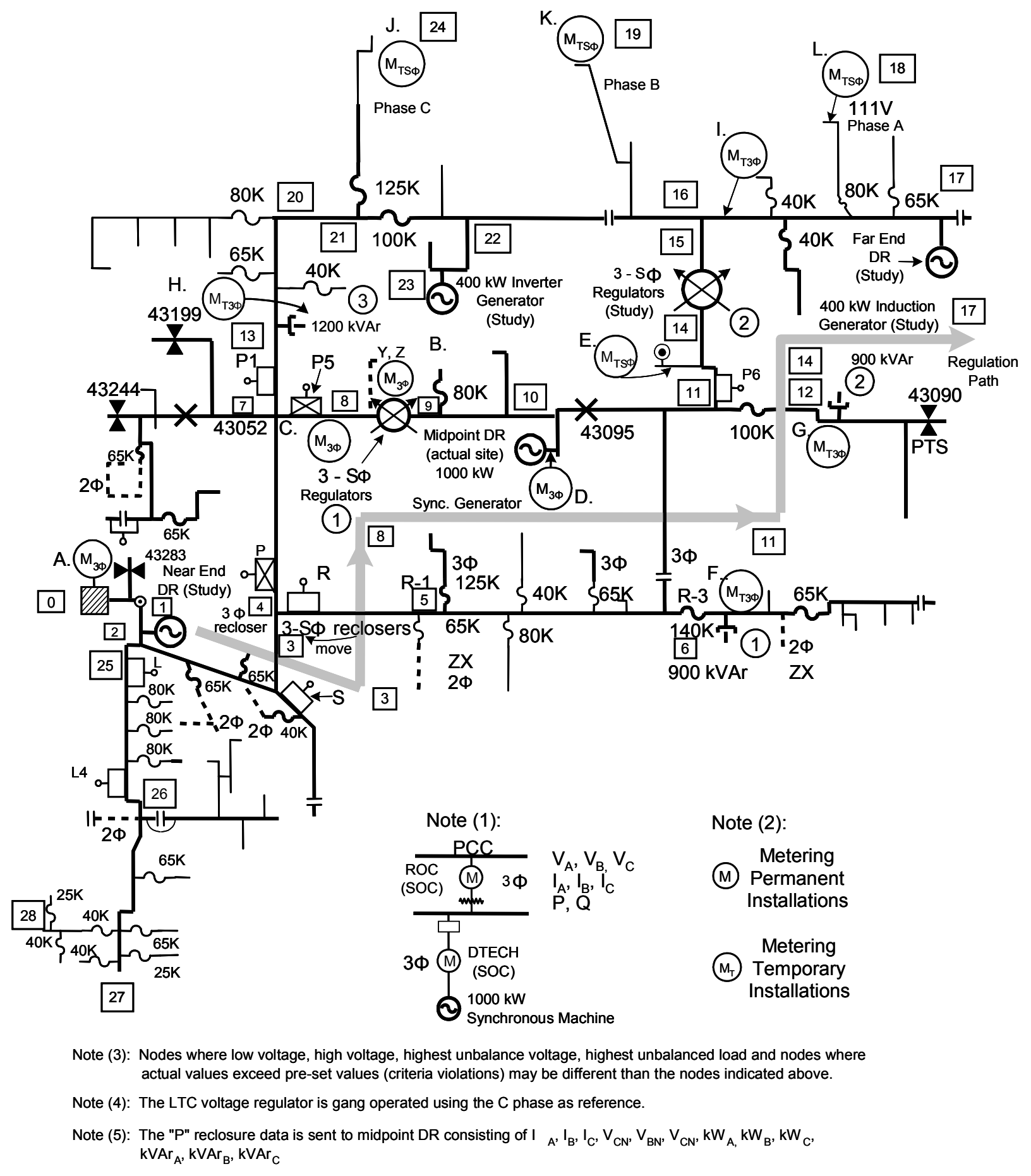

Figure 102. Milford Circuit DC 8103 
A number of circuit protection devices are shown on the circuit. These include a three-phase

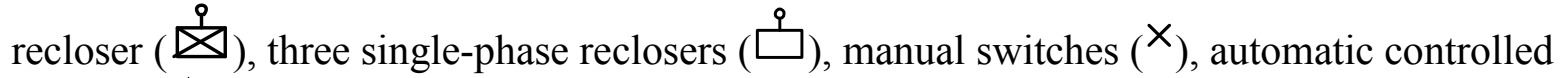

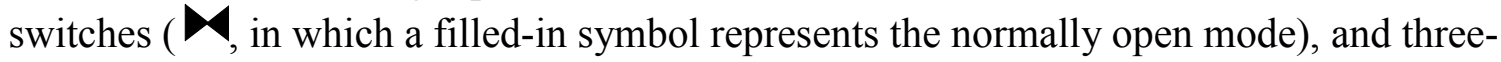
phase and single-phase fuses ( $\sim \sim$ ), which are identified with a number size and Type K.

Finally, there are three DR generators, a 1,000-kW synchronous generator at the midpoint denoted as Node [10], a 400-kW induction generator at Node [17] for study purposes, and, at the tag end of the circuit, and a 400-kW inverter-based generator at Node [23] for study purposes. The generation simulations and their resultant voltage profiles with and without traditional voltage regulation methods are provided in Section 10.

As noted earlier, nodes are placed throughout the circuit. These are points at which the real and reactive power phase quantities, phase power factors, phase currents, and phase voltages are calculated. Nodes are placed on each side of the step VRs to provide calculated values of voltage before and after regulation and at each capacitor location to provide the calculated values of voltage.

Table 18 provides the permanent metering points and quantities measured and the temporary metering points and quantities measured. All metering points are designated by a letter (e.g., $\mathrm{A}, \mathrm{B}$, and C) that corresponds to the circuit one-line diagram of Figure 102. Those shown with the letter $\mathrm{T}$ are temporarily installed for this project. Also, the metering may be three-

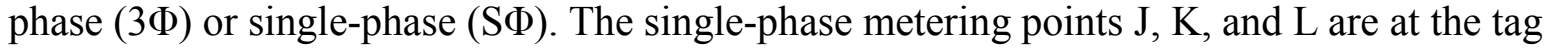
ends of the circuit. These measured quantities and the three-phase quantities were compared to the calculated values determined from the simulations, and adjustments were made to the circuit models and loads to ensure the simulations agree with field measurements. 
Table 18. Measurement Locations and Data Collection

\begin{tabular}{|c|c|c|c|c|c|}
\hline \multicolumn{6}{|c|}{ Permanent Metering Locations } \\
\hline A. & Substation & $\mathrm{M}_{3 \Phi}$ & $\mathrm{V}_{\mathrm{AN}}, \mathrm{V}_{\mathrm{BN}}, \mathrm{V}_{\mathrm{CN}}$ & $I_{A}, I_{B}, I_{C}$ & P, Q, P.F. \\
\hline B. & 3 SФ Bidirectional regulators & $M_{3 \Phi}$ & $\mathrm{V}_{\mathrm{AN}}, \mathrm{V}_{\mathrm{BN}}, \mathrm{V}_{\mathrm{CN}}$ & $\mathrm{I}_{\mathrm{A}}, \mathrm{I}_{\mathrm{B}}, \mathrm{I}_{\mathrm{C}}$ & P, Q, P.F. \\
\hline C. & $3 S \Phi$ reclosures & $\mathrm{M}_{3 \Phi}$ & $\mathrm{V}_{\mathrm{AN}}, \mathrm{V}_{\mathrm{BN}}, \mathrm{V}_{\mathrm{CN}}$ & $I_{A}, I_{B}, I_{C}$ & P, Q, P.F. \\
\hline D. & 1,000-kW Synchronous DR & $M_{3 \Phi}$ & $\mathrm{V}_{\mathrm{AN}}, \mathrm{V}_{\mathrm{BN}}, \mathrm{V}_{\mathrm{CN}}$ & $\mathrm{I}_{\mathrm{A}}, \mathrm{I}_{\mathrm{B}}, \mathrm{I}_{\mathrm{C}}$ & P, Q, P.F. \\
\hline \multicolumn{6}{|c|}{ Temporary Metering Locations } \\
\hline E. & $\begin{array}{l}\text { Customer } \\
\text { (LV unbalanced voltage) }\end{array}$ & $\mathrm{M}_{\mathrm{TS} \Phi}$ & $\mathrm{V}_{\mathrm{AN}}$ & $\mathrm{I}_{\mathrm{A}}$ & P, Q, P.F. \\
\hline $\mathrm{F}$. & 900-kVAr capacitor & $\mathrm{M}_{\mathrm{T} 3 \Phi}$ & $\mathrm{V}_{\mathrm{AN}}, \mathrm{V}_{\mathrm{BN}}, \mathrm{V}_{\mathrm{CN}}$ & $I_{A}, I_{B}, I_{C}$ & P, Q, P.F. \\
\hline G. & 900-kVAr capacitor & $\mathrm{M}_{\mathrm{T} 3 \Phi}$ & $\mathrm{V}_{\mathrm{AN}}, \mathrm{V}_{\mathrm{BN}}, \mathrm{V}_{\mathrm{CN}}$ & $\mathrm{I}_{\mathrm{A}}, \mathrm{I}_{\mathrm{B}}, \mathrm{I}_{\mathrm{C}}$ & P, Q, P.F. \\
\hline $\mathrm{H}$. & 1,200-kVAr capacitor & $\mathrm{M}_{\mathrm{T} \ \Phi}$ & $\mathrm{V}_{\mathrm{AN}}, \mathrm{V}_{\mathrm{BN}}, \mathrm{V}_{\mathrm{CN}}$ & $I_{A}, I_{B}, I_{C}$ & P, Q, P.F. \\
\hline I. & $\begin{array}{l}3 \Phi \text { circuit tag end } \\
\text { (LV unbalanced voltage) }\end{array}$ & $\mathrm{M}_{\mathrm{T} 3 \Phi}$ & $\mathrm{V}_{\mathrm{AN}}, \mathrm{V}_{\mathrm{BN}}, \mathrm{V}_{\mathrm{CN}}$ & $\mathrm{I}_{\mathrm{A}}, \mathrm{I}_{\mathrm{B}}, \mathrm{I}_{\mathrm{C}}$ & P, Q, P.F. \\
\hline J.K.L. & Single-phase tag ends & $\mathrm{M}_{\mathrm{TS} \Phi}$ & $\mathrm{V}_{\mathrm{CN}}$ & $\mathrm{V}_{\mathrm{BN}}$ & $\mathrm{V}_{\mathrm{AN}}$ \\
\hline
\end{tabular}

\subsection{Circuit Simulations and Voltage Profiles}

The simulations were extensive. To simplify the explanation of how they were conducted, Table 19 describes the tests run. These tests were organized into four groups. The first group consisted of tests $1-8$, to which traditional voltage regulation (i.e., LTCs) was applied. The second group consisted of tests 9-11, to which DG voltage regulation strategy tests were applied. The third group consisted of tests $12-17$, to which the combined traditional LTC and circuit regulation tests as well as DG regulation strategy tests were applied. The fourth group consisted of all traditional voltage regulation and all generation voltage regulation strategies (see tests 18-19). 
Table 19. Matrix of Voltage Regulation Simulations

\begin{tabular}{|c|c|c|c|c|c|c|c|c|c|c|c|c|c|}
\hline \multirow{2}{*}{ 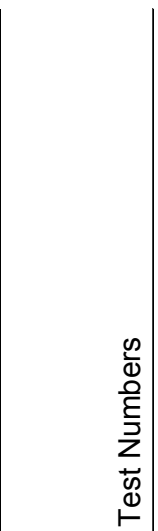 } & \multirow{2}{*}{ 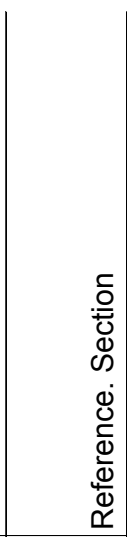 } & \multirow{2}{*}{$\begin{array}{l}\text { Primary } \\
\text { Voltage } \\
\text { Spread } \\
\text { (a) } 87 \% \\
\text { (b) } 92 \% \\
\text { (c) } 93 \% \\
\text { (d) } 95 \% \\
\text { (e) } 98 \% \\
\text { (f) } 105 \% \\
\end{array}$} & \multirow[t]{2}{*}{ 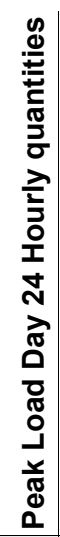 } & \multirow{2}{*}{ 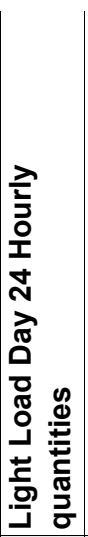 } & \multirow{2}{*}{ 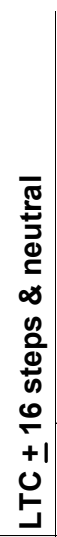 } & \multicolumn{2}{|c|}{$\begin{array}{c}\text { Line } \\
\text { Regulator } \\
\end{array}$} & \multicolumn{3}{|c|}{ Capacitors } & \multicolumn{3}{|c|}{$\begin{array}{c}\text { DR (Synchronous Machine) } \\
\text { Locations (1) } \\
\text { (a)Peak shave, P fixed } \\
\text { (b) P.F.= Unity, P variable } \\
\text { (c) } \pm \text { P.F. Constant, Variable P \& } \\
Q \\
\text { (d) } \pm \text { Q (Vars Volt reg) P Min } \\
\text { (e) P } \pm \text { jQ (Optimizing) } \\
\text { (f) Fixed P.F. Anti-island }\end{array}$} \\
\hline & & & & & & \#1 & \#2 & \#1 & \#2 & \#3 & Near End & $\begin{array}{l}\text { Midpoint } \\
\text { (Actual } \\
\text { Site) }\end{array}$ & Far End \\
\hline 1 & I.A. & (a) (d) (f) & $x$ & $\mathrm{x}$ & & & & & & & & & \\
\hline 2 & I.B. & (d) (f) & $x$ & $x$ & $x$ & & & & & & & & \\
\hline 3 & I.C. & (d) (f) & $x$ & $x$ & $x$ & $x$ & & & & & & & \\
\hline 4 & I.C'. & (d) (f) & $\mathrm{x}$ & $\mathrm{x}$ & $\mathrm{x}$ & $\mathrm{x}$ & $\mathrm{x}$ & & & & & & \\
\hline 5 & I.D & (d) (f) & $x$ & $x$ & $x$ & & & $x$ & & & & & \\
\hline 6 & I.D ' & (d) (f) & $x$ & $x$ & $\mathrm{x}$ & & & $\mathrm{x}$ & $x$ & & & & \\
\hline 7 & I.D." & (d) (f) & $x$ & $x$ & $\mathrm{x}$ & & & $x$ & $x$ & $x$ & & & \\
\hline 8 & I.E. & (d) (f) & $x$ & $x$ & $x$ & $x$ & $x$ & $x$ & $x$ & $x$ & & & \\
\hline 9 & II.A. & $\mathrm{x}$ & $\mathrm{x}$ & $\mathrm{x}$ & & & & & & & (b) (d) (e) & & \\
\hline 10 & II.B. & $x$ & $x$ & $x$ & & & & & & & & (b) (d) (e) & \\
\hline 11 & II.C. & $\mathrm{x}$ & $\mathrm{x}$ & $\mathrm{x}$ & & & & & & & & & (b) (d) (e) \\
\hline 12 & II.D. & \multicolumn{6}{|c|}{\begin{tabular}{|l|l|} 
Repeat IIA., IIB., IIC. turn on LTC & \\
\end{tabular}} & & & & & & \\
\hline 13 & II.E. & \multicolumn{9}{|c|}{ Repeat IIA., IIB., IIC. turn on LTC, and Regulator (1) } & & & \\
\hline 14 & II.F. & \multicolumn{10}{|c|}{ Repeat IIA., IIB., IIC. turn on LTC, and Regulator (1) and (2) } & & \\
\hline 15 & II.G. & \multicolumn{11}{|c|}{ Repeat IIA., IIB., IIC. turn on LTC, and Regulator (1) and (2) and Cap. (1) } & \\
\hline 16 & II.H. & \multicolumn{11}{|c|}{ Repeat IIA., IIB., IIC. turn on LTC, and Regulator (1) and (2) and Cap. (1), (2) } & \\
\hline 17 & II.I. & \multicolumn{12}{|c|}{ Repeat IIA., IIB., IIC. turn on LTC, and Regulator (1) and (2) and Cap. (1), (2), (3) } \\
\hline \multicolumn{14}{|c|}{ (9) } \\
\hline \multicolumn{14}{|l|}{19} \\
\hline \multicolumn{14}{|l|}{20} \\
\hline \multicolumn{14}{|l|}{21} \\
\hline \multicolumn{14}{|l|}{22} \\
\hline \multicolumn{14}{|l|}{23} \\
\hline \multicolumn{14}{|l|}{24} \\
\hline \multicolumn{14}{|l|}{25} \\
\hline & & & & & & & & & & & & & \\
\hline \multicolumn{14}{|c|}{ Note (1): DR Control Strategies } \\
\hline & \multicolumn{13}{|c|}{$\begin{array}{l}\text { (a.) The DR is operated at fixed real power output and normally Is scheduled to run during peak periods when } \\
\text { the cost of DR generation is lower than host utility cost. The Q is zero at the PCC. } 984 \mathrm{~kW} \text { rated }\end{array}$} \\
\hline & (b.) The & $\mathrm{R}$ is operat & $\mathrm{d}$ at $u$ & unity P.F & with & varia & le pow & er ou & put. & $492 \mathrm{kl}$ & $V(50 \%)$ to 1 & $050 \mathrm{~kW} \mathrm{ma}$ & \\
\hline & $\begin{array}{l}\text { (c.) DR is } \\
\text { not violat }\end{array}$ & $\begin{array}{l}\text { operated at } \\
\text { e voltage cri }\end{array}$ & $\begin{array}{l}\text { a fixe } \\
\text { aria. }\end{array}$ & d powe & facto & or eith & rlead & or lag & & xampl & \pm 0.8 P.F.) & with variable & $P$ and $Q$ to \\
\hline & $\begin{array}{l}\text { (d.) The } \\
\text { voltage. }\end{array}$ & $\begin{array}{l}\mathrm{R} \text { is operat } \\
246 \mathrm{~kW}(25\end{array}$ & $\begin{array}{l}\mathrm{d} \text { as } \\
\text { of ra }\end{array}$ & $\begin{array}{l}\text { a synch } \\
\text { ated) }\end{array}$ & onou & is con & enser & with $r$ & inim & m wa & ts and variab & ble VArs to $r$ & gulate \\
\hline & $\begin{array}{l}\text { (e.) DR is } \\
\text { real losse }\end{array}$ & $\begin{array}{l}\text { operated w } \\
\text { s, minimize }\end{array}$ & e vari & $\begin{array}{l}\text { iable } P \\
\text { ve loss }\end{array}$ & $\begin{array}{l}\text { and } \mathrm{C} \\
\mathrm{s}, \mathrm{reg}\end{array}$ & $\begin{array}{l}\text { and } \\
\text { gulate }\end{array}$ & $\begin{array}{l}\text { used } \\
\text { oltage }\end{array}$ & $\begin{array}{l}\text { to op } \\
\text { and }\end{array}$ & $\begin{array}{l}\text { imize } \\
\text { naxin }\end{array}$ & $\begin{array}{l}\text { to a s } \\
\text { iize re }\end{array}$ & $\begin{array}{l}\text { pecific set of } \\
\text { leased capac }\end{array}$ & $\begin{array}{l}\text { f criteria such } \\
\text { city. }\end{array}$ & as minimize \\
\hline
\end{tabular}




\begin{tabular}{|c|c|c|c|c|c|c|c|c|c|c|c|c|c|}
\hline \multirow{2}{*}{ 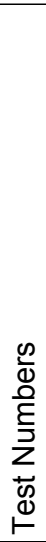 } & \multirow{2}{*}{ 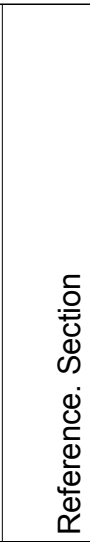 } & \multirow{2}{*}{$\begin{array}{l}\text { Primary } \\
\text { Voltage } \\
\text { Spread } \\
\text { (a) } 87 \% \\
\text { (b) } 92 \% \\
\text { (c) } 93 \% \\
\text { (d) } 95 \% \\
\text { (e) } 98 \% \\
\text { (f) } 105 \%\end{array}$} & \multirow[t]{2}{*}{ 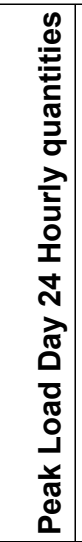 } & \multirow{2}{*}{ 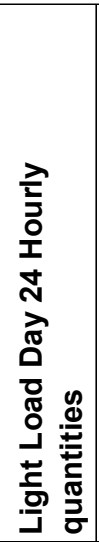 } & \multirow{2}{*}{ 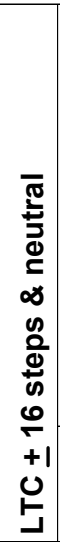 } & \multicolumn{2}{|c|}{$\begin{array}{c}\text { Line } \\
\text { Regulator }\end{array}$} & \multicolumn{3}{|c|}{ Capacitors } & \multicolumn{3}{|c|}{$\begin{array}{l}\text { DR (H.S. Generator and Inverter) } \\
\text { Locations } \\
\text { (a)Peak shave, P fixed } \\
\text { (b) P.F.= Unity, P variable } \\
\text { (c) } \pm \text { P.F. Constant, Variable P } \\
\text { (d) } \pm Q \text { (Vars Volt Reg) P }=0 \\
\text { (e) } P \pm \text { jQ (Optimizing) } \\
\text { (f) Frequency Dithering Anti-Island }\end{array}$} \\
\hline & & & & & & $\# 1$ & \#2 & \#1 & \#2 & \#3 & Near End & $\begin{array}{l}\text { Midpoint } \\
\text { (Actual } \\
\text { Site) } \\
\end{array}$ & Far End \\
\hline 1 & & (a) (d) (f) & & & & & & & & & & & \\
\hline 2 & & (d) (f) & & & & & & & & & & & \\
\hline 3 & & (d) (f) & & & & & & & & & & & \\
\hline 4 & & (d) (f) & & & & & & & & & & & \\
\hline 5 & & (d) (f) & & & & & & & & & & & \\
\hline 6 & & (d) (f) & & & & & & & & & & & \\
\hline 7 & & (d) (f) & & & & & & & & & & & \\
\hline 8 & & (d) $(f)$ & & & & & & & & & & & \\
\hline 9 & II.A. & $\mathrm{x}$ & $x$ & $x$ & & & & & & & (b) (d) (e) & & \\
\hline 10 & II.B. & $x$ & $x$ & $x$ & & & & & & & & (b) (d) (e) & \\
\hline 11 & II.C. & $\mathrm{x}$ & $\mathrm{x}$ & $\mathrm{x}$ & & & & & & & & & (b) (d) (e) \\
\hline 12 & II.D. & \multicolumn{6}{|c|}{\begin{tabular}{|l|l|} 
Repeat II.A., II.B., II.C. turn on LTC & \\
\end{tabular}} & & & & & & \\
\hline 13 & II.E. & \multicolumn{9}{|c|}{\begin{tabular}{|l|l|} 
Repeat II.A., II.B., II.C. turn on LTC and Regulator (1) & \\
\end{tabular}} & & & \\
\hline 14 & II.F. & \multicolumn{10}{|c|}{ Repeat II.A., II.B., II.C. turn on LTC and Regulator (1) and (2) } & & \\
\hline 15 & II.G. & \multicolumn{11}{|c|}{ Repeat II.A., II.B., II.C. turn on LTC and Regulator (1) and (2) and Cap. (1) } & \\
\hline 16 & II.H. & \multicolumn{11}{|c|}{ Repeat II.A., II.B., II.C. turn on LTC and Regulator (1) and (2) and Cap. (1) and (2) } & \\
\hline 17 & II.I. & \multicolumn{12}{|c|}{ Repeat II.A., II.B., II.C. turn on LTC and Regulator (1) and (2) and Cap. (1), (2) and (3) } \\
\hline 18 & & & & & & & & & & & & & \\
\hline 19 & & & & & & & & & & & & & \\
\hline 20 & & & & & & & & & & & & & \\
\hline 21 & & & & & & & & & & & & & \\
\hline 22 & & & & & & & & & & & & & \\
\hline 23 & & & & & & & & & & & & & \\
\hline 24 & & & & & & & & & & & & & \\
\hline 25 & & & & & & & & & & & & & \\
\hline 26 & & & & & & & & & & & & & \\
\hline 27 & & & & & & & & & & & & & \\
\hline 28 & & & & & & & & & & & & & \\
\hline 29 & & & & & & & & & & & & & \\
\hline
\end{tabular}




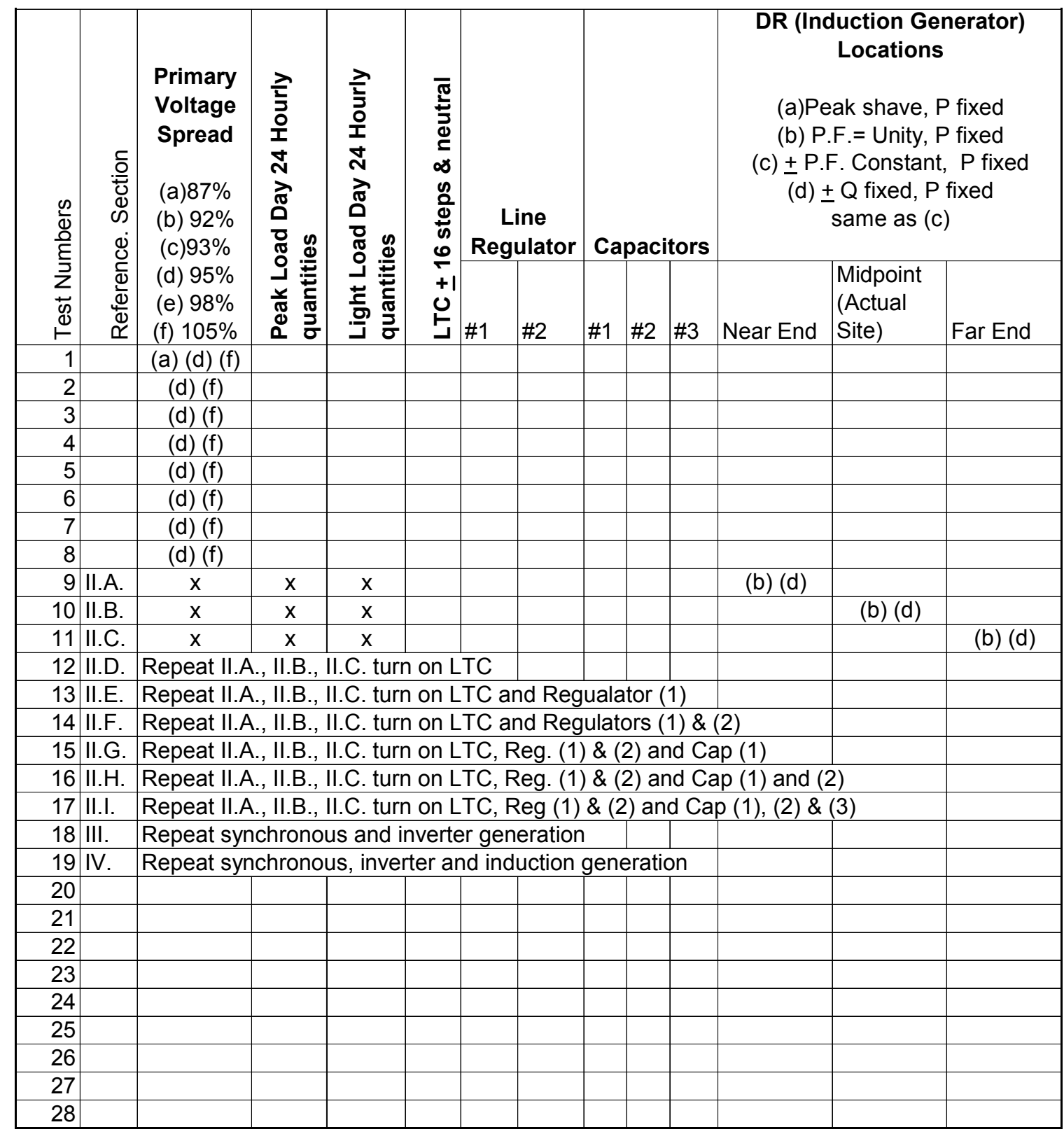

The first step of properly setting up the simulations was to ensure the simulated data matched the actual circuit conditions. Two conditions were modeled: the peak load day and the light load day. The Milford Circuit DC 8103 had a maximum load of 15.3 MVA on the peak day and a minimum load of 5.91 MVA on the peak day. 
To verify the accuracy of the model, simulation results for a given time were compared with recorded data. The modeled circuit was placed in a state closely resembling the conditions of the actual system when the measurement was made; specifically, the LTC, VR 1, and capacitor banks at nodes 6 and 12 (900 kVAr each) were enabled while at 100\% primary voltage. The simulation HL base case feeder currents were $578 \mathrm{~A}, 654 \mathrm{~A}$, and 637 A for phases A, B, and C, respectively, compared with actual recorded currents of $565 \mathrm{~A}$, $651 \mathrm{~A}$, and $637 \mathrm{~A}$. The simulated megavolt-amperes of the circuit were 14.89, compared to a calculated 15.3 derived from the measured feeder currents and voltages. This was an error of $2.42 \%$.

The program employed to perform the modeling was the Distribution Engineering Workstation. With it, load can be modeled as a constant current (CC), CP (P + j Q), or VDC. At first, $\mathrm{CC}$ was selected to best represent the load characteristics of Milford DC 8103. But, as discussed later (see Appendix B.1), all three models were tested by comparing the actual phase currents at Node 0 with the simulated phase currents at Node 0 . The VDC model yielded the most accurate results, with only $2 \%$ error for Test 8 . The CC model had an error of $5.3 \%$, compared with $12.3 \%$ error for the $\mathrm{CP}$ model. The circuit regulation path shown in Figure 102 illustrates the nodes on this path from the first circuit (Node 1) to the major junction (Node 3) to the first step regulator (VR 1) to the next major junction (Node 8) and then through the second step regulator (VR 2) to the next major junction node (Node 11) and the following major junction (Node 14) and, finally, to the circuit tag end (Node 17). Voltage profiles are shown through each of these major nodes. Base case simulations were conducted for $100 \%$ voltage on the primary of the substation using only the LTC transformer regulation for both HL and LL conditions. This case was used to determine the released capacity and the real loss reduction when the $2 / 3$ s rule and $2 / 5$ s rule was applied for capacitive compensation and when the optimized size and locations were determined by the Distribution Engineering Workstation.

The following circuit voltage profiles are shown for each test (i.e., tests 1-8 with the primary substation voltage set at $95 \%$ and $105 \%$ ). The first set of profiles is for HL conditions at $95 \%$ and $105 \%$ primary voltage with different traditional voltage regulation methods applied. Simulated voltage data as a function of circuit distance in thousands of meters are given in Table 20 for the HL conditions. Simulated voltage data versus circuit length are given in Table 21 for LL. 
Table 20. Voltage Profile Data for HL Tests 1 Through 8 at $95 \%$ and $105 \%$ Substation Primary Voltage

\begin{tabular}{|c|c|c|c|c|c|c|}
\hline Distance & $\begin{array}{c}\text { Test } 8105 \\
\text { HL }\end{array}$ & $\begin{array}{c}\text { Test } 895 \\
\text { HL }\end{array}$ & $\begin{array}{c}\text { Test } 7105 \\
\text { HL }\end{array}$ & $\begin{array}{c}\text { Test } 795 \\
\text { HL }\end{array}$ & $\begin{array}{c}\text { Test } 6105 \\
\text { HL }\end{array}$ & $\begin{array}{c}\text { Test } 695 \\
\text { HL }\end{array}$ \\
\hline 0.1034 & 123.4557 & 111.3062 & 123.6329 & 111.5378 & 123.2305 & 111.1696 \\
\hline 0.2378 & 125.708 & 123.0442 & 126.1231 & 123.5816 & 125.9826 & 122.6563 \\
\hline 0.3218 & 125.6375 & 122.9755 & 126.0558 & 123.5167 & 125.9165 & 122.5933 \\
\hline 0.3218 & 125.6375 & 122.9755 & 126.0558 & 123.5167 & 125.9165 & 122.5933 \\
\hline 0.5288 & 125.5127 & 122.8549 & 125.9412 & 123.4071 & 125.784 & 122.4678 \\
\hline 0.7648 & 125.3684 & 122.7151 & 125.8093 & 123.2809 & 125.6336 & 122.3253 \\
\hline 1.3788 & 124.9925 & 122.3509 & 125.4657 & 122.9521 & 125.2414 & 121.9537 \\
\hline 2.583 & 124.2592 & 121.6403 & 124.7952 & 122.3106 & 124.4755 & 121.228 \\
\hline 2.666 & 124.2088 & 121.5915 & 124.7492 & 122.2665 & 124.4228 & 121.1781 \\
\hline 3.206 & 123.896 & 121.2882 & 124.4645 & 121.9942 & 124.095 & 120.8676 \\
\hline 3.429 & 123.7671 & 121.1633 & 124.3472 & 121.8821 & 123.9599 & 120.7396 \\
\hline 3.716 & 123.6015 & 121.0028 & 124.1965 & 121.7379 & 123.7862 & 120.5751 \\
\hline 5.754 & 122.4322 & 119.8693 & 123.1323 & 120.72 & 122.5577 & 119.4114 \\
\hline 6.798 & 121.8696 & 119.3236 & 122.6233 & 120.2335 & 121.9637 & 118.8488 \\
\hline 6.9262 & 121.8012 & 119.2573 & 122.5615 & 120.1744 & 121.8913 & 118.7803 \\
\hline 7.0603 & 121.7295 & 119.1876 & 122.4967 & 120.1123 & 121.8155 & 118.7084 \\
\hline 7.2034 & 121.6533 & 119.1135 & 122.4279 & 120.0464 & 121.7349 & 118.6319 \\
\hline 7.3784 & 121.5602 & 119.023 & 122.3438 & 119.9659 & 121.6365 & 118.5384 \\
\hline 7.7094 & 121.3851 & 118.8527 & 122.1858 & 119.8145 & 121.4511 & 118.3625 \\
\hline 7.9064 & 121.2815 & 118.7519 & 122.0923 & 119.7249 & 121.3413 & 118.2582 \\
\hline 7.9064 & 121.2815 & 118.7519 & 122.0923 & 119.7249 & 121.3413 & 118.2582 \\
\hline 8.2464 & 121.1003 & 118.5758 & 121.9286 & 119.5681 & 121.1495 & 118.0761 \\
\hline 8.4174 & 121.0092 & 118.4872 & 121.8462 & 119.4892 & 121.053 & 117.9845 \\
\hline 8.8571 & 120.7771 & 118.2615 & 121.6366 & 119.2883 & 120.8069 & 117.7508 \\
\hline 9.4411 & 120.4697 & 117.9625 & 121.3589 & 119.0223 & 120.4807 & 117.4411 \\
\hline 9.6082 & 120.3851 & 117.8803 & 121.2829 & 118.9495 & 120.3907 & 117.3557 \\
\hline 10.0174 & 120.1793 & 117.68 & 121.098 & 118.7724 & 120.1715 & 117.1476 \\
\hline 10.3374 & 120.0189 & 117.5239 & 120.9538 & 118.6343 & 120.0005 & 116.9853 \\
\hline 10.5075 & 119.9332 & 117.4406 & 120.8768 & 118.5605 & 119.9092 & 116.8986 \\
\hline 10.8129 & 119.78 & 117.2915 & 120.739 & 118.4286 & 119.7458 & 116.7434 \\
\hline 10.9501 & 119.7113 & 117.2247 & 120.6773 & 118.3695 & 119.6725 & 116.6739 \\
\hline 11.0961 & 119.6384 & 117.1537 & 120.6118 & 118.3067 & 119.5947 & 116.6 \\
\hline 11.6971 & 119.3406 & 116.8639 & 120.3442 & 118.0505 & 119.2764 & 116.2978 \\
\hline 12.0081 & 119.1878 & 116.7151 & 120.2071 & 117.9191 & 119.113 & 116.1427 \\
\hline 12.0291 & 119.1799 & 116.7074 & 120.2002 & 117.9126 & 119.1063 & 116.1363 \\
\hline 12.0292 & 119.1799 & 116.7074 & 120.2002 & 117.9126 & 119.1063 & 116.1363 \\
\hline 12.2671 & 119.0908 & 116.6202 & 120.123 & 117.8384 & 119.0306 & 116.0647 \\
\hline 12.3884 & 119.0454 & 116.5758 & 120.0836 & 117.8006 & 118.992 & 116.0282 \\
\hline 12.5244 & 118.9949 & 116.5264 & 120.0398 & 117.7586 & 118.9492 & 115.9876 \\
\hline 12.6619 & 123.5833 & 121.719 & 119.6677 & 117.4023 & 118.5847 & 115.6436 \\
\hline 12.7589 & 123.5494 & 121.6858 & 119.6369 & 117.3728 & 118.5546 & 115.6152 \\
\hline 12.9495 & 123.4828 & 121.6208 & 119.5766 & 117.3149 & 118.4955 & 115.5593 \\
\hline 13.0549 & 123.4461 & 121.5849 & 119.5434 & 117.283 & 118.4629 & 115.5284 \\
\hline
\end{tabular}




\begin{tabular}{|c|c|c|c|c|c|c|}
\hline Distance & $\begin{array}{c}\text { Test } 8105 \\
\text { HL }\end{array}$ & $\begin{array}{c}\text { Test } 895 \\
\text { HL }\end{array}$ & $\begin{array}{c}\text { Test } 7105 \\
\text { HL }\end{array}$ & $\begin{array}{c}\text { Test } 795 \\
\text { HL }\end{array}$ & $\begin{array}{c}\text { Test } 6105 \\
\text { HL }\end{array}$ & $\begin{array}{c}\text { Test } 695 \\
\text { HL }\end{array}$ \\
\hline 13.3859 & 123.3272 & 121.4686 & 119.4355 & 117.1794 & 118.3572 & 115.4284 \\
\hline 13.6541 & 123.2372 & 121.3807 & 119.3541 & 117.1013 & 118.2775 & 115.3529 \\
\hline 13.9121 & 123.1493 & 121.2948 & 119.2746 & 117.025 & 118.1997 & 115.2793 \\
\hline 14.1148 & 123.0811 & 121.2281 & 119.2129 & 116.9659 & 118.1393 & 115.2221 \\
\hline 14.5219 & 122.9253 & 121.0757 & 119.0711 & 116.8297 & 118.0004 & 115.0905 \\
\hline 15.0699 & 122.7163 & 120.8712 & 118.8808 & 116.647 & 117.814 & 114.9139 \\
\hline 15.2599 & 122.644 & 120.8006 & 118.8151 & 116.5839 & 117.7496 & 114.8529 \\
\hline 15.4019 & 122.5915 & 120.7492 & 118.7674 & 116.538 & 117.7029 & 114.8087 \\
\hline 15.5403 & 122.5407 & 120.6994 & 118.7212 & 116.4937 & 117.6576 & 114.7658 \\
\hline 15.8453 & 122.4272 & 120.5885 & 118.618 & 116.3947 & 117.5566 & 114.6701 \\
\hline 16.1153 & 122.3263 & 120.4897 & 118.5262 & 116.3065 & 117.4666 & 114.5849 \\
\hline 17.4453 & 121.8485 & 120.0221 & 118.0925 & 115.8901 & 117.0417 & 114.1825 \\
\hline 17.6035 & 121.7941 & 119.9687 & 118.0429 & 115.8424 & 116.9931 & 114.1362 \\
\hline 17.8465 & 121.7104 & 119.8865 & 117.9665 & 115.7688 & 116.9182 & 114.0649 \\
\hline 18.2915 & 121.5581 & 119.737 & 117.8278 & 115.6351 & 116.782 & 113.9354 \\
\hline 18.4488 & 121.5041 & 119.684 & 117.7785 & 115.5877 & 116.7337 & 113.8895 \\
\hline 18.6148 & 121.4471 & 119.628 & 117.7265 & 115.5376 & 116.6827 & 113.8409 \\
\hline 18.7818 & 121.3896 & 119.5716 & 117.6742 & 115.4872 & 116.6313 & 113.7921 \\
\hline 19.1128 & 121.2759 & 119.46 & 117.5705 & 115.3873 & 116.5296 & 113.6953 \\
\hline 19.4183 & 121.1659 & 119.352 & 117.47 & 115.2905 & 116.431 & 113.6015 \\
\hline 20.0144 & 120.9738 & 119.1627 & 117.2951 & 115.1221 & 116.2595 & 113.4383 \\
\hline 20.3923 & 120.8534 & 119.0441 & 117.1854 & 115.0165 & 116.152 & 113.3361 \\
\hline 20.7652 & 120.7479 & 118.94 & 117.09 & 114.9246 & 116.0583 & 113.2471 \\
\hline 20.9525 & 120.6938 & 118.8866 & 117.0408 & 114.8773 & 116.0102 & 113.2013 \\
\hline 21.4666 & 120.5452 & 118.74 & 116.9062 & 114.7477 & 115.8781 & 113.0758 \\
\hline 21.7311 & 120.4684 & 118.6642 & 116.8365 & 114.6806 & 115.8098 & 113.0109 \\
\hline 21.9173 & 120.4176 & 118.614 & 116.7906 & 114.6364 & 115.7648 & 112.9681 \\
\hline 22.0473 & 120.3945 & 118.591 & 116.77 & 114.6166 & 115.7445 & 112.9487 \\
\hline 22.1883 & 120.3691 & 118.5657 & 116.7473 & 114.5946 & 115.7222 & 112.9274 \\
\hline 22.3543 & 120.3392 & 118.5358 & 116.7206 & 114.5688 & 115.6959 & 112.9022 \\
\hline 22.4763 & 120.3188 & 118.5153 & 116.7024 & 114.5512 & 115.6779 & 112.8851 \\
\hline 22.8133 & 120.2615 & 118.458 & 116.6513 & 114.5019 & 115.6277 & 112.8371 \\
\hline 22.9733 & 120.2611 & 118.457 & 116.6524 & 114.5029 & 115.6288 & 112.8381 \\
\hline 23.1957 & 120.2593 & 118.4543 & 116.6526 & 114.5031 & 115.6289 & 112.8383 \\
\hline 23.3361 & 120.2518 & 118.4463 & 116.6469 & 114.4976 & 115.6234 & 112.833 \\
\hline 23.3575 & 120.2507 & 118.4451 & 116.6461 & 114.4968 & 115.6225 & 112.8321 \\
\hline 23.5515 & 120.2404 & 118.4341 & 116.6383 & 114.4892 & 115.6149 & 112.8248 \\
\hline 23.7088 & 120.2324 & 118.4255 & 116.6323 & 114.4834 & 115.6089 & 112.8191 \\
\hline 24.0118 & 120.2169 & 118.4089 & 116.6207 & 114.4722 & 115.5975 & 112.8082 \\
\hline 24.1251 & 120.2111 & 118.4026 & 116.6163 & 114.4679 & 115.5932 & 112.8041 \\
\hline 24.3851 & 120.1984 & 118.3889 & 116.6069 & 114.4588 & 115.5839 & 112.7952 \\
\hline
\end{tabular}




\begin{tabular}{|l|l|l|l|l|l|l|}
\hline Distance & $\begin{array}{c}\text { Test 8 105 } \\
\text { HL }\end{array}$ & $\begin{array}{c}\text { Test 8 95 } \\
\text { HL }\end{array}$ & $\begin{array}{c}\text { Test 7 105 } \\
\text { HL }\end{array}$ & $\begin{array}{c}\text { Test 7 95 } \\
\text { HL }\end{array}$ & $\begin{array}{c}\text { Test 6 105 } \\
\text { HL }\end{array}$ & $\begin{array}{c}\text { Test 6 95 } \\
\text { HL }\end{array}$ \\
\hline & & & & & & \\
\hline 24.6292 & 120.1855 & 118.3751 & 116.5971 & 114.4493 & 115.5743 & 112.786 \\
\hline 24.8032 & 120.1775 & 118.3664 & 116.5912 & 114.4437 & 115.5685 & 112.7805 \\
\hline 25.0212 & 120.1669 & 118.3549 & 116.5833 & 114.4361 & 115.5608 & 112.7731 \\
\hline 25.1302 & 120.1616 & 118.3492 & 116.5794 & 114.4323 & 115.5569 & 112.7694 \\
\hline 25.3432 & 120.1602 & 118.3468 & 116.5803 & 114.4331 & 115.5578 & 112.7702 \\
\hline 26.3495 & 120.1169 & 118.2991 & 116.5492 & 114.403 & 115.5272 & 112.741 \\
\hline 26.5235 & 120.1087 & 118.2901 & 116.5431 & 114.3971 & 115.5212 & 112.7352 \\
\hline 26.9295 & 120.0867 & 118.2664 & 116.5263 & 114.3809 & 115.5047 & 112.7195 \\
\hline 27.0567 & 120.0802 & 118.2593 & 116.5213 & 114.3761 & 115.4998 & 112.7148 \\
\hline 27.3222 & 120.0613 & 118.2394 & 116.506 & 114.3613 & 115.4848 & 112.7004 \\
\hline 27.3813 & 120.0555 & 118.2334 & 116.5011 & 114.3566 & 115.48 & 112.6959 \\
\hline 27.6828 & 120.0258 & 118.2027 & 116.4759 & 114.3322 & 115.4553 & 112.6722 \\
\hline 27.8391 & 124.8328 & 125.3121 & 116.46 & 114.3168 & 115.4396 & 112.6572 \\
\hline 27.9641 & 124.8208 & 125.3 & 116.4494 & 114.3066 & 115.4292 & 112.6473 \\
\hline 28.1491 & 124.8031 & 125.2822 & 116.4338 & 114.2915 & 115.4138 & 112.6326 \\
\hline 28.4312 & 124.7764 & 125.2551 & 116.4102 & 114.2687 & 115.3906 & 112.6105 \\
\hline 28.6137 & 124.7643 & 125.2429 & 116.3995 & 114.2584 & 115.3802 & 112.6004 \\
\hline 28.983 & 124.7407 & 125.2191 & 116.3787 & 114.2383 & 115.3598 & 112.581 \\
\hline 29.633 & 124.7009 & 125.1789 & 116.3436 & 114.2044 & 115.3253 & 112.548 \\
\hline 29.925 & 124.6853 & 125.1632 & 116.3299 & 114.1911 & 115.3118 & 112.5351 \\
\hline 30.264 & 124.6764 & 125.1541 & 116.322 & 114.1834 & 115.3041 & 112.5277 \\
\hline 30.904 & 124.6626 & 125.14 & 116.3098 & 114.1716 & 115.2922 & 112.5163 \\
\hline 31.183 & 124.66 & 125.1373 & 116.3076 & 114.1695 & 115.29 & 112.5143 \\
\hline 31.4215 & 124.6582 & 125.1354 & 116.3061 & 114.1681 & 115.2885 & 112.5128 \\
\hline 31.6365 & 124.6578 & 125.1349 & 116.3059 & 114.1679 & 115.2883 & 112.5126 \\
\hline 31.7505 & 124.6579 & 125.1349 & 116.306 & 114.168 & 115.2884 & 112.5127 \\
\hline 31.8455 & 124.6567 & 125.1337 & 116.305 & 114.167 & 115.2874 & 112.5117 \\
\hline 32.0297 & 124.6513 & 125.1283 & 116.3002 & 114.1624 & 115.2827 & 112.5073 \\
\hline
\end{tabular}




\begin{tabular}{|c|c|c|c|c|c|c|}
\hline Distance & $\begin{array}{c}\text { Test } 5105 \\
\text { HL }\end{array}$ & $\begin{array}{c}\text { Test } 595 \\
\text { HL }\end{array}$ & $\begin{array}{c}\text { Test } 4105 \\
\text { HL }\end{array}$ & $\begin{array}{c}\text { Test } 495 \\
\text { HL }\end{array}$ & $\begin{array}{c}\text { Test } 3105 \\
\text { HL }\end{array}$ & $\begin{array}{c}\text { Test } 395 \\
\text { HL }\end{array}$ \\
\hline 0.1034 & 122.938 & 110.9229 & 122.3679 & 110.3743 & 122.402 & 110.4374 \\
\hline 0.2378 & 126.1191 & 122.0339 & 125.6008 & 120.704 & 125.6804 & 120.8505 \\
\hline 0.3218 & 126.054 & 121.9726 & 125.5325 & 120.6399 & 125.6127 & 120.7874 \\
\hline 0.3218 & 126.054 & 121.9726 & 125.5325 & 120.6399 & 125.6127 & 120.7874 \\
\hline 0.5288 & 125.9091 & 121.8367 & 125.3614 & 120.4788 & 125.444 & 120.6303 \\
\hline 0.7648 & 125.7461 & 121.6837 & 125.1687 & 120.2971 & 125.254 & 120.4534 \\
\hline 1.3788 & 125.3209 & 121.2847 & 124.6664 & 119.8238 & 124.759 & 119.9924 \\
\hline 2.583 & 124.4898 & 120.5049 & 123.6842 & 118.898 & 123.7909 & 119.0909 \\
\hline 2.666 & 124.4327 & 120.4512 & 123.6166 & 118.8343 & 123.7243 & 119.0289 \\
\hline 3.206 & 124.0755 & 120.1161 & 123.1915 & 118.4334 & 123.3056 & 118.6389 \\
\hline 3.429 & 123.9283 & 119.978 & 123.0162 & 118.2681 & 123.1329 & 118.4781 \\
\hline 3.716 & 123.739 & 119.8004 & 122.7908 & 118.0555 & 122.9109 & 118.2713 \\
\hline 5.754 & 122.3989 & 118.5429 & 121.1944 & 116.55 & 121.3383 & 116.8066 \\
\hline 6.798 & 121.7472 & 117.9315 & 120.4109 & 115.8106 & 120.5671 & 116.0882 \\
\hline 6.9262 & 121.6678 & 117.857 & 120.3152 & 115.7203 & 120.473 & 116.0005 \\
\hline 7.0603 & 121.5846 & 117.7787 & 120.2245 & 115.6346 & 120.3839 & 115.9175 \\
\hline 7.2034 & 121.496 & 117.6954 & 120.128 & 115.5434 & 120.2891 & 115.8292 \\
\hline 7.3784 & 121.3878 & 117.5936 & 120.01 & 115.4319 & 120.1732 & 115.7213 \\
\hline 7.7094 & 121.1841 & 117.4019 & 119.7878 & 115.2219 & 119.955 & 115.5181 \\
\hline 7.9064 & 121.0633 & 117.2882 & 119.656 & 115.0973 & 119.8256 & 115.3976 \\
\hline 7.9064 & 121.0633 & 117.2882 & 119.656 & 115.0973 & 119.8256 & 115.3976 \\
\hline 8.2464 & 120.8525 & 117.0899 & 119.4264 & 114.8803 & 119.6 & 115.1876 \\
\hline 8.4174 & 120.7464 & 116.9901 & 119.3108 & 114.7711 & 119.4865 & 115.0818 \\
\hline 8.8571 & 120.4757 & 116.7354 & 119.0157 & 114.4921 & 119.1966 & 114.8119 \\
\hline 9.4411 & 120.1168 & 116.3976 & 118.6245 & 114.1223 & 118.8124 & 114.454 \\
\hline 9.6082 & 120.0174 & 116.3041 & 118.5158 & 114.0195 & 118.7057 & 114.3547 \\
\hline 10.0174 & 119.7751 & 116.0761 & 118.2508 & 113.7688 & 118.4456 & 114.1124 \\
\hline 10.3374 & 119.586 & 115.8982 & 118.044 & 113.5732 & 118.2426 & 113.9233 \\
\hline 10.5075 & 119.4851 & 115.8032 & 117.9336 & 113.4688 & 118.1343 & 113.8224 \\
\hline 10.8129 & 119.3044 & 115.6332 & 117.736 & 113.2819 & 117.9403 & 113.6417 \\
\hline 10.9501 & 119.2234 & 115.5569 & 117.6474 & 113.198 & 117.8533 & 113.5607 \\
\hline 11.0961 & 119.1373 & 115.4759 & 117.5532 & 113.109 & 117.7609 & 113.4746 \\
\hline 11.6971 & 118.7849 & 115.1443 & 117.1676 & 112.7441 & 117.3825 & 113.1221 \\
\hline 12.0081 & 118.6037 & 114.9737 & 116.9693 & 112.5565 & 117.1878 & 112.9408 \\
\hline 12.0291 & 118.5958 & 114.9664 & 116.9603 & 112.5479 & 117.1791 & 112.9326 \\
\hline 12.0292 & 118.5958 & 114.9664 & 116.9603 & 112.5479 & 117.1791 & 112.9326 \\
\hline 12.2671 & 118.507 & 114.8829 & 116.8581 & 112.4503 & 117.0798 & 112.8402 \\
\hline 12.3884 & 118.4617 & 114.8403 & 116.806 & 112.4006 & 117.0292 & 112.7931 \\
\hline 12.5244 & 118.4113 & 114.7929 & 116.7479 & 112.3453 & 116.9729 & 112.7407 \\
\hline 12.6619 & 117.9452 & 114.355 & 121.8413 & 117.2531 & 122.089 & 117.6882 \\
\hline 12.7589 & 117.9097 & 114.3217 & 121.8023 & 117.2159 & 122.0512 & 117.653 \\
\hline 12.9495 & 117.84 & 114.2562 & 121.7257 & 117.1428 & 121.9769 & 117.5838 \\
\hline 13.0549 & 117.8016 & 114.22 & 121.6834 & 117.1024 & 121.9359 & 117.5456 \\
\hline
\end{tabular}




\begin{tabular}{|c|c|c|c|c|c|c|}
\hline Distance & $\begin{array}{c}\text { Test } 5105 \\
\text { HL }\end{array}$ & $\begin{array}{c}\text { Test } 595 \\
\text { HL }\end{array}$ & $\begin{array}{c}\text { Test } 4105 \\
\text { HL }\end{array}$ & $\begin{array}{c}\text { Test } 495 \\
\text { HL }\end{array}$ & $\begin{array}{c}\text { Test } 3105 \\
\text { HL }\end{array}$ & $\begin{array}{c}\text { Test } 395 \\
\text { HL }\end{array}$ \\
\hline 13.3859 & 117.6775 & 114.1034 & 121.5472 & 116.9724 & 121.8035 & 117.4223 \\
\hline 13.6541 & 117.5828 & 114.0144 & 121.443 & 116.8729 & 121.7026 & 117.3283 \\
\hline 13.9121 & 117.4906 & 113.9277 & 121.3415 & 116.776 & 121.6042 & 117.2367 \\
\hline 14.1148 & 117.4189 & 113.8603 & 121.2625 & 116.7006 & 121.5277 & 117.1654 \\
\hline 14.5219 & 117.2574 & 113.7085 & 121.0856 & 116.5319 & 121.3555 & 117.005 \\
\hline 15.0699 & 117.0407 & 113.5047 & 120.8479 & 116.3053 & 121.1244 & 116.7897 \\
\hline 15.2599 & 116.9658 & 113.4342 & 120.7657 & 116.227 & 121.0445 & 116.7153 \\
\hline 15.4019 & 116.9111 & 113.3828 & 120.7058 & 116.1698 & 120.9862 & 116.661 \\
\hline 15.5403 & 116.8582 & 113.3331 & 120.6477 & 116.1144 & 120.9298 & 116.6084 \\
\hline 15.8453 & 116.7402 & 113.2221 & 120.5182 & 115.9909 & 120.8039 & 116.4911 \\
\hline 16.1153 & 116.6352 & 113.1234 & 120.403 & 115.8811 & 120.692 & 116.3868 \\
\hline 17.4453 & 116.1359 & 112.6539 & 119.8544 & 115.3575 & 120.1593 & 115.8906 \\
\hline 17.6035 & 116.0783 & 112.5995 & 119.7913 & 115.2969 & 120.0982 & 115.8334 \\
\hline 17.8465 & 115.9897 & 112.5157 & 119.6943 & 115.2037 & 120.0041 & 115.7453 \\
\hline 18.2915 & 115.8284 & 112.3633 & 119.5176 & 115.034 & 119.8329 & 115.5849 \\
\hline 18.4488 & 115.7711 & 112.3092 & 119.455 & 114.9738 & 119.7722 & 115.528 \\
\hline 18.6148 & 115.7107 & 112.2521 & 119.3889 & 114.9103 & 119.7081 & 115.468 \\
\hline 18.7818 & 115.6499 & 112.1947 & 119.3223 & 114.8464 & 119.6435 & 115.4076 \\
\hline 19.1128 & 115.5294 & 112.0808 & 119.1904 & 114.7197 & 119.5157 & 115.2878 \\
\hline 19.4183 & 115.4136 & 111.9713 & 119.0638 & 114.5982 & 119.3928 & 115.1727 \\
\hline 20.0144 & 115.2081 & 111.7771 & 118.8381 & 114.381 & 119.1747 & 114.9684 \\
\hline 20.3923 & 115.0791 & 111.6552 & 118.6963 & 114.2444 & 119.0377 & 114.8401 \\
\hline 20.7652 & 114.964 & 111.5464 & 118.5694 & 114.1218 & 118.9155 & 114.7257 \\
\hline 20.9525 & 114.9051 & 111.4907 & 118.5045 & 114.0591 & 118.853 & 114.6671 \\
\hline 21.4666 & 114.7434 & 111.338 & 118.3264 & 113.887 & 118.6815 & 114.5064 \\
\hline 21.7311 & 114.6599 & 111.259 & 118.2343 & 113.7982 & 118.5928 & 114.4234 \\
\hline 21.9173 & 114.604 & 111.2063 & 118.1726 & 113.7385 & 118.5335 & 114.3678 \\
\hline 22.0473 & 114.5842 & 111.1875 & 118.1498 & 113.7158 & 118.5124 & 114.348 \\
\hline 22.1883 & 114.5622 & 111.1667 & 118.1246 & 113.6908 & 118.489 & 114.3261 \\
\hline 22.3543 & 114.5363 & 111.1422 & 118.095 & 113.6614 & 118.4615 & 114.3004 \\
\hline 22.4763 & 114.5187 & 111.1256 & 118.0747 & 113.6412 & 118.4427 & 114.2828 \\
\hline 22.8133 & 114.4693 & 111.0789 & 118.0178 & 113.5846 & 118.3902 & 114.2337 \\
\hline 22.9733 & 114.4702 & 111.0798 & 118.0168 & 113.5821 & 118.3913 & 114.2347 \\
\hline 23.1957 & 114.4703 & 111.0799 & 118.0142 & 113.5775 & 118.3914 & 114.2348 \\
\hline 23.3361 & 114.4648 & 111.0747 & 118.0063 & 113.5684 & 118.3856 & 114.2294 \\
\hline 23.3575 & 114.464 & 111.0739 & 118.0051 & 113.567 & 118.3847 & 114.2285 \\
\hline 23.5515 & 114.4564 & 111.0668 & 117.9942 & 113.5546 & 118.3767 & 114.221 \\
\hline 23.7088 & 114.4505 & 111.0612 & 117.9856 & 113.5447 & 118.3705 & 114.2152 \\
\hline 24.0118 & 114.4392 & 111.0505 & 117.9692 & 113.5258 & 118.3586 & 114.204 \\
\hline 24.1251 & 114.435 & 111.0465 & 117.963 & 113.5187 & 118.3541 & 114.1998 \\
\hline 24.3851 & 114.4258 & 111.0378 & 117.9494 & 113.5029 & 118.3444 & 114.1907 \\
\hline 24.6292 & 114.4163 & 111.0288 & 117.9357 & 113.4872 & 118.3343 & 114.1813 \\
\hline 24.8032 & 114.4106 & 111.0235 & 117.9271 & 113.4771 & 118.3283 & 114.1757 \\
\hline
\end{tabular}




\begin{tabular}{|l|l|l|l|l|l|l|}
\hline Distance & $\begin{array}{c}\text { Test 5 105 } \\
\text { HL }\end{array}$ & $\begin{array}{c}\text { Test 5 95 } \\
\text { HL }\end{array}$ & $\begin{array}{c}\text { Test 4 105 } \\
\text { HL }\end{array}$ & $\begin{array}{c}\text { Test 4 95 } \\
\text { HL }\end{array}$ & $\begin{array}{c}\text { Test 3 105 } \\
\text { HL }\end{array}$ & $\begin{array}{c}\text { Test 3 95 } \\
\text { HL }\end{array}$ \\
\hline 25.0212 & 114.403 & 111.0162 & 117.9158 & 113.4639 & 118.3202 & 114.1681 \\
\hline 25.1302 & 114.3992 & 111.0126 & 117.9102 & 113.4573 & 118.3162 & 114.1643 \\
\hline 25.3432 & 114.3999 & 111.0133 & 117.9078 & 113.4526 & 118.3171 & 114.1651 \\
\hline 26.3495 & 114.3698 & 110.9849 & 117.8608 & 113.3966 & 118.2853 & 114.1353 \\
\hline 26.5235 & 114.3639 & 110.9793 & 117.8519 & 113.3862 & 118.279 & 114.1294 \\
\hline 26.9295 & 114.3477 & 110.9639 & 117.8286 & 113.3595 & 118.2618 & 114.1133 \\
\hline 27.0567 & 114.3429 & 110.9594 & 117.8217 & 113.3515 & 118.2568 & 114.1086 \\
\hline 27.3222 & 114.3281 & 110.9454 & 117.802 & 113.3299 & 118.2411 & 114.0939 \\
\hline 27.3813 & 114.3234 & 110.941 & 117.7961 & 113.3237 & 118.2361 & 114.0893 \\
\hline 27.6828 & 114.2992 & 110.9181 & 117.7659 & 113.2918 & 118.2104 & 114.0651 \\
\hline 27.8391 & 114.2838 & 110.9035 & 124.8491 & 125.4185 & 118.194 & 114.0498 \\
\hline 27.9641 & 114.2736 & 110.8939 & 124.8371 & 125.4064 & 118.1832 & 114.0397 \\
\hline 28.1491 & 114.2585 & 110.8796 & 124.8195 & 125.3886 & 118.1672 & 114.0247 \\
\hline 28.4312 & 114.2358 & 110.8582 & 124.7928 & 125.3617 & 118.1431 & 114.0021 \\
\hline 28.6137 & 114.2255 & 110.8484 & 124.7807 & 125.3496 & 118.1322 & 113.9919 \\
\hline 28.983 & 114.2055 & 110.8296 & 124.7573 & 125.3259 & 118.111 & 113.972 \\
\hline 29.633 & 114.1717 & 110.7976 & 124.7177 & 125.286 & 118.0752 & 113.9385 \\
\hline 29.925 & 114.1585 & 110.7851 & 124.7022 & 125.2703 & 118.0612 & 113.9253 \\
\hline 30.264 & 114.1509 & 110.7779 & 124.6933 & 125.2614 & 118.0532 & 113.9178 \\
\hline 30.904 & 114.1393 & 110.7669 & 124.6796 & 125.2476 & 118.0409 & 113.9063 \\
\hline 31.183 & 114.1371 & 110.7649 & 124.677 & 125.2448 & 118.0386 & 113.9042 \\
\hline 31.4215 & 114.1357 & 110.7635 & 124.6751 & 125.2429 & 118.0371 & 113.9027 \\
\hline 31.6365 & 114.1354 & 110.7632 & 124.6747 & 125.2425 & 118.0368 & 113.9025 \\
\hline 31.7505 & 114.1355 & 110.7633 & 124.6747 & 125.2425 & 118.0369 & 113.9025 \\
\hline 31.8455 & 114.1345 & 110.7624 & 124.6735 & 125.2413 & 118.0358 & 113.9016 \\
\hline 32.0297 & 114.1299 & 110.7581 & 124.6682 & 125.2358 & 118.031 & 113.897 \\
\hline
\end{tabular}




\begin{tabular}{|c|c|c|c|c|}
\hline Distance & $\begin{array}{c}\text { Test } 2105 \\
\mathrm{HL}\end{array}$ & $\begin{array}{c}\text { Test } 295 \\
\text { HL }\end{array}$ & $\begin{array}{c}\text { Test } 1105 \\
\text { HL }\end{array}$ & $\begin{array}{c}\text { Test } 195 \\
\text { HL }\end{array}$ \\
\hline 0.1034 & 122.5967 & 110.633 & 122.9494 & 111.4887 \\
\hline 0.2378 & 126.1397 & 121.3093 & 121.7467 & 110.7418 \\
\hline 0.3218 & 126.075 & 121.2491 & 121.6862 & 110.6914 \\
\hline 0.3218 & 126.075 & 121.2491 & 121.6862 & 110.6914 \\
\hline 0.5288 & 125.9154 & 121.1006 & 121.5367 & 110.5674 \\
\hline 0.7648 & 125.7367 & 120.9344 & 121.3694 & 110.4285 \\
\hline 1.3788 & 125.271 & 120.5011 & 120.9332 & 110.0664 \\
\hline 2.583 & 124.3601 & 119.6537 & 120.0801 & 109.3582 \\
\hline 2.666 & 124.2974 & 119.5954 & 120.0214 & 109.3095 \\
\hline 3.206 & 123.9043 & 119.2297 & 119.6533 & 109.0039 \\
\hline 3.429 & 123.7422 & 119.0789 & 119.5015 & 108.8779 \\
\hline 3.716 & 123.5337 & 118.885 & 119.3062 & 108.7158 \\
\hline 5.754 & 122.0572 & 117.5112 & 117.9233 & 107.5674 \\
\hline 6.798 & 121.3351 & 116.8394 & 117.247 & 107.0059 \\
\hline 6.9262 & 121.247 & 116.7574 & 117.1644 & 106.9374 \\
\hline 7.0603 & 121.1643 & 116.6805 & 117.0871 & 106.8733 \\
\hline 7.2034 & 121.0764 & 116.5988 & 117.0048 & 106.8051 \\
\hline 7.3784 & 120.9689 & 116.4988 & 116.9042 & 106.7217 \\
\hline 7.7094 & 120.7665 & 116.3106 & 116.7147 & 106.5647 \\
\hline 7.9064 & 120.6465 & 116.1991 & 116.6024 & 106.4716 \\
\hline 7.9064 & 120.6465 & 116.1991 & 116.6024 & 106.4716 \\
\hline 8.2464 & 120.4372 & 116.0044 & 116.4064 & 106.3092 \\
\hline 8.4174 & 120.3318 & 115.9064 & 116.3078 & 106.2274 \\
\hline 8.8571 & 120.0629 & 115.6563 & 116.056 & 106.0187 \\
\hline 9.4411 & 119.7063 & 115.3247 & 115.7222 & 105.7419 \\
\hline 9.6082 & 119.6076 & 115.2329 & 115.6298 & 105.6653 \\
\hline 10.0174 & 119.367 & 115.0091 & 115.4045 & 105.4786 \\
\hline 10.3374 & 119.1791 & 114.8344 & 115.2287 & 105.3328 \\
\hline 10.5075 & 119.0789 & 114.7412 & 115.1349 & 105.255 \\
\hline 10.8129 & 118.8994 & 114.5743 & 114.9668 & 105.1156 \\
\hline 10.9501 & 118.8189 & 114.4994 & 114.8914 & 105.0532 \\
\hline 11.0961 & 118.7334 & 114.4199 & 114.8114 & 104.9868 \\
\hline 11.6971 & 118.3833 & 114.0942 & 114.4836 & 104.7149 \\
\hline 12.0081 & 118.2033 & 113.9268 & 114.3151 & 104.5752 \\
\hline 12.0291 & 118.1955 & 113.9196 & 114.3078 & 104.5692 \\
\hline 12.0292 & 118.1955 & 113.9196 & 114.3078 & 104.5692 \\
\hline 12.2671 & 118.1073 & 113.8377 & 114.2253 & 104.5011 \\
\hline 12.3884 & 118.0623 & 113.7959 & 114.1832 & 104.4664 \\
\hline 12.5244 & 118.0123 & 113.7494 & 114.1365 & 104.4278 \\
\hline 12.6619 & 117.5493 & 113.3197 & 113.7037 & 104.0708 \\
\hline 12.7589 & 117.514 & 113.2869 & 113.6707 & 104.0436 \\
\hline 12.9495 & 117.4448 & 113.2227 & 113.606 & 103.9902 \\
\hline 13.0549 & 117.4066 & 113.1872 & 113.5703 & 103.9607 \\
\hline
\end{tabular}




\begin{tabular}{|l|l|l|l|l|}
\hline Distance & $\begin{array}{c}\text { Test 2 105 } \\
\text { HL }\end{array}$ & $\begin{array}{c}\text { Test 2 95 } \\
\text { HL }\end{array}$ & $\begin{array}{c}\text { Test 1 105 } \\
\text { HL }\end{array}$ & \multicolumn{1}{|c|}{ Test 1 95 } \\
\hline & & & & \\
\hline 13.3859 & 117.2834 & 113.0727 & 113.4551 & 103.8655 \\
\hline 13.6541 & 117.1894 & 112.9854 & 113.3672 & 103.793 \\
\hline 13.9121 & 117.0978 & 112.9003 & 113.2815 & 103.7223 \\
\hline 14.1148 & 117.0265 & 112.8342 & 113.2149 & 103.6673 \\
\hline 14.5219 & 116.8662 & 112.6852 & 113.0649 & 103.5433 \\
\hline 15.0699 & 116.6509 & 112.4851 & 112.8635 & 103.3767 \\
\hline 15.2599 & 116.5765 & 112.416 & 112.7939 & 103.3191 \\
\hline 15.4019 & 116.5222 & 112.3656 & 112.7431 & 103.2771 \\
\hline 15.5403 & 116.4697 & 112.3167 & 112.6939 & 103.2365 \\
\hline 15.8453 & 116.3525 & 112.2078 & 112.5843 & 103.1458 \\
\hline 16.1153 & 116.2482 & 112.1109 & 112.4867 & 103.0652 \\
\hline 17.4453 & 115.7523 & 111.6502 & 112.0228 & 102.6819 \\
\hline 17.6035 & 115.695 & 111.5967 & 111.969 & 102.6366 \\
\hline 17.8465 & 115.6069 & 111.5143 & 111.8861 & 102.5668 \\
\hline 18.2915 & 115.4466 & 111.3645 & 111.7353 & 102.4398 \\
\hline 18.4488 & 115.3898 & 111.3113 & 111.6818 & 102.3947 \\
\hline 18.6148 & 115.3298 & 111.2552 & 111.6254 & 102.3472 \\
\hline 18.7818 & 115.2693 & 111.1987 & 111.5685 & 102.2993 \\
\hline 19.1128 & 115.1496 & 111.0868 & 111.4559 & 102.2045 \\
\hline 19.4183 & 115.0345 & 110.9792 & 111.3476 & 102.1133 \\
\hline 20.0144 & 114.8303 & 110.7883 & 111.1555 & 101.9515 \\
\hline 20.3923 & 114.7021 & 110.6684 & 111.0349 & 101.8499 \\
\hline 20.7652 & 114.5877 & 110.5615 & 110.9273 & 101.7593 \\
\hline 20.9525 & 114.5292 & 110.5068 & 110.8722 & 101.7128 \\
\hline 21.4666 & 114.3686 & 110.3566 & 110.7211 & 101.5855 \\
\hline 21.7311 & 114.2856 & 110.279 & 110.643 & 101.5198 \\
\hline 21.9173 & 114.2301 & 110.2271 & 110.5908 & 101.4758 \\
\hline 22.0473 & 114.2103 & 110.2087 & 110.5722 & 101.4602 \\
\hline 22.1883 & 114.1885 & 110.1882 & 110.5517 & 101.4429 \\
\hline 22.3543 & 114.1627 & 110.1642 & 110.5275 & 101.4226 \\
\hline 22.4763 & 114.1452 & 110.1478 & 110.511 & 101.4088 \\
\hline 22.8133 & 114.0961 & 110.102 & 110.4649 & 101.37 \\
\hline 22.9733 & 114.097 & 110.1028 & 110.4658 & 101.3708 \\
\hline 23.1957 & 114.0971 & 110.1029 & 110.4659 & 101.3709 \\
\hline 23.3361 & 114.0917 & 110.0978 & 110.4607 & 101.3666 \\
\hline 23.3575 & 114.0908 & 110.097 & 110.46 & 101.3659 \\
\hline 23.5515 & 114.0833 & 110.09 & 110.4529 & 101.36 \\
\hline 23.7088 & 114.0775 & 110.0845 & 110.4474 & 101.3554 \\
\hline 24.0118 & 114.0662 & 110.074 & 110.4369 & 101.3465 \\
\hline 24.1251 & 114.062 & 110.0701 & 110.4329 & 101.3432 \\
\hline 24.3851 & 114.0529 & 110.0615 & 110.4243 & 101.336 \\
\hline 24.6292 & 114.0434 & 110.0527 & 110.4154 & 101.3285 \\
\hline
\end{tabular}




\begin{tabular}{|c|c|c|c|c|}
\hline Distance & $\begin{array}{c}\text { Test } 2105 \\
\text { HL }\end{array}$ & $\begin{array}{c}\text { Test } 295 \\
\text { HL }\end{array}$ & $\begin{array}{c}\text { Test } 1105 \\
\text { HL }\end{array}$ & $\begin{array}{c}\text { Test } 195 \\
\text { HL }\end{array}$ \\
\hline 24.8032 & 114.0378 & 110.0474 & 110.4101 & 101.324 \\
\hline 25.0212 & 114.0302 & 110.0403 & 110.403 & 101.318 \\
\hline 25.1302 & 114.0264 & 110.0367 & 110.3994 & 101.315 \\
\hline 25.3432 & 114.0271 & 110.0374 & 110.4001 & 101.3156 \\
\hline 26.3495 & 113.9972 & 110.0094 & 110.372 & 101.2919 \\
\hline 26.5235 & 113.9913 & 110.0039 & 110.3664 & 101.2872 \\
\hline 26.9295 & 113.9752 & 109.9888 & 110.3513 & 101.2744 \\
\hline 27.0567 & 113.9705 & 109.9844 & 110.3468 & 101.2706 \\
\hline 27.3222 & 113.9558 & 109.9707 & 110.333 & 101.259 \\
\hline 27.3813 & 113.9511 & 109.9663 & 110.3286 & 101.2553 \\
\hline 27.6828 & 113.927 & 109.9438 & 110.3059 & 101.2362 \\
\hline 27.8391 & 113.9117 & 109.9295 & 110.2915 & 101.224 \\
\hline 27.9641 & 113.9016 & 109.92 & 110.282 & 101.216 \\
\hline 28.1491 & 113.8866 & 109.906 & 110.2679 & 101.2041 \\
\hline 28.4312 & 113.864 & 109.8849 & 110.2466 & 101.1862 \\
\hline 28.6137 & 113.8538 & 109.8753 & 110.237 & 101.1781 \\
\hline 28.983 & 113.834 & 109.8568 & 110.2183 & 101.1624 \\
\hline 29.633 & 113.8004 & 109.8254 & 110.1867 & 101.1357 \\
\hline 29.925 & 113.7873 & 109.8131 & 110.1744 & 101.1253 \\
\hline 30.264 & 113.7798 & 109.8061 & 110.1673 & 101.1193 \\
\hline 30.904 & 113.7682 & 109.7952 & 110.1564 & 101.11 \\
\hline 31.183 & 113.7661 & 109.7932 & 110.1544 & 101.1083 \\
\hline 31.4215 & 113.7646 & 109.7919 & 110.153 & 101.1072 \\
\hline 31.6365 & 113.7643 & 109.7916 & 110.1528 & 101.107 \\
\hline 31.7505 & 113.7644 & 109.7917 & 110.1528 & 101.1071 \\
\hline 31.8455 & 113.7634 & 109.7908 & 110.1519 & 101.1063 \\
\hline 32.0297 & 113.7589 & 109.7865 & 110.1477 & 101.1027 \\
\hline
\end{tabular}


Table 21. Voltage Profile Data for Light Load Tests 1 Through 8 at $95 \%$ and $105 \%$ Substation Primary Voltage

\begin{tabular}{|c|c|c|c|c|c|c|}
\hline Distance & $\begin{array}{c}\text { Test } 8105 \\
\text { LL }\end{array}$ & $\begin{array}{c}\text { Test } 895 \\
\text { LL }\end{array}$ & $\begin{array}{c}\text { Test } 7105 \\
\text { LL }\end{array}$ & $\begin{array}{c}\text { Test } 795 \\
\text { LL }\end{array}$ & $\begin{array}{c}\text { Test } 6105 \\
\text { LL }\end{array}$ & $\begin{array}{c}\text { Test } 695 \\
\text { LL }\end{array}$ \\
\hline 0.1034 & 125.9754 & 113.9697 & 125.9744 & 113.9687 & 125.5215 & 113.4715 \\
\hline 0.2378 & 125.6501 & 126.1978 & 125.648 & 126.1954 & 126.246 & 125.7513 \\
\hline 0.3218 & 125.6232 & 126.1708 & 125.6211 & 126.1683 & 126.2194 & 125.7247 \\
\hline 0.3218 & 125.6232 & 126.1708 & 125.6211 & 126.1683 & 126.2194 & 125.7247 \\
\hline 0.5288 & 125.617 & 126.1647 & 125.6149 & 126.1621 & 126.1918 & 125.6973 \\
\hline 0.7648 & 125.6046 & 126.1523 & 125.6024 & 126.1496 & 126.1567 & 125.6622 \\
\hline 1.3788 & 125.572 & 126.1198 & 125.5696 & 126.1168 & 126.0648 & 125.5707 \\
\hline 2.583 & 125.5091 & 126.057 & 125.5064 & 126.0535 & 125.8856 & 125.392 \\
\hline 2.666 & 125.5048 & 126.0527 & 125.5021 & 126.0492 & 125.8732 & 125.3798 \\
\hline 3.206 & 125.4822 & 126.0303 & 125.4794 & 126.0265 & 125.7984 & 125.3052 \\
\hline 3.429 & 125.4729 & 126.0211 & 125.4701 & 126.0172 & 125.7676 & 125.2745 \\
\hline 3.716 & 125.4611 & 126.0093 & 125.4582 & 126.0053 & 125.7279 & 125.2349 \\
\hline 5.754 & 125.3787 & 125.9272 & 125.3753 & 125.9223 & 125.4476 & 124.9556 \\
\hline 6.798 & 125.3473 & 125.8961 & 125.3437 & 125.8907 & 125.3146 & 124.823 \\
\hline 6.9262 & 125.3436 & 125.8925 & 125.34 & 125.887 & 125.2984 & 124.807 \\
\hline 7.0603 & 125.3329 & 125.8817 & 125.3292 & 125.8762 & 125.2746 & 124.7832 \\
\hline 7.2034 & 125.3214 & 125.8703 & 125.3177 & 125.8647 & 125.2492 & 124.7579 \\
\hline 7.3784 & 125.3075 & 125.8563 & 125.3037 & 125.8506 & 125.2181 & 124.727 \\
\hline 7.7094 & 125.2813 & 125.8302 & 125.2776 & 125.8244 & 125.1597 & 124.6687 \\
\hline 7.9064 & 125.2659 & 125.8148 & 125.2621 & 125.8089 & 125.1251 & 124.6342 \\
\hline 7.9064 & 125.2659 & 125.8148 & 125.2621 & 125.8089 & 125.1251 & 124.6342 \\
\hline 8.2464 & 125.2381 & 125.787 & 125.2342 & 125.7809 & 125.064 & 124.5734 \\
\hline 8.4174 & 125.2241 & 125.7729 & 125.2201 & 125.7668 & 125.0333 & 124.5427 \\
\hline 8.8571 & 125.1886 & 125.7375 & 125.1846 & 125.7312 & 124.9548 & 124.4646 \\
\hline 9.4411 & 125.1418 & 125.6906 & 125.1376 & 125.684 & 124.8508 & 124.3609 \\
\hline 9.6082 & 125.1295 & 125.6784 & 125.1253 & 125.6717 & 124.8222 & 124.3324 \\
\hline 10.0174 & 125.1 & 125.6488 & 125.0957 & 125.642 & 124.7525 & 124.263 \\
\hline 10.3374 & 125.077 & 125.6259 & 125.0727 & 125.6189 & 124.6981 & 124.2088 \\
\hline 10.5075 & 125.0646 & 125.6135 & 125.0602 & 125.6064 & 124.6691 & 124.1798 \\
\hline 10.8129 & 125.0425 & 125.5914 & 125.038 & 125.5842 & 124.617 & 124.1279 \\
\hline 10.9501 & 125.0326 & 125.5815 & 125.0281 & 125.5742 & 124.5936 & 124.1046 \\
\hline 11.0961 & 125.0222 & 125.5711 & 125.0177 & 125.5637 & 124.5688 & 124.0799 \\
\hline 11.6971 & 124.9798 & 125.5287 & 124.9752 & 125.5211 & 124.4674 & 123.9789 \\
\hline 12.0081 & 124.9582 & 125.5072 & 124.9536 & 125.4994 & 124.4152 & 123.9269 \\
\hline 12.0291 & 124.9564 & 125.5054 & 124.9517 & 125.4976 & 124.4134 & 123.9251 \\
\hline 12.0292 & 124.9564 & 125.5054 & 124.9517 & 125.4976 & 124.4134 & 123.9251 \\
\hline 12.2671 & 124.9358 & 125.4847 & 124.9311 & 125.4769 & 124.3929 & 123.9047 \\
\hline 12.3884 & 124.9253 & 125.4742 & 124.9205 & 125.4663 & 124.3825 & 123.8942 \\
\hline 12.5244 & 124.9136 & 125.4626 & 124.9089 & 125.4546 & 124.3709 & 123.8827 \\
\hline 12.6619 & 124.284 & 125.3955 & 124.8416 & 125.3871 & 124.3038 & 123.8159 \\
\hline 12.7589 & 124.2758 & 125.3873 & 124.8334 & 125.3789 & 124.2957 & 123.8078 \\
\hline 12.9495 & 124.2597 & 125.3713 & 124.8173 & 125.3628 & 124.2798 & 123.7919 \\
\hline
\end{tabular}




\begin{tabular}{|c|c|c|c|c|c|c|}
\hline Distance & $\begin{array}{c}\text { Test } 8105 \\
\text { LL }\end{array}$ & $\begin{array}{c}\text { Test } 895 \\
\text { LL }\end{array}$ & $\begin{array}{c}\text { Test } 7105 \\
\text { LL }\end{array}$ & $\begin{array}{c}\text { Test } 795 \\
\text { LL }\end{array}$ & $\begin{array}{c}\text { Test } 6105 \\
\text { LL }\end{array}$ & $\begin{array}{c}\text { Test } 695 \\
\text { LL }\end{array}$ \\
\hline 13.0549 & 124.2508 & 125.3624 & 124.8085 & 125.3539 & 124.271 & 123.7831 \\
\hline 13.3859 & 124.2215 & 125.3332 & 124.7792 & 125.3245 & 124.2419 & 123.7542 \\
\hline 13.6541 & 124.2 & 125.3118 & 124.7578 & 125.303 & 124.2207 & 123.733 \\
\hline 13.9121 & 124.1789 & 125.2909 & 124.7368 & 125.282 & 124.1998 & 123.7122 \\
\hline 14.1148 & 124.1626 & 125.2747 & 124.7206 & 125.2656 & 124.1837 & 123.6961 \\
\hline 14.5219 & 124.1228 & 125.2349 & 124.6808 & 125.2257 & 124.1442 & 123.6568 \\
\hline 15.0699 & 124.0693 & 125.1816 & 124.6274 & 125.1722 & 124.0912 & 123.604 \\
\hline 15.2599 & 124.0508 & 125.1632 & 124.609 & 125.1537 & 124.0729 & 123.5858 \\
\hline 15.4019 & 124.0375 & 125.1499 & 124.5957 & 125.1404 & 124.0597 & 123.5726 \\
\hline 15.5403 & 124.0247 & 125.1372 & 124.5829 & 125.1275 & 124.047 & 123.56 \\
\hline 15.8453 & 123.9958 & 125.1084 & 124.5541 & 125.0987 & 124.0184 & 123.5315 \\
\hline 16.1153 & 123.9701 & 125.0828 & 124.5285 & 125.0729 & 123.9929 & 123.5061 \\
\hline 17.4453 & 123.8498 & 124.9629 & 124.4085 & 124.9525 & 123.8737 & 123.3873 \\
\hline 17.6035 & 123.8375 & 124.9507 & 124.3962 & 124.9401 & 123.8615 & 123.3751 \\
\hline 17.8465 & 123.8185 & 124.9317 & 124.3772 & 124.9211 & 123.8427 & 123.3563 \\
\hline 18.2915 & 123.7842 & 124.8977 & 124.3431 & 124.8869 & 123.8088 & 123.3226 \\
\hline 18.4488 & 123.772 & 124.8855 & 124.3309 & 124.8747 & 123.7967 & 123.3105 \\
\hline 18.6148 & 123.7591 & 124.8727 & 124.3181 & 124.8618 & 123.7839 & 123.2978 \\
\hline 18.7818 & 123.7461 & 124.8597 & 124.3051 & 124.8487 & 123.771 & 123.2849 \\
\hline 19.1128 & 123.7203 & 124.8341 & 124.2794 & 124.823 & 123.7455 & 123.2595 \\
\hline 19.4183 & 123.6947 & 124.8086 & 124.2538 & 124.7973 & 123.7202 & 123.2342 \\
\hline 20.0144 & 123.6585 & 124.7728 & 124.2179 & 124.7613 & 123.6845 & 123.1987 \\
\hline 20.3923 & 123.6362 & 124.7507 & 124.1957 & 124.739 & 123.6624 & 123.1767 \\
\hline 20.7652 & 123.6182 & 124.733 & 124.1779 & 124.7211 & 123.6447 & 123.1591 \\
\hline 20.9525 & 123.6088 & 124.7237 & 124.1686 & 124.7118 & 123.6355 & 123.1499 \\
\hline 21.4666 & 123.583 & 124.6983 & 124.143 & 124.6861 & 123.6101 & 123.1245 \\
\hline 21.7311 & 123.5696 & 124.685 & 124.1297 & 124.6727 & 123.5969 & 123.1114 \\
\hline 21.9173 & 123.5612 & 124.6768 & 124.1214 & 124.6644 & 123.5886 & 123.1032 \\
\hline 22.0473 & 123.5534 & 124.669 & 124.1136 & 124.6566 & 123.5809 & 123.0955 \\
\hline 22.1883 & 123.5448 & 124.6605 & 124.105 & 124.648 & 123.5723 & 123.0869 \\
\hline 22.3543 & 123.5347 & 124.6504 & 124.0949 & 124.6379 & 123.5623 & 123.0769 \\
\hline 22.4763 & 123.5277 & 124.6435 & 124.088 & 124.6309 & 123.5554 & 123.07 \\
\hline 22.8133 & 123.5082 & 124.6242 & 124.0686 & 124.6114 & 123.5361 & 123.0508 \\
\hline 22.9733 & 123.5073 & 124.6234 & 124.0678 & 124.6106 & 123.5353 & 123.05 \\
\hline 23.1957 & 123.5057 & 124.622 & 124.0663 & 124.6091 & 123.5337 & 123.0485 \\
\hline 23.3361 & 123.5027 & 124.619 & 124.0633 & 124.6061 & 123.5308 & 123.0455 \\
\hline 23.3575 & 123.5022 & 124.6186 & 124.0628 & 124.6057 & 123.5304 & 123.0451 \\
\hline 23.5515 & 123.498 & 124.6146 & 124.0588 & 124.6016 & 123.5263 & 123.041 \\
\hline 23.7088 & 123.4948 & 124.6114 & 124.0556 & 124.5983 & 123.5231 & 123.0379 \\
\hline
\end{tabular}




\begin{tabular}{|c|c|c|c|c|c|c|}
\hline Distance & $\begin{array}{c}\text { Test } 8105 \\
\text { LL }\end{array}$ & $\begin{array}{c}\text { Test } 895 \\
\text { LL }\end{array}$ & $\begin{array}{c}\text { Test } 7105 \\
\text { LL }\end{array}$ & $\begin{array}{c}\text { Test } 795 \\
\text { LL }\end{array}$ & $\begin{array}{c}\text { Test } 6105 \\
\text { LL }\end{array}$ & $\begin{array}{c}\text { Test } 695 \\
\text { LL }\end{array}$ \\
\hline 24.0118 & 123.4884 & 124.6053 & 124.0494 & 124.5921 & 123.5169 & 123.0317 \\
\hline 24.1251 & 123.4861 & 124.6031 & 124.047 & 124.5898 & 123.5146 & 123.0294 \\
\hline 24.3851 & 123.4808 & 124.598 & 124.0419 & 124.5846 & 123.5095 & 123.0243 \\
\hline 24.6292 & 123.4755 & 124.5929 & 124.0367 & 124.5795 & 123.5044 & 123.0192 \\
\hline 24.8032 & 123.4722 & 124.5897 & 124.0335 & 124.5762 & 123.5011 & 123.016 \\
\hline 25.0212 & 123.4678 & 124.5855 & 124.0292 & 124.5719 & 123.4969 & 123.0117 \\
\hline 25.1302 & 123.4656 & 124.5834 & 124.027 & 124.5697 & 123.4947 & 123.0096 \\
\hline 25.3432 & 123.4642 & 124.5823 & 124.0258 & 124.5684 & 123.4935 & 123.0083 \\
\hline 26.3495 & 123.4459 & 124.5648 & 124.0079 & 124.5505 & 123.4757 & 122.9907 \\
\hline 26.5235 & 123.4425 & 124.5615 & 124.0046 & 124.5472 & 123.4724 & 122.9873 \\
\hline 26.9295 & 123.4337 & 124.553 & 123.996 & 124.5385 & 123.4639 & 122.9788 \\
\hline 27.0567 & 123.4311 & 124.5505 & 123.9934 & 124.5359 & 123.4613 & 122.9763 \\
\hline 27.3222 & 123.4239 & 124.5435 & 123.9864 & 124.5289 & 123.4543 & 122.9693 \\
\hline 27.3813 & 123.4218 & 124.5415 & 123.9843 & 124.5268 & 123.4523 & 122.9673 \\
\hline 27.6828 & 123.4112 & 124.5311 & 123.9738 & 124.5162 & 123.4418 & 122.9568 \\
\hline 27.8391 & 124.232 & 123.6907 & 123.9678 & 124.5102 & 123.4358 & 122.9509 \\
\hline 27.9641 & 124.2276 & 123.6863 & 123.9634 & 124.5058 & 123.4314 & 122.9465 \\
\hline 28.1491 & 124.221 & 123.6798 & 123.9568 & 124.4992 & 123.4249 & 122.9401 \\
\hline 28.4312 & 124.2111 & 123.67 & 123.947 & 124.4893 & 123.4151 & 122.9303 \\
\hline 28.6137 & 124.2065 & 123.6654 & 123.9423 & 124.4847 & 123.4105 & 122.9257 \\
\hline 28.983 & 124.1976 & 123.6565 & 123.9335 & 124.4758 & 123.4018 & 122.917 \\
\hline 29.633 & 124.1829 & 123.6419 & 123.9189 & 124.4611 & 123.3872 & 122.9025 \\
\hline 29.925 & 124.1772 & 123.6362 & 123.9131 & 124.4553 & 123.3815 & 122.8968 \\
\hline 30.264 & 124.1739 & 123.6329 & 123.9098 & 124.452 & 123.3782 & 122.8935 \\
\hline 30.904 & 124.1685 & 123.6276 & 123.9045 & 124.4467 & 123.373 & 122.8883 \\
\hline 31.183 & 124.1674 & 123.6265 & 123.9034 & 124.4456 & 123.3718 & 122.8872 \\
\hline 31.4215 & 124.1666 & 123.6257 & 123.9026 & 124.4448 & 123.3711 & 122.8864 \\
\hline 31.6365 & 124.1662 & 123.6253 & 123.9022 & 124.4444 & 123.3707 & 122.886 \\
\hline 31.7505 & 124.1662 & 123.6253 & 123.9022 & 124.4444 & 123.3707 & 122.886 \\
\hline 31.8455 & 124.1658 & 123.6249 & 123.9018 & 124.444 & 123.3703 & 122.8856 \\
\hline 32.0297 & 124.1639 & 123.6231 & 123.9 & 124.4422 & 123.3685 & 122.8838 \\
\hline
\end{tabular}




\begin{tabular}{|c|c|c|c|c|c|c|}
\hline Distance & $\begin{array}{c}\text { Test } 5105 \\
\text { LL }\end{array}$ & $\begin{array}{c}\text { Test } 595 \\
\text { LL }\end{array}$ & $\begin{array}{c}\text { Test } 4105 \\
\text { LL }\end{array}$ & $\begin{array}{c}\text { Test } 495 \\
\text { LL }\end{array}$ & $\begin{array}{c}\text { Test } 3105 \\
\text { LL }\end{array}$ & $\begin{array}{c}\text { Test } 395 \\
\text { LL }\end{array}$ \\
\hline 0.1034 & 125.1863 & 113.1052 & 124.8272 & 112.684 & 124.8297 & 112.6858 \\
\hline 0.2378 & 126.275 & 125.6313 & 126.2478 & 126.1644 & 126.2539 & 126.1689 \\
\hline 0.3218 & 126.2487 & 125.6051 & 126.2214 & 126.1377 & 126.2276 & 126.1422 \\
\hline 0.3218 & 126.2487 & 125.6051 & 126.2214 & 126.1377 & 126.2276 & 126.1422 \\
\hline 0.5288 & 126.2059 & 125.5625 & 126.162 & 126.0775 & 126.1685 & 126.0822 \\
\hline 0.7648 & 126.1545 & 125.5114 & 126.0928 & 126.0072 & 126.0996 & 126.0122 \\
\hline 1.3788 & 126.0206 & 125.378 & 125.9124 & 125.8242 & 125.9201 & 125.8297 \\
\hline 2.583 & 125.7585 & 125.1171 & 125.5592 & 125.4657 & 125.5685 & 125.4725 \\
\hline 2.666 & 125.7404 & 125.0992 & 125.5349 & 125.441 & 125.5443 & 125.4478 \\
\hline 3.206 & 125.6284 & 124.9877 & 125.3819 & 125.2857 & 125.3921 & 125.2931 \\
\hline 3.429 & 125.5822 & 124.9417 & 125.3188 & 125.2216 & 125.3293 & 125.2292 \\
\hline 3.716 & 125.5227 & 124.8825 & 125.2376 & 125.1391 & 125.2485 & 125.147 \\
\hline 5.754 & 125.1016 & 124.4633 & 124.6618 & 124.5544 & 124.6755 & 124.5643 \\
\hline 6.798 & 124.8962 & 124.2589 & 124.377 & 124.2651 & 124.3921 & 124.276 \\
\hline 6.9262 & 124.8712 & 124.234 & 124.3422 & 124.2297 & 124.3575 & 124.2408 \\
\hline 7.0603 & 124.838 & 124.201 & 124.3088 & 124.1957 & 124.3243 & 124.2069 \\
\hline 7.2034 & 124.8027 & 124.1659 & 124.2732 & 124.1595 & 124.289 & 124.1709 \\
\hline 7.3784 & 124.7596 & 124.1229 & 124.2297 & 124.1153 & 124.2457 & 124.1268 \\
\hline 7.7094 & 124.6782 & 124.0419 & 124.1477 & 124.0318 & 124.1642 & 124.0437 \\
\hline 7.9064 & 124.6299 & 123.9939 & 124.0991 & 123.9823 & 124.1158 & 123.9943 \\
\hline 7.9064 & 124.6299 & 123.9939 & 124.0991 & 123.9823 & 124.1158 & 123.9943 \\
\hline 8.2464 & 124.5453 & 123.9096 & 124.0138 & 123.8955 & 124.031 & 123.9079 \\
\hline 8.4174 & 124.5026 & 123.8672 & 123.9709 & 123.8519 & 123.9883 & 123.8644 \\
\hline 8.8571 & 124.3937 & 123.7588 & 123.8611 & 123.7402 & 123.8791 & 123.7532 \\
\hline 9.4411 & 124.2491 & 123.6149 & 123.7154 & 123.592 & 123.7343 & 123.6055 \\
\hline 9.6082 & 124.2089 & 123.5748 & 123.6749 & 123.5507 & 123.694 & 123.5644 \\
\hline 10.0174 & 124.1107 & 123.4772 & 123.576 & 123.45 & 123.5956 & 123.4641 \\
\hline 10.3374 & 124.0341 & 123.4009 & 123.4987 & 123.3714 & 123.5188 & 123.3858 \\
\hline 10.5075 & 123.9932 & 123.3602 & 123.4575 & 123.3294 & 123.4778 & 123.344 \\
\hline 10.8129 & 123.9198 & 123.2871 & 123.3836 & 123.2541 & 123.4043 & 123.269 \\
\hline 10.9501 & 123.8869 & 123.2544 & 123.3504 & 123.2203 & 123.3713 & 123.2354 \\
\hline 11.0961 & 123.8519 & 123.2196 & 123.3151 & 123.1845 & 123.3363 & 123.1996 \\
\hline 11.6971 & 123.7086 & 123.0769 & 123.1707 & 123.0374 & 123.1926 & 123.0531 \\
\hline 12.0081 & 123.6347 & 123.0034 & 123.0962 & 122.9616 & 123.1186 & 122.9776 \\
\hline 12.0291 & 123.6315 & 123.0002 & 123.093 & 122.9583 & 123.1154 & 122.9743 \\
\hline 12.0292 & 123.6315 & 123.0002 & 123.093 & 122.9583 & 123.1154 & 122.9743 \\
\hline 12.2671 & 123.5952 & 122.9641 & 123.0562 & 122.9205 & 123.079 & 122.9368 \\
\hline 12.3884 & 123.5767 & 122.9457 & 123.0374 & 122.9012 & 123.0604 & 122.9176 \\
\hline 12.5244 & 123.5561 & 122.9251 & 123.0165 & 122.8797 & 123.0396 & 122.8963 \\
\hline 12.6619 & 123.3715 & 122.7414 & 123.5686 & 125.2723 & 123.5931 & 125.29 \\
\hline 12.7589 & 123.3569 & 122.7269 & 123.5539 & 125.2574 & 123.5785 & 125.2752 \\
\hline 12.9495 & 123.3283 & 122.6984 & 123.525 & 125.2282 & 123.5499 & 125.2462 \\
\hline
\end{tabular}




\begin{tabular}{|c|c|c|c|c|c|c|}
\hline Distance & $\begin{array}{c}\text { Test } 5105 \\
\text { LL }\end{array}$ & $\begin{array}{c}\text { Test } 595 \\
\text { LL }\end{array}$ & $\begin{array}{c}\text { Test } 4105 \\
\text { LL }\end{array}$ & $\begin{array}{c}\text { Test } 495 \\
\text { LL }\end{array}$ & $\begin{array}{c}\text { Test } 3105 \\
\text { LL }\end{array}$ & $\begin{array}{c}\text { Test } 395 \\
\text { LL }\end{array}$ \\
\hline 13.0549 & 123.3125 & 122.6827 & 123.5091 & 125.2121 & 123.5341 & 125.2302 \\
\hline 13.3859 & 123.2614 & 122.6319 & 123.4576 & 125.1601 & 123.483 & 125.1785 \\
\hline 13.6541 & 123.2223 & 122.5929 & 123.4181 & 125.1202 & 123.4439 & 125.1388 \\
\hline 13.9121 & 123.1843 & 122.5551 & 123.3797 & 125.0814 & 123.4059 & 125.1003 \\
\hline 14.1148 & 123.1546 & 122.5256 & 123.3498 & 125.0512 & 123.3762 & 125.0703 \\
\hline 14.5219 & 123.0881 & 122.4594 & 123.2827 & 124.9834 & 123.3097 & 125.0029 \\
\hline 15.0699 & 122.9986 & 122.3704 & 123.1925 & 124.8923 & 123.2202 & 124.9123 \\
\hline 15.2599 & 122.9677 & 122.3396 & 123.1613 & 124.8607 & 123.1893 & 124.8809 \\
\hline 15.4019 & 122.9451 & 122.3171 & 123.1385 & 124.8377 & 123.1667 & 124.858 \\
\hline 15.5403 & 122.9231 & 122.2952 & 123.1164 & 124.8153 & 123.1447 & 124.8358 \\
\hline 15.8453 & 122.8743 & 122.2466 & 123.0671 & 124.7655 & 123.0959 & 124.7863 \\
\hline 16.1153 & 122.8308 & 122.2033 & 123.0233 & 124.7212 & 123.0524 & 124.7422 \\
\hline 17.4453 & 122.6228 & 121.9964 & 122.8135 & 124.5093 & 122.8444 & 124.5315 \\
\hline 17.6035 & 122.6001 & 121.9737 & 122.7905 & 124.486 & 122.8216 & 124.5085 \\
\hline 17.8465 & 122.565 & 121.9388 & 122.7551 & 124.4503 & 122.7866 & 124.4729 \\
\hline 18.2915 & 122.5014 & 121.8755 & 122.6909 & 124.3854 & 122.7229 & 124.4085 \\
\hline 18.4488 & 122.4787 & 121.8529 & 122.668 & 124.3623 & 122.7003 & 124.3855 \\
\hline 18.6148 & 122.4548 & 121.8292 & 122.6439 & 124.338 & 122.6764 & 124.3613 \\
\hline 18.7818 & 122.4308 & 121.8052 & 122.6196 & 124.3134 & 122.6524 & 124.337 \\
\hline 19.1128 & 122.3831 & 121.7578 & 122.5715 & 124.2648 & 122.6047 & 124.2887 \\
\hline 19.4183 & 122.3373 & 121.7122 & 122.5253 & 124.2181 & 122.5589 & 124.2423 \\
\hline 20.0144 & 122.2616 & 121.6369 & 122.4487 & 124.1408 & 122.4831 & 124.1656 \\
\hline 20.3923 & 122.2142 & 121.5896 & 122.4007 & 124.0924 & 122.4357 & 124.1175 \\
\hline 20.7652 & 122.1714 & 121.5471 & 122.3574 & 124.0487 & 122.3929 & 124.0742 \\
\hline 20.9525 & 122.1495 & 121.5253 & 122.3353 & 124.0264 & 122.371 & 124.052 \\
\hline 21.4666 & 122.0895 & 121.4656 & 122.2746 & 123.9651 & 122.311 & 123.9912 \\
\hline 21.7311 & 122.0585 & 121.4347 & 122.2432 & 123.9334 & 122.28 & 123.9598 \\
\hline 21.9173 & 122.0377 & 121.414 & 122.2221 & 123.9122 & 122.2592 & 123.9387 \\
\hline 22.0473 & 122.0301 & 121.4064 & 122.2143 & 123.9043 & 122.2515 & 123.9309 \\
\hline 22.1883 & 122.0216 & 121.398 & 122.2057 & 123.8956 & 122.2431 & 123.9224 \\
\hline 22.3543 & 122.0117 & 121.3881 & 122.1955 & 123.8854 & 122.2331 & 123.9123 \\
\hline 22.4763 & 122.0048 & 121.3813 & 122.1885 & 123.8783 & 122.2263 & 123.9054 \\
\hline 22.8133 & 121.9857 & 121.3623 & 122.169 & 123.8586 & 122.2072 & 123.886 \\
\hline 22.9733 & 121.9849 & 121.3615 & 122.168 & 123.8577 & 122.2064 & 123.8852 \\
\hline 23.1957 & 121.9834 & 121.36 & 122.1662 & 123.856 & 122.2049 & 123.8837 \\
\hline 23.3361 & 121.9805 & 121.3571 & 122.1631 & 123.8529 & 122.202 & 123.8807 \\
\hline 23.3575 & 121.9801 & 121.3567 & 122.1626 & 123.8524 & 122.2015 & 123.8803 \\
\hline 23.5515 & 121.9761 & 121.3527 & 122.1583 & 123.8481 & 122.1975 & 123.8762 \\
\hline 23.7088 & 121.9729 & 121.3495 & 122.155 & 123.8448 & 122.1944 & 123.873 \\
\hline 24.0118 & 121.9668 & 121.3435 & 122.1485 & 123.8383 & 122.1883 & 123.8668 \\
\hline 24.1251 & 121.9645 & 121.3412 & 122.146 & 123.8359 & 122.186 & 123.8645 \\
\hline 24.3851 & 121.9595 & 121.3362 & 122.1406 & 123.8305 & 122.181 & 123.8594 \\
\hline
\end{tabular}




\begin{tabular}{|l|l|l|l|l|l|l|}
\hline Distance & $\begin{array}{c}\text { Test 5 105 } \\
\text { LL }\end{array}$ & $\begin{array}{c}\text { Test 5 95 } \\
\text { LL }\end{array}$ & $\begin{array}{c}\text { Test 4 105 } \\
\text { LL }\end{array}$ & $\begin{array}{c}\text { Test 4 95 } \\
\text { LL }\end{array}$ & $\begin{array}{c}\text { Test 3 105 } \\
\text { LL }\end{array}$ & $\begin{array}{c}\text { Test 3 95 } \\
\text { LL }\end{array}$ \\
\hline & & & & & & \\
\hline 24.6292 & 121.9544 & 121.3311 & 122.1352 & 123.8252 & 122.1759 & 123.8543 \\
\hline 24.8032 & 121.9512 & 121.3279 & 122.1318 & 123.8217 & 122.1727 & 123.851 \\
\hline 25.0212 & 121.947 & 121.3237 & 122.1273 & 123.8172 & 122.1685 & 123.8467 \\
\hline 25.1302 & 121.9449 & 121.3216 & 122.125 & 123.815 & 122.1664 & 123.8446 \\
\hline 25.3432 & 121.9437 & 121.3204 & 122.1235 & 123.8136 & 122.1652 & 123.8434 \\
\hline 26.3495 & 121.9262 & 121.303 & 122.1046 & 123.7948 & 122.1477 & 123.8256 \\
\hline 26.5235 & 121.9229 & 121.2997 & 122.1011 & 123.7914 & 122.1444 & 123.8223 \\
\hline 26.9295 & 121.9145 & 121.2913 & 122.0921 & 123.7824 & 122.136 & 123.8138 \\
\hline 27.0567 & 121.9119 & 121.2888 & 122.0894 & 123.7797 & 122.1335 & 123.8112 \\
\hline 27.3222 & 121.905 & 121.2819 & 122.0821 & 123.7725 & 122.1266 & 123.8042 \\
\hline 27.3813 & 121.903 & 121.2799 & 122.08 & 123.7704 & 122.1246 & 123.8022 \\
\hline 27.6828 & 121.8927 & 121.2697 & 122.0693 & 123.7596 & 122.1142 & 123.7917 \\
\hline 27.8391 & 121.8868 & 121.2638 & 124.5168 & 125.412 & 122.1083 & 123.7857 \\
\hline 27.9641 & 121.8825 & 121.2595 & 124.5124 & 125.4075 & 122.104 & 123.7813 \\
\hline 28.1491 & 121.8761 & 121.2531 & 124.5059 & 125.4009 & 122.0976 & 123.7749 \\
\hline 28.4312 & 121.8664 & 121.2435 & 124.496 & 125.391 & 122.0879 & 123.7651 \\
\hline 28.6137 & 121.8619 & 121.239 & 124.4914 & 125.3864 & 122.0834 & 123.7605 \\
\hline 28.983 & 121.8532 & 121.2304 & 124.4826 & 125.3775 & 122.0747 & 123.7517 \\
\hline 29.633 & 121.8389 & 121.2161 & 124.468 & 125.3628 & 122.0604 & 123.7372 \\
\hline 29.925 & 121.8332 & 121.2105 & 124.4622 & 125.357 & 122.0548 & 123.7315 \\
\hline 30.264 & 121.83 & 121.2073 & 124.459 & 125.3537 & 122.0516 & 123.7283 \\
\hline 30.904 & 121.8249 & 121.2022 & 124.4537 & 125.3484 & 122.0464 & 123.723 \\
\hline 31.183 & 121.8238 & 121.2011 & 124.4526 & 125.3472 & 122.0453 & 123.7219 \\
\hline 31.4215 & 121.823 & 121.2003 & 124.4517 & 125.3464 & 122.0445 & 123.7211 \\
\hline 31.6365 & 121.8226 & 121.1999 & 124.4514 & 125.346 & 122.0442 & 123.7208 \\
\hline 31.7505 & 121.8226 & 121.1999 & 124.4513 & 125.346 & 122.0441 & 123.7207 \\
\hline 31.8455 & 121.8222 & 121.1995 & 124.4509 & 125.3456 & 122.0437 & 123.7203 \\
\hline 32.0297 & 121.8204 & 121.1977 & 124.4491 & 125.3438 & 122.0419 & 123.7185 \\
\hline
\end{tabular}




\begin{tabular}{|l|l|l|l|l|}
\hline Distance & $\begin{array}{c}\text { Test 2 105 } \\
\text { LL }\end{array}$ & $\begin{array}{c}\text { Test 2 95 } \\
\text { LL }\end{array}$ & $\begin{array}{c}\text { Test 1 105 } \\
\text { LL }\end{array}$ & $\begin{array}{c}\text { Test 1 95 } \\
\text { LL }\end{array}$ \\
\hline & & & & \\
\hline 0.1034 & 124.8365 & 112.7094 & 124.8365 & 112.9372 \\
\hline 0.2378 & 126.2705 & 126.2252 & 126.2705 & 114.2202 \\
\hline 0.3218 & 126.2443 & 126.199 & 126.2443 & 114.1963 \\
\hline 0.3218 & 126.2443 & 126.199 & 126.2443 & 114.1963 \\
\hline 0.5288 & 126.1855 & 126.1402 & 126.1855 & 114.1426 \\
\hline 0.7648 & 126.1169 & 126.0717 & 126.1169 & 114.08 \\
\hline 1.3788 & 125.9383 & 125.8932 & 125.9383 & 113.917 \\
\hline 2.583 & 125.5886 & 125.5436 & 125.5886 & 113.5978 \\
\hline 2.666 & 125.5645 & 125.5195 & 125.5645 & 113.5758 \\
\hline 3.206 & 125.4131 & 125.3682 & 125.4131 & 113.4376 \\
\hline 3.429 & 125.3507 & 125.3058 & 125.3507 & 113.3806 \\
\hline 3.716 & 125.2703 & 125.2255 & 125.2703 & 113.3072 \\
\hline 5.754 & 124.7003 & 124.6557 & 124.7003 & 112.7869 \\
\hline 6.798 & 124.4185 & 124.3741 & 124.4185 & 112.5297 \\
\hline 6.9262 & 124.3841 & 124.3397 & 124.3841 & 112.4983 \\
\hline 7.0603 & 124.3511 & 124.3067 & 124.3511 & 112.4681 \\
\hline 7.2034 & 124.3159 & 124.2715 & 124.3159 & 112.436 \\
\hline 7.3784 & 124.2729 & 124.2286 & 124.2729 & 112.3968 \\
\hline 7.7094 & 124.1919 & 124.1475 & 124.1919 & 112.3228 \\
\hline 7.9064 & 124.1438 & 124.0995 & 124.1438 & 112.2789 \\
\hline 7.9064 & 124.1438 & 124.0995 & 124.1438 & 112.2789 \\
\hline 8.2464 & 124.0595 & 124.0152 & 124.0595 & 112.202 \\
\hline 8.4174 & 124.0171 & 123.9728 & 124.0171 & 112.1632 \\
\hline 8.8571 & 123.9086 & 123.8643 & 123.9086 & 112.0642 \\
\hline 9.4411 & 123.7646 & 123.7204 & 123.7646 & 111.9327 \\
\hline 9.6082 & 123.7245 & 123.6803 & 123.7245 & 111.8962 \\
\hline 10.0174 & 123.6268 & 123.5827 & 123.6268 & 111.807 \\
\hline 10.3374 & 123.5505 & 123.5064 & 123.5505 & 111.7373 \\
\hline 10.5075 & 123.5097 & 123.4656 & 123.5097 & 111.7001 \\
\hline 10.8129 & 123.4366 & 123.3926 & 123.4366 & 111.6334 \\
\hline 10.9501 & 123.4038 & 123.3598 & 123.4038 & 111.6035 \\
\hline 11.0961 & 123.369 & 123.325 & 123.369 & 111.5717 \\
\hline 11.6971 & 123.2263 & 123.1823 & 123.2263 & 111.4414 \\
\hline 12.0081 & 123.1527 & 123.1088 & 123.1527 & 111.3743 \\
\hline 12.0291 & 123.1495 & 123.1056 & 123.1495 & 111.3714 \\
\hline 12.0292 & 123.1495 & 123.1056 & 123.1495 & 111.3714 \\
\hline 12.2671 & 123.1134 & 123.0695 & 123.1134 & 111.3383 \\
\hline 12.3884 & 123.0949 & 123.051 & 123.0949 & 111.3215 \\
\hline 12.5244 & 123.0744 & 123.0305 & 123.0744 & 111.3028 \\
\hline 12.6619 & 122.8905 & 122.8467 & 122.8905 & 111.1349 \\
\hline 12.7589 & 122.876 & 122.8322 & 122.876 & 111.1217 \\
\hline 12.9495 & 122.8475 & 122.8037 & 122.8475 & 111.0956 \\
\hline & & & & \\
\hline
\end{tabular}




\begin{tabular}{|l|l|l|l|l|}
\hline Distance & $\begin{array}{c}\text { Test 2 105 } \\
\text { LL }\end{array}$ & $\begin{array}{c}\text { Test 2 95 } \\
\text { LL }\end{array}$ & $\begin{array}{c}\text { Test 1 105 } \\
\text { LL }\end{array}$ & \multicolumn{1}{|c|}{\begin{tabular}{c} 
Lest 1 95 \\
\hline
\end{tabular}} \\
\hline 13.0549 & 122.8317 & 122.7879 & 122.8317 & 111.0813 \\
\hline 13.3859 & 122.7809 & 122.7371 & 122.7809 & 111.0349 \\
\hline 13.6541 & 122.7419 & 122.6982 & 122.7419 & 110.9993 \\
\hline 13.9121 & 122.7041 & 122.6603 & 122.7041 & 110.9647 \\
\hline 14.1148 & 122.6746 & 122.6308 & 122.6746 & 110.9378 \\
\hline 14.5219 & 122.6083 & 122.5646 & 122.6083 & 110.8773 \\
\hline 15.0699 & 122.5192 & 122.4756 & 122.5192 & 110.796 \\
\hline 15.2599 & 122.4884 & 122.4448 & 122.4884 & 110.7679 \\
\hline 15.4019 & 122.4659 & 122.4223 & 122.4659 & 110.7473 \\
\hline 15.5403 & 122.4441 & 122.4004 & 122.4441 & 110.7274 \\
\hline 15.8453 & 122.3954 & 122.3518 & 122.3954 & 110.683 \\
\hline 16.1153 & 122.3521 & 122.3085 & 122.3521 & 110.6435 \\
\hline 17.4453 & 122.145 & 122.1015 & 122.145 & 110.4545 \\
\hline 17.6035 & 122.1223 & 122.0788 & 122.1223 & 110.4338 \\
\hline 17.8465 & 122.0874 & 122.0439 & 122.0874 & 110.4019 \\
\hline 18.2915 & 122.024 & 121.9806 & 122.024 & 110.3441 \\
\hline 18.4488 & 122.0015 & 121.9581 & 122.0015 & 110.3235 \\
\hline 18.6148 & 121.9777 & 121.9343 & 121.9777 & 110.3018 \\
\hline 18.7818 & 121.9537 & 121.9103 & 121.9537 & 110.2799 \\
\hline 19.1128 & 121.9063 & 121.8629 & 121.9063 & 110.2366 \\
\hline 19.4183 & 121.8607 & 121.8173 & 121.8607 & 110.195 \\
\hline 20.0144 & 121.7853 & 121.7419 & 121.7853 & 110.1261 \\
\hline 20.3923 & 121.738 & 121.6947 & 121.738 & 110.083 \\
\hline 20.7652 & 121.6954 & 121.6522 & 121.6954 & 110.0442 \\
\hline 20.9525 & 121.6736 & 121.6304 & 121.6736 & 110.0243 \\
\hline 21.4666 & 121.6139 & 121.5707 & 121.6139 & 109.9697 \\
\hline 21.7311 & 121.583 & 121.5398 & 121.583 & 109.9416 \\
\hline 21.9173 & 121.5623 & 121.5191 & 121.5623 & 109.9226 \\
\hline 22.0473 & 121.5547 & 121.5114 & 121.5547 & 109.9157 \\
\hline 22.1883 & 121.5462 & 121.503 & 121.5462 & 109.908 \\
\hline 22.3543 & 121.5363 & 121.4931 & 121.5363 & 109.899 \\
\hline 22.4763 & 121.5295 & 121.4863 & 121.5295 & 109.8928 \\
\hline 22.8133 & 121.5105 & 121.4673 & 121.5105 & 109.8754 \\
\hline 22.9733 & 121.5097 & 121.4665 & 121.5097 & 109.8747 \\
\hline 23.1957 & 121.5082 & 121.465 & 121.5082 & 109.8733 \\
\hline 23.3361 & 121.5053 & 121.4621 & 121.5053 & 109.8706 \\
\hline 23.3575 & 121.5049 & 121.4617 & 121.5049 & 109.8702 \\
\hline 23.5515 & 121.5009 & 121.4577 & 121.5009 & 109.8666 \\
\hline 23.7088 & 121.4977 & 121.4545 & 121.4977 & 109.8637 \\
\hline 24.0118 & 121.4916 & 121.4484 & 121.4916 & 109.8582 \\
\hline 24.1251 & 121.4894 & 121.4462 & 121.4894 & 109.8561 \\
\hline 24.3851 & 121.4843 & 121.4411 & 121.4843 & 109.8515 \\
\hline & & & & \\
\hline
\end{tabular}




\begin{tabular}{|l|l|l|l|l|}
\hline Distance & $\begin{array}{c}\text { Test 2 105 } \\
\text { LL }\end{array}$ & $\begin{array}{c}\text { Test 2 95 } \\
\text { LL }\end{array}$ & $\begin{array}{c}\text { Test 1 105 } \\
\text { LL }\end{array}$ & $\begin{array}{c}\text { Test 1 95 } \\
\text { LL }\end{array}$ \\
\hline 24.6292 & 121.4793 & 121.4361 & 121.4793 & 109.8469 \\
\hline 24.8032 & 121.4761 & 121.4329 & 121.4761 & 109.844 \\
\hline 25.0212 & 121.4719 & 121.4287 & 121.4719 & 109.8401 \\
\hline 25.1302 & 121.4698 & 121.4266 & 121.4698 & 109.8382 \\
\hline 25.3432 & 121.4686 & 121.4254 & 121.4686 & 109.8371 \\
\hline 26.3495 & 121.4512 & 121.408 & 121.4512 & 109.8212 \\
\hline 26.5235 & 121.4479 & 121.4047 & 121.4479 & 109.8182 \\
\hline 26.9295 & 121.4395 & 121.3963 & 121.4395 & 109.8106 \\
\hline 27.0567 & 121.437 & 121.3938 & 121.437 & 109.8083 \\
\hline 27.3222 & 121.4301 & 121.3869 & 121.4301 & 109.802 \\
\hline 27.3813 & 121.4281 & 121.3849 & 121.4281 & 109.8002 \\
\hline 27.6828 & 121.4178 & 121.3746 & 121.4178 & 109.7908 \\
\hline 27.8391 & 121.4119 & 121.3688 & 121.4119 & 109.7854 \\
\hline 27.9641 & 121.4076 & 121.3645 & 121.4076 & 109.7815 \\
\hline 28.1491 & 121.4013 & 121.3581 & 121.4013 & 109.7757 \\
\hline 28.4312 & 121.3916 & 121.3485 & 121.3916 & 109.7669 \\
\hline 28.6137 & 121.3871 & 121.344 & 121.3871 & 109.7628 \\
\hline 28.983 & 121.3785 & 121.3353 & 121.3785 & 109.7549 \\
\hline 29.633 & 121.3642 & 121.3211 & 121.3642 & 109.7419 \\
\hline 29.925 & 121.3586 & 121.3155 & 121.3586 & 109.7368 \\
\hline 30.264 & 121.3554 & 121.3123 & 121.3554 & 109.7339 \\
\hline 30.904 & 121.3503 & 121.3072 & 121.3503 & 109.7292 \\
\hline 31.183 & 121.3492 & 121.3061 & 121.3492 & 109.7282 \\
\hline 31.4215 & 121.3484 & 121.3053 & 121.3484 & 109.7274 \\
\hline 31.6365 & 121.3481 & 121.3049 & 121.3481 & 109.7271 \\
\hline 31.7505 & 121.348 & 121.3049 & 121.348 & 109.7271 \\
\hline 31.8455 & 121.3476 & 121.3045 & 121.3476 & 109.7267 \\
\hline 32.0297 & 121.3459 & 121.3027 & 121.3459 & 109.7251 \\
\hline & & & & \\
\hline
\end{tabular}

A summary of simulation information for each test, load condition, primary substation voltage, and traditional voltage regulation method is displayed in Table 22. This table summarizes the significant information collected from each simulation to show how these significant data change for each simulation. The data given for each test are:

- Test number

- Primary voltage: $95 \%$ or $105 \%$

- Load condition: HL or LL

- Substation transformer LTC tap setting $( \pm 16)$

- $\quad$ Step VR tap settings $( \pm 32)$

- Capacitor kVArc applied

- Line currents $\left(\mathrm{I}_{\mathrm{A}}, \mathrm{I}_{\mathrm{B}}, \mathrm{I}_{\mathrm{C}}\right)$ at Node [0]
- Lowest three-phase voltage: $\mathrm{V}_{\mathrm{A}}$, $\mathrm{V}_{\mathrm{B}}, \mathrm{V}_{\mathrm{C}}$

- Lowest single-phase voltage

- Highest unbalanced current: $\mathrm{I}_{2} / \mathrm{I}_{1} \%$

- Highest unbalanced voltage: $\mathrm{V}_{2} / \mathrm{V}_{1} \%$

- Kilowatt losses per phase and total

- Kilovar losses per phase and total

- Released capacity per phase and total. 
Table 22. Simulation Summary Data for Tests 1 Through 8

\begin{tabular}{|c|c|c|c|c|c|c|c|c|c|c|}
\hline \multirow[b]{2}{*}{ Test No. } & \multirow[b]{2}{*}{$\begin{array}{c}\text { Pri. Volt } \\
\%\end{array}$} & \multirow[b]{2}{*}{ Load } & \multirow[b]{2}{*}{$\begin{array}{c}\text { LTC Tap } \\
\text { / Reg } \\
\text { Tap }\end{array}$} & \multicolumn{3}{|c|}{ Node 01} & \multicolumn{3}{|c|}{ Lowest $3 \Phi$ Voltage } & \multirow[t]{2}{*}{$\begin{array}{c}\text { Lowest } \\
1 \Phi \\
\text { Voltage }\end{array}$} \\
\hline & & & & $\mathrm{IA}$ & IB & IC & VA & VB & VC & \\
\hline 1 & 95 & $\mathrm{HL}$ & NA & 451.10 & 511.63 & 498.20 & 101.10 & 102.32 & 102.04 & 100.81 \\
\hline 1 & 95 & LL & NA & 208.36 & 240.56 & 234.39 & 109.72 & 110.18 & 110.01 & 109.59 \\
\hline 1 & 105 & $\mathrm{HL}$ & NA & 542.98 & 614.92 & 598.73 & 110.14 & 111.60 & 111.22 & 109.80 \\
\hline 1 & 105 & $\mathrm{LL}$ & $\mathrm{NA}$ & 228.26 & 263.55 & 256.78 & 123.06 & 118.60 & 117.22 & 121.20 \\
\hline 2 & 95 & $\mathrm{HL}$ & 16 & 539.33 & 610.54 & 594.13 & 109.78 & 111.21 & 110.80 & 109.44 \\
\hline 2 & 95 & $\mathrm{LL}$ & 16 & 228.19 & 263.43 & 256.63 & 123.02 & 118.60 & 117.22 & 121.16 \\
\hline 2 & 105 & $\mathrm{HL}$ & 6 & 579.58 & 655.94 & 638.52 & 113.75 & 115.29 & 114.86 & 113.39 \\
\hline 2 & 105 & LL & 0 & 228.26 & 263.55 & 256.78 & 123.06 & 118.60 & 117.22 & 121.20 \\
\hline 3 & 95 & $\mathrm{HL}$ & 16 & 566.73 & 640.04 & 620.83 & 112.87 & 113.35 & 111.81 & 111.25 \\
\hline & $\operatorname{VR}(1) 3$ & 32,3 & & & & & & & & \\
\hline 3 & 95 & LL & 16 & 232.26 & 267.90 & 260.58 & 122.89 & 118.60 & 117.22 & 121.79 \\
\hline & VR (1) & $, 14,12$ & & & & & & & & \\
\hline 3 & 105 & $\mathrm{HL}$ & 6 & 608.51 & 687.11 & 666.73 & 117.11 & 117.64 & 116.00 & 115.40 \\
\hline & $\operatorname{VR}(1) 3$ & 32,32 & & & & & & & & \\
\hline 3 & 105 & LL & 0 & 229.42 & 264.82 & 258.19 & 123.03 & 118.60 & 117.22 & 121.89 \\
\hline & VR (1) & $, 4,5$ & & & & & & & & \\
\hline 4 & 95 & $\mathrm{HL}$ & 16 & 576.90 & 650.25 & 626.60 & 112.49 & 112.98 & 111.65 & 111.10 \\
\hline & $\operatorname{VR}(1) 3$ & 32,32 & & & & & & & & \\
\hline & VR (2) 1 & 13,13 & & & & & & & & \\
\hline 4 & 95 & LL & 16 & 232.70 & 268.15 & 260.71 & 122.87 & 118.60 & 117.22 & 121.78 \\
\hline & VR (1) 1 & $, 14,14$ & & & & & & & & \\
\hline & VR (2) & $2,1,1$ & & & & & & & & \\
\hline 4 & 105 & $\mathrm{HL}$ & 6 & 614.37 & 692.81 & 669.94 & 116.90 & 117.44 & 115.92 & 115.32 \\
\hline & $\operatorname{VR}(1) 3$ & 32,32 & & & & & & & & \\
\hline & VR $(2$ & $9,7,7$ & & & & & & & & \\
\hline 4 & 105 & LL & 0 & 230.08 & 265.30 & 258.44 & 123.00 & 118.60 & 117.22 & 121.93 \\
\hline & VR (1) & , 4,5 & & & & & & & & \\
\hline & VR $(2$ & $3,2,2$ & & & & & & & & \\
\hline 5 & 95 & $\mathrm{HL}$ & 16 & 529.93 & 603.50 & 586.65 & 110.75 & 112.16 & 111.80 & 110.40 \\
\hline & Cap (1) & $\overline{0 \mathrm{kVA}}$ & & & & & & & & \\
\hline 5 & 95 & LL & 14 & 211.04 & 245.95 & 238.81 & 122.91 & 118.60 & 117.22 & 121.05 \\
\hline & Cap (1) & $\overline{00 \mathrm{kVA}}$ & & & & & & & & \\
\hline 5 & 105 & $\mathrm{HL}$ & 5 & 563.43 & 641.46 & 623.81 & 114.12 & 115.63 & 115.26 & 113.75 \\
\hline & Cap (1) & $0 \mathrm{kVA}$ & & & & & & & & \\
\hline 5 & 105 & LL & -1 & 212.02 & 247.12 & 239.99 & 123.54 & 118.60 & 117.22 & 121.67 \\
\hline & Cap (1) & $\overline{0 \mathrm{kVA}}$ & & & & & & & & \\
\hline
\end{tabular}




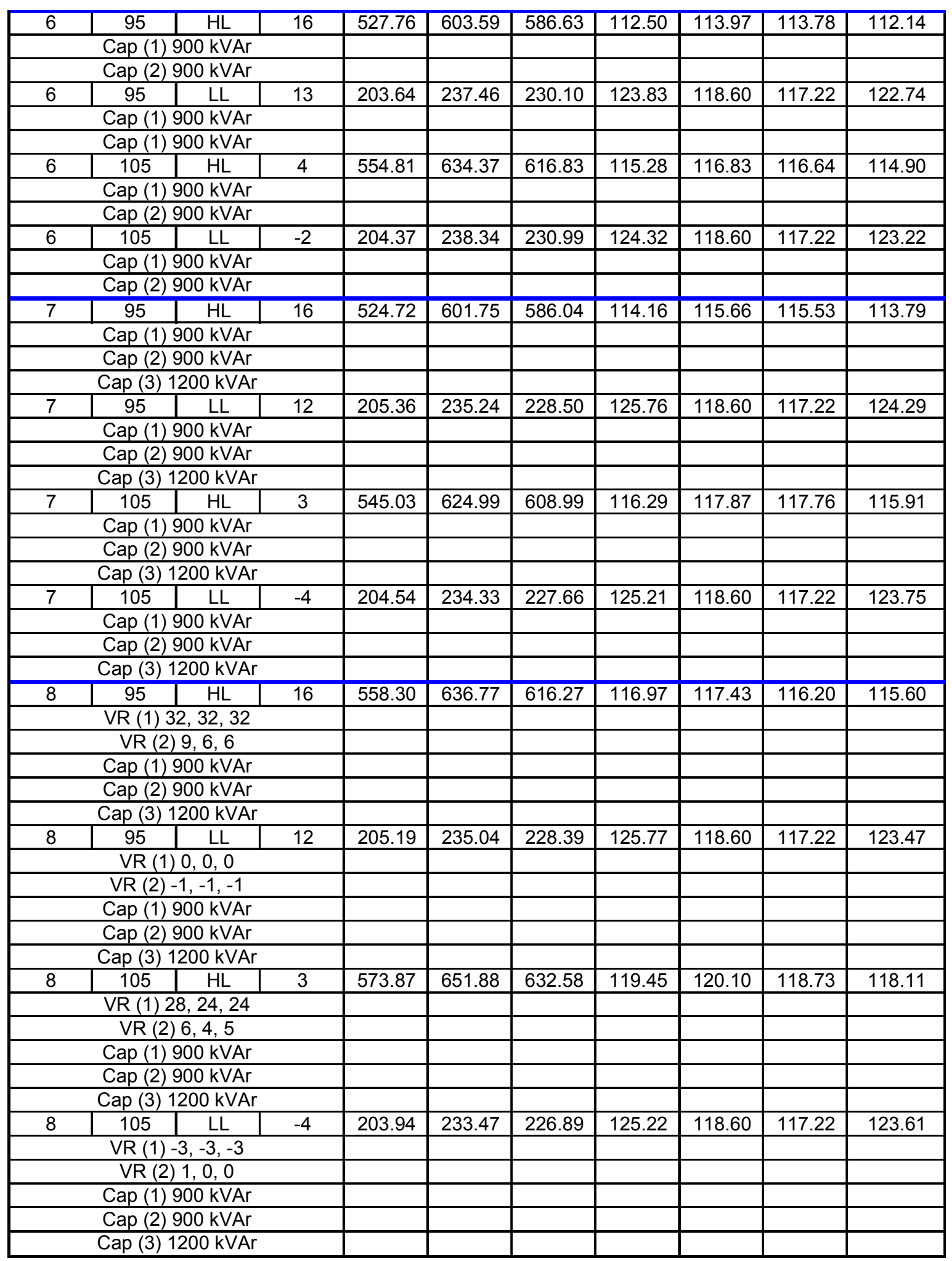




\begin{tabular}{|c|c|c|c|c|c|c|c|c|c|c|}
\hline \multirow{2}{*}{$\begin{array}{l}\text { Highest } \\
\text { |2/l1\% }\end{array}$} & \multirow{2}{*}{$\begin{array}{l}\text { Highest } \\
\text { V2/V1 \% }\end{array}$} & \multicolumn{3}{|c|}{ kW Losses/Ф \& Total } & \multicolumn{3}{|c|}{ kVAr Losses/Ф \& Total } & \multicolumn{3}{|c|}{$\begin{array}{c}\text { Released Capacity \% / } \\
\text { Total }\end{array}$} \\
\hline & & A & $\mathrm{B}$ & $\mathrm{C}$ & $A$ & $\mathrm{~B}$ & C & A & $\mathrm{B}$ & C \\
\hline \multirow[t]{2}{*}{100.06} & 1.32 & 159.26 & 131.82 & 144.29 & 509.98 & 664.33 & 637.60 & 32.23 & 32.07 & 32.03 \\
\hline & & & 435.37 & & & 1811.91 & & & 32.11 & \\
\hline \multirow[t]{2}{*}{100.15} & 1.26 & 53.79 & 43.90 & 53.40 & 111.47 & 150.07 & 146.52 & 16.88 & 16.87 & 16.87 \\
\hline & & & 151.09 & & & 408.06 & & & 16.87 & \\
\hline \multirow[t]{2}{*}{100.06} & 1.43 & 218.89 & 180.17 & 195.86 & 739.94 & 959.69 & 921.89 & 9.84 & 9.76 & 9.72 \\
\hline & & & 594.92 & & & 2621.52 & & & 9.77 & \\
\hline \multirow[t]{2}{*}{100.15} & 1.26 & 59.67 & 48.78 & 58.90 & 133.79 & 180.12 & 175.86 & -0.65 & -0.66 & -0.66 \\
\hline & & & 167.35 & & & 489.77 & & & -0.66 & \\
\hline \multirow[t]{2}{*}{100.06} & 1.44 & 218.28 & 179.34 & 197.37 & 768.13 & 992.13 & 951.65 & 10.29 & 10.25 & 10.25 \\
\hline & & & 594.99 & & & 2711.91 & & & 10.26 & \\
\hline \multirow[t]{2}{*}{100.15} & 1.26 & 60.03 & 48.98 & 59.62 & 140.56 & 188.56 & 183.80 & -0.79 & -0.79 & -0.78 \\
\hline & & & 168.63 & & & 512.92 & & & -0.79 & \\
\hline \multirow[t]{2}{*}{100.06} & 1.48 & 246.50 & 202.37 & 220.58 & 859.41 & 1111.33 & 1067.28 & -0.10 & -0.13 & -0.15 \\
\hline & & & 669.45 & & & 3038.02 & & & -0.13 & \\
\hline \multirow[t]{2}{*}{100.15} & 1.26 & 59.67 & 48.78 & 58.90 & 133.79 & 180.12 & 175.86 & -0.65 & -0.66 & -0.66 \\
\hline & & & 167.35 & & & 489.77 & & & -0.66 & \\
\hline \multirow[t]{2}{*}{100.06} & 1.49 & 240.17 & 192.88 & 208.79 & 856.27 & 1101.07 & 1048.32 & 5.73 & 5.91 & 6.22 \\
\hline & & & 641.84 & & & 3005.66 & & & 5.96 & \\
\hline \multirow[t]{2}{*}{100.15} & 1.26 & 61.32 & 49.78 & 60.28 & 145.82 & 195.31 & 189.69 & -2.59 & -2.50 & -2.33 \\
\hline & & & 171.38 & & & 530.82 & & & -2.47 & \\
\hline \multirow[t]{2}{*}{100.06} & 1.52 & 271.04 & 217.50 & 233.26 & 956.33 & 1231.29 & 1173.85 & -5.10 & -4.88 & -4.58 \\
\hline & & & 721.80 & & & 3361.47 & & & -4.85 & \\
\hline \multirow[t]{2}{*}{100.15} & 1.26 & 59.99 & 49.02 & 59.15 & 135.22 & 181.91 & 177.88 & -1.16 & -1.14 & -1.21 \\
\hline & & & 168.16 & & & 495.01 & & & -1.17 & \\
\hline \multirow[t]{2}{*}{100.06} & 1.44 & 251.98 & 196.59 & 210.46 & 894.06 & 1146.18 & 1070.86 & 4.04 & 4.41 & 5.35 \\
\hline & & & 659.03 & & & 3111.10 & & & 4.62 & \\
\hline & & & & & & & & & & \\
\hline \multirow[t]{2}{*}{100.15} & 1.26 & 61.52 & 49.75 & 60.31 & 146.51 & 195.74 & 189.85 & -2.79 & -2.59 & -2.38 \\
\hline & & & 171.58 & & & 532.10 & & & -2.58 & \\
\hline \multirow{2}{*}{100.06} & 1.48 & 278.24 & 219.52 & 234.25 & 979.06 & 1257.47 & 1186.79 & -6.11 & -5.75 & -5.08 \\
\hline & & & 732.01 & & & 3423.32 & & & -5.64 & \\
\hline & & & & & & & & & & \\
\hline \multirow[t]{3}{*}{100.15} & 1.26 & 60.29 & 49.03 & 59.19 & 136.17 & 182.72 & 178.21 & -1.45 & -1.33 & -1.31 \\
\hline & & & 168.51 & & & 497.10 & & & -1.36 & \\
\hline & & & & & & & & & & \\
\hline 100.06 & 1.42 & 215.79 & 182.39 & 200.36 & 752.38 & 977.98 & 941.40 & 11.85 & 11.28 & 11.38 \\
\hline & & & 598.54 & & & 2671.76 & & & 11.49 & \\
\hline 100.15 & 1.26 & 56.68 & 47.82 & 58.36 & 124.56 & 167.85 & 165.15 & 7.91 & 7.04 & 7.35 \\
\hline & & & 162.86 & & & 457.56 & & & 7.41 & \\
\hline 100.06 & 1.45 & 238.77 & 201.82 & 219.42 & 821.34 & 1069.08 & 1030.55 & 3.31 & 2.72 & 2.78 \\
\hline & & & 660.01 & & & 2920.97 & & & 2.92 & \\
\hline 100.15 & 1.26 & 56.63 & 47.95 & 58.06 & 120.21 & 162.41 & 160.21 & 7.14 & 6.25 & 6.55 \\
\hline & & & 162.64 & & & 442.83 & & & 6.62 & \\
\hline
\end{tabular}




\begin{tabular}{|c|c|c|c|c|c|c|c|c|c|c|}
\hline 100.06 & 1.36 & 213.16 & 187.86 & 201.94 & 739.70 & 973.85 & 939.19 & 12.21 & 11.27 & 11.39 \\
\hline & & & 602.96 & & & 2652.74 & & & 11.60 & \\
\hline 10 & 100 & 5211 & 1790 & 5706 & 11221 & 15078 & 1527 & 1168 & 1080 & 1120 \\
\hline 100.15 & $1 . \angle 0$ & 53.44 & $\frac{41.09}{158.19}$ & 57.06 & 113.31 & $\frac{153.12}{419.79}$ & 152.16 & 17.68 & $\frac{10.80}{11.23}$ & 11.28 \\
\hline & & & & & & & & & & \\
\hline 100.06 & 1.38 & 231.00 & 203.74 & 216.63 & 786.79 & 1037.76 & 1002.45 & 5.41 & 4.41 & 4.50 \\
\hline & & & 651.37 & & & 2827.00 & & & 4.75 & \\
\hline & & & & & & & & & & \\
\hline 100.15 & 1.26 & 53.28 & 47.79 & 56.74 & 109.03 & 148.31 & 147.93 & 11.09 & 10.19 & 10.66 \\
\hline & & & 157.81 & & & 405.27 & & & 10.63 & \\
\hline & & & & & & & & & & \\
\hline 100.06 & 1.31 & 214.50 & 191.42 & 207.62 & 735.61 & 974.26 & 940.81 & 12.72 & 11.54 & 11.48 \\
\hline & & & 613.54 & & & 2650.68 & & & 11.88 & \\
\hline & & & & & & & & & & \\
\hline 10015 & 190 & 5100 & 1720 & 5700 & 11620 & 15160 & 151 & 1110 & 1210 & 1211 \\
\hline & 1.26 & 54.09 & $\frac{47.32}{159.27}$ & 57.86 & 116.29 & $\frac{151.68}{419.28}$ & 151.31 & 11.49 & $\frac{12.18}{12.06}$ & 12.44 \\
\hline & & & & & & & & & & \\
\hline 10005 & & & & & & & & & & \\
\hline 100.05 & 1.33 & 227.32 & $\frac{203.40}{648.82}$ & 218.10 & 761.52 & $\frac{1011.00}{27506}$ & 978.17 & 7.68 & $\frac{6.44}{6.79}$ & 6.32 \\
\hline & & & & & & & & & & \\
\hline & & & & & & & & & & \\
\hline 100.15 & 1.26 & 53.41 & 47.01 & 57.10 & 109.99 & 143.76 & 144.07 & 12.23 & 12.90 & 13.15 \\
\hline & & & 157.52 & & & 397.82 & & & 12.78 & \\
\hline & & & & & & & & & & \\
\hline & & & & & & & & & & \\
\hline 100.06 & 1.31 & 242.97 & 207.48 & 220.18 & 843.99 & 1105.93 & 1049.85 & 7.13 & 6.39 & 6.91 \\
\hline & & & 670.63 & & & 2999.77 & & & 6.80 & \\
\hline & & & & & & & & & & \\
\hline & & & & & & & & & & \\
\hline & & & & & & & & & & \\
\hline 100.15 & 1.26 & 54.02 & 47.28 & 57.86 & 116.06 & 151.36 & 151.16 & 11.56 & 12.25 & 12.48 \\
\hline & & & 159.16 & & & 418.58 & & & 12.12 & \\
\hline & & & & & & & & & & \\
\hline & & & & & & & & & & \\
\hline & & & & & & & & & & \\
\hline 10005 & 197 & 2522 & 211410 & 2002 & 0510 & 11115 & 100100 & 200 & 28 & 270 \\
\hline & & & $\frac{214.40}{695.05}$ & & & 3028.18 & & & $\frac{2.42}{2.63}$ & \\
\hline & & & & & & & & & & \\
\hline & & & & & & & & & & \\
\hline & & & & & & & & & & \\
\hline 100.15 & 1.26 & 53.29 & 46.80 & 56.98 & 109.40 & 142.70 & 143.05 & 12.49 & 13.22 & 13.44 \\
\hline & & & 157.07 & & & 395.15 & & & 13.07 & \\
\hline & & & & & & & & & & \\
\hline & & & & & & & & & & \\
\hline & & & & & & & & & & \\
\hline & & & & & & & & & & \\
\hline
\end{tabular}


Because line-to-line loads and line-to-neutral single-phase loads create a $100 \%$ load imbalance at that node on the circuit, the highest load imbalance data are not very meaningful. For proof of $100 \%$ load imbalance, see Appendix B.2.

Therefore, a separate load imbalance, Table 23, was assembled to show the imbalance at Node 0 and at the source of the circuit and the range of imbalance throughout the circuit (low to high excluding $100 \%$ ). 
Table 23. Simulation Summary Data for Unbalanced Loading (Tests 1 Through 8)

\begin{tabular}{|c|c|c|c|c|c|c|c|}
\hline \multirow[b]{2}{*}{ Test No. } & \multirow[b]{2}{*}{ Pri. Volt $\%$} & \multirow[b]{2}{*}{ Load } & \multirow[b]{2}{*}{$\begin{array}{l}\text { LTC Tap / } \\
\text { Reg Tap }\end{array}$} & \multirow[b]{2}{*}{ Node 01} & \multicolumn{3}{|c|}{$I_{2} / I_{1} \%$} \\
\hline & & & & & Low & $\begin{array}{l}\text { High } \\
(<100)\end{array}$ & Max \\
\hline 1 & \begin{tabular}{|l|}
95 \\
\end{tabular} & $\mathrm{HL}$ & NA & 3.77 & 1.0444 & 88.45 & 100.06 \\
\hline & & & & & & & \\
\hline 1 & 95 & LL & NA & 4.72 & 2.4117 & 85.38 & 100.15 \\
\hline 1 & 105 & $\overline{\mathrm{HL}}$ & NA & 3.73 & 1.1119 & 88.42 & 100.06 \\
\hline & & & & & & & \\
\hline 1 & 105 & $\mathrm{LL}$ & NA & 4.72 & 2.4123 & 85.38 & 100.15 \\
\hline & & & & & & & \\
\hline 2 & 95 & $\mathrm{HL}$ & 16 & 3.70 & 1.1098 & 88.41 & 100.06 \\
\hline & & & & & & & \\
\hline 2 & 95 & LL & 16 & 4.71 & 2.399 & 85.38 & 100.15 \\
\hline & & & & & & & \\
\hline 2 & 105 & $\mathrm{HL}$ & 6 & 3.71 & 1.1348 & 88.40 & 100.06 \\
\hline & & & & & & & \\
\hline 2 & 105 & $\mathrm{LL}$ & 0 & 4.72 & 2.4123 & 85.38 & 100.15 \\
\hline & & & & & & & \\
\hline 3 & 95 & $\mathrm{HL}$ & 16 & 3.59 & 1.1817 & 88.42 & 100.06 \\
\hline & $\operatorname{VR}(1) 3$ & 32,32 & & & & & \\
\hline 3 & 95 & $\mathrm{LL}$ & 16 & 4.66 & 2.3741 & 85.38 & 100.15 \\
\hline & VR (1) 1 & 14,14 & & & & & \\
\hline 3 & \begin{tabular}{l|l}
105 \\
\end{tabular} & $\mathrm{HL}$ & 6 & 3.60 & 1.1907 & 88.41 & 100.06 \\
\hline & VR (1) 3 & 32,32 & & & & & \\
\hline 3 & 105 & $\mathrm{LL}$ & 0 & 4.71 & 2.4308 & 85.38 & 100.15 \\
\hline & VR (1) & 4,5 & & & & & \\
\hline 4 & 95 & $\mathrm{HL}$ & 16 & 3.44 & 0.9068 & 88.46 & 100.06 \\
\hline & $\operatorname{VR}(1) 3$ & 32,32 & & & & & \\
\hline & VR (2) 1 & 13,13 & & & & & \\
\hline 4 & 95 & LL & 16 & 4.63 & 2.3226 & 85.38 & 100.15 \\
\hline & VR (1) 1 & 14,14 & & & & & \\
\hline & VR (2) & 1,1 & & & & & \\
\hline 4 & 105 & $\mathrm{HL}$ & 6 & 3.51 & 1.0169 & 88.44 & 100.06 \\
\hline & VR (1) 3 & 32,32 & & & & & \\
\hline & VR (2) & 7,7 & & & & & \\
\hline 4 & 105 & LL & 0 & 4.67 & 2.3681 & 85.38 & 100.15 \\
\hline & VR (1) & 4,5 & & & & & \\
\hline & VR (2) & 2,2 & & & & & \\
\hline 5 & 95 & $\mathrm{HL}$ & 16 & 3.87 & 0.7447 & 88.41 & 100.06 \\
\hline & Cap (1) & $0 \mathrm{kVAr}$ & & & & & \\
\hline 5 & 95 & $\mathrm{LL}$ & 14 & 5.11 & 0.291 & 85.39 & 100.15 \\
\hline & Cap (1) & $0 \mathrm{kVAr}$ & & & & & \\
\hline 5 & 105 & $\mathrm{HL}$ & 5 & 3.88 & 0.747 & 88.41 & 100.06 \\
\hline & Cap (1) & $0 \mathrm{kVAr}$ & & & & & \\
\hline 5 & 105 & $\mathrm{LL}$ & -1 & 5.12 & 0.2758 & 85.39 & 100.15 \\
\hline
\end{tabular}




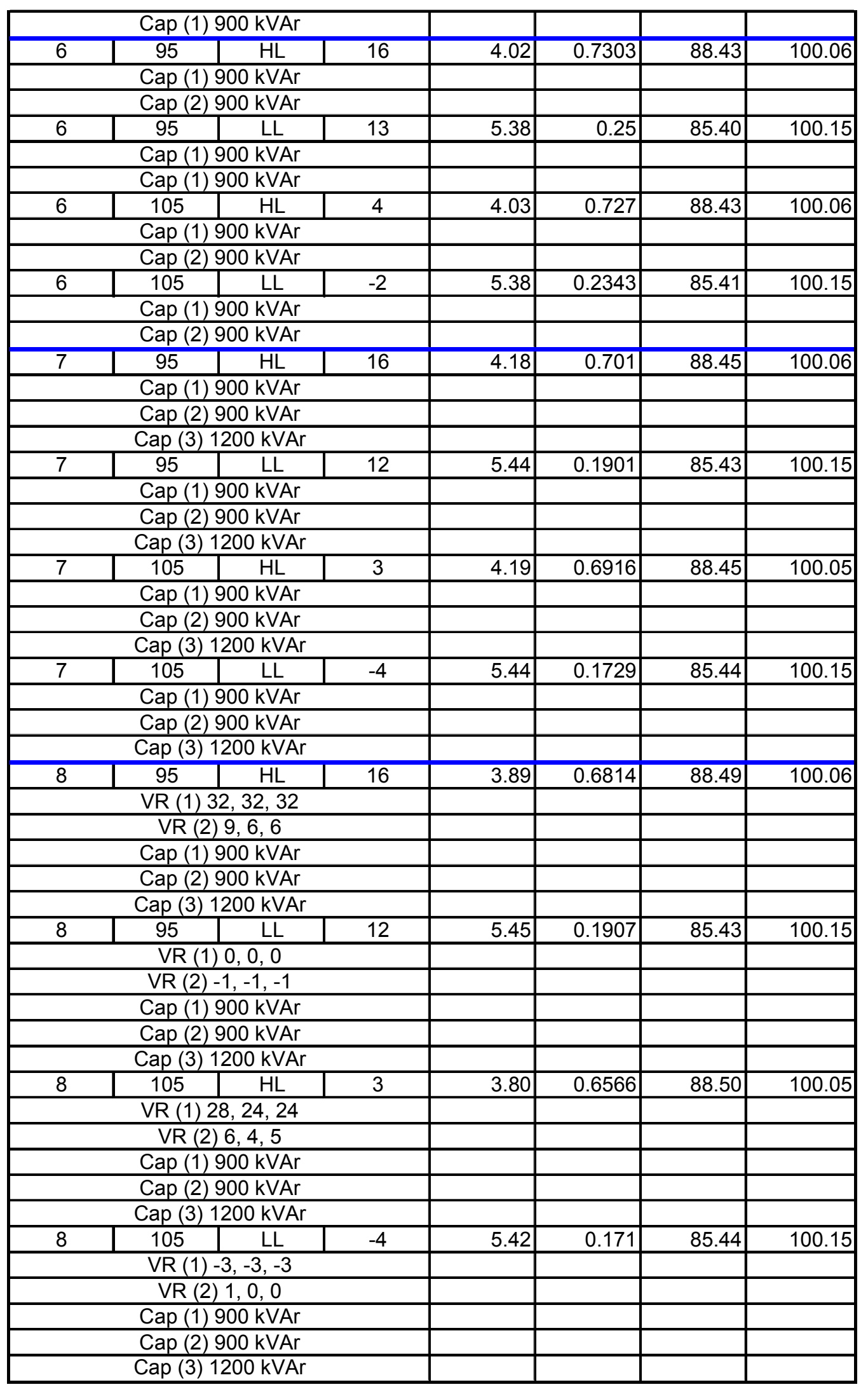


Finally, Appendix B contains selected simulation data by node for each item listed.

\subsubsection{Heavy Load Circuit Voltage Profiles (First Set 95\% and 105\% Primary Voltage)}

Figure 103 shows the voltage profiles for each test, 1 through 8, at 105\% and $95 \%$ substation primary voltage and HL condition. The figure shows a vast range of voltage spread (highest to lowest) throughout the circuit. The voltages shown are located at the secondary of the circuit distribution transformer. Therefore, an additional $3 \mathrm{~V}$ have been added to Range A (i.e., 114 $\mathrm{V}+3 \mathrm{~V}=117 \mathrm{~V})$ for a minimum acceptable level of $117 \mathrm{~V}$ and a maximum acceptable level of $129 \mathrm{~V}(126 \mathrm{~V}+3 \mathrm{~V}=129 \mathrm{~V})$. This addition accounts for the voltage drop in the secondary and service drop to the customer because Range A of ANSI Standard C84.1 is the voltage at the customer's meter. The regulated voltage is to remain within these maximum and minimum values of $129 \mathrm{~V}$ and $117 \mathrm{~V}$.

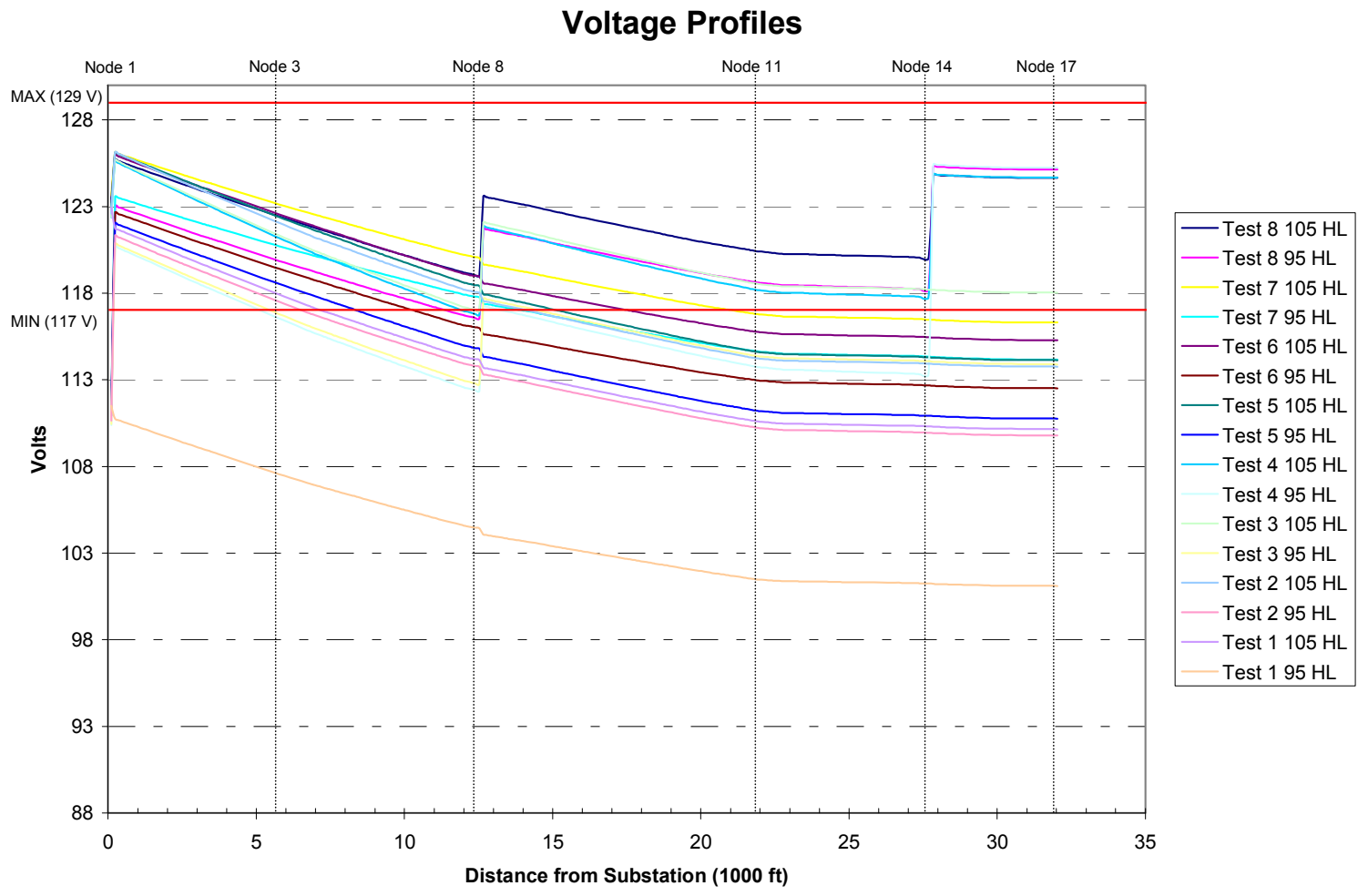

Figure 103. HL circuit voltage profiles for all tests (first set $-95 \%$ and $105 \%$ substation primary voltage)

In Table 24, the voltage spread throughout the circuit for Test 1 has a range of $25.2 \mathrm{~V}$ with no LTC regulation. For Test 8 , it is only $10.4 \mathrm{~V}$ with all methods of regulation. As more regulation is sequentially added to the circuit, the voltage spread reduces. 
Table 24. HL Circuit Voltage Profiles (First Set)

\begin{tabular}{|c|c|c|c|c|}
\hline \multirow[b]{2}{*}{ Test No. } & \multirow[b]{2}{*}{$\begin{array}{l}\text { Substation Primary Voltage } \\
\text { Spread and Load Condition }\end{array}$} & \multirow[b]{2}{*}{ Voltage Regulation Method } & \multicolumn{2}{|c|}{ Voltage Spread } \\
\hline & & & $\begin{array}{c}\text { Highest } \\
\text { Three- } \\
\text { Phase }\end{array}$ & $\begin{array}{l}\text { Lowest } \\
\text { Single- } \\
\text { Phase }\end{array}$ \\
\hline Test 1 & $95 \%, 105 \%, \mathrm{HL}$ & No LTC & 126.01 & 100.81 \\
\hline Test 2 & $95 \%, 105 \%, \mathrm{HL}$ & LTC & 126.08 & 109.44 \\
\hline Test 3 & $95 \%, 105 \%, \mathrm{HL}$ & LTC, VR 1 & 126.00 & 111.25 \\
\hline Test 4 & $95 \%, 105 \%, \mathrm{HL}$ & LTC, VR 1, VR 2 & 126.00 & 111.10 \\
\hline Test 5 & $95 \%, 105 \%, \mathrm{HL}$ & LTC, CAP 1 & 126.05 & 110.40 \\
\hline Test 6 & $95 \%, 105 \%, \mathrm{HL}$ & LTC, CAP 1, CAP 2 & 126.00 & 112.14 \\
\hline Test 7 & $95 \%, 105 \%, \mathrm{HL}$ & LTC, CAP 1, CAP 2, CAP 3 & 126.20 & 113.79 \\
\hline Test 8 & $95 \%, 105 \%, \mathrm{HL}$ & $\begin{array}{l}\text { LTC, VR 1, VR } 2 \\
\text { CAP 1, CAP 2, CAP } 3\end{array}$ & 126.00 & 115.6 \\
\hline
\end{tabular}

In the individual voltage profiles for Test 1, Figure 104, the voltage spread because of the $10 \%$ change ( $95 \%$ to $105 \%)$ in the substation primary voltage is $9.05 \mathrm{~V}$ (or $110.15-101.1 \mathrm{~V}$ ).

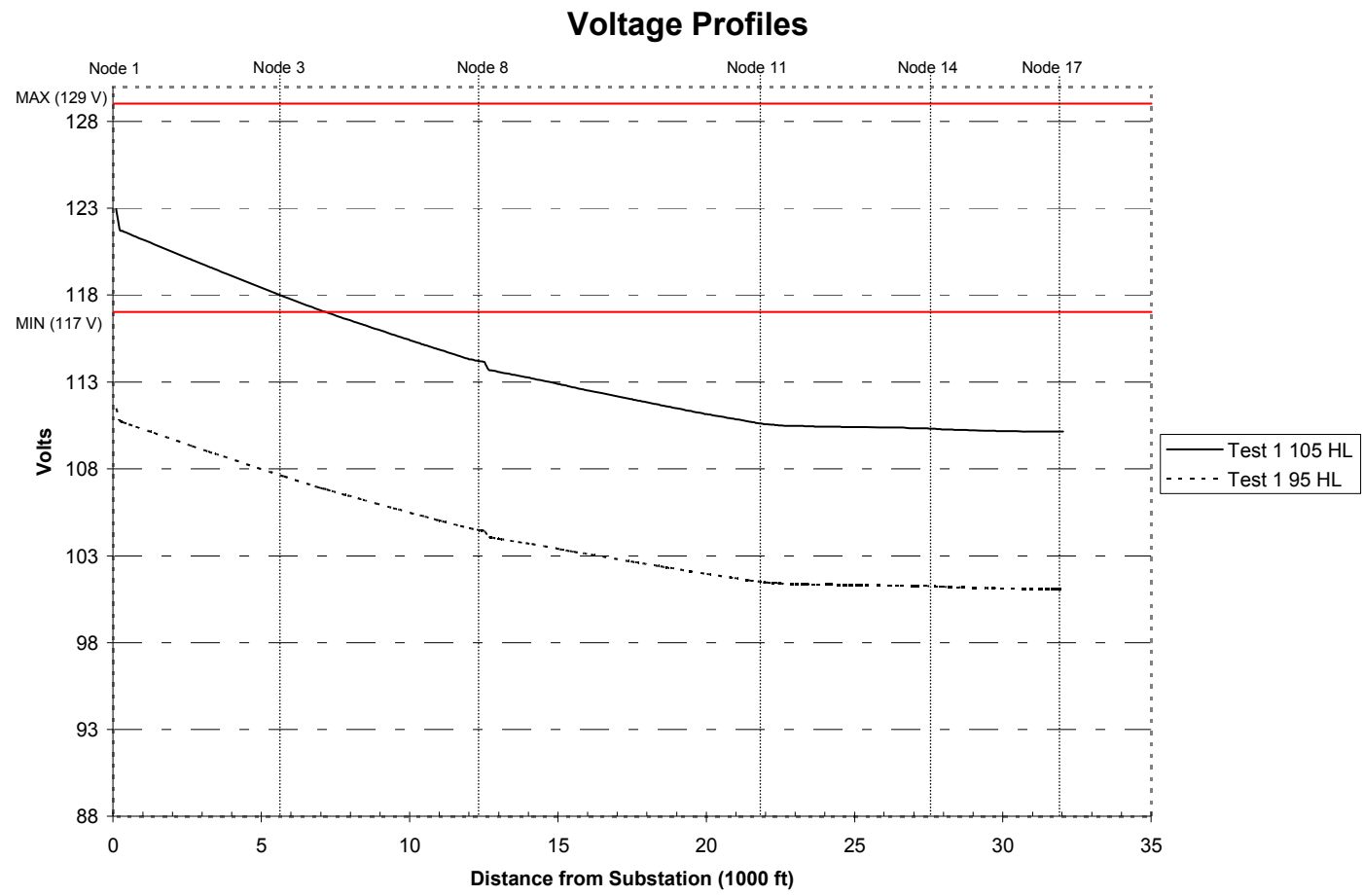

Figure 104. Test $1 \mathrm{HL}$ circuit voltage profiles for $95 \%$ and $105 \%$ substation primary voltage (no LTC) 
For Test 2, Figure 105 shows a significant improvement in voltage spread with the addition of the LTC at the substation, even though the primary substation voltage spread is $10 \%$. Here, the spread is only $3.97 \mathrm{~V}$ (or $113.76 \mathrm{~V}-109.79 \mathrm{~V}$ ). Because the deadband for the LTC is $\pm 1 \mathrm{~V}$, the spread could be as much as $5.97 \mathrm{~V}$.

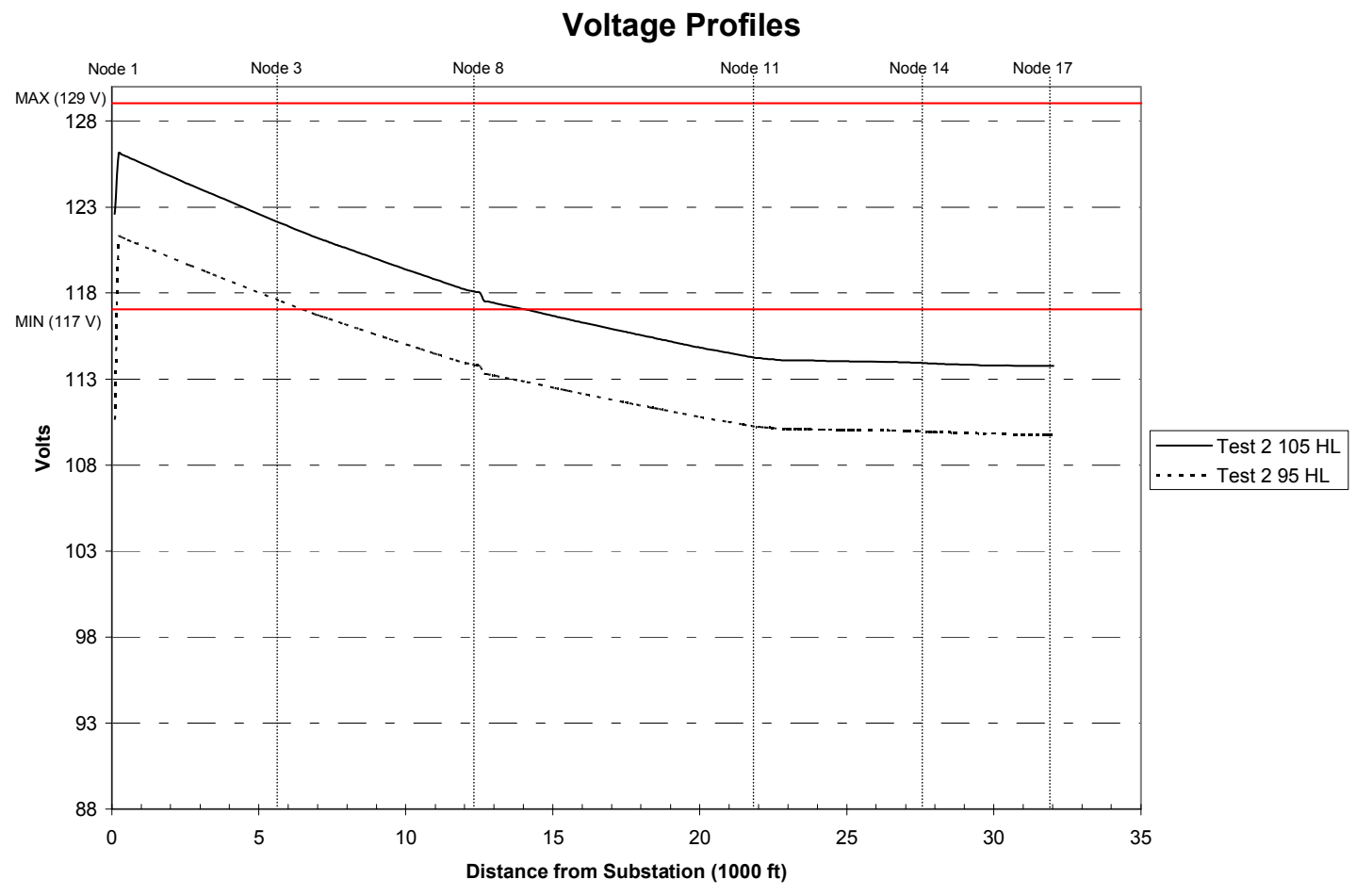

Figure 105. Test $2 \mathrm{HL}$ circuit voltage profiles for $95 \%$ and $105 \%$ substation primary voltage (LTC) 
Test 3, Figure 106, shows the benefit of adding the first step VR. The voltage profile with $105 \%$ primary voltage bumps the tag end voltage up to $118.04 \mathrm{~V}$, and with a $95 \%$ primary voltage, the tag end voltage is $113.9 \mathrm{~V}$.

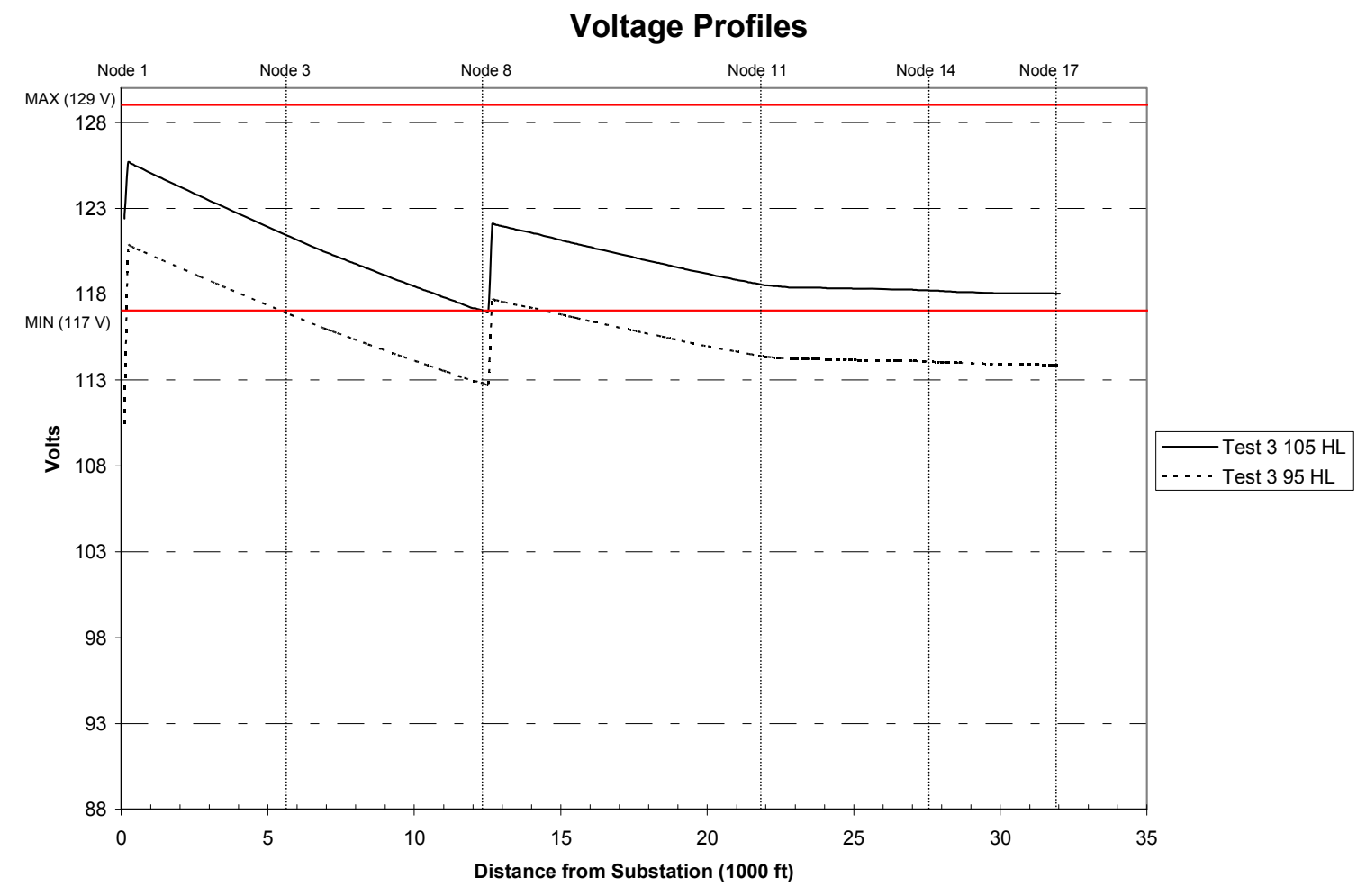

Figure 106. Test $3 \mathrm{HL}$ circuit voltage profiles for $95 \%$ and $105 \%$ substation primary voltage (LTC and VR 1) 
When two step regulators are added, as in Test 4 (Figure 107), the tag end voltage at Node 17 reaches almost $124.67 \mathrm{~V}$ with $105 \%$ primary voltage, but at $95 \%$ primary voltage, this becomes $125.24 \mathrm{~V}$. At Node 8 with a primary voltage of $95 \%$, the voltage gets down to $112.34 \mathrm{~V}$.

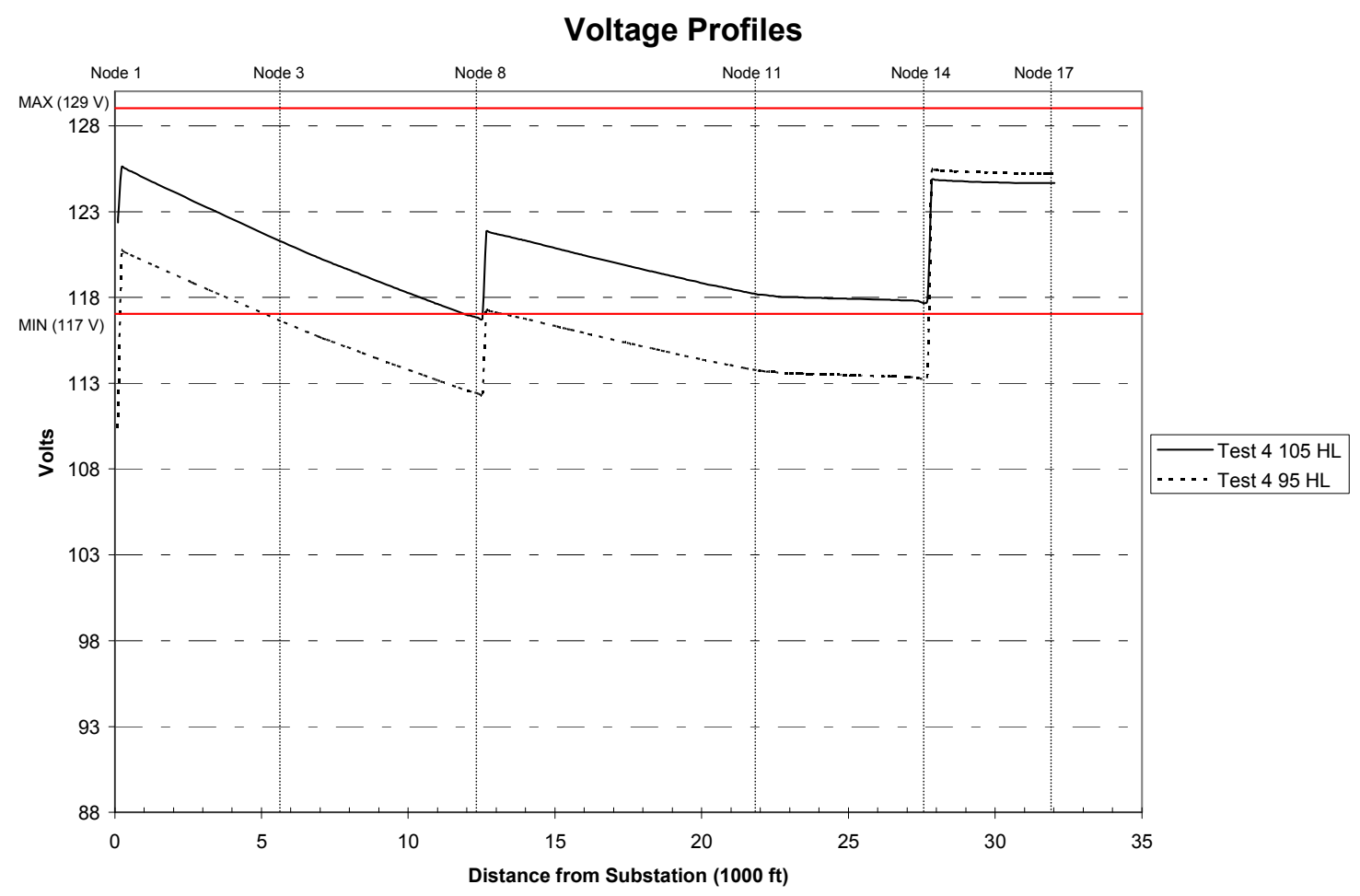

Figure 107. Test $4 \mathrm{HL}$ circuit voltage profiles for $95 \%$ and $105 \%$ substation primary voltage (LTC, VR 1, and VR 2) 
Test 5 uses the LTC at the substation transformer and Capacitor 1, located at Node 6, which has a reactive capability of $900 \mathrm{kVArc}$ to improve the voltage regulation. Notice that the rate of change of voltage drop (or slope) in Figure 108 is less than that of Test 4 from the substation to Node 8 because of the addition of the capacitor and less reactive current. However, the voltage improvement compared with the two-step regulator test is considerably less. The tag end voltage at Node 17 is $114.13 \mathrm{~V}$ for $105 \%$ primary voltage and $110.76 \mathrm{~V}$ for $95 \%$ primary voltage.

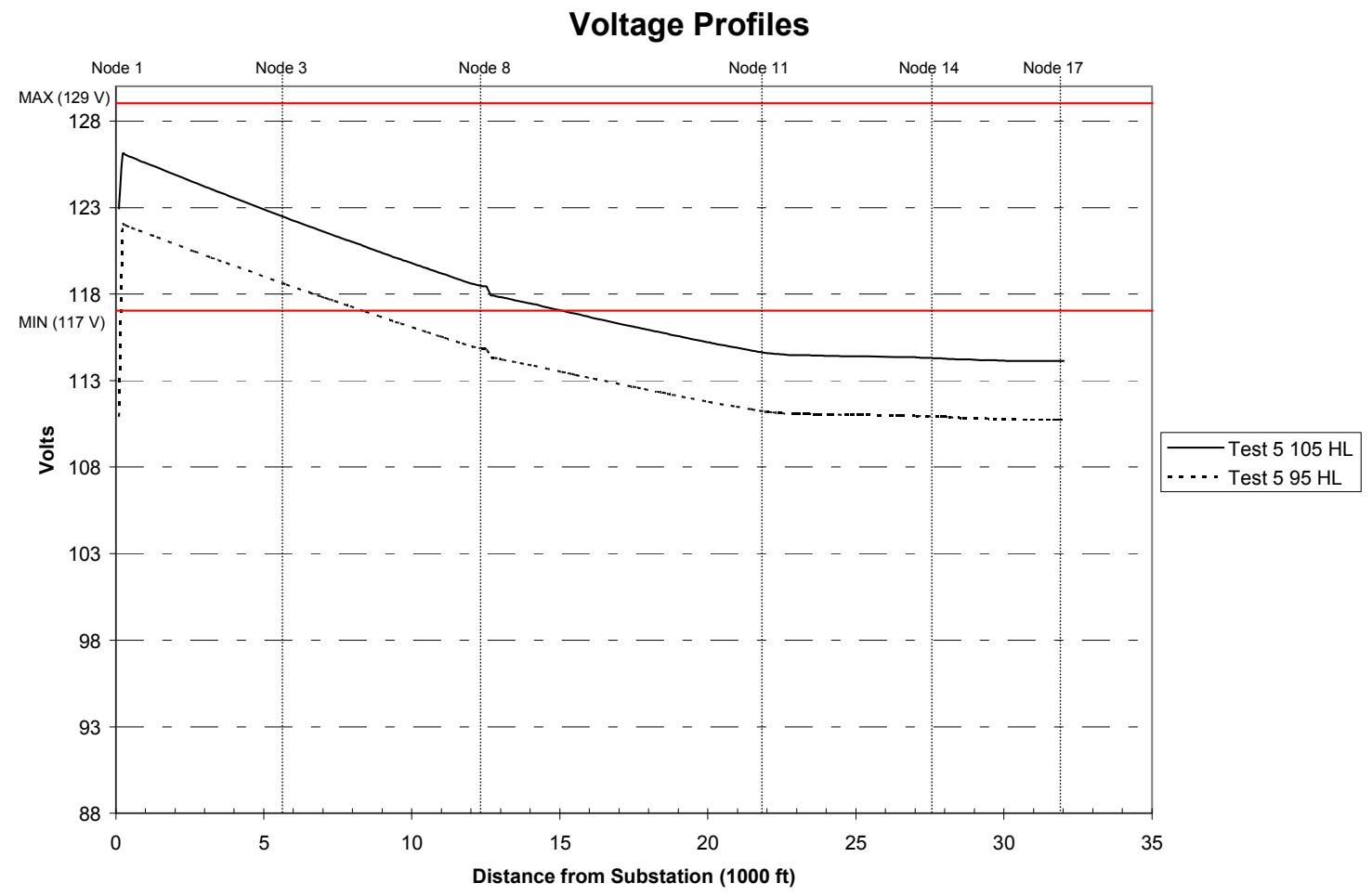

Figure 108. Test $5 \mathrm{HL}$ circuit voltage profiles for $95 \%$ and $105 \%$ substation primary voltage (LTC and CAP 1) 
Test 6 results, shown in Figure 109, are based on two capacitors (1 and 2) located at nodes 6 and 12 of Figure 102 and the LTC transformer to regulate the circuit voltage. Here, the voltage gradient is less than that of Test 5 because of the additional capacitor (2), and the tag end voltage at Node 17 is $112.51 \mathrm{~V}$ compared with $110.76 \mathrm{~V}$ for Test 5 at $95 \%$ primary voltage. It is common to gain about $2 \mathrm{~V}$ with the addition of the second capacitor.

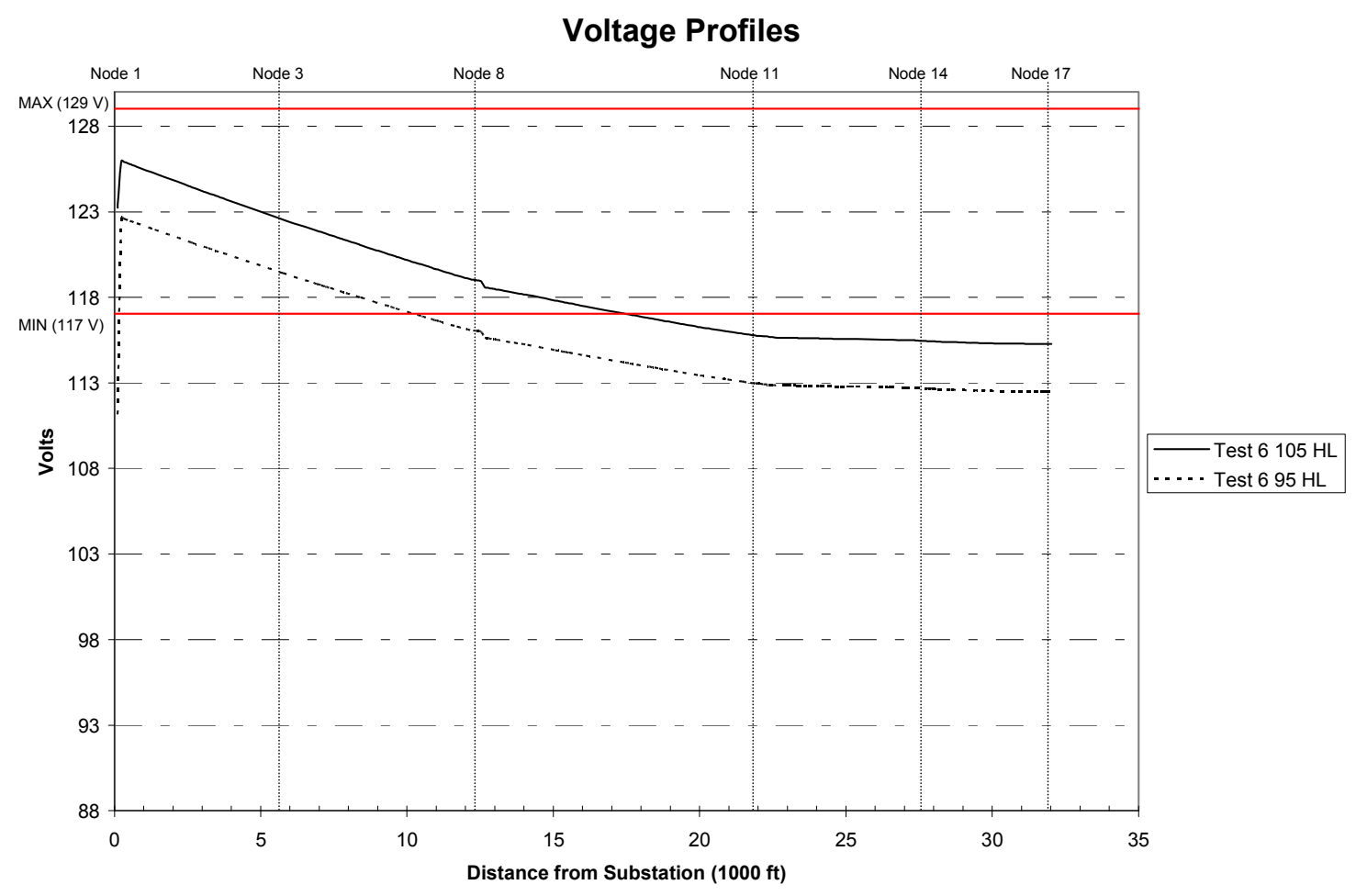

Figure 109. Test 6 HL circuit voltage profiles for $95 \%$ and $105 \%$ substation primary voltage (LTC, CAP 1, and CAP 2) 
Test 7 involves the LTC and three capacitors. Its results are shown in Figure 110. These capacitors consist of a $900 \mathrm{kVArc}$ at Node 6 (Capacitor 1), a $900 \mathrm{kVArc}$ at Node 12

(Capacitor 2), and a 1,200 kVArc at Node 13 (Capacitor 3). Now, the tag end voltage is up to $114.17 \mathrm{~V}$ at $95 \%$ primary voltage.

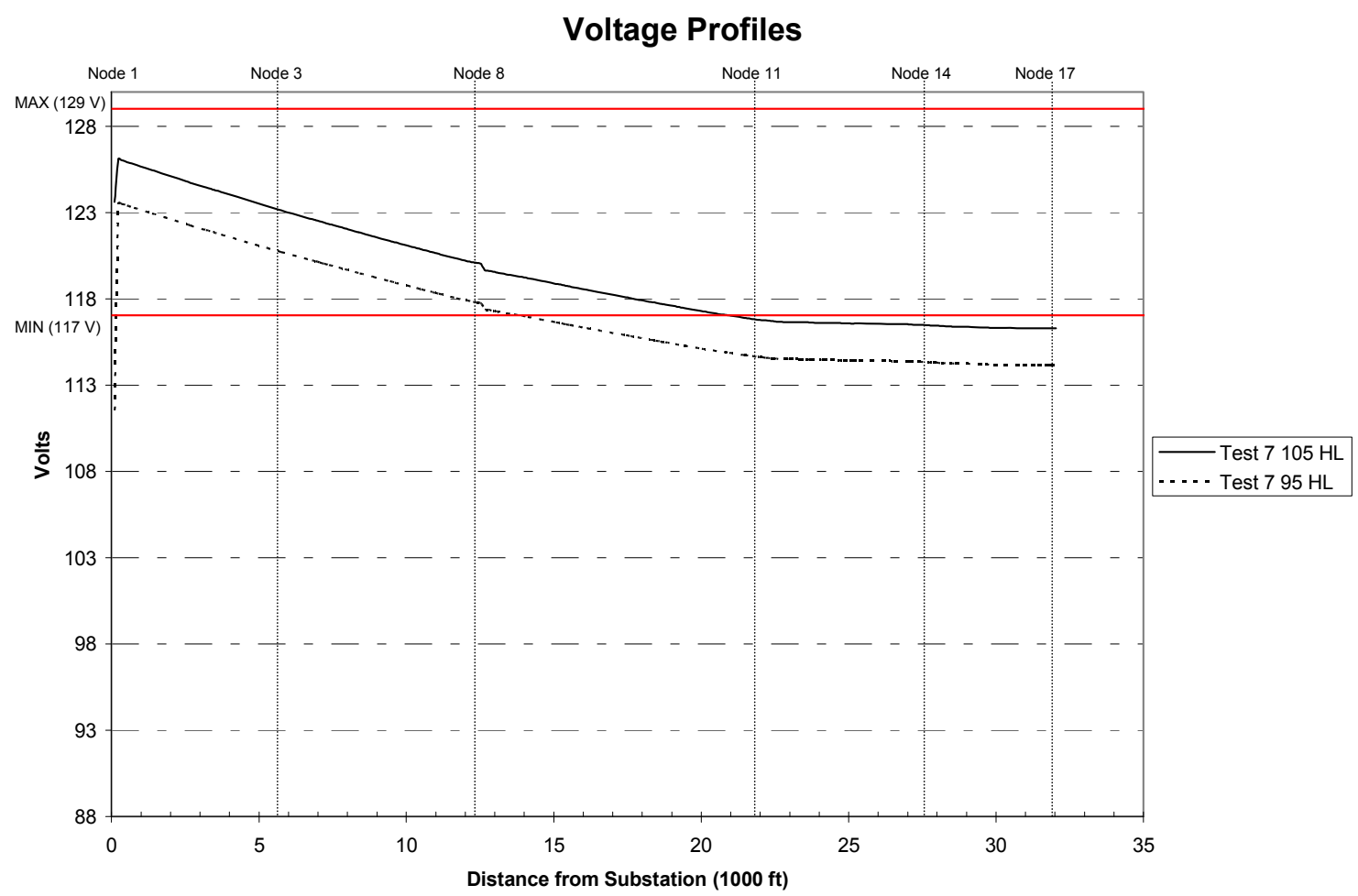

Figure 110. Test $7 \mathrm{HL}$ circuit voltage profiles for $95 \%$ and $105 \%$ substation primary voltage (LTC, CAP 1, CAP 2, and CAP 3 ) 
Test 8, which uses all forms of regulation (i.e., the LTC, two step regulators, and three capacitors), yields the best overall voltage profile with the lowest voltage at Node 8 of 116.53 $\mathrm{V}$. The tag end voltage of $125.47 \mathrm{~V}$ is almost $11 \mathrm{~V}(10.96 \mathrm{~V})$ more than that of Test 7 with three capacitors and a primary voltage of $95 \%$. See Figure 111 . With $105 \%$ primary voltage, the lowest voltage of $118.99 \mathrm{~V}$ occurs at Node 8 , just ahead of the first step regulator. Test 8 is the only voltage profile under HL conditions that almost meets a minimum voltage criteria of $117 \mathrm{~V}$ (actual minimum $=116.53 \mathrm{~V}$ ); all other profiles fail. This indicates a need for DG to add more regulation at Node 10 , where the $1,000-\mathrm{kW}$ synchronous generator is sited.

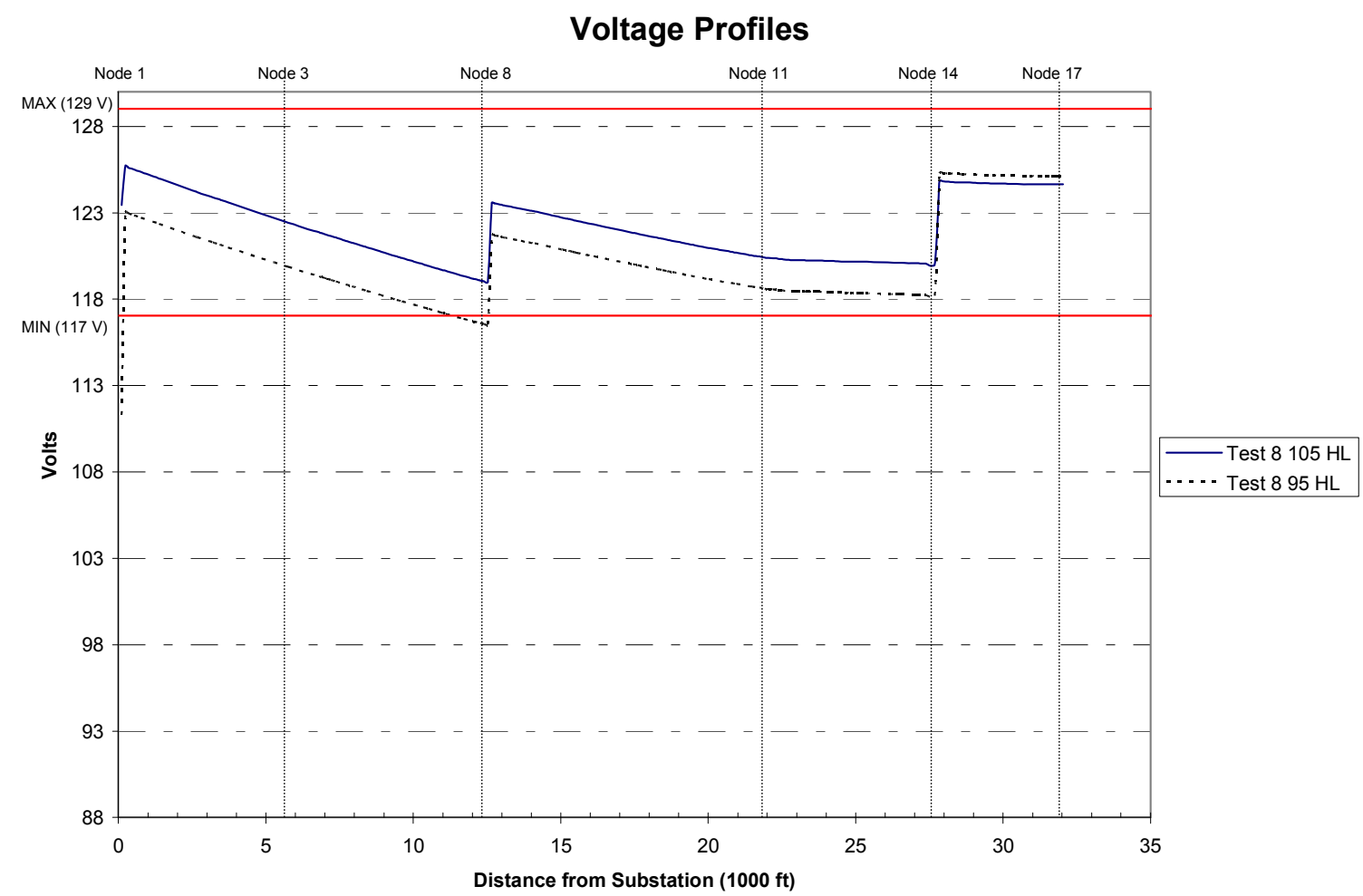

Figure 111. Test $8 \mathrm{HL}$ circuit voltage profiles for $95 \%$ and $105 \%$ substation primary voltage (LTC, VR 1, VR 2, CAP 1, CAP 2, and CAP 3)

\subsubsection{Heavy Load Comparison Circuit Voltage Profiles (Second Set 95\% Primary Voltage)}

Because it is difficult to see how each voltage regulation method taken in succession improves the voltage profile, voltage profile comparisons $\mathrm{A}$ through $\mathrm{F}$ are given in Figure 112 through Figure 118. The comparisons are also provided in Table 25 at 95\% primary voltage. 
Table 25. Voltage Profile Comparison for HL and 95\% Primary Voltage

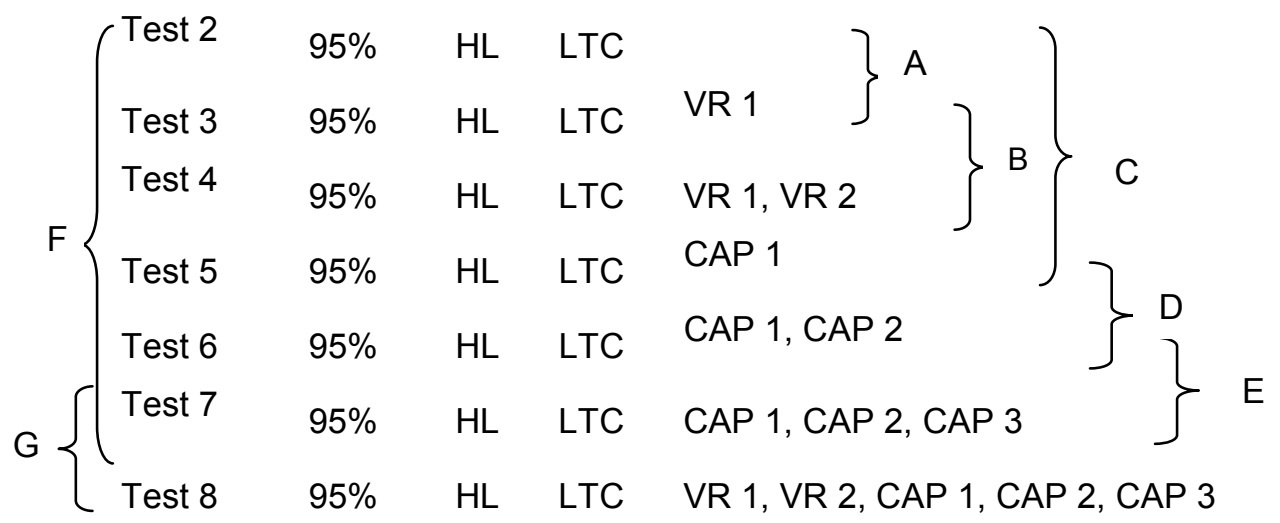

Comparison A, of Tests 2 and 3, is depicted in Figure 112. The Node 17 voltage improvement, from adding the first step regulator, is $4.11 \mathrm{~V}$.

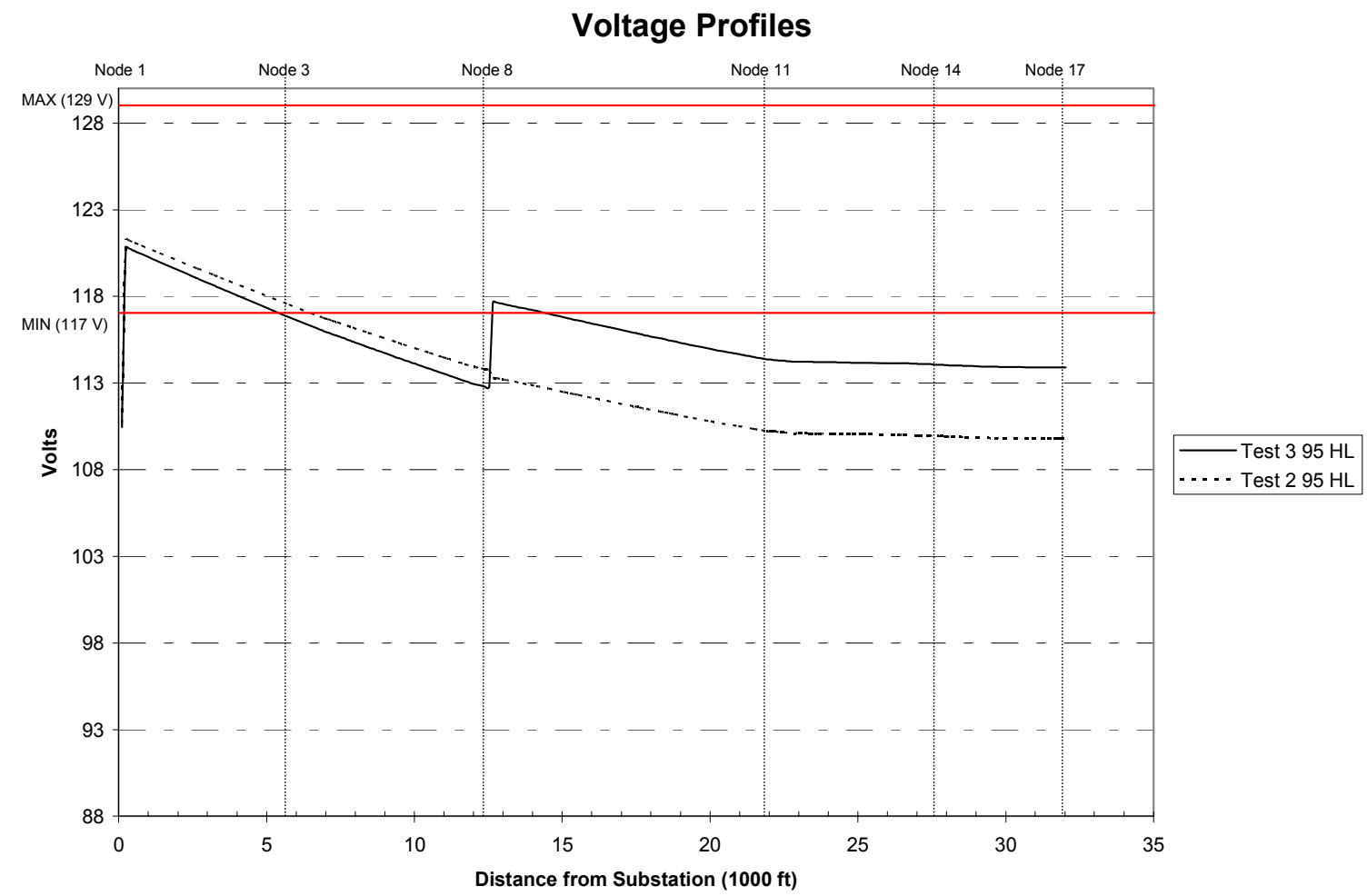

Figure 112. Comparison A: Test 2 and Test $3 \mathrm{HL}$ circuit voltage profiles for $95 \%$ substation primary voltage (LTC versus LTC and VR 1 ) 
When the second regulator is added (Comparison B), the tag end voltage, shown in Figure 113 at Node 17, goes up to $125.24 \mathrm{~V}$, for a gain of $11.34 \mathrm{~V}$.

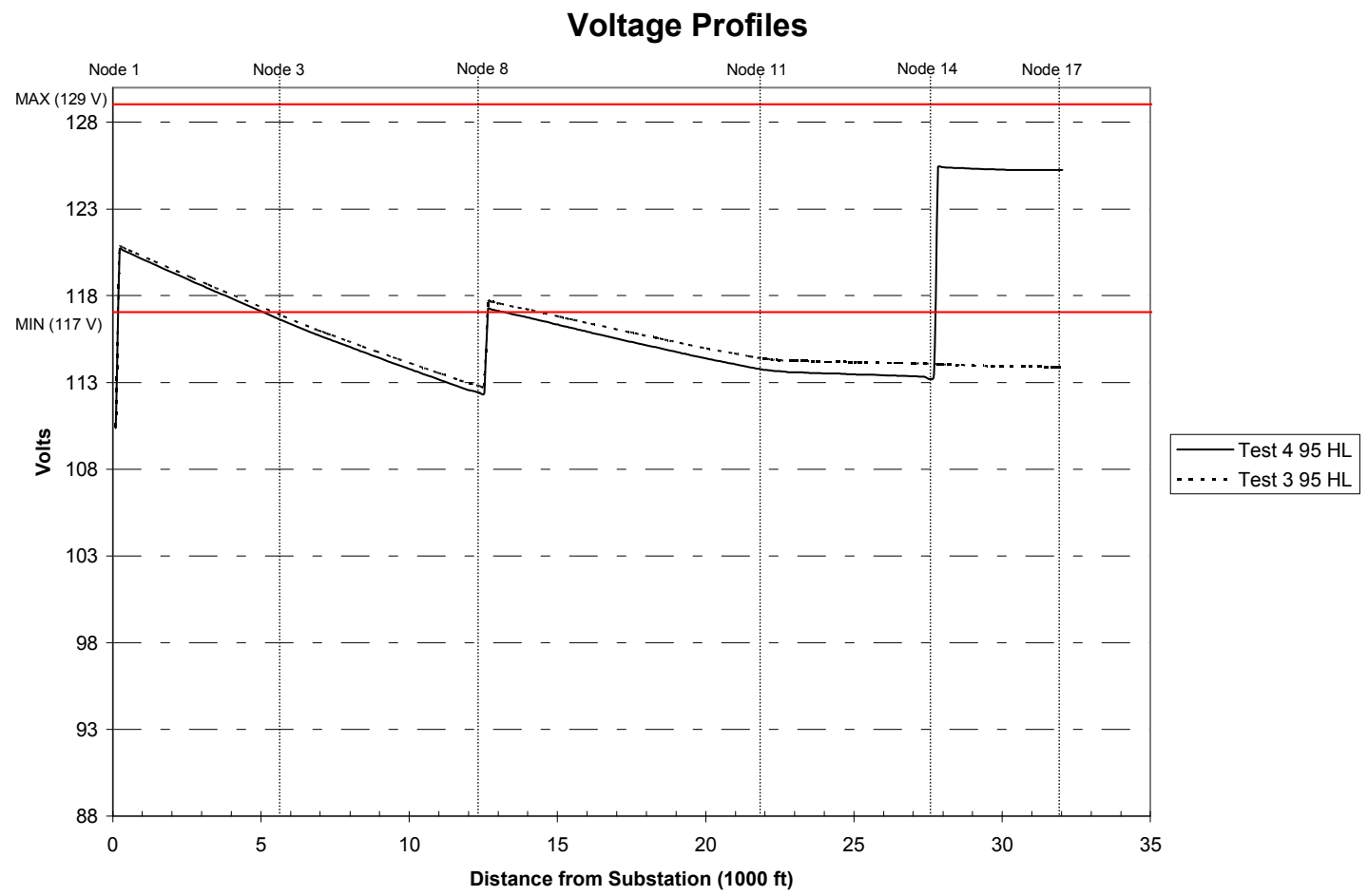

Figure 113. Comparison B: Test 3 and Test $4 \mathrm{HL}$ circuit voltage profiles for $95 \%$ substation primary voltage (LTC and VR 1 versus LTC, VR 1 , and VR 2) 
As seen from Comparison $\mathrm{C}$ in Figure 114, the addition of the first capacitor for Test 5 marginally increases the voltage at Node 17 by $0.97 \mathrm{~V}(110.76-109.79 \mathrm{~V})$.

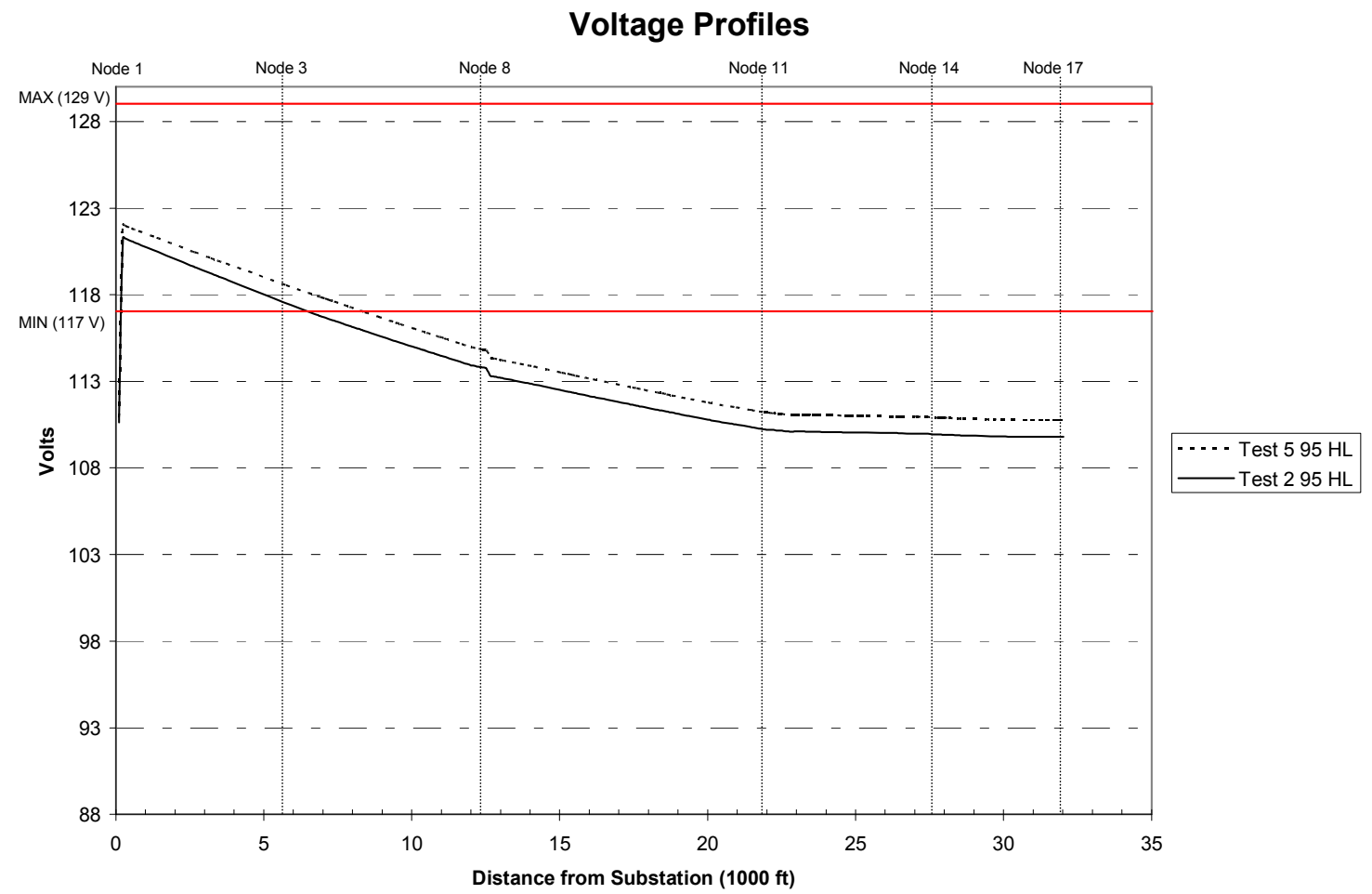

Figure 114. Comparison C: Test 2 and Test $5 \mathrm{HL}$ circuit voltage profiles for $95 \%$ substation primary voltage (LTC versus LTC and CAP 1) 
Comparison D, for Tests 5 and 6, shows the voltage improvement from adding the second capacitor. Figure 115 at Node 17 shows a $1.75-\mathrm{V}$ improvement.

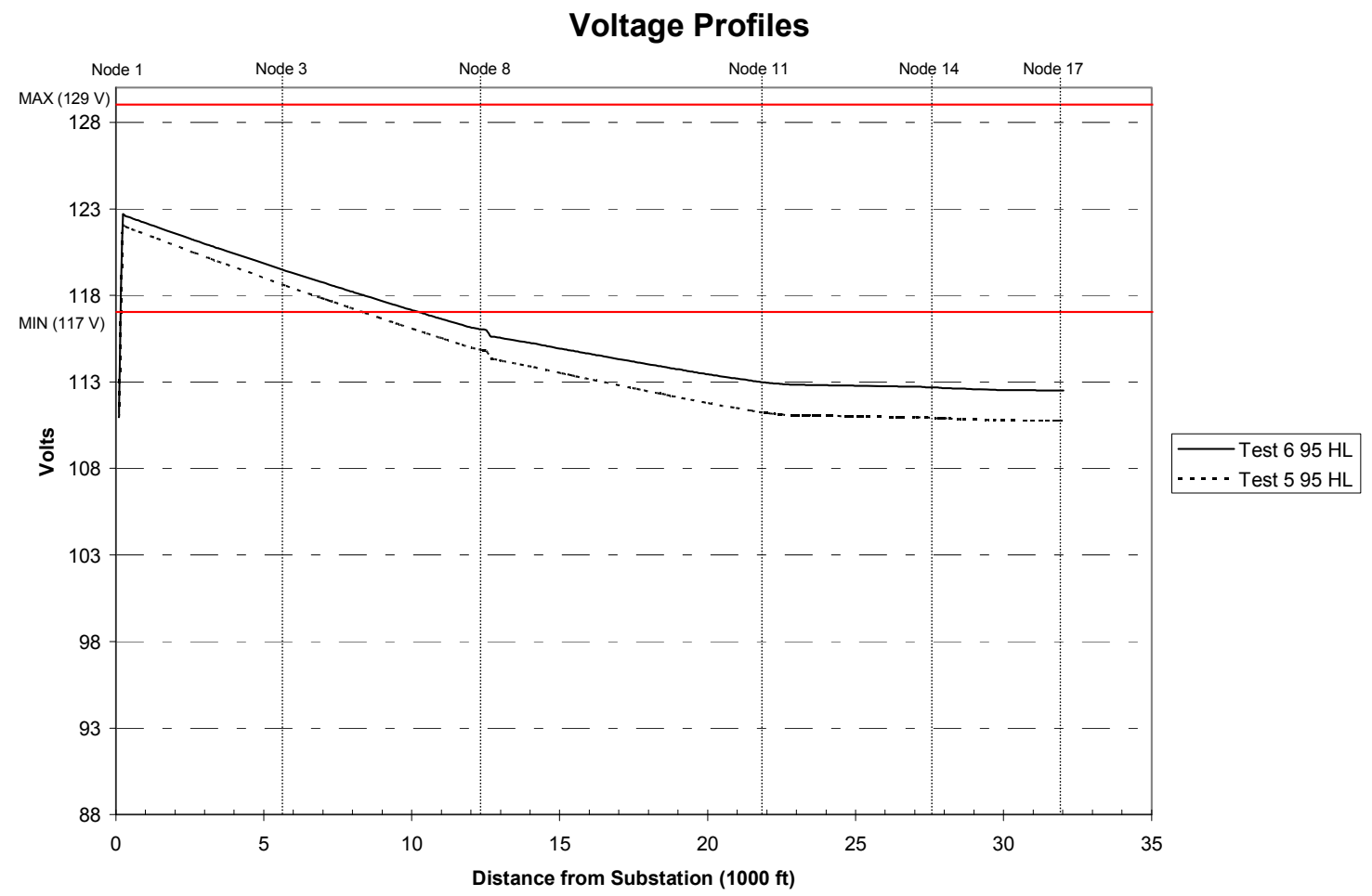

Figure 115. Comparison D: Test 5 and Test $6 \mathrm{HL}$ circuit voltage profiles for $95 \%$ substation primary voltage (LTC and CAP 1 versus LTC, CAP 1, and CAP 2) 
Comparison E, Figure 116, shows a voltage rise at Node 17 of only $1.66 \mathrm{~V}$ with the addition of the third capacitor.

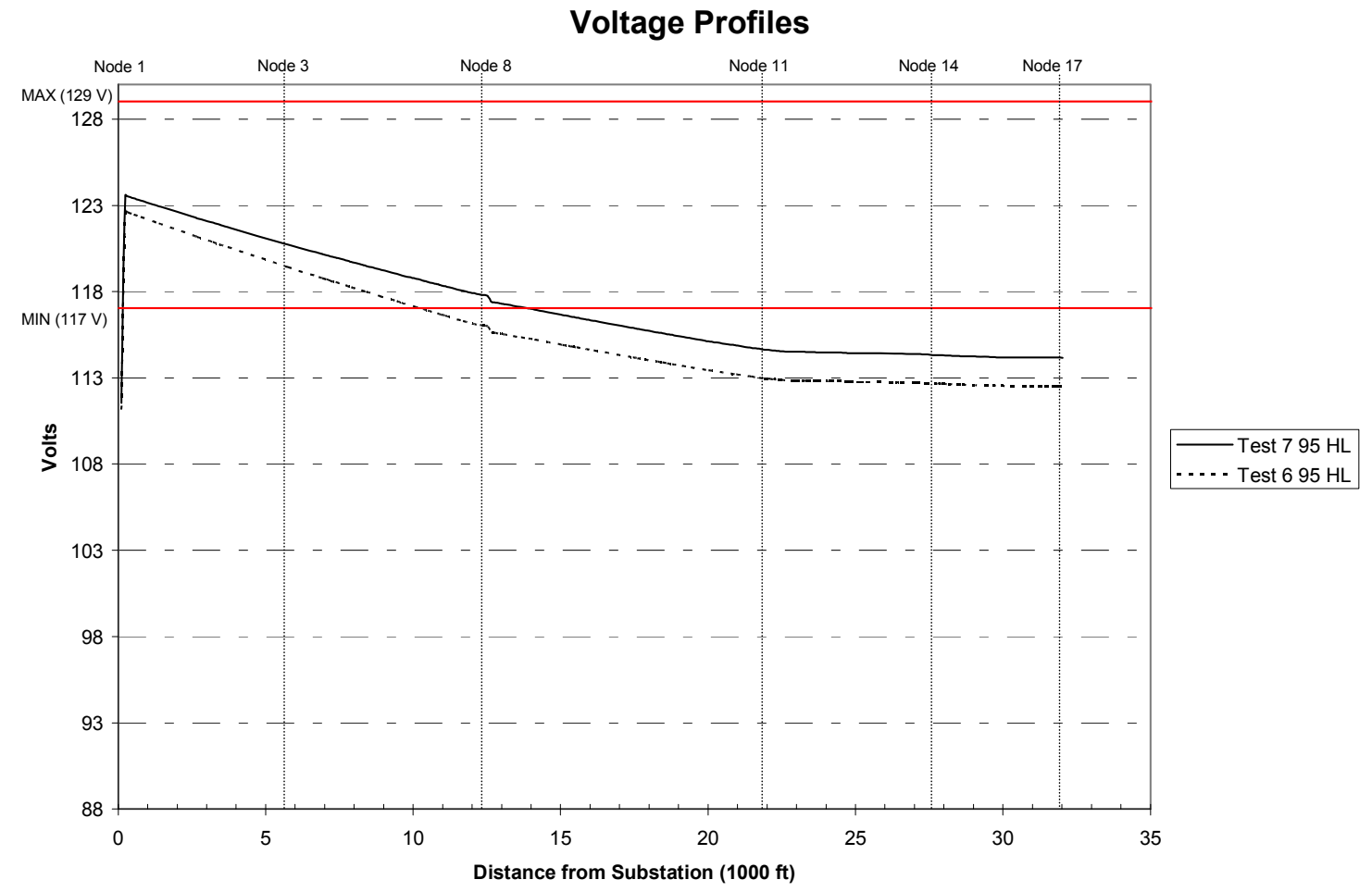

Figure 116. Comparison E: Test 6 and Test $7 \mathrm{HL}$ circuit voltage profiles for $95 \%$ substation primary voltage (LTC, CAP 1, and CAP 2 versus LTC, CAP 1, CAP 2, and CAP 3) 
Comparison F shows the greatest improvement when Test 2, with the LTC, is compared with Test 8, with all methods of regulation.

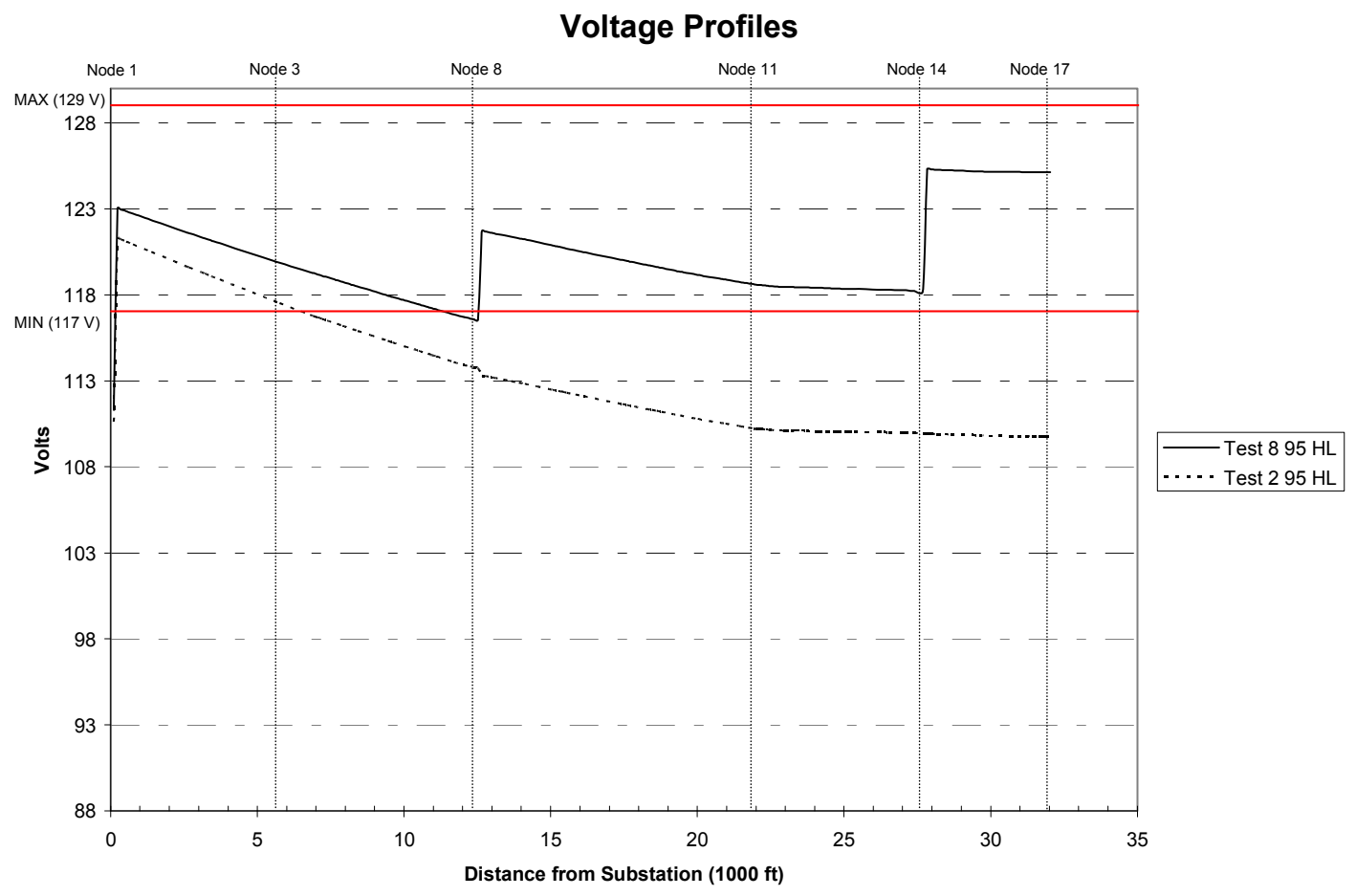

Figure 117. Comparison F: Test 2 and Test $8 \mathrm{HL}$ circuit voltage profiles for $95 \%$ substation primary voltage (LTC vs. LTC, VR 1, VR 2, CAP 1, CAP 2, and CAP 3)

At Node 17 of Figure 117, the voltage difference between the two tests is $15.35 \mathrm{~V}$ (125.14109.79 V). Of course, there are lower voltage gains at Node 8 and Node 14, which are the voltages at the points just before the first and second step regulators. 
Comparison $\mathrm{G}$ of Figure 118 shows the difference of voltage between Test 7 (with the LTC and all capacitors on) and Test 8 (with all methods of regulation on). The voltage improvement at Node 17 is $10.97 \mathrm{~V}(125.14-114.17 \mathrm{~V})$.

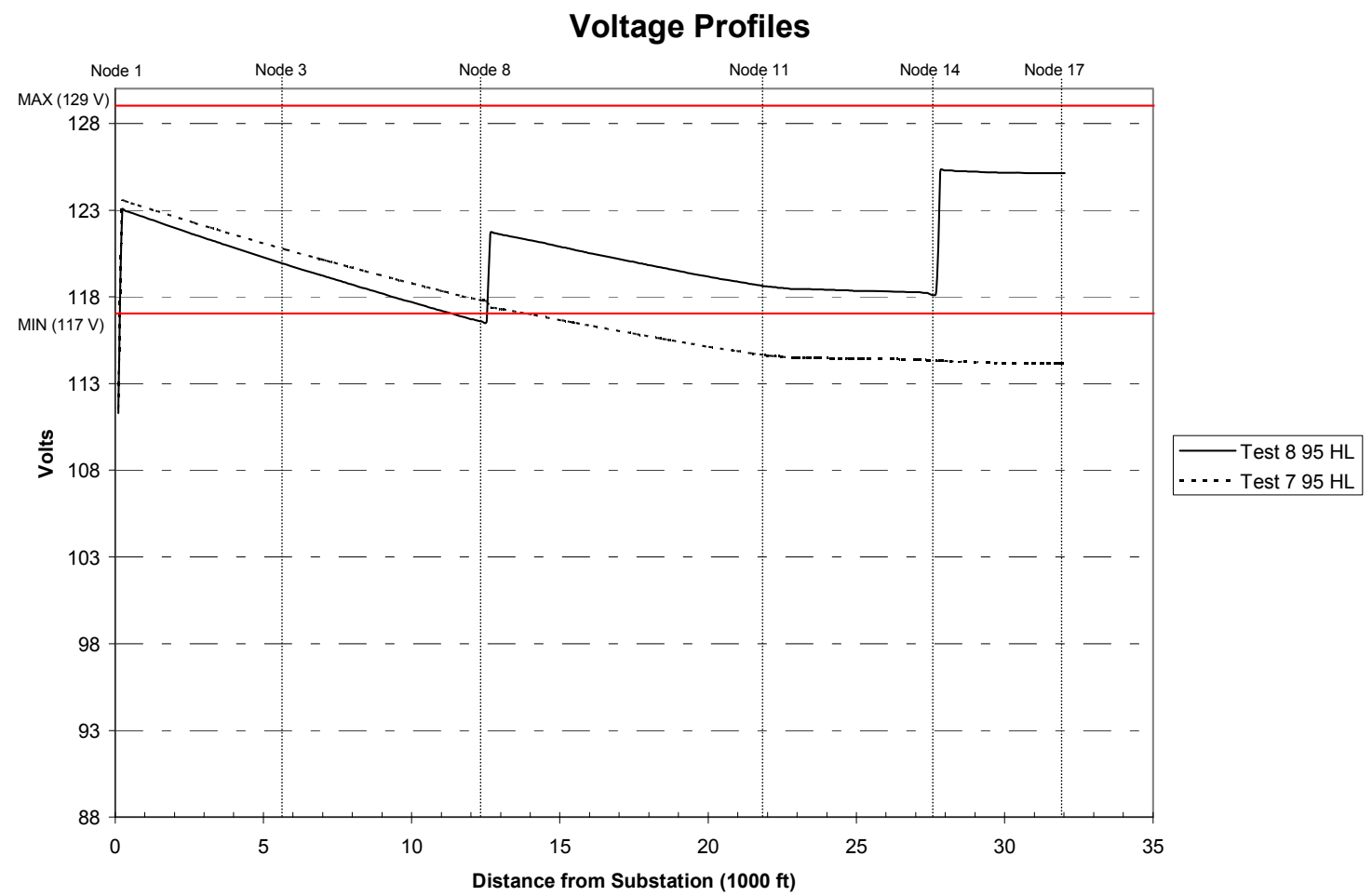

Figure 118. Comparison G: Test 7 and Test 8 HL circuit voltage profiles for $95 \%$ substation primary voltage (LTC, CAP 1, CAP 2, and CAP 3 versus LTC, VR 1, VR 2, CAP 1, CAP 2, and CAP 3)

\subsubsection{Voltage Profile Data as a Function of Distance from Substation to Tag End}

Specific data for the highest three-phase and lowest single-phase voltage were provided in Table 24 for tests $1-8$ at $95 \%$ and $105 \%$ primary voltage during HL conditions. The distance is given in thousands of feet from the substation to the end of the regulation path in Table 20 .

\subsubsection{Light Load Circuit Voltage Profiles (Third Set 95\% and 105\% Primary Voltage)}

A process similar to that for the HL voltage profiles is repeated for the LL voltage profiles beginning with Figure 119 and ending with Figure 134. The LL circuit is 5.91 MVA compared with the LL base case of 5.96 MVA. Even though the LL base case is 5.96 MVA compared with the HL base case of 14.89 MVA, there is one test, Test 1 at $95 \%$ with no LTC regulation, in which the voltage is below the minimum acceptable level of $114.7 \mathrm{~V}$. All the rest of the test voltage profiles are above the minimum voltage level. 
The voltage spread throughout the circuit for Test 1 is shown in Table 26 to be only $16.65 \mathrm{~V}$ (126.24-109.59 V). This compares to $25.2 \mathrm{~V}$ for the same test during HL conditions and no LTC regulation. As additional regulation is added to the circuit, the voltage spread diminishes to its lowest value of only $2.91 \mathrm{~V}$ for Test 8 . During the LL period, the highest voltage on the circuit reaches $126.38 \mathrm{~V}$. Care should be exercised not to exceed $129 \mathrm{~V}$ when operating distribution generation near the substation bus or near the load side of any of the two step regulators. It may be necessary to operate DG with a strategy to absorb volt-amperes reactive to prevent $\mathrm{HV}$ during light load.

Table 26. LL Circuit Voltage Profiles (Third Set)

\begin{tabular}{cllll}
\hline Test & $\begin{array}{c}\text { Substation Primary } \\
\text { Voltage Spread and Load } \\
\text { Condition }\end{array}$ & \multicolumn{1}{c}{ Voltage Regulation } \\
Method & \multicolumn{2}{c}{$\begin{array}{c}\text { Voltage Spread } \\
\text { Lowest } \\
\text { Highest } \\
\text { Single-Phase }\end{array}$} \\
\cline { 2 - 5 } Test 1 & $95 \%, 105 \%$, LL & No LTC & 126.24 & 109.59 \\
Test 2 & $95 \%, 105 \%$, LL & LTC & 126.24 & 121.16 \\
Test 3 & $95 \%, 105 \%$, LL & LTC, VR 1 & 126.23 & 121.79 \\
Test 4 & $95 \%, 105 \%$, LL & LTC, VR 1, VR 2 & 126.22 & 121.78 \\
Test 5 & $95 \%, 105 \%$, LL & LTC, CAP 1 & 126.25 & 121.05 \\
Test 6 & $95 \%, 105 \%$, LL & LTC, CAP 1, CAP 2 & 126.22 & 122.74 \\
Test 7 & $95 \%, 105 \%$, LL & LTC, CAP 1, CAP 2, CAP 3 & 126.37 & 123.75 \\
Test 8 & $95 \%, 105 \%$, LL & LTC, VR 1, VR 2, CAP 1, & 126.38 & 123.47 \\
& & CAP 2, CAP 3 & & \\
\hline
\end{tabular}


Voltage Profiles

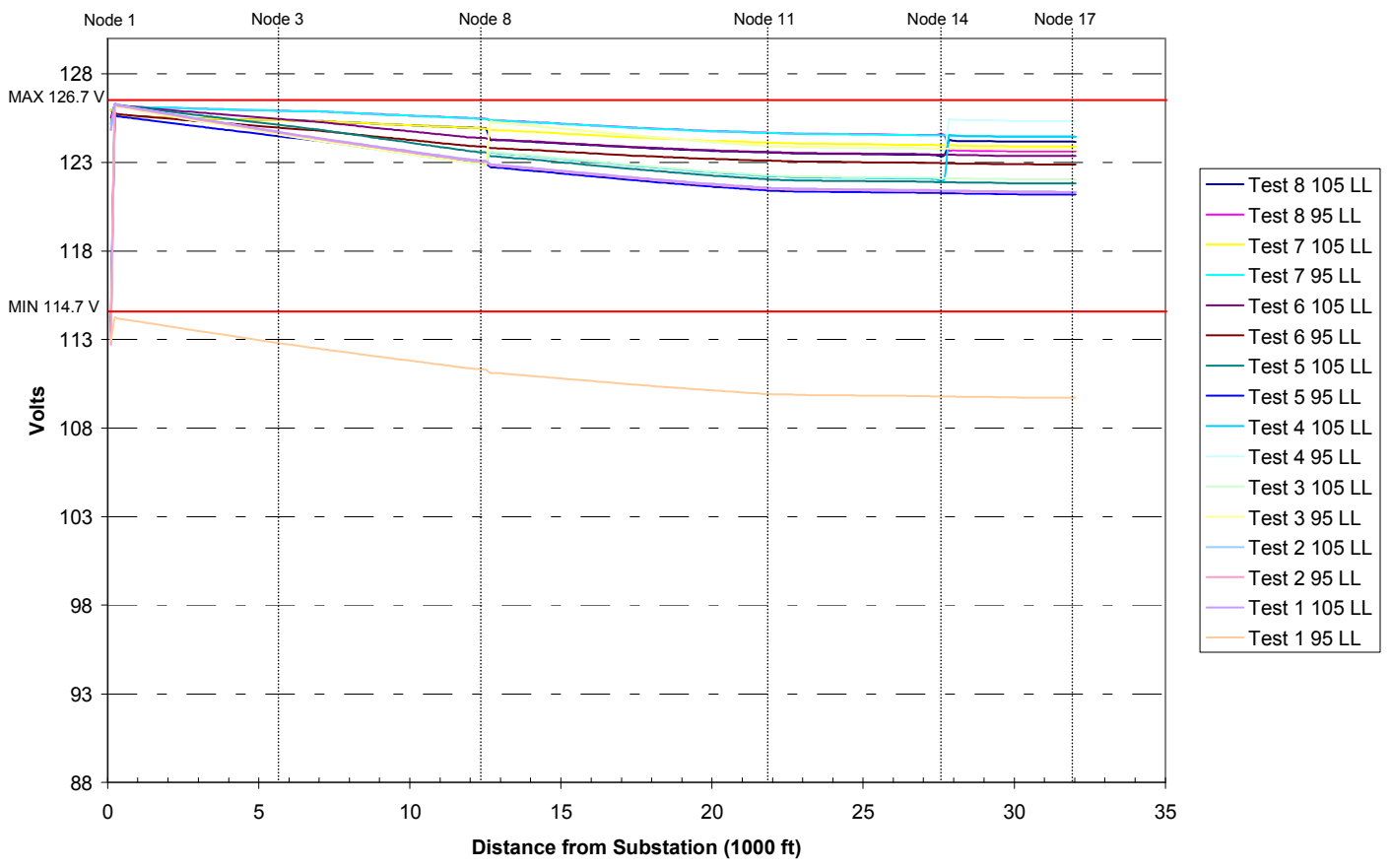

Figure 119. LL circuit voltage profiles for all tests (third set $-95 \%$ and $105 \%$ substation primary voltage) 
The Test 1 voltage profile shown in Figure 120 shows a uniformly wide voltage spread at each node on the circuit as the primary voltage ranges from $95 \%$ to $105 \%$. The voltage spread at the tag end is $11.62 \mathrm{~V}(121.35-109.73 \mathrm{~V})$.

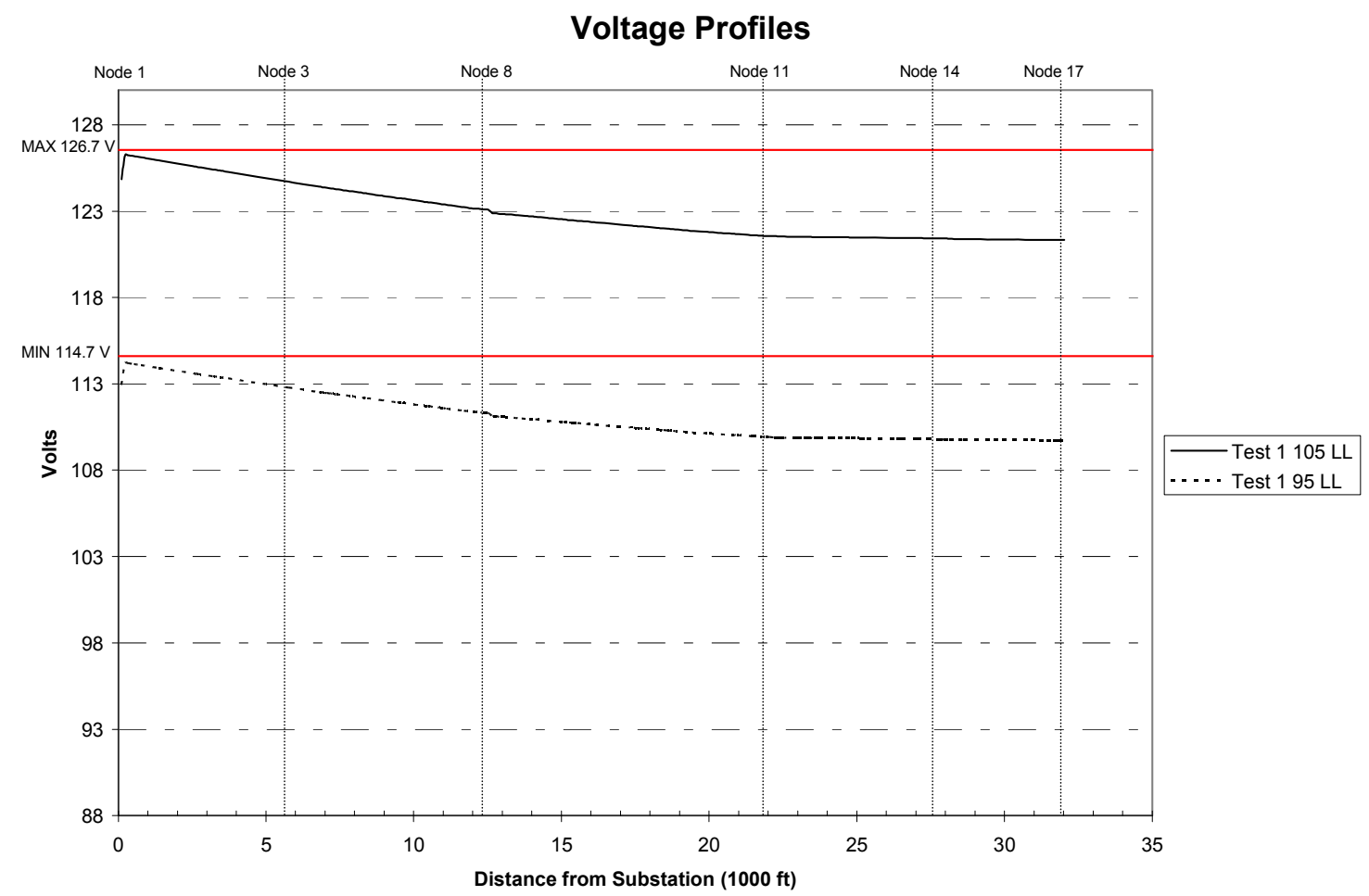

Figure 120. Test $1 \mathrm{LL}$ circuit voltage profiles for $95 \%$ and $105 \%$ substation primary voltage (no LTC) 
When the LTC is added in Test 2, Figure 121, the voltage spread is still uniform, but it is reduced considerably to only $0.05 \mathrm{~V}(121.35-121.3 \mathrm{~V})$ at Node 17.

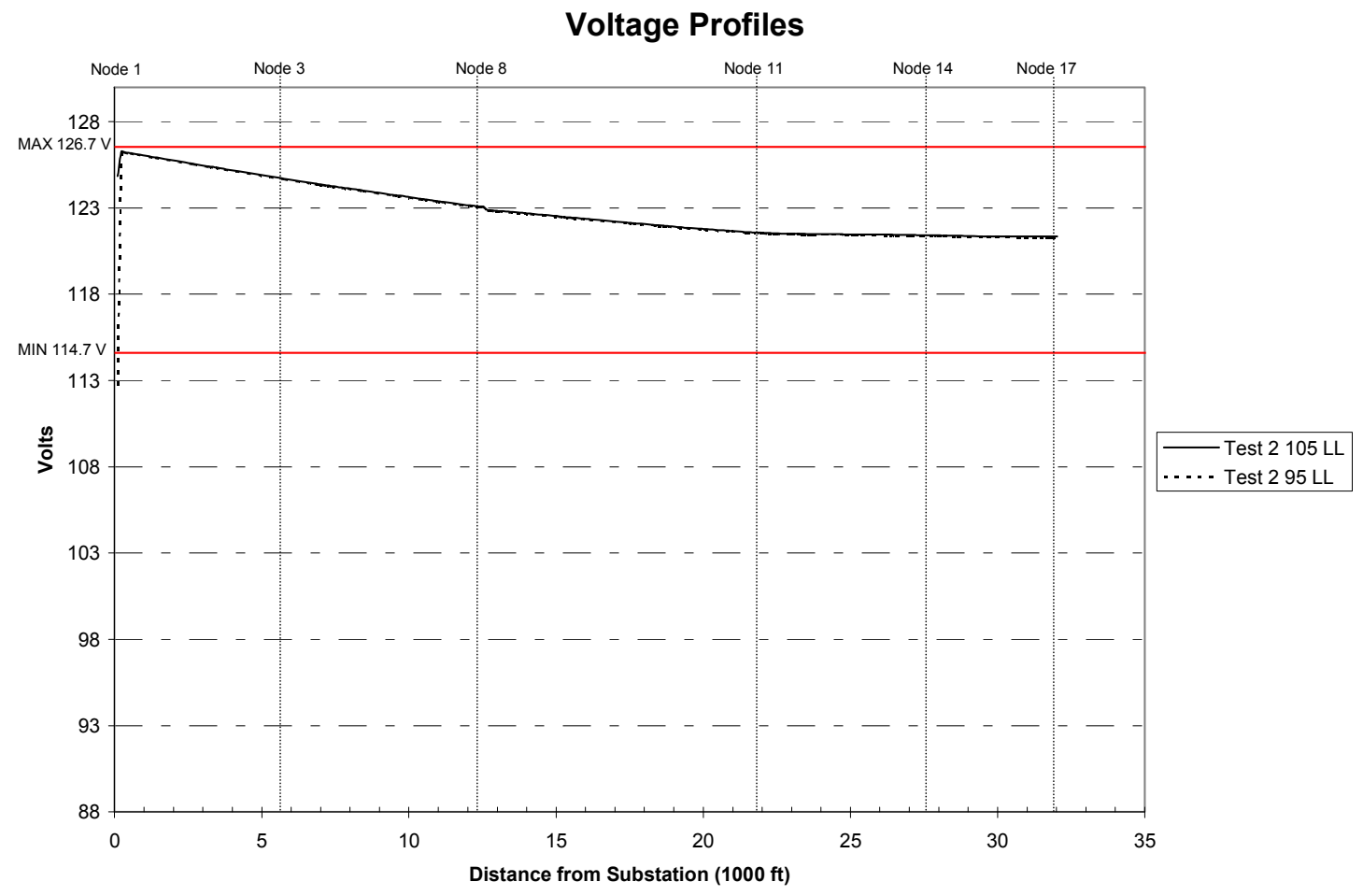

Figure 121. Test 2 LL circuit voltage profiles for $95 \%$ and $105 \%$ substation primary voltage (LTC) 
The Test 3 profile, with the LTC and the first step VR, of Figure 122 shows a $1.68-\mathrm{V}$ spread beginning at Node 8 and continuing to the tag end of the circuit.

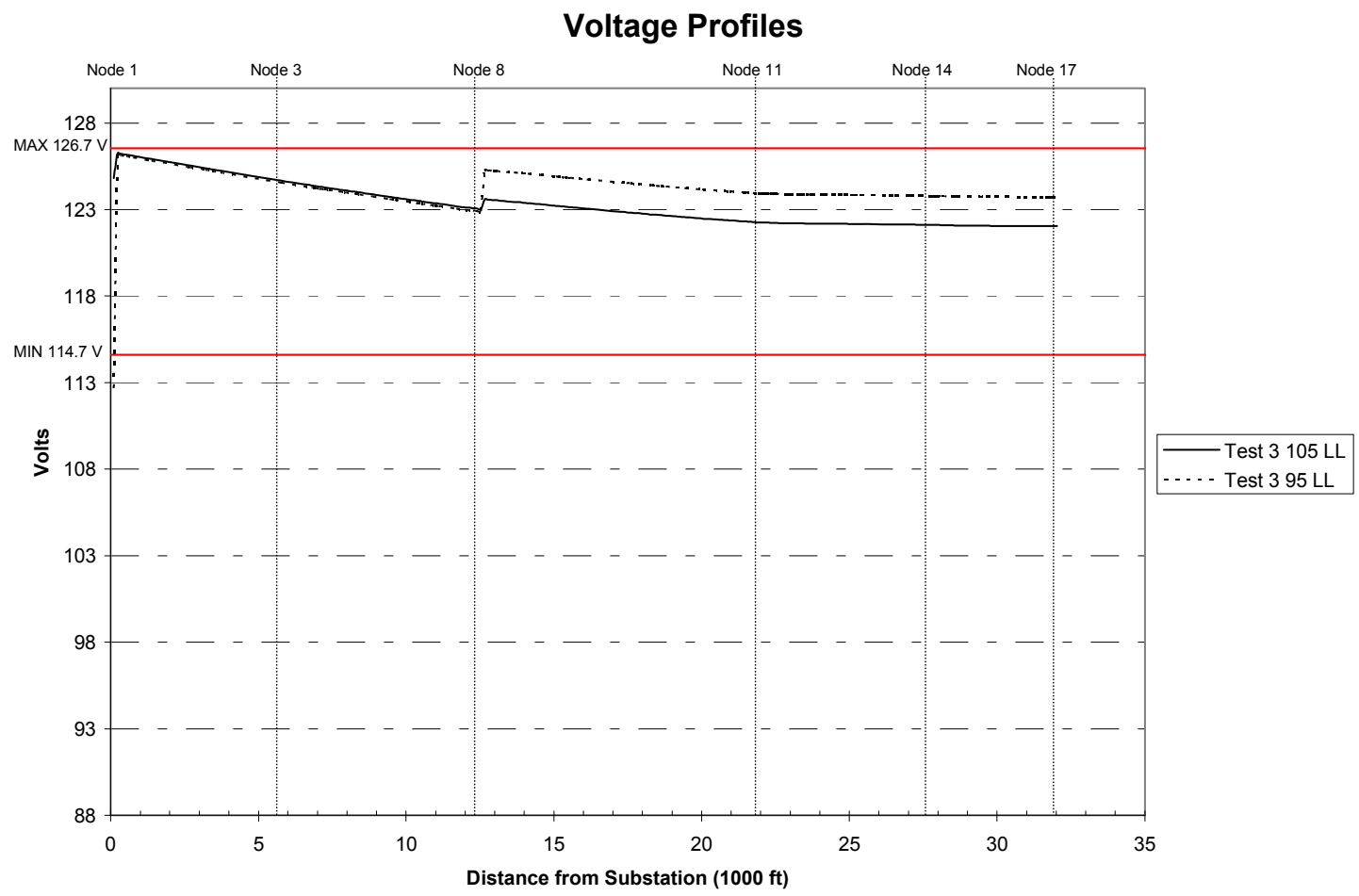

Figure 122. Test 3 LL circuit voltage profiles for $95 \%$ and $105 \%$ substation primary voltage (LTC and VR 1) 
Test 4 shows a similar condition when the second regulator is added. As shown in Figure 123, there is no voltage spread up to Node 8 , and at the tag end, the voltage spread is only $0.9 \mathrm{~V}$.

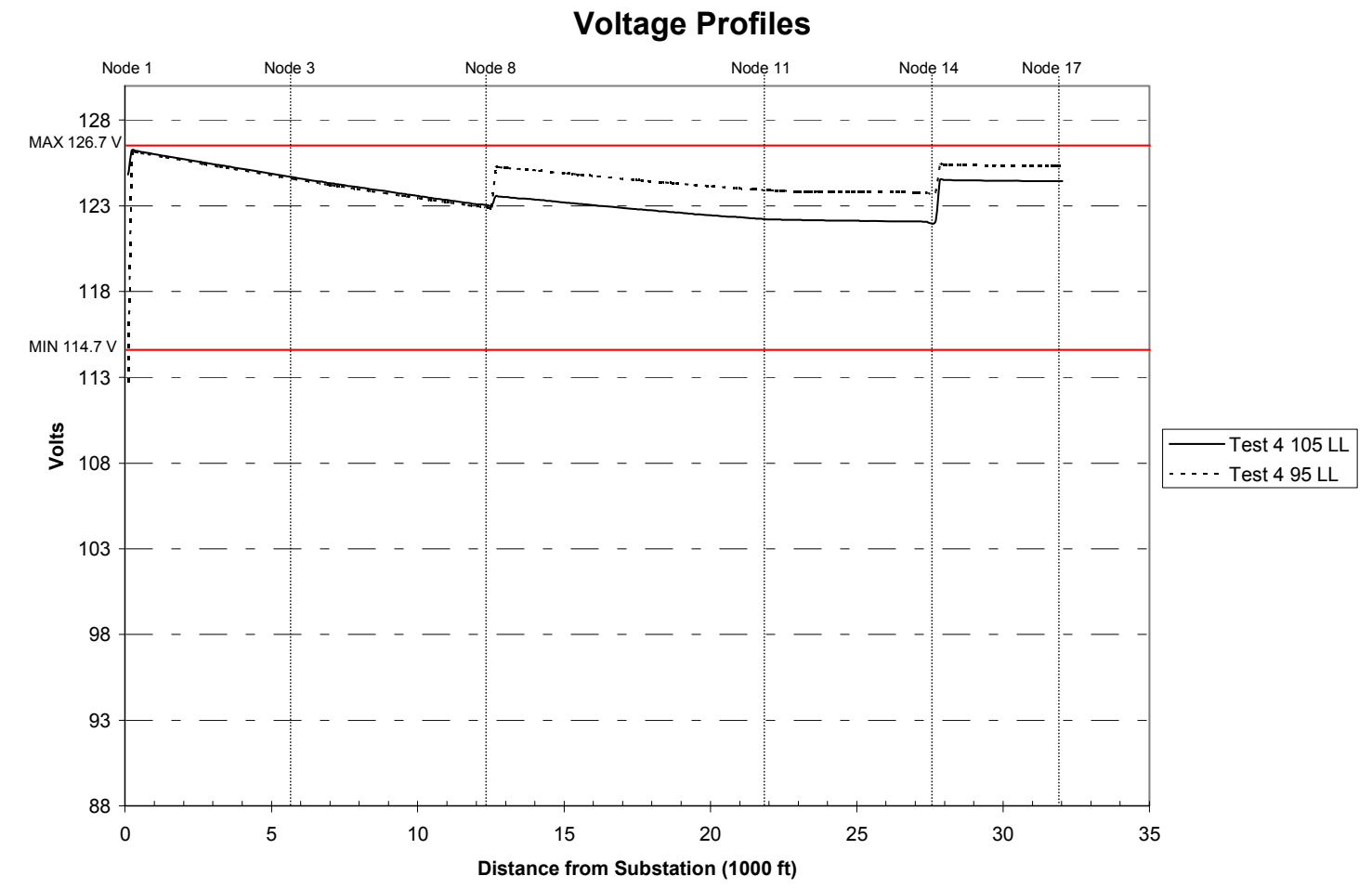

Figure 123. Test 4 LL circuit voltage profiles for $95 \%$ and $105 \%$ substation primary voltage (LTC, VR 1, and VR 2) 
Test 5, with the first capacitor on, shows a worsening condition in voltage spread. But the voltage spread at any node of Figure 124 is still very small. It is only $0.62 \mathrm{~V}$

(121.82-121.2 V).

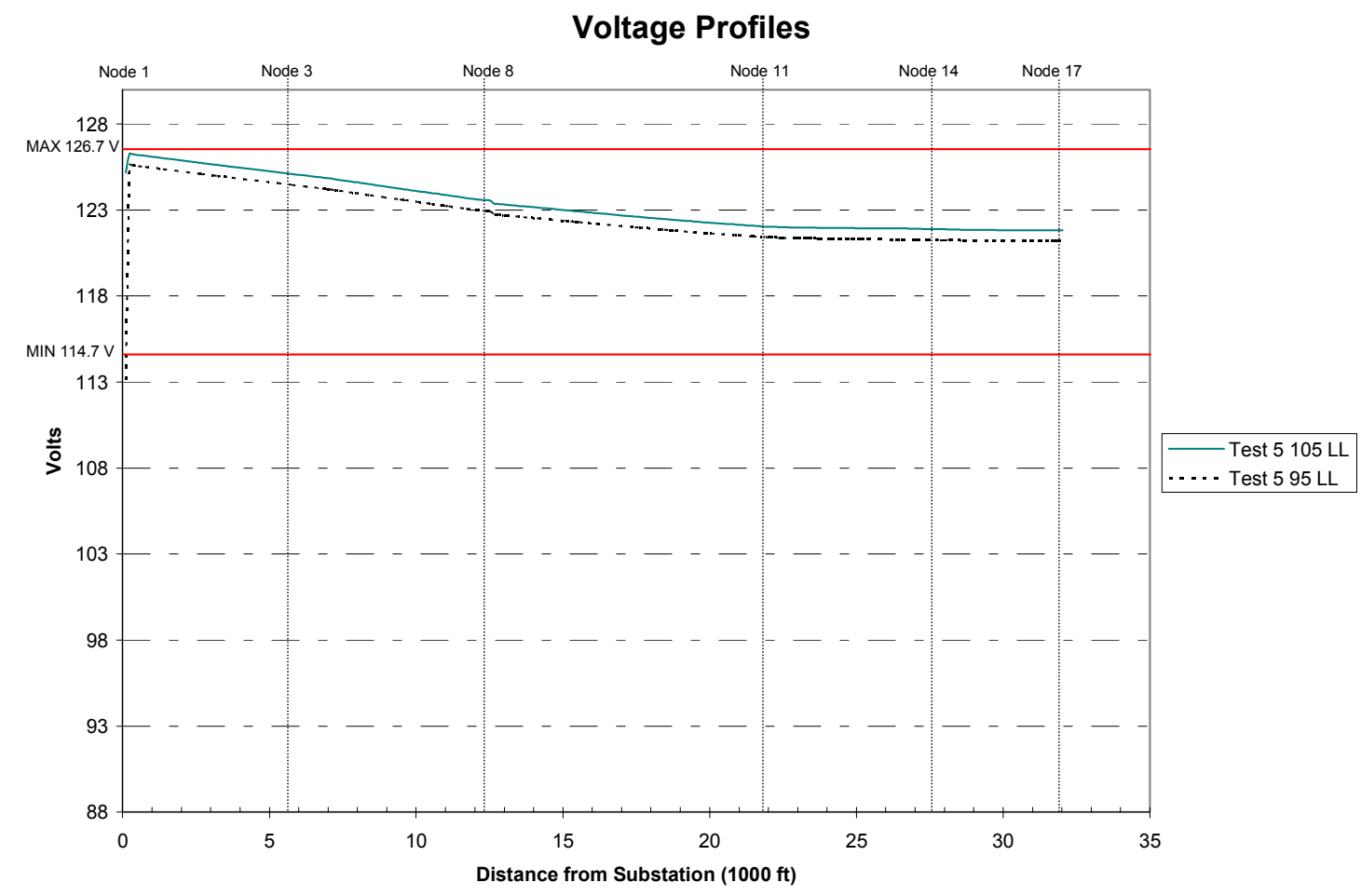

Figure 124. Test 5 LL circuit voltage profiles for $95 \%$ and $105 \%$ substation primary voltage (LTC and CAP 1) 
When the second capacitor is added in Test 6 , the voltage profile of Figure 125 shows little change throughout the length of the circuit, and the voltage spread is very low-only $0.48 \mathrm{~V}$ $(123.37-122.98 \mathrm{~V})$ at Node 17.

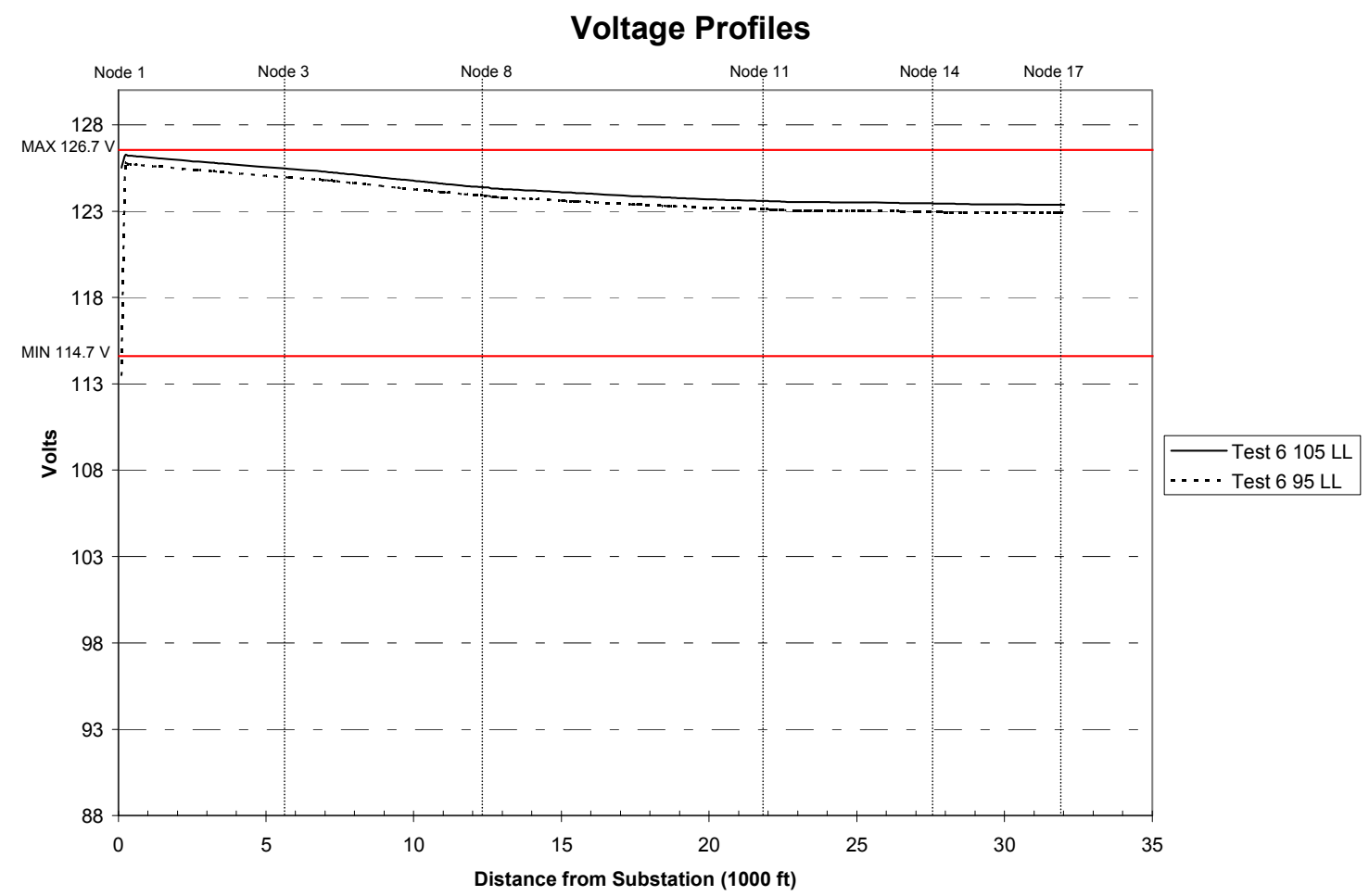

Figure 125. Test 6 LL circuit voltage profiles for $95 \%$ and $105 \%$ substation primary voltage (LTC, CAP 1, and CAP 2) 
Test 7, shown in Figure 126 and with the LTC and three capacitors on, is near the ultimate voltage regulation test. The voltage profile is nearly at the same level throughout the circuit, and the spread is only $0.54 \mathrm{~V}(124.44 \mathrm{~V}-123.9 \mathrm{~V}=0.54 \mathrm{~V})$ at Node 17 .

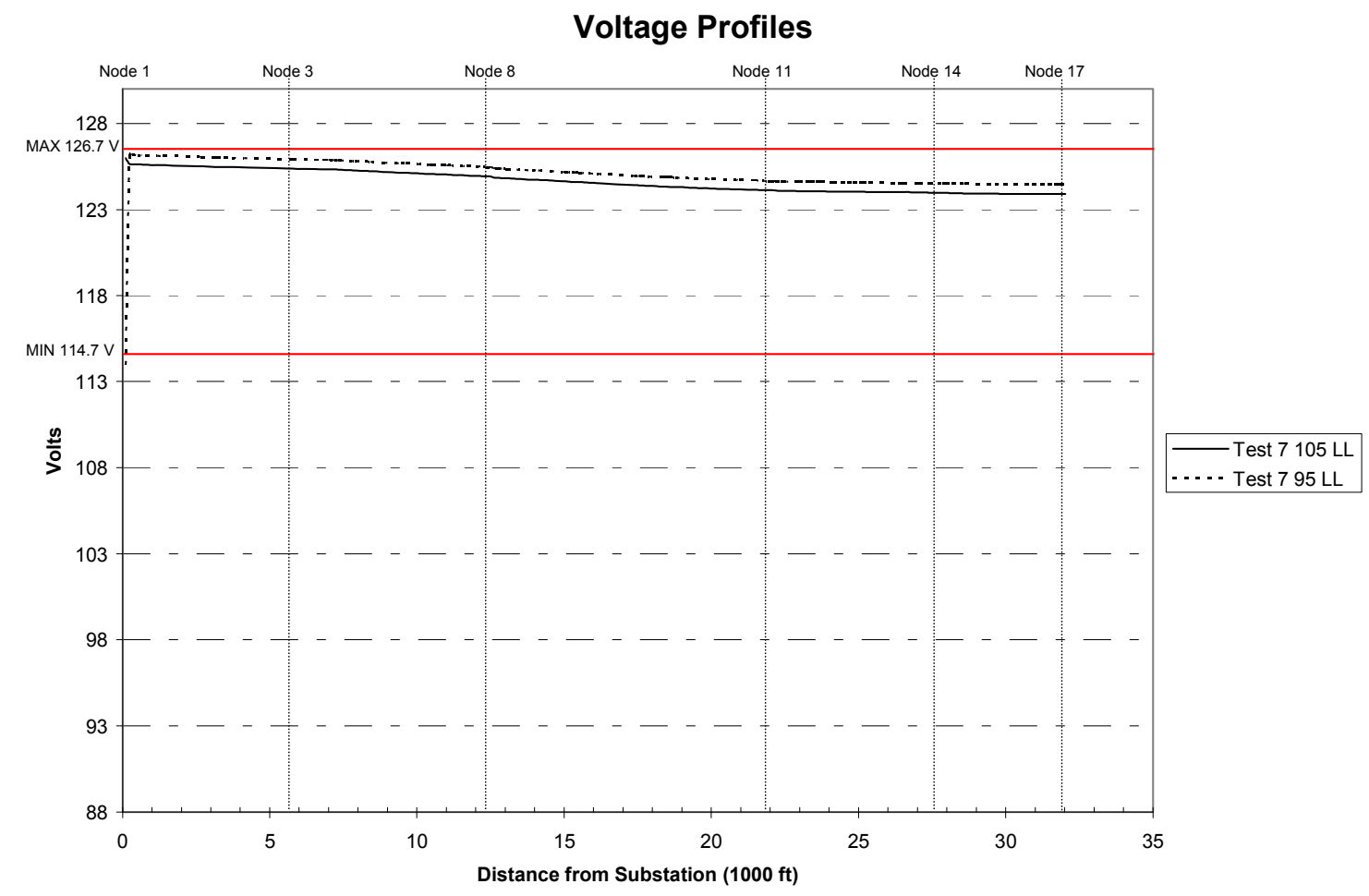

Figure 126. Test 7 LL circuit voltage profiles for $95 \%$ and $105 \%$ substation primary voltage (LTC, CAP 1, CAP 2, and CAP 3) 
Test 8, with all regulation and shown in Figure 127, is the ultimate test. It has no voltage spread from Node 8 to Node 17 and a spread of only $0.54 \mathrm{~V}(124.17-123.63 \mathrm{~V})$ at Node 17.

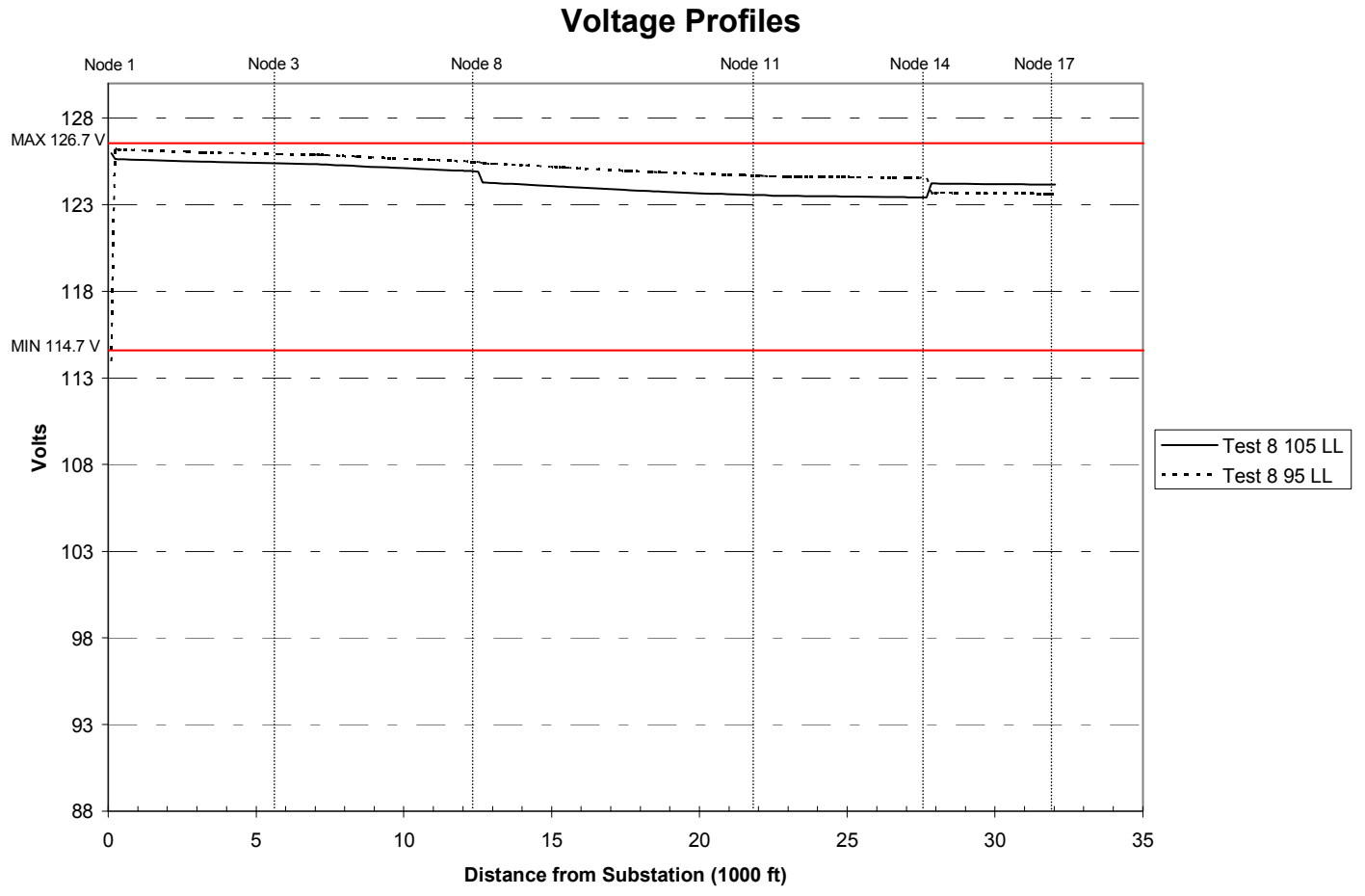

Figure 127. Test 8 LL circuit voltage profiles for $95 \%$ and $105 \%$ substation primary voltage (LTC, VR 1, VR 2, CAP 1, CAP 2, and CAP 3) 


\subsubsection{Light Load Comparison Circuit Voltage Profiles (Fourth Set 95\% Primary Voltage)}

The voltage comparison Test A of Figure 128 shows the improvement in voltage throughout the circuit when the first step regulator is added. The voltage profile drops from $126.07 \mathrm{~V}$ at the source (with the LTC) to $121.31 \mathrm{~V}$ at Node 17 for Test 2 . When the regulator is added in Test 3, the voltage rise at Node 17 is $2.41 \mathrm{~V}(123.72-121.81 \mathrm{~V})$.

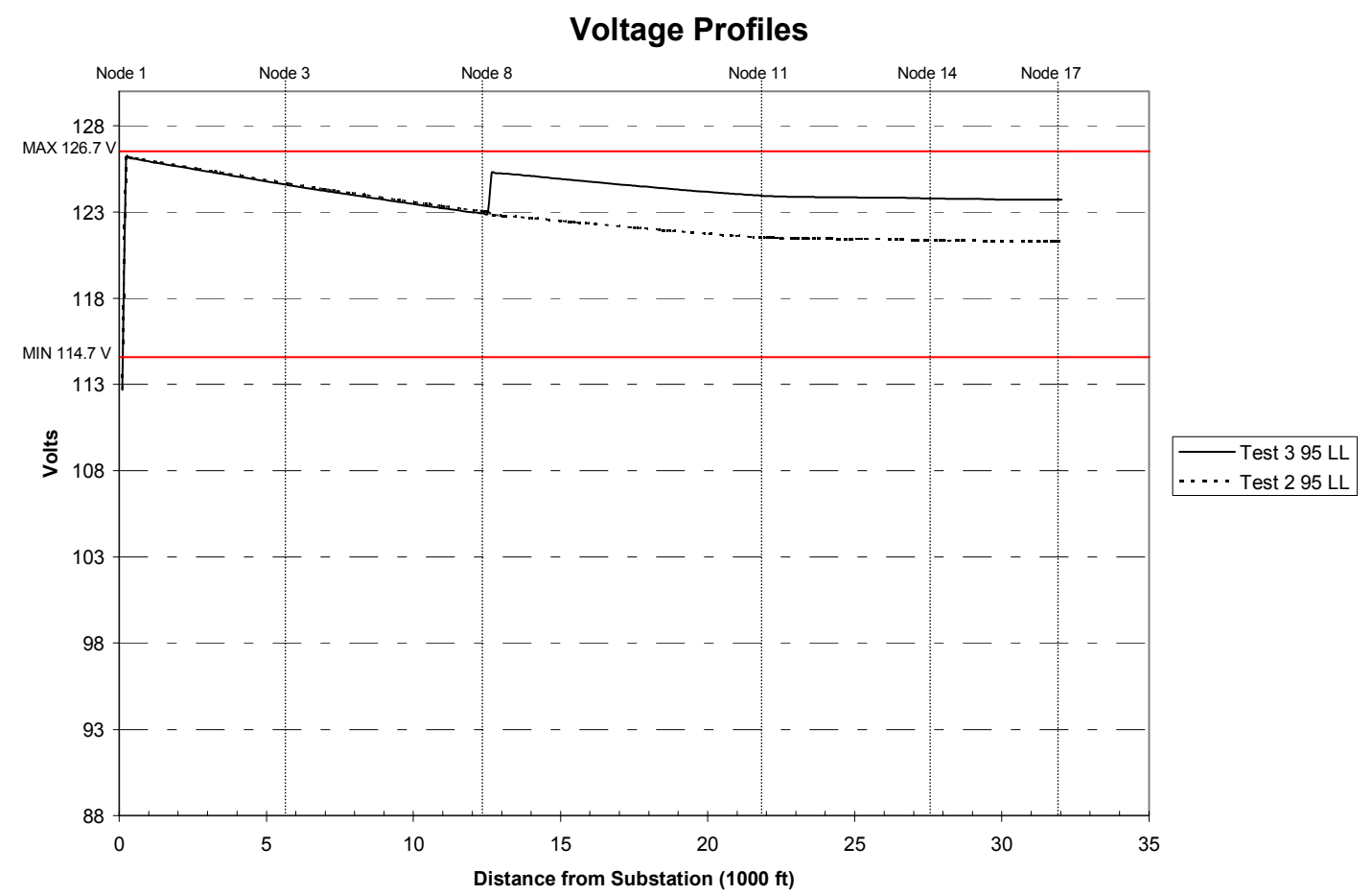

Figure 128. Comparison A: Test 2 and Test 3 LL circuit voltage profiles for $95 \%$ substation primary voltage (LTC versus LTC and VR 1) 
Comparison B, for Tests 3 and 4, is shown in Figure 129. The profiles of Tests 3 and 4 are the same until the second regulator is added. Beyond Node 14, there is a slight voltage spread of $1.63 \mathrm{~V}(125.35-123.72 \mathrm{~V})$.

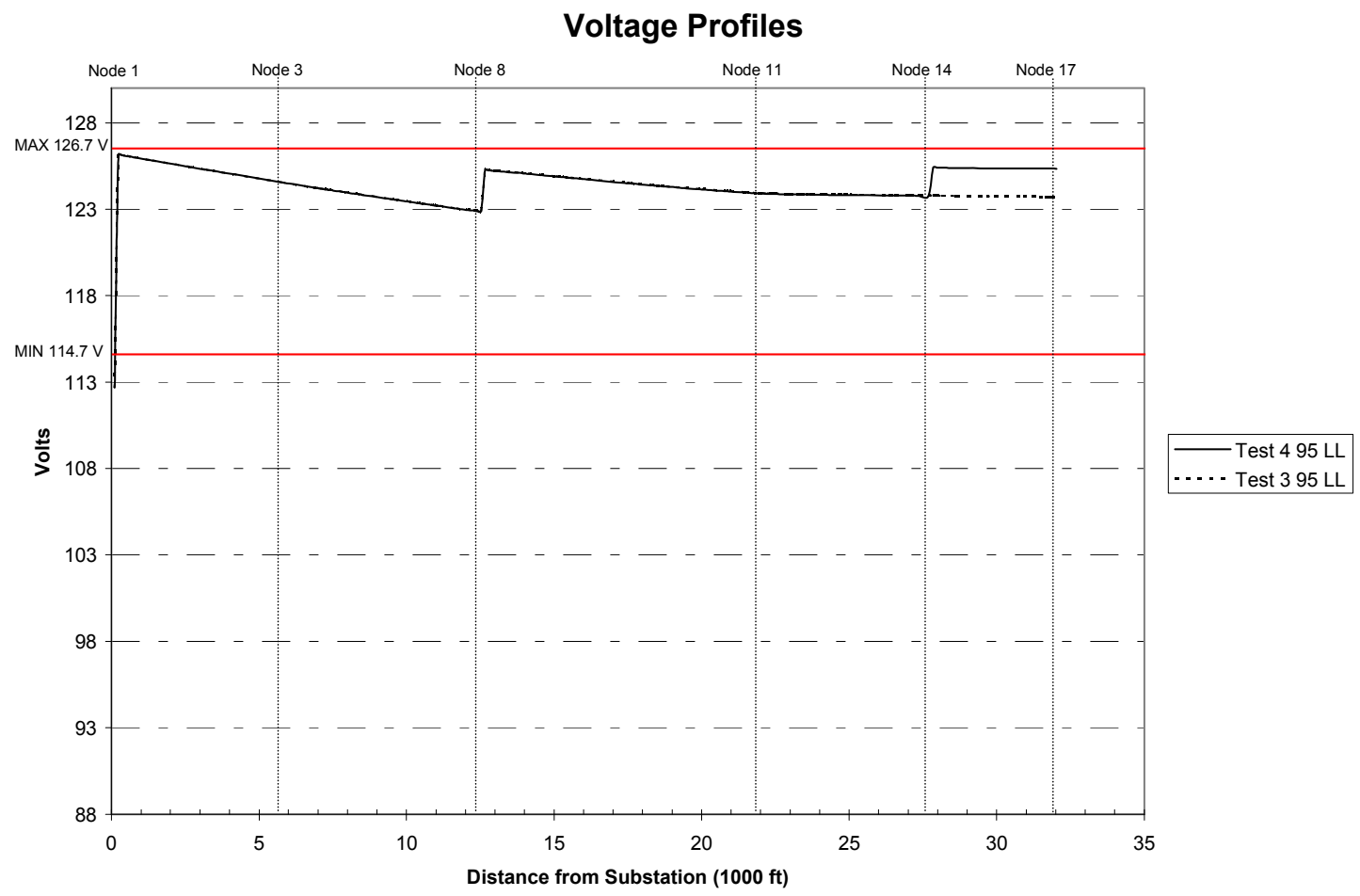

Figure 129. Comparison B: Test 3 and Test 4 LL circuit voltage profiles for $95 \%$ substation primary voltage (LTC versus LTC, VR 1, and VR 2) 
Figure 130 shows Comparison $\mathrm{C}$, in which a slight voltage spread exists throughout the circuit.

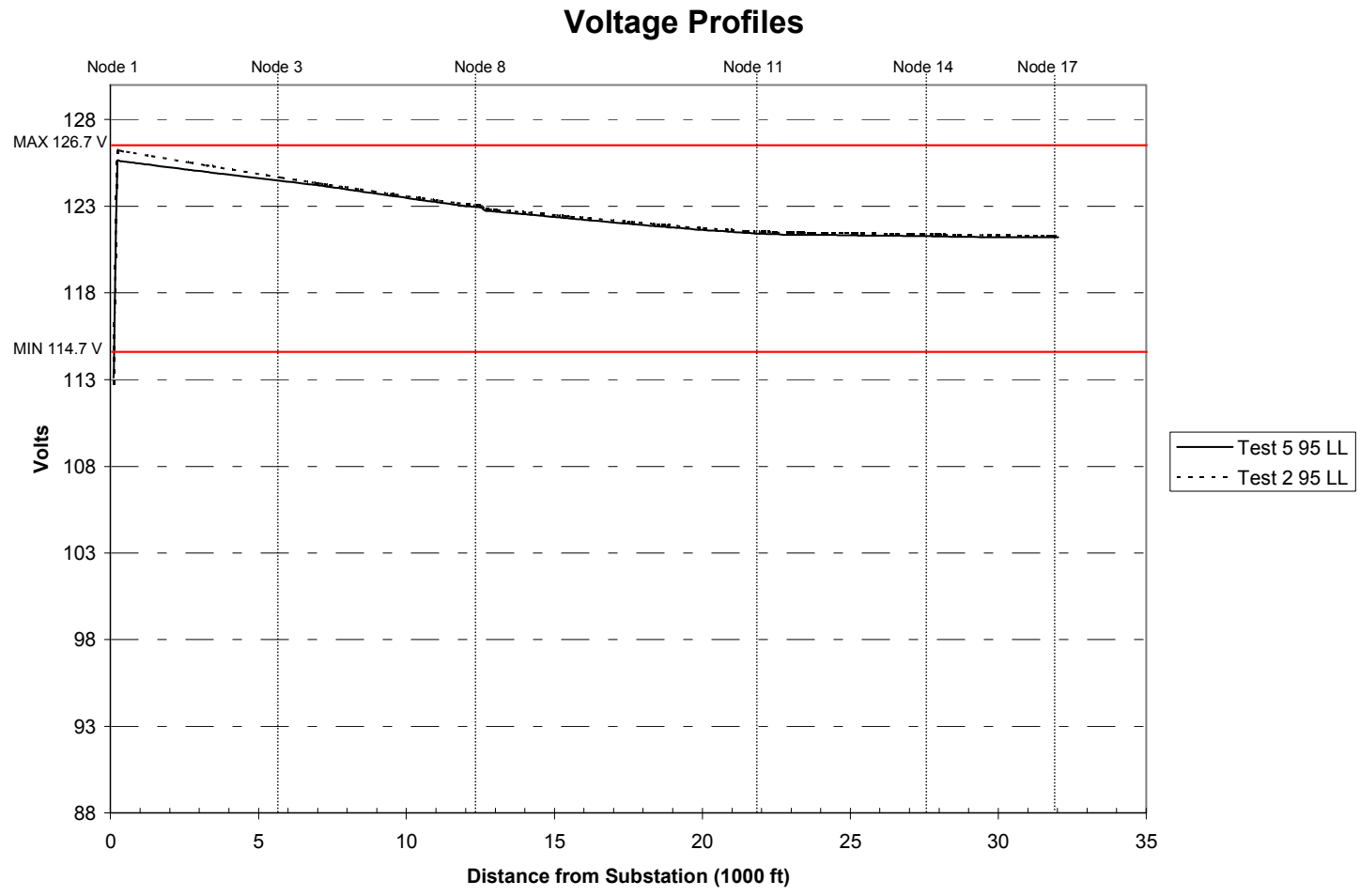

Figure 130. Comparison C: Test 2 and Test 5 LL circuit voltage profiles for $95 \%$ substation primary voltage (LTC versus LTC and CAP 1) 
The tests 6 and 5 comparison (D) of Figure 131 shows the rise in voltage at the tag end is greater than the rise at the source with the addition of the second capacitor. This is because this capacitor reduces the reactive and total current from its location back to the source.

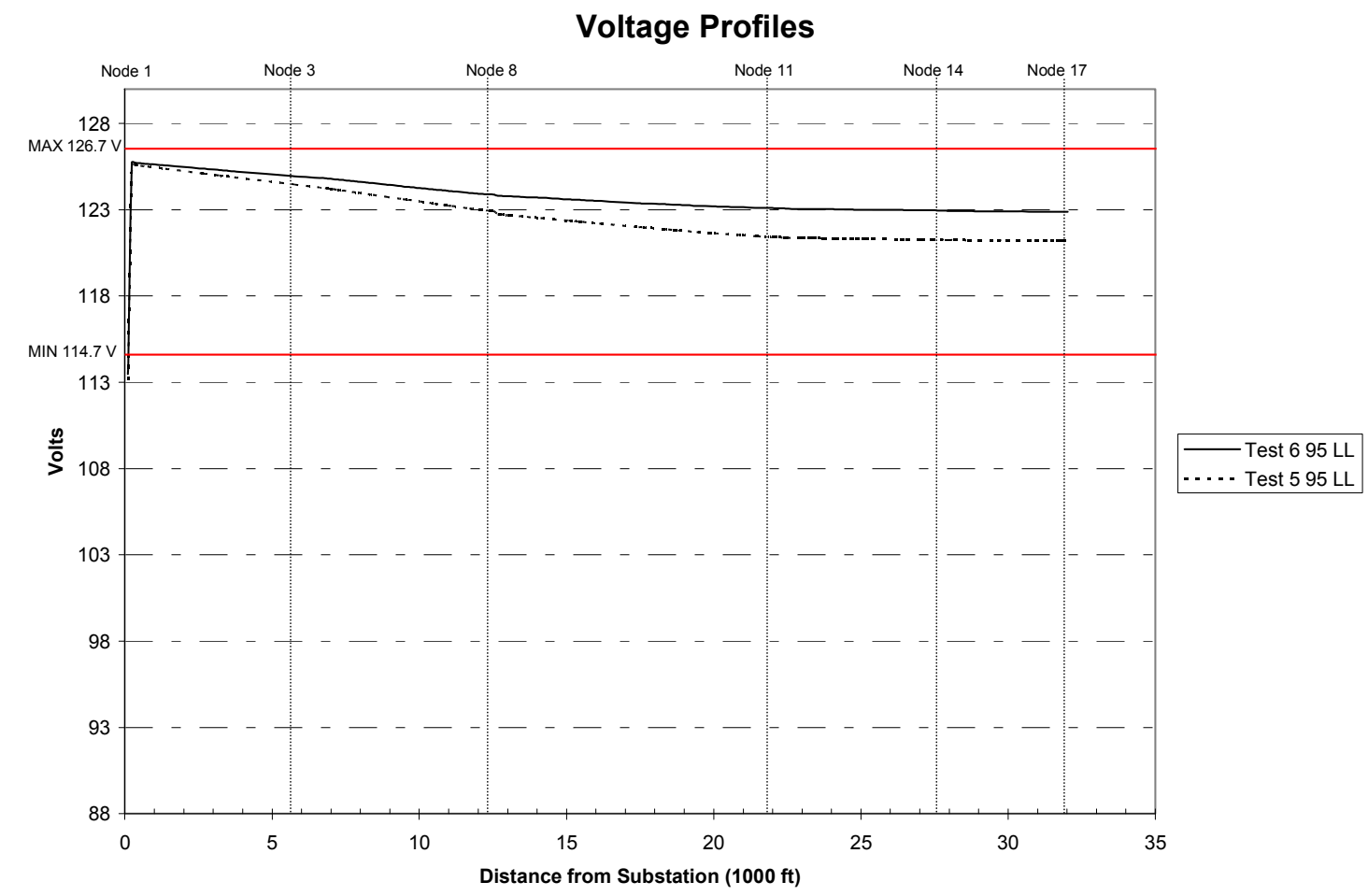

Figure 131. Comparison D: Test 5 and Test 6 LL circuit voltage profiles for $95 \%$ substation primary voltage (LTC and CAP 1 versus LTC, CAP 1, and CAP 2) 
When all three capacitors are added, as in Comparison E of Figure 132, the voltage rise (less voltage drop) effect is even greater. It shows a nearly level voltage throughout the circuit for Test 7.

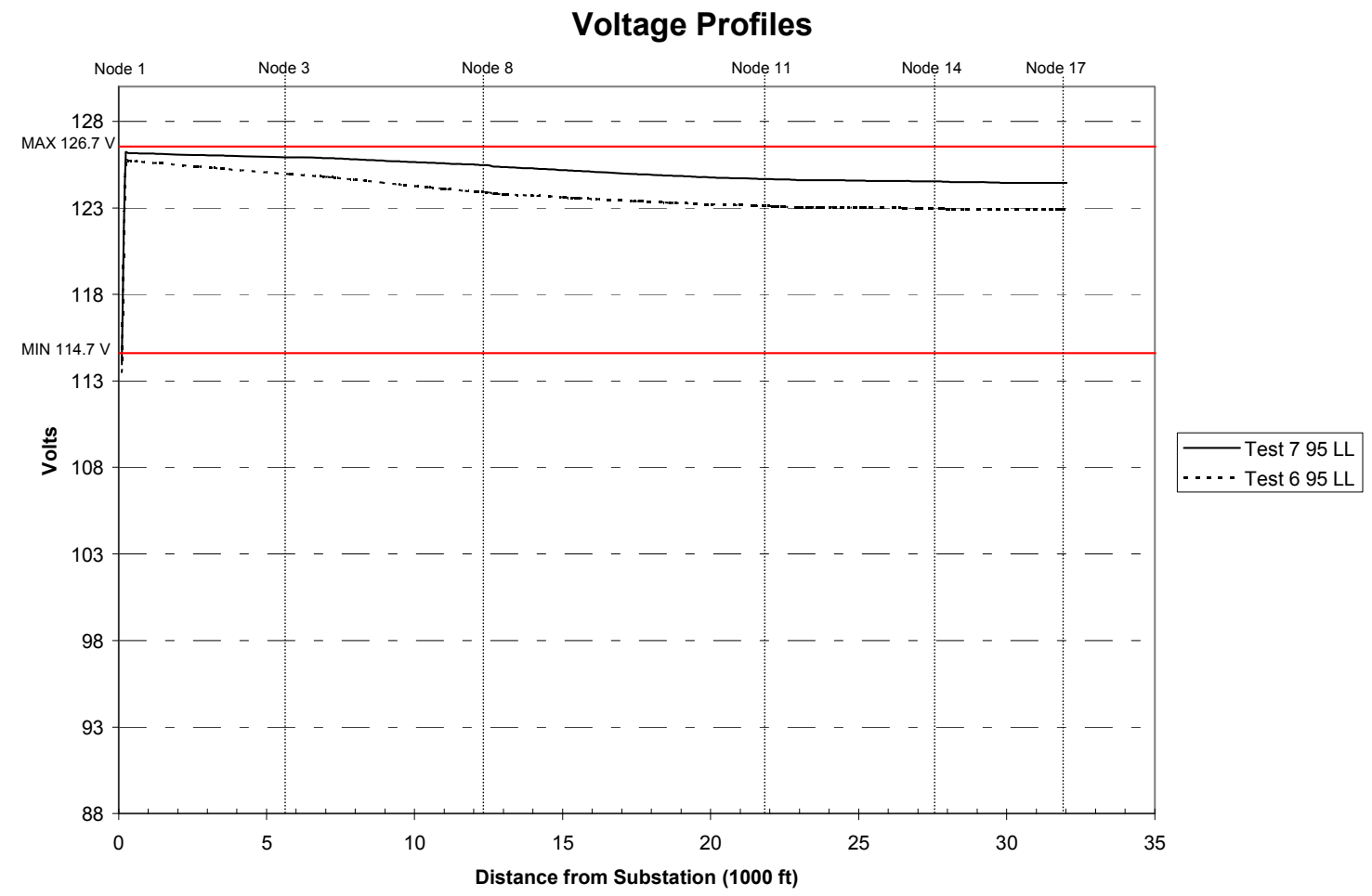

Figure 132. Comparison E: Test 6 and Test 7 LL circuit voltage profiles for $95 \%$ substation primary voltage (LTC, CAP 1, and CAP 2 versus LTC, CAP 1, CAP 2, and CAP 3) 
Comparison F shows the improvement in regulation between using only the LTC of Test 2 and all the regulation of Test 8 . The difference in voltage at Node 17 in Figure 133 is $2.32 \mathrm{~V}$ (123.72-121.3 V).

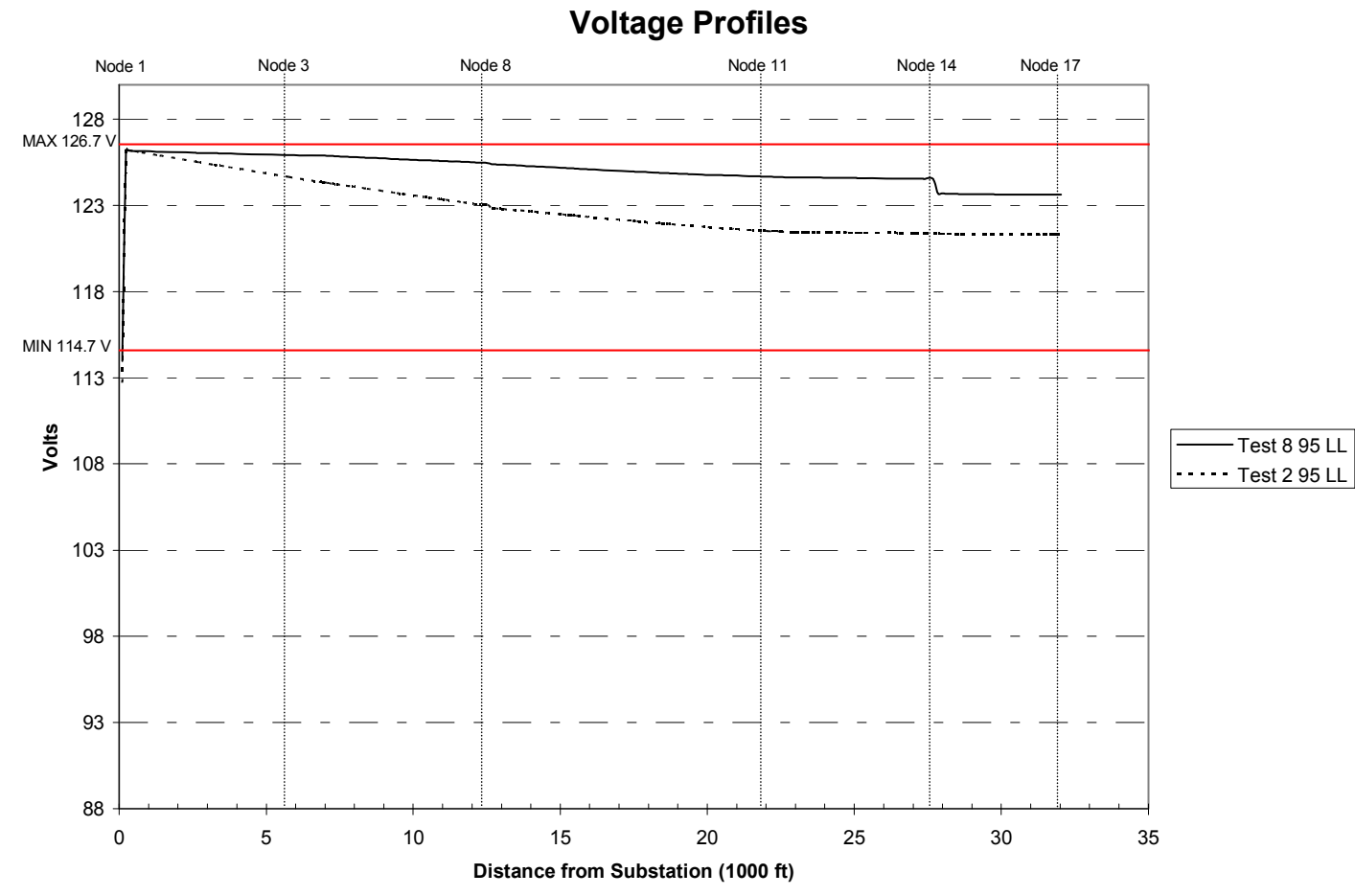

Figure 133. Comparison F: Test 2 and Test 8 LL circuit voltage profiles for $95 \%$ substation primary voltage (LTC versus LTC, VR 1, VR 2, CAP 1, CAP 2, and CAP 3) 
Comparison G, shown in Figure 134, shows the effect of adding the two regulators to the three capacitors of Test 7 . The voltage is more uniform throughout the circuit for Test 7 than it is with the two regulators added in Test 8 . But, as stated earlier, the voltage spread is better with the two regulators turned on when the primary voltage ranges from $95 \%$ to $105 \%$.

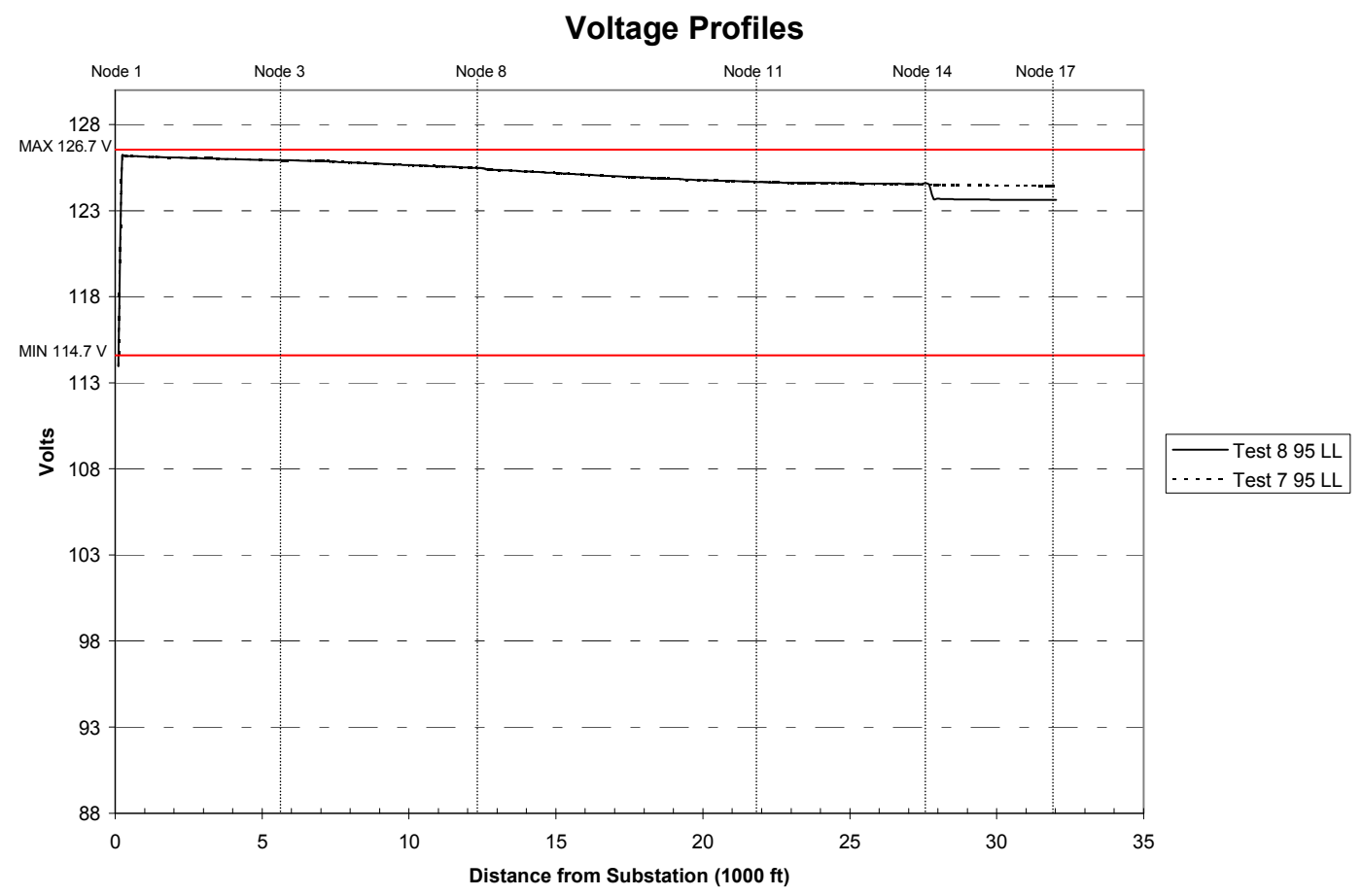

Figure 134. Comparison G: Test 7 and Test 8 LL circuit voltage profiles for $95 \%$ substation primary voltage (LTC, CAP 1, CAP 2, and CAP 3 versus LTC, VR 1, VR 2, CAP 1, CAP 2, and CAP 3)

\subsection{Distribution Circuit Simulation Summary Data for Tests 1- 8}

As noted earlier, for each test, summary data were assembled (see Table 22). These data consist of the primary voltage (either $95 \%$ or $105 \%$ ), load condition (either HL or LL), tap settings for the LTC and step regulators, line currents at Node 0, the lowest three-phase voltage, the lowest single-phase voltage, the highest load imbalance, the highest voltage imbalance, the kilowatt losses per phase and total, the kilovar losses per phase and total, and the release capacity per phase and total when the capacitors are applied. Because the maximum imbalance load can be $100 \%$ for any line-to-line load connected to the wye system, Table 23 is provided to show the range of unbalance other than $100 \%$.

\subsubsection{Unbalanced Voltage}

Unbalanced voltage is determined by calculating the negative sequence $V_{2}$ and positive sequence $V_{1}$ and dividing $V_{2}$ by $V_{1}$. Table 22 shows the highest unbalanced voltage in percent for each test. The voltage unbalance is very important for siting DG because most synchronous generators trip when the unbalance reaches 3\%. The highest unbalanced voltages on the circuit range from $1.52 \%$ to $1.26 \%$. The highest unbalanced voltages for each test are shown in Table 27. 
It is interesting to note that the highest measured imbalance from the unbalance voltage survey of Section 4.5.1.2 and Table 9 was 5.94\% with the average being $0.83 \%$ for wye and closed delta transformer connections. Although these measured data are taken on the secondary side of the distribution transformers, whereas the simulated data are for the primary in Table 27, there is reasonably close agreement - especially when one considers the data in Table 27 are for one circuit while the measured data are for a large population of 1,209 tests across a multiplicity of circuits from 13 utilities.

Table 27. Highest Unbalanced Voltages

\begin{tabular}{|c|c|c|c|c|c|}
\hline & Regulation Method & $95 \% \mathrm{HL}$ & $95 \%$ LL & $105 \% \mathrm{HL}$ & $105 \% \mathrm{LL}$ \\
\hline Test 1 & No LTC & $1.32 \%$ & $1.26 \%$ & $1.43 \%$ & $1.26 \%$ \\
\hline Test 2 & LTC & $1.44 \%$ & $1.26 \%$ & $1.48 \%$ & $1.26 \%$ \\
\hline Test 3 & LTC, VR 1 & $1.49 \%$ & $1.26 \%$ & $1.52 \%$ & $1.26 \%$ \\
\hline Test 4 & LTC, VR 1, VR 2 & $1.44 \%$ & $1.26 \%$ & $1.48 \%$ & $1.26 \%$ \\
\hline Test 5 & LTC, CAP 1 & $1.42 \%$ & $1.26 \%$ & $1.45 \%$ & $1.26 \%$ \\
\hline Test 6 & LTC, CAP 1, CAP 2 & $1.36 \%$ & $1.26 \%$ & $1.38 \%$ & $1.26 \%$ \\
\hline Test 7 & LTC, CAP 1, CAP 2, CAP 3 & $1.31 \%$ & $1.26 \%$ & $1.33 \%$ & $1.26 \%$ \\
\hline Test 8 & LTC, VR 1, VR 2, CAP 1, CAP 2, CAP 3 & $1.31 \%$ & $1.26 \%$ & $1.27 \%$ & $1.26 \%$ \\
\hline
\end{tabular}

Notice that all the unbalanced voltages are greater during HL conditions. Increasing the voltage increases the load, and thus, the unbalance increases. Adding an LTC increased the voltage unbalance from $1.32 \%$ to $1.44 \%$ at $95 \% \mathrm{HL}$. Another finding was that the highest unbalanced voltages occur at $105 \%$ primary voltage, as would be expected. In fact, with no LTC, the voltage unbalance at $105 \%$ primary voltage was $1.43 \%$ versus $1.32 \%$ at $95 \%$ primary voltage. Lowering the primary voltage to $95 \%$ reduced the voltage unbalance because of the effect of the VDC source model. Adding capacitors reduced the unbalanced voltage, and adding a step VR increased the unbalance from $1.44 \%$ with the LTC up to $1.49 \%$ with one step regulator and a $95 \%$ primary voltage.

When the LTC and all three capacitors were on, the voltage unbalanced was reduced to $1.31 \%$ at $95 \%$ primary voltage and down to $1.33 \%$ at $105 \%$ primary voltage. The highest unbalanced voltage on the circuit was reduced to $1.31 \%$ with all regulation. It is obvious from these results that the voltage regulation method and the amount of regulation on the circuit are important in reducing the imbalance to acceptable levels. 


\subsubsection{Unbalanced Loading}

Table 23 summarizes the load imbalance for each test. It is calculated as the negative sequence load $\mathrm{I}_{2}$ divided by the positive sequence load $\mathrm{I}_{1}$ in percent. Most synchronous generators trip when the load imbalance becomes more than $10 \%-20 \%$. However, inverter-based generation has been tested and successfully operated even when the unbalance reached $100 \%$. However, the total capability was reduced to $75 \%$. The data in Table 23 show there are locations on the circuit where the unbalanced load was greater than $10 \%-20 \%$. Care should be given to discover the magnitude of unbalanced load before a synchronous generator is sited on the circuit. At Node 10, where the $1,000-\mathrm{kW}$ generator is interconnected to the distribution circuit, the load unbalance is $1.22 \%$ for $95 \%$ primary voltage and HL, and when all regulation is turned on, the voltage unbalance is $1.29 \%$. Notice from Table 23 that the load unbalance is always higher at LL than HL. This is good because most DR operate during peak load conditions.

The load imbalance at the substation Node 01 ranges from about $4 \%$ for $\mathrm{HL}$ and about $5.45 \%$ at LL. This is an excellent location to site synchronous generators. There are points on the circuit where the load unbalance is lower than at the substation, but there are many points where the imbalance is up to $90 \%$. Adding regulation did not necessarily improve load imbalance. In fact, with all regulation on, the Node 10 value was $3.89 \%$ at $95 \%$ primary voltage versus $3.77 \%$ with no regulation and HL conditions.

\subsection{Significant Results and Conclusions}

The Milford Circuit DC 8103 is typical of residential and light commercial load characteristics.

- The CC load model, the CP load model, and the VDC load model were applied in tests 7 and 8 at 95\% source voltages for HL conditions. However, as shown in Appendix B.1, the VDC model was better than the $\mathrm{CC}$ model (i.e., $2.0 \%$ error versus $5.3 \%$ ). Therefore, the best overall method to represent the load characteristics as a function of source voltage changes was the VDC model.

\section{Heavy Load}

- The highest voltage spread, measured as the difference between the highest threephase voltage and the lowest single-phase voltage, was $25.2 \mathrm{~V}$ and occurred with no LTC regulation at the substation transformer during HL conditions and with the primary voltage on the substation transformer ranging from $95 \%$ to $105 \%$. This spread was reduced to $10.4 \mathrm{~V}$ with all regulation methods turned on.

- When the LTC and first step regulator were added, the tag end voltage improved 4.11 $\mathrm{V}$, and with the second regulator turned on, the voltage increased another $11.34 \mathrm{~V}$.

- When the LTC and first capacitor were added, the voltage increased only $0.97 \mathrm{~V}$, and with the addition of the second capacitor, the voltage gain was $1.75 \mathrm{~V}$ at the tag end. The third capacitor added another $1.66 \mathrm{~V}$ rise at the tag end.

- When all regulation was turned on, the tag end voltage improvement was $15.35 \mathrm{~V}$ over only the LTC transformer to regulate voltage. Also, when DR are added to the circuit, this method of voltage regulation reduces the voltage spread even further. 


\section{Light Load}

- During LL and no LTC regulation, the voltage spread, as determined by subtracting the highest three-phase voltage from the lowest single-phase voltage, was $16.65 \mathrm{~V}$ compared to $25.2 \mathrm{~V}$ for HL. With all regulation turned on, this difference diminished to $2.91 \mathrm{~V}$.

- Care should be exercised when operating DR during LL because the highest threephase voltage on the Milford Circuit was $126.24 \mathrm{~V}$. It may be necessary to operate DR to absorb volt-amperes reactive to prevent $\mathrm{HV}$.

\section{Unbalanced Conditions}

- Raising the source voltage during HL conditions with the LTC and a step regulator turned on created the highest unbalance voltage of $1.52 \%$. Most synchronous generators trip at unbalanced voltages more than $3 \%$. Studies should always be conducted to ensure the unbalanced voltage does not exceed $3 \%$ at the point of interconnection (not point of common coupling) for the DR. Of course, if an isolation transformer is installed at the point of interconnection with a delta-wye transformer connection, it will improve the voltage unbalance seen by the generator.

- Adding the LTC regulation at HL and 95\% primary substation voltage lowered the maximum unbalance voltage to $1.44 \%$. Adding a step regulator worsened the unbalanced voltage by raising it to $1.49 \%$, but adding a second step regulator reduced the unbalanced voltage to $1.44 \%$.

- Adding capacitors reduced the maximum unbalanced voltage. The first capacitor turned on lowered this unbalanced voltage to $1.42 \%$. With both capacitors turned on, the unbalanced voltage went down to $1.36 \%$. All three capacitors turned on lowered the unbalance to $1.31 \%$.

- Having all regulation turned on lowered the maximum unbalanced voltage to $1.31 \%$ for $95 \%$ primary voltage and HL (worst-case condition). The 13-utility unbalanced voltage survey showed the maximum measured voltage unbalance of 5.94\%.

- The highest voltage unbalance during LL was $1.26 \%$, even though the primary voltage on the transformer ranged from $95 \%$ to $105 \%$.

- At many locations on the circuit, the current imbalance exceeded 20\%. Most synchronous generators trip between $10 \%$ and $20 \%$ current imbalance. Inverter-based generation can operate under $100 \%$ load imbalance, but typically, they do so at $75 \%$ of rated capacity. The greatest unbalance current was 100\% at locations where line-toline loads or line-to-neutral loads were connected to the three-phase, wye-grounded system. Unbalances of 20\%-90\% were apparent at many nodes, but, generally, the load magnitudes were small and, thus, not causing the generator to trip. The greater load imbalances occurred at LL. 
- The current imbalance at Node 10 , where the $1,000-\mathrm{kW}$ synchronous generator is interconnected, was $1.22 \%$ during HL conditions, and the voltage unbalance was $1.29 \%$. Even the load imbalance at the substation was about $4 \%$ at HL but reached about $6 \%$ at LL. If a DR is installed at the substation and operated in an isolated mode (i.e. as a microgrid), it may see $10 \%$ current imbalance during LL. However, on other circuits on the system, the load imbalance at the substation could be as high as $20 \%$. It is important to determine the imbalanced current at the point of interconnection before ever siting a synchronous DR generator.

- The zero sequence current ranged from 45.43 A to 50.24 A for the simulations conducted. See Table B-3. This level of zero sequence current is not a problem from a system standpoint, but when the system is lost, a portion of the circuit is operated as a microgrid or island, and there are line-to-neutral loads and line-to-line loads, high negative sequence currents can be expected. When the wye ungrounded synchronous generator windings are connected to a delta-wye high-side transformer with neutral solidly grounded, zero sequence currents from a line-to-ground load on one phase of the system will create $100 \%$ negative sequence currents on the generator windings, or the negative sequence current will be equal to the positive sequence current. Most small generators are wye ungrounded with a delta-wye high-side transformer and grounded neutral connection, so this represents the most common installation. Appendix A.3 shows that a generator with only 33.34 A of zero sequence current on the high side will see $100 \%$ unbalanced currents on the generator windings. The negative sequence current is $33 \%$ of the full rated three-phase capacity of the generator. This will cause the generator negative sequence relays to trip at $10 \%$ negative sequence current. This means the generator will trip if it serves only $30 \mathrm{~A}$ of single-phase load, even though the rated current is 100 A per phase of load current. Because the other two phases are unloaded, the generator can produce only $1 / 10$ of its full rated three-phase machine capacity (i.e., $30 \mathrm{~A}$ single-phase load $\div 300 \mathrm{~A}$ threephase capacity). 


\section{Project Results - Design of Field Voltage Regulation and Metering Equipment}

\subsection{Introduction}

This section defines the equipment installed on Milford Circuit DC 8103 to control voltage and the metering equipment used to validate the models.

\subsection{Voltage Regulation Equipment}

Figure 135 shows the locations of the voltage regulation equipment on Milford Circuit DC 8103. Table 28 describes this equipment.

Table 28. Voltage Regulation Equipment

\begin{tabular}{|c|c|}
\hline Location & Description \\
\hline A. & $\begin{array}{l}\text { 10-MVA delta-wye transformer } \# 1 \\
Z=7.02 \%, 41.57 \mathrm{kV} / 13.8 \mathrm{kV}, 0, \pm 2.5, \pm 5.0 \text { high-side setting }=0 \text {, low-side setting }= \pm 5 \text {, } \\
\text { LTC }+16 \text { steps }+10 \%, a_{N}=3 \text { (See Figure } 136 \text { ) }\end{array}$ \\
\hline B. & Three 167-kVA single-phase step VRs, 32 steps \\
\hline D. & $\begin{array}{l}1,000-\mathrm{kW} \text { synchronous generator, } 1,050-\mathrm{kW} \text { power prime mover, rated output }=1,312 \mathrm{kVA} \\
@ \mathrm{PF}=0.8,1,800 \mathrm{rpm}, 480 \mathrm{~V}, 60 \mathrm{~Hz} \text {, voltage regulation } \pm 5 \% \text {. }\end{array}$ \\
\hline F. & (1) 900-kVAr three-phase capacitor, Y-connected, neutral grounded \\
\hline $\begin{array}{l}\text { G. } \\
\mathrm{H} \text {. }\end{array}$ & $\begin{array}{l}\text { (2) 900-kVAr three-phase capacitor, Y-connected, neutral grounded } \\
\text { (3) 1,200-kVAr three-phase capacitor, Y-connected, neutral grounded }\end{array}$ \\
\hline
\end{tabular}




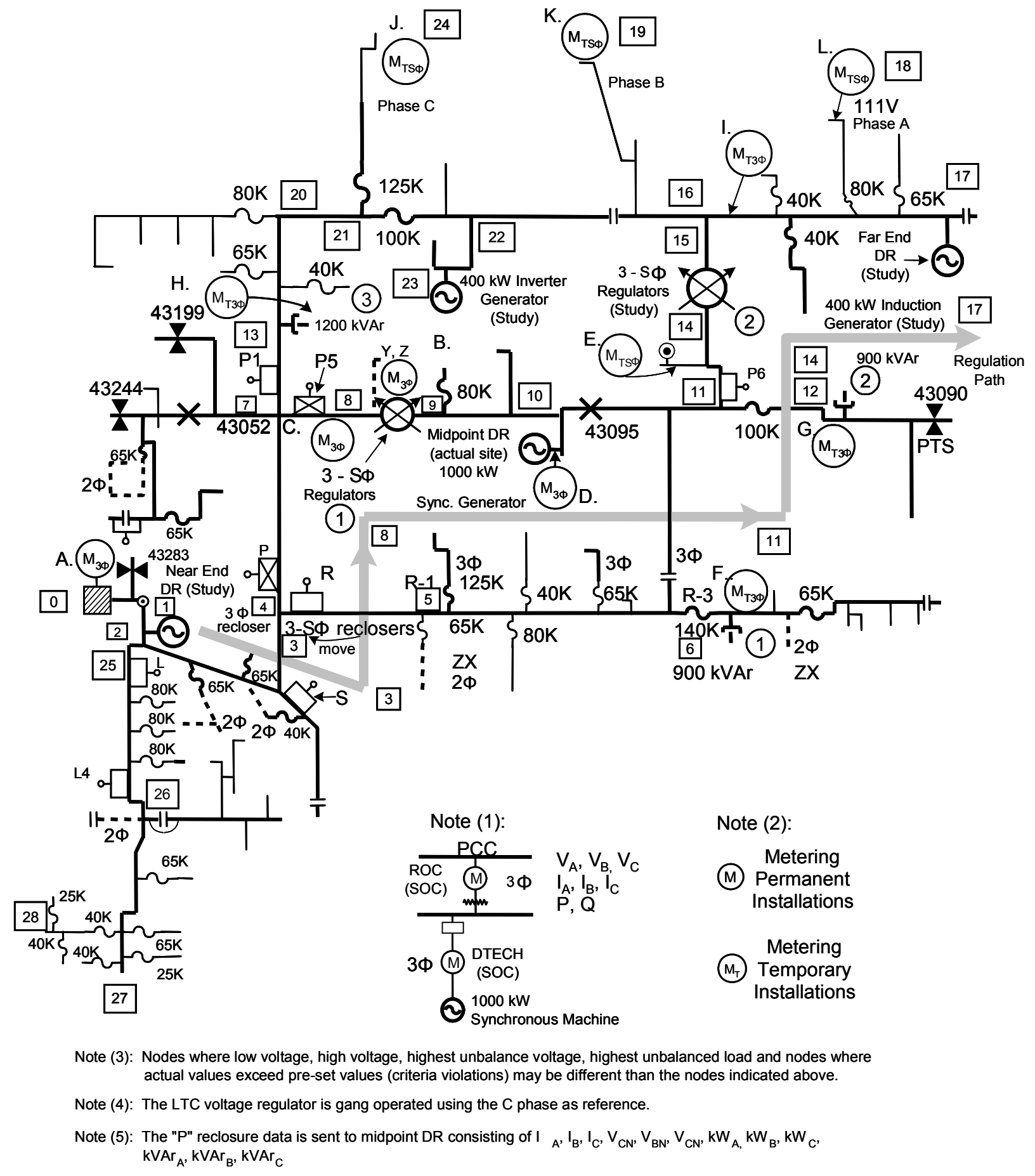

Figure 135. Milford Circuit DC 8103 


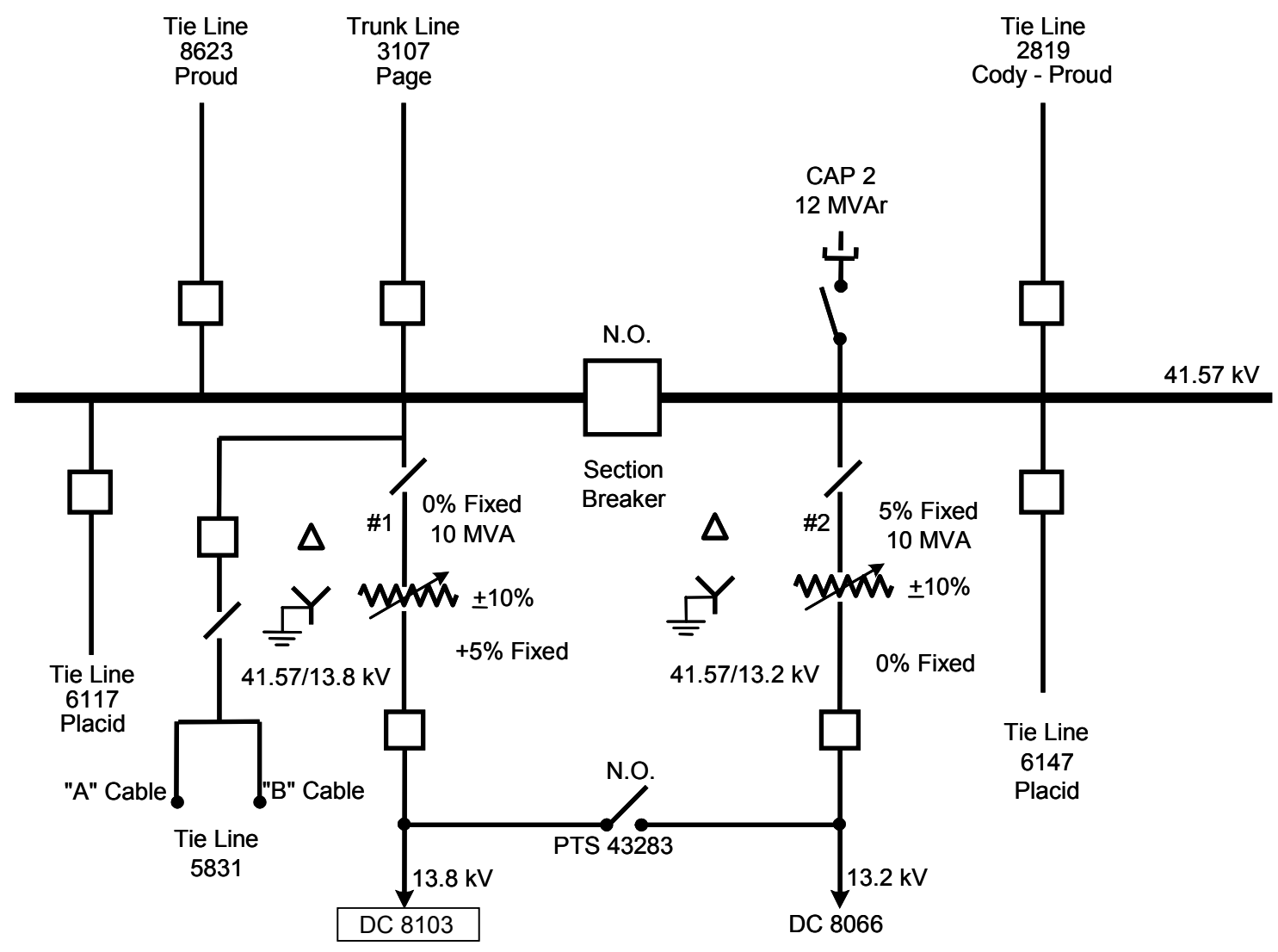

Figure 136. Milford substation one-line diagram

\subsection{Major System Protection Equipment}

The major system protection equipment is listed in Table 29. It consists of three-phase and single-phase reclosers and single-phase sectionalizers.

Table 29. Major System Protection Equipment

\section{Location}

1. $\mathrm{R}$

2. $\mathrm{P}$

3. $\mathrm{P5}$

4. $\mathrm{P} 1$

5. S

6. L

7. L4

8. P6

\section{Description}

Three three-phase reclosers $-280 \mathrm{~A}$ vacuum $\mathrm{V} 4 \mathrm{~L}, 2 \mathrm{~A}, 2 \mathrm{D}$

One three-phase recloser -680 A vacuum VWE, R, C

Three single-phase reclosers $-280 \mathrm{~A}$ vacuum $\mathrm{V} 4 \mathrm{~L}, 2 \mathrm{~A}, 2 \mathrm{D}$

Three single-phase reclosers $-280 \mathrm{~A}$ vacuum $\mathrm{V} 4 \mathrm{~L}, 2 \mathrm{~A}, 2 \mathrm{D}$

Three single-phase reclosers $-140 \mathrm{~A}$ vacuum $\mathrm{V} 4 \mathrm{~L}, 1 \mathrm{~A}, 3 \mathrm{D}$

Three single-phase reclosers - $140 \mathrm{~A}$ vacuum $\mathrm{V} 4 \mathrm{~L}, 1 \mathrm{~A}, 3 \mathrm{D}$

Three single-phase sectionalizers $-140 \mathrm{~A}$ hydraulic

Three single-phase reclosers - 140 A V4L, 2A, 2D 
Table 30. Measurement Locations and Data Collection

Permanent Metering Locations

\begin{tabular}{llllll}
\hline A. & Substation & $M_{3 \Phi}$ & $V_{A N}, V_{B N}, V_{C N}$ & $I_{A}, I_{B}, I_{C}$ & $P, Q, P F$ \\
B. & Regulators (1) & $M_{3 \Phi}$ & $V_{A N}, V_{B N}, V_{C N}$ & $I_{A}, I_{B}, I_{C}$ & $P, Q, P F$ \\
D. & 1,000-kW synchronous DR & $M_{3 \Phi}$ & $V_{A N}, V_{B N}, V_{C N}$ & $I_{A}, I_{B}, I_{C}$ & $P, Q, P F$ \\
\hline \multicolumn{5}{c}{ Temporary Metering Locations } \\
F. & 900-kVAr capacitor & $M_{T 3 \Phi}$ & $V_{A N}, V_{B N}, V_{C N}$ & $I_{A}, I_{B}, I_{C}$ & \\
G. & 900-kVAr capacitor & $M_{T 3 \Phi}$ & $V_{A N}, V_{B N}, V_{C N}$ & $I_{A}, I_{B}, I_{C}$ & \\
H. & 1,200-kVAr capacitor & $M_{T 3 \Phi}$ & $V_{A N}, V_{B N}, V_{C N}$ & $I_{A}, I_{B}, I_{C}$ & \\
I. & Three-phase circuit tag end & $M_{T 3 \Phi}$ & & $I_{A}, I_{B}, I_{C}$ & \\
K.L. & Single-phase tag ends & $M_{T S \Phi}$ & $V_{A N}$ & $V_{B N}$ & \\
\hline
\end{tabular}

\subsection{Metering Equipment and Accuracy of Measurements}

\subsubsection{Substation Metering}

The substation metering consists of an ION 7600 Power Measurements Meter, which measures the three-phase voltages, three line currents, and phase power factors and calculates the kilowatts per phase and total kilowatts, kilovars per phase and total kilovars, kilovoltamperes per phase and total kilovolt-amperes, and unbalanced voltage and current. The integration period is 15 minutes. The accuracy of the metered data is $0.1 \%$ for voltage, $0.1 \%$ for current, $\pm 0.01 \mathrm{~Hz}$ for frequency, and $0.5 \%$ for power factor. The kilowatt, kilovar, and kilovolt-ampere accuracy is Class 0.2 .

\subsubsection{Synchronous Generator Metering}

The generator metering is a 3720 ACM meter that measures the three-phase voltages, three line currents, three-phase kilowatts, three-phase kilovars, three-phase kilovolt-amperes, power factor, frequency, and circuit load including generation. The data are 30 -second samples integrated over 5 minutes. The SOC data are 5-minute samples averaged over 15 minutes. The accuracy of the measured data is shown in Table 31.

Table 31. Accuracy of 3720 ACM

\begin{tabular}{lc}
\hline \multicolumn{1}{c}{ Parameter } & Accuracy \\
\cline { 2 - 2 } Current & \\
Kilowatts & $0.2 \%$ \\
Kilovars & $0.4 \%$ \\
Kilovolt-amperes & $0.4 \%$ \\
Voltage & $0.4 \%$ \\
Power factor & $0.2 \%$ \\
Frequency & $1.0 \%$ \\
\hline
\end{tabular}




\subsubsection{Capacitor Location Metering}

The metering at the capacitor locations (nodes F, G, and H) is the Line Tracker LT40 from Grid Sense. These metering devices measure the three-phase voltages and three line currents. The voltage is sampled at 600 samples per second, and the current is sampled at 1,200 samples per second. The accuracy of the current measurement is $\pm 5 \%$. The integration period is 15 minutes.

\subsubsection{Voltage Regulator Locations}

The CL-6 control of the VRs at Location B measures the load and source phase voltages, line currents, phase kilowatts, phase kilovars, phase kilovolt-amperes, and phase power factor. The data are integrated over 15 minutes, and the accuracy is Class 1 metering or $1 \%$ for all power quantities (i.e., voltage, current, kilowatts, kilovars, and kilovolt-amperes).

\subsubsection{Single-Phase Voltage Customer Metering}

The Rustrak measured voltage and current. The accuracy is $0.25 \%$ for voltage and $0.5 \%$ for current. Each is in percent of reading. The integration period is 15 minutes. 


\section{Project Results - Distributed Generation Control Strategies for Field Verification}

\subsection{Introduction}

This section defines the generation equipment voltage regulation capability and the control strategies used to regulate voltage on the Milford Circuit DC 8103.

\subsection{1,000-kW Synchronous Generator}

The voltage regulation control capabilities for the $1,000-\mathrm{kW}$ synchronous generator located at the midpoint on the circuit Node D is given in Figure 137.

\subsection{0-kW High-Speed Generator and Inverter}

The voltage regulation control capability for the $400-\mathrm{kW}$ high-speed generator and inverter is shown in Figure 138.

\subsection{0-kW Self-Excited Induction Generator}

The voltage regulation control capability for the $400-\mathrm{kW}$ self-excited induction generator is shown in Figure 139.

\subsection{Voltage Regulation Simulations and Field Verification Strategy 17}

The voltage regulation simulations and Strategy 17 used for field verification is outlined in Table 32. 


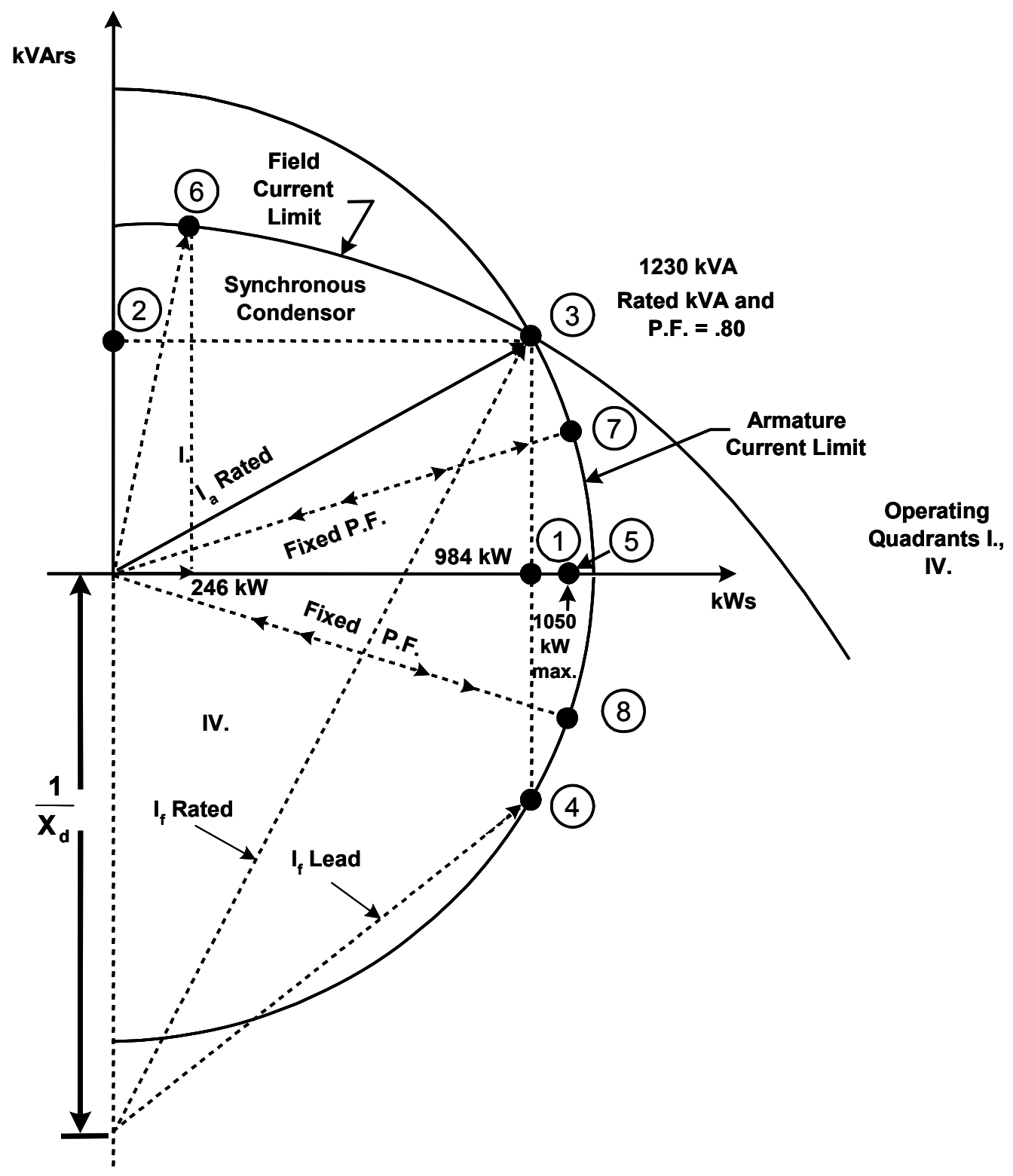

\section{Operating Points}

(a) points (1) and (2) is rated $\mathrm{kW}$ and rated KVArs

(b) point (3) rated kVA and rated lagging P.F.

(c) point (4) leading P.F.

(d) point (5) maximum kW

(e) point 6)minimum kW and maximum kVArs (synchronous condensor)

(f) points (7) and (8)fixed power factor lagging and leading

Figure 137. DR control strategies for a 1,000-kW synchronous machine 


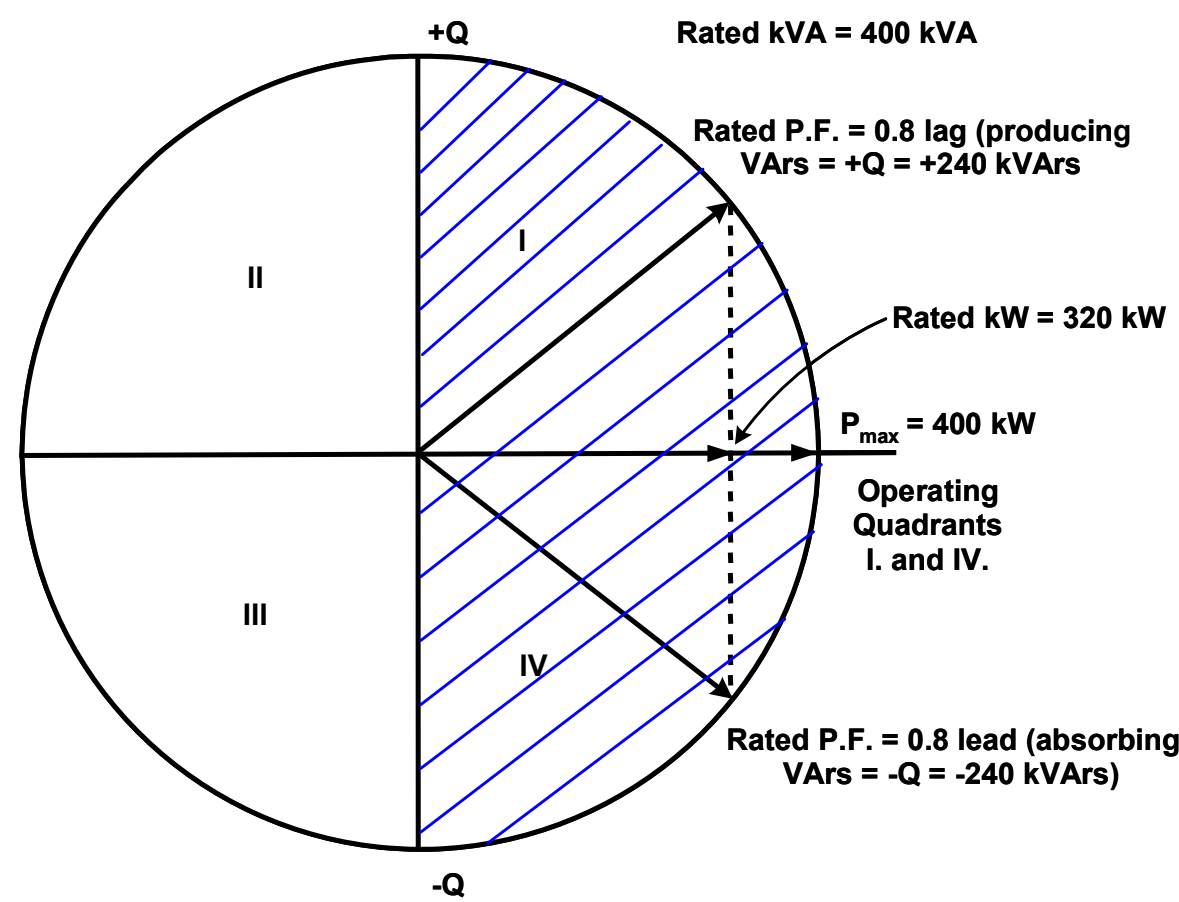

Note (4): DR Control Strategies

(a) Peak Shave P fixed

(b) P.F. = unity, P variable

(c) \pm P.F. constant, $P$ variable

(d) $\pm Q$ (VArs volt. reg.) $P=0$

(e) $P \pm j Q$ (optimizing) - see synchronous machine

(f) Frequency Dithering (anti-islanding)

Note (5): (f) not included in model

Figure 138. DR control strategies for a 400-kW high-speed generator and inverter - current mode 


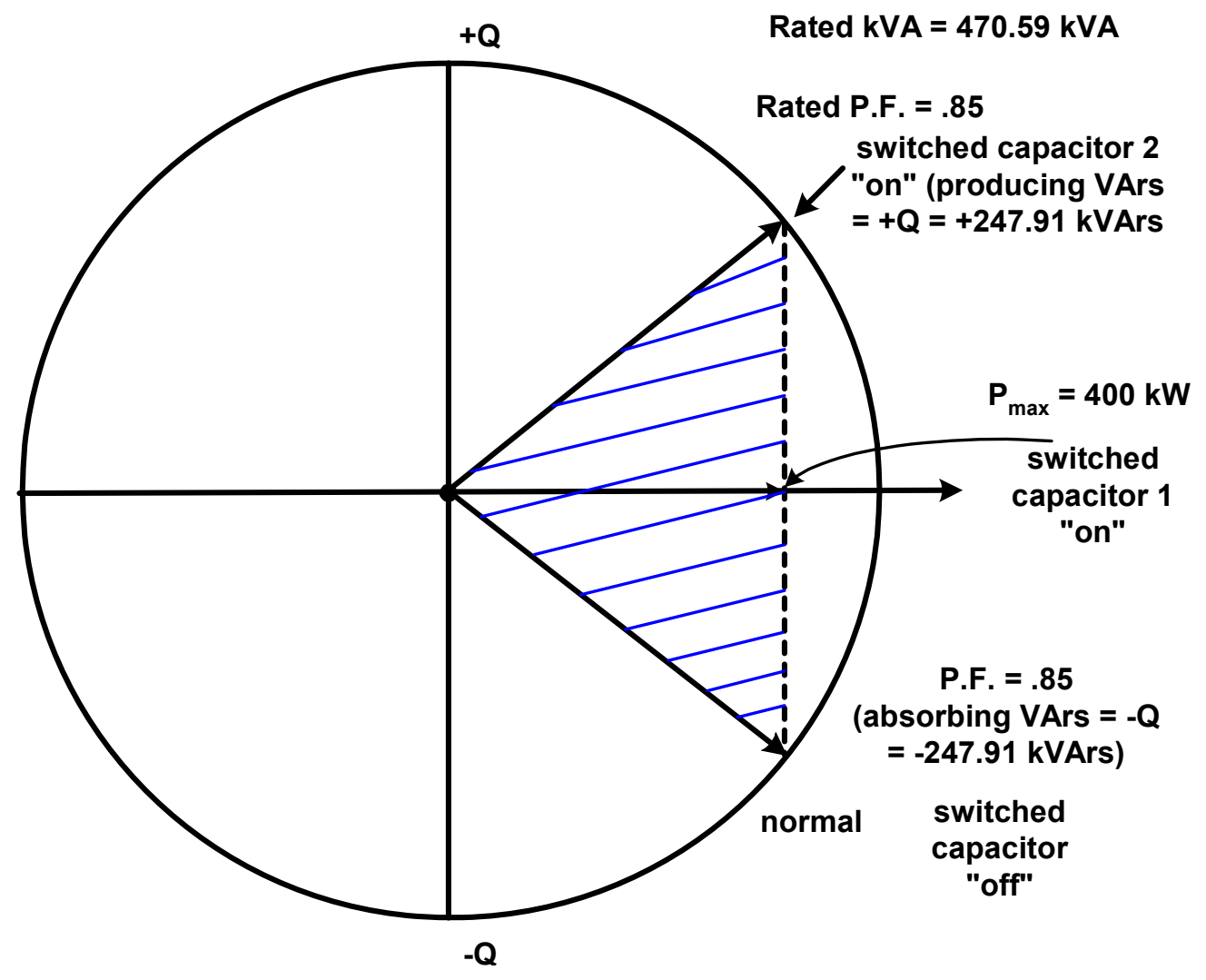

(a) Peak Shave $P$ fixed

(b) P.F. = unity, $P$ fixed

(c) \pm P.F. constant at $P$ fixed

(d) $\pm Q$ fixed, $P$ fixed same as (c)

Note (4) Induction machine with inverter is the same as Inverter where the speed is varied to create variable dc output after the rectifier and the ac current output from the inverter is phase shifted (switching) to produce a variable P.F. with respect to the system voltage.

Figure 139. DR control strategies for a $400-\mathrm{kW}$ self-excited induction generator 


\section{Table 32. Matrix of Voltage Regulation Simulations and Control Strategy 17 for Field Verification}

\begin{tabular}{|c|c|c|c|c|c|c|c|c|c|c|c|c|c|}
\hline \multirow{2}{*}{ 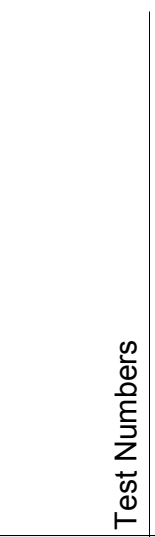 } & \multirow{2}{*}{ 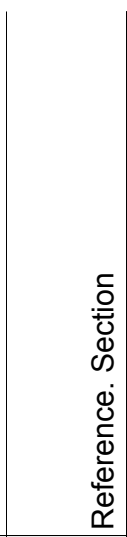 } & \multirow{2}{*}{$\begin{array}{l}\text { Primary } \\
\text { Voltage } \\
\text { Spread } \\
\text { (a) } 87 \% \\
\text { (b) } 92 \% \\
\text { (c) } 93 \% \\
\text { (d) } 95 \% \\
\text { (e) } 98 \% \\
\text { (f) } 105 \%\end{array}$} & \multirow[t]{2}{*}{ 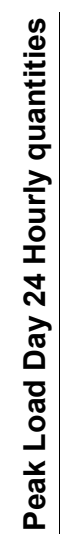 } & \multirow[t]{2}{*}{ 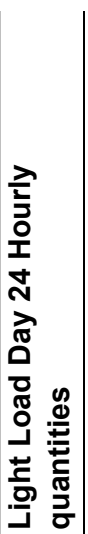 } & \multirow{2}{*}{ 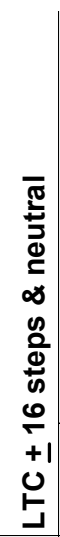 } & \multicolumn{2}{|c|}{$\begin{array}{c}\text { Line } \\
\text { Regulator }\end{array}$} & \multicolumn{3}{|c|}{ Capacitors } & \multicolumn{3}{|c|}{$\begin{array}{c}\text { DR (Synchronous Machine) } \\
\text { Locations (1) } \\
\text { (a)Peak shave, P fixed } \\
\text { (b) P.F.= Unity, P variable } \\
\text { (c) } \pm \text { P.F. Constant, Variable P \& } \\
\text { Q } \\
\text { (d) } \pm \text { Q (Vars Volt reg) P Min } \\
\text { (e) P } \pm \text { jQ (Optimizing) } \\
\text { (f) Fixed P.F. Anti-island }\end{array}$} \\
\hline & & & & & & \#1 & \#2 & \#1 & \#2 & \#3 & Near End & $\begin{array}{l}\text { Midpoint } \\
\text { (Actual } \\
\text { Site) }\end{array}$ & Far End \\
\hline 1 & I.A. & (a) (d) (f) & $x$ & $\mathrm{x}$ & & & & & & & & & \\
\hline 2 & I.B. & (d) (f) & $\mathrm{x}$ & $x$ & $x$ & & & & & & & & \\
\hline 3 & I.C. & (d) (f) & $x$ & $x$ & $x$ & $x$ & & & & & & & \\
\hline 4 & I.C'. & (d) (f) & $x$ & $x$ & $x$ & $x$ & $x$ & & & & & & \\
\hline 5 & I.D & (d) (f) & $x$ & $x$ & $x$ & & & $x$ & & & & & \\
\hline 6 & I.D $^{\prime}$ & (d) (f) & $x$ & $x$ & $x$ & & & $x$ & $x$ & & & & \\
\hline 7 & I.D." & (d) (f) & $x$ & $x$ & $x$ & & & $x$ & $x$ & $x$ & & & \\
\hline 8 & I.E. & (d) (f) & $x$ & $x$ & $x$ & $x$ & $x$ & $x$ & $x$ & $x$ & & & \\
\hline 9 & II.A. & $\mathrm{x}$ & $x$ & $x$ & & & & & & & (b) (d) (e) & & \\
\hline 10 & II.B. & $\mathrm{x}$ & $x$ & $x$ & & & & & & & & (b) (d) (e) & \\
\hline 11 & II.C. & $\mathrm{x}$ & $x$ & $\mathrm{x}$ & & & & & & & & & (b) (d) (e) \\
\hline 12 & II.D. & \multicolumn{6}{|c|}{\begin{tabular}{|l|l|} 
Repeat IIA., IIB., IIC. turn on LTC & \\
\end{tabular}} & & & & & & \\
\hline 13 & II.E. & \multicolumn{9}{|c|}{ Repeat IIA., IIB., IIC. turn on LTC, and Regulator (1) } & & & \\
\hline 14 & II.F. & \multicolumn{10}{|c|}{ Repeat IIA., IIB., IIC. turn on LTC, and Regulator (1) and (2) } & & \\
\hline 15 & II.G. & \multicolumn{11}{|c|}{ Repeat IIA., IIB., IIC. turn on LTC, and Regulator (1) and (2) and Cap. (1) } & \\
\hline 16 & II.H. & \multicolumn{11}{|c|}{ Repeat IIA., IIB., IIC. turn on LTC, and Regulator (1) and (2) and Cap. (1), (2) } & \\
\hline 17 & II.I. & \multicolumn{12}{|c|}{ Repeat IIA., IIB., IIC. turn on LTC, and Regulator (1) and (2) and Cap. (1), (2), (3) } \\
\hline \multicolumn{14}{|c|}{ 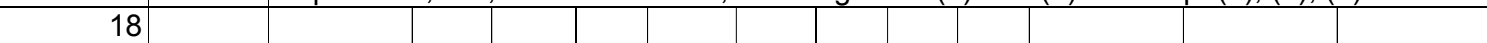 } \\
\hline \multicolumn{14}{|l|}{19} \\
\hline \multicolumn{14}{|l|}{20} \\
\hline \multicolumn{14}{|l|}{21} \\
\hline \multicolumn{14}{|l|}{22} \\
\hline \multicolumn{14}{|l|}{23} \\
\hline \multicolumn{14}{|l|}{24} \\
\hline \multicolumn{14}{|l|}{25} \\
\hline & & & & & & & & & & & & & \\
\hline \multicolumn{14}{|c|}{ Note (1): DR Control Strategies } \\
\hline & \multicolumn{13}{|c|}{$\begin{array}{l}\text { (a.) The DR is operated at fixed real power output and normally Is scheduled to run during peak periods when } \\
\text { the cost of DR generation is lower than host utility cost. The } Q \text { is zero at the PCC. } 984 \mathrm{~kW} \text { rated }\end{array}$} \\
\hline & (b.) The & $\mathrm{DR}$ is operat & $\mathrm{d}$ at u & Inity P.F & with & varia & le pow & er ou & put. & $492 \mathrm{k}$ & $V(50 \%)$ to 1 & $050 \mathrm{~kW} \mathrm{ma}$ & \\
\hline & $\begin{array}{l}\text { (c.) DR is } \\
\text { not violat }\end{array}$ & $\begin{array}{l}\text { operated at } \\
\text { e voltage cri }\end{array}$ & $\begin{array}{l}\text { a fixe } \\
\text { eria. }\end{array}$ & d power & facto & or eith & rlead & or lag & (for $€$ & xamp & \pm 0.8 P.F.) & with variable & $P$ and $Q$ to \\
\hline & $\begin{array}{l}\text { (d.) The } \mathrm{L} \\
\text { voltage. }\end{array}$ & $\begin{array}{l}\mathrm{R} \text { is operat } \\
246 \mathrm{~kW}(25\end{array}$ & $\begin{array}{l}d \text { as } \\
\text { of ra }\end{array}$ & $\begin{array}{l}\text { a synch } \\
\text { ted) }\end{array}$ & onou & is con & enser & with $n$ & inim & m wa & ts and variak & ble VArs to $r$ & gulate \\
\hline & $\begin{array}{l}\text { (e.) DR is } \\
\text { real losse }\end{array}$ & $\begin{array}{l}\text { operated w } \\
\text { s, minimize }\end{array}$ & $\begin{array}{l}\text { h vari } \\
\text { eacti }\end{array}$ & $\begin{array}{l}\text { iable } \mathrm{P} \\
\text { ve losse }\end{array}$ & $\begin{array}{l}\text { and } Q \\
\mathrm{~s}, \mathrm{reg}\end{array}$ & $\begin{array}{l}\text { 2 and } \\
\text { gulate }\end{array}$ & $\begin{array}{l}\text { used } \\
\text { oltage }\end{array}$ & $\begin{array}{l}\text { to opt } \\
\text { and } 1\end{array}$ & $\begin{array}{l}\text { imize } \\
\text { naxin }\end{array}$ & $\begin{array}{l}\text { to a s } \\
\text { ize re }\end{array}$ & $\begin{array}{l}\text { ecific set of } \\
\text { eased capac }\end{array}$ & $\begin{array}{l}\text { criteria such } \\
\text { city. }\end{array}$ & as minimize \\
\hline
\end{tabular}




\begin{tabular}{|c|c|c|c|c|c|c|c|c|c|c|c|c|c|}
\hline \multirow{2}{*}{ 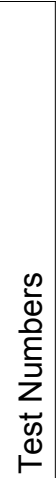 } & \multirow{2}{*}{ 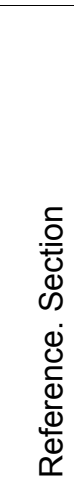 } & \multirow{2}{*}{$\begin{array}{l}\text { Primary } \\
\text { Voltage } \\
\text { Spread } \\
\\
\text { (a) } 87 \% \\
\text { (b) } 92 \% \\
\text { (c) } 93 \% \\
\text { (d) } 95 \% \\
\text { (e) } 98 \% \\
\text { (f) } 105 \%\end{array}$} & \multirow{2}{*}{ 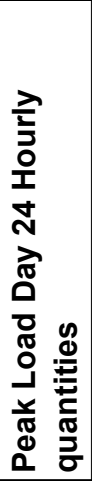 } & \multirow{2}{*}{ 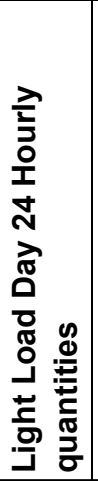 } & \multirow{2}{*}{ 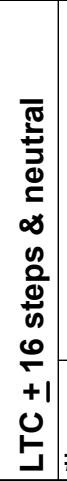 } & \multicolumn{2}{|c|}{$\begin{array}{c}\text { Line } \\
\text { Regulator }\end{array}$} & \multicolumn{3}{|c|}{ Capacitors } & \multicolumn{3}{|c|}{$\begin{array}{c}\text { DR (Induction Generator) } \\
\text { Locations } \\
\\
\text { (a)Peak shave, P fixed } \\
\text { (b) P.F.= Unity, P fixed } \\
\text { (c) } \pm \text { P.F. Constant, P fixed } \\
\text { (d) } \pm \text { Q fixed, P fixed } \\
\text { same as (c) }\end{array}$} \\
\hline & & & & & & $\# 1$ & \#2 & \#1 & \#2 & \#3 & Near End & \begin{tabular}{|l} 
Midpoint \\
(Actual \\
Site)
\end{tabular} & Far End \\
\hline 1 & & (a) (d) (f) & & & & & & & & & & & \\
\hline 2 & & (d) (f) & & & & & & & & & & & \\
\hline 3 & & (d) (f) & & & & & & & & & & & \\
\hline 4 & & (d) (f) & & & & & & & & & & & \\
\hline 5 & & (d) (f) & & & & & & & & & & & \\
\hline 6 & & (d) (f) & & & & & & & & & & & \\
\hline 7 & & (d) (f) & & & & & & & & & & & \\
\hline 8 & & (d) (f) & & & & & & & & & & & \\
\hline 9 & II.A. & $\mathrm{x}$ & $x$ & $x$ & & & & & & & (b) (d) & & \\
\hline 10 & II.B. & $x$ & $x$ & $x$ & & & & & & & & (b) (d) & \\
\hline 11 & II.C. & $\mathrm{x}$ & $\mathrm{x}$ & $x$ & & & & & & & & & (b) (d) \\
\hline 12 & II.D. & \multirow{2}{*}{\multicolumn{12}{|c|}{$\begin{array}{l}\text { Repeat II.A., II.B., II.C. turn on LTC } \\
\text { Repeat II.A., II.B., II.C. turn on LTC and Regualator (1) }\end{array}$}} \\
\hline 13 & II.E. & & & & & & & & & & & & \\
\hline 14 & II.F. & \multicolumn{12}{|c|}{ Repeat II.A., II.B., II.C. turn on LTC and Regulators (1) \& (2) } \\
\hline 15 & II.G. & \multicolumn{12}{|c|}{ 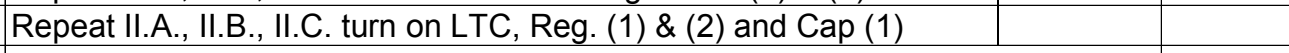 } \\
\hline 16 & II.H. & \multirow{2}{*}{\multicolumn{12}{|c|}{ Repeat II.A., II.B., II.C. turn on LTC, Reg. (1) \& (2) and Cap (1) and (2) }} \\
\hline 17 & II.I. & \multicolumn{8}{|c|}{ Repeat II.A., II.B., II.C. turn on LTC, Reg (1) \& (2) and Cap (1), (2) \& (3) } & & & & \\
\hline 18 & III. & Repeat syr & chronol & $s$ and ir & verte & er ger & eration & & & & & & \\
\hline 19 & IV. & \multicolumn{12}{|c|}{ Repeat synchronous, inverter and induction generation } \\
\hline 20 & & & & & & & & & & & & & \\
\hline 21 & & & & & & & & & & & & & \\
\hline 22 & & & & & & & & & & & & & \\
\hline 23 & & & & & & & & & & & & & \\
\hline 24 & & & & & & & & & & & & & \\
\hline 25 & & & & & & & & & & & & & \\
\hline 26 & & & & & & & & & & & & & \\
\hline 27 & & & & & & & & & & & & & \\
\hline 28 & & & & & & & & & & & & & \\
\hline 29 & & & & & & & & & & & & & \\
\hline
\end{tabular}




\begin{tabular}{|c|c|c|c|c|c|c|c|c|c|c|c|c|c|}
\hline \multirow{2}{*}{ 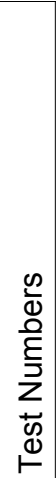 } & \multirow{2}{*}{ 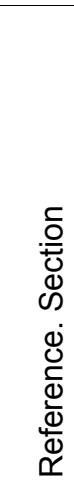 } & \multirow{2}{*}{$\begin{array}{l}\text { Primary } \\
\text { Voltage } \\
\text { Spread } \\
\\
\text { (a) } 87 \% \\
\text { (b) } 92 \% \\
\text { (c) } 93 \% \\
\text { (d) } 95 \% \\
\text { (e) } 98 \% \\
\text { (f) } 105 \%\end{array}$} & \multirow{2}{*}{ 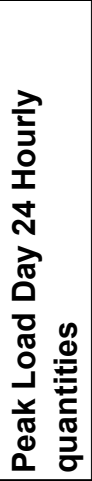 } & \multirow{2}{*}{ 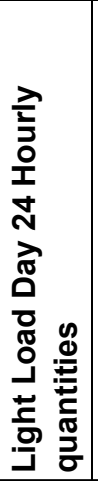 } & \multirow{2}{*}{ 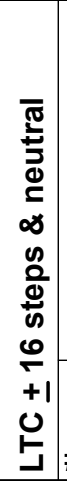 } & \multicolumn{2}{|c|}{$\begin{array}{c}\text { Line } \\
\text { Regulator }\end{array}$} & \multicolumn{3}{|c|}{ Capacitors } & \multicolumn{3}{|c|}{$\begin{array}{c}\text { DR (Induction Generator) } \\
\text { Locations } \\
\\
\text { (a)Peak shave, P fixed } \\
\text { (b) P.F.= Unity, P fixed } \\
\text { (c) } \pm \text { P.F. Constant, P fixed } \\
\text { (d) } \pm \text { Q fixed, P fixed } \\
\text { same as (c) }\end{array}$} \\
\hline & & & & & & $\# 1$ & \#2 & \#1 & \#2 & \#3 & Near End & \begin{tabular}{|l} 
Midpoint \\
(Actual \\
Site)
\end{tabular} & Far End \\
\hline 1 & & (a) (d) (f) & & & & & & & & & & & \\
\hline 2 & & (d) (f) & & & & & & & & & & & \\
\hline 3 & & (d) (f) & & & & & & & & & & & \\
\hline 4 & & (d) (f) & & & & & & & & & & & \\
\hline 5 & & (d) (f) & & & & & & & & & & & \\
\hline 6 & & (d) (f) & & & & & & & & & & & \\
\hline 7 & & (d) (f) & & & & & & & & & & & \\
\hline 8 & & (d) (f) & & & & & & & & & & & \\
\hline 9 & II.A. & $\mathrm{x}$ & $x$ & $x$ & & & & & & & (b) (d) & & \\
\hline 10 & II.B. & $x$ & $x$ & $x$ & & & & & & & & (b) (d) & \\
\hline 11 & II.C. & $\mathrm{x}$ & $\mathrm{x}$ & $x$ & & & & & & & & & (b) (d) \\
\hline 12 & II.D. & \multirow{2}{*}{\multicolumn{12}{|c|}{$\begin{array}{l}\text { Repeat II.A., II.B., II.C. turn on LTC } \\
\text { Repeat II.A., II.B., II.C. turn on LTC and Regualator (1) }\end{array}$}} \\
\hline 13 & II.E. & & & & & & & & & & & & \\
\hline 14 & II.F. & \multicolumn{12}{|c|}{ Repeat II.A., II.B., II.C. turn on LTC and Regulators (1) \& (2) } \\
\hline 15 & II.G. & \multicolumn{12}{|c|}{ 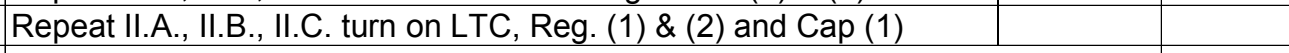 } \\
\hline 16 & II.H. & \multirow{2}{*}{\multicolumn{12}{|c|}{ Repeat II.A., II.B., II.C. turn on LTC, Reg. (1) \& (2) and Cap (1) and (2) }} \\
\hline 17 & II.I. & \multicolumn{8}{|c|}{ Repeat II.A., II.B., II.C. turn on LTC, Reg (1) \& (2) and Cap (1), (2) \& (3) } & & & & \\
\hline 18 & III. & Repeat syr & chronol & $s$ and ir & verte & er ger & eration & & & & & & \\
\hline 19 & IV. & \multicolumn{12}{|c|}{ Repeat synchronous, inverter and induction generation } \\
\hline 20 & & & & & & & & & & & & & \\
\hline 21 & & & & & & & & & & & & & \\
\hline 22 & & & & & & & & & & & & & \\
\hline 23 & & & & & & & & & & & & & \\
\hline 24 & & & & & & & & & & & & & \\
\hline 25 & & & & & & & & & & & & & \\
\hline 26 & & & & & & & & & & & & & \\
\hline 27 & & & & & & & & & & & & & \\
\hline 28 & & & & & & & & & & & & & \\
\hline 29 & & & & & & & & & & & & & \\
\hline
\end{tabular}




\section{Project Results - Field Verification of Models}

\subsection{Introduction}

This section verifies the models using field test data. Voltage regulation models were developed for the LTC transformer at the substation, the bidirectional step regulators, the capacitors, all the distribution circuit transformer connections, and the line sections. In addition, models for synchronous, induction, and inverter generators were created.

The field verification was conducted using the metering equipment defined in Section 7 and the DG control for the 1,000-kW synchronous generator defined in Section 8. The Strategy 17 control strategy was used. In it, all three capacitors $(1,2$, and 3$)$, the regulator (1), and the substation LTC were turned on.

The field verification studies were conducted for the circuit peak days of July 17, 2006, at 17:43 and July 29, 2006, at 17:11, when the 1,000-kW DR was running, and on July 31, 2006 at 17:56, when this DR was turned off.

\subsection{Circuit and Generation Measured Data}

The nodes at which measured data were taken on the circuit and Node D (Node 10), where the generation is interconnected with the circuit, are described in Figure 135. The test dates and data collection periods are shown in Table 33.

Table 33. Test Dates and Data Collection Periods

\begin{tabular}{rccccc}
\hline Date & $\begin{array}{c}\text { Circuit } \\
\text { Start }\end{array}$ & $\begin{array}{c}\text { Circuit } \\
\text { Peak }\end{array}$ & $\begin{array}{c}\text { Circuit } \\
\text { End }\end{array}$ & $\begin{array}{c}\text { Generation Start } \\
\text { (Post-Generation } \\
\text { Start) }\end{array}$ & $\begin{array}{c}\text { Generation End } \\
\text { (Pre-Generation } \\
\text { Off) }\end{array}$ \\
\cline { 2 - 5 } $7 / 17 / 06$ & $10: 42$ & $17: 43$ & $23: 58$ & $17: 28$ & $21: 23$ \\
$7 / 29 / 06$ & $00: 11$ & $17: 11$ & $23: 56$ & $17: 11$ & generator \\
$7 / 31 / 06$ & $00: 11$ & $17: 56$ & $23: 56$ & $\stackrel{2}{\longrightarrow}$ \\
$8 / 02 / 06$ & $00: 11$ & $17: 56$ & $23: 56$ & $10: 26$ & $12: 16$ \\
\hline
\end{tabular}

Because there is a time delay between the initiation of the "start" generator command and when the unit is synchronized and carrying load and a delay between the initiation of the "stop" generator command and when the generator is not carrying load, the times given in the Table 33 are the post-generation start (the time the unit is carrying load) and the pre-generation off (the time the unit is still carrying load but has received the "stop" generator command). 


\section{Illustrative Example of Generation Run Time and Distribution Circuit Time Stamps for Simulation Studies and Field Verification}

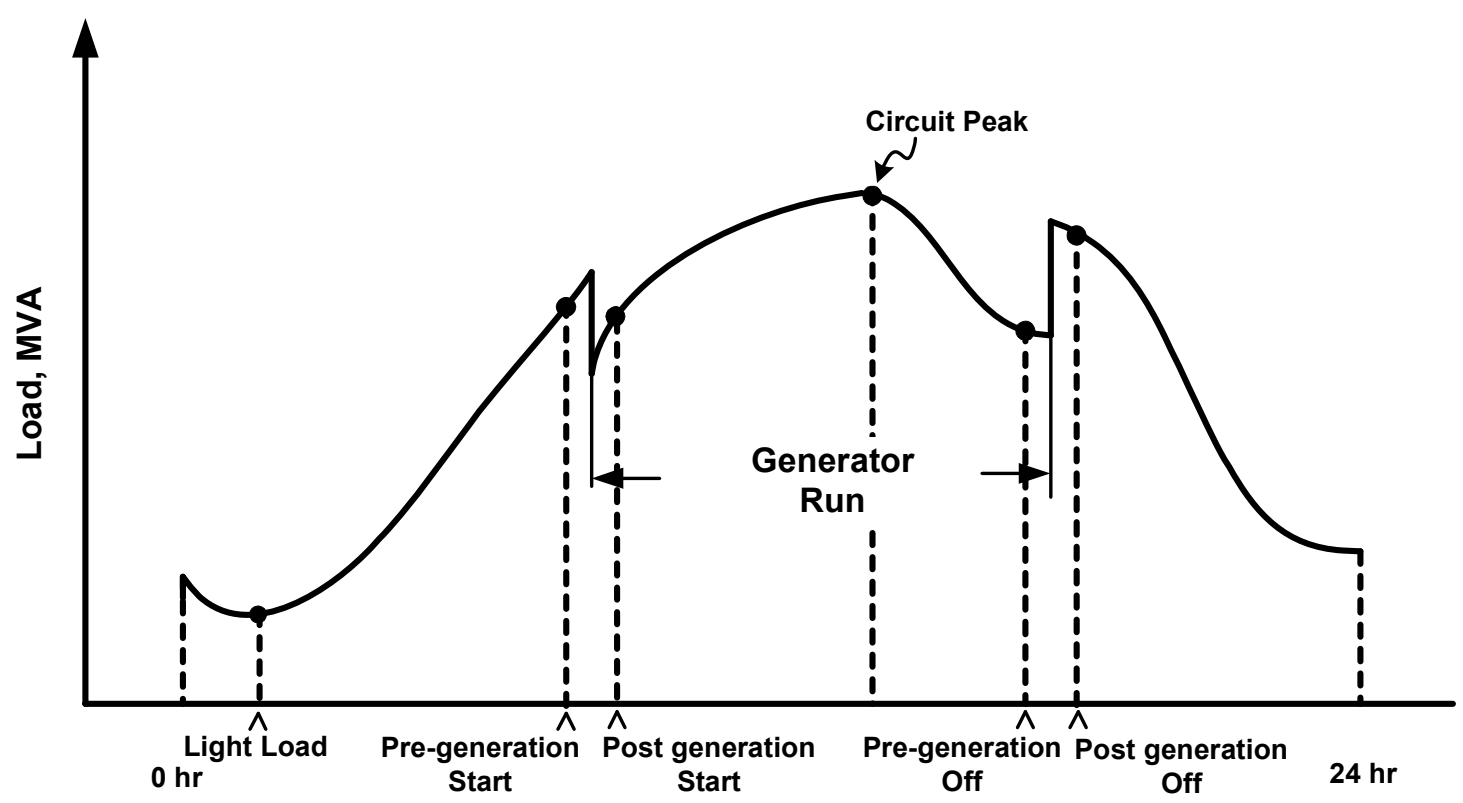

Figure 140. Daily circuit load profile and time stamps

These times are described in Figure 140. As shown in Table 33, there were four days during the summer when tests were conducted and four days when measured data were collected. The first two days, July 17 and July 29, 2006, the 1,000-kW DG was turned on to offset the circuit peak load. On July 31, 2006, this DG was turned off. This was to determine if there is a difference in the variance between simulation and field-measured data when the DG was running and when it was not. Simulations and comparisons with field-measured data were not conducted on the last test day (Aug. 2, 2006) because the load of the South Branch of the Milford Circuit DC 8103 was cut over to a circuit fed from the Page Substation on Aug. 1 at 19:00. This removed approximately 2 MVA of load (i.e., a 15.92 MVA peak prior to cut-over versus a 13.98-MVA peak after the cut-over) from the Milford Circuit DC 8103. See Table 34.

The times of circuit peak data and generation data are given in Table 34 for the four test days. The measured data consist of:

- Circuit peak kilovolt-amperes

- Circuit phase currents at Substation Node 1

- Generation line currents at Node 10

- Generation line-to-line voltages at Node 10

- Generation three-phase PF at Node 10

- Generation three-phase kilowatts, kilovars, and kilovolt-amperes at Node 10. 


\subsection{Bidirectional Voltage Regulator Measured Data}

The bidirectional VR measured data for the circuit peak time of each test day are given in Table 35 and listed below for each phase regulator at Node 9 .

- Load current

- Load PF

- Kilowatt load

- Kilovolt-ampere load

- Kilovar load

- Voltage $120-\mathrm{V}$ base

\subsection{Circuit Equipment (Capacitors) and Customer-Measured Data}

The measured data at the time of circuit peak for each of the three capacitors at nodes 6,12 , and 13 on the respective test days are given in Table 36. They consist of the phase currents and phase voltages. Also, the customer-measured data for Node $I$, which consist of $I_{a}$ and $I_{c}$ phase currents; Node $\mathrm{L}$ and the $\mathrm{V}_{\mathrm{C}}$ phase voltage; and Node $\mathrm{K}$ and the $\mathrm{V}_{\mathrm{A}}$ phase voltage are given in this table.

\subsection{Percent Variance Between Simulated and Field-Measured Data}

Table 37 and Table 38 compare simulated data and field-measured data for the two test days when DG was running. Table 39 shows similar data for when the DG was turned off. The field data for the phase currents, phase voltages, and phase power factors of the circuit, generator, and the VR1 bidirectional regulator are given for Node 1, Node 10, and Node 9 on the left. The corresponding simulation data are shown on the right. The measured phase currents and phase voltages for the three capacitors and their respective simulation data are also given in these tables, but the simulated voltage data have been reduced by $3.67 \mathrm{~V}$ to reduce the measured primary voltage to secondary voltage values. Finally, customermeasured current and voltage data and simulation data for Nodes I and L are listed.

Table 40 summarizes the differences between measured and simulated values. For three-phase quantities, the percent variance is calculated as the absolute value of the sum of the differences between the actual and simulated values divided by three and then divided by the average of the actual three-phase values. The result is multiplied by 100 to represent the percent difference using the average of the actuals as the base. For single-phase quantities, the percent variance is the absolute value of the difference divided by the actual as the base, multiplied by 100 . 


\section{Table 34. Circuit and Generation Measured Data}

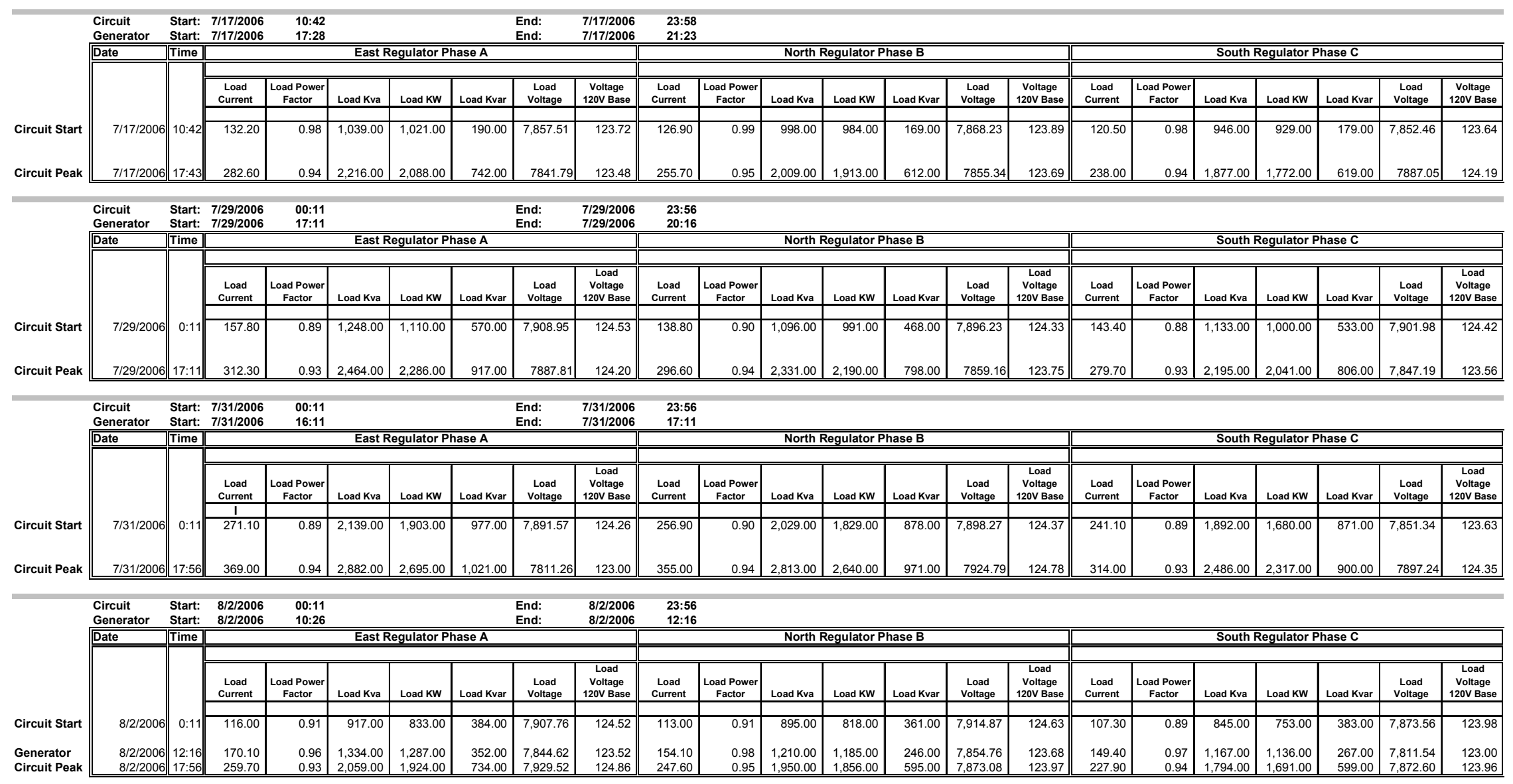

August 1st moved south branch to Page Substation at 19:00 


\section{Table 35. Bidirectional VR Measured Data}

\begin{tabular}{|c|c|c|c|c|c|c|c|c|c|c|c|c|c|c|c|c|c|c|c|c|c|c|c|}
\hline \multirow[b]{4}{*}{ Circuit Start } & \multirow{4}{*}{$\begin{array}{l}\text { Circuit } \\
\text { Generator } \\
\begin{array}{|l|}\text { Date } \\
7 / 17 / 2006\end{array}\end{array}$} & \multirow{3}{*}{$\begin{array}{l}\text { Start: } \\
\text { Start: } \\
\text { Time } \\
\end{array}$} & \multirow{2}{*}{\multicolumn{7}{|c|}{$\begin{array}{l}\text { East Regulator Phase } \mathrm{A} \\
\end{array}$}} & \multicolumn{14}{|l|}{$\begin{array}{r}23: 58 \\
21: 23 \\
\end{array}$} \\
\hline & & & & & & & & & & \multicolumn{7}{|c|}{$\begin{array}{l}\text { North Regulator Phase B } \\
\end{array}$} & \multicolumn{7}{|c|}{ 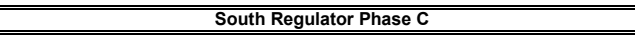 } \\
\hline & & & $\begin{array}{l}\text { Load } \\
\text { Current }\end{array}$ & \begin{tabular}{|c|}
$\begin{array}{c}\text { Load Power } \\
\text { Factor }\end{array}$ \\
\end{tabular} & Load Kva & Load KW & Load Kvar & $\begin{array}{c}\text { Load } \\
\text { Voltage }\end{array}$ & $\begin{array}{c}\text { Voltage } \\
\text { 120V Base }\end{array}$ & $\begin{array}{c}\text { Load } \\
\text { Current }\end{array}$ & \begin{tabular}{|c|} 
Load Power \\
Factor \\
\end{tabular} & Load Kva & Load KW & Load Kvar & $\begin{array}{c}\text { Load } \\
\text { Voltage }\end{array}$ & $\begin{array}{c}\text { Voltage } \\
\text { 120V Base }\end{array}$ & $\begin{array}{l}\text { Load } \\
\text { Current }\end{array}$ & $\begin{array}{c}\text { Load Power } \\
\text { Factor } \\
\end{array}$ & Load Kva & Load KW & Load Kvar & $\begin{array}{c}\text { Load } \\
\text { voltage }\end{array}$ & $\begin{array}{c}\text { Voltage } \\
120 V \text { Base } \\
\end{array}$ \\
\hline & & $10: 42$ & 132.20 & 0.98 & $1,039.00$ & $1,021.00$ & $\begin{array}{l}190.00 \\
\end{array}$ & $7,857.51$ & 123.72 & 126.90 & 0.99 & 998.00 & 984.00 & 169.00 & 7,868.23 & 123.89 & 120.50 & 0.98 & $\begin{array}{l}946.00 \\
\end{array}$ & 929.00 & 179.00 & $7,852.46$ & 123.64 \\
\hline \multirow[t]{4}{*}{ Circuit Peak } & 7/17/2006 & $17: 43$ & 282.60 & 0.94 & $2,216.00$ & $2,088.00$ & 742.00 & 7841.79 & 123.48 & 255.70 & 0.95 & $2,009.00$ & $1,913.00$ & 612.00 & 7855.34 & 123.69 & 238.00 & 0.94 & $1,877.00$ & $1,772.00$ & 619.00 & 7887.05 & 124.19 \\
\hline & $\begin{array}{l}\text { Circuit } \\
\text { Generator }\end{array}$ & $\begin{array}{l}\text { Start: } \\
\text { Start: }\end{array}$ & $\begin{array}{l}7 / 29 / 2006 \\
7 / 29 / 2006\end{array}$ & $\begin{array}{l}00: 11 \\
17: 11\end{array}$ & & & & $\begin{array}{l}\text { End: } \\
\text { End: }\end{array}$ & $\begin{array}{l}7 / 29 / 2006 \\
7 / 29 / 2006\end{array}$ & $\begin{array}{l}23: 56 \\
20: 16 \\
\end{array}$ & & & & & & & & & & & & & \\
\hline & Date & Time & \multicolumn{7}{|c|}{ East Regulator Phase $\mathrm{A}$} & \multicolumn{7}{|c|}{ 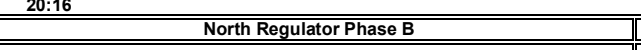 } & \multicolumn{7}{|c|}{$\begin{array}{l}\text { South Regulator Phase } \mathrm{C} \\
\end{array}$} \\
\hline & & & $\begin{array}{c}\text { Load } \\
\text { Current }\end{array}$ & $\begin{array}{c}\text { Load Power } \\
\text { Factor }\end{array}$ & Load Kva & Load KW & Load Kvar & $\begin{array}{c}\text { Load } \\
\text { Voltage }\end{array}$ & $\begin{array}{c}\text { Load } \\
\text { Voltage } \\
\text { 120V Base } \\
\end{array}$ & $\begin{array}{l}\text { Load } \\
\text { Current } \\
\end{array}$ & $\begin{array}{c}\text { Load Power } \\
\text { Factor }\end{array}$ & Load Kva & Load KW & Load Kvar & $\begin{array}{c}\text { Load } \\
\text { Voltage }\end{array}$ & \begin{tabular}{c|c|} 
Load \\
Voltage \\
120V Base \\
\end{tabular} & $\begin{array}{l}\text { Load } \\
\text { Current }\end{array}$ & \begin{tabular}{|c} 
Load Power \\
Factor \\
\end{tabular} & Load Kva & Load KW & Load Kvar & $\begin{array}{c}\text { Load } \\
\text { voltage }\end{array}$ & $\begin{array}{c}\begin{array}{c}\text { Load } \\
\text { Voltage } \\
\text { 120V Base }\end{array} \\
\end{array}$ \\
\hline Circuit Start & $7 / 29 / 2006$ & $0: 11$ & 157.80 & 0.89 & $1,248.00$ & $1,110.00$ & 570.00 & $\begin{array}{l}7,908.95 \\
\end{array}$ & \begin{tabular}{|c||}
124.53 \\
\end{tabular} & 138.80 & 0.90 & \begin{tabular}{|l|}
$1,096.00$ \\
\end{tabular} & 991.00 & 468.00 & $\begin{array}{ll}7,896.23 \\
\end{array}$ & 124.33 & 143.40 & 0.88 & $1,133.00$ & $1,000.00$ & 533.00 & $7,901.98$ & 124.42 \\
\hline \multirow[t]{4}{*}{ Circuit Peak } & $7 / 29 / 2006$ & 17:11 & 312.30 & 0.93 & 2,464.00 & $2,286.00$ & 917.00 & 7887.81 & 124.20 & 296.60 & 0.94 & $2,331.00$ & $2,190.00$ & 798.00 & 7859.16 & 123.75 & 279.70 & 0.93 & $2,195.00$ & $2,041.00$ & 806.00 & $7,847.19$ & 123.56 \\
\hline & $\begin{array}{l}\text { Circuit } \\
\text { Generator }\end{array}$ & $\begin{array}{l}\text { Start: } \\
\text { Start: }\end{array}$ & $\begin{array}{l}7 / 31 / 2006 \\
7 / 31 / 2006\end{array}$ & $\begin{array}{l}00: 11 \\
16: 11\end{array}$ & & & & $\begin{array}{l}\text { End: } \\
\text { End: }\end{array}$ & $\begin{array}{l}7 / 31 / 2006 \\
7 / 31 / 2006\end{array}$ & $\begin{array}{l}\text { 23:56 } \\
\text { 17:11 }\end{array}$ & & & & & & & & & & & & & \\
\hline & Date & Time & \multicolumn{7}{|c|}{$\begin{array}{l}\text { East Regulator Phase A } \\
\end{array}$} & \multicolumn{7}{|c|}{ 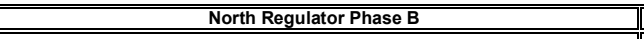 } & \multicolumn{7}{|c|}{ South Regulator Phase $\mathrm{C}$} \\
\hline & & & $\begin{array}{c}\text { Load } \\
\text { Current }\end{array}$ & \begin{tabular}{|c|}
$\begin{array}{c}\text { Load Power } \\
\text { Factor }\end{array}$ \\
\end{tabular} & \begin{tabular}{|l} 
Load Kva \\
\end{tabular} & Load KW & Load Kvar & $\begin{array}{c}\text { Load } \\
\text { Voltage }\end{array}$ & $\begin{array}{c}\text { Load } \\
\text { Voltage } \\
\text { 120V Base } \\
\end{array}$ & $\begin{array}{c}\text { Load } \\
\text { Current }\end{array}$ & \begin{tabular}{|c} 
Load Power \\
Factor \\
\end{tabular} & $\begin{array}{l}\text { Load Kva } \\
\end{array}$ & Load KW & Load Kvar & $\begin{array}{c}\text { Load } \\
\text { Voltage }\end{array}$ & \begin{tabular}{c|c|} 
Load \\
Voltage \\
120V Base \\
\end{tabular} & $\begin{array}{c}\text { Load } \\
\text { Current }\end{array}$ & \begin{tabular}{|c} 
Load Power \\
Factor \\
\end{tabular} & \begin{tabular}{|l} 
Load Kva \\
\end{tabular} & Load KW & Load Kvar & $\begin{array}{c}\text { Load } \\
\text { Voltage }\end{array}$ & $\begin{array}{c}\text { Load } \\
\text { Voltage } \\
120 \mathrm{~V} \text { Base }\end{array}$ \\
\hline Circuit Start & 7/31/2006 & $0: 11$ & $\frac{1}{271.10}$ & 0.89 & $2,139.00$ & $1,903.00$ & 977.00 & \begin{tabular}{|l|l|}
$7,891.57$ \\
\end{tabular} & \begin{tabular}{|c|}
124.26 \\
\end{tabular} & 256.90 & 0.90 & \begin{tabular}{|l|}
$2,029.00$ \\
\end{tabular} & $1,829.00$ & 878.00 & \begin{tabular}{|l|}
$7,898.27$ \\
\end{tabular} & \begin{tabular}{l|l|}
124.37 \\
\end{tabular} & 241.10 & 0.89 & $1,892.00$ & $1,680.00$ & 871.00 & $7,851.34$ & 123.63 \\
\hline \multirow[t]{4}{*}{ Circuit Peak } & $7 / 31 / 2006$ & $17: 56$ & 369.00 & 0.94 & $2,882.00$ & $2,695.00$ & $1,021.00$ & 7811.26 & 123.00 & 355.00 & 0.94 & $2,813.00$ & $2,640.00$ & 971.00 & 7924.79 & 124.78 & 314.00 & 0.93 & $2,486.00$ & $2,317.00$ & 900.00 & 7897.24 & 124.35 \\
\hline & $\begin{array}{l}\text { Circuit } \\
\text { Generator }\end{array}$ & $\begin{array}{l}\text { Start: } \\
\text { Start: }\end{array}$ & $\begin{array}{l}8 / 2 / 2006 \\
8 / 2 / 2006\end{array}$ & $\begin{array}{l}00: 11 \\
10: 26\end{array}$ & & & & $\begin{array}{l}\text { End: } \\
\text { End: }\end{array}$ & $\begin{array}{l}8 / 2 / 2006 \\
8 / 2 / 2006\end{array}$ & $\begin{array}{l}23: 56 \\
12: 16\end{array}$ & & & & & & & & & & & & & \\
\hline & Date & Time & \multicolumn{7}{|c|}{$\begin{array}{l}\text { East Regulator Phase A } \\
\end{array}$} & \multicolumn{7}{|c|}{ 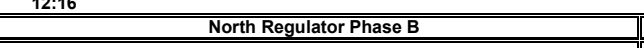 } & \multicolumn{7}{|c|}{$\begin{array}{l}\text { South Regulator Phase } \mathrm{C} \\
\end{array}$} \\
\hline & & & $\begin{array}{c}\text { Load } \\
\text { Current }\end{array}$ & $\begin{array}{c}\text { Load Power } \\
\text { Factor } \\
\end{array}$ & Load Kva & Load KW & Load Kvar & $\begin{array}{c}\text { Load } \\
\text { Voltage }\end{array}$ & $\begin{array}{c}\text { Load } \\
\text { Vottage } \\
\text { 120V Base }\end{array}$ & $\begin{array}{l}\text { Load } \\
\text { Current }\end{array}$ & $\begin{array}{c}\text { Load Power } \\
\text { Factor } \\
\end{array}$ & \begin{tabular}{|l} 
Load Kva \\
\end{tabular} & Load KW & Load Kvar & $\begin{array}{c}\text { Load } \\
\text { Voltage }\end{array}$ & $\begin{array}{c}\text { Load } \\
\text { Voltage } \\
120 \mathrm{~V} \text { Base }\end{array}$ & $\begin{array}{c}\text { Load } \\
\text { Current }\end{array}$ & \begin{tabular}{|c|} 
Load Power \\
Factor \\
\end{tabular} & Load Kva & Load KW & Load Kvar & $\begin{array}{c}\text { Load } \\
\text { Voltage }\end{array}$ & $\begin{array}{c}\text { Load } \\
\text { Vottage } \\
120 \mathrm{~V} \text { Base }\end{array}$ \\
\hline Circuit Start & 8/2/2006 & $0: 11$ & 116.00 & 0.91 & 917.00 & 833.00 & 384.00 & $7,907.76$ & \begin{tabular}{l|l}
124.52 \\
\end{tabular} & 113.00 & 0.91 & 895.00 & 818.00 & 361.00 & $7,914.87$ & 124.63 & 107.30 & 0.89 & 845.00 & 753.00 & 383.00 & $7,873.56$ & 123.98 \\
\hline $\begin{array}{l}\text { Generator } \\
\text { Circuit Peak }\end{array}$ & $\begin{array}{l}8 / 2 / 2006 \\
8 / 2 / 2006\end{array}$ & $\mid$\begin{tabular}{|l|}
$12: 16$ \\
$17: 56$
\end{tabular} & $\begin{array}{l}170.10 \\
259.70\end{array}$ & $\begin{array}{l}0.96 \\
0.93\end{array}$ & $\begin{array}{l}1,334.00 \\
2,059.00\end{array}$ & $\begin{array}{l}1,287.00 \\
1,924.00\end{array}$ & $\begin{array}{l}352.00 \\
734.00\end{array}$ & $\begin{array}{l}7,844.62 \\
7,929.52\end{array}$ & $\begin{array}{l}123.52 \\
124.86\end{array}$ & $\begin{array}{l}154.10 \\
247.60\end{array}$ & $\begin{array}{l}0.98 \\
0.95\end{array}$ & \begin{tabular}{|l|}
$1,210.00$ \\
$1,950.00$
\end{tabular} & $\begin{array}{l}1,185.00 \\
1,856.00\end{array}$ & $\begin{array}{l}246.00 \\
595.00\end{array}$ & \begin{tabular}{|l|}
$7,854.76$ \\
$7,873.08$ \\
\end{tabular} & $\begin{array}{l}123.68 \\
123.97\end{array}$ & $\begin{array}{l}149.40 \\
227.90\end{array}$ & $\begin{array}{l}0.97 \\
0.94\end{array}$ & $\begin{array}{l}1,167.00 \\
1,794.00\end{array}$ & $\begin{array}{l}1,136.00 \\
1,691.00\end{array}$ & $\begin{array}{l}267.00 \\
599.00\end{array}$ & $\begin{array}{l}7,811.54 \\
7,872.60\end{array}$ & $\begin{array}{l}123.00 \\
123.96\end{array}$ \\
\hline
\end{tabular}

August 1st moved south branch to Page Substation at 19:00 
Table 36. Circuit Equipment and Customer-Measured Data

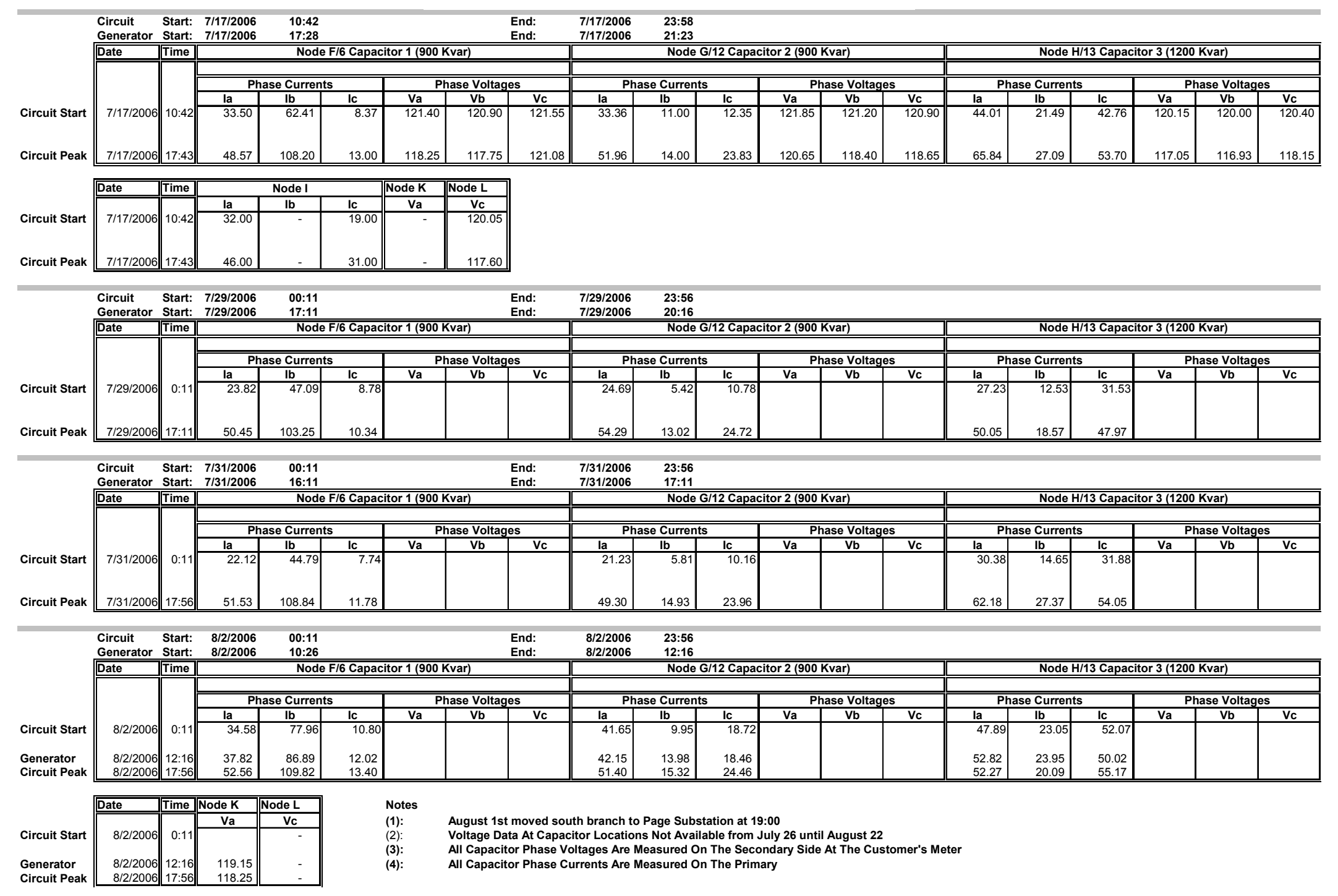


Table 37. Field Verification Data - July 17, 2006 - DR Generation On

\begin{tabular}{|c|c|c|c|c|c|c|c|c|c|c|c|c|c|c|c|c|c|c|c|}
\hline & \multicolumn{9}{|c|}{ Field Data - 7/17/06 - Circuit Peak 17:43 } & \multicolumn{9}{|c|}{ Simulation } \\
\hline & & \multicolumn{3}{|c|}{ Current } & \multicolumn{3}{|c|}{ Voltage } & \multicolumn{3}{|c|}{$\overline{\mathrm{PF}}$} & \multicolumn{3}{|c|}{ Current } & \multicolumn{3}{|c|}{ Voltage } & \multicolumn{3}{|c|}{$\overline{\mathrm{PF}}$} \\
\hline \begin{tabular}{l|l} 
Location \\
\end{tabular} & Node & $\mathbf{A}$ & $\mathrm{B}$ & $\mathrm{C}$ & $\mathbf{A}$ & $\mathrm{B}$ & $\mathrm{C}$ & $\mathbf{A}$ & $\mathrm{B}$ & $\mathrm{C}$ & A & $\mathrm{B}$ & $\mathrm{C}$ & A & $\mathrm{B}$ & $\mathrm{C}$ & $\mathbf{A}$ & B & $\begin{array}{c}\mathrm{C} \\
\mathrm{C}\end{array}$ \\
\hline \multirow[t]{2}{*}{ Start of Circuit } & 1 & 565.00 & 651.00 & 637.00 & 126.00 & 126.00 & 126.00 & 0.95 & 0.95 & 0.95 & 554.54 & 637.77 & 619.97 & 125.86 & 125.80 & 125.39 & 0.93 & 0.92 & 0.93 \\
\hline & & & $2.2 \%$ & & & $0.3 \%$ & & & $5.7 \%$ & & & & & & & & & & \\
\hline \multirow[t]{2}{*}{ Generator } & 10 & 18.31 & 17.24 & 17.00 & 122.50 & 122.50 & 120.25 & 0.96 & 0.96 & 0.96 & 17.49 & 17.25 & 17.36 & 121.68 & 123.38 & 122.62 & 0.96 & 0.96 & 0.96 \\
\hline & & & $2.3 \%$ & & & $1.1 \%$ & & & $0.0 \%$ & & 11.45 & & & & & & & & \\
\hline \multirow[t]{2}{*}{ VR 1} & 9 & 282.60 & 255.70 & 238.00 & 123.48 & 123.69 & 124.19 & 0.94 & 0.95 & 0.94 & 274.07 & 246.07 & 227.56 & 123.60 & 123.82 & 123.62 & 0.93 & 0.94 & 0.93 \\
\hline & & & $3.7 \%$ & & & $0.2 \%$ & & & $2.4 \%$ & & & & & & & & & & \\
\hline \multirow[t]{2}{*}{ Cap 1* } & 6 & 48.57 & 108.20 & 13.00 & 118.25 & 117.75 & 121.08 & & & & 48.03 & 104.47 & 13.77 & 121.25 & 121.14 & 123.29 & & & \\
\hline & & & $3.0 \%$ & & & $0.7 \%$ & & & & & & & & 117.58 & 117.47 & 119.62 & & & \\
\hline \multirow{2}{*}{ Cap $2^{*}$} & 12 & 51.96 & 14.00 & 23.83 & 120.65 & 118.40 & 118.65 & & & & 52.18 & 14.87 & 24.53 & 120.51 & 123.09 & 121.87 & & & \\
\hline & & & $2.0 \%$ & & & $1.5 \%$ & & & & & & & & 116.84 & 119.42 & 118.20 & & & \\
\hline \multirow[t]{2}{*}{ Сap 3* } & 13 & 65.84 & 27.09 & 53.70 & 117.05 & 116.93 & 118.15 & & & & 64.44 & 27.92 & 50.18 & 119.04 & 121.44 & 120.03 & & & \\
\hline & & & $3.9 \%$ & & & $1.2 \%$ & & & & & & & & 115.37 & 117.77 & 116.36 & & & \\
\hline \multirow{2}{*}{ Node I } & 16 & 460 & & 3100 & & & & & & & 3273 & & 2294 & & & & & & \\
\hline & & & $38.3 \%$ & & & & & & & & & & & & & & & & \\
\hline \multirow[t]{2}{*}{ Node $L^{*}$} & 18 & & & & 117.60 & & & & & & & & & 121.42 & & & & & \\
\hline & & & & & & $0.1 \%$ & & & & & & & & 117.75 & & & & & \\
\hline
\end{tabular}

*The voltage measurements were taken at the customer's meter. Therefore, an average voltage drop of $3.67 \mathrm{~V}$ was included to reduce the primary voltage to the secondary voltage for the comparison shown in the table.

Table 38. Field Verification Data - July 29, 2006 - DR Generation On

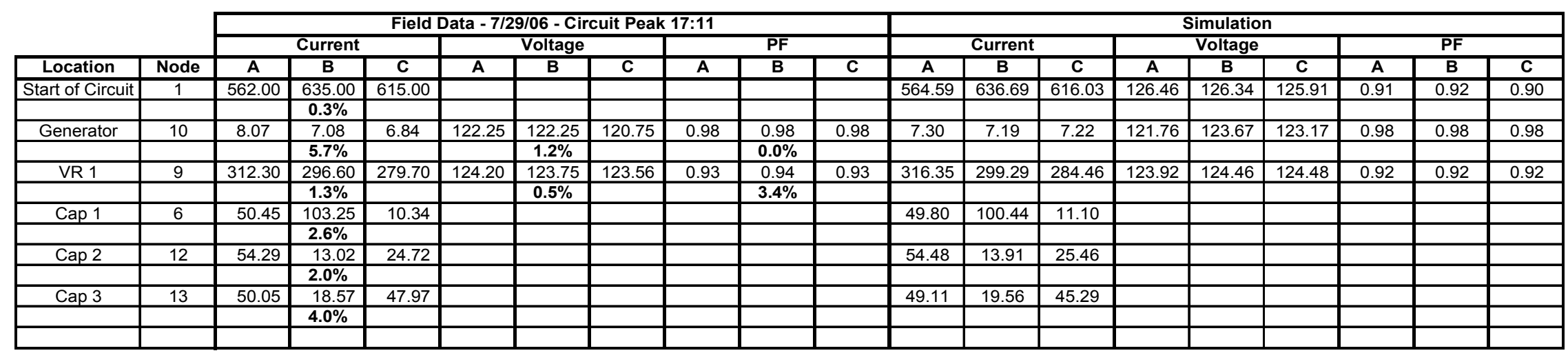


Table 39. Field Verification Data - July 31, 2006 - DR Generation Off

\begin{tabular}{|c|c|c|c|c|c|c|c|c|c|c|c|c|c|c|c|c|c|c|c|}
\hline & \multicolumn{9}{|c|}{ Field Data - 7/31/06 - Circuit Peak 17:56 } & \multicolumn{9}{|c|}{ Simulation } \\
\hline & & \multicolumn{3}{|c|}{ Current } & \multicolumn{3}{|c|}{ Voltage } & \multicolumn{3}{|c|}{$\overline{P F}$} & \multicolumn{3}{|c|}{ Current } & \multicolumn{3}{|c|}{ Voltage } & \multicolumn{3}{|c|}{$\overline{\mathrm{PF}}$} \\
\hline Location & Node & A & $\mathrm{B}$ & $\mathrm{C}$ & $\bar{A}$ & $\mathrm{~B}$ & $\mathbf{C}$ & $\overline{\mathbf{A}}$ & $B$ & $\mathbf{C}$ & A & $\mathrm{B}$ & $\mathrm{C}$ & A & $\mathrm{B}$ & $\mathrm{C}$ & A & B & C \\
\hline Start of Circuit & 1 & 604.00 & 696.00 & 678.00 & & & & & & & 608.76 & 699.59 & 678.97 & 126.26 & 126.14 & 125.58 & 0.92 & 0.92 & 0.91 \\
\hline & & & $0.5 \%$ & & & & & & & & & & & & & & & & \\
\hline Generator & 10 & $\mathrm{~N} / \mathrm{A}$ & $\mathrm{N} / \mathrm{A}$ & $\mathrm{N} / \mathrm{A}$ & $\mathrm{N} / \mathrm{A}$ & $\mathrm{N} / \mathrm{A}$ & $\mathrm{N} / \mathrm{A}$ & $\mathrm{N} / \mathrm{A}$ & $\mathrm{N} / \mathrm{A}$ & $\mathrm{N} / \mathrm{A}$ & & & & & & & & & \\
\hline VR 1 & 9 & 369.00 & 355.00 & 314.00 & 123.00 & 124.78 & 124.35 & 0.94 & 0.94 & 0.93 & 376.11 & 362.02 & 324.22 & 122.93 & 123.63 & 123.61 & 0.93 & 0.92 & 0.92 \\
\hline & & & $2.3 \%$ & & & $0.5 \%$ & & & $3.4 \%$ & & & & & & & & & & \\
\hline Cap 1 & 6 & 51.53 & 108.84 & 11.78 & & & & & & & 50.82 & 105.52 & 12.49 & & & & & & \\
\hline & & & $2.8 \%$ & & & & & & & & & & & & & & & & \\
\hline Cap 2 & 12 & 49.30 & 14.93 & 23.96 & & & & & & & 48.74 & 15.69 & 24.55 & & & & & & \\
\hline & & & $2.2 \%$ & & & & & & & & & & & & & & & & \\
\hline Cap 3 & 13 & 62.18 & 27.37 & 54.05 & & & & & & & 60.45 & 28.03 & 49.14 & & & & & & \\
\hline & & & $5.1 \%$ & & & & & & & & & & & & & & & & \\
\hline & & & & & & & & & & & & & & & & & & & \\
\hline
\end{tabular}


Table 40. Percent Variance Between Actual and Simulated Currents, Voltages, and Power Factors

\begin{tabular}{|c|c|c|c|c|}
\hline Location & Node & Current & Voltage & PF \\
\hline \multicolumn{5}{|c|}{ Test Day 7/17/06 DR Generation Running } \\
\hline Start of circuit & 1 & 2.2 & \begin{tabular}{l|l|}
0.3 \\
\end{tabular} & 5.7 \\
\hline Generator & 10 & 2.3 & 1.1 & 0.0 \\
\hline VR & 9 & 3.7 & 0.2 & 2.4 \\
\hline Capacitor 1 & 6 & 3.0 & 0.7 & -- \\
\hline Capacitor 2 & 12 & 2.0 & 1.5 & -- \\
\hline Capacitor 3 & 13 & 3.9 & 1.2 & -- \\
\hline Node I & 16 & 38.3 & -- & -- \\
\hline Node L & 18 & -- & 0.1 & -- \\
\hline \multicolumn{5}{|c|}{ Test Day 7/29/06 DR Generation Running } \\
\hline Start of circuit & 1 & 0.3 & -- & -- \\
\hline Generator & 10 & 5.7 & 1.2 & 0.0 \\
\hline VR & 9 & 1.3 & 0.5 & 3.4 \\
\hline Capacitor 1 & 6 & 2.6 & -- & -- \\
\hline Capacitor 2 & 12 & 2.0 & -- & -- \\
\hline Capacitor 3 & 13 & 4.0 & -- & -- \\
\hline \multicolumn{5}{|c|}{ Test Day 7/31/06 DR Generation Off } \\
\hline Start of circuit & 1 & 0.5 & -- & -- \\
\hline Generator & N/A & N/A & $\mathrm{N} / \mathrm{A}$ & N/A \\
\hline VR & 9 & 2.3 & 0.5 & 3.4 \\
\hline Capacitor 1 & 6 & 2.8 & -- & -- \\
\hline Capacitor 2 & 12 & 2.2 & -- & -- \\
\hline Capacitor 3 & 13 & 5.1 & -- & -- \\
\hline
\end{tabular}

\subsection{Summary of Variance Results}

From Table 40, the variance between actual field-measured data and simulated data is less than $6 \%$ (i.e., 5.7\% actual) for the phase currents throughout the circuit (except for Node I, where the percent unbalanced current is very high). The highest variance for the phase voltages throughout the circuit is $1.5 \%$ percent, whereas the highest variance for the $\mathrm{PF}$ is $5.7 \%$ at the start of the circuit. It should be noted that the lowest variances occur on the load side of the step regulators because these regulators are individually phase-controlled and not gang-operated as the LTC at the substation.

As shown in the variance data in Table 40, the simulated data closely match the actual fieldmeasured data in most cases, and the models developed in Section 5 can be used to estimate phase currents, phase voltages, and power factors without the use of extensive circuit metering. However, knowing the phasing of the loads is paramount to accurate simulated data. No significant difference in percent variance occurred when the DR was turned on or off. 


\section{Project Results - Distributed Generation Penetration Limits Using Maximum Real Power and Maximum Real and Reactive Power Methods of Voltage Control}

\subsection{Introduction}

In this section, the largest synchronous generator that can be installed on the distribution circuit is determined. This is determined based on three criteria:

- The lowest single-phase voltage must not be less than 114 meter V or $117.67 \mathrm{~V}$ on the secondary of the distribution transformer, and the highest single-phase voltage cannot exceed 126 meter $\mathrm{V}$.

- The generator must not exceed $100 \%$ of the thermal rating on the most limiting element.

- The generator must not create a reverse-power condition (i.e., there can be no flow back through the substation transformer).

Although these criteria were used for testing, a fourth criterion was that no system protection device could misoperate. This criterion was not applied because it was the subject of a prior Department of Energy contract.

\subsection{Real Power Distributed Generation Size Limitation}

Table 41 summarizes the results of applying the highest acceptable real power injection at the beginning point, BP, Node 2; the middle point, Node 10; and the end point, Node 17. The limiting criterion in all cases was reverse power. The optimum location was at the midpoint, which had the lowest single-phase voltage improvement of $1.7 \%$ and a real power loss savings of $2.04 \%$. The base case real power losses were $5.4 \%$. The largest real power injection was $13,980 \mathrm{~kW}$ at the end point; the beginning and midpoints were slightly less at $13,290 \mathrm{~kW}$ and $13,740 \mathrm{~kW}$, respectively. 
Table 41. Maximum Limit for Real Power Injection

\begin{tabular}{|c|c|c|c|c|c|c|c|c|c|c|c|c|c|c|c|c|c|c|c|c|}
\hline \multirow[b]{2}{*}{$\begin{array}{c}\text { Machine } \\
\text { Type }\end{array}$} & \multirow[b]{2}{*}{$\begin{array}{l}\text { Real } \\
\text { Power }\end{array}$} & \multirow[b]{2}{*}{$\begin{array}{c}\text { Reactive } \\
\text { Power }\end{array}$} & \multirow[b]{2}{*}{$\begin{array}{l}\text { Reason for } \\
\text { Failure }\end{array}$} & \multirow[b]{2}{*}{ Location } & \multirow[b]{2}{*}{ LTC Tap } & \multicolumn{3}{|c|}{ Node 01} & \multicolumn{3}{|c|}{ Lowest 3థ Voltage } & \multirow{2}{*}{\begin{tabular}{c|} 
Lowest \\
$1 \Phi$ \\
Voltage
\end{tabular}} & \multirow[b]{2}{*}{$\begin{array}{l}\text { Highest } \\
\text { I2/11 \% }\end{array}$} & \multirow[b]{2}{*}{$\begin{array}{l}\text { Highest } \\
\text { V2/V1 \% }\end{array}$} & \multirow[b]{2}{*}{$\begin{array}{l}\% \text { kW } \\
\text { Loss }\end{array}$} & \multirow[b]{2}{*}{$\begin{array}{c}\text { Total } \\
\text { kVar Loss }\end{array}$} & \multicolumn{3}{|c|}{$12 / 1 \%$} & \multirow[b]{2}{*}{$\begin{array}{l}\text { Node } 1 \\
\text { Capacity }\end{array}$} \\
\hline & & & & & & IA & IB & IC & VA & VB & vc & & & & & & Node 0 & Low & $\begin{array}{c}\text { High } \\
(<100)\end{array}$ & \\
\hline SG & 13290 & 0 & Reverse Power & $\mathrm{BP}$ & 10 & 220.1696 & \begin{tabular}{|l|}
271.2878 \\
\end{tabular} & 260.5665 & 119.6138 & 120.2031 & \begin{tabular}{|l|}
118.6864 \\
\end{tabular} & 118.0618 & 100.0552 & 1.3408 & $4.5 \%$ & 1826.3 & 10.089 & 0.0029 & 88.4603 & 59.5696 \\
\hline & & $\operatorname{VR}(1) 32$, & 30,32 & & & & & & & & & & & & & & & & & \\
\hline SG & 13740 & 0 & Reverse Power & $\mathrm{MP}$ & 9 & 209.6296 & 236.8509 & 231.5352 & \begin{tabular}{|l|}
123.4694 \\
\end{tabular} & 121.8682 & \begin{tabular}{|l|}
120.5033 \\
\end{tabular} & 119.8612 & 100.0533 & 0.9978 & $3.4 \%$ & 1035.66 & 7.8879 & 0.7686 & 88.2549 & 76.4327 \\
\hline & & $\operatorname{VR}(1) 14$, & 23,22 & & & & & & & & & & & & & & & & & \\
\hline SG & 13980 & 0 & Reverse Power & $E P$ & 11 & 259.8293 & 283.9373 & 288.2117 & \begin{tabular}{|l|}
122.7994 \\
\end{tabular} & 121.6083 & \begin{tabular}{|l|}
119.4167 \\
\end{tabular} & 1118.7864 & 100.0536 & 1.3867 & $5.4 \%$ & 2511.05 & 6.7255 & 0.6844 & 88.1865 & 71.7475 \\
\hline & & VR (1) 11, & 17,21 & & & & & & & & & & & & & & & & & \\
\hline
\end{tabular}

\begin{tabular}{|c|c|c|c|c|c|c|c|}
\hline \multicolumn{8}{|c|}{ Improvement } \\
\hline Machine & Real & $\begin{array}{l}\text { Reactive } \\
\text { Power }\end{array}$ & Reason for & & Lowest & & Node 1 \\
\hline & Power & Power & Failure & Location & Voltage & Losses & Capacity \\
\hline SG & 13290 & 0 & Reverse Power & $\mathrm{BP}$ & $0.18 \%$ & $0.93 \%$ & $59.78 \%$ \\
\hline & & $\operatorname{VR}(1) 32$, & 30,32 & & & & \\
\hline$S G$ & 13740 & 0 & Reverse Power & $\mathrm{MP}$ & $1.70 \%$ & $2.04 \%$ & $76.64 \%$ \\
\hline & & VR (1) 14, & 23,22 & & & & \\
\hline SG & 13980 & 0 & Reverse Power & $\mathrm{EP}$ & $0.79 \%$ & $0.00 \%$ & $71.95 \%$ \\
\hline & & VR (1) 11, & & & & & \\
\hline
\end{tabular}

Table 42. Maximum Limit for Real and Reactive Power Injection

\begin{tabular}{|c|c|c|c|c|c|c|c|c|c|c|c|c|c|c|c|c|c|c|c|c|}
\hline \multirow[b]{2}{*}{$\begin{array}{l}\text { Machine } \\
\text { Type }\end{array}$} & \multirow[b]{2}{*}{$\begin{array}{l}\text { Real } \\
\text { Power }\end{array}$} & \multirow[b]{2}{*}{$\begin{array}{c}\text { Reactive } \\
\text { Power }\end{array}$} & \multirow[b]{2}{*}{$\begin{array}{l}\text { Reason for } \\
\text { Failure }\end{array}$} & \multirow[b]{2}{*}{ Location } & \multirow[b]{2}{*}{ LTC Tap } & \multicolumn{3}{|c|}{ Node 01} & \multicolumn{3}{|c|}{ Lowest $3 \Phi$ Voltage } & \multirow{2}{*}{$\begin{array}{c}\text { Lowest } \\
1 \Phi \\
\text { Voltage }\end{array}$} & \multirow[b]{2}{*}{$\begin{array}{l}\text { Highest } \\
12 / 11 \%\end{array}$} & \multirow[b]{2}{*}{$\begin{array}{l}\text { Highest } \\
\text { V2/V1 \% }\end{array}$} & \multirow[b]{2}{*}{$\begin{array}{l}\% \text { kW } \\
\text { Loss }\end{array}$} & \multirow[b]{2}{*}{$\begin{array}{c}\text { Total } \\
\text { kVar Loss }\end{array}$} & \multicolumn{3}{|c|}{$12 / 11 \%$} & \multirow[b]{2}{*}{$\begin{array}{l}\text { Node } 1 \\
\text { Capacity }\end{array}$} \\
\hline & & & & & & IA & IB & IC & VA & VB & vc & & & & & & Node 0 & Low & $\begin{array}{c}\text { High } \\
(<100)\end{array}$ & \\
\hline SG & 13220 & 1893 & Reverse Power & $\mathrm{BP}$ & \begin{tabular}{|l|}
8 \\
\end{tabular} & 142.0642 & \begin{tabular}{|l|}
196.0875 \\
\end{tabular} & 183.4428 & \begin{tabular}{|l|}
119.7491 \\
\end{tabular} & 120.385 & 118.8633 & 118.2371 & 100.0551 & 1.3197 & $4.4 \%$ & 1687.92 & 14.4753 & 0.0028 & 88.4668 & 70.7768 \\
\hline & & $\operatorname{VR}(1) 32$ & 29,31 & & & & & & & & & & & & & & & & & \\
\hline$S G$ & 14250 & 3480 & Reverse Power & $\mathrm{MP}$ & 6 & 67.2904 & 105.9963 & 101.8954 & 126.4554 & 124.0628 & 126.3554 & 123.2218 & 100.0518 & 0.7465 & $3.1 \%$ & 748.35 & 22.1997 & 0.4043 & 88.3036 & 84.2032 \\
\hline & & $\operatorname{VR}(1)-10$ & $0,-2$ & & & & & & & & & & & & & & & & & \\
\hline SG & 14490 & 2007 & Reverse Power & EP & 8 & 177.3008 & 200.7446 & 203.7936 & 124.1336 & 122.7252 & 120.8493 & 120.205 & 100.0529 & 1.2065 & $5.1 \%$ & 2227.32 & 9.7905 & 0.5588 & 88.2301 & 70.0828 \\
\hline & & $\operatorname{VR}(1) 1$, & 9,10 & & & & & & & & & & & & & & & & & \\
\hline
\end{tabular}

\begin{tabular}{|c|c|c|c|c|c|c|c|}
\hline \multicolumn{8}{|c|}{ Improvement } \\
\hline $\begin{array}{c}\text { Machine } \\
\text { Type }\end{array}$ & $\begin{array}{l}\text { Real } \\
\text { Power }\end{array}$ & $\begin{array}{c}\text { Reactive } \\
\text { Power }\end{array}$ & $\begin{array}{l}\text { Reason for } \\
\text { Failure }\end{array}$ & Location & $\begin{array}{l}\text { Lowest } \\
\text { Voltage }\end{array}$ & Losses & $\begin{array}{l}\text { Node } 1 \\
\text { Capacity }\end{array}$ \\
\hline SG & 13220 & 1893 & Reverse Power & $\mathrm{BP}$ & $0.32 \%$ & $1.00 \%$ & $70.98 \%$ \\
\hline & & $\operatorname{VR}(1) 32$ & 29,31 & & & & \\
\hline SG & 14250 & 3480 & Reverse Power & MP & $4.55 \%$ & $2.30 \%$ & $84.41 \%$ \\
\hline & & $\operatorname{VR}(1)-10$ & $0,-2$ & & & & \\
\hline SG & 14490 & 2007 & Reverse Power & $E P$ & $1.99 \%$ & $0.28 \%$ & $70.29 \%$ \\
\hline & & VR (1) 1, & & & & & \\
\hline
\end{tabular}




\subsection{Real and Reactive Distributed Generation Size Limitation}

Table 42 shows the results of applying the maximum real and reactive power without exceeding study criteria. Notice that a much larger DG can be used when injecting both real and reactive power because, now, the real power limit is $14,490 \mathrm{~kW}$ (versus 13,980 $\mathrm{kW}$ for real power injection only). Again, the optimum location is at the midpoint. However, in this case, the lowest single-phase voltage was improved $4.55 \%$ versus only $1.7 \%$ in the real power case. The real power loss savings were marginally better $(2.3 \%$ versus $2.04 \%)$ than the optimum real power injection case.

\subsection{Findings}

It should be remembered that the three-phase feeder conductor size of $636 \mathrm{kcmil}$ aluminum is uniform throughout the Milford Circuit. Therefore, maximum size was not limited because of location of DG on the circuit. The optimum location for the greatest voltage improvement and real power loss savings is the midpoint. 


\section{Project Results - Distributed Generation Voltage Regulation and Optimum Generator Conditions for Maximum Improvement of Voltage Regulation, Loss Reduction, and Released Capacity}

\subsection{Introduction}

In this section, three generation types are employed to determine their effect on distribution circuit voltage regulation when the magnitude of the real power injection and the reactive power export or import (absorption) is varied. The three types of generation are a $400-\mathrm{kW}$ induction generator with two fixed reactive values, a $400-\mathrm{kW}$ inverter-based generator with a \pm 0.8 power factor, and a $1,000-\mathrm{kW}$ synchronous machine with a \pm 0.8 power factor.

Three effects may occur when DG is operated on a distribution circuit: voltage regulation improvement, released capacity savings, and real power loss reduction. These improvements were tabulated for each generator type for both high (105\%) and low (95\%) substation primary voltage. The data are summarized for the actual locations of the induction generator and the high-speed generator and inverter; the data for the synchronous machine are summarized for its actual location on the distribution circuit or the midlocation (ML, Node 10), at the beginning location (BL, Node 1), on the secondary of the substation after the LTC, and at the end location (EL, Node 17) near the tag end of the circuit.

This analysis was conducted for the actual peak-load, or HL, condition at 17:43 on July 17, 2006, and at the LL condition at 05:00 the same day. The results are tabulated and displayed in graphical form for voltage improvement, released capacity savings, and real energy loss reduction.

\subsection{Factors to Consider for Test Result Evaluation}

Two factors must be considered for simulation test result evaluation: (1) the dead band of the VR and (2) the effect the VDC source model on the increase or decrease in kilowatt and kilovar load as the voltage changes.

\subsubsection{Regulator Dead Ban}

The regulator dead band is set for $1 \mathrm{~V}$. Because the regulator has a $+5 \%$ boost in voltage over 32 steps and a $-5 \%$ buck in voltage over 32 steps, the number of steps within the dead band is

$$
\frac{5 \% \text { voltage change }}{32 \text { steps }}=0.15625 \% / \text { step x } 120 \text { volts, or } 0.1875 \text { volts } / \text { tap. }
$$

The number of steps within the deadband is then

$$
\frac{1 \text { volt deadband }}{0.1875 \text { volts } / \text { tap }}=5 \text { steps. }
$$


So the voltage can change as much as 1 volt and not result in a change of the VR tap setting. When DG is run during an HV condition on the primary of the substation and at different real and plus or minus reactive power flows, the voltage will decrease or increase. This will cause the kilowatt and kilovar loads to decrease or increase. The change in voltage may not be enough to cause a reduction in the regulator settings (see Figure 141, Figure 155, and Figure 161). During the LV condition on the primary of the substation, the LTC and step regulator taps are typically set at the highest tap settings, and there is less effect because of the step regulator step-changing interventions.

\subsubsection{Voltage-Dependent Current Source}

The model that best represents how load characteristics change with a change in voltage is the VDC source model. Adding real and positive reactive power from DG sources will increase the voltage and cause an increase in current or kilovolt-ampere load, which, in turn, increases the real power energy losses and reduces the released capacity (see Figure 144). The model will cause a $1.8 \%$ increase in current with a $1 \%$ increase in voltage and a $1.8 \%$ decrease in current with a $1 \%$ decrease in voltage.

\subsection{Interpreting the Distributed Generation Voltage Regulation Application Results}

Two sets of tables summarize the results of the DG applications. The first set, Table 44 through Table 51, summarizes the results of applying each of the three generation types for both HV and LV substation primary conditions. Table 43 explains how to interpret the results. In the second set, Table 52 shows a summary of the benefits of running each type of DG. In the cases of the induction and inverter-based generators, the results are given for the location where the units are installed on the circuit. The induction generator is located at Node 17, or the EL, and the inverter generator is located at Node 22, or the midpoint. For the synchronous generator, the findings are summarized for generator locations at the beginning (Node 1), middle or actual location site (Node 10), and end (Node 17).

\subsubsection{Induction Generator Voltage Regulation Application (High Voltage) - Heavy Load, Table 44}

Each of the generator applications consists of different real power and reactive power injections (+) or absorption (-) for both HV (105\%) and LV (95\%) conditions on the primary of the substation. The LTC tap setting at the secondary of the substation transformer is recorded for each real and reactive power case considered, as well as the tap settings of the step regulator for each case. The phase currents $\mathrm{I}_{\mathrm{A}}, \mathrm{I}_{\mathrm{B}}$, and $\mathrm{I}_{\mathrm{C}}$ at Node 1 ; the lowest three phase voltages $\mathrm{V}_{\mathrm{A}}, \mathrm{V}_{\mathrm{B}}$, and $\mathrm{V}_{\mathrm{C}}$; and the lowest single-phase voltage on the circuit are recorded along with the highest current unbalance $\mathrm{I}_{2} / \mathrm{I}_{1} \%$, the highest voltage unbalance $\mathrm{V}_{2} / \mathrm{V}_{1} \%$, the percent circuit losses (excluding the substation transformer), kilovar losses, and capacity savings.

Six cases were studied for the real power and reactive power. These are: 


\begin{tabular}{|c|c|c|c|}
\hline & $P(k W)$ & $Q$ (kVAr) & $\begin{array}{c}\text { Substation Primary } \\
\text { Voltage }\end{array}$ \\
\hline 1 & 400 & 0 & LV \\
\hline 2 & 400 & 247.91 & LV \\
\hline 3 & 400 & -247.91 & LV \\
\hline 4 & 400 & 0 & HV \\
\hline 5 & 400 & 247.91 & HV \\
\hline 6 & 400 & -247.91 & HV \\
\hline
\end{tabular}

Table 43 explains the meaning of each improvement from the base case with no DG application. For the substation primary LV case, notice that when plus reactive and real power are injected $(400 \mathrm{~kW},+247.91 \mathrm{kVAr})$, the lowest three-phase voltages increase, the lowest single-phase voltage increases, and the percent losses on the circuit are slightly lower (i.e., $5.145 \%$ compared with $5.4 \%$ for the base case). The percent loss improvement is then $0.25 \%$, and the released capacity savings are $6.10 \%$. For the substation primary HV case, the phase currents $\mathrm{I}_{\mathrm{A}}, \mathrm{I}_{\mathrm{B}}$, and $\mathrm{I}_{\mathrm{C}}$ are about 20 A more because the higher voltage levels cause an increase in the load. Here, when the DG injects $400 \mathrm{~kW}$ of real power and $+247.91 \mathrm{kVArs}$ of reactive power, the lowest single-phase voltage improves by $0.12 \%$, the percent energy loss savings improves $0.30 \%$, and the released capacity improves only by $3.80 \%$. This reduction in released capacity is due to the higher voltage and increased load. For the LV case, the released capacity was $6.10 \%$. For the LV case, when the generator is absorbing volt-amperes reactive (i.e., -247.91 Vars), there is a drop in voltage. The lowest single-phase voltage is now $2.63 \%$ less than the base case. The loss savings is $0.2 \%$, but the released capacity is $6.44 \%$. For the HV case and the same P and $-\mathrm{Q}$, the lowest voltage drops only $0.06 \%$, the loss savings are $0.19 \%$, and the released capacity is only $2.31 \%$. Again, the higher the voltage, the greater the load and less released capacity. 
Table 43. Explaining the Meaning of

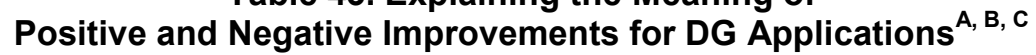

\begin{tabular}{lll} 
Improvement Parameter & \multicolumn{1}{c}{ Negative \% Numbers } & \multicolumn{1}{c}{ Positive \% Numbers } \\
\cline { 2 - 3 } $\begin{array}{l}\text { \% Lowest single-phase } \\
\text { voltage }\end{array}$ & $\begin{array}{l}\text { Voltage drop from base case } \\
\text { (negative improvement) }\end{array}$ & $\begin{array}{l}\text { Voltage rise from base case } \\
\text { (positive improvement) }\end{array}$ \\
\% Real energy losses & $\begin{array}{l}\text { Higher losses than base case } \\
\text { (negative improvement) }\end{array}$ & $\begin{array}{l}\text { Lower losses than base case } \\
\text { (positive improvement) }\end{array}$ \\
\% Released capacity & $\begin{array}{l}\text { Higher capacity needed to } \\
\text { serve the load } \\
\text { (negative improvement) }\end{array}$ & $\begin{array}{l}\text { Lower capacity needed to } \\
\text { serve load } \\
\text { (positive improvement) }\end{array}$
\end{tabular}

Notes:

A. The percent voltage improvement is the percent difference between the lowest single-phase voltage on the circuit for the base case and the lowest single-phase voltage for the DG application being considered divided by the base case and multiplied by $100 \%$.

B. The energy losses are the sum of all feeder lateral distribution transformer, secondary, and service losses, and the percent improvement is the difference between the base case percent losses for the circuit and the percent losses for the DG application.

C. The kilovolt-ampere released capacity percent improvement is the difference between the percent capacity needed to serve the load at Node 1 and the percent base case capacity.

\subsubsection{Inverter Generator Voltage Regulation Application (High Voltage) - Heavy Load, Table 45}

Because the real and reactive power capability of the high-speed generator inverter is about the same as that of the induction generator, the results are very similar to the induction generator voltage regulation application.

Six cases were studied for the inverter-based generation. These represent a full range of real and reactive capability.

\begin{tabular}{cccc}
\hline & $\mathbf{P}(\mathbf{k W})$ & $\mathbf{Q}(\mathbf{k V A r})$ & $\begin{array}{c}\text { Substation Primary } \\
\text { Voltage }\end{array}$ \\
\cline { 2 - 4 } 1 & 400 & 0 & $\mathrm{LV}$ \\
2 & 320 & 240 & $\mathrm{LV}$ \\
3 & 320 & -240 & $\mathrm{LV}$ \\
4 & 400 & 0 & $\mathrm{HV}$ \\
5 & 320 & 240 & $\mathrm{HV}$ \\
6 & 320 & -240 & $\mathrm{HV}$ \\
\hline
\end{tabular}

The savings in losses, voltage improvement, and released capacity are, in general, less than those of the induction generator application because the real power at maximum +Var output is less. 


\subsubsection{Synchronous Generator Voltage Regulation Application (Beginning Location, High Voltage) - Heavy Load, Table 46}

Thirteen cases, with the range of real and reactive power shown below, were studied for the synchronous generator. Because regulation results differ depending on location, three locations were selected: the BL, ML, and EL.

\begin{tabular}{|c|c|c|c|c|}
\hline & Location & $\mathbf{P}$ & $\mathbf{Q}$ & $\begin{array}{c}\text { Substation Primary } \\
\text { Voltage }\end{array}$ \\
\hline 1 & $B L$ & $50 \%$ & 0 & HV \\
\hline 2 & $B L$ & $75 \%$ & 0 & HV \\
\hline 3 & $\mathrm{BL}$ & $100 \%$ & 0 & $\mathrm{HV}$ \\
\hline 4 & $\mathrm{BL}$ & $106.7 \%$ & 0 & $\mathrm{HV}$ \\
\hline 5 & $\mathrm{BL}$ & $100 \%$ & $100 \%$ & HV \\
\hline 6 & $\mathrm{BL}$ & $100 \%$ & $-100 \%$ & HV \\
\hline 7 & $\mathrm{BL}$ & $25 \%$ & $100 \%$ & HV \\
\hline 8 & $\mathrm{BL}$ & $25 \%$ & $75 \%$ & HV \\
\hline 9 & $\mathrm{BL}$ & $25 \%$ & $50 \%$ & HV \\
\hline 10 & $\mathrm{BL}$ & $25 \%$ & $25 \%$ & $\mathrm{HV}$ \\
\hline 11 & $\mathrm{BL}$ & $25 \%$ & $0 \%$ & HV \\
\hline 12 & $\mathrm{BL}$ & $25 \%$ & $-50 \%$ & HV \\
\hline 13 & $\mathrm{BL}$ & $25 \%$ & $-100 \%$ & HV \\
\hline
\end{tabular}

When the synchronous generator is located at the source end (near the substation), its maximum generation of real and reactive power reduces these requirements from the source and, as such, results in the highest released capacity of $7.36 \%$ when operated at $\mathrm{P}=100 \%$ and $\mathrm{Q}=+100 \%$. Although the losses are less than those of the base case, the loss savings are not as good as when the generation is closer to the load. The lowest single-phase voltage is, in general, better than the previous cases but still less than the base case.

\subsubsection{Synchronous Generator Voltage Regulation Application (End Location, High Voltage) - Heavy Load, Table 47}

Installing generation at the end of the circuit (EL) resulted in a released capacity savings of $6.85 \%$, a loss savings of $0.53 \%$, and near-base case conditions for voltage when the real power was $106.7 \%$ of nameplate and the reactive was $0 \%$. When $100 \%$ reactive and $100 \%$ real power were applied, the voltage improved above the base case by $0.82 \%$, and the released capacity was $5.97 \%$. The worst-case voltage occurred when the unit was operated at $-100 \%$ reactive and $100 \%$ real power. The lowest single-phase voltage dropped $0.44 \%$ below the base case. Both the voltage and the released capacity improvement parameters were worse than those of the base case when the unit was operated at $25 \%$ real power and $-100 \%$ reactive. Here, the voltage dropped $-0.7 \%$, and the released capacity was only $1.5 \%$. 


\subsubsection{Synchronous Generator Voltage Regulation Application (Midlocation, High Voltage) - Heavy Load, Table 48}

The mid-location is the actual site of the 1,000-kW synchronous generator. The maximum released capacity of $6.88 \%$ occurs when the machine is operated at its maximum real power output of $106.7 \%$ and zero reactive power. This is to be expected because this input power to the circuit just offsets the same required power plus losses that the source provides to the circuit. Also, this case results in the second-highest loss savings of $0.48 \%$, the highest loss saving being when the real power and reactive power are $100 \%$ each. The minimum released capacity of $1.15 \%$, the lowest loss saving of $0.03 \%$, and the worst-case single-phase voltage improvement of $-0.81 \%$ occurs when the real power is only $25 \%$ and the reactive is $-100 \%$. Here, the voltage is reduced because of the high value of the generation reactive absorption. The best-case voltage improvement is $0.5 \%$ when the reactive is $100 \%$ export.

\subsubsection{Synchronous Generator Voltage Regulation Application (Beginning Location, Low Voltage) Heavy Load, Table 49}

When the primary of the substation is operating at the LV condition of $95 \%$, the LTC at the substation is in the all-raise position (16), and the step regulator on the circuit operates at the all-raise position on all three phases $(32,32,32)$. The released capacity savings reached their highest levels in this case with a $10.44 \%$ savings occurring at $100 \%$ real power and $100 \%$ reactive power. The voltages are lower than those of the base case by about $2 \%$ to $3 \%$. The worst case occurred at $-2.96 \%$ with $25 \%$ real and $-100 \%$ reactive. This is obvious because the negative reactive is reducing the voltage. The best-case voltage of $-1.93 \%$ occurred when $100 \%$ real power and $100 \%$ reactive export power were delivered at the BL. The loss improvements are quite low and range from only $0.02 \%$ to $0.13 \%$.

\subsubsection{Synchronous Generator Voltage Regulation Application (End Location, Low Voltage) - Heavy Load, Table 50}

As before (see 11.3.6), the LTC regulator and the step regulator are operating at full-raise position during this LV condition on the primary of the substation. The capacity savings of $9.98 \%$ are slightly lower than those of the previous case, with the maximum real power output of $106.7 \%$. All the voltage improvements are still negative, with the worst case occurring at $3.33 \%$ when $\mathrm{P}=25 \%$ and $\mathrm{Q}-100 \%$. The loss savings were better than the previous cases, with the maximum of $0.56 \%$ at $\mathrm{P}=100 \%$ and $\mathrm{Q}=100 \%$. This is because a portion of the tag end load of the circuit is now being served by the DG. Again, this LV condition results in less kilovolt-amperes of load and, hence, lower losses.

\subsubsection{Synchronous Generator Voltage Regulation Application (Midlocation, Low Voltage) - Heavy Load, Table 51}

The midlocation performance is somewhat between the BL and EL performances. The released capacity is now $10.01 \%$ at $106.7 \%$ real power, whereas the released capacity was $10.44 \%$ at the $\mathrm{BL}$ and $9.98 \%$ at the EL. The greatest loss savings of $0.51 \%$ occurred at $\mathrm{P}=100 \%$ and $\mathrm{Q}=$ $100 \%$, which is slightly less than the maximum loss savings of $0.56 \%$ when the DG is located at the EL. The worst-case lowest single-phase voltage was $-3.36 \%$, compared with the best case of $-2.96 \%$ when the $\mathrm{DG}$ is located at the $\mathrm{BL}$ and operating at $\mathrm{P}=25 \%$ and $\mathrm{Q}=-100 \%$. The best-case lowest single-phase voltage is $-1.14 \%$ when $\mathrm{P}=100 \%$ and $\mathrm{Q}=100 \%$, which is almost the same result as when the unit was located at the EL (-1.15\%). 
Table 44. Induction Generator Voltage Regulation Application (LV, HV) - HL

\begin{tabular}{|c|c|c|c|c|c|c|c|c|c|c|c|c|c|c|c|c|c|c|c|c|}
\hline & & & & & & & & & & & & & & & & & \multirow{2}{*}{\multicolumn{3}{|c|}{$12 / 11 \%$}} & \multirow[b]{3}{*}{$\begin{array}{l}\text { Node 1 } \\
\text { Capacity }\end{array}$} \\
\hline \multirow{2}{*}{$\begin{array}{c}\text { Machine } \\
\text { Type }\end{array}$} & \multirow[b]{2}{*}{$\begin{array}{l}\text { Real } \\
\text { Power }\end{array}$} & \multirow[b]{2}{*}{\begin{tabular}{|c|} 
Reactive \\
Power
\end{tabular}} & \multirow[b]{2}{*}{ HV / LV } & \multirow[b]{2}{*}{ Location } & \multirow[b]{2}{*}{ LTC Tap } & \multicolumn{3}{|c|}{ Node 01} & \multicolumn{3}{|c|}{ Lowest $3 \Phi$ Voltage } & \multirow{2}{*}{$\begin{array}{c}\text { Lowest } \\
1 \Phi \\
\text { Voltage } \\
\end{array}$} & \multirow[b]{2}{*}{$\begin{array}{l}\text { Highest } \\
12 / 11 \%\end{array}$} & \multirow[b]{2}{*}{$\begin{array}{l}\text { Highest } \\
\text { V2/N1 }\end{array}$} & \multirow[b]{2}{*}{$\begin{array}{c}\% \text { kW } \\
\text { Loss }\end{array}$} & \multirow[b]{2}{*}{$\begin{array}{c}\text { Total } \\
\text { kVar Loss }\end{array}$} & & & & \\
\hline & & & & & & IA & IB & IC & VA & VB & vc & & & & & & Node 0 & Low & $\begin{array}{l}\text { Pigh } \\
(<100)\end{array}$ & \\
\hline IG & 400 & 0 & LV & $N / A$ & 16 & 552.457 & 630.2362 & 612.8234 & 116.9241 & 117.2165 & 115.8406 & 115.1259 & 100.0563 & 1.3398 & $5.2 \%$ & 2924.33 & 3.9985 & 0.7005 & 88.4458 & 6.0751 \\
\hline & & & & & & & & & & & & & & & & & & & & \\
\hline IG & 400 & 247.91 & $\mathrm{LV}$ & $\mathrm{N} / \mathrm{A}$ & 16 & \begin{tabular}{|l|}
553.1808 \\
\end{tabular} & 631.4567 & \begin{tabular}{|l}
614.1055 \\
\end{tabular} & 117.2827 & 117.5672 & 116.2048 & 115.4859 & 100.0561 & 1.3216 & $5.1 \%$ & 2931.47 & 4.0372 & 0.6957 & 88.4485 & 5.8932 \\
\hline & & 2 (1) 32,32 & & & & & & & & & & & & & & & & & & \\
\hline IG & 400 & -247.91 & LV & N/A & 16 & 551.9444 & 629.1895 & \begin{tabular}{|l|l|}
611.7199 \\
\end{tabular} & 116.5597 & 116.8611 & 1115.4707 & 114.7603 & 100.0564 & 1.3594 & $5.2 \%$ & 2920.77 & 3.9577 & 0.7054 & 88.443 & 6.2311 \\
\hline & & & & & & & & & & & & & & & & & & & & \\
\hline IG & 400 & 0 & $\mathrm{HV}$ & $\mathrm{N} / \mathrm{A}$ & 3 & 571.6509 & 648.8639 & 632.2462 & 119.2617 & 119.7564 & 118.2793 & 117.5369 & 100.0553 & 1.2632 & $5.1 \%$ & 2990.79 & 3.8551 & 0.6694 & 88.4629 & 3.299 \\
\hline & & & & & & & & & & & & & & & & & & & & \\
\hline IG & 400 & 247.91 & $\mathrm{HV}$ & $\mathrm{N} / \mathrm{A}$ & 3 & 568.6085 & 646.8681 & \begin{tabular}{|l|l|}
629.8217 \\
\end{tabular} & 119.7302 & 120.1709 & 118.7389 & 117.9912 & 100.0551 & 1.2666 & $5.1 \%$ & 2960.5 & 3.9362 & 0.6629 & 88.4648 & 3.5964 \\
\hline & & R (1) 26,23 & & & & & & & & & & & & & & & & & & \\
\hline IG & 400 & -247.91 & $\mathrm{HV}$ & $\mathrm{N} / \mathrm{A}$ & 4 & 579.2854 & 656.8888 & 639.831 & 119.523 & 120.0307 & 118.5256 & 117.7804 & 100.0552 & 1.2836 & $5.2 \%$ & 3082.02 & 3.8059 & 0.6799 & 88.4585 & 2.103 \\
\hline & $\frac{400}{v}$ & $\frac{1-241.91}{2(1)} 32,28$ & MV & & & 00.29054 & 050.0000 & 009.001 & 119.020 & 120.0501 & 110.0200 & 111.1004 & & 1.20000 & & & & 0.0799 & 00.4500 & 2.100 \\
\hline
\end{tabular}

\begin{tabular}{|c|c|c|c|c|c|c|c|}
\hline \multicolumn{8}{|c|}{ Improvement } \\
\hline $\begin{array}{c}\text { Machine } \\
\text { Type }\end{array}$ & $\begin{array}{l}\text { Real } \\
\text { Power }\end{array}$ & $\begin{array}{c}\text { Reactive } \\
\text { Power }\end{array}$ & HV / LV & Location & $\begin{array}{c}\text { Lowest } \\
1 \Phi \\
\text { Voltage }\end{array}$ & $\begin{array}{c}\text { kW } \\
\text { Losses }\end{array}$ & $\begin{array}{c}\text { Node } 1 \\
\text { Capacity }\end{array}$ \\
\hline IG & 400 & & $\mathrm{LV}$ & $\mathrm{N} / \mathrm{A}$ & $-2.32 \%$ & $0.23 \%$ & $6.28 \%$ \\
\hline & & (1) 32,32 & & & & & \\
\hline$\overline{I G}$ & 400 & 247.91 & LV & $\mathrm{N} / \mathrm{A}$ & $-2.01 \%$ & $0.25 \%$ & $6.10 \%$ \\
\hline & $\mathrm{C}_{\mathrm{s}}$ & & & & & & \\
\hline IG & 400 & \begin{tabular}{|l|}
-247.91 \\
\end{tabular} & $\mathrm{LV}$ & $\mathrm{N} / \mathrm{A}$ & $-2.63 \%$ & $0.20 \%$ & $6.44 \%$ \\
\hline & & R(1) 32,32 & 32 & & & & \\
\hline IG & 400 & \begin{tabular}{|l|}
0 \\
\end{tabular} & $\mathrm{HV}$ & $\mathrm{N} / \mathrm{A}$ & $-0.27 \%$ & $0.25 \%$ & $3.51 \%$ \\
\hline & & R (1) 30,26 & 27 & & & & \\
\hline IG & 400 & \begin{tabular}{|l|l|}
247.91 \\
\end{tabular} & $\mathrm{HV}$ & $\mathrm{N} / \mathrm{A}$ & $0.12 \%$ & $0.30 \%$ & $3.80 \%$ \\
\hline & & & & & & & \\
\hline IG & 400 & \begin{tabular}{|l|}
-247.91 \\
\end{tabular} & $\mathrm{HV}$ & $\mathrm{N} / \mathrm{A}$ & $-0.06 \%$ & $0.19 \%$ & $2.31 \%$ \\
\hline & & (1) 32,28 & & & & & \\
\hline
\end{tabular}


Table 45. Inverter Generator Voltage Regulation Application (LV, HV) - HL

Table 11.2 Inverter Generator Voltage Regulation Application (LV, HV) - Heavy Load

\begin{tabular}{|c|c|c|c|c|c|c|c|c|c|c|c|c|c|c|c|c|c|c|c|c|}
\hline \multirow[b]{2}{*}{$\begin{array}{c}\text { Machine } \\
\text { Type }\end{array}$} & \multirow[b]{2}{*}{$\begin{array}{l}\text { Real } \\
\text { Power }\end{array}$} & \multirow[b]{2}{*}{$\begin{array}{l}\text { Reactive } \\
\text { Power }\end{array}$} & \multirow[b]{2}{*}{ HV /LV } & \multirow[b]{2}{*}{ Location } & \multirow[b]{2}{*}{ LTC Tap } & \multicolumn{3}{|c|}{ Node 01} & \multicolumn{3}{|c|}{ Lowest $3 \Phi$ Voltage } & \multirow{2}{*}{\begin{tabular}{|c|} 
Lowest \\
$1 \Phi$ \\
Voltage
\end{tabular}} & \multirow[b]{2}{*}{$\begin{array}{l}\text { Highest } \\
12 / 11 \%\end{array}$} & \multirow[b]{2}{*}{$\begin{array}{l}\text { Highest } \\
\text { V2/N1 }\end{array}$} & \multirow[b]{2}{*}{$\begin{array}{c}\% \mathrm{~kW} \\
\text { Loss }\end{array}$} & \multirow[b]{2}{*}{$\begin{array}{c}\text { Total } \\
\text { kVar Loss }\end{array}$} & \multicolumn{3}{|c|}{$12 / 11 \%$} & \multirow[b]{2}{*}{\begin{tabular}{|c|} 
Node 1 \\
Capacity
\end{tabular}} \\
\hline & & & & & & IA & IB & IC & VA & , & vc & & & & & & Node 0 & Low & $\begin{array}{l}\text { High } \\
(<100)\end{array}$ & \\
\hline InvG & 400 & 0 & $\mathrm{LV}$ & $\mathrm{N} / \mathrm{A}$ & 16 & 552.4406 & 630.1595 & 612.4781 & 117.0235 & $\begin{array}{l}117.1949 \\
\end{array}$ & 115.8796 & \begin{tabular}{|l|}
115.1541 \\
\end{tabular} & 100.0562 & 1.3684 & $5.2 \%$ & 2952.59 & 3.9782 & 0.6943 & 82.3537 & \begin{tabular}{|l}
6.0865 \\
\end{tabular} \\
\hline & & (1) 32,32 & & & & & & & & & & & & & & & & & & \\
\hline $\operatorname{lnvG}$ & 320 & 240 & $\mathrm{LV}$ & $\mathrm{N} / \mathrm{A}$ & 16 & 555.3521 & 633.2756 & 616.0219 & 117.5087 & 117.6895 & 116.3973 & 115.6369 & 107.5141 & 1.363 & $5.2 \%$ & 2985.68 & 3.9892 & 0.6889 & 80.9469 & 5.6221 \\
\hline & & (1) 32,32 & 32 & & & & & & & & & & & & & & & & & \\
\hline $\operatorname{lnvG}$ & 320 & -240 & $\mathrm{LV}$ & $\mathrm{N} / \mathrm{A}$ & 16 & 555.7432 & 633.3703 & 615.2559 & 116.3862 & 116.6189 & 115.2498 & 114.5651 & 100.0565 & 1.3826 & $5.3 \%$ & 2978.01 & 3.9493 & 0.7025 & 68.8583 & 5.608 \\
\hline & & 2(1) 32,32 & & & & & & & & & & & & & & & & & & \\
\hline InvG & 400 & $\begin{array}{c}0 \\
\text { (1) } 29,26\end{array}$ & $\frac{H V}{26}$ & N/A & 3 & 570.7684 & 648.7027 & 631.0691 & 119.3939 & 119.7258 & \begin{tabular}{|l|l|}
118.3498 \\
\end{tabular} & \begin{tabular}{|l|l|}
117.5964 \\
\end{tabular} & 100.0552 & 1.3194 & $5.2 \%$ & 3013.17 & 3.8672 & 0.6628 & 90.3889 & 3.323 \\
\hline $\operatorname{lnvG}$ & 320 & 240 & $\mathrm{HV}$ & $\mathrm{N} / \mathrm{A}$ & 3 & \begin{tabular}{|l|}
576.2127 \\
\end{tabular} & 653.6868 & \begin{tabular}{|l|l|}
637.0627 \\
\end{tabular} & 119.765 & 120.1514 & 118.7634 & 117.9769 & 122.1077 & 1.2764 & $5.2 \%$ & 3070.41 & 3.8373 & 0.6587 & 77.7744 & 2.5802 \\
\hline InvG & 32 & $\begin{array}{l}\frac{2}{2}(1) 32,28 \\
-240\end{array}$ & $\frac{29}{H V}$ & N/A & 4 & 583.018 & 660.9927 & $\mid 6$ & 119.355 & & & & & 13048 & $52 \%$ & 313933 & 37977 & 06769 & 688948 & 14914 \\
\hline & & $\frac{-240}{1) 32,2}$ & & & & & & & & & & & & & & & & & & \\
\hline
\end{tabular}

\begin{tabular}{|c|c|c|c|c|c|c|c|}
\hline \multicolumn{8}{|c|}{ Improvement } \\
\hline $\begin{array}{l}\text { Machine } \\
\text { Type }\end{array}$ & $\begin{array}{l}\text { Real } \\
\text { Power }\end{array}$ & $\begin{array}{l}\text { Reactive } \\
\text { Power }\end{array}$ & $\mathrm{HV} / \mathrm{LV}$ & Location & $\begin{array}{l}\text { Lowest } \\
\text { Voltage }\end{array}$ & Losses & $\begin{array}{l}\text { Node } 1 \\
\text { Capacity }\end{array}$ \\
\hline $\operatorname{lnvG}$ & 400 & 0 & $\mathrm{LV}$ & $\mathrm{N} / \mathrm{A}$ & $-2.29 \%$ & $0.22 \%$ & $6.29 \%$ \\
\hline & & (1) 32,32 & & & & & \\
\hline $\operatorname{lnvG}$ & 320 & 240 & LV & $\mathrm{N} / \mathrm{A}$ & $-1.88 \%$ & $0.16 \%$ & $5.83 \%$ \\
\hline & & (1) 32,32 & 32 & & & & \\
\hline $\operatorname{InvG}$ & 320 & -240 & $\mathrm{LV}$ & N/A & $-2.79 \%$ & $0.13 \%$ & $5.81 \%$ \\
\hline & & (1) 32,32 & 32 & & & & \\
\hline $\operatorname{lnvG}$ & 400 & 0 & $\mathrm{HV}$ & $\mathrm{N} / \mathrm{A}$ & $-0.22 \%$ & $0.24 \%$ & $3.53 \%$ \\
\hline & & & & & & & \\
\hline $\operatorname{lnvG}$ & 320 & 240 & $\mathrm{HV}$ & N/A & $0.10 \%$ & $0.21 \%$ & $2.79 \%$ \\
\hline InvG & $\frac{1}{320}$ & $\frac{1(1) 32,28}{-240}$ & $\frac{29}{H V}$ & & & & \\
\hline & & & & & & & \\
\hline
\end{tabular}


Table 46. Synchronous Generator Voltage Regulation Application (BL, HV) - HL

\begin{tabular}{|c|c|c|c|c|c|c|c|c|c|c|c|c|c|c|c|c|c|c|c|c|}
\hline \multirow[b]{2}{*}{$\begin{array}{l}\text { Machine } \\
\text { Type }\end{array}$} & \multirow[b]{2}{*}{$\begin{array}{l}\text { Real } \\
\text { Power }\end{array}$} & \multirow[b]{2}{*}{$\begin{array}{l}\text { Reactive } \\
\text { Power }\end{array}$} & \multirow[b]{2}{*}{ HV /LV } & \multirow[b]{2}{*}{ Location } & \multirow[b]{2}{*}{ LTC Tap } & \multicolumn{3}{|c|}{ Node 01} & \multicolumn{3}{|c|}{ Lowest $3 \Phi$ Voltage } & \multirow{2}{*}{$\begin{array}{c}\text { Lowest } \\
1 \Phi \\
\text { Voltage } \\
\end{array}$} & \multirow[b]{2}{*}{$\begin{array}{c}\text { Highest } \\
12 / 11 \%\end{array}$} & \multirow[b]{2}{*}{$\begin{array}{l}\text { Highest } \\
\text { V2/V1 }\end{array}$} & \multirow[b]{2}{*}{$\begin{array}{c}\% \text { kW } \\
\text { Loss }\end{array}$} & \multirow[b]{2}{*}{$\begin{array}{c}\text { Total } \\
\text { kVar Loss }\end{array}$} & \multicolumn{3}{|c|}{$12 / 1 \%$} & \multirow[b]{2}{*}{$\begin{array}{l}\text { Node } 1 \\
\text { Capacity }\end{array}$} \\
\hline & & & & & & IA & IB & IC & VA & VB & vc & & & & & & Node 0 & Low & $\begin{array}{c}\text { High } \\
(<100)\end{array}$ & \\
\hline SG & $50 \%$ & 0 & $\mathrm{HV}$ & $\mathrm{BL}$ & 3 & 569.7675 & 647.5676 & 630.3888 & 118.9988 & 119.5496 & 118.0643 & 117.3244 & 100.0554 & 1.3032 & $5.3 \%$ & 3085.62 & 3.9085 & 0.6717 & 88.4676 & 3.4922 \\
\hline & & $\lcm{32,28}$ & 20 & & & & & & & & & & & & & & & & & \\
\hline$S G$ & $75 \%$ & 0 & $\mathrm{HV}$ & $\mathrm{BL}$ & 3 & 560.5307 & 638.4113 & 621.1837 & 119.0267 & 119.5778 & 118.0917 & 117.3515 & 100.0554 & 1.3034 & $5.3 \%$ & 3040.39 & 3.9695 & 0.6718 & 88.4675 & 4.8567 \\
\hline & $100 \%$ & 1) 32,28 , & 29 & & & & & & & & & & & & & & & & & \\
\hline SG & $\frac{100 \%}{v}$ & $\frac{0}{32.28}$ & HV & BL & 3 & 551.3242 & 629.2837 & 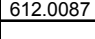 & 119.0544 & 119.6056 & 118.1188 & 117.3782 & 100.0554 & 1.3037 & $5.3 \%$ & 2995.99 & 4.0321 & 0.0027 & 88.4674 & 6.217 \\
\hline SG & $106.7 \%$ & $\frac{3 L, \angle 3}{0}$ & $\mathrm{HV}$ & $\mathrm{BL}$ & 3 & 548.8595 & 626.8399 & 609.5523 & 119.0618 & 119.613 & 118.126 & 117.3854 & 100.0554 & 1.3037 & $5.3 \%$ & 2984.23 & 4.0492 & 0.0027 & 88.4674 & 6.5812 \\
\hline & & (1) 32,28 , & 29 & & & & & & & & & & & & & & & & & \\
\hline SG & $100 \%$ & $100 \%$ & HV & $\mathrm{BL}$ & 3 & 545.0327 & 623.0061 & 605.5145 & 119.5898 & 120.1441 & 118.643 & 117.8965 & 100.0552 & 1.3085 & $5.3 \%$ & 2989.46 & 4.1082 & 0.0027 & 88.4658 & 7.1526 \\
\hline & & (1) 32,28 , & 29 & & & & & & & & & & & & & & & & & \\
\hline SG & $100 \%$ & $-100 \%$ & $\mathrm{HV}$ & $\mathrm{BL}$ & 3 & 565.4395 & 644.8146 & 627.551 & 119.1903 & 119.6974 & 118.2112 & 117.4696 & 100.0554 & 1.3253 & $5.3 \%$ & 3089.67 & 3.9899 & 0.0029 & 88.4606 & 3.9024 \\
\hline & & (1) 32,29 & 30 & & & & & & & & & & & & & & & & & \\
\hline$S G$ & $25 \%$ & $100 \%$ & $\mathrm{HV}$ & $\mathrm{BL}$ & 3 & 573.1587 & 650.8478 & 633.5213 & 119.5061 & 120.0599 & 118.561 & 117.8155 & 100.0552 & 1.3078 & $5.3 \%$ & 3125.69 & 3.9176 & 0.6742 & 88.466 & 3.0033 \\
\hline 00 & $\mathrm{~V}$ & (1) 32,28 & 29 & & & 370.1001 & & & & & 110.001 & 111.0100 & & & 0.510 & & 0.9710 & & & 0.0000 \\
\hline$S G$ & $25 \%$ & $75 \%$ & $\mathrm{HV}$ & $B L$ & 3 & 574.5047 & 652.2184 & 634.9375 & 119.3716 & 119.9246 & 118.4293 & 117.6853 & 100.0553 & 1.3066 & $5.3 \%$ & 3126.54 & 3.901 & 0.6735 & 88.4664 & 2.799 \\
\hline & & 1) 32,28 & & & & & & & & & & & & & & & & & & 2.100 \\
\hline SG & $25 \%$ & $50 \%$ & $\mathrm{HV}$ & $\mathrm{BL}$ & 3 & 575.9175 & 653.6441 & 636.4107 & 119.2389 & 119.7912 & 118.2994 & 117.5568 & 100.0553 & 1.3054 & $5.3 \%$ & 3127.81 & 3.8843 & 0.6729 & 88.4668 & 2.5866 \\
\hline & & (1) 32,28 & 29 & & & & & & & & & & & & & & & & & \\
\hline$S G$ & $25 \%$ & $25 \%$ & $\mathrm{HV}$ & $\mathrm{BL}$ & 3 & 577.4435 & 655.1716 & 637.9895 & 119.1038 & 119.6552 & 118.1671 & 117.426 & 100.0554 & 1.3041 & $5.3 \%$ & 3129.55 & 3.8668 & 0.6722 & 88.4672 & 2.3589 \\
\hline & & (1) 32,28 , & 29 & & & 5790334 & 6567516 & & & & & & & & & & & & & \\
\hline$S G$ & $25 \%$ & $\frac{0 \%}{1(1) 32.28}$ & $\mathrm{HV}$ & $\mathrm{BL}$ & 3 & 579.0334 & 656.7516 & 639.6228 & 118.9705 & 119.5212 & 118.0366 & $\begin{array}{l}117.297 \\
\end{array}$ & 100.0554 & 1.3029 & $5.3 \%$ & 3131.69 & 3.8492 & 0.6715 & 88.4676 & 2.1235 \\
\hline SG & $25 \%$ & $-50 \%$ & $\mathrm{HV}$ & BL & 4 & 588.6396 & 667.0585 & \begin{tabular}{|l}
649.8389 \\
\end{tabular} & 119.3703 & 1119.9216 & 118.4242 & 117.6801 & 100.0553 & 13072 & $54 \%$ & 3211.77 & 3.8093 & 0.6749 & 884658 & 05874 \\
\hline & & (1) 32,28 & 29 & & & & & & & & & & & & & & & & & 0.5814 \\
\hline SG & $25 \%$ & $-100 \%$ & HV & $\mathrm{BL}$ & 4 & 592.5017 & 671.6892 & 654.5498 & 119.1057 & 119.6124 & 118.1285 & 117.3878 & 100.0554 & 1.3245 & $5.4 \%$ & 3226.28 & 3.8167 & 0.6809 & 88.4608 & -0.1027 \\
\hline & $\mathrm{V}$ & (1) 32,29 & 30 & & & & & & & & & & & & & & & & & \\
\hline
\end{tabular}

\begin{tabular}{|c|c|c|c|c|c|c|c|}
\hline \multicolumn{8}{|c|}{ Improvement } \\
\hline $\begin{array}{c}\text { Machine } \\
\text { Type }\end{array}$ & $\begin{array}{l}\text { Real } \\
\text { Power }\end{array}$ & $\begin{array}{l}\text { Reactive } \\
\text { Power }\end{array}$ & HV / LV & Location & $\begin{array}{l}\text { Lowest } \\
\text { Voltage }\end{array}$ & Losses & \begin{tabular}{|l|} 
Node 1 \\
Capacity
\end{tabular} \\
\hline$S G$ & $50 \%$ & 0 & $\mathrm{HV}$ & $\mathrm{BL}$ & $-0.45 \%$ & $0.08 \%$ & $3.70 \%$ \\
\hline & & 32,28 & & & & & \\
\hline$S G$ & $75 \%$ & 0 & $\mathrm{HV}$ & $\mathrm{BL}$ & $-0.43 \%$ & $0.11 \%$ & $5.06 \%$ \\
\hline$S G$ & $\frac{\mathrm{V}}{100 \%}$ & (1) 32,28 & 29 & $\mathrm{Bl}$ & & & $642 \%$ \\
\hline & & & 29 & & $-0.40 \%$ & $0.13 \%$ & $6.42 \%$ \\
\hline$\overline{S G}$ & $106.7 \%$ & 0 & $\mathrm{HV}$ & $\mathrm{BL}$ & $-0.40 \%$ & $0.13 \%$ & $6.79 \%$ \\
\hline & V & (1) 32,28 , & 29 & & & & \\
\hline SG & $100 \%$ & $100 \%$ & HV & $\mathrm{BL}$ & $0.04 \%$ & $0.14 \%$ & $7.36 \%$ \\
\hline & & (1) 32,28 & 29 & & & & \\
\hline SG & $100 \%$ & $-100 \%$ & $\mathrm{HV}$ & $\begin{array}{ll}\mathrm{BL} \\
\end{array}$ & $-0.33 \%$ & $0.08 \%$ & $4.11 \%$ \\
\hline & & (1) 32,29 & 30 & & & & \\
\hline$S G$ & $25 \%$ & $100 \%$ & $\mathrm{HV}$ & $\mathrm{BL}$ & $-0.03 \%$ & $0.08 \%$ & $3.21 \%$ \\
\hline & & 1) 32,28 , & & & & & \\
\hline$S G$ & $25 \%$ & $75 \%$ & $\mathrm{HV}$ & $\mathrm{BL}$ & $-0.14 \%$ & $0.07 \%$ & $3.01 \%$ \\
\hline & & 1) 32,28 & & & & & \\
\hline$S G$ & $25 \%$ & $50 \%$ & $\mathrm{HV}$ & $\mathrm{BL}$ & $-0.25 \%$ & $0.07 \%$ & $2.79 \%$ \\
\hline & & (1) 32,28 & 29 & & & & \\
\hline$S G$ & $25 \%$ & $25 \%$ & $\mathrm{HV}$ & $\mathrm{BL}$ & $-0.36 \%$ & $0.07 \%$ & $2.57 \%$ \\
\hline & & & 29 & & & & \\
\hline$S G$ & $25 \%$ & $0 \%$ & HV & $\mathrm{BL}$ & $-0.47 \%$ & $0.06 \%$ & $2.33 \%$ \\
\hline & & & & & & & \\
\hline$S G$ & $25 \%$ & $-50 \%$ & $\mathrm{HV}$ & $\overline{B L}$ & $-0.15 \%$ & $0.03 \%$ & $0.79 \%$ \\
\hline & $\mathrm{V}$ & (1) 32,28, & 29 & & & & \\
\hline$S G$ & $25 \%$ & \begin{tabular}{|l|}
$-100 \%$ \\
(1) 32,29
\end{tabular} & \begin{tabular}{|l|} 
HV \\
30
\end{tabular} & $B L$ & $-0.40 \%$ & $0.02 \%$ & $0.10 \%$ \\
\hline
\end{tabular}


Table 47. Synchronous Generator Voltage Regulation Application (EL, HV) - HL

\begin{tabular}{|c|c|c|c|c|c|c|c|c|c|c|c|c|c|c|c|c|c|c|c|c|}
\hline \multirow[b]{2}{*}{$\begin{array}{l}\text { Machine } \\
\text { Type }\end{array}$} & \multirow[b]{2}{*}{$\begin{array}{l}\text { Real } \\
\text { Power }\end{array}$} & \multirow[b]{2}{*}{\begin{tabular}{|c|} 
Reactive \\
Power
\end{tabular}} & \multirow[b]{2}{*}{ HV / LV } & \multirow[b]{2}{*}{ Location } & \multirow[b]{2}{*}{ LTC Tap } & \multicolumn{3}{|c|}{ Node 01} & \multicolumn{3}{|c|}{ Lowest $3 \Phi$ Voltage } & \multirow{2}{*}{$\begin{array}{c}\text { Lowest } \\
1 \Phi \\
\text { Voltage } \\
\end{array}$} & \multirow[b]{2}{*}{$\begin{array}{l}\text { Highest } \\
12 / 11 \% \\
\end{array}$} & \multirow[b]{2}{*}{$\begin{array}{l}\text { Highest } \\
\text { V2/V1 }\end{array}$} & \multirow[b]{2}{*}{$\begin{array}{l}\% \text { kW } \\
\text { Loss }\end{array}$} & \multirow[b]{2}{*}{$\begin{array}{c}\text { Total } \\
\text { kVar Loss }\end{array}$} & \multicolumn{3}{|c|}{$2 / 11 \%$} & \multirow[b]{2}{*}{$\begin{array}{l}\text { Node } 1 \\
\text { Capacity }\end{array}$} \\
\hline & & & & & & IA & IB & IC & VA & VB & vc & & & & & & Node 0 & Low & $\begin{array}{l}\text { High } \\
(<100)\end{array}$ & \\
\hline$S G$ & $50 \%$ & 0 & $\mathrm{HV}$ & $E L$ & 3 & 570.0817 & 647.3645 & 630.642 & 119.2577 & 119.742 & 118.2744 & 117.532 & 100.0553 & 1.2618 & $5.1 \%$ & 2973.1 & 3.8604 & 0.6697 & 88.4628 & 3.5224 \\
\hline & & 1) 32,28 , & 29 & & & & & & & & & & & & & & & & & \\
\hline SG & $75 \%$ & & $\mathrm{HV}$ & EL & 3 & 561.0321 & 638.1117 & 621.5711 & 119.4095 & 119.8616 & 118.401 & 117.6572 & 100.0552 & 1.2525 & $5.0 \%$ & 2875.42 & 3.8957 & 0.6689 & 88.4604 & 4.9014 \\
\hline SG & $100 \%$ & $\frac{32,2}{0}$ & $\mathrm{HV}$ & EL & 3 & 552.0319 & 628.8905 & 612.534 & 119.5571 & 119.9779 & 118.5236 & \begin{tabular}{|l|l|}
117.7784 \\
\end{tabular} & 100.0552 & 1.2434 & $4.9 \%$ & 2781.08 & 3.9317 & 0.6683 & 38.4579 & 6.2756 \\
\hline & & 32,28, & & & & & & & & & & & & & & & & & & \\
\hline SG & $106.7 \%$ & 0 & $\mathrm{HV}$ & $E L$ & 3 & 549.6257 & 626.4219 & 610.115 & 119.596 & 120.0085 & 118.5558 & 117.8102 & 100.0551 & 1.241 & $4.9 \%$ & 2756.33 & 3.9415 & 0.6681 & 88.4573 & 6.6435 \\
\hline & & (1) 32,28 , & & & & & & & & & & & & & & & & & & \\
\hline SG & $100 \%$ & $\frac{100 \%}{(1) 3128}$ & $\mathrm{HV}$ & EL & 3 & 553.2643 & 632.3135 & 615.4063 & 120.6019 & 120.9582 & 119.5848 & 118.8273 & 100.0547 & 1.2115 & $4.8 \%$ & 2797.7 & 4.0748 & 0.6528 & 94.3303 & 5.7655 \\
\hline & & (1) 31,28 , & $\mathrm{HV}$ & $E \mathrm{EL}$ & 4 & 558,1387 & 634.894 & 618.1221 & 119.1788 & 1195887 & 118.0735 & 1173335 & 100.0554 & 12939 & $50 \%$ & 286719 & 38478 & 06961 & 884414 & \\
\hline SG & $100 \%$ & 1) 32,29 & & & & & & 010.1242 & 715.1100 & 115.0001 & 100.0700 & 111.0000 & 10.0034 & 1.2505 & $0.07 /$ & 2007.11 & 0.0410 & 0.0001 & 00.4414 & 5.3809 \\
\hline SG & $25 \%$ & $100 \%$ & $\mathrm{HV}$ & $E L$ & 3 & 581.5285 & 660.3106 & 643.6109 & 120.1129 & 120.6085 & 119.1723 & 118.4197 & 100.0549 & 1.2474 & $5.2 \%$ & 3101.21 & 3.9285 & 0.6554 & 88.473 & 1.593 \\
\hline & & (1) 32,28 , & & & & & & & & & & & & & & & & & & \\
\hline SG & $25 \%$ & $75 \%$ & $\mathrm{HV}$ & $E L$ & 3 & 580.7804 & 659.2589 & 642.5056 & 119.8622 & 120.3626 & 118.9172 & 118.1675 & 100.055 & 1.2532 & $5.2 \%$ & 3091.66 & 3.9041 & 0.6591 & 88.4711 & 1.7498 \\
\hline & & 32,28 & & & & & & & & & & & & & & & & & & \\
\hline$S G$ & $25 \%$ & $50 \%$ & $\mathrm{HV}$ & $E \mathrm{EL}$ & 3 & 580.1452 & 658.3071 & 641.5027 & 119.6129 & 120.1184 & 118.6635 & 117.9167 & 100.0551 & 1.259 & $5.2 \%$ & 3084.02 & 3.879 & 0.6629 & 88.4692 & 1.8916 \\
\hline & & & & & & & & & & & & & & & & & & & & \\
\hline SG & $25 \%$ & $25 \%$ & HV & $E L$ & 3 & 579.605 & 657.4265 & 640.572 & 119.3567 & 119.8678 & 118.4028 & \begin{tabular}{|l|l}
117.659 \\
\end{tabular} & 100.0552 & 1.2651 & $5.2 \%$ & 3078.1 & 3.8526 & 0.6667 & 88.4673 & 2.0229 \\
\hline SG & $25 \%$ & (1) 32,28 , & $\frac{29}{\mathrm{HV}}$ & $\overline{E L}$ & 3 & 579.1803 & 656.6483 & 639.7466 & 119.1019 & 119.6189 & 118.1435 & 117.4027 & 100.0554 & 1.2734 & $5.2 \%$ & 3074.16 & 38256 & 0.6705 & 88.4653 & 21389 \\
\hline & 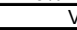 & (1) 32,28 & 9 & & & & & & & & & & & & & & & & & 2.1000 \\
\hline SG & $25 \%$ & $-50 \%$ & $\mathrm{HV}$ & $E L$ & 4 & 584.876 & 663.1896 & 645.9419 & 119.2565 & 119.7462 & 118.2349 & 117.493 & 100.0554 & 1.3302 & $5.3 \%$ & 3152.87 & 3.8112 & 0.6902 & 88.4529 & 1.164 \\
\hline & $\frac{V}{250}$ & (1) 32,29 , & $0_{\mathrm{H}}^{0}$ & $\mathrm{FI}$ & 4 & 5848512 & 6 & 7803 & 7268 & 1192323 & 1176578 & 9226 & 0556 & 13404 & $5.4 \%$ & 6199 & 3768 & 07057 & 88442 & \\
\hline & 2070 & & IIV & 드 & 4 & 584.8512 & 602.3715 & .8003 & 7268 & $\angle 3<3$ & & & & 1.3404 & $5.4 \%$ & 61.99 & 3.168 & $0.105 /$ & 88.442 & . \\
\hline
\end{tabular}

\begin{tabular}{|c|c|c|c|c|c|c|c|}
\hline \multicolumn{8}{|c|}{ Improvement } \\
\hline $\begin{array}{l}\text { Machine } \\
\text { Type }\end{array}$ & $\begin{array}{l}\text { Real } \\
\text { Power }\end{array}$ & $\begin{array}{l}\text { Reactive } \\
\text { Power }\end{array}$ & HV / LV & Location & $\begin{array}{l}\text { Lowest } \\
\text { Voltage }\end{array}$ & Losses & $\begin{array}{l}\text { Node 1 } \\
\text { Capacity }\end{array}$ \\
\hline SG & $50 \%$ & 0 & $\mathrm{HV}$ & $E L$ & $-0.27 \%$ & $0.28 \%$ & $3.73 \%$ \\
\hline & & & & & & & \\
\hline$S G$ & $75 \%$ & 0 & $\mathrm{HV}$ & EL & $-0.17 \%$ & $0.39 \%$ & $5.11 \%$ \\
\hline & $\frac{v}{100 \%}$ & (1) $32,2 \varepsilon$ & 29 & & & & \\
\hline SG & $100 \%$ & $\frac{0}{(1) 32,2 \varepsilon}$ & HV & EL & $-0.06 \%$ & $0.50 \%$ & $6.48 \%$ \\
\hline$S G$ & $106.7 \%$ & 0 & $\mathrm{HV}$ & EL & $-0.04 \%$ & $0.53 \%$ & $6.85 \%$ \\
\hline & & & 29 & & & & \\
\hline SG & $100 \%$ & $100 \%$ & $\mathrm{HV}$ & $E L$ & $0.82 \%$ & $0.56 \%$ & $5.97 \%$ \\
\hline & & (1) 31,28 & 28 & & & & \\
\hline SG & $100 \%$ & $\frac{-100 \%}{(1+32 x}$ & $\mathrm{HV}$ & EL & $-0.44 \%$ & $0.37 \%$ & $5.59 \%$ \\
\hline$S G$ & $25 \%$ & $100 \%$ & HV & EL & $0.48 \%$ & $0.22 \%$ & $180 \%$ \\
\hline & $\mathrm{v}$ & (1) $32,2 \varepsilon$ & & & & & 1.000 \\
\hline SG & $25 \%$ & $75 \%$ & $\mathrm{HV}$ & EL & $0.27 \%$ & $0.21 \%$ & $1.96 \%$ \\
\hline & & & & & & & \\
\hline$S G$ & $25 \%$ & $50 \%$ & $\mathrm{HV}$ & EL & $0.05 \%$ & $0.20 \%$ & $2.10 \%$ \\
\hline & & & & & & & \\
\hline SG & $25 \%$ & $25 \%$ & $\mathrm{HV}$ & EL & $-0.17 \%$ & $0.18 \%$ & $2.23 \%$ \\
\hline & & 1) $32,2 \varepsilon$ & & & & & \\
\hline SG & $25 \%$ & $0 \%$ & $\mathrm{HV}$ & EL & $-0.38 \%$ & $0.16 \%$ & $2.35 \%$ \\
\hline & & 32 & & & & & \\
\hline$S G$ & $25 \%$ & $-50 \%$ & $\mathrm{HV}$ & EL & $-0.31 \%$ & $0.09 \%$ & $1.37 \%$ \\
\hline$\overline{S G}$ & $25 \%$ & & $\mathrm{HV}$ & $\mathrm{EL}$ & $-0.79 \%$ & $0.03 \%$ & $1.50 \%$ \\
\hline & 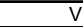 & & & & & & \\
\hline
\end{tabular}


Table 48. Synchronous Generator Voltage Regulation Application (ML, HV) - HL

\begin{tabular}{|c|c|c|c|c|c|c|c|c|c|c|c|c|c|c|c|c|c|c|c|c|}
\hline \multirow{2}{*}{$\begin{array}{c}\text { Machine } \\
\text { Type }\end{array}$} & \multirow{2}{*}{$\begin{array}{l}\text { Real } \\
\text { Power }\end{array}$} & \multirow[b]{2}{*}{$\begin{array}{l}\text { Reactive } \\
\text { Power }\end{array}$} & \multirow[b]{2}{*}{ HV /LV } & \multirow[b]{2}{*}{ Location } & \multirow{2}{*}{ LTC Tap } & \multicolumn{3}{|c|}{$\begin{array}{l}\text { Node } 01 \\
\end{array}$} & \multicolumn{3}{|c|}{ Lowest $3 \Phi$ Voltage } & \multirow{3}{*}{$\begin{array}{l}\text { Lowest } \\
1 \Phi \\
\text { voltage } \\
118.1577\end{array}$} & \multirow{2}{*}{$\begin{array}{l}\text { Highest } \\
\text { |2/11\% }\end{array}$} & \multirow{2}{*}{$\begin{array}{l}\text { Highest } \\
\text { V2N1 \% }\end{array}$} & \multirow[b]{2}{*}{$\begin{array}{c}\% \mathrm{~kW} \\
\text { Loss }\end{array}$} & \multirow{2}{*}{$\begin{array}{c}\text { Total } \\
\text { KVar Loss }\end{array}$} & \multicolumn{3}{|c|}{$12 / 1 \% \%$} & \multirow{2}{*}{\begin{tabular}{|c|} 
Node 1 \\
Capacity \\
\end{tabular}} \\
\hline & & & & & & IA & IB & IC & VA & VB & vc & & & & & & Node 0 & Low & $\begin{array}{l}\text { High } \\
(<100)\end{array}$ & \\
\hline & $50 \%$ & 0 & $\mathrm{HV}$ & $\mathrm{ML}$ & 4 & 576.1912 & 654.2454 & 637.2163 & 119.9124 & 120.3898 & 118.9073 & & & & & & 3.8547 & 0.6735 & 88.4599 & $\begin{array}{l}.497 \\
\end{array}$ \\
\hline & & (1) 32,28, & 29 & & & & & & & & & & & & & & & & & \\
\hline SG & $75 \%$ & 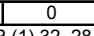 & HV & ML & 4 & 567.1353 & 645.0019 & 628.1048 & 120.0596 & 120.5 & 119.0274 & 118.2764 & 100.055 & 1.2599 & $5.1 \%$ & 2970.4 & 3.8887 & 0.6722 & 88.4574 & 3.8745 \\
\hline & $100 \%$ & (1) 32,28 & 29 & $M I$ & 3 & 5517973 & 6286983 & 6121472 & 1195362 & 119.0369 & 1184938 & & 100052 & & & & & & & \\
\hline 56 & 000 & $\frac{0}{R(1) 32,28}$ & $\frac{1}{29} \mathrm{HV}$ & IVL & 3 & $551.19 / 3$ & 628.6983 & 612.1442 & 179.5302 & $T 19.9309$ & 118.4938 & 117.7489 & 100.0552 & 1.2387 & $5.0 \%$ & 2808.4 & 3.9286 & 0.6662 & 88.4572 & 6.3043 \\
\hline SG & $106.7 \%$ & 0 & $\mathrm{HV}$ & $\mathrm{ML}$ & 3 & 549.37 & 626.2141 & 609.6999 & \begin{tabular}{|l|}
119.5748 \\
\end{tabular} & 119.9656 & 118.525 & 117.7798 & 100.0551 & 1.236 & $4.9 \%$ & 2784.82 & 3.9382 & 0.6659 & 88.4565 & 6.6745 \\
\hline & $1100 \%$ & $\begin{array}{l}2(1) 32,28 \\
100 \%\end{array}$ & 29 & & & & & & & & 1195915 & & & & & & & & & \\
\hline SG & $100 \%$ & $\frac{100 \%}{3(1)}$ & HV & ML & 3 & $551.66 \mathrm{6t}$ & 630.5463 & 613.5866 & $120.61 / 6$ & 120.9517 & 119.5915 & 118.8339 & 100.0547 & 1.231 & $4.9 \%$ & 2817.49 & 4.0656 & 0.6485 & 88.466 & 6.0289 \\
\hline SG & $100 \%$ & $-100 \%$ & $\mathrm{HV}$ & $\mathrm{ML}$ & 4 & 559.1494 & 636.2131 & 619.1058 & 119.1376 & 119.526 & 118.0228 & 117.2833 & 100.0554 & 1.3092 & $5.1 \%$ & 2891.46 & 3.8543 & 0.696 & 88.4403 & 5.1843 \\
\hline & & रे (1) 32,29 & 30 & & & & & & & & & & & & & & & & & 5.1843 \\
\hline SG & $25 \%$ & $100 \%$ & $\mathrm{HV}$ & $\mathrm{ML}$ & 3 & 580.1401 & 658.7236 & 642.1133 & 120.143 & 120.6317 & 119.2001 & 118.4472 & 100.0549 & 1.2438 & $5.2 \%$ & 3101 & 3.9212 & 0.6527 & 88.4733 & 1.8296 \\
\hline & & र (1) 32,28 & 29 & & & & & & & & & & & & & & & & & \\
\hline SG & $25 \%$ & $75 \%$ & $\mathrm{HV}$ & $\mathrm{ML}$ & 3 & 579.7367 & 658.0634 & 641.3639 & 119.8813 & 120.3756 & 118.9341 & 118.1842 & 100.055 & 1.2525 & $5.2 \%$ & 3094.6 & 3.898 & 0.657 & 88.4713 & 1.928 \\
\hline & & $\frac{R(1) 32,28}{50 \%}$ & 29 & & & & & & & & & & & & & & & & & \\
\hline SG & $25 \%$ & $50 \%$ & HV & ML & 3 & 579.4374 & 657.4961 & 640.7111 & 119.6221 & 120.1221 & 118.6705 & 117.9236 & 100.0551 & 1.2632 & $5.2 \%$ & 3089.47 & 3.8745 & 0.6613 & 88.4693 & 2.0125 \\
\hline$S G$ & $25 \%$ & $\begin{array}{l}\text { (1) } 32,28 \\
25 \% \\
\end{array}$ & $\begin{array}{ll}29 \\
\end{array}$ & ML & 3 & 579.2344 & 657.005 & 640.1354 & 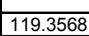 & 119.8629 & 118.4008 & 117.657 & 100.0552 & 1.2744 & $52 \%$ & 308546 & 38498 & 0.6656 & 88.4672 & 20857 \\
\hline & & र(1) 32,28 & 29 & & & & & & & & & & & & & & & & & 2.0031 \\
\hline$S G$ & $25 \%$ & $0 \%$ & HV & $\mathrm{ML}$ & 4 & 585.2859 & 663.5147 & 646.3563 & 119.7628 & 120.2777 & 118.7848 & 118.0366 & 100.0551 & 1.2926 & $5.3 \%$ & 3156.84 & 3.8214 & 0.6748 & 88.4625 & 1.1155 \\
\hline & & $\begin{array}{r}2(1) 32,28 \\
-50 \%\end{array}$ & 29 & & & & & & & & & & & & & & & & & \\
\hline SG & $25 \%$ & & HV & ML & 4 & 585.4652 & 663.9017 & 646.5384 & 119.2355 & 119.7207 & 118.2116 & 117.47 & 100.0554 & 1.3345 & $5.3 \%$ & 3161.93 & 3.8146 & 0.6908 & 88.4525 & 1.0579 \\
\hline$S G$ & $25 \%$ & $\begin{array}{l}2 \text { (1) } 32,29 \\
-100 \%\end{array}$ & 30 & $\mathrm{ML}$ & 4 & 586.0652 & $\begin{array}{l}664.643 \\
\end{array}$ & 647.0848 & 118.7006 & 119.1573 & 117.6306 & 116.8956 & 100.0556 & 1.3769 & $5.4 \%$ & 3172.1 & 3.8039 & 0.7068 & 88.4424 & 0.9474 \\
\hline & & (1) 32,30 & & & & & & & & & & & & & & & & & & . \\
\hline
\end{tabular}

\begin{tabular}{|c|c|c|c|c|c|c|c|}
\hline \multicolumn{8}{|c|}{ Improvement } \\
\hline $\begin{array}{l}\text { Machine } \\
\text { Type }\end{array}$ & $\begin{array}{l}\text { Real } \\
\text { Power }\end{array}$ & $\begin{array}{l}\text { Reactive } \\
\text { Power }\end{array}$ & HV /LV & Location & $\begin{array}{l}\text { Lowest } \\
\text { Voltage }\end{array}$ & Losses & $\begin{array}{l}\text { Node } 1 \\
\text { Capacity }\end{array}$ \\
\hline SG & $50 \%$ & 0 & $\mathrm{HV}$ & ML & $0.26^{\circ}$ & $0.23 \%$ & \\
\hline SG & $75 \%$ & & $\mathrm{HV}$ & $\begin{array}{l}\mathrm{ML} \\
\end{array}$ & $0.36^{\circ}$ & & \\
\hline & & & & & & & \\
\hline SG & $100 \%$ & 0 & $\mathrm{HV}$ & $\overline{M L}$ & $-0.09 \%$ & $0.45 \%$ & 6.51 \\
\hline$S G$ & $106.7 \%$ & $\frac{724}{0}$ & $\mathrm{HV}$ & $\begin{array}{l}\mathrm{ML} \\
\end{array}$ & $-0.06^{\circ}$ & $0.48 \%$ & $\begin{array}{ll}6.8 \\
\end{array}$ \\
\hline & & 32,28 & & & & & \\
\hline SG & $100 \%$ & & $\mathrm{HV}$ & $\mathrm{ML}$ & $0.83^{\circ}$ & $0.51 \%$ & 6.24 \\
\hline SG & $100 \%$ & (1) 31, & HV & $\overline{M L}$ & $-0.49^{\circ}$ & $0.34 \%$ & 5.36 \\
\hline & & (1) 32,29 & & & & & \\
\hline$S G$ & $25 \%$ & & $\mathrm{HV}$ & $\mathrm{ML}$ & $0.50^{\circ}$ & $0.20 \%$ & 2.04 \\
\hline SG & $25 \%$ & (1) 75 & $\mathrm{HV}$ & $\overline{\mathrm{ML}}$ & $0.28 \%$ & $0.19 \%$ & 2.13 \\
\hline & $\mathrm{v}$ & (1) 32 & & & & & \\
\hline SG & $25 \%$ & 5 & $\mathrm{HV}$ & $\mathrm{ML}$ & $0.06^{\circ}$ & $0.18 \%$ & 2.22 \\
\hline SG & $25 \%$ & 25 & $\mathrm{HV}$ & $\overline{M L}$ & $-0.17^{\circ}$ & $0.16 \%$ & 2.29 \\
\hline & & 12328 & & & & & \\
\hline$S G$ & $25 \%$ & $0 \%$ & $\mathrm{HV}$ & $\mathrm{ML}$ & $0.15^{\circ}$ & $0.13 \%$ & $1.32 \%$ \\
\hline SG & $25 \%$ & $-50 \%$ & $\mathrm{HV}$ & $\overline{M L}$ & $-0.33^{\circ}$ & $0.08 \%$ & $1.26^{\circ}$ \\
\hline 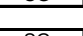 & 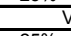 & 1) 32,29 & & & & & \\
\hline SG & $25 \%$ & & $\mathrm{HV}$ & $\mathrm{ML}$ & $-0.81^{\circ}$ & $0.03 \%$ & $1.15^{\circ}$ \\
\hline
\end{tabular}


Table 49. Synchronous Generator Voltage Regulation Application (BL, LV) - HL

\begin{tabular}{|c|c|c|c|c|c|c|c|c|c|c|c|c|c|c|c|c|c|c|c|c|}
\hline \multirow[b]{2}{*}{$\begin{array}{l}\text { Thachine } \\
\text { Type }\end{array}$} & \multirow[b]{2}{*}{$\begin{array}{l}\text { Real } \\
\text { Power }\end{array}$} & \multirow[b]{2}{*}{$\begin{array}{l}\text { Reactive } \\
\text { Power }\end{array}$} & \multirow[b]{2}{*}{ IILV } & \multirow[b]{2}{*}{ Location } & \multirow[b]{2}{*}{ LTC Tap } & \multicolumn{3}{|c|}{ Node 01} & \multicolumn{3}{|c|}{ Lowest $3 \Phi$ Voltage } & \multirow{2}{*}{$\begin{array}{c}\text { Lowest } \\
1 \Phi \\
\text { Voltage }\end{array}$} & \multirow[b]{2}{*}{$\begin{array}{c}\text { Highest } \\
12 / 11 \%\end{array}$} & \multirow[b]{2}{*}{$\begin{array}{l}\text { Highest } \\
\text { V2/V1 \% }\end{array}$} & \multirow[b]{2}{*}{$\begin{array}{l}\% \mathrm{~kW} \\
\text { Loss }\end{array}$} & \multirow[b]{2}{*}{$\begin{array}{c}\text { Total } \\
\text { kVar Loss }\end{array}$} & \multicolumn{3}{|c|}{ |2/11\% } & \multirow[b]{2}{*}{$\begin{array}{l}\text { Node 1 } \\
\text { Capacity }\end{array}$} \\
\hline & & & & & & IA & IB & IC & VA & VB & vc & & & & & & Node 0 & Low & $\begin{array}{l}\text { High } \\
(<100)\end{array}$ & \\
\hline SG & $50 \%$ & 0 & LV & $\mathrm{BL}$ & 16 & 548.6123 & 626.86 & 609.0336 & 116.7192 & \begin{tabular}{|l|l}
117.0647 \\
\end{tabular} & 115.6745 & 114.9617 & 100.0564 & 1.3905 & $5.3 \%$ & 2996.76 & 4.0662 & 0.7018 & 88.4496 & 6.5782 \\
\hline & & 32,32 & & & & & & & & & & & & & & & & & & \\
\hline SG & $75 \%$ & 0 & LV & $\mathrm{BL}$ & 16 & 539.1941 & 617.5306 & 599.6492 & 116.7506 & 117.0963 & 115.7052 & 114.9921 & 100.0563 & 1.3908 & $5.3 \%$ & 2948.95 & 4.1333 & 0.702 & 88.4495 & 7.9686 \\
\hline & $100 \%$ & 8 & IV & $B$ & 16 & 98083 & 82319 & 0297 & 67817 & 1274 & 57356 & & & & & 0208 & 2024 & & & \\
\hline 30 & $100 \%$ & 0 & LV & & & 9.8083 & 8.2319 & & 6.1817 & & 115.7356 & .0221 & 0.0563 & 3911 & $3 \%$ & 2902.08 & .2024 & 0.0031 & 3.4494 & 9.3544 \\
\hline SG & $106.7 \%$ & 0 & $\mathrm{LV}$ & $\overline{B L}$ & 16 & 527.2958 & 605.7425 & 587.7935 & 116.79 & 117.1357 & 115.7437 & 115.0301 & 100.0563 & 1.3912 & $5.3 \%$ & 2889.65 & 4.2212 & 0.0031 & 88.4494 & 9.7254 \\
\hline & & 32,32 & 32 & & & & & & & & & & & & & & & & & \\
\hline$S G$ & $100 \%$ & $100 \%$ & LV & $\overline{B L}$ & 16 & 523.8771 & 602.3187 & 584.1404 & 117.3644 & 7.7121 & 116.3049 & 115.585 & 100.0561 & 1.3965 & $5.3 \%$ & 2897.59 & 4.2846 & 0.0031 & 88.4476 & 10.2357 \\
\hline & & (1) 32,32 , & 32 & & & & & & & & & & & & & & & & & \\
\hline$S G$ & $100 \%$ & $-100 \%$ & LV & $B L$ & 16 & 537.3505 & 615.5354 & 597.8991 & 116.1926 & 116.5363 & 115.1599 & 4.453 & 100.0566 & 1.3855 & $5.3 \%$ & 2914.53 & 4.111 & 0.0031 & 88.4514 & 8.266 \\
\hline & & (1) 32,32, & & & & & & & & & & & & & & & & & & \\
\hline SG & $25 \%$ & $100 \%$ & LV & $\mathrm{BL}$ & 16 & 552.5664 & 630.6959 & 612.7041 & 117.2703 & 117.6177 & 116.213 & 115.4941 & 100.0561 & 1.3957 & $5.3 \%$ & 3041.67 & 4.0744 & 0.0031 & 88.4479 & 6.0066 \\
\hline & & 1) 32,32 , & & & & & & & & & & & & & & & & & & \\
\hline SG & $25 \%$ & $75 \%$ & $\mathrm{LV}$ & $\overline{B L}$ & 16 & 553.8063 & 631.9622 & 614.0214 & 117.124 & 117.4709 & 116.07 & 115.3527 & 100.0562 & 1.3943 & $5.3 \%$ & 3041.9 & 4.0567 & 0.7039 & 88.4483 & 5.8179 \\
\hline & & & & & & & & & & & & & & & & & & & & \\
\hline$S G$ & $25 \%$ & $50 \%$ & $\mathrm{LV}$ & $\mathrm{BL}$ & 16 & 555.1215 & 633.2907 & 615.4032 & 116.9796 & 17.326 & 115.929 & 115.2133 & 100.0563 & 1.393 & $5.3 \%$ & 3042.61 & 4.038 & 0.7032 & 88.4488 & 6199 \\
\hline & & (1) 32,32 , & 32 & & & & & & & & & & & & & & & & & \\
\hline$S G$ & $25 \%$ & $25 \%$ & LV & $B \mathrm{BL}$ & 16 & 556.5554 & 634.725 & 616.8951 & 116.8325 & 1117.1784 & 115.7852 & 115.0712 & 100.0563 & 1.3916 & $5.3 \%$ & 3043.81 & 4.0199 & 0.7024 & 88.4493 & 5.4061 \\
\hline & & 1) 32,32 , & 32 & & & & & & & & & & & & & & 10009 & & 884497 & \\
\hline 56 & $25 \%$ & $\frac{0 \%}{32,32}$ & $\begin{array}{l}\text { LV } \\
32\end{array}$ & BL & 16 & 558.0616 & 636.2191 & 618.449 & 116.6874 & 117.0328 & 115.6434 & 114.931 & 100.0564 & 1.3902 & $5.4 \%$ & 3045.5 & 4.0009 & 0.7016 & 88.4497 & 5.1834 \\
\hline SG & $25 \%$ & $-50 \%$ & $\mathrm{LV}$ & $\mathrm{BL}$ & 16 & 561.387 & 639.4819 & 621.8422 & 116.3935 & 116.7379 & 115.3562 & 114.6471 & 100.0565 & 1.3875 & $5.4 \%$ & 3050.38 & 3.961 & 0.7 & 88.4507 & 4.6972 \\
\hline SG & $\frac{V}{25 \%}$ & $\frac{1) 32,32}{-100 \%}$ & LV & $\mathrm{BL}$ & 16 & 565.0973 & 643.0796 & 625.5829 & 6.098 & & 115.0675 & 114.3616 & 100.0566 & 1.3847 & & 3057.28 & 3.9192 & 0.0031 & 88.4517 & \\
\hline 30 & $20 \%$ & $-100 \%$ & $L V$ & $B L$ & & & & & & 116.4414 & $715.06 / 5$ & 114.3616 & 100.0566 & 1.3847 & $5.4 \%$ & 3057.28 & 3.9192 & 0.0031 & $88.451 /$ & \\
\hline
\end{tabular}

\begin{tabular}{|c|c|c|c|c|c|c|c|}
\hline \multicolumn{8}{|c|}{ Improvement } \\
\hline $\begin{array}{l}\text { Machine } \\
\text { Type }\end{array}$ & $\begin{array}{l}\text { Real } \\
\text { Power }\end{array}$ & $\begin{array}{l}\text { Reactive } \\
\text { Power }\end{array}$ & HV /LV & Location & $\begin{array}{l}\text { Lowest } \\
\text { Voltage }\end{array}$ & Losses & $\begin{array}{l}\text { Node } 1 \\
\text { Capacity }\end{array}$ \\
\hline$S G$ & $50 \%$ & 0 & $\mathrm{LV}$ & $\mathrm{BL}$ & $-2.45 \%$ & $0.07 \%$ & $6.78 \%$ \\
\hline & & & & & & & \\
\hline SG & $75 \%$ & 0 & LV & $\mathrm{BL}$ & $-2.43 \%$ & $0.09 \%$ & $8.17 \%$ \\
\hline & 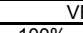 & $\begin{array}{r}32,3 \\
\end{array}$ & & & & & \\
\hline$S G$ & $100 \%$ & $\frac{0}{323}$ & $\mathrm{LV}$ & $B L$ & $-2.40 \%$ & $0.12 \%$ & $9.56 \%$ \\
\hline SG & $106.7 \%$ & $\frac{102,02}{0}$ & LV & BL & $-2.40 \%$ & $0.12 \%$ & $9.93 \%$ \\
\hline & & (1) 32,32 & 32 & & & & \\
\hline SG & $100 \%$ & $100 \%$ & LV & $\mathrm{BL}$ & $-1.93 \%$ & $0.13 \%$ & $10.44 \%$ \\
\hline & & (1) 32,32 & 32 & & & & \\
\hline SG & $100 \%$ & $-100 \%$ & LV & $\overline{B L}$ & $-2.89 \%$ & $0.10 \%$ & $8.47 \%$ \\
\hline & & (1) 32,32 & 32 & & & & \\
\hline SG & $25 \%$ & $100 \%$ & LV & $\mathrm{BL}$ & $-2.00 \%$ & $0.06 \%$ & $6.21 \%$ \\
\hline & 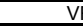 & & & & & & \\
\hline$S G$ & $25 \%$ & $75 \%$ & LV & $B L$ & $-2.12 \%$ & $0.06 \%$ & $6.02 \%$ \\
\hline SG & $25 \%$ & (1) 32,32 & LV & BL & $-2.24 \%$ & $0.05 \%$ & $5.83 \%$ \\
\hline & 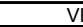 & & & & & & \\
\hline SG & $25 \%$ & $25 \%$ & LV & $\mathrm{BL}$ & $-2.36 \%$ & $0.05 \%$ & $5.61 \%$ \\
\hline & & (1) 32,32 & 32 & & & & \\
\hline$\overline{S G}$ & $25 \%$ & $0 \%$ & LV & $\mathrm{BL}$ & $-2.48 \%$ & $0.05 \%$ & $5.39 \%$ \\
\hline Se & $\frac{\mathrm{V}}{25 \%}$ & & 2 & D. & $272 \%$ & $004 \%$ & 10 \\
\hline & & & 32 & & & & \\
\hline$S G$ & $25 \%$ & $-100 \%$ & $\mathrm{LV}$ & $\mathrm{BL}$ & $-2.96 \%$ & $0.02 \%$ & $4.37 \%$ \\
\hline & & (1) 32,32 & & & & & \\
\hline
\end{tabular}


Table 50. Synchronous Generator Voltage Regulation Application (EL, LV) - HL

\begin{tabular}{|c|c|c|c|c|c|c|c|c|c|c|c|c|c|c|c|c|c|c|c|c|}
\hline \multirow{3}{*}{$\begin{array}{l}\text { Type } \\
\text { SG }\end{array}$} & \multirow[b]{2}{*}{ Power } & \multirow[b]{2}{*}{ Power } & \multirow{2}{*}{ HVILV } & \multicolumn{2}{|c|}{ 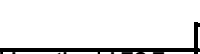 } & \multicolumn{3}{|c|}{ Node 01} & \multicolumn{3}{|c|}{ Lowest 3\$ Voltage } & \multirow{2}{*}{$\begin{array}{l}\text { Lowest } \\
1 \Phi\end{array}$} & \multirow{2}{*}{\begin{tabular}{|l} 
Highest \\
\end{tabular}} & \multirow{2}{*}{ Highest } & \multirow{2}{*}{$\begin{array}{c}\begin{array}{c}\% \text { kW } \\
\text { Loss }\end{array} \\
\end{array}$} & \multirow{2}{*}{ Total } & \multicolumn{3}{|c|}{$12 / 1 \%$} & \multirow[b]{2}{*}{ Capacity } \\
\hline & & & & Locatio & LTC Tap & IA & IB & IC & VA & VB & $\mathrm{VC}$ & & & & & & Node 0 & Low & (<100) & \\
\hline & $50 \%$ & 0 & $\mathrm{LV}$ & $\mathrm{EL}$ & 16 & 549.006 & \begin{tabular}{|l|}
626.7122 \\
\end{tabular} & 609.3697 & 116.9842 & \begin{tabular}{|l|}
117.2648 \\
\end{tabular} & 115.8915 & 115.1763 & 100.0562 & 1.3285 & $5.1 \%$ & \begin{tabular}{|l|}
2886.35 \\
\end{tabular} & 4.0128 & 0.7003 & 88.4449 & 6.6003 \\
\hline & & & & & & & & & & & & & & & & & & & & \\
\hline SG & $75 \%$ & 1) 32 & $\mathrm{LV}$ & EL & 16 & 539.8146 & 617.3121 & 600.1594 & 117.1419 & 117.3913 & 116.0245 & 115.3077 & 100.0561 & 1.2987 & $5.0 \%$ & 2787.26 & 4.0515 & 0.6999 & 88.4424 & 8.0012 \\
\hline SG & $100 \%$ & $\frac{32,}{0}$ & $\overline{L V}$ & $\overline{E L}$ & 16 & 530.6745 & 607.9443 & 590.9841 & 117.2951 & 117.5141 & 116.1531 & 115.4348 & 100.0561 & 1.2695 & $4.9 \%$ & 2691.66 & 4.0909 & 0.6994 & 88.44 & 93973 \\
\hline & & & & & & & & & & & & & & & & & & & & \\
\hline SG & $106.7 \%$ & 0 & LV & EL & 16 & 528.2311 & \begin{tabular}{|l|l|}
605.4366 \\
\end{tabular} & 588.5284 & 117.3354 & \begin{tabular}{|l|l|}
117.5464 \\
\end{tabular} & \begin{tabular}{|l|}
116.1868 \\
\end{tabular} & 115.4681 & 100.0561 & 1.2617 & $4.9 \%$ & 2666.6 & 4.1016 & 0.6993 & 88.4393 & 9.771 \\
\hline SG & $\begin{array}{r}\mathrm{V} \\
100 \%\end{array}$ & $\frac{(1) 32,32}{100 \%}$ & $\frac{32}{\mathrm{LVV}}$ & EL & 16 & 5332555 & 6119855 & 595217 & 1183551 & 118.5527 & 1172303 & 1164097 & 1000556 & 12174 & $48 \%$ & 271958 & 42068 & 0685 & 884481 & 8795 \\
\hline & $\mathrm{VF}$ & $\frac{10 \%}{(1) 32,32}$ & 32 & & & & & & & & & & & & & & & & & 8.195 \\
\hline$S G$ & $100 \%$ & $-100 \%$ & $\mathrm{LV}$ & $E L$ & 16 & \begin{tabular}{|l|}
530.07555 \\
\end{tabular} & \begin{tabular}{|l|l|}
605.5359 \\
\end{tabular} & 588.4268 & 116.1839 & 116.4322 & 115.0243 & 114.3189 & 100.0566 & 1.3493 & $5.0 \%$ & 2696.18 & 3.9554 & 0.7147 & 88.4312 & 9.7562 \\
\hline & $\mathrm{V}$ & (1) 32,32 & 32 & & & & & & & & & & & & & & & & & \\
\hline$S G$ & $25 \%$ & $100 \%$ & $\mathrm{LV}$ & EL & 16 & \begin{tabular}{|l|}
561.0616 \\
\end{tabular} & 640.3213 & 622.989 & 117.8818 & 118.1728 & 116.8302 & 116.1042 & 100.0558 & 1.3084 & $5.2 \%$ & 3021.61 & 4.0834 & 0.6866 & 88.4553 & 4.5721 \\
\hline & & 1) 32,32 & 32 & & & & & & & & & & & & & & & & & \\
\hline SG & $25 \%$ & $75 \%$ & $\mathrm{LV}$ & EL & 16 & 560.1873 & 639.133 & 621.7479 & 117.6192 & 117.915 & 116.5635 & 115.8405 & 100.056 & 1.32 & $5.2 \%$ & 3010.52 & 4.0577 & 0.6901 & 88.4534 & 4.7492 \\
\hline & $\mathrm{V}$ & 1) 32,32 & & & & & & & & & & & & & & & & & & \\
\hline SG & $25 \%$ & $\frac{50 \%}{11) 32,32}$ & LV & EL & 16 & 559.4335 & 638.051 & 620.6159 & 117.358 & 117.6588 & 116.2982 & 115.5783 & 100.0561 & 1.3322 & $5.2 \%$ & 3001.46 & 4.0314 & 0.6936 & 88.4514 & 4.9104 \\
\hline$S G$ & $25 \%$ & $25 \%$ & LV & $E L$ & 16 & 558.779 & 637.0428 & \begin{tabular}{|l|l|}
619.5591 \\
\end{tabular} & \begin{tabular}{|l|l}
117.0894 \\
\end{tabular} & \begin{tabular}{|l|l|}
117.3959 \\
\end{tabular} & \begin{tabular}{|l|l|}
116.0254 \\
\end{tabular} & \begin{tabular}{|l|l}
115.3086 \\
\end{tabular} & \begin{tabular}{|l|l|}
100662 \\
\end{tabular} & 1.3453 & $5.2 \%$ & 2994.18 & 4.0034 & 0.6973 & 88.4494 & 5.0607 \\
\hline & $V_{1}$ & (1) 32,32 & 32 & & & & & & & & & & & & & & & & & \\
\hline$S G$ & $25 \%$ & $0 \%$ & LV & $E L$ & 16 & \begin{tabular}{|l|l}
558.2482 \\
\end{tabular} & \begin{tabular}{|l|l|}
636.1438 \\
\end{tabular} & 618.6145 & 116.822 & \begin{tabular}{|l|l}
117.1345 \\
\end{tabular} & \begin{tabular}{|l|l|}
115.754 \\
\end{tabular} & 115.0403 & 100.0563 & 1.3589 & $5.2 \%$ & 2988.98 & 3.9748 & 0.7009 & 88.4474 & 5.1947 \\
\hline SG & $25 \%$ & $\frac{(1) 32,32}{-50 \%}$ & LV & EL & 16 & \begin{tabular}{|l}
557.5336 \\
\end{tabular} & 634.6244 & 617.0118 & \begin{tabular}{|l|l|}
116.2731 \\
\end{tabular} & \begin{tabular}{|l|}
116.59994 \\
\end{tabular} & 1151969 & 1144896 & \begin{tabular}{|c|}
1000565 \\
\end{tabular} & 13886 & $53 \%$ & 298461 & 39137 & 07083 & 88.4432 & 5,4211 \\
\hline & & (1) 32,32 & & & & & & & & & & & & & & & & & & \\
\hline$S G$ & $25 \%$ & $-100 \%$ & LV & $\mathrm{EL}$ & 16 & 557.3193 & \begin{tabular}{|l|l|}
633.5188 \\
\end{tabular} & 615.8361 & 115.7105 & 116.0528 & 114.626 & 113.9253 & 100.0568 & 1.4216 & $5.4 \%$ & 2988.86 & 3.8481 & 0.7159 & 88.4389 & 5.5859 \\
\hline & & (1) 32,32 & & & & & & & & & & & & & & & & & & \\
\hline
\end{tabular}

\begin{tabular}{|c|c|c|c|c|c|c|c|}
\hline \multicolumn{8}{|c|}{ Improvement } \\
\hline $\begin{array}{l}\text { Machine } \\
\text { Type }\end{array}$ & $\begin{array}{l}\text { Real } \\
\text { Power }\end{array}$ & $\begin{array}{l}\text { Reactive } \\
\text { Power }\end{array}$ & HV ILV & Location & $\begin{array}{l}\text { Lowest } \\
\text { Voltage }\end{array}$ & Losses & $\begin{array}{l}\text { Node } 1 \\
\text { Capacity }\end{array}$ \\
\hline SG & $50 \%$ & 0 & LV & $\mathrm{EL}$ & $-2.27 \%$ & $0.28 \%$ & $6.81 \%$ \\
\hline & & 1) 32,3 & & & & & \\
\hline$S G$ & $75 \%$ & 0 & LV & $E \mathrm{EL}$ & $-2.16 \%$ & $0.39 \%$ & $8.21 \%$ \\
\hline & $v_{v}$ & & - & & $2050 \%$ & & \\
\hline & & (1)32,32 & & & & & $9.60 \%$ \\
\hline SG & $106.7 \%$ & 1) 0 & LV & EL & $-2.03 \%$ & $0.54 \%$ & $9.98 \%$ \\
\hline & & (1) 32,32 & 32 & & & & \\
\hline SG & $100 \%$ & $100 \%$ & LV & $E \mathrm{EL}$ & $-1.15 \%$ & $0.56 \%$ & $9.00 \%$ \\
\hline$\overline{S G}$ & $100 \%$ & (1) 32,32 & 2 & & $300 \%$ & $040 \%$ & \\
\hline & $100 \% \mathrm{~V}$ & $\frac{-100 \%}{(1) 32,32}$ & 32 & & & & $9.96 \%$ \\
\hline SG & $25 \%$ & $100 \%$ & $\overline{L V}$ & $\overline{E L}$ & $-1.49 \%$ & $0.21 \%$ & $4.78 \%$ \\
\hline & & (1) 32,32 & & & & & \\
\hline SG & $25 \%$ & $75 \%$ & LV & EL & $-1.71 \%$ & $0.20 \%$ & $4.96 \%$ \\
\hline & $\mathrm{v}$ & |) 32,3 & & & & & \\
\hline SG & $25 \%$ & $50 \%$ & LV & $E L$ & $-1.93 \%$ & $0.19 \%$ & $5.12 \%$ \\
\hline SG & $25 \%$ & $25 \%$ & IV & $E L$ & $-216 \%$ & $0,17 \%$ & $5.27 \%$ \\
\hline & & (1) 32,32 & & & & & \\
\hline SG & $25 \%$ & $0 \%$ & LV & EL & $-2.39 \%$ & $0.15 \%$ & $5.40 \%$ \\
\hline & & & & & & & \\
\hline SG & $25 \%$ & & LV & $\overline{E L}$ & $-2.86 \%$ & $0.10 \%$ & $5.63 \%$ \\
\hline$S G$ & & 32,34 & Ty & $F=$ & $3220 \%$ & $040 \%$ & $570 \%$ \\
\hline & $\frac{20 \%}{\mathrm{v}}$ & 40070 & & $\underline{L L}$ & & $0.04 \%$ & $5.19 \%$ \\
\hline & & (1) 32,28 & & & & & \\
\hline
\end{tabular}


Table 51. Synchronous Generator Voltage Regulation Application (ML, LV) - HL

\begin{tabular}{|c|c|c|c|c|c|c|c|c|c|c|c|c|c|c|c|c|c|c|c|c|}
\hline \multirow[b]{2}{*}{$\begin{array}{l}\text { Machine } \\
\text { Type }\end{array}$} & \multirow[b]{2}{*}{$\begin{array}{l}\text { Real } \\
\text { Power }\end{array}$} & \multirow[b]{2}{*}{$\begin{array}{l}\text { Reactive } \\
\text { Power }\end{array}$} & \multirow[b]{2}{*}{ HV/LV } & \multirow[b]{2}{*}{ Location } & \multirow[b]{2}{*}{ LTC Tap } & \multicolumn{3}{|c|}{ Node 01} & \multicolumn{3}{|c|}{ Lowest 3 $\Phi$ Voltage } & \multirow{3}{*}{$\begin{array}{c}\text { Lowest } \\
1 \Phi \\
\text { Voltage } \\
1151577\end{array}$} & \multirow{2}{*}{$\begin{array}{c}\text { Highest } \\
12 / 11 \%\end{array}$} & \multirow[b]{2}{*}{$\begin{array}{l}\text { Highest } \\
\text { V2N1 \% }\end{array}$} & \multirow[b]{2}{*}{$\begin{array}{l}\% \mathrm{~kW} \\
\text { Loss }\end{array}$} & \multirow[b]{2}{*}{$\begin{array}{c}\text { Total } \\
\text { kVar Loss }\end{array}$} & \multicolumn{3}{|c|}{$12 / 1 \%$} & \multirow[b]{2}{*}{$\begin{array}{l}\text { Node 1 } \\
\text { Capacity }\end{array}$} \\
\hline & & & & & & IA & IB & IC & VA & VB & vc & & & & & & Node 0 & Low & $\begin{array}{l}\text { High } \\
(<100)\end{array}$ & \\
\hline SG & $50 \%$ & 0 & $\mathrm{LV}$ & $\begin{array}{ll}\mathrm{ML} \\
\end{array}$ & \begin{tabular}{|l|}
16 \\
\end{tabular} & 548.8954 & 626.6189 & 609.1722 & 116.97 & 117.2405 & 115.8728 & & & 1.3534 & $5.2 \%$ & $\begin{array}{ll}2901.89 \\
\end{array}$ & 4.0115 & 0.6993 & 88.4445 & \\
\hline & & & & & & & & & & & & & & & & & & & & \\
\hline SG & $75 \%$ & 0 & LV & $\mathrm{ML}$ & 16 & 539.6345 & 617.1659 & 599.8566 & 117.1235 & 117.3573 & 115.9993 & 115.2827 & 100.0562 & 1.3356 & $5.0 \%$ & 2808.77 & 4.0498 & 0.6983 & 88.4419 & 8.023 \\
\hline$S G$ & $100 \%$ & 1) 32, & LV & ML & 16 & 530.4157 & 6077407 & 590.5718 & 117.2744 & 117472 & 116.1231 & 115.4052 & 100.0561 & 13181 & $49 \%$ & 271796 & 4.089 & 0.6974 & 88.4392 & 94276 \\
\hline . & $\mathrm{v}$ & (1) 32,32 & & NVLL & & & 001.1401 & 090.0110 & & & 110.1201 & 110.4002 & 100.0567 & 1.3181 & $4.9 \%$ & 2717.96 & 4.089 & $0.69 / 4$ & 88.4392 & $\begin{array}{l}9.4216 \\
\end{array}$ \\
\hline SG & $106.7 \%$ & 0 & LV & $\mathrm{ML}$ & 16 & 527.9495 & 605.2168 & 588.0861 & 117.3145 & 117.5024 & 116.1559 & 115.4376 & 100.0561 & 1.3134 & $4.9 \%$ & 2694 & 4.0996 & 0.6971 & 88.4385 & 9.8037 \\
\hline$S G$ & $100 \%$ & 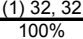 & $\overline{\mathrm{LV}}$ & $\overline{M L}$ & 16 & 531.6213 & $\overline{610.1927}$ & 593.3617 & 118.373 & 118.547 & 117.2388 & 116.508 & 100.0556 & 1.277 & $4.9 \%$ & 2737.94 & 4.1987 & 0.6806 & $\begin{array}{l}88.4478 \\
\end{array}$ & 9.0622 \\
\hline & & 1) 32,32 & & & & & & & & & & & & & & & & & & \\
\hline$S G$ & $100 \%$ & $\frac{-100 \%}{(1) 32,32}$ & $\begin{array}{l}\mathrm{LV} \\
32\end{array}$ & $\mathrm{ML}$ & 16 & 531.0778 & 606.8617 & 589.4042 & 116.1427 & 116.3682 & 114.973 & 114.2682 & 100.0566 & 1.364 & $5.0 \%$ & 2719.09 & 3.9633 & 0.7148 & 88.43 & 9.5586 \\
\hline SG & $25 \%$ & $100 \%$ & LV & $\mathrm{ML}$ & 16 & 559.6553 & 638.7202 & 621.4732 & 117.9141 & 118.1974 & 116.8601 & 116.1337 & 100.0558 & 1.3311 & $5.2 \%$ & 3020.68 & 4.0764 & 0.6836 & 88.4556 & 4.8107 \\
\hline SG & $25 \%$ & (1) 32,32 & LV & $\mathrm{ML}$ & 16 & 559.128 & 637.9253 & 620.5903 & 117.6398 & 117.9289 & 116.5818 & 1158586 & 100.0559 & 1.3409 & $5.2 \%$ & 301289 & 4052 & 0.6878 & 88.4535 & 4.9292 \\
\hline & & & & & & & & & & & & & & & & & & & & $4.929 \mathrm{C}$ \\
\hline$S G$ & $25 \%$ & $50 \%$ & LV & $\mathrm{ML}$ & 16 & 558.713 & 637.2304 & 619.8111 & $\begin{array}{l}117.368 \\
\end{array}$ & 117.663 & 116.306 & 1115.586 & 100.0561 & 1.3507 & $5.2 \%$ & $\begin{array}{l}3006.48 \\
\end{array}$ & 4.0271 & 0.6919 & 88.4515 & 5.0327 \\
\hline SG & $25 \%$ & (1) 32,32 & $\frac{2}{L V}$ & $\mathrm{ML}$ & 16 & 558.3989 & 636.6144 & 619.1121 & 117.0897 & 117.3909 & 116.0236 & 115.3068 & 100.0562 & 1.3611 & $5.2 \%$ & 3001.22 & 4.0008 & 0.6961 & 88.4493 & 5.1245 \\
\hline & & & & & & & & & & & & & & & & & & & & \\
\hline SG & $25 \%$ & $0 \%$ & LV & $\mathrm{ML}$ & 16 & 558.1977 & 636.0994 & 618.5182 & 116.8139 & 117.1215 & 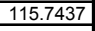 & 115.0301 & 100.0563 & 1.3715 & $5.3 \%$ & 2997.36 & 3.9741 & 0.7003 & 88.4472 & 5.2013 \\
\hline$S G$ & & 1) 32,32 & LV & $\overline{M L}$ & 16 & 550100 & 635.3429 & 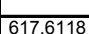 & 116.2514 & 116.5729 & 115.1727 & 114.4656 & 100.0566 & 13936 & $53 \%$ & 2993.51 & 39175 & 07089 & & \\
\hline$S G$ & $\frac{25 \%}{v}$ & $\frac{-50 \%}{(1) 32,32}$ & 32 & $\mathrm{ML}$ & 16 & 558.1264 & 635.3429 & 617.6118 & 110.2514 & & & 114.4656 & 100.0566 & 1.3936 & $5.3 \%$ & 2993.51 & 3.9175 & 0.7089 & 88.4428 & 5.314 \\
\hline SG & $25 \%$ & $-100 \%$ & LV & $\mathrm{ML}$ & 16 & 558.5165 & 634.977 & 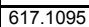 & 115.6802 & 116.0168 & 114.5926 & 113.8923 & 100.0568 & 1.4169 & $5.4 \%$ & 2995.16 & 3.8573 & 0.7177 & 88.4382 & 5.3686 \\
\hline & & (1) 32,32 & & & & & & & & & & & & & & & & & & \\
\hline
\end{tabular}

\begin{tabular}{|c|c|c|c|c|c|c|c|}
\hline \multicolumn{8}{|c|}{ Improvement } \\
\hline $\begin{array}{l}\text { Machin } \\
\text { Mype }\end{array}$ & $\begin{array}{l}\text { Real } \\
\text { Power }\end{array}$ & $\begin{array}{l}\text { Reactive } \\
\text { Power }\end{array}$ & HV/LV & Location & Lowest & Losses & $\begin{array}{l}\text { Node } 1 \\
\text { Capacity }\end{array}$ \\
\hline$S G$ & $50 \%$ & & LV & $\mathrm{ML}$ & $-2.29 \%$ & $0.24 \%$ & $6.82 \%$ \\
\hline & & & & & & & \\
\hline$S G$ & $75 \%$ & 0 & LV & ML & $-2.18 \%$ & $0.35^{\circ}$ & $8.23 \%$ \\
\hline SG & $100 \%$ & & LV & $\overline{M L}$ & $-2.08^{\circ}$ & $0.45^{\circ}$ & 96 \\
\hline & & & & & & & \\
\hline$S G$ & $106.7 \%$ & 0 & LV & $\mathrm{ML}$ & $-2.05 \%$ & $0.48 \%$ & $10.01 \%$ \\
\hline & & & & & & & \\
\hline$S G$ & $100 \%$ & $\frac{100 \%}{1132}$ & LV & $\mathrm{ML}$ & $-1.14 \%$ & $0.51 \%$ & $9.27 \%$ \\
\hline SG & $100 \%$ & $-100 \%$ & LV & $\begin{array}{l}\mathrm{ML} \\
\end{array}$ & $-3.04 \%$ & $0.37 \%$ & $9.76 \%$ \\
\hline & & & & & & & \\
\hline$S G$ & $25 \%$ & $100 \%$ & LV & $\mathrm{ML}$ & $-1.46 \%$ & $0.19 \%$ & $5.02 \%$ \\
\hline & & (1) 32,32 & & & & & \\
\hline SG & $25 \%$ & $\frac{75 \%}{(1) 32.32}$ & LV LV & ML & $-1.69 \%$ & $0.18 \%$ & $5.14 \%$ \\
\hline SG & $25 \%$ & $50 \%$ & LV & $\mathrm{ML}$ & $-1.93 \%$ & $0.17 \%$ & $5.24 \%$ \\
\hline$\overline{S G}$ & $25 \%$ & $\frac{(1) 32,32}{25 \%}$ & LV & ML & $-2.16 \%$ & $0.15 \%$ & $5.33 \%$ \\
\hline & & (1) 32,32 & & & & & \\
\hline$S G$ & $25 \%$ & $0 \%$ & LV & $\mathrm{ML}$ & $-2.40 \%$ & $0.13 \%$ & $5.41 \%$ \\
\hline SG & $25 \%$ & $\frac{10<, 0<}{-50 \%}$ & LV & $\begin{array}{l}\mathrm{ML} \\
\end{array}$ & $-2.88 \%$ & $0.09 \%$ & $5.52 \%$ \\
\hline & & & & & & & \\
\hline SG & $\frac{25 \%}{V}$ & $\frac{-100 \%}{(1) 32,32}$ & LV & ML & $-3.36 \%$ & $0.04 \%$ & $5.57 \%$ \\
\hline
\end{tabular}




\subsection{Plotted Performance Data}

To help the reader follow the evaluation of the data, Table 52 and Table 53 show the results of each generator application and its improvement for HL and LL conditions. These data are plotted in Figure 141 through Figure 164 for the HL circuit condition. 
Table 52. Distributed Generator Voltage Regulation Application Summary - HL

\begin{tabular}{|c|c|c|c|c|c|c|c|c|}
\hline \multicolumn{5}{|c|}{ LV } & \multicolumn{4}{|c|}{$\mathrm{HV}$} \\
\hline $\mathrm{Q}$ & $\mathrm{V}$ & V Imp & Loss & Cap & $\mathrm{V}$ & V Imp & Loss & Cap \\
\hline 247.91 & 115.4859 & $-2.01 \%$ & $0.25 \%$ & $6.10 \%$ & 117.9912 & $0.12 \%$ & $0.30 \%$ & $3.80 \%$ \\
\hline 0 & 115.1259 & $-2.32 \%$ & $0.23 \%$ & $6.28 \%$ & 117.5369 & $-0.27 \%$ & $0.25 \%$ & $3.51 \%$ \\
\hline-247.91 & 114.7603 & $-2.63 \%$ & $0.20 \%$ & $6.44 \%$ & 117.7804 & $-0.06 \%$ & $0.19 \%$ & $2.31 \%$ \\
\hline
\end{tabular}

Inv Gen - P $=400$

\begin{tabular}{|c|c|c|c|c|c|c|c|c|c|}
\hline \multicolumn{1}{|c}{ HV } \\
\hline $\mathrm{Q}$ & $\mathrm{V}$ & $\mathrm{V} \operatorname{Imp}$ & Loss & Cap & & $\mathrm{V}$ & $\mathrm{V}$ Imp & Loss & Cap \\
\hline 0 & 115.1541 & $-2.29 \%$ & $0.22 \%$ & $6.29 \%$ & & 117.5964 & $-0.22 \%$ & $0.24 \%$ & $3.53 \%$ \\
\hline
\end{tabular}

\begin{tabular}{|c|c|c|c|c|c|c|r|r|r|}
\hline \multicolumn{1}{|c|}{ InV Gen - P 320} \\
\hline Q & \multicolumn{3}{|c|}{ LV } & \multicolumn{4}{|c|}{ HV } \\
\hline 240 & V & V Imp & Loss & Cap & & V & V Imp & Loss & Cap \\
\hline-240 & 115.6369 & $-1.88 \%$ & $0.16 \%$ & $5.83 \%$ & & 117.9769 & $0.10 \%$ & $0.21 \%$ & $2.79 \%$ \\
\hline
\end{tabular}

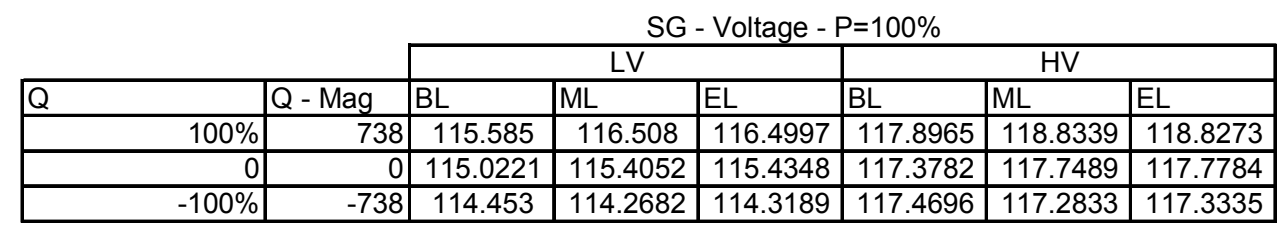

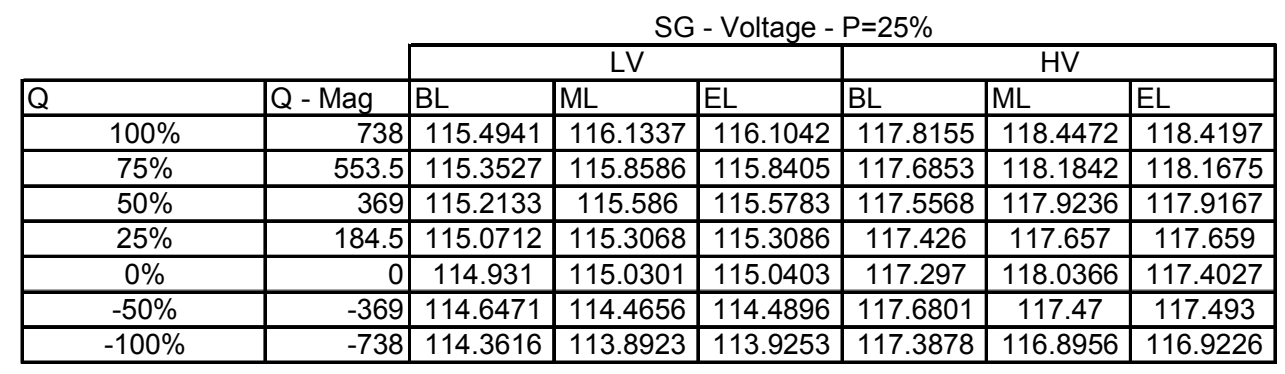

\begin{tabular}{|r|r|r|r|r|r|r|r|}
\multicolumn{9}{c|}{ SG - Loss - P=100\% } \\
\cline { 2 - 9 } \multicolumn{2}{c|}{} & \multicolumn{3}{c|}{ LV } & \multicolumn{3}{c|}{ HV } \\
\hline $100 \%$ & 738 & $0.13 \%$ & $0.51 \%$ & $0.56 \%$ & $0.14 \%$ & $0.51 \%$ & $0.56 \%$ \\
\hline$-100 \%$ & -738 & $0.10 \%$ & $0.37 \%$ & $0.40 \%$ & $0.08 \%$ & $0.34 \%$ & $0.37 \%$ \\
\hline
\end{tabular}

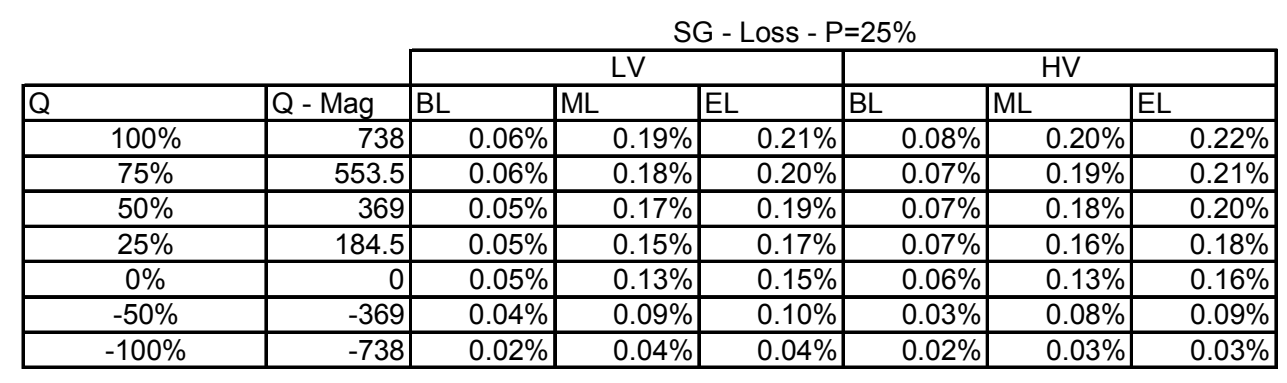




\begin{tabular}{|r|r|r|r|r|r|r|r|}
\multicolumn{2}{c|}{} & \multicolumn{5}{c|}{ SG - Cap - P=100\% } \\
\cline { 3 - 9 } \multicolumn{2}{c|}{} & \multicolumn{3}{c|}{ LV } & \multicolumn{3}{c|}{ HV } \\
\hline $100 \%$ & 738 & $10.44 \%$ & $9.27 \%$ & $9.00 \%$ & $7.36 \%$ & $6.24 \%$ & $5.97 \%$ \\
\hline$-100 \%$ & -738 & $8.47 \%$ & $9.76 \%$ & $9.96 \%$ & $4.11 \%$ & $5.39 \%$ & $5.59 \%$ \\
\hline
\end{tabular}

\begin{tabular}{|c|r|r|r|r|r|r|r|}
\multicolumn{2}{c|}{} & \multicolumn{7}{c|}{ SG - Cap - P=25\% } \\
\cline { 3 - 9 } & \multicolumn{3}{c|}{ LV } & \multicolumn{3}{c|}{ HV } \\
\hline & Q - Mag & BL & ML & EL & BL & ML & EL \\
\hline $100 \%$ & 738 & $6.21 \%$ & $5.02 \%$ & $4.78 \%$ & $3.21 \%$ & $2.04 \%$ & $1.80 \%$ \\
\hline $75 \%$ & 553.5 & $6.02 \%$ & $5.14 \%$ & $4.96 \%$ & $3.01 \%$ & $2.13 \%$ & $1.96 \%$ \\
\hline $50 \%$ & 369 & $5.83 \%$ & $5.24 \%$ & $5.12 \%$ & $2.79 \%$ & $2.22 \%$ & $2.10 \%$ \\
\hline $25 \%$ & 184.5 & $5.61 \%$ & $5.33 \%$ & $5.27 \%$ & $2.57 \%$ & $2.29 \%$ & $2.23 \%$ \\
\hline $0 \%$ & 0 & $5.39 \%$ & $5.41 \%$ & $5.40 \%$ & $2.33 \%$ & $1.32 \%$ & $2.35 \%$ \\
\hline$-50 \%$ & -369 & $4.90 \%$ & $5.52 \%$ & $5.63 \%$ & $0.79 \%$ & $1.26 \%$ & $1.37 \%$ \\
\hline$-100 \%$ & -738 & $4.37 \%$ & $5.57 \%$ & $5.79 \%$ & $0.10 \%$ & $1.15 \%$ & $1.50 \%$ \\
\hline
\end{tabular}

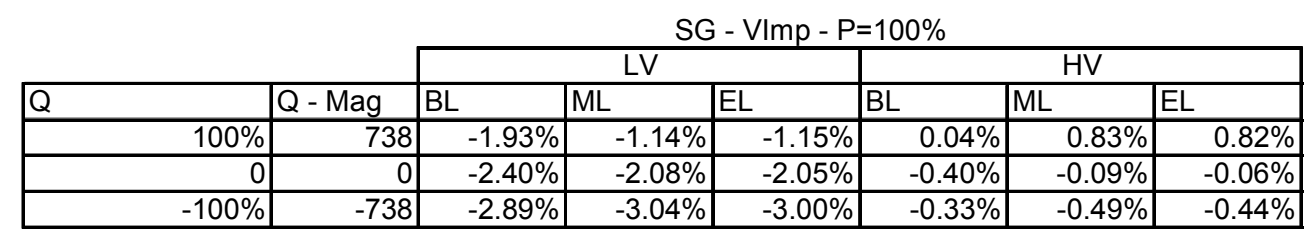

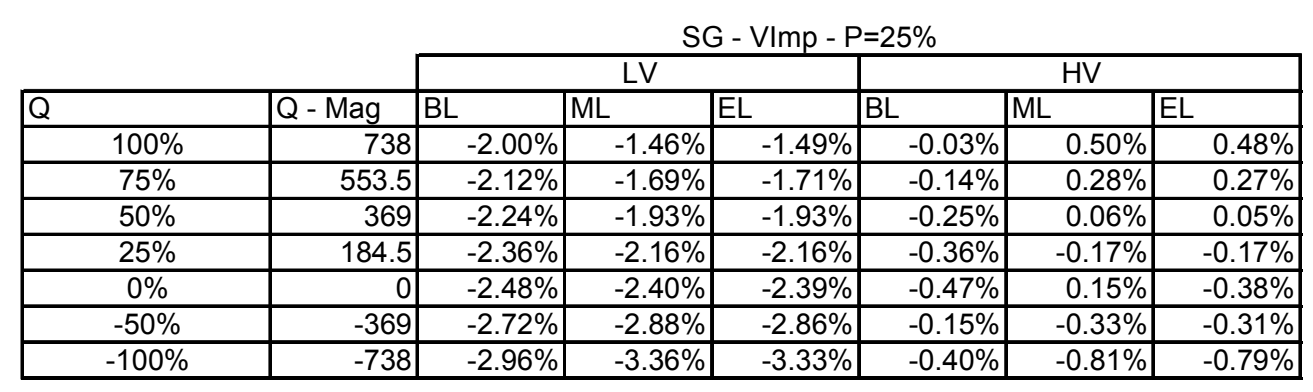

\begin{tabular}{|l|r|}
\hline \multicolumn{2}{|c|}{ Base Case: } \\
\hline Lowest Voltage: & 117.855 \\
\hline Losses: & $5.40 \%$ \\
\hline Node 1 Capacity: & $-0.21 \%$ \\
\hline
\end{tabular}


Table 53. Distributed Generator Voltage Regulation Application Summary - LL

\begin{tabular}{|c|c|c|c|c|c|c|c|c|}
\hline \multicolumn{5}{|c|}{ LV } & \multicolumn{4}{|c|}{ HV } \\
\hline $\mathrm{Q}$ & $\mathrm{V}$ & V Imp & Loss & Cap & $\mathrm{V}$ & VImp & Loss & Cap \\
\hline 247.91 & 122.4196 & $0.98 \%$ & $0.37 \%$ & $2.64 \%$ & 121.6783 & $0.37 \%$ & $0.38 \%$ & $2.89 \%$ \\
\hline 0 & 121.9696 & $0.61 \%$ & $0.31 \%$ & $2.04 \%$ & 122.048 & $0.67 \%$ & $0.33 \%$ & $2.02 \%$ \\
\hline-247.91 & 121.5126 & $0.23 \%$ & $0.23 \%$ & $1.38 \%$ & 121.6023 & $0.30 \%$ & $0.25 \%$ & $1.31 \%$ \\
\hline
\end{tabular}

Inv Gen - P $=400$

\begin{tabular}{|c|c|c|c|c|c|c|c|c|c|}
\multicolumn{1}{|c}{ HV } \\
\hline Q & V & V Imp & Loss & Cap & & V & V Imp & Loss & Cap \\
\hline 0 & 122.0019 & $0.63 \%$ & $0.28 \%$ & $2.01 \%$ & & 122.0808 & $0.70 \%$ & $0.30 \%$ & $1.99 \%$ \\
\hline
\end{tabular}

Inv Gen - P = 320

\begin{tabular}{|c|c|c|c|c|c|c|c|c|c|}
\hline & \multicolumn{3}{|c|}{ IV Gen - P 320} & \multicolumn{3}{|c|}{ HV } \\
\hline $\mathrm{Q}$ & $\mathrm{V}$ & $\mathrm{V}$ Imp & Loss & Cap & & V & V Imp & Loss & Cap \\
\hline 240 & 122.5691 & $1.10 \%$ & $0.29 \%$ & $2.20 \%$ & & 122.6307 & $1.15 \%$ & $0.33 \%$ & $2.19 \%$ \\
\hline-240 & 121.361 & $0.10 \%$ & $0.18 \%$ & $0.90 \%$ & & 121.4598 & $0.19 \%$ & $0.22 \%$ & $0.87 \%$ \\
\hline
\end{tabular}

\begin{tabular}{|r|r|l|l|l|l|l|l|}
\multicolumn{1}{c|}{ SG - Voltage - P=100\% } \\
\cline { 2 - 9 } & \multicolumn{5}{c|}{ LV } & \multicolumn{3}{c|}{ HV } \\
\hline & Q - Mag & BL & ML & EL & BL & ML & EL \\
\hline $100 \%$ & 738 & 121.8718 & 122.7779 & 122.7609 & 121.8101 & 122.7128 & 122.7038 \\
\hline$-100 \%$ & -738 & 121.2282 & 120.7316 & 120.7239 & 121.3707 & 120.8687 & 120.8612 \\
\hline
\end{tabular}

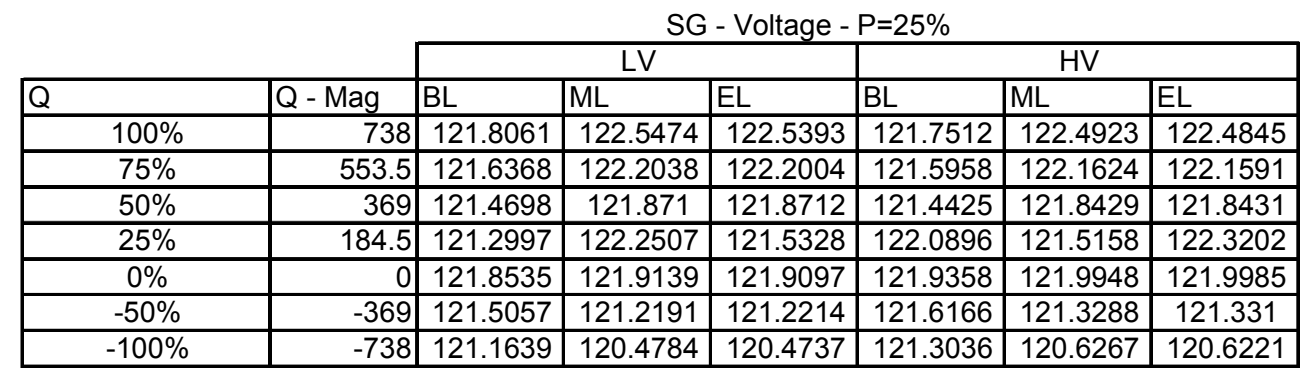

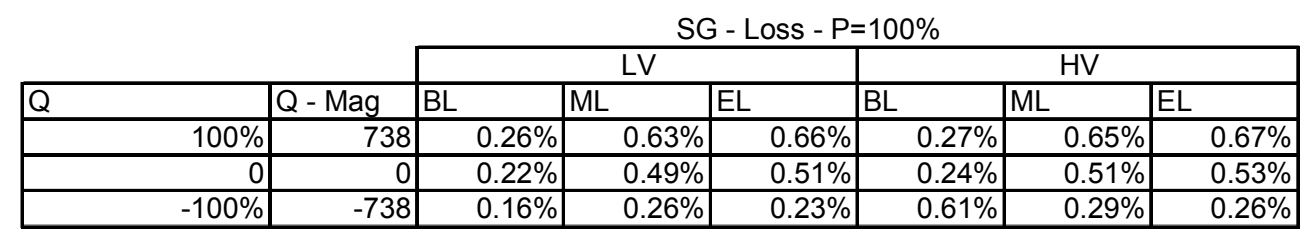

\begin{tabular}{|c|r|r|r|r|r|r|r|}
\multicolumn{2}{c|}{} & \multicolumn{7}{c|}{ SG - Loss - P=25\% } \\
\cline { 3 - 9 } \multicolumn{2}{c|}{} & \multicolumn{3}{|c|}{ LV } & \multicolumn{3}{c|}{ HV } \\
\hline & Q - Mag & BL & ML & EL & BL & ML & EL \\
\hline $100 \%$ & 738 & $0.19 \%$ & $0.38 \%$ & $0.39 \%$ & $0.21 \%$ & $0.40 \%$ & $0.41 \%$ \\
\hline $75 \%$ & 553.5 & $0.18 \%$ & $0.34 \%$ & $0.36 \%$ & $0.20 \%$ & $0.37 \%$ & $0.38 \%$ \\
\hline $50 \%$ & 369 & $0.17 \%$ & $0.31 \%$ & $0.32 \%$ & $0.19 \%$ & $0.33 \%$ & $0.35 \%$ \\
\hline $25 \%$ & 184.5 & $0.15 \%$ & $0.28 \%$ & $0.28 \%$ & $0.19 \%$ & $0.29 \%$ & $0.32 \%$ \\
\hline $0 \%$ & 0 & $0.16 \%$ & $0.23 \%$ & $0.24 \%$ & $0.18 \%$ & $0.26 \%$ & $0.27 \%$ \\
\hline$-50 \%$ & -369 & $0.12 \%$ & $0.12 \%$ & $0.12 \%$ & $0.15 \%$ & $0.15 \%$ & $0.15 \%$ \\
\hline$-100 \%$ & -738 & $0.09 \%$ & $0.00 \%$ & $-0.04 \%$ & $0.12 \%$ & $0.03 \%$ & $0.00 \%$ \\
\hline
\end{tabular}




\begin{tabular}{|r|r|r|r|r|r|r|r|}
\multicolumn{2}{c|}{ SG - Cap - P=100\% } \\
\cline { 3 - 9 } \multicolumn{2}{c|}{} & \multicolumn{3}{c|}{ LV } & \multicolumn{3}{c|}{ HV } \\
\hline $100 \%$ & 738 & $7.40 \%$ & $7.32 \%$ & $7.20 \%$ & $7.43 \%$ & $7.35 \%$ & $7.28 \%$ \\
\hline$-100 \%$ & -738 & $2.29 \%$ & $2.56 \%$ & $2.59 \%$ & $2.25 \%$ & $2.57 \%$ & $2.61 \%$ \\
\hline
\end{tabular}

\begin{tabular}{|c|r|r|r|r|r|r|r|}
\multicolumn{2}{c|}{} & \multicolumn{7}{c|}{ SG - Cap - P=25\% } \\
\cline { 3 - 9 } & \multicolumn{3}{c|}{ LV } & \multicolumn{3}{c|}{ HV } \\
\hline & Q - Mag & BL & ML & EL & BL & ML & EL \\
\hline $100 \%$ & 738 & $3.34 \%$ & $3.13 \%$ & $3.06 \%$ & $3.37 \%$ & $3.15 \%$ & $3.09 \%$ \\
\hline $75 \%$ & 553.5 & $2.88 \%$ & $2.71 \%$ & $2.66 \%$ & $2.91 \%$ & $2.73 \%$ & $2.68 \%$ \\
\hline $50 \%$ & 369 & $2.41 \%$ & $2.31 \%$ & $2.29 \%$ & $2.42 \%$ & $2.33 \%$ & $2.30 \%$ \\
\hline $25 \%$ & 184.5 & $1.89 \%$ & $1.65 \%$ & $1.87 \%$ & $1.65 \%$ & $1.89 \%$ & $1.63 \%$ \\
\hline $0 \%$ & 0 & $1.14 \%$ & $1.19 \%$ & $1.15 \%$ & $1.12 \%$ & $1.17 \%$ & $1.18 \%$ \\
\hline$-50 \%$ & -369 & $-0.06 \%$ & $0.13 \%$ & $0.15 \%$ & $-0.09 \%$ & $0.10 \%$ & $0.13 \%$ \\
\hline$-100 \%$ & -738 & $-1.31 \%$ & $-1.20 \%$ & $-1.16 \%$ & $-1.34 \%$ & $-1.19 \%$ & $-1.15 \%$ \\
\hline
\end{tabular}

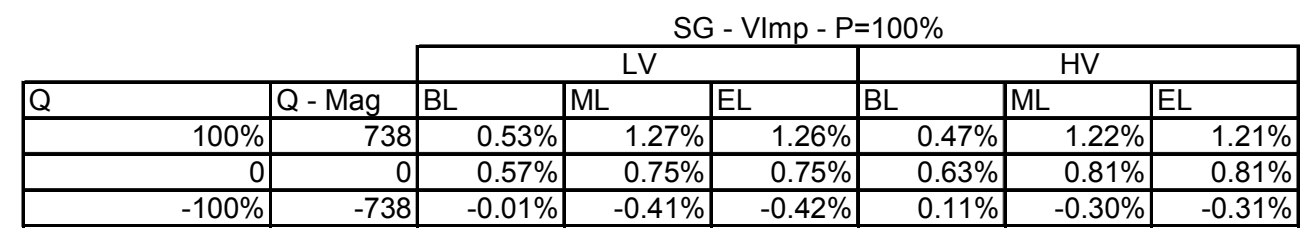

\begin{tabular}{|c|r|r|r|r|r|r|r|}
\multicolumn{2}{c|}{} & \multicolumn{7}{c|}{ SG - VImp - P=25\% } \\
\cline { 3 - 9 } \multicolumn{2}{c|}{} & \multicolumn{3}{c|}{ LV } & \multicolumn{3}{c|}{ HV } \\
\hline Q & Q - Mag & BL & ML & EL & BL & ML & EL \\
\hline $100 \%$ & 738 & $0.47 \%$ & $1.08 \%$ & $1.08 \%$ & $0.43 \%$ & $1.04 \%$ & $1.03 \%$ \\
\hline $75 \%$ & 553.5 & $0.33 \%$ & $0.80 \%$ & $0.80 \%$ & $0.30 \%$ & $0.77 \%$ & $0.76 \%$ \\
\hline $50 \%$ & 369 & $0.19 \%$ & $0.53 \%$ & $0.53 \%$ & $0.17 \%$ & $0.50 \%$ & $0.50 \%$ \\
\hline $25 \%$ & 184.5 & $0.05 \%$ & $0.84 \%$ & $0.25 \%$ & $0.71 \%$ & $0.23 \%$ & $0.90 \%$ \\
\hline $0 \%$ & 0 & $0.51 \%$ & $0.56 \%$ & $0.56 \%$ & $0.58 \%$ & $0.63 \%$ & $0.63 \%$ \\
\hline$-50 \%$ & -369 & $0.22 \%$ & $-0.01 \%$ & $-0.01 \%$ & $0.32 \%$ & $0.08 \%$ & $0.08 \%$ \\
\hline$-100 \%$ & -738 & $-0.06 \%$ & $-0.62 \%$ & $-0.63 \%$ & $0.06 \%$ & $-0.50 \%$ & $-0.50 \%$ \\
\hline
\end{tabular}

\begin{tabular}{|l|r|}
\hline \multicolumn{2}{|c|}{ Base Case: } \\
\hline Lowest Voltage: & 121.2343 \\
\hline Losses: & $3.40 \%$ \\
\hline Node 1 Capacity: & $60.58 \%$ \\
\hline
\end{tabular}




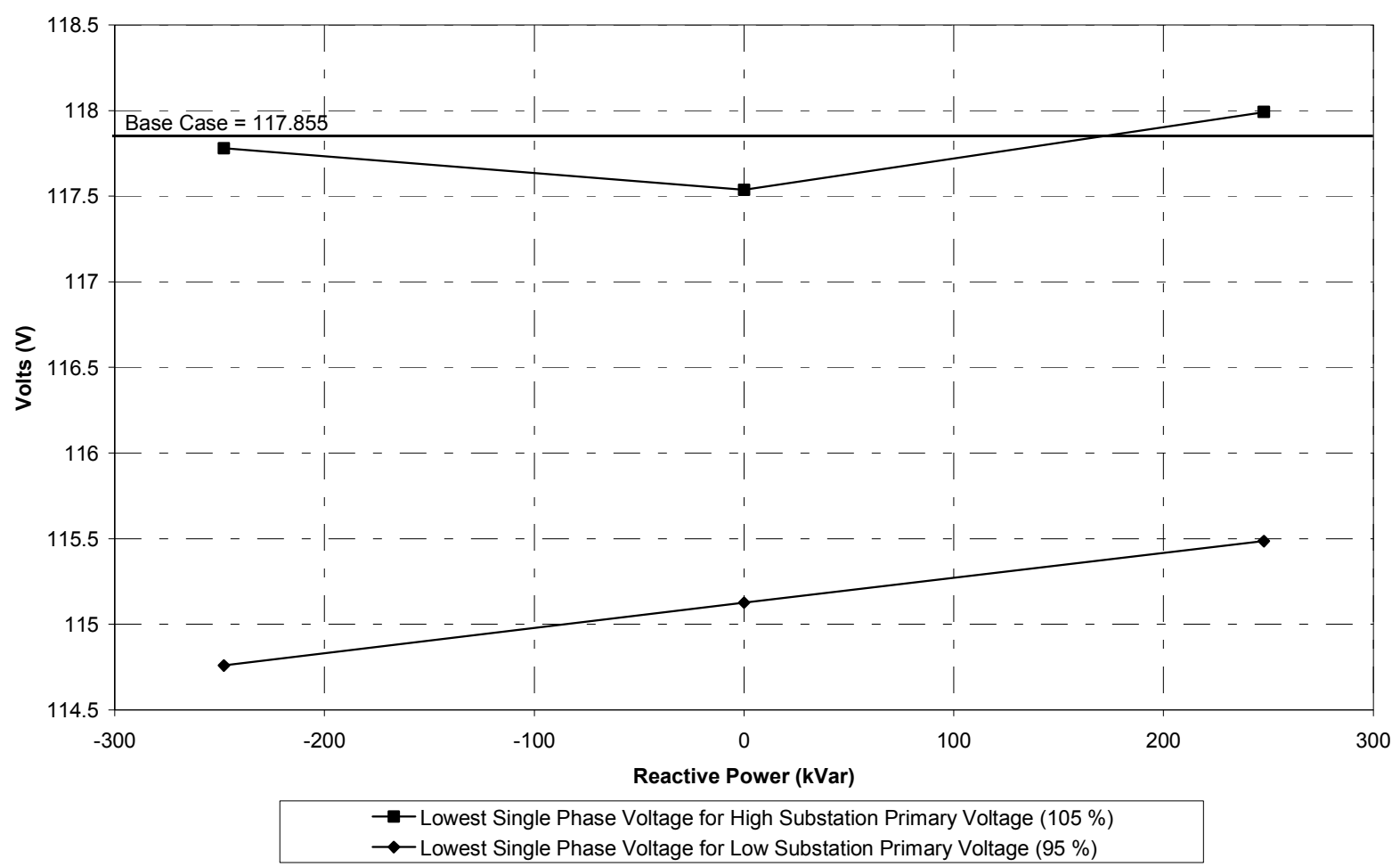

Figure 141. HL condition - voltage rise or drop (in volts on a 120-V base) as a function of induction generator $+/$ - reactive power with a constant real power output of $400 \mathrm{~kW}$

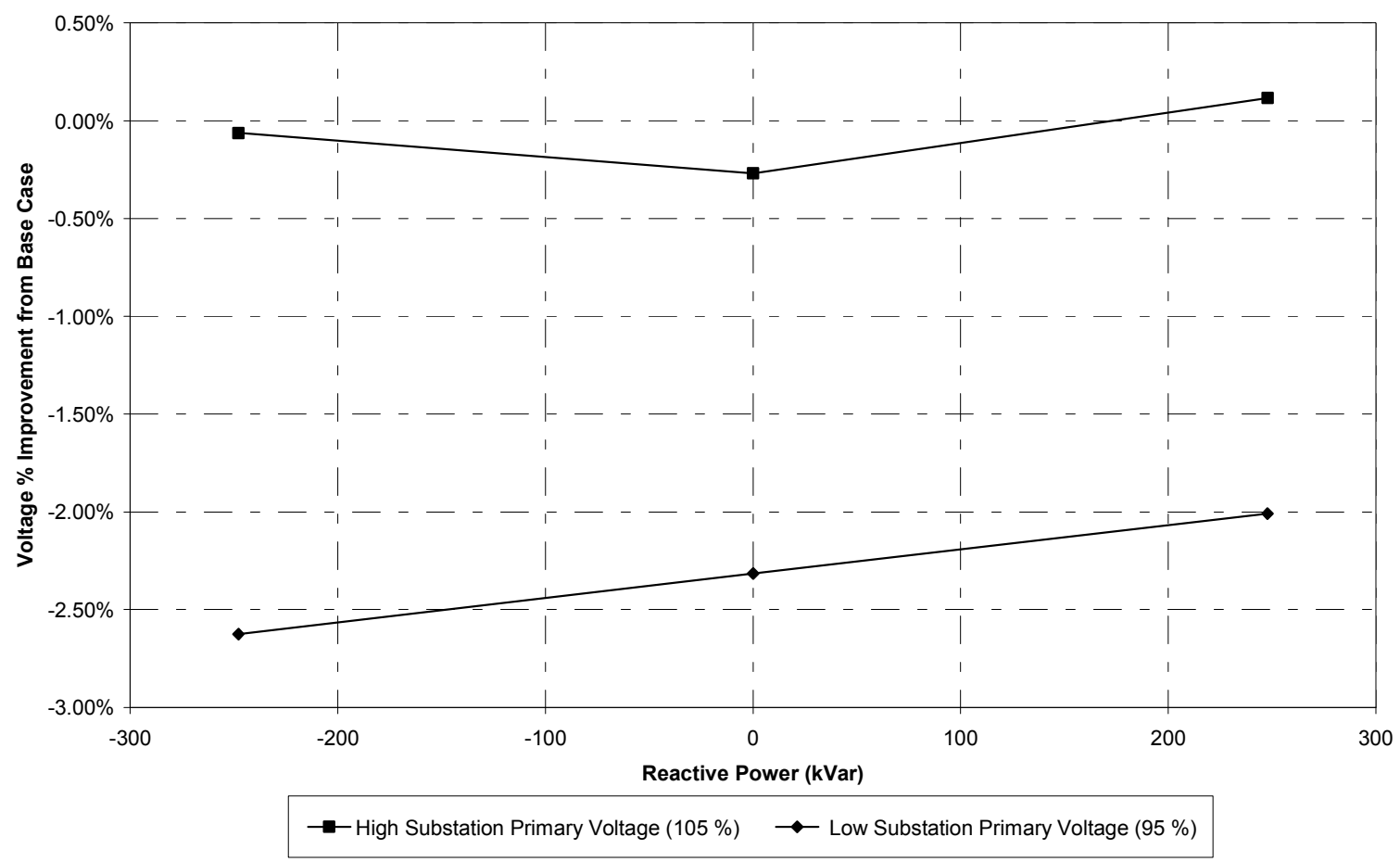

Figure 142. HL condition - reduction or increase in voltage (in percent on a $120-\mathrm{V}$ base) as a function of induction generator $+/$ - reactive power with a constant real power output of $400 \mathrm{~kW}$ 


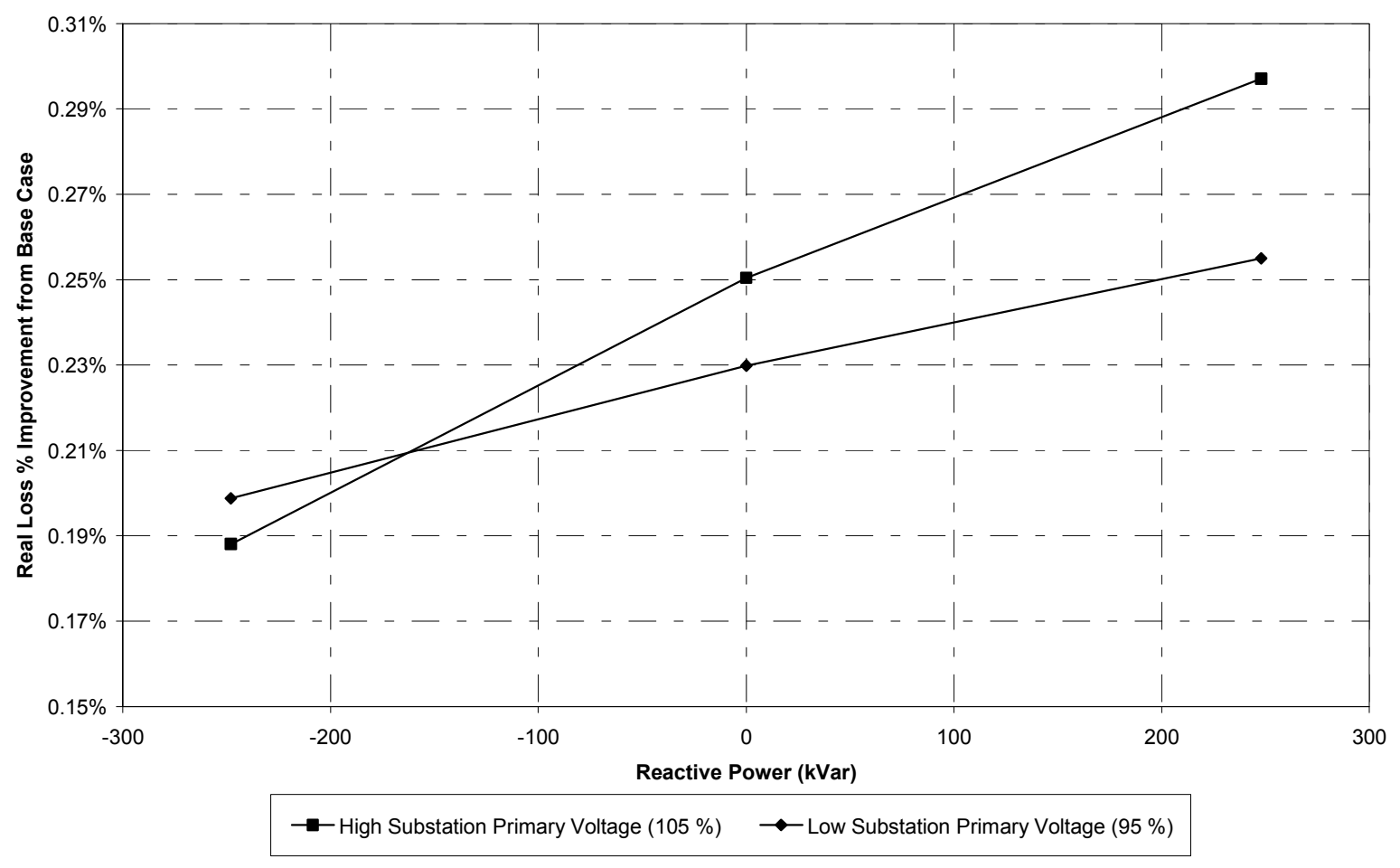

Figure 143. HL condition - reduction or increase of real power (in percent of total circuit kilowatts) as a function of induction generator $+/$ - reactive power with a constant real power output of $400 \mathrm{~kW}$

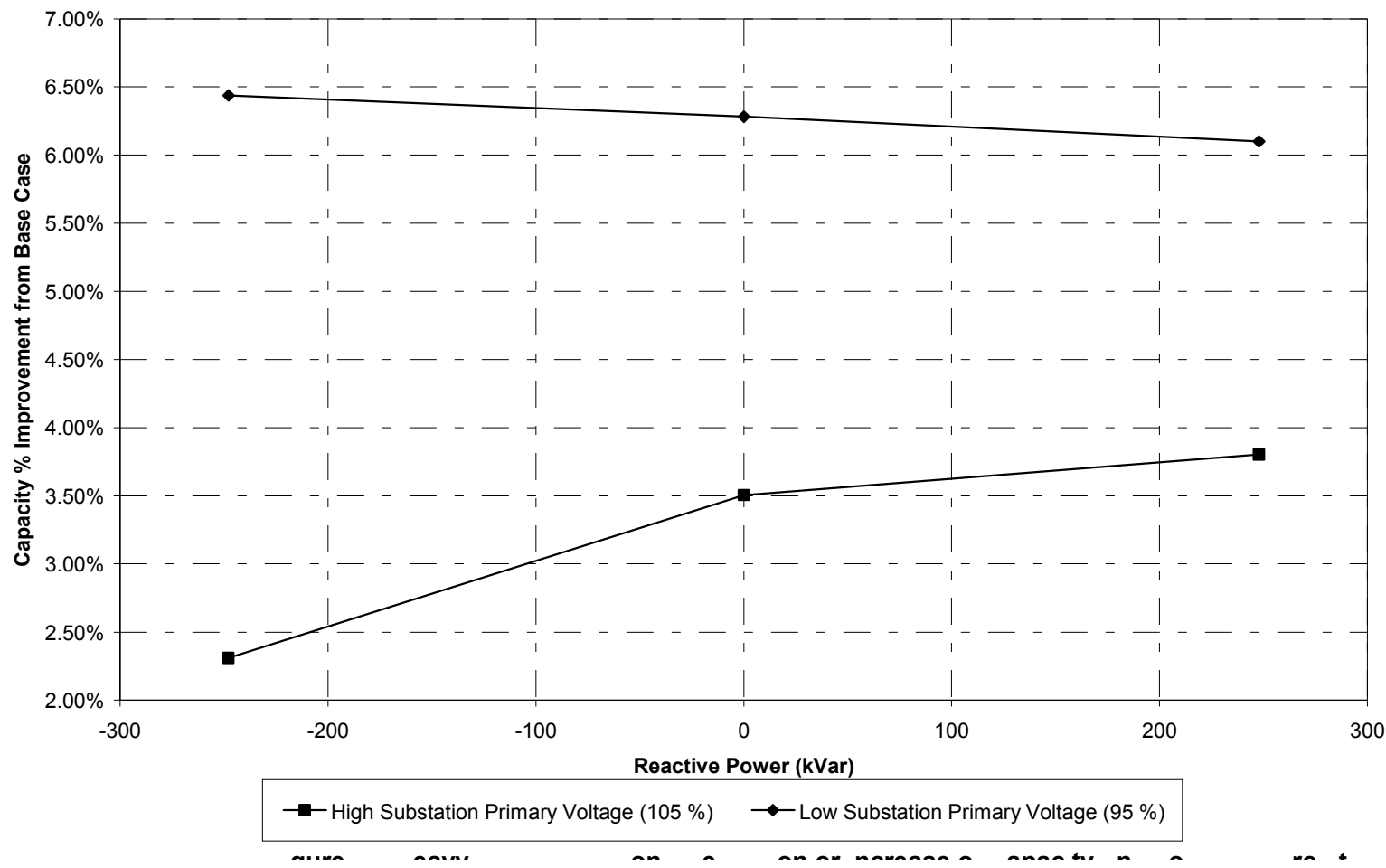

Figure 144. HL load condition - reduction or increase of capacity (in percent of total circuit kilovolt-amperes) as a function of induction generator +/- reactive power with a CP output of $400 \mathrm{~kW}$ 


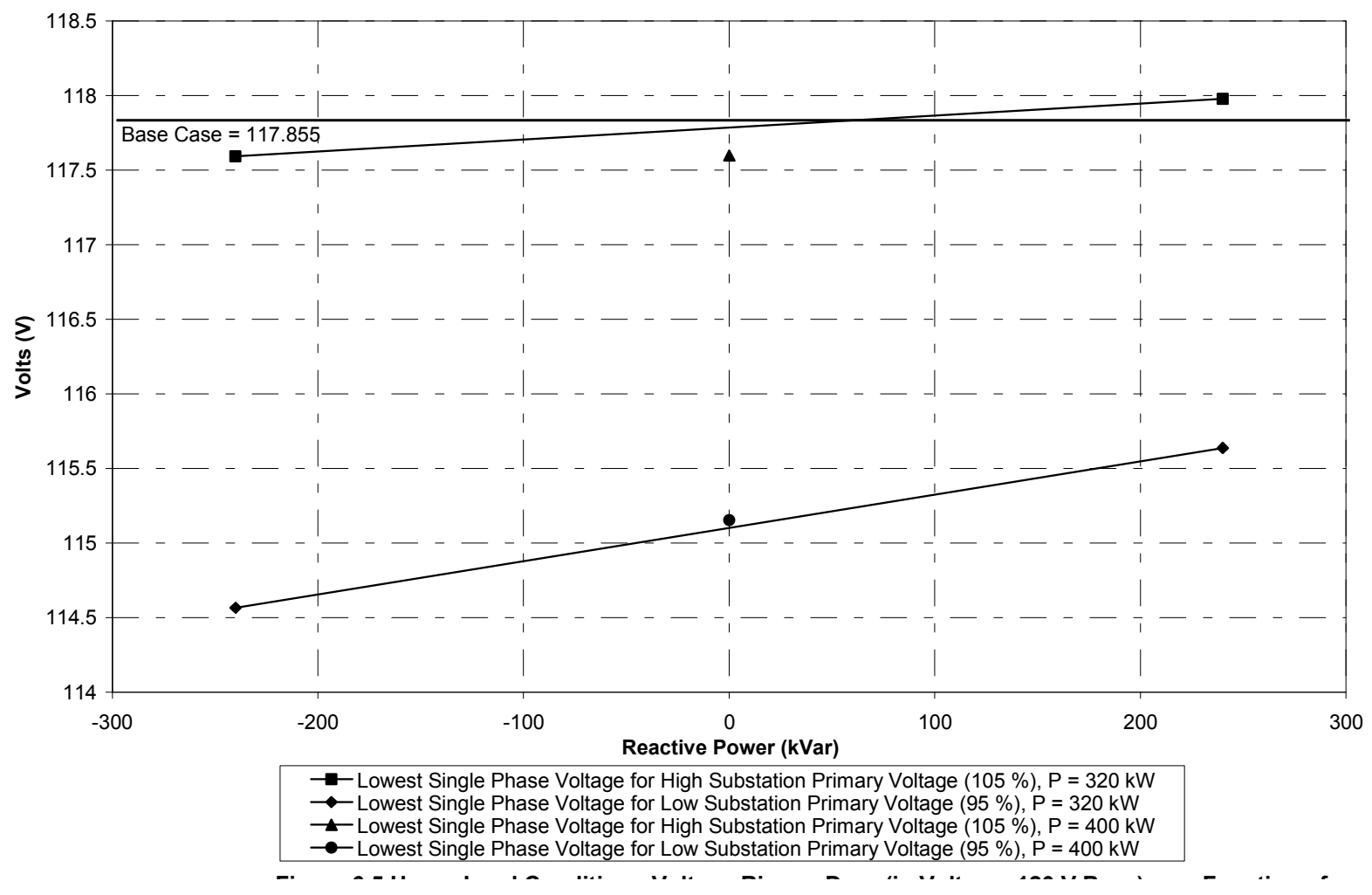

Figure 145. HL condition - voltage rise or drop (in volts on a 120-V base) as a function of inverter generator $+/$ - reactive power with a constant real power output of $400 \mathrm{~kW}$

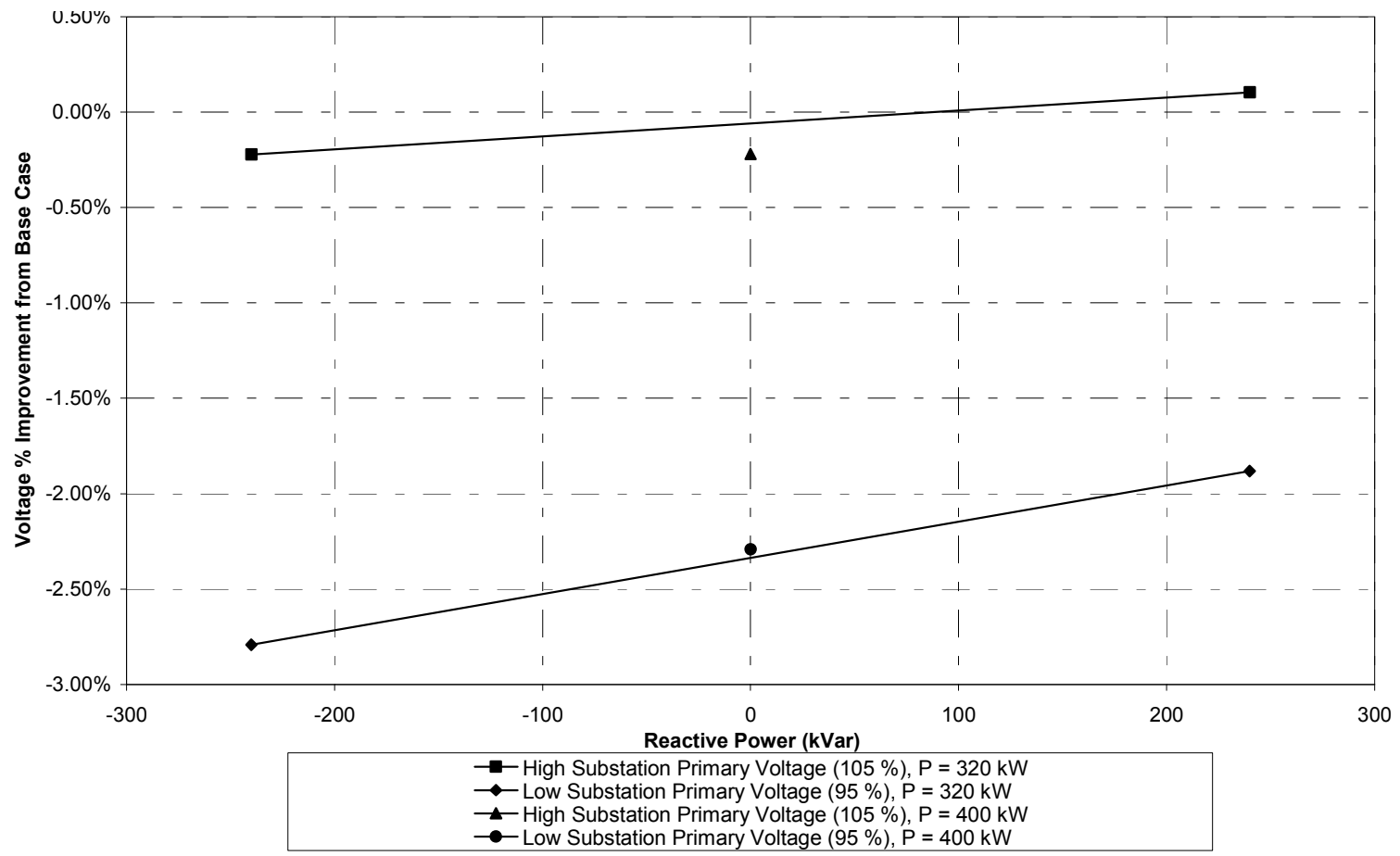

Figure 146. HL condition - reduction or increase in voltage (in percent on a 120-V base) as a function of inverter generator $+/$ - reactive power with a constant real power output of $320 \mathrm{~kW}$ or $400 \mathrm{~kW}$ 


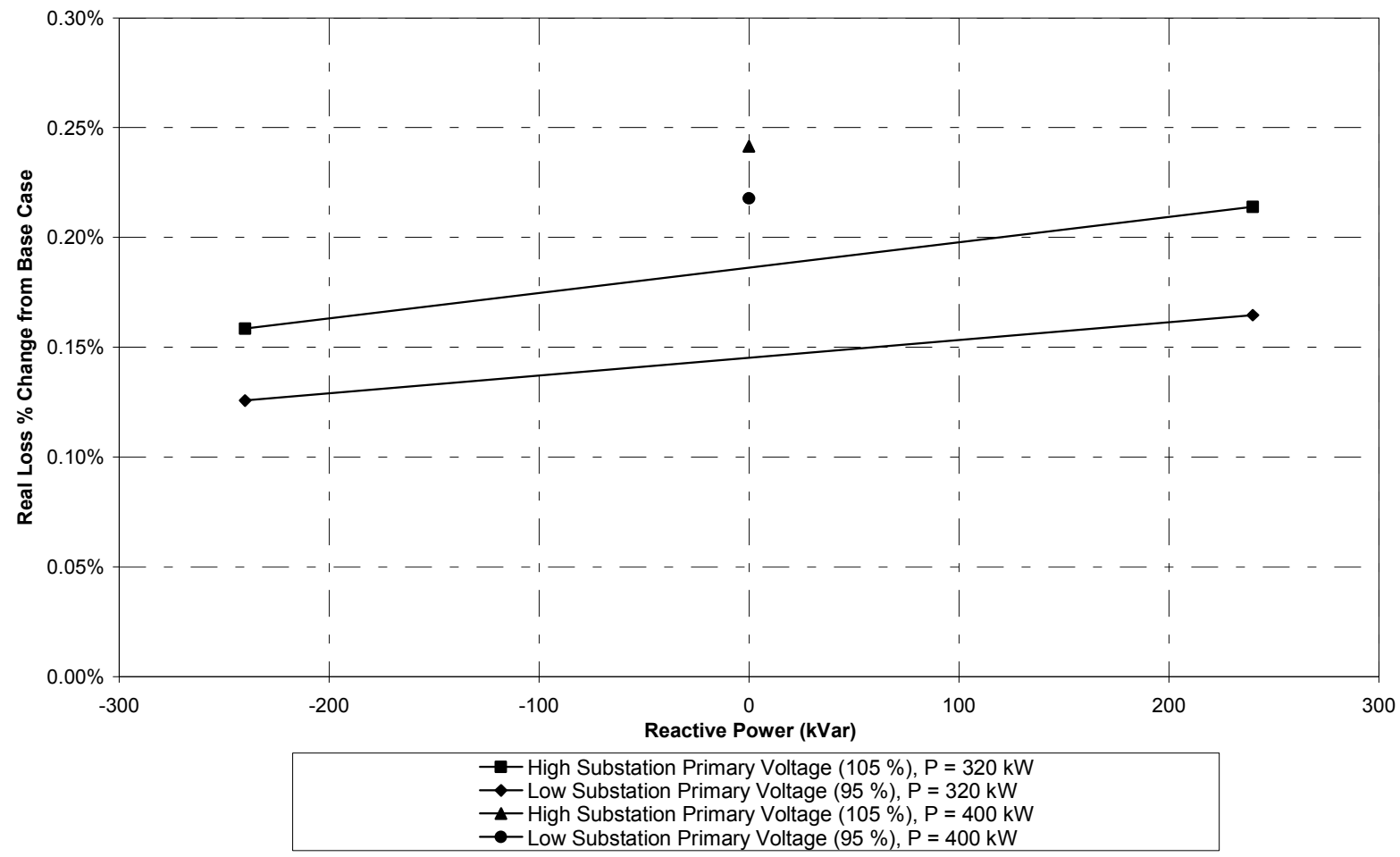

Figure 147. HL condition - reduction or increase of real power (in percent of total circuit kilowatts) as a function of induction generator +/- reactive power with a constant real power output of $320 \mathrm{~kW}$ or $400 \mathrm{~kW}$

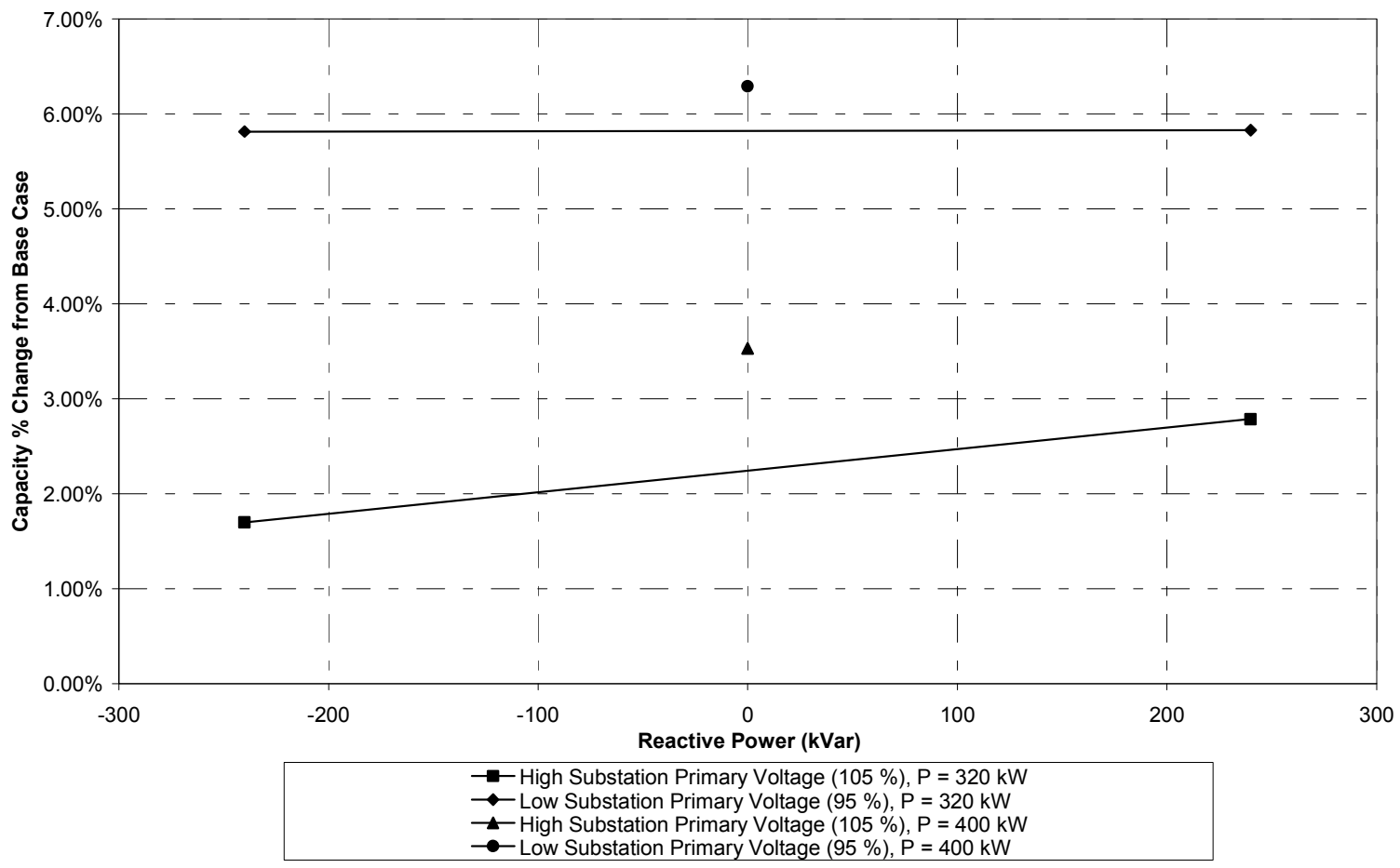

Figure 148. HL condition - reduction or increase of capacity (in percent of total circuit kilovolt-amperes) as a function of induction generator $+/$ - reactive power with a CP output of $320 \mathrm{~kW}$ or $400 \mathrm{~kW}$ 


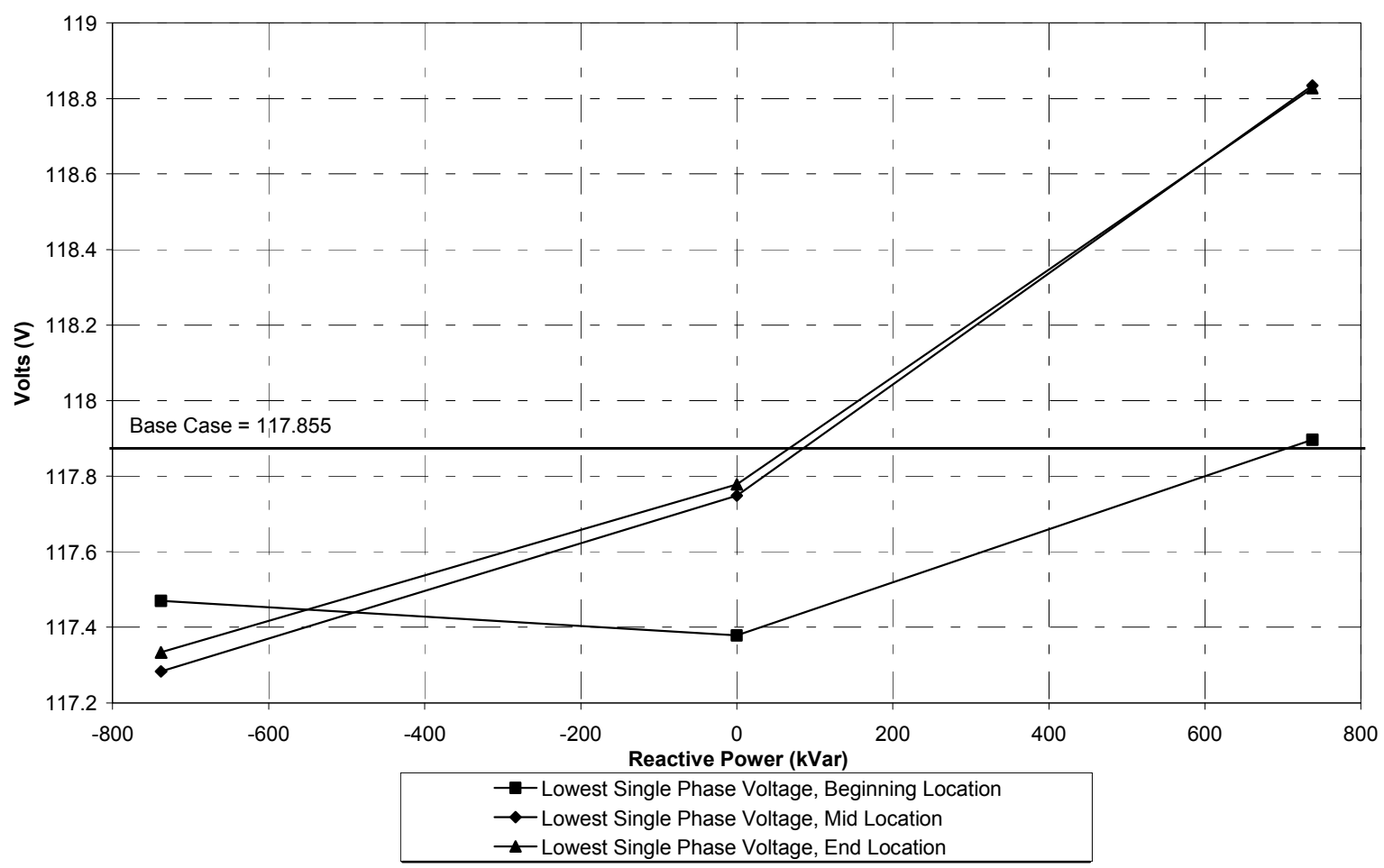

Figure 149. High substation primary voltage, HL condition - voltage rise or drop (in volts on a $120-\mathrm{V}$ base) as a function of location and synchronous generator $+/$ - reactive power with a constant real power output of $984 \mathrm{~kW}$

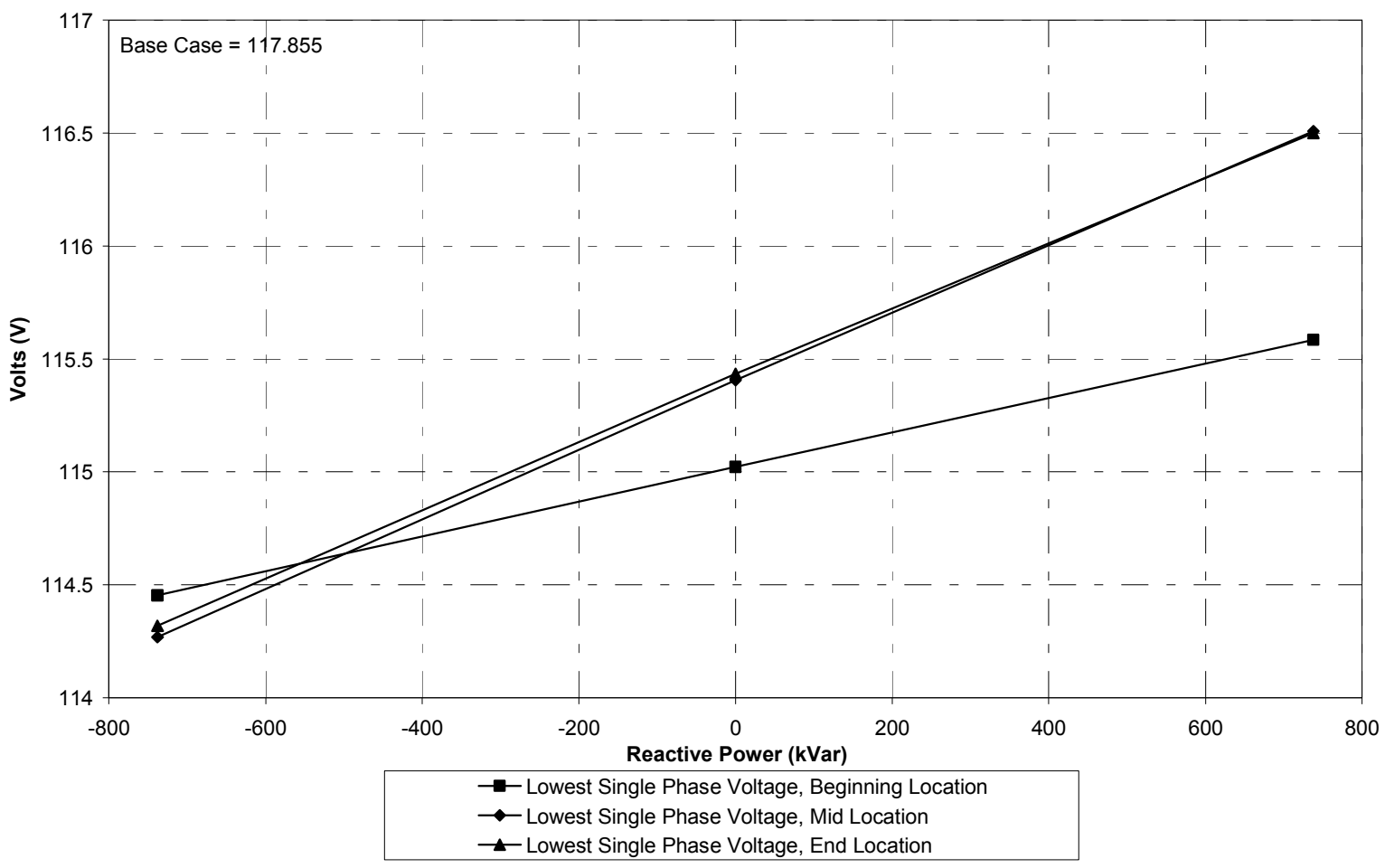

Figure 150. Low substation primary voltage, $\mathrm{HL}$ condition - voltage rise or drop (in volts on a 120-V base) as a function of location and synchronous generator + /- reactive power with a constant real power output of 984 kW 


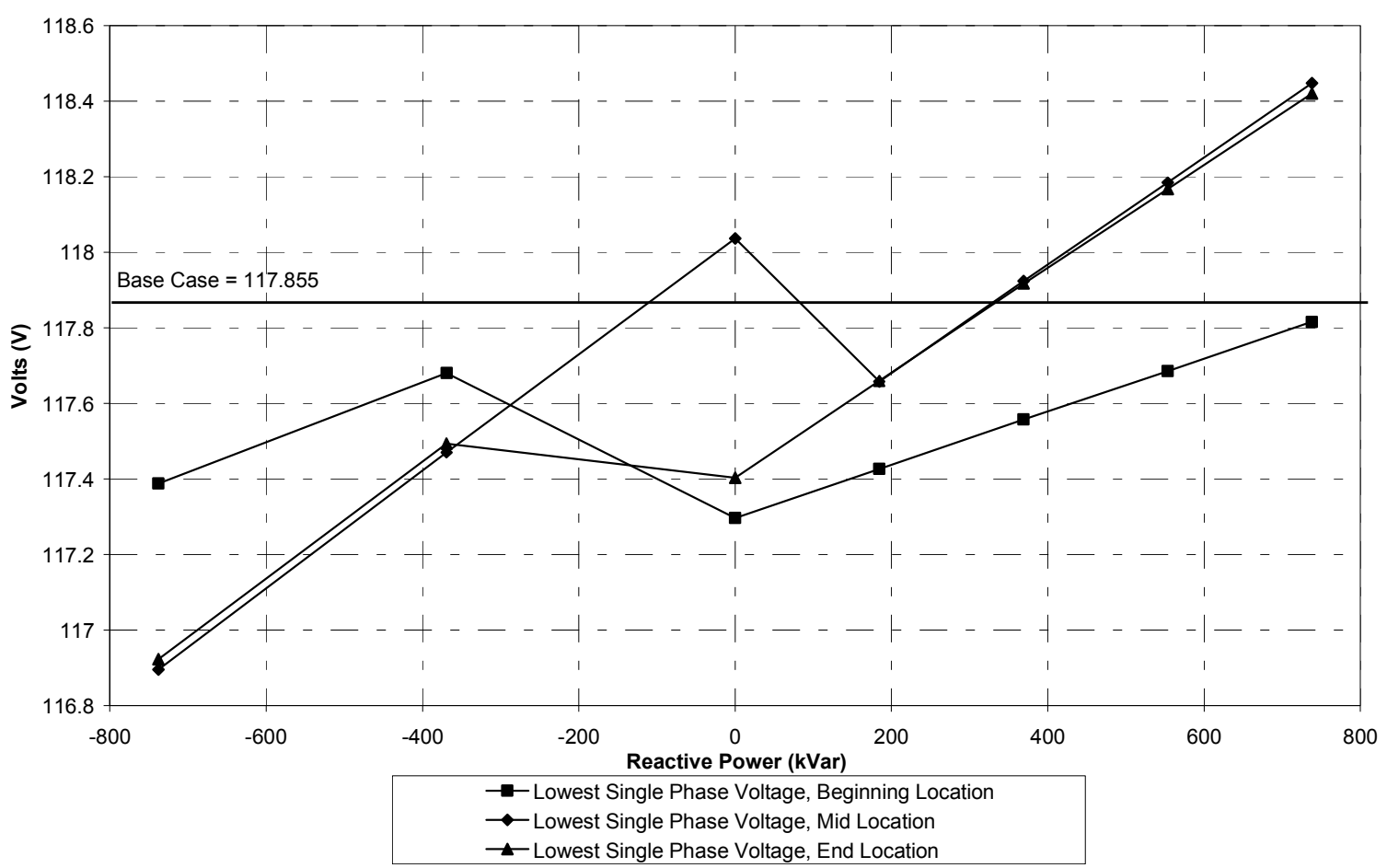

Figure 151. High substation primary voltage, HL condition - voltage rise or drop (in volts on a $120-\mathrm{V}$ base) as a function of location and synchronous generator $+/$ - reactive power with a constant real power output of $246 \mathrm{~kW}$

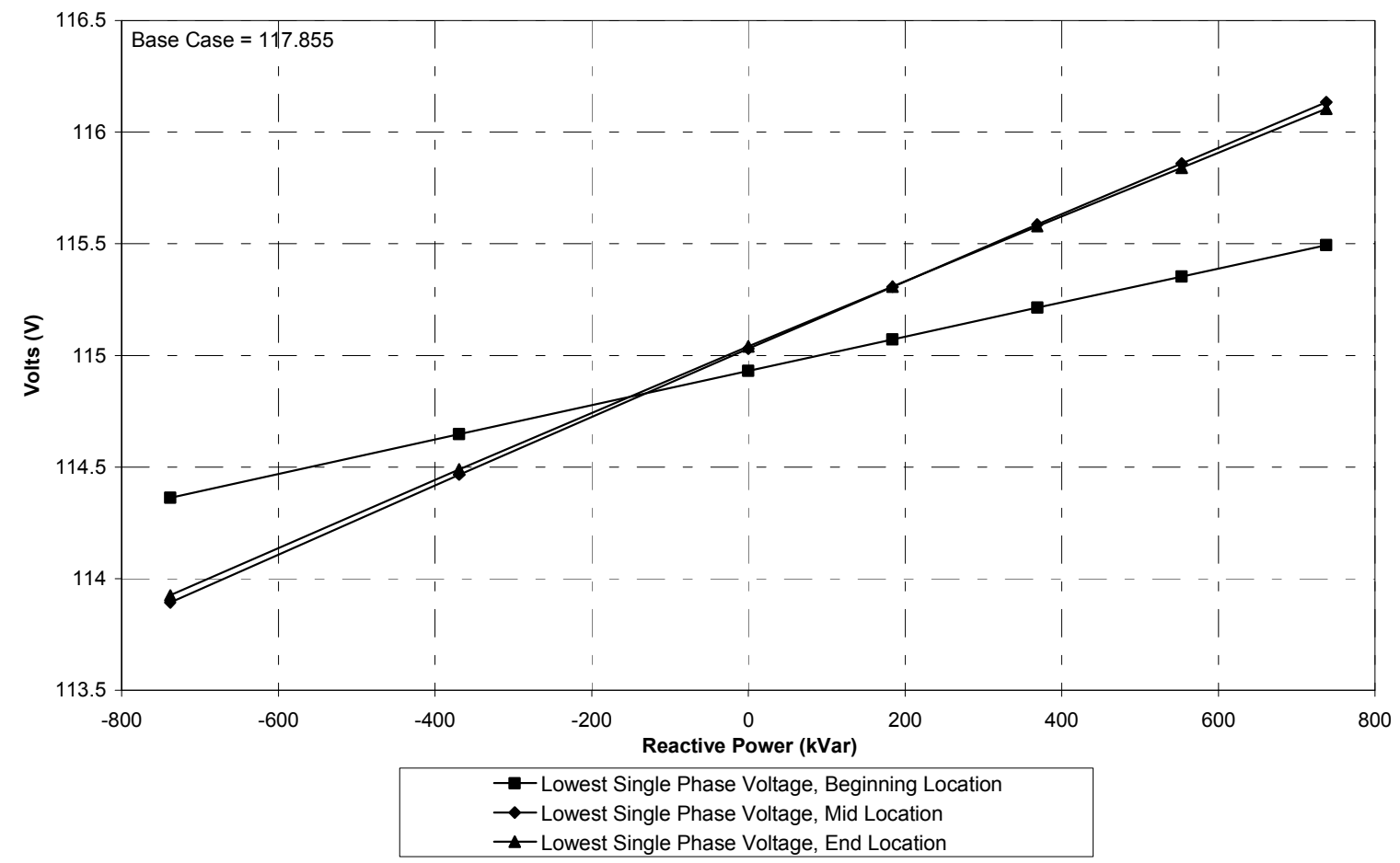

Figure 152. Low substation primary voltage, $\mathrm{HL}$ condition - voltage rise or drop (in volts on a 120-V base) as a function of location and synchronous generator +/- reactive power with a constant real power output of 246 kW 


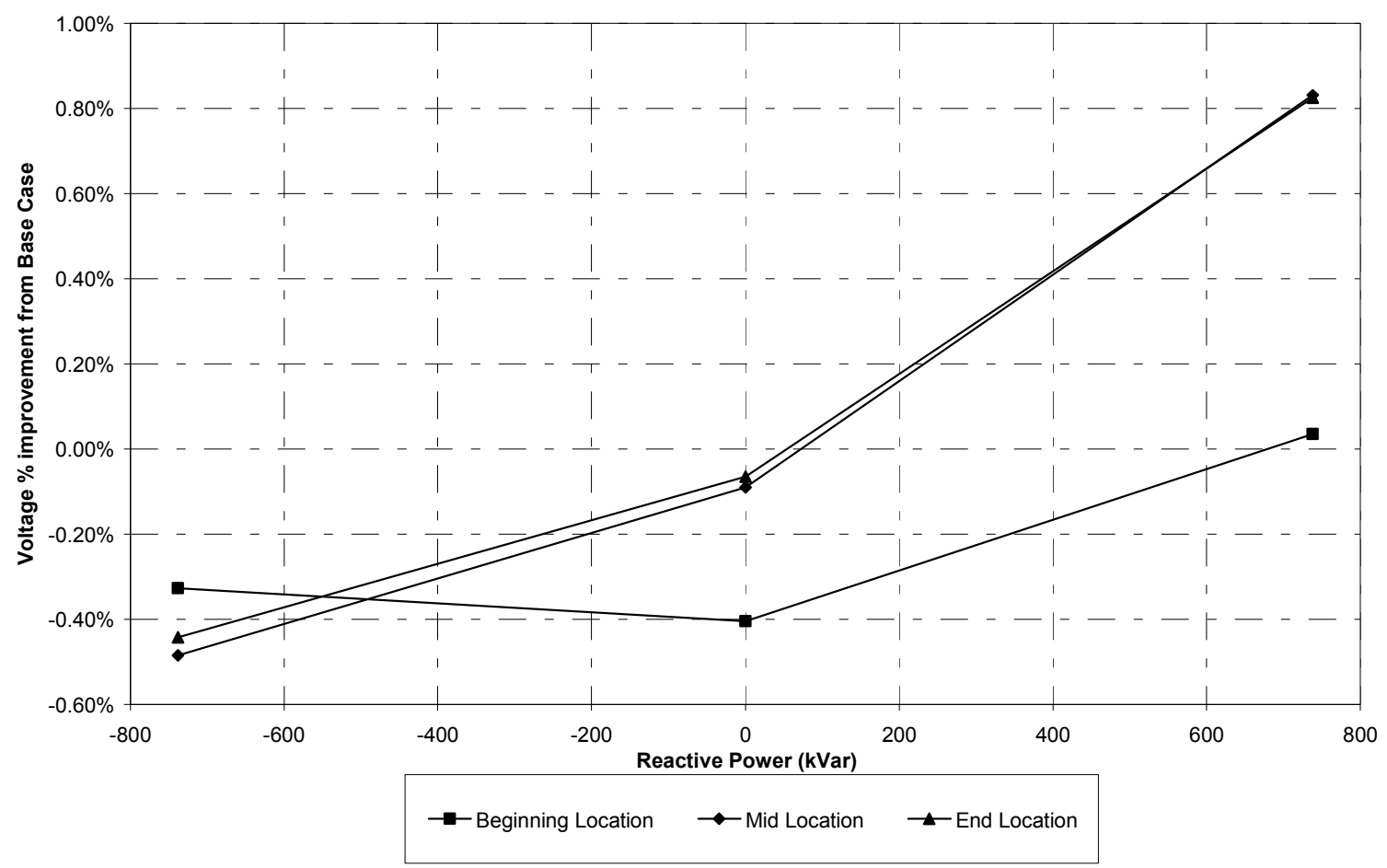

Figure 153. High substation primary voltage, HL condition - reduction or increase in voltage (in volts on a $120-\mathrm{V}$ base) as a function of location and synchronous generator $+/$ - reactive power with a constant real power output of $984 \mathrm{~kW}$

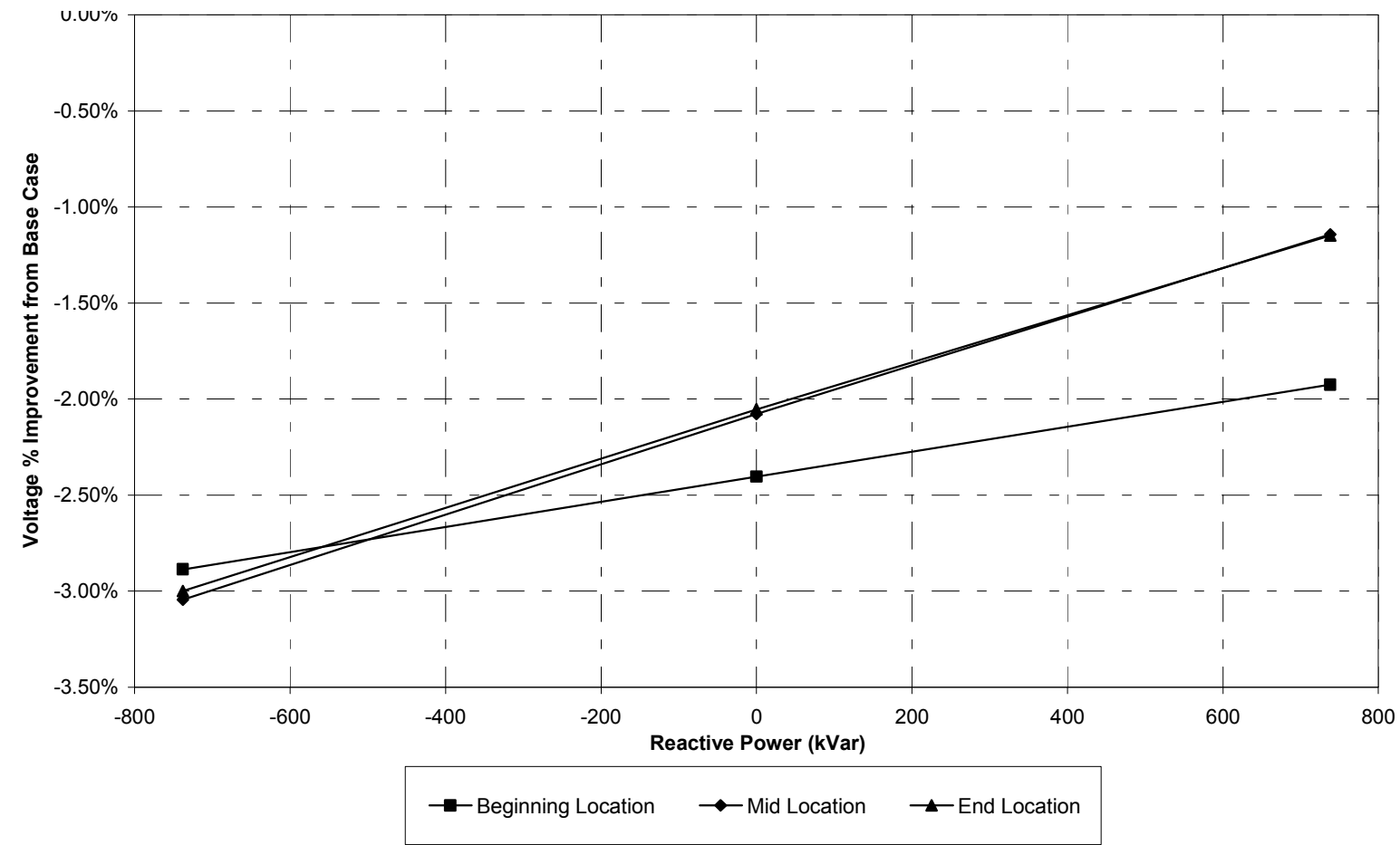

Figure 154. Low substation primary voltage, HL condition - voltage rise or drop (in volts on a $120-\mathrm{V}$ base) as a function of location and synchronous generator $+/$ - reactive power with a constant real power output of $984 \mathrm{~kW}$ 


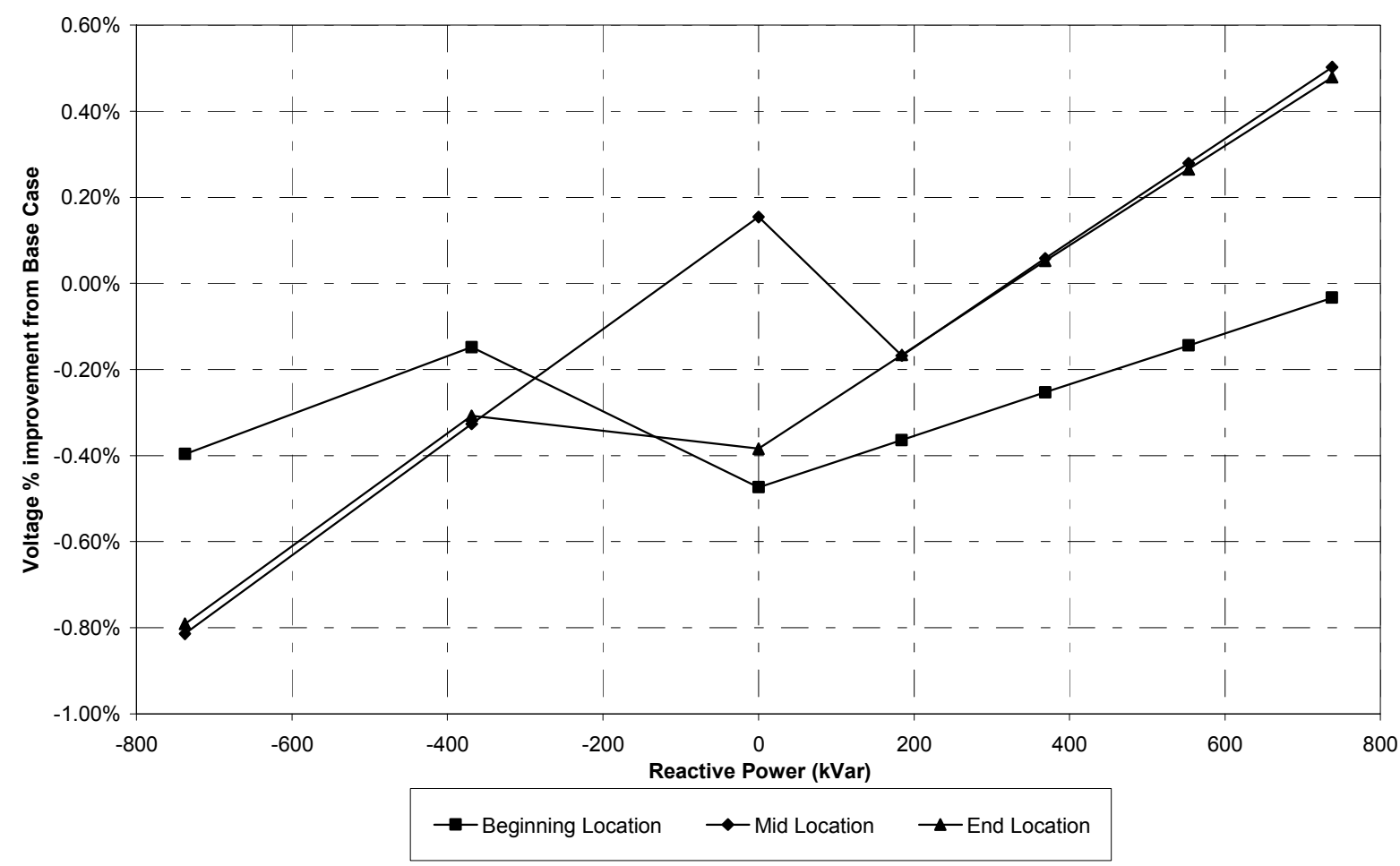

Figure 155. High substation primary voltage, HL condition - reduction or increase in voltage (in volts on $120-\mathrm{V}$ base) as a function of location and synchronous generator +/- reactive power with a constant real power output of $246 \mathrm{~kW}$

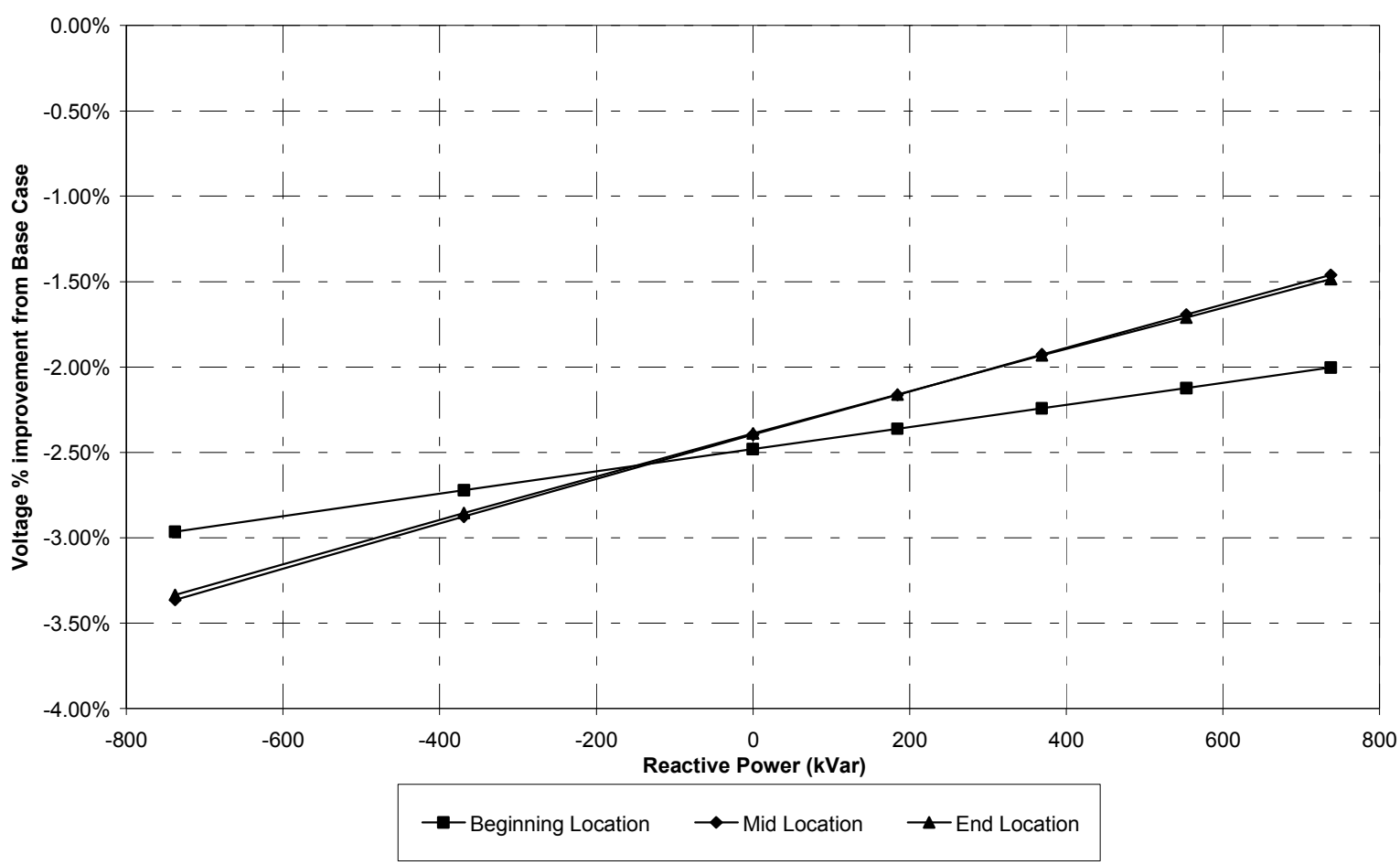

Figure 156. Low substation primary voltage, HL condition - reduction or increase in voltage (in volts on a $120-\mathrm{V}$ base) as a function of location and synchronous generator + /- reactive power with a constant real power output of 246 kW 


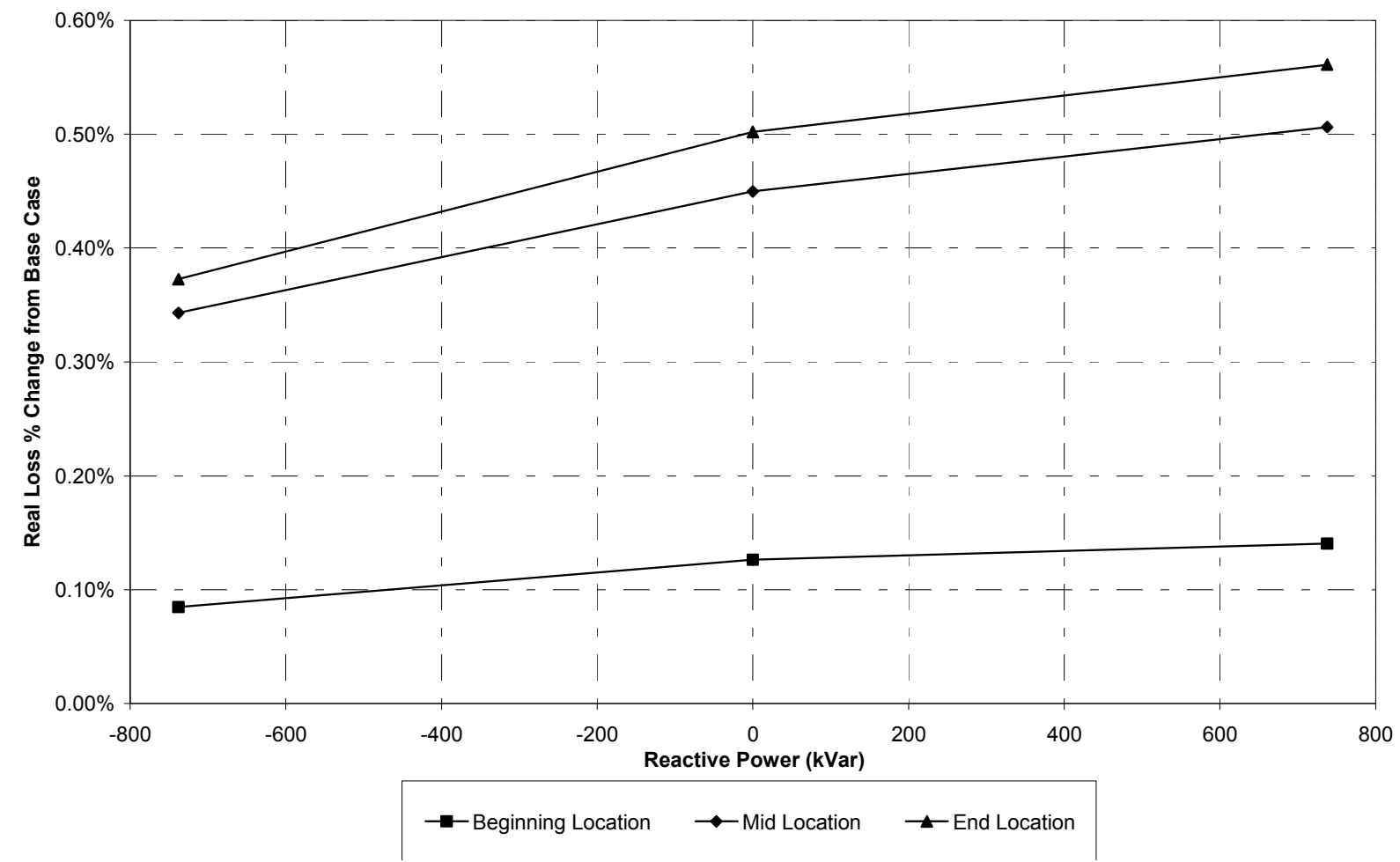

Figure 157. High substation primary voltage, HL condition - reduction or increase of real power loss (in percent total circuit kilowatts) as a function of location and synchronous generator +/- reactive power with a constant real power output of $984 \mathbf{k W}$

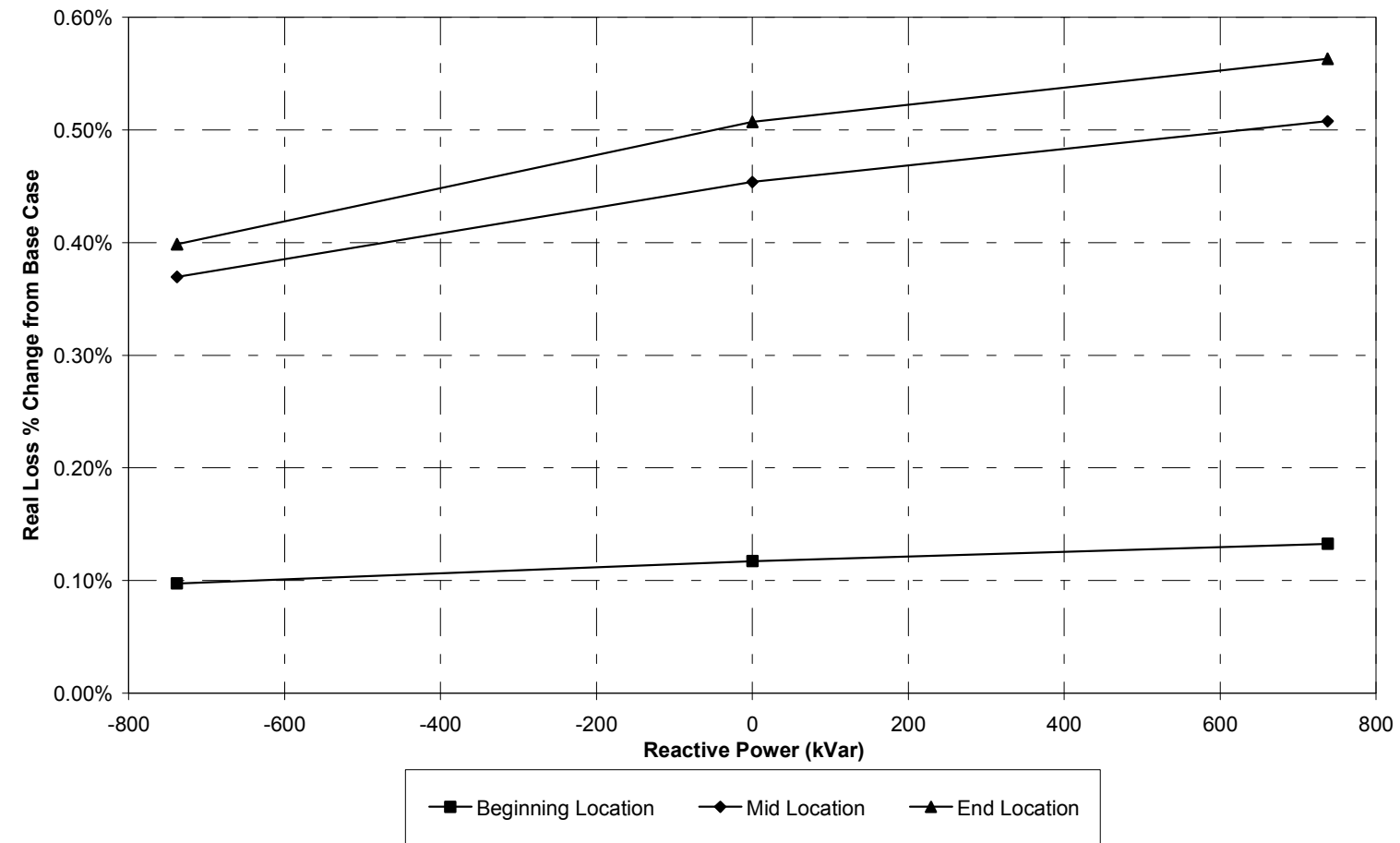

Figure 158. Low substation primary voltage, HL condition - reduction or increase of real power loss (in percent total circuit kilowatts) as a function of location and synchronous generator $+/$ - reactive power with a constant real power output of $984 \mathrm{~kW}$ 


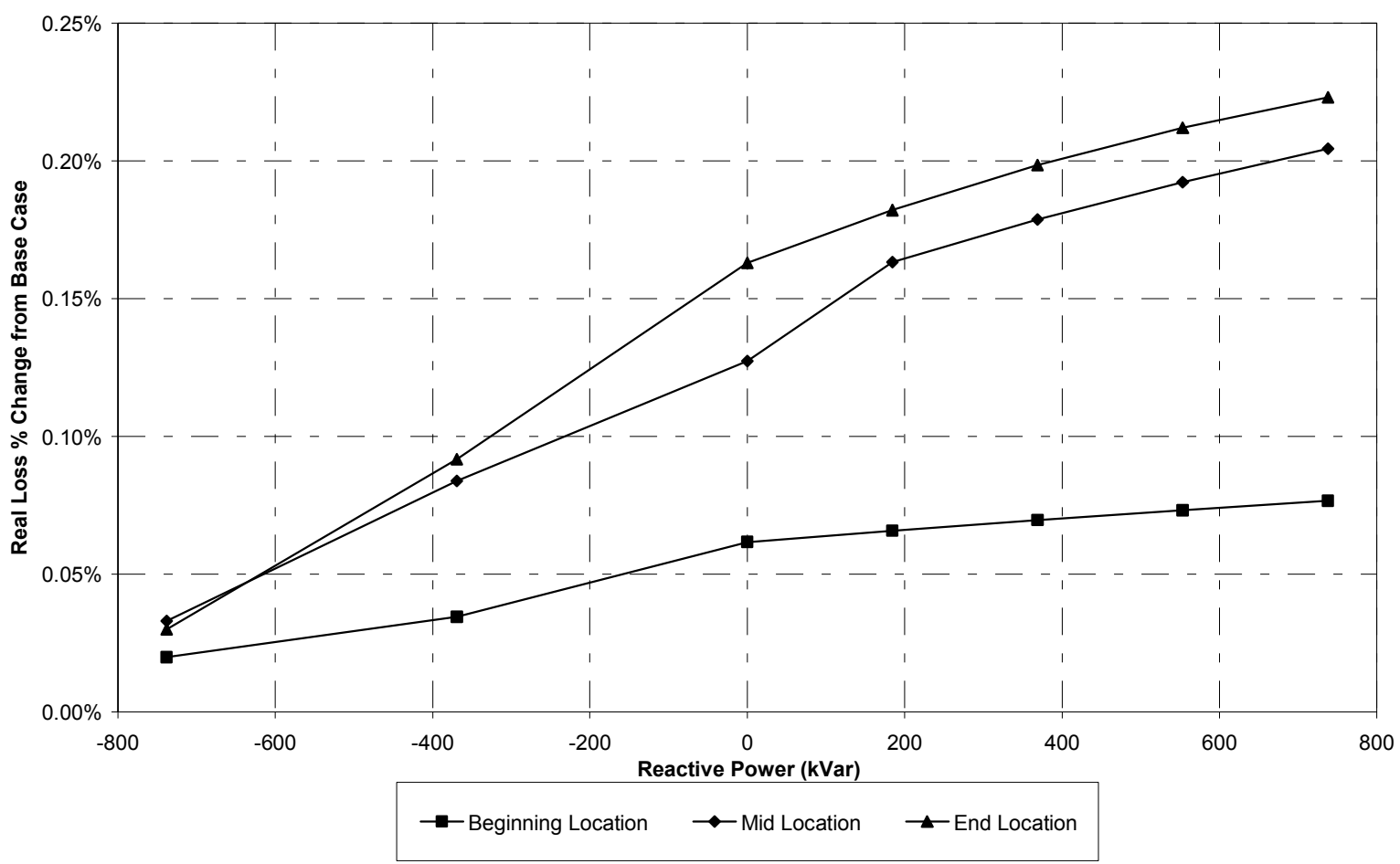

Figure 159. High substation primary voltage, $\mathrm{HL}$ condition - reduction or increase of real power loss (in percent total circuit kilowatts) as a function of location and synchronous generator +/- reactive power with a constant real power output of $246 \mathrm{~kW}$

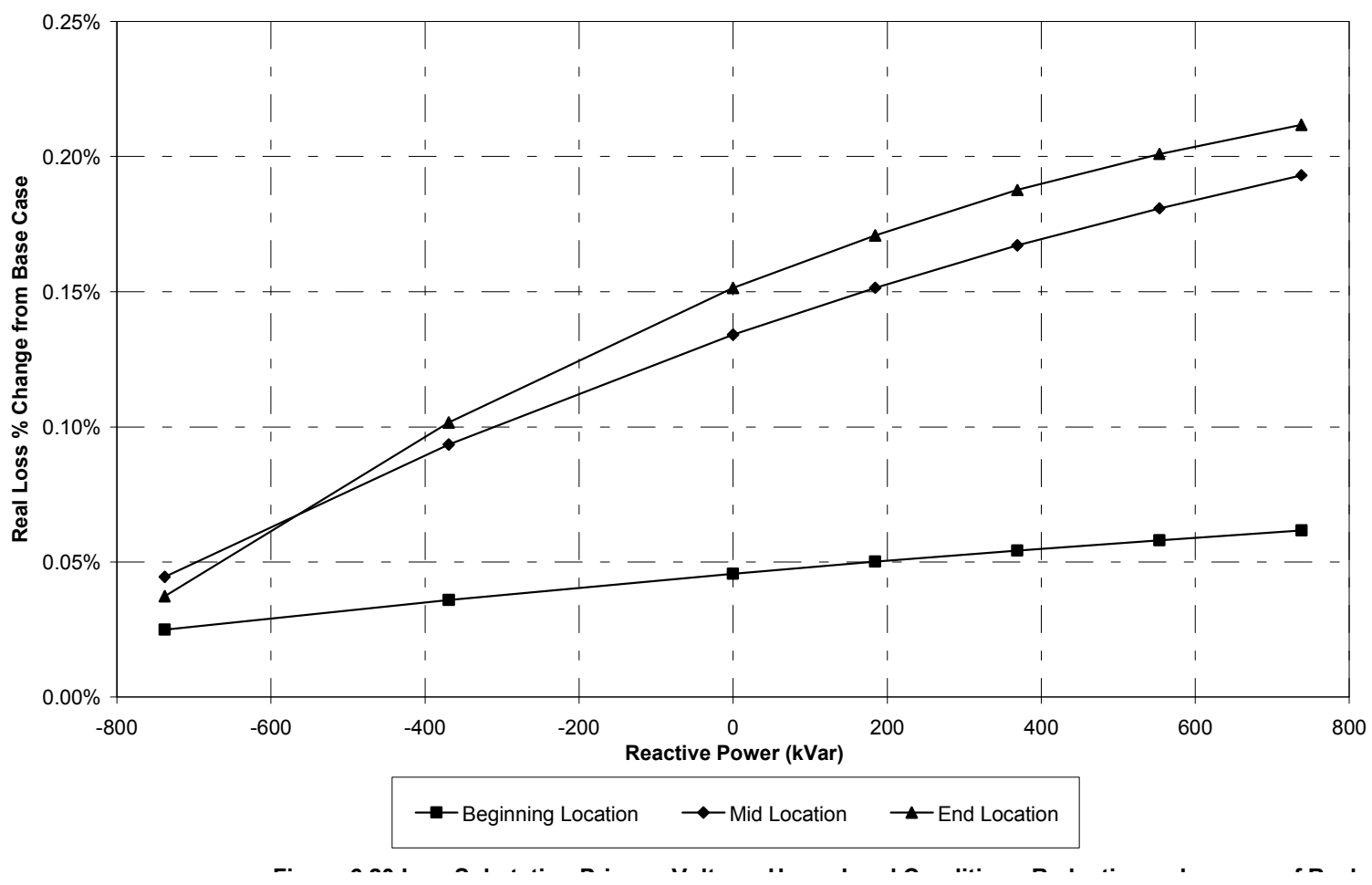

Figure 160. Low substation primary voltage, HL condition - reduction or increase of real power loss (in percent total circuit kilowatts) as a function of location and synchronous generator +/- reactive power with a constant real power output of $246 \mathrm{~kW}$ 


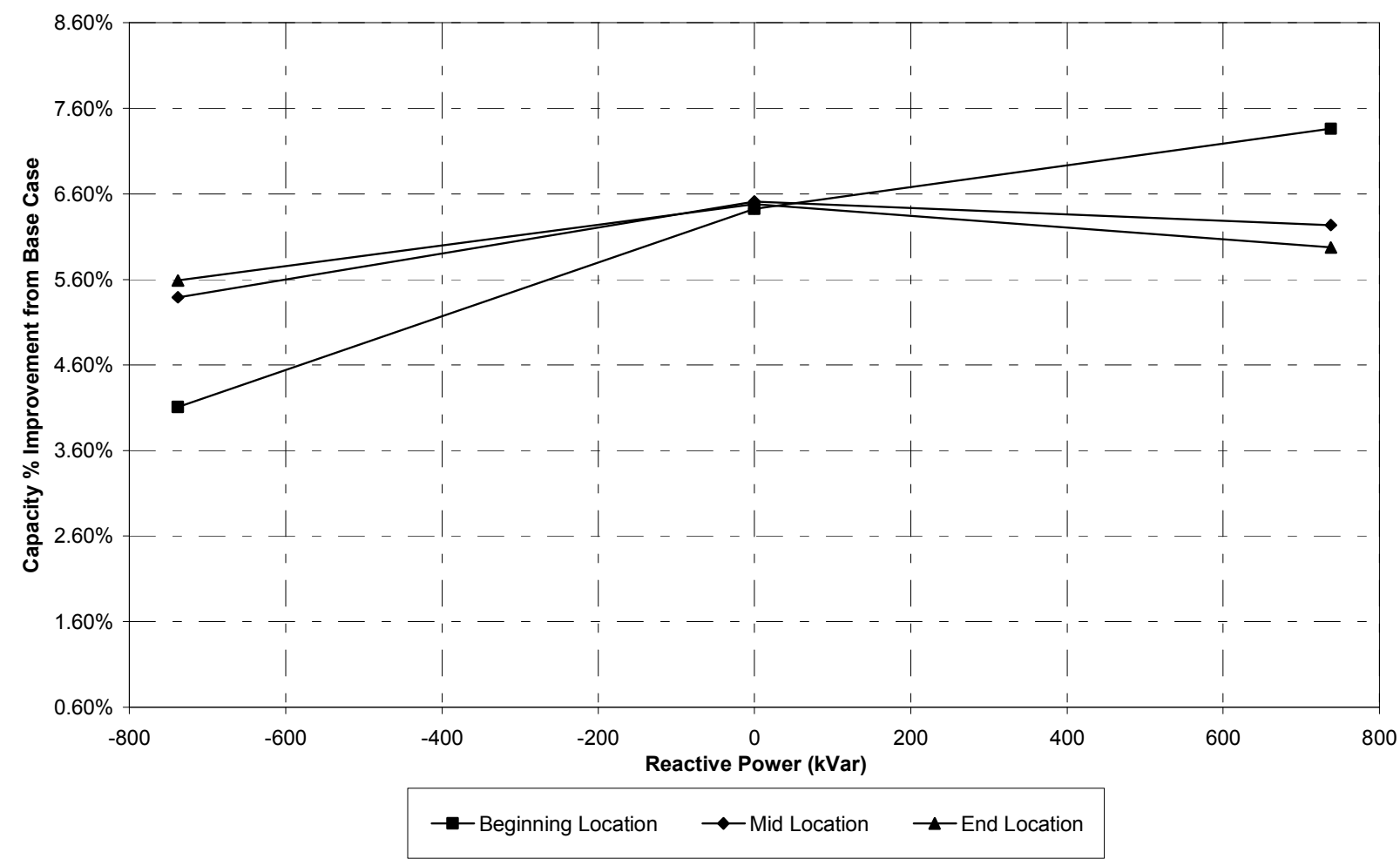

Figure 161. High substation primary voltage, HL condition - reduction or increase of capacity (in percent total circuit kilowatts) as a function of location and synchronous generator +/- reactive power with a constant real power output of 984 kW

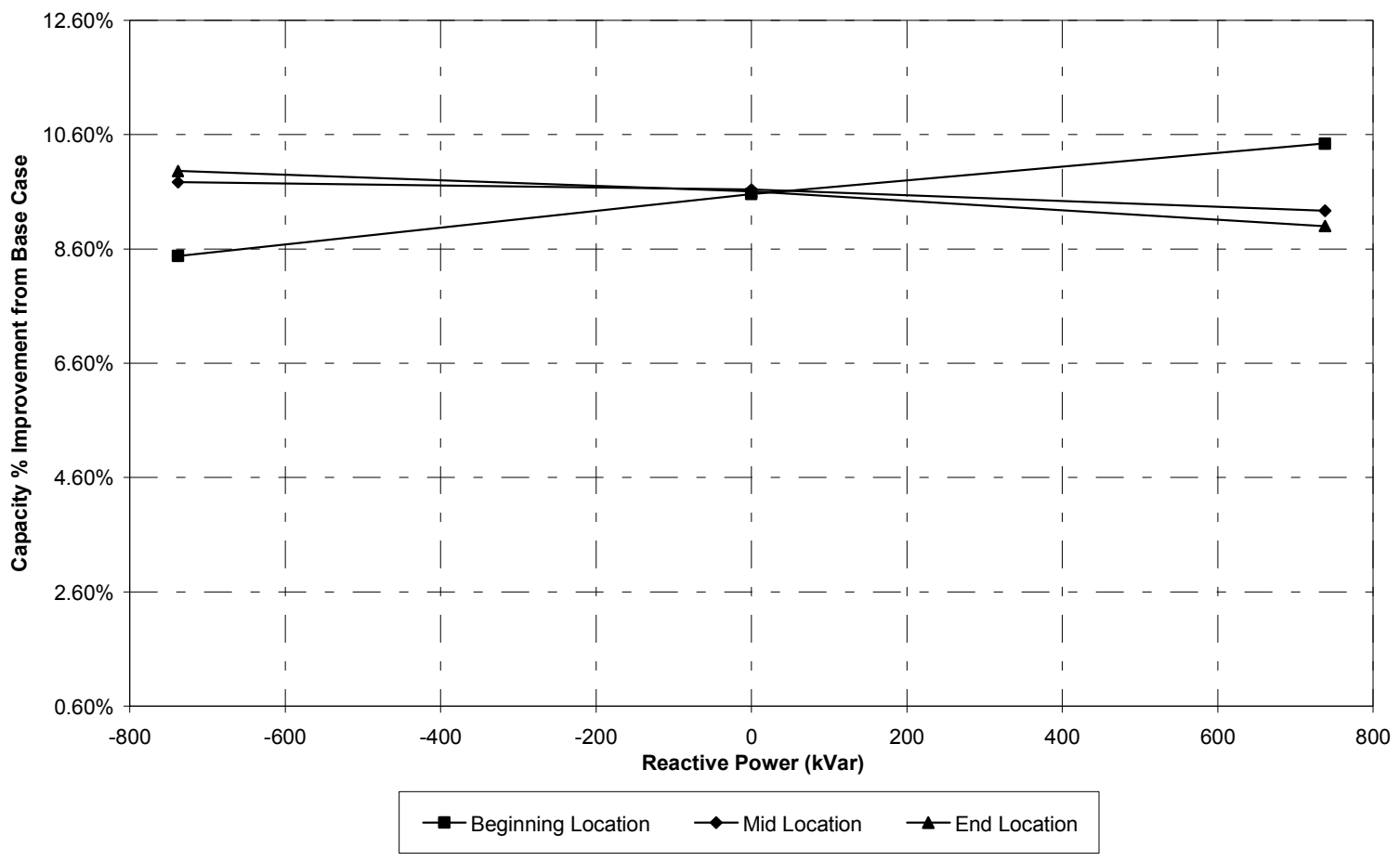

Figure 162. Low substation primary voltage, HL condition - reduction or increase of capacity (in percent total circuit kilowatts) as a function of location and synchronous generator $+/$ - reactive power with a constant real power output of $984 \mathrm{~kW}$ 


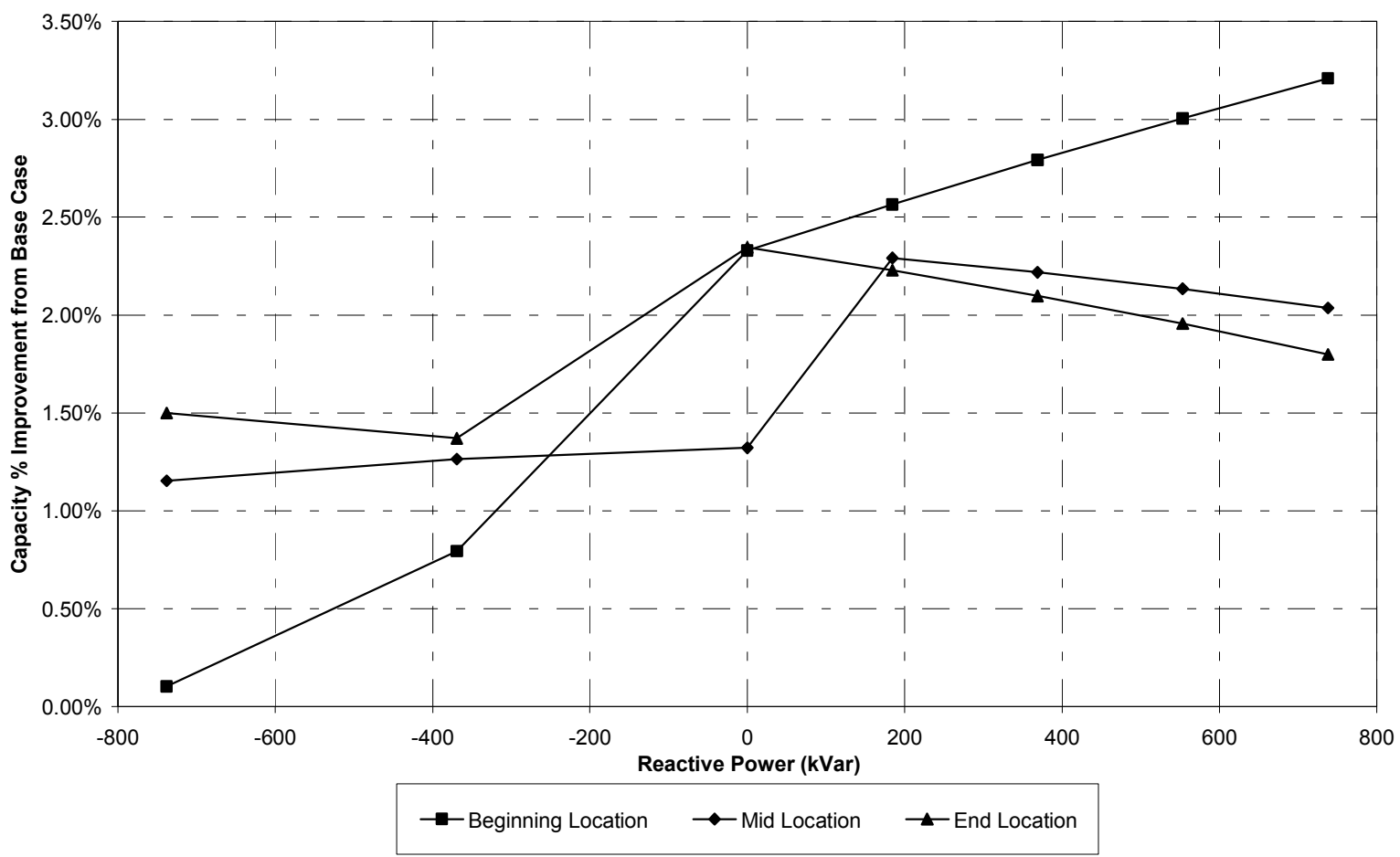

Figure 163. High substation primary voltage, HL condition - reduction or increase of capacity (in percent total circuit kilowatts) as a function of location and synchronous generator $+/$ - reactive power with a constant real power output of $246 \mathrm{~kW}$

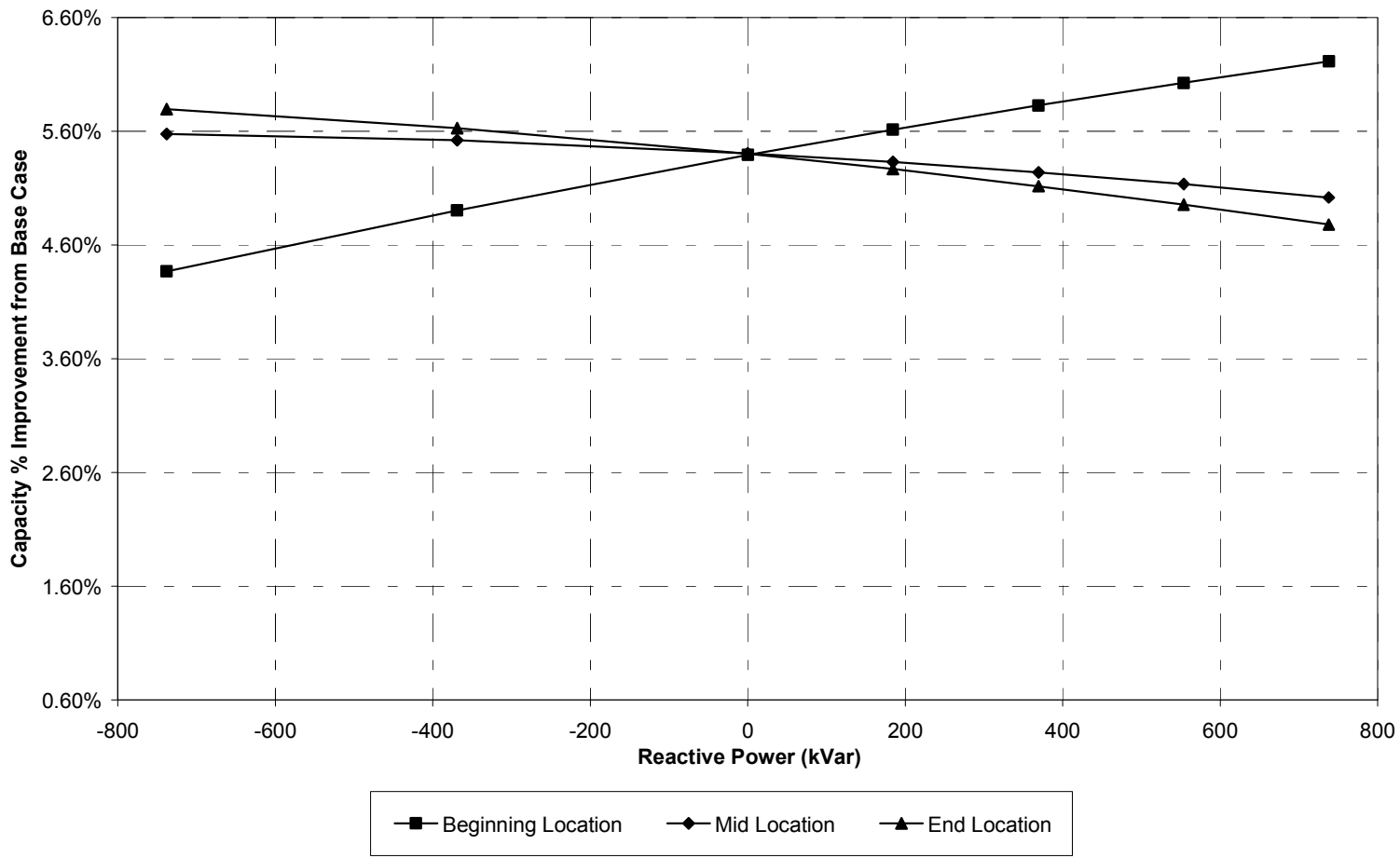

Figure 164. Low substation primary voltage, HL condition - reduction or increase of capacity (in percent total circuit kilowatts) as a function of location and synchronous generator $+/$ - reactive power with a constant real power output of $246 \mathrm{~kW}$ 


\subsection{Findings and Conclusions}

\subsubsection{Synchronous Generator (Low Voltage on Primary of Substation) - Heavy Load Condition}

- Released capacity improvement - BL

The best-case released capacity improvement occurred when the DG was located at the BL of the circuit, with a $10.44 \%$ savings when the synchronous generator was operating at its highest real and reactive power output. This occurred with all LTC regulator taps and step regulator taps in their full-raise position because of the LV condition of $95 \%$ on the primary of the substation. Adding reactive from the generator improves the voltage but causes an increase in kilovolt-ampere load that reduces the released capacity.

There is a $1.8 \%$ change in current for a $1 \%$ change in voltage. When the primary of the substation is operating at $\mathrm{LV}$, or $95 \%$, there is a $5 \%$ drop from nominal $(100 \%)$ that causes a $9 \%(1.8 \% \times 5 \%)$ drop in current. The base case peak load kilovolt-amperes at the start of the circuit without generation are $14,187 \mathrm{~kW}+\mathrm{j} 5846 \mathrm{kVAr}$, or 15,344 $\mathrm{kVA}$. When the generator is running at $984 \mathrm{~kW}+\mathrm{j} 738 \mathrm{kVAr}$, or $1,230 \mathrm{kVA}$, the circuit load drops to $12,347 \mathrm{~kW}+\mathrm{j} 5415 \mathrm{kVArs}$, or $13,482 \mathrm{kVA}$. This example shows a released capacity of

$$
\begin{aligned}
& 15,344 \mathrm{kVA}(\mathrm{w} / \mathrm{o} \text { generation })-13,482 \mathrm{kVA}(\text { with generation })=1,862 \mathrm{kVA} \text { load } \\
& \text { reduction. }
\end{aligned}
$$

The percent load reduction is then

$$
\frac{1,862 \mathrm{kVA}}{15,344 \mathrm{kVA}} \times 100 \%=12.14 \% \text { road reduction. }
$$

The example given is based on balanced circuit loading, so the maximum released capacity of $10.44 \%$ indicates an unbalanced condition exists and, as such, the released capacity will be less because one of the phases has a lower load current than the other two before the generation is added. Therefore, the reduction in load or released capacity will be less on the phase with the lowest load. 
- Loss savings - EL

The greatest reduction in real power losses occurs when the synchronous generator is located at the EL on the circuit, $\mathrm{P}=100 \%$, and $\mathrm{Q}=100 \%$. Here, the loss reduction was $0.56 \%$ better than the base case $(5.7 \%)$. There were marginal loss savings (a range of only $0.02 \%$ to $0.13 \%$ ) when the DG was applied at the head-end of the circuit. Locating the generation at the EL with $100 \%$ reactive and $100 \%$ real power reduces the current magnitude and, hence, the $I^{2} \mathrm{R}$ losses.

- Voltage improvement - ML

The best case for voltage improvement occurs when the synchronous generator is at the ML of the circuit and delivering $100 \%$ real power and $100 \%$ reactive power. The lowest single-phase voltage was $-1.14 \%$ (down from the base case). The EL DG voltage improvement is almost the same (-1.15\% from the base case). The BL DG had voltages about $2 \%-3 \%$ less than the base case. It should be noted that the criteria of $114 \mathrm{~V}$ could not be met under the LV condition on the primary of the substation for customers with secondary and services.

\subsubsection{Synchronous Generator (High Voltage on Primary of Generator Substation) - Heavy Load Condition}

- Released capacity improvement - BL

During HV conditions on the primary of the substation, the highest released capacity of $7.36 \%$ occurs when the synchronous generator is located at the head-end of the circuit, $\mathrm{P}=100 \%$, and $\mathrm{Q}=100 \%$. When the HV condition exists, the load increases so that reactive has to be at its highest value to reduce the load. Notice that the released capacity is lower for the HV case than for the LV case, in which the value was $10.44 \%$. This is because when the voltage increases, the load increases because of the VDC source model, which best represents how the load magnitude changes with changes in voltage.

- Loss savings - EL

The greatest loss savings of $0.56 \%$ was obtained at the EL for the $\mathrm{HV}$ case when $\mathrm{P}=$ $100 \%$ and $\mathrm{Q}=100 \%$. This is the same result as for the LV case. For the LV case, the step regulator taps and the LTC tap were at full raise, and the magnitude of the current being served from the source was reduced because of the added generation. In addition, the $I^{2} \mathrm{R}$ losses are less. In the HV case, the step regulator tap settings and the LTC tap setting were low, which reduced the voltage and, thus, the load. The worst-case loss savings at the BL is only $0.02 \%$, whereas the best case at the BL is $0.14 \%$ at full real and reactive power. This is because the load being served is just offset by the DG power output at the source end, and the load seen by the circuit is essentially the same. 
- Voltage improvement - ML

The best voltage improvement of $0.83 \%$ occurs at the ML when the unit is operated at $\mathrm{P}=100 \%$ and $\mathrm{Q}=100 \%$. The EL had about the same improvement of $0.82 \%$. Notice these voltage improvement levels are better than the LV case, in which the substation primary voltage is $10 \%$ less than the HV level of $105 \%$. This is because the LTC and step regulator are not in the full-raise position, as is the case for the LV condition, and, thus, the step regulator and the LTC raise the voltage. The generator is delivering $100 \%$ reactive power, thus further reducing the voltage drop. It should be noted that the voltage criteria of $114 \mathrm{~V}$ was met during the HV condition.

\subsubsection{Induction Generator for Heavy Load Condition}

- Released capacity improvement

The induction generator is located at the tag end of the circuit, so its released capacity is less than the synchronous generator at the BL. The highest released capacity of $6.44 \%$ occurs during the $\mathrm{LV}$ case when $\mathrm{P}=400 \mathrm{~kW}$ and $\mathrm{Q}=-247.91 \mathrm{kVAr}$. The absorbed kilovars cause the voltage to drop, which causes a lower circuit load, which produces the highest released capacity for the induction generator case. The capacity savings is less than that of the synchronous generator $(10.44 \%)$ because the maximum real power capability of the induction generator is only $400 \mathrm{~kW}$ with a reactive capability of $\pm 247.91 \mathrm{kVAr}$. The maximum kilovolt-ampere capacity is $1,230 \mathrm{kVA}$, and the maximum real power output of the synchronous generator is much bigger$1,050 \mathrm{~kW}(106.7 \%)$ with a reactive capability of $\pm 738 \mathrm{kVAr}$. Obviously, the larger machine will realize a greater released capacity, especially because it is located at the optimum location on the circuit (the BL) for the greatest released capacity.

The induction generator is located at the tag end, which is the least optimum location for released capacity. With the induction generator operating at $400 \mathrm{~kW}$ and a-Q of $247.91 \mathrm{kVAr}$, this caused the voltage to drop and thus lowered the circuit load. The synchronous generator under the LV case had its highest released capacity at the BL of $10.44 \%$ with full-rated real power and full-rated-plus reactive power. Notice that the released capacity for the synchronous generator with zero reactive and maximum real power of $106.7 \%$ was $9.93 \%$, which is not that different from the maximum released capacity of $10.44 \%$. The released capacity for the HV case was only $3.8 \%$ for the induction generator.

- Loss savings

The maximum loss savings of $0.3 \%$ was achieved at $\mathrm{P}=400 \mathrm{~kW}$ and $\mathrm{Q}=247.91$ kVAr during the HV condition because the LTC tap setting was down to 3 and the step regulator tap settings were low $(24,21,19)$. The current on the circuit was lowered with the reactive injection, which caused the losses to be at their lowest. 
- Voltage improvement

The best voltage is obtained under $\mathrm{HV}$ conditions with full reactive and real power, which is $0.12 \%$ better than the base case. With the synchronous machine, the best voltage improvement during $\mathrm{HV}$ conditions is $0.83 \%$ at the ML with full real and reactive power output. Again, the synchronous machine's better improvement in voltage is due to its ability to produce more real and reactive power.

\subsubsection{Inverter Generator for Heavy Load Condition}

- Released capacity improvement

The inverter generator is located near the midpoint of the circuit; therefore, like the induction generator (at the tag end), it will not produce high values of released capacity. Its highest released capacity was $6.29 \%$ at $P=400 \mathrm{~kW}$ and $\mathrm{Q}=0$ under LV conditions, which is essentially the same as the induction generator capacity saving of $6.44 \%$, because their real power capabilities are the same. At HV, the released capacity is about half (3.53\%) because the load increased because of the higher primary voltage.

- Loss savings

It would be expected that the inverter generator would have the highest loss savings at full real power and full reactive to reduce the magnitude of the current to its lowest value. But this is not the case because the real power capability drops down to $320 \mathrm{~kW}$ when the full reactive capability is $240 \mathrm{kVAr}$. Here, the greatest loss saving of $0.24 \%$ occurs at full rated real power of $400 \mathrm{~kW}$ at the $\mathrm{HV}$ condition. This is because the LTC and the step regulator reduced the voltage and lowered the current.

- Voltage improvement

As expected, the best voltage improvement of $0.10 \%$ is realized at full real power of 320 $\mathrm{kW}$ and full reactive power of $240 \mathrm{kVAr}$ for the $\mathrm{HV}$ condition. The voltage improvement is $-1.88 \%$ at the LV condition with full-rated real (320) and reactive (240).

\subsection{Optimum Generator Conditions for Maximum Improvements - Heavy Load Conditions}

To summarize the findings and conclusions, Table 54 shows the optimum generator conditions for released capacity, loss savings, and voltage improvement.

\subsection{Optimum Generator Conditions for Maximum Improvements - Light Load Conditions}

The optimum generator conditions for improvements during light load conditions are given in Table 55. 
Table 54. Optimum Generator Conditions for Maximum Improvements - HL

\begin{tabular}{|c|c|c|c|c|c|c|c|}
\hline \multirow{2}{*}{$\begin{array}{l}\begin{array}{c}\text { Generator } \\
\text { Type }\end{array} \\
\text { Synchronous } \\
\text { generator }\end{array}$} & \multirow{2}{*}{$\begin{array}{l}\begin{array}{c}\text { Substation } \\
\text { Primary } \\
\text { Voltage }\end{array} \\
\text { LV }\end{array}$} & \multicolumn{3}{|c|}{ Optimum Condition } & \multirow{2}{*}{$\begin{array}{c}\begin{array}{c}\text { Released } \\
\text { Capacity } \\
\%\end{array} \\
10.44 \\
\end{array}$} & \multirow[t]{2}{*}{$\begin{array}{l}\text { Loss }^{\mathrm{E}} \\
\text { Reduction \% }\end{array}$} & \multirow[t]{2}{*}{$\begin{array}{c}\text { Voltage }^{\mathrm{E}} \\
\text { Improvement } \%\end{array}$} \\
\hline & & $\mathrm{BL}$ & 100 & 100 & & & \\
\hline & LV & EL & 100 & 100 & & 0.56 & \\
\hline & LV & ML & 100 & 100 & & & $-1.14^{\mathrm{A}}$ \\
\hline & $\mathrm{HV}$ & $\mathrm{BL}$ & 100 & 100 & 7.36 & & \\
\hline & HV & EL & 100 & 100 & & 0.56 & \\
\hline & HV & $\mathrm{ML}$ & 100 & 100 & & & 0.82 \\
\hline & & & $\begin{array}{l}\mathrm{P}, \\
\mathrm{kW}\end{array}$ & $\begin{array}{l}\text { Q, } \\
\text { kVAr }\end{array}$ & & & \\
\hline \multirow[t]{6}{*}{$\begin{array}{l}\text { Induction } \\
\text { generator }\end{array}$} & LV & $\mathrm{EL}^{\mathrm{B}}$ & 400 & -247.9 & 6.44 & & \\
\hline & LV & EL & $400^{\mathrm{B}}$ & $247.9^{B}$ & & 0.25 & \\
\hline & LV & EL & 400 & 247.9 & & & $-2.01^{A}$ \\
\hline & $\mathrm{HV}$ & EL & 400 & 247.9 & 3.80 & & \\
\hline & HV & EL & 400 & 247.9 & & 0.30 & \\
\hline & $\mathrm{HV}$ & EL & 400 & 247.9 & & & 0.12 \\
\hline \multirow[t]{6}{*}{$\begin{array}{l}\text { Inverter } \\
\text { generator }\end{array}$} & LV & $\mathrm{ML}^{\mathrm{C}}$ & 400 & 0 & 6.29 & & \\
\hline & LV & $\mathrm{ML}$ & $320^{\mathrm{D}}$ & $240^{\mathrm{D}}$ & & 0.16 & \\
\hline & LV & $M L$ & 320 & 240 & & & $-1.88^{A}$ \\
\hline & HV & $M L$ & 400 & 0 & 3.53 & & \\
\hline & HV & $\mathrm{ML}$ & 400 & 0 & & 0.24 & \\
\hline & HV & $\mathrm{ML}$ & 320 & 240 & & & 0.10 \\
\hline
\end{tabular}

Notes:

A. The voltage criterion of $114 \mathrm{~V}$ is not met for customers with secondary and services (3.67 $\mathrm{V}$ drop). Only for customers with services is the voltage criterion met.

B. The kilovolt-ampere capability of the induction generator is $400+\mathrm{j} 247.9=470.6 \mathrm{kVA}$. It is located at the tag end of circuit, or EL.

C. The inverter generator location is at the midpoint of the circuit, or ML.

D. The kilovolt-ampere capability of the inverter generator is $320+\mathrm{j} 240=400 \mathrm{kVA}$.

E. The base case energy losses are $5.4 \%$, and the base case lowest single-phase voltage is $117.855 \mathrm{~V}$.

F. The kilovolt-ampere capability of the synchronous machine is $984+\mathrm{j} 738=1,236 \mathrm{kVA}$. The unit is located at midpoint. 
Table 55. Optimum Generator Conditions for Maximum Improvement - LL

\begin{tabular}{|c|c|c|c|c|c|c|c|}
\hline \multirow{2}{*}{$\begin{array}{l}\begin{array}{c}\text { Generator } \\
\text { Type }\end{array} \\
\begin{array}{l}\text { Synchronous } \\
\text { generator }^{\mathrm{E}}\end{array}\end{array}$} & \multirow{2}{*}{$\begin{array}{c}\begin{array}{c}\text { Substation } \\
\text { Primary } \\
\text { Voltage }\end{array} \\
\text { LV }\end{array}$} & Optimum Condition & $\begin{array}{l}\mathrm{Im} \mathrm{Co} \\
\mathrm{P}, \%\end{array}$ & $\begin{array}{r}\text { dition } \\
\text { Q, \% }\end{array}$ & \multirow{2}{*}{$\begin{array}{c}\begin{array}{c}\text { Released } \\
\text { Capacity } \\
\%\end{array} \\
7.40 \\
\end{array}$} & \multirow[t]{2}{*}{$\begin{array}{c}\text { Loss }^{\mathrm{D}} \\
\text { Reduction } \%\end{array}$} & \multirow[t]{2}{*}{$\begin{array}{c}\text { Voltage }^{\mathrm{D}} \\
\text { Improvement } \%\end{array}$} \\
\hline & & $\mathrm{BL}$ & 100 & 100 & & & \\
\hline & LV & EL & 100 & 100 & & 0.66 & \\
\hline & LV & $M L$ & 100 & 100 & & & 1.27 \\
\hline & HV & $\mathrm{BL}$ & 100 & 100 & 7.43 & & \\
\hline & HV & EL & 100 & 100 & & 0.67 & \\
\hline & $\mathrm{HV}$ & ML & 100 & 100 & & & 1.22 \\
\hline & & & $\begin{array}{l}\mathrm{P}, \\
\mathrm{kW}\end{array}$ & $\begin{array}{c}\text { Q, } \\
\text { kVAr }\end{array}$ & & & \\
\hline \multirow[t]{6}{*}{$\begin{array}{l}\text { Induction } \\
\text { generator }\end{array}$} & LV & $E L^{A}$ & 400 & +247.9 & 2.64 & & \\
\hline & LV & EL & $400^{A}$ & $247.9^{\mathrm{A}}$ & & 0.37 & \\
\hline & LV & EL & 400 & 247.9 & & & 0.98 \\
\hline & HV & EL & 400 & 247.9 & 2.89 & & \\
\hline & HV & EL & 400 & 247.9 & & 0.38 & \\
\hline & HV & EL & 400 & 0 & & & 0.67 \\
\hline \multirow[t]{6}{*}{$\begin{array}{l}\text { Inverter } \\
\text { generator }\end{array}$} & LV & $\mathrm{ML}^{\mathrm{B}}$ & 320 & 240 & 2.20 & & \\
\hline & LV & ML & $320^{C}$ & $240^{C}$ & & 0.29 & \\
\hline & LV & ML & 320 & 240 & & & 1.10 \\
\hline & HV & ML & 320 & 0 & 2.19 & & \\
\hline & HV & ML & 320 & 240 & & 0.33 & \\
\hline & HV & ML & 400 & 0 & & & 1.15 \\
\hline
\end{tabular}

Notes:

A. The kilovolt-ampere capability of the induction generator is $400+\mathrm{j} 247.9=470.6 \mathrm{kVA}$. It is located at the tag end of the circuit, or EL.

B. The inverter generator is at the midpoint of the circuit, or ML.

C. The kilovolt-ampere capability of the inverter generator is $320+\mathrm{j} 240=400 \mathrm{kVA}$.

D. The base case energy losses are $3.4 \%$, and the base case lowest single-phase voltage is $121.33 \mathrm{~V}$.

E. The kilovolt-ampere capability of the synchronous machine is $984+j 738=1,236 \mathrm{kVA}$. The unit is located at midpoint. 
Table 56. Induction Generator Voltage Regulation Application (LV, HV) - LL

\begin{tabular}{|c|c|c|c|c|c|c|c|c|c|c|c|c|c|c|c|c|c|c|c|c|}
\hline \multirow[b]{2}{*}{$\begin{array}{c}\text { Machine } \\
\text { Type }\end{array}$} & \multirow[b]{2}{*}{$\begin{array}{l}\text { Real } \\
\text { Power }\end{array}$} & \multirow[b]{2}{*}{\begin{tabular}{|c|} 
Reactive \\
Power
\end{tabular}} & \multirow[b]{2}{*}{ HV / LV } & \multirow[b]{2}{*}{ Location } & \multirow[b]{2}{*}{ LTC Tap } & \multicolumn{3}{|c|}{ Node 01} & \multicolumn{3}{|c|}{ Lowest $3 \Phi$ Voltage } & \multirow{2}{*}{$\begin{array}{c}\text { Lowest } \\
1 \Phi \\
\text { Voltage } \\
\end{array}$} & \multirow[b]{2}{*}{$\begin{array}{l}\text { Highest } \\
12 / 11 \% \\
\end{array}$} & \multirow[b]{2}{*}{$\begin{array}{l}\text { Highest } \\
\text { V2/V1 \% }\end{array}$} & \multirow[b]{2}{*}{$\begin{array}{l}\% \text { kW } \\
\text { Loss }\end{array}$} & \multirow[b]{2}{*}{$\begin{array}{c}\text { Total } \\
\text { kVar Loss }\end{array}$} & \multicolumn{3}{|c|}{ |2/11\% } & \multirow[b]{2}{*}{$\begin{array}{l}\text { Node } 1 \\
\text { Capacity }\end{array}$} \\
\hline & & & & & & IA & IB & IC & VA & VB & vc & & & & & & Node 0 & Low & $\begin{array}{c}\text { High } \\
(<100)\end{array}$ & \\
\hline IG & 400 & 0 & LV & N/A & 16 & $215.5865 \mid$ & 250.8556 & 244.2324 & 123.2152 & 123.0762 & 122.3465 & 121.9696 & 100.1499 & 0.5791 & $3.1 \%$ & 457.01 & 4.9459 & 0.5876 & 85.371 & 62.6147 \\
\hline & & $\frac{1}{\operatorname{VR}(1) 7,7,8}$ & & & & & & & & & & & & & & & & & & \\
\hline IG & 400 & 247.91 & LV & N/A & 16 & 211.3691 & 246.811 & 240.07 & 123.6507 & 123.4998 & 122.7978 & 122.4196 & 100.1498 & 0.5601 & $3.0 \%$ & 438.8 & 5.0728 & 0.5724 & 85.3752 & 63.2174 \\
\hline & & $\operatorname{VR}(1) 7,7,8$ & & & & & & & & & & & & & & & & & & \\
\hline IG & 400 & -247.91 & $\mathrm{LV}$ & & 16 & 220.2762 & 255.3078 & 248.8162 & 122.7733 & 122.6469 & 121.8883 & 121.5126 & 100.1499 & 0.5984 & $3.2 \%$ & 478.96 & 4.8132 & 0.6007 & 85.3667 & 61.9511 \\
\hline & & $\operatorname{VR}(1) 7,7,8$ & & & & & & & & & & & & & & & & & & \\
\hline IG & 400 & 0 & $\mathrm{HV}$ & $\mathrm{N} / \mathrm{A}$ & 0 & 215.6606 & 250.9707 & 244.3936 & 123.2559 & 123.1314 & 122.4251 & 122.048 & 100.1499 & 0.57 & $3.1 \%$ & 436.17 & 4.9564 & 0.5879 & 85.3752 & 62.5975 \\
\hline & & $\mathrm{VR}(1) 7$ & & & & & & & & & & & & & & & & & & \\
\hline IG & 400 & \begin{tabular}{|l|}
247.91 \\
\end{tabular} & $\mathrm{HV}$ & $\mathrm{N} / \mathrm{A}$ & -1 & 209.897 & 245.1622 & 238.5137 & 122.8626 & 122.7266 & 122.0544 & 121.6783 & 100.1497 & 0.5505 & $3.0 \%$ & 411.4 & 5.0894 & 0.5725 & 85.3793 & 63.4632 \\
\hline & & $\operatorname{VR}(1) 7,7$, & & & & & & & & & & & & & & & & & & \\
\hline IG & 400 & $|-247.91|$ & $\mathrm{HV}$ & $\mathrm{N} / \mathrm{A}$ & 0 & 220.6714 & 255.7775 & 249.293 & 122.8241 & 122.7128 & 121.9782 & 121.6023 & 100.1499 & 0.5895 & $3.1 \%$ & 458.58 & 4.8202 & 0.6019 & 85.371 & 61.8811 \\
\hline & & & & & & & & & & & & & & & & & & & & \\
\hline
\end{tabular}

\begin{tabular}{|c|c|c|c|c|c|c|c|}
\hline \multicolumn{8}{|c|}{ Improvement } \\
\hline $\begin{array}{c}\text { Machine } \\
\text { Type }\end{array}$ & $\begin{array}{l}\text { Real } \\
\text { Power }\end{array}$ & $\begin{array}{c}\text { Reactive } \\
\text { Power }\end{array}$ & HV / LV & Location & $\begin{array}{l}\text { Lowest } \\
\text { Voltage }\end{array}$ & $\begin{array}{c}\text { kW } \\
\text { Losses }\end{array}$ & $\begin{array}{l}\text { Node } 1 \\
\text { Capacity }\end{array}$ \\
\hline IG & 400 & 0 & $\mathrm{LV}$ & N/A & $0.61 \%$ & $0.31 \%$ & $2.04 \%$ \\
\hline & & $\operatorname{VR}(1) 7,7$, & & & & & \\
\hline IG & 400 & 247.91 & LV & $\mathrm{N} / \mathrm{A}$ & $0.98 \%$ & $0.37 \%$ & $2.64 \%$ \\
\hline & & VR (1) 7, 7, & & & & & \\
\hline IG & 400 & \begin{tabular}{|l|}
-247.91 \\
\end{tabular} & LV & $\mathrm{N} / \mathrm{A}$ & $0.23 \%$ & $0.23 \%$ & $1.38 \%$ \\
\hline & & $\operatorname{VR}(1) 7,7$, & & & & & \\
\hline IG & 400 & 0 & $\mathrm{HV}$ & $\mathrm{N} / \mathrm{A}$ & $0.67 \%$ & $0.33 \%$ & $2.02 \%$ \\
\hline & & VR (1) 7,7, & & & & & \\
\hline IG & 400 & \begin{tabular}{|l|l|}
247.91 \\
\end{tabular} & $\mathrm{HV}$ & N/A & $0.37 \%$ & $0.38 \%$ & $2.89 \%$ \\
\hline & & VR (1) 7,7, & & & & & \\
\hline IG & 400 & \begin{tabular}{|l|}
-247.91 \\
\end{tabular} & $\mathrm{HV}$ & $\mathrm{N} / \mathrm{A}$ & $0.30 \%$ & $0.25 \%$ & $1.31 \%$ \\
\hline & & $\operatorname{VR}(1) 8,8$, & & & & & \\
\hline
\end{tabular}


Table 57. Inverter Generator Voltage Regulation Application (LV, HV) - LL

\begin{tabular}{|c|c|c|c|c|c|c|c|c|c|c|c|c|c|c|c|c|c|c|c|c|}
\hline \multirow[b]{2}{*}{$\begin{array}{c}\text { Machine } \\
\text { Type }\end{array}$} & \multirow[b]{2}{*}{$\begin{array}{l}\text { Real } \\
\text { Power }\end{array}$} & \multirow[b]{2}{*}{$\begin{array}{c}\text { Reactive } \\
\text { Power }\end{array}$} & \multirow[b]{2}{*}{ HV / LV } & \multirow[b]{2}{*}{ Location } & \multirow[b]{2}{*}{ LTC Tap } & \multicolumn{3}{|c|}{ Node 01} & \multicolumn{3}{|c|}{ Lowest $3 \Phi$ Voltage } & \multirow{2}{*}{\begin{tabular}{c|} 
Lowest \\
$1 \Phi$ \\
Voltage \\
\end{tabular}} & \multirow[b]{2}{*}{$\begin{array}{c}\text { Highest } \\
\text { |2/11\% }\end{array}$} & \multirow[b]{2}{*}{$\begin{array}{l}\text { Highest } \\
\text { V2N1 }\end{array}$} & \multirow[b]{2}{*}{$\begin{array}{l}\% \text { kW } \\
\text { Loss }\end{array}$} & \multirow[b]{2}{*}{$\begin{array}{c}\text { Total } \\
\text { kVar Loss }\end{array}$} & \multicolumn{3}{|c|}{$12 / 1 \%$} & \multirow[b]{2}{*}{$\begin{array}{l}\text { Node } 1 \\
\text { Capacity }\end{array}$} \\
\hline & & & & & & IA & IB & IC & VA & 然 & vc & & & & & & Node 0 & Low & $\begin{array}{l}\text { High } \\
(<100)\end{array}$ & \\
\hline InvG & 400 & \begin{tabular}{l|l}
0 \\
0
\end{tabular} & $\mathrm{LV}$ & $\mathrm{N} / \mathrm{A}$ & 16 & 215.7648 & 251.0805 & 244.2842 & 123.3158 & 123.0805 & 122.3867 & 122.0019 & 100.1498 & 0.5704 & $3.1 \%$ & 467.04 & 4.9222 & 0.4231 & 75.2233 & 62.5812 \\
\hline & & $\mathrm{R}(1) 7,7,8$ & & & & & & & & & & & & & & & & & & \\
\hline $\operatorname{lnvG}$ & 320 & 240 & $\overline{\mathrm{LV}}$ & $\mathrm{N} / \mathrm{A}$ & $\frac{16}{16}$ & 214.3897 & 249.7643 & 242.9851 & 123.8584 & 123.6347 & 122.9837 & 122.5691 & 189.5674 & 0.5357 & $3.1 \%$ & 461.97 & 4.9736 & 0.4169 & 99.0905 & 62.7773 \\
\hline & & $R(1) 7,7,8$ & & & & & & & & & & & & & & & & & & \\
\hline InvG & 320 & -240 & LV & N/A & 16 & 223.2678 & 258.5274 & 251.7314 & 122.6213 & 122.4607 & 121.6974 & 121.361 & 100.1499 & 0.6131 & $3.2 \%$ & 495.7 & 4.7719 & 0.4153 & 73.2693 & 61.4713 \\
\hline . mive & $0 \angle 0$ & $\frac{1}{R(1) 7,7,8}$ & & & & & & 201.1014 & & 122.4007 & 127.0574 & 121.001 & 100.1495 & 0.0101 & 3.210 & & 4.1715 & 0.4150 & 13.2090 & 01.4710 \\
\hline InvG & 400 & \begin{tabular}{|l|l|}
0 \\
\end{tabular} & $\mathrm{HV}$ & N/A & 0 & 215.8399 & 251.1973 & 244.4464 & 123.3571 & 123.1364 & 122.4658 & 122.0808 & 100.1498 & 0.5606 & $3.1 \%$ & 446.17 & 4.9327 & 0.4232 & 75.2366 & 62.5637 \\
\hline & & $R(1) 7,7,8$ & & & & & & & & & & & & & & & & & & \\
\hline $\operatorname{InvG}$ & 320 & \begin{tabular}{|l|l|}
240 & \\
\end{tabular} & $\mathrm{HV}$ & $\mathrm{N} / \mathrm{A}$ & 0 & 214.4339 & 249.8456 & 243.1133 & 123.8825 & 123.6733 & 123.0455 & 122.6307 & 189.2126 & 0.5253 & $3.1 \%$ & 441.2 & 4.9842 & 0.4169 & 98.9889 & 62.7652 \\
\hline & & $\mathrm{R}(1) 7,7,8$ & & & & & & & & & & & & & & & & & & \\
\hline $\operatorname{InvG}$ & 320 & \begin{tabular}{|l|l|l|}
-240 & \\
\end{tabular} & $\mathrm{HV}$ & N/A & 0 & 223.374 & 258.6802 & 251.9266 & 122.6821 & 122.5365 & 121.7965 & 121.4598 & 100.1499 & 0.6034 & $3.2 \%$ & 473.61 & 4.7821 & 0.4166 & 73.2757 & 61.4486 \\
\hline & & $/ \mathrm{R}(1) 7,7,8$ & & & & & & & & & & & & & & & & & & \\
\hline
\end{tabular}

\begin{tabular}{|c|c|c|c|c|c|c|c|}
\hline \multicolumn{8}{|c|}{ Improvement } \\
\hline $\begin{array}{l}\text { Machine } \\
\text { Type }\end{array}$ & $\begin{array}{l}\text { Real } \\
\text { Power }\end{array}$ & $\begin{array}{l}\text { Reactive } \\
\text { Power }\end{array}$ & HV / LV & Location & $\begin{array}{l}\text { Lowest } \\
\text { Voltage }\end{array}$ & Losses & $\begin{array}{l}\text { Node 1 } \\
\text { Capacity }\end{array}$ \\
\hline $\ln v G$ & 400 & 0 & $\mathrm{LV}$ & N/A & $0.63 \%$ & $0.28 \%$ & $2.01 \%$ \\
\hline & & $\operatorname{VR}(1) 7,7$, & & & & & \\
\hline $\operatorname{lnvG}$ & 320 & 240 & LV & $\mathrm{N} / \mathrm{A}$ & $1.10 \%$ & $0.29 \%$ & $2.20 \%$ \\
\hline & & VR (1) 7,7, & & & & & \\
\hline $\operatorname{lnvG}$ & 320 & $\begin{array}{l}-240 \\
\end{array}$ & LV & N/A & $0.10 \%$ & $0.18 \%$ & $0.90 \%$ \\
\hline & & $\operatorname{VR}(1) 7,7$ & & & & & \\
\hline $\operatorname{lnvG}$ & 400 & 0 & $\mathrm{HV}$ & $\mathrm{N} / \mathrm{A}$ & $0.70 \%$ & $0.30 \%$ & $1.99 \%$ \\
\hline & & VR (1) 7,7, & & & & & \\
\hline $\operatorname{lnvG}$ & 320 & 240 & $\mathrm{HV}$ & $\mathrm{N} / \mathrm{A}$ & $1.15 \%$ & $0.33 \%$ & $2.19 \%$ \\
\hline & & $\operatorname{VR}(1) 7,7$, & & & & & \\
\hline $\operatorname{lnvG}$ & 320 & -240 & $\mathrm{HV}$ & N/A & $0.19 \%$ & $0.22 \%$ & $0.87 \%$ \\
\hline & & VR (1) 7,7, & & & & & \\
\hline
\end{tabular}


Table 58. Synchronous Generator Voltage Regulation Application (BL, HV) - LL

\begin{tabular}{|c|c|c|c|c|c|c|c|c|c|c|c|c|c|c|c|c|c|c|c|c|}
\hline \multirow[b]{2}{*}{$\begin{array}{c}\text { Machine } \\
\text { Type }\end{array}$} & \multirow[b]{2}{*}{$\begin{array}{l}\text { Real } \\
\text { Power }\end{array}$} & \multirow[b]{2}{*}{$\begin{array}{c}\text { Reactive } \\
\text { Power }\end{array}$} & \multirow[b]{2}{*}{ HV / LV } & \multirow[b]{2}{*}{ Location } & \multirow[b]{2}{*}{ LTC Tap } & \multicolumn{3}{|c|}{ Node 01} & \multicolumn{3}{|c|}{ Lowest $3 \Phi$ Voltage } & \multirow{2}{*}{$\begin{array}{c}\text { Lowest } \\
1 \Phi \\
\text { Voltage } \\
\end{array}$} & \multirow[b]{2}{*}{$\begin{array}{l}\text { Highest } \\
12 / 11 \%\end{array}$} & \multirow[b]{2}{*}{$\begin{array}{l}\text { Highest } \\
\text { V2/V1\% }\end{array}$} & \multirow[b]{2}{*}{$\begin{array}{l}\% \mathrm{~kW} \\
\text { Loss }\end{array}$} & \multirow[b]{2}{*}{$\begin{array}{c}\text { Total } \\
\text { kVar Loss }\end{array}$} & \multicolumn{3}{|c|}{$12 / 1 \%$} & \multirow[b]{2}{*}{$\begin{array}{l}\text { Node 1 } \\
\text { Capacity }\end{array}$} \\
\hline & & & & & & IA & IB & IC & VA & VB & vc & & & & & & Node 0 & Low & $\begin{array}{l}\text { High } \\
(<100)\end{array}$ & \\
\hline SG & $50 \%$ & 0 & $\mathrm{HV}$ & $\mathrm{BL}$ & 0 & 212.8733 & 248.4197 & 241.7729 & 123.0971 & 123.0572 & 122.3328 & 121.9559 & 100.15 & 0.5814 & $3.2 \%$ & 464.51 & 5.0537 & 2.4249 & 85.3796 & 62.9777 \\
\hline & & (1) 7,7 , & & & & & & & & & & & & & & & & & & \\
\hline SG & $75 \%$ & 0 & HV & $\mathrm{BL}$ & 0 & 204.3709 & 239.9243 & \begin{tabular}{|l|l|}
233.3379 \\
\end{tabular} & 123.117 & 123.0771 & 122.3526 & 121.9756 & 100.15 & 0.5814 & $3.2 \%$ & 448.7 & 5.2441 & 2.4249 & 85.3796 & 64.2438 \\
\hline & & $\frac{\operatorname{VR}(1) 7,7,8}{0}$ & & & & & 2315685 & & & & & & & & & & & & & \\
\hline SG & $100 \%$ & 0 & HV & $\mathrm{BL}$ & 0 & 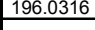 & & 225.0531 & 123.1365 & \begin{tabular}{|l|l}
123.0966 \\
\end{tabular} & 122.372 & 121.995 & 100.15 & 0.5813 & $3.2 \%$ & 433.75 & 5.4457 & 2.4249 & 85.3797 & 65.489 \\
\hline SG & $106.7 \%$ & 0 & HV & BL & 0 & 193.8247 & 229.3523 & \begin{tabular}{|l|l|}
222.8581 \\
\end{tabular} & 123.1417 & 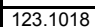 & \begin{tabular}{|l|}
122.372 \\
\end{tabular} & 122.0001 & 100.15 & 0.5813 & $3.2 \%$ & 429.88 & 5.5017 & 2.4249 & 85.3797 & 65.8193 \\
\hline & & $\mathrm{VR}(1) 7,7$ & & & & & & & & & & & & & & & & & & \\
\hline SG & $100 \%$ & $100 \%$ & $\mathrm{HV}$ & $\mathrm{BL}$ & -1 & 179.4087 & 214.7065 & 207.7869 & 122.9478 & 122.9088 & 122.1866 & 121.8101 & 100.1499 & 0.5809 & $3.1 \%$ & 403.36 & 5.8977 & 2.4257 & 85.3798 & 68.002 \\
\hline & & & & & & & & & & & & & & & & & & & & \\
\hline SG & $100 \%$ & $\frac{-100 \%}{11) 7}$ & HV & $\mathrm{BL}$ & 0 & 214.3268 & 249.4135 & 243.3793 & 122.507 & 122.4672 & 121.746 & 121.3707 & 100.1499 & 0.5816 & $2.8 \%$ & 464.99 & 5.0029 & 2.4248 & 85.3794 & 62.8296 \\
\hline SG & $25 \%$ & $100 \%$ & $\mathrm{HV}$ & BL & $\begin{array}{ll}-1 \\
\end{array}$ & 206.9496 & 241.9569 & 234.9388 & 122.8884 & \begin{tabular}{|l|}
122.8494 \\
\end{tabular} & 122.1275 & 121.7512 & 100.1499 & 0.581 & $3.2 \%$ & 450.51 & 5.1888 & 2.4257 & 85.3798 & 63.9408 \\
\hline & & (1) 7,7 & & & & & & & & & & & & & & & & & & \\
\hline$S G$ & $25 \%$ & $75 \%$ & HV & $\mathrm{BL}$ & -1 & 209.9137 & 245.0483 & 238.1157 & 122.7317 & 122.6927 & 121.9717 & 121.5958 & 100.1499 & 0.581 & $3.2 \%$ & 455.68 & 5.1136 & 2.4256 & 85.3797 & 63.4801 \\
\hline & & $\operatorname{VR}(1) 7,7,8$ & & & & & & & & & & & & & & & & & & \\
\hline$S G$ & $25 \%$ & $50 \%$ & $\mathrm{HV}$ & $\mathrm{BL}$ & -1 & 213.0619 & 248.2864 & 241.4423 & 122.5771 & 122.5382 & 121.818 & 121.4425 & 100.1499 & 0.5811 & $3.2 \%$ & 461.23 & 5.037 & 2.4256 & 85.3797 & 62.9976 \\
\hline$\overline{S G}$ & $25 \%$ & $25 \%$ & HV & $\overline{B L}$ & 0 & 2179593 & 253.4542 & 2 & 123232 & 123192 & 1224669 & 1220896 & 100,15 & 0.5813 & $32 \%$ & 47463 & 4953 & 24249 & 85,3797 & 622274 \\
\hline & & VR (1) 7 78 & & & & & & & & & & & & & & & & & & \\
\hline SG & $25 \%$ & $0 \%$ & $\mathrm{HV}$ & $\mathrm{BL}$ & 0 & 221.5205 & 257.0412 & 250.3433 & 123.0768 & 123.0369 & 122.3126 & 121.9358 & 100.15 & 0.5814 & $3.2 \%$ & 481.16 & 4.8738 & 2.4249 & 85.3796 & 61.6928 \\
\hline & & & & & & & & & & & & & & & & & & & & \\
\hline SG & $25 \%$ & \begin{tabular}{|c|}
$-50 \%$ \\
\end{tabular} & $\mathrm{HV}$ & $B L$ & 0 & 229.5958 & 265.1166 & 258.5824 & 122.7537 & \begin{tabular}{|l|l|}
122.7113 \\
\end{tabular} & 121.9926 & 121.6166 & 100.1499 & 0.5818 & $3.2 \%$ & 497.01 & 4.7075 & 2.423 & 85.3795 & 60.4893 \\
\hline SG & $25 \%$ & $-100 \%$ & HV & $\overline{B L}$ & 0 & 238.1321 & 273.5304 & 267.2042 & 122,438 & 1223987 & 121.6786 & 121,3036 & 100,1499 & 0.5819 & $33 \%$ & 5136 & 45422 & 2.4229 & 853794 & 592354 \\
\hline & & VR (1) 8,8, & & & & & & & & & & & & & & & & & & \\
\hline
\end{tabular}

\begin{tabular}{|c|c|c|c|c|c|c|c|}
\hline \multicolumn{8}{|c|}{ Improvement } \\
\hline $\begin{array}{l}\text { Machine } \\
\text { Type }\end{array}$ & $\begin{array}{l}\text { Real } \\
\text { Power }\end{array}$ & $\begin{array}{l}\text { Reactive } \\
\text { Power }\end{array}$ & HV / LV & Location & $\begin{array}{l}\text { Lowest } \\
\text { Voltage }\end{array}$ & Losses & $\begin{array}{l}\text { Node } 1 \\
\text { Capacity }\end{array}$ \\
\hline SG & $50 \%$ & 0 & $\mathrm{HV}$ & $\mathrm{BL}$ & $0.60 \%$ & $0.20 \%$ & $2.40 \%$ \\
\hline & & $\operatorname{VR}(1) 7,7$ & & & & & \\
\hline$S G$ & $75 \%$ & & $\mathrm{HV}$ & $B L$ & $0.61 \%$ & $0.22 \%$ & $3.67 \%$ \\
\hline$S G$ & $100 \%$ & $\frac{\mathrm{VR}(1) T, 7}{0}$ & HV & BI & $063 \%$ & $024 \%$ & $491 \%$ \\
\hline & & $\frac{1}{\operatorname{VR}(1) 7,7}$ & & & & & $4.91 \%$ \\
\hline SG & $106.7^{\circ}$ & 0 & HV & $\mathrm{BL}$ & $0.63 \%$ & $0.25 \%$ & $5.24 \%$ \\
\hline & & $\operatorname{VR}(1) 7,7$ & & 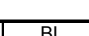 & $0.47 \%$ & $027 \%$ & \\
\hline SG & $100 \%$ & $100 \%$ & HV & & & $0.27 \%$ & $7.43 \%$ \\
\hline SG & $100 \%$ & $-100 \%$ & $\mathrm{HV}$ & $\mathrm{BL}$ & $0.11 \%$ & $0.61 \%$ & $2.25 \%$ \\
\hline & 250 & $\operatorname{VR}(1) 7,7$ & $\overline{H Y}$ & Rin & $043^{\circ}$ & $021 \%$ & 2,270 \\
\hline & 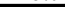 & $18(1) 77$ & & & & & $0.01 / 10$ \\
\hline SG & $25 \%$ & $75 \%$ & $\mathrm{HV}$ & $\mathrm{BL}$ & $0.30 \%$ & $0.20 \%$ & $2.91 \%$ \\
\hline & & & & & & 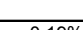 & \\
\hline SG & $25 \%$ & $50 \%$ & $\mathrm{HV}$ & BL & $0.17 \%$ & $0.19 \%$ & $2.42 \%$ \\
\hline$S G$ & $25 \%$ & $\frac{N R(1), 1}{25 \%}$ & HV & $\overline{B L}$ & $0.71 \%$ & $0.19 \%$ & $165 \%$ \\
\hline & & $\mathrm{VR}(1) 7,7$ & & & & & \\
\hline SG & $25 \%$ & $0 \%$ & $\mathrm{HV}$ & $\mathrm{BL}$ & $0.58 \%$ & $0.18 \%$ & $1.12 \%$ \\
\hline & & $\operatorname{VR}(1) 7,7$ & & & 80 & 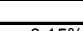 & \\
\hline$S G$ & $25 \%$ & $-50 \%$ & HV & BL & $0.32 \%$ & $0.15 \%$ & $-0.09 \%$ \\
\hline$S G$ & $25 \%$ & $\operatorname{VR}(1) 8,8$ & HV & $\mathrm{BL}_{\mathrm{s}}$ & $006 \%$ & $012 \%$ & $-134 \%$ \\
\hline & & $\frac{1}{\operatorname{VR}(1)} 8,8$ & & & & & \\
\hline
\end{tabular}


Table 59. Synchronous Generator Voltage Regulation Application (EL, HV) - LL

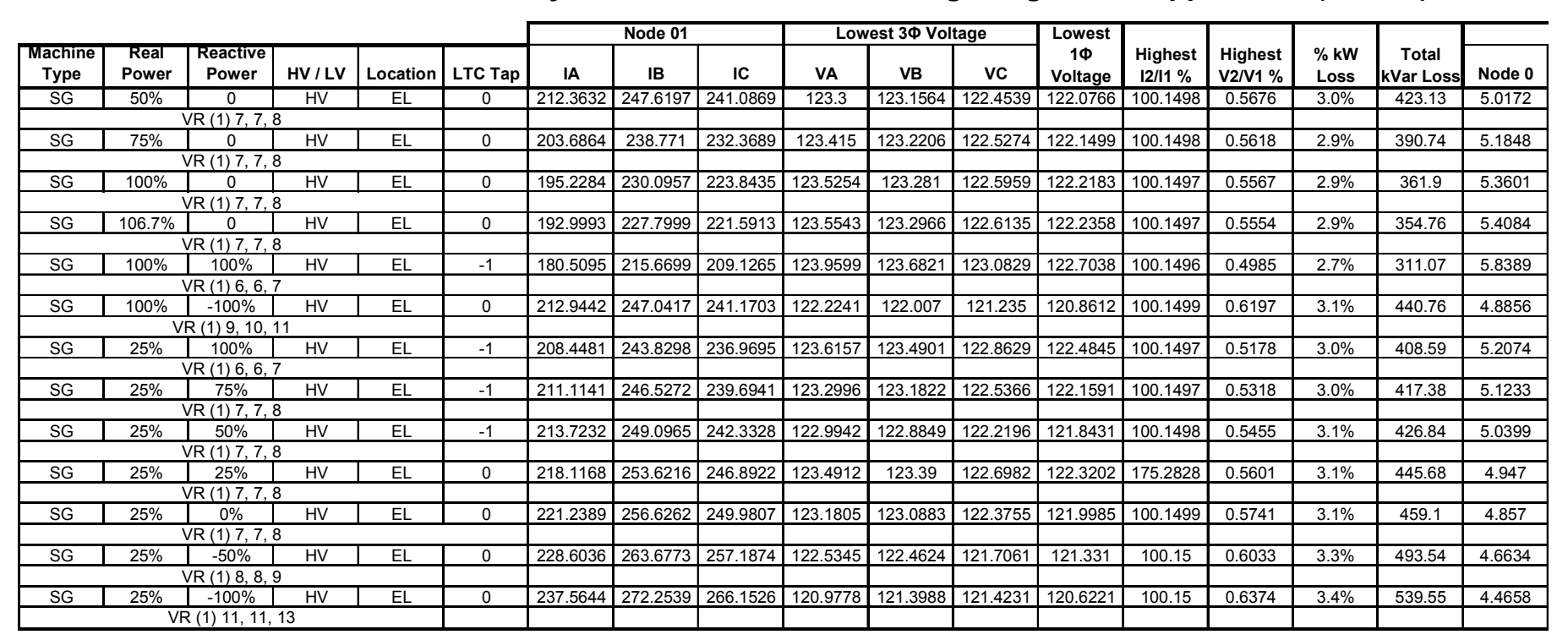

\begin{tabular}{|c|c|c|c|c|c|c|c|}
\hline \multicolumn{8}{|c|}{ Improvement } \\
\hline $\begin{array}{l}\text { Machine } \\
\text { Type }\end{array}$ & $\begin{array}{l}\text { Real } \\
\text { Power }\end{array}$ & $\begin{array}{l}\text { Reactive } \\
\text { Power }\end{array}$ & HV/LV & Location & $\begin{array}{l}\text { Lowest } \\
\text { Voltage }\end{array}$ & Losses & $\begin{array}{l}\text { Node } 1 \\
\text { Capacity }\end{array}$ \\
\hline$S G$ & $50 \%$ & 0 & $\mathrm{HV}$ & $E L$ & $0.69 \%$ & $0.37 \%$ & $2.52 \%$ \\
\hline & & $\operatorname{VR}(1) 7,7$ & & & & & \\
\hline SG & $75 \%$ & $\frac{0}{0}$ & $\mathrm{HV}$ & EL & $.76 \%$ & $0.45 \%$ & $3.84 \%$ \\
\hline$\overline{S G}$ & $100 \%$ & $\frac{V R(1) T, 7,}{0}$ & HV & 51 & $81 \%$ & $053 \%$ & $513 \%$ \\
\hline & & $\operatorname{VR}(1) 7,7$ & & & & & \\
\hline$S G$ & $106.7 \%$ & 0 & $\mathrm{HV}$ & $\mathrm{EL}$ & $0.83 \%$ & $0.55 \%$ & $5.48 \%$ \\
\hline & & $\operatorname{VR}(1) 7,7$ & & & & & \\
\hline$S G$ & $100 \%$ & $100 \%$ & $\mathrm{HV}$ & EL & $1.21 \%$ & $0.67 \%$ & $7.28 \%$ \\
\hline & & $\operatorname{VRR}(1) 6,6$ & & & & & \\
\hline$S G$ & $100 \%$ & \begin{tabular}{|l|}
$-100 \%$ \\
\end{tabular} & HV & EL & $-0.31 \%$ & $0.26^{\circ}$ & $2.61 \%$ \\
\hline & & $\mathrm{R}(1) 9,10$, & 11 & & & & \\
\hline$S G$ & $25 \%$ & $100 \%$ & $\mathrm{HV}$ & EL & $1.03 \%$ & $0.41 \%$ & $3.09 \%$ \\
\hline & & $\mathrm{VR}(1) 6,6$ & & & & & \\
\hline SG & $25 \%$ & $75 \%$ & $\mathrm{HV}$ & EL & $0.76 \%$ & $0.38 \%$ & $2.68 \%$ \\
\hline & & $\mathrm{VR}(1) 7,7$ & & & & & \\
\hline SG & $25 \%$ & $50 \%$ & HV & $\mathrm{EL}$ & $0.50 \%$ & $0.35 \%$ & $2.30 \%$ \\
\hline & & $\operatorname{VR}(1) 7,7$ & & & & & \\
\hline$S G$ & $25 \%$ & $25 \%$ & $\mathrm{HV}$ & EL & $0.90 \%$ & $0.32 \%$ & $1.63 \%$ \\
\hline & & $\operatorname{VR~}(1) 7,7$ & & & & & \\
\hline SG & $25 \%$ & $0 \%$ & $\mathrm{HV}$ & $E L$ & $0.63 \%$ & $0.27 \%$ & $1.18 \%$ \\
\hline & & & & & & & \\
\hline$S G$ & $25 \%$ & -5 & $\mathrm{HV}$ & EL & $0.08 \%$ & $0.15 \%$ & $0.13 \%$ \\
\hline & & & & & & & \\
\hline SG & $25 \%$ & $-100 \%$ & $\mathrm{HV}$ & $E L$ & $-0.50 \%$ & $0.00 \%$ & $-1.15 \%$ \\
\hline & & (I) 11,11 & & & & & \\
\hline
\end{tabular}


Table 60. Synchronous Generator Voltage Regulation Application (ML, HV) - LL

\begin{tabular}{|c|c|c|c|c|c|c|c|c|c|c|c|c|c|c|c|c|c|c|c|c|}
\hline \multirow{2}{*}{$\begin{array}{c}\text { Machine } \\
\text { Type }\end{array}$} & \multirow{2}{*}{$\begin{array}{l}\text { Real } \\
\text { Power }\end{array}$} & \multirow[b]{2}{*}{\begin{tabular}{|c|} 
Reactive \\
Power
\end{tabular}} & \multirow[b]{2}{*}{ HV / LV } & \multirow[b]{2}{*}{ Location } & \multirow[b]{2}{*}{ LTC Tap } & \multicolumn{3}{|c|}{ Node 01} & \multicolumn{3}{|c|}{ Lowest $3 \Phi$ Voltage } & \multirow{3}{*}{$\begin{array}{c}\begin{array}{c}\text { Lowest } \\
\mathbf{1} \\
\text { Voltage }\end{array} \\
122.0716\end{array}$} & \multirow{3}{*}{$\begin{array}{l}\text { Highest } \\
\text { 12/11\% } \\
\end{array}$} & \multirow{2}{*}{$\begin{array}{l}\text { Highest } \\
\text { V2/V1 \% }\end{array}$} & \multirow{2}{*}{$\begin{array}{l}\% \mathrm{~kW} \\
\text { Loss }\end{array}$} & \multirow{2}{*}{$\begin{array}{c}\text { Total } \\
\text { kVar Loss }\end{array}$} & \multicolumn{3}{|c|}{$12 / 1 \%$} & \multirow[b]{2}{*}{$\begin{array}{l}\text { Node 1 } \\
\text { Capacity }\end{array}$} \\
\hline & & & & & & IA & IB & IC & VA & VB & vc & & & & & & Node 0 & Low & $\begin{array}{l}\text { High } \\
(<100)\end{array}$ & \\
\hline SG & $50 \%$ & 0 & $\mathrm{HV}$ & $\mathrm{ML}$ & 0 & 212.4129 & 247.6931 & 241.1176 & 123.2956 & 123.148 & 22.4488 & & & 0.5669 & $3.1 \%$ & \begin{tabular}{|l|}
427.69 \\
\end{tabular} & 5.0118 & 0.5212 & 85.3742 & 63.086 \\
\hline & & $\operatorname{VR}(1) 7,7,8$ & & & & & & & & & & & & & & & & & & \\
\hline SG & $75 \%$ & \begin{tabular}{l|l} 
& 0 \\
\end{tabular} & $\mathrm{HV}$ & $\mathrm{ML}$ & 0 & 203.7158 & 238.8462 & 232.3743 & 123.4114 & 123.2107 & 122.523 & 122.1455 & 100.1498 & 0.5606 & $3.0 \%$ & 395.73 & 5.1779 & 0.5246 & 85.3715 & 64.4044 \\
\hline & & $\operatorname{VR}(1) 7,7,6$ & & & & & & & & & & & & & & & & & & \\
\hline SG & $100 \%$ & 0 & HV & $\mathrm{ML}$ & 0 & 195.2055 & 230.1473 & 223.7941 & 123.5246 & 123.2713 & 122.5943 & 122.2167 & 100.1497 & 0.555 & $2.9 \%$ & 366.13 & 5.3526 & 0.5277 & 85.3688 & 65.7008 \\
\hline & & $\operatorname{VR}(1) 7,7,8$ & & & & & & & & & & & & & & & & & & \\
\hline SG & $106.7 \%$ & \begin{tabular}{|l|}
0 \\
\end{tabular} & $\mathrm{HV}$ & $\mathrm{ML}$ & 0 & 192.9567 & 227.8406 & 221.522 & 123.5546 & 123.2872 & 122.613 & 122.2353 & 100.1497 & 0.5536 & $2.9 \%$ & 358.59 & 5.4009 & 0.5285 & 85.3681 & 66.0446 \\
\hline & & $R(1) 7,7,8$ & & & & & & & & & & & & & & & & & & \\
\hline$S G$ & $100 \%$ & $100 \%$ & $\mathrm{HV}$ & $\mathrm{ML}$ & -1 & 180.0864 & 215.2308 & 208.6781 & 123.9702 & 123.6826 & 123.0919 & 122.7128 & 100.1496 & 0.4961 & $2.7 \%$ & 314.73 & 5.8244 & 0.4985 & 85.3812 & 67.9239 \\
\hline 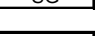 & 10070 & $\frac{1}{\operatorname{VR}(1) 6,6,}$ & inv & TWL & & 700.0007 & 210.2000 & 200.00 & 120.0702 & & 120.0070 & 122.1720 & $100.1+700$ & 年 & 2.10 & 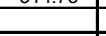 & 0.0647 & 0.7000 & 00.0012 & \\
\hline SG & $100 \%$ & $-100 \%$ & $\mathrm{HV}$ & $\mathrm{ML}$ & 0 & 212.9588 & 247.2812 & 241.2013 & 122.231 & 122.0028 & 121.2425 & 120.8687 & 100.1499 & 0.6183 & $3.1 \%$ & 434.31 & 4.8977 & 0.5685 & 85.3543 & 63.1474 \\
\hline & & $R(1) 9,10$, & & & & & & & & & & & & & & & & & & \\
\hline SG & $25 \%$ & $100 \%$ & $\mathrm{HV}$ & $\mathrm{ML}$ & -1 & 208.0818 & 243.3935 & 236.593 & \begin{tabular}{|l|}
123.6243 \\
\end{tabular} & 123.4957 & 122.8707 & 122.4923 & 100.1497 & 0.5168 & $3.0 \%$ & 410.4 & 5.1974 & 0.4874 & 85.3893 & 63.7267 \\
\hline & & $\operatorname{VR}(1) 6,6,7$ & & & & & & & & & & & & & & & & & & \\
\hline SG & $25 \%$ & | $75 \%$ & HV & $\mathrm{ML}$ & -1 & 210.8721 & 246.2299 & 239.4376 & \begin{tabular}{|l|l|l}
123.3036 \\
\end{tabular} & 123.1838 & 122.5398 & 122.1624 & 100.1497 & 0.531 & $3.0 \%$ & 420.44 & 5.114 & 0.4958 & 85.3863 & 63.304 \\
\hline & & $R(1) 7,7,8$ & & & & & 248.9264 & 242.1842 & 1229947 & & 122.2195 & & 100.1498 & & & & & & & 629022 \\
\hline SG & $25 \%$ & $50 \%$ & $\mathrm{HV}$ & IML & & & & & & & & & 100.1498 & 0.5449 & $3.1 \%$ & 430.49 & 5.0319 & 0.5032 & 85.3831 & 62.9022 \\
\hline SG & $25 \%$ & $25 \%$ & $\mathrm{HV}$ & $\mathrm{ML}$ & -1 & 216.6113 & 251.8874 & 245.2029 & 122.6785 & 122.5761 & 121.8915 & 121.5158 & 100.1498 & 0.5592 & $3.1 \%$ & 442.03 & 4.945 & 0.5106 & 85.3799 & 62.4609 \\
\hline & & $\operatorname{VR}(1) 7,7,8$ & & & & & & & & & & & & & & & & & & \\
\hline$S G$ & $25 \%$ & $0 \%$ & $\mathrm{HV}$ & $\mathrm{ML}$ & 0 & 221.2784 & 256.6741 & 250.0092 & 123.1772 & 123.0832 & 122.3719 & 121.9948 & 100.1499 & 0.5738 & $3.1 \%$ & 462 & 4.854 & 0.5172 & 85.3769 & 61.7475 \\
\hline & & $\operatorname{VR}(1) 7,7,8$ & & & & & & & & & & & & & & & & & & \\
\hline$S G$ & $25 \%$ & $-50 \%$ & $\mathrm{HV}$ & $\mathrm{ML}$ & 0 & 228.7312 & 263.8744 & 257.3197 & 122.5323 & 122.4578 & 121.704 & 121.3288 & 100.15 & 0.6032 & $3.2 \%$ & 492.86 & 4.6678 & 0.5316 & 85.3705 & 60.6745 \\
\hline & & $\frac{\mathrm{VR}(1) 8,8,}{\mathrm{~V}}$ & & & & & & & & & & & & & & & & & & \\
\hline SG & $25 \%$ & $-100 \%$ & $\mathrm{HV}$ & $\mathrm{ML}$ & 0 & 237.6779 & 272.5148 & 266.2969 & \begin{tabular}{|l|l|}
121.8619 \\
\end{tabular} & 121.8078 & 120.9998 & 120.6267 & 100.15 & 0.6372 & $3.4 \%$ & 532.26 & 4.4801 & 0.59 & 85.3626 & 59.3868 \\
\hline & 2070 & (1) 11,11 , & & & & & & & & & & & & 0.0012 & 0.710 & & & 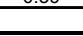 & & 0.000 \\
\hline
\end{tabular}

\begin{tabular}{|c|c|c|c|c|c|c|c|}
\hline \multicolumn{8}{|c|}{ Improvement } \\
\hline Machine & $\begin{array}{l}\text { Real } \\
\text { Power }\end{array}$ & $\begin{array}{l}\text { Reactive } \\
\text { Power }\end{array}$ & HV /LY & L ocation & $\begin{array}{l}\text { Lowest } \\
\text { Voltage }\end{array}$ & Losses & $\begin{array}{c}\text { Node } 1 \\
\text { Capacity }\end{array}$ \\
\hline SG & $50 \%$ & 0 & $\mathrm{HV}$ & ML & $0.69 \%$ & $0.35 \%$ & $2.51 \%$ \\
\hline & & $\operatorname{VR}(1) 7,7$, & & & & & \\
\hline SG & $75 \%$ & 0 & $\mathrm{HV}$ & $\mathrm{ML}$ & $0.75 \%$ & $0.43 \%$ & $3.83 \%$ \\
\hline & & 1) 7,7 , & & & & & \\
\hline SG & $100 \%$ & 0 & $\mathrm{HV}$ & ML & $0.81 \%$ & $0.51 \%$ & $5.13 \%$ \\
\hline & & $\operatorname{VR}(1) 7,7$, & & & & & \\
\hline SG & $106.7 \%$ & 0 & $\mathrm{HV}$ & $\mathrm{ML}$ & $0.83 \%$ & $0.53 \%$ & $5.47 \%$ \\
\hline$S G$ & $100 \%$ & $100 \%$ & $\mathrm{HV}$ & $\overline{M L}$ & $122 \%$ & $065 \%$ & $735 \%$ \\
\hline & & $\mathrm{VR}(1) 6,6$, & & & & & \\
\hline SG & $100 \%$ & $-100 \%$ & HV & $\begin{array}{l}M L \\
\end{array}$ & $-0.30 \%$ & $0.29 \%$ & $2.57 \%$ \\
\hline & & IR (1) 9,10 & 11 & & & & \\
\hline$S G$ & $25 \%$ & $100 \%$ & $\mathrm{HV}$ & $\mathrm{ML}$ & $1.04 \%$ & $0.40 \%$ & $3.15 \%$ \\
\hline & & R (1) 6,6 , & & & & & \\
\hline SG & $25 \%$ & $75 \%$ & $\mathrm{HV}$ & ML & $0.77 \%$ & $0.37 \%$ & $2.73 \%$ \\
\hline & & $\frac{\mathrm{VR}(1) 7,7}{5 \mathrm{~V}^{\prime}}$ & & & & & \\
\hline$S G$ & $25 \%$ & $50 \%$ & $\mathrm{HV}$ & $\mathrm{ML}$ & $0.50 \%$ & $0.33 \%$ & $2.33 \%$ \\
\hline & & $\operatorname{VR}(1) 7,7$, & & & (n) & 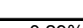 & \\
\hline$S G$ & $25 \%$ & $25 \%$ & $\mathrm{HV}$ & $\mathrm{ML}$ & $0.23 \%$ & $0.29 \%$ & $1.89 \%$ \\
\hline$S G$ & $25 \%$ & $0 \%$ & $\mathrm{HV}$ & $\overline{M L}$ & $0.63 \%$ & $0.26 \%$ & $1.17 \%$ \\
\hline & & (1) 7,7 & & & & & \\
\hline SG & $25 \%$ & $-50 \%$ & $\mathrm{HV}$ & $\mathrm{ML}$ & $0.08 \%$ & $0.15 \%$ & $0.10 \%$ \\
\hline & & (1) 8,8, & & & & & \\
\hline SG & $25 \%$ & $-100 \%$ & $\mathrm{HV}$ & ML & $-0.50 \%$ & $0.03 \%$ & $-1.19 \%$ \\
\hline
\end{tabular}


Table 61. Synchronous Generator Voltage Regulation Application (BL, LV) - LL

\begin{tabular}{|c|c|c|c|c|c|c|c|c|c|c|c|c|c|c|c|c|c|c|c|c|}
\hline \multirow[b]{2}{*}{$\begin{array}{c}\text { Machine } \\
\text { Type }\end{array}$} & \multirow[b]{2}{*}{$\begin{array}{l}\text { Real } \\
\text { Power }\end{array}$} & \multirow[b]{2}{*}{$\begin{array}{c}\text { Reactive } \\
\text { Power }\end{array}$} & \multirow[b]{2}{*}{ HV / LV } & \multirow[b]{2}{*}{ Location } & \multirow[b]{2}{*}{ LTC Tap } & \multicolumn{3}{|c|}{ Node 01} & \multicolumn{3}{|c|}{ Lowest $3 \Phi$ Voltage } & \multirow{2}{*}{\begin{tabular}{c|} 
Lowest \\
$1 \Phi$ \\
Voltage \\
\end{tabular}} & \multirow[b]{2}{*}{$\begin{array}{c}\text { Highest } \\
12 / 11 \%\end{array}$} & \multirow[b]{2}{*}{$\begin{array}{l}\text { Highest } \\
\text { V2/V1\% }\end{array}$} & \multirow[b]{2}{*}{$\begin{array}{c}\% \mathrm{~kW} \\
\text { Loss }\end{array}$} & \multirow[b]{2}{*}{$\begin{array}{c}\text { Total } \\
\text { kVar Loss }\end{array}$} & \multicolumn{3}{|c|}{$\mid 2 / 11 \%$} & \multirow[b]{2}{*}{$\begin{array}{l}\text { Node 1 } \\
\text { Capacity }\end{array}$} \\
\hline & & & & & & IA & IB & IC & VA & VB & vc & & & & & & Node 0 & Low & $\begin{array}{l}\text { High } \\
(<100)\end{array}$ & \\
\hline SG & $50 \%$ & 0 & $\mathrm{LV}$ & $\mathrm{BL}$ & 16 & 212.7966 & 248.3 & 241.6071 & 123.0554 & 123.0003 & 122.2526 & \begin{tabular}{l|l}
121.876 \\
\end{tabular} & 100.15 & 0.5896 & $3.2 \%$ & \begin{tabular}{|l|l|l|l|}
484.87 \\
\end{tabular} & 5.0431 & 2.4115 & 85.3754 & 62.9955 \\
\hline & & 1) $7,7,8$ & & & & & & & & & & & & & & & & & & \\
\hline SG & $75 \%$ & 0 & LV & $\mathrm{BL}$ & 16 & 204.296 & \begin{tabular}{|l|}
239.806 \\
\end{tabular} & 233.172 & 123.0777 & 123.0225 & 122.2747 & 121.898 & 100.15 & 0.5896 & $3.2 \%$ & 467.61 & 5.2333 & 2.4115 & 85.3754 & 64.2614 \\
\hline$S G$ & $100 \%$ & R (1) $7,7,8$ & IV & BI & 16 & 1959588 & 2314519 & 2248876 & 1230995 & 1230443 & 1222964 & 1219196 & 10015 & 0,5896 & $32 \%$ & 45128 & 54346 & 24115 & 853754 & 655064 \\
\hline & & /R (1) $7,7,8$ & & & & & & & & & & & & & & & & & & \\
\hline SG & $106.7 \%$ & \begin{tabular}{l|l|l|}
0 & \\
\end{tabular} & LV & $\mathrm{BL}$ & 16 & 193.7525 & 229.2363 & 222.6927 & 123.1053 & 123.0501 & 122.3022 & 121.9254 & 100.15 & 0.5896 & $3.2 \%$ & 447.05 & 5.4905 & 2.4115 & 85.3754 & 65.8366 \\
\hline & & $\frac{1 \mathrm{R}(1) 7,7,8}{100}$ & NV & $\mathrm{Bl}$ & 15 & 1796220 & 2140111 & 2079320 & 1230487 & 1229945 & 1222484 & 121,8718 & & & & & & & 853756 & 679715 \\
\hline$S G$ & $100 \%$ & $100 \%$ & & & & & & & & & & 121.8718 & 100.15 & 0.589 & $3.1 \%$ & 419.17 & 5.8832 & 2.4124 & 85.3756 & 67.9715 \\
\hline SG & $100 \%$ & $\mid-100 \%$ & LV & BL & 16 & 214.4479 & 249.197 & 243.3917 & 122.4026 & 122.3609 & 121.603 & 121.2282 & 100.1499 & 0.5913 & $3.2 \%$ & 485.98 & 4.961 & 2.3827 & 85.3752 & 62.8618 \\
\hline & & R (1) $8,7,9$ & & & & & & & & & & & & & & & & & & \\
\hline SG & $25 \%$ & \begin{tabular}{|l|l|}
$100 \%$ \\
\end{tabular} & LV & BL & 15 & 207.126 & 242.1265 & 235.0549 & 122.9824 & 122.9283 & 122.1826 & 121.8061 & 100.15 & 0.5891 & $3.2 \%$ & 470.68 & 5.177 & 2.4124 & 85.3756 & 63.9156 \\
\hline & & R (1) $7,7,8$ & & & & & & & & & & & & & & & & & & \\
\hline SG & $25 \%$ & $75 \%$ & LV & BL & 15 & 210.0627 & 245.1867 & 238.2018 & 122.8117 & 122.7576 & 122.0128 & 121.6368 & 100.1499 & 0.5891 & $3.2 \%$ & 476.27 & 5.1021 & 2.4124 & 85.3755 & 63.4595 \\
\hline & & $\operatorname{Re}(1) 7,7,8$ & & & & & & & & & & & & & & & & & & \\
\hline SG & $25 \%$ & \begin{tabular}{|l|l|}
$50 \%$ \\
\end{tabular} & LV & $\mathrm{BL}$ & 15 & 213.1836 & 248.3942 & 241.499 & 122.6432 & 122.5892 & 121.8454 & 121.4698 & 100.1499 & 0.5892 & $3.2 \%$ & 482.29 & 5.0259 & 2.4123 & 85.3755 & 62.9815 \\
\hline & $20 \%$ & $\frac{5}{2(1) 7,7,8}$ & & & & 270.1000 & & 241.499 & 122.0432 & & 121.0404 & 121.4090 & 100.1499 & & $0.2 \%$ & & 0.0209 & & 05.0705 & 02.9010 \\
\hline SG & $25 \%$ & $25 \%$ & LV & $B L$ & 15 & 216.5831 & 251.8481 & 245.0485 & 122.4716 & 122.4176 & 121.6747 & \begin{tabular}{|l|}
121.2997 \\
\end{tabular} & 100.1499 & 0.5893 & $3.2 \%$ & 488.93 & 4.9462 & 2.4123 & 85.3754 & 62.4667 \\
\hline & & (1) $7,7,8$ & & & & & & & & & & & & & & & & & & \\
\hline$S G$ & $25 \%$ & \begin{tabular}{|l|}
$0 \%$ \\
\end{tabular} & LV & $B \mathrm{BL}$ & 16 & 221.4424 & \begin{tabular}{|l|l}
256.9205 \\
\end{tabular} & 250.1782 & 123.0328 & 122.9777 & 122.2301 & 121.8535 & 100.15 & 0.5896 & $3.2 \%$ & 503.07 & 4.8634 & 2.4115 & 85.3753 & 61.7108 \\
\hline & & R R (1) $7,7,8$ & IV & & & 2294716 & & & 1226806 & 122626 & 1218813 & 1215057 & 110144 & 059 & & 52022 & 46972 & 24006 & 85375 & 605152 \\
\hline 30 & $25 \%$ & \begin{tabular}{|c|}
$-50 \%$ \\
$R(1) 88$
\end{tabular} & & & & & & 258.3683 & 122.6806 & & & 121.5057 & 100.1499 & 0.59 & $3.3 \%$ & 520.22 & 4.6972 & 2.4096 & 85.3752 & 60.5152 \\
\hline SG & $25 \%$ & \begin{tabular}{|l|l|}
$-100 \%$ \\
\end{tabular} & LV & $\mathrm{BL}$ & 16 & 237.9682 & 273.3106 & 266.9479 & 122.3359 & 122.2815 & 121.5386 & 121.1639 & 100.1499 & 0.5901 & $3.3 \%$ & 538.25 & 4.532 & 2.4095 & 85.3751 & 59.2682 \\
\hline & & $\operatorname{VR}(1) 8,8,9$ & & & & & & & & & & & & & & & & & & \\
\hline
\end{tabular}

\begin{tabular}{|c|c|c|c|c|c|c|c|}
\hline \multicolumn{8}{|c|}{ Improvement } \\
\hline $\begin{array}{l}\text { Machine } \\
\text { Type }\end{array}$ & $\begin{array}{l}\text { Real } \\
\text { Power }\end{array}$ & \begin{tabular}{|c|} 
Reactive \\
Power
\end{tabular} & HV /LV & Location & $\begin{array}{l}\text { Lowest } \\
\text { Voltage }\end{array}$ & Losses & $\begin{array}{l}\text { Node 1 } 1 \\
\text { Capacity }\end{array}$ \\
\hline SG & $50 \%$ & 0 & $\mathrm{LV}$ & $\mathrm{BL}$ & $0.53 \%$ & $0.18 \%$ & $2.42 \%$ \\
\hline & & $\operatorname{VR}(1) 7,7$, & & & & & \\
\hline$S G$ & $75 \%$ & 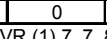 & LV & $\mathrm{BL}$ & $0.55 \%$ & $0.20 \%$ & $3.69 \%$ \\
\hline$\overline{S G}$ & $100 \%$ & 0 & $\begin{array}{l}\text { LV } \\
\end{array}$ & $\overline{B L}$ & $0.57 \%$ & $0.22 \%$ & $4.93 \%$ \\
\hline & & $\frac{1}{\operatorname{VR}(1) 7,}$ & & & & & \\
\hline SG & $106.7 \%$ & 0 & LV & BL & $0.57 \%$ & $0.23 \%$ & $5.26 \%$ \\
\hline & & $\operatorname{VR}(1) 7,7$, & & & & & \\
\hline$S G$ & $100 \%$ & $100 \%$ & LV & $\mathrm{BL}$ & $0.53 \%$ & $0.26 \%$ & $7.40 \%$ \\
\hline & & $\operatorname{VR}(1) 7,7$, & & & & & \\
\hline SG & $100 \%$ & $-100 \%$ & LV & $B L$ & $-0.01 \%$ & $0.16 \%$ & $2.29 \%$ \\
\hline & & $\operatorname{VR}(1) 8,7$, & & & & & \\
\hline SG & $25 \%$ & $100 \%$ & LV & $\mathrm{BL}$ & $0.47 \%$ & $0.19 \%$ & $3.34 \%$ \\
\hline & & $\frac{1}{\operatorname{VR}(1) 7,7,}$ & & & & & \\
\hline SG & $25 \%$ & $75 \%$ & LV & $\mathrm{BL}$ & $0.33 \%$ & $0.18 \%$ & $2.88 \%$ \\
\hline & & $\frac{1}{\operatorname{VR}(1) 7,7,}$ & & & & & \\
\hline SG & $25 \%$ & $50 \%$ & LV & $\mathrm{BL}$ & $0.19 \%$ & $0.17 \%$ & $2.41 \%$ \\
\hline & & $\operatorname{VR}(1) 7,7$, & & & & & \\
\hline SG & $25 \%$ & $25 \%$ & LV & $B L$ & $0.05 \%$ & $0.15 \%$ & $1.89 \%$ \\
\hline & & & & & & & \\
\hline SG & $25 \%$ & $0 \%$ & LV & $B L$ & $0.51 \%$ & $0.16 \%$ & $1.14 \%$ \\
\hline$S G$ & $25 \%$ & $\operatorname{VR}(1) l, \ell$, & NV & $\overline{\mathrm{BI}}$ & $022 \%$ & 0120 & 0 \\
\hline & & & & & & & $-0.06 \%$ \\
\hline SG & $25 \%$ & \begin{tabular}{|l|}
$-100 \%$ \\
\end{tabular} & LV & BL & $-0.06 \%$ & $0.09 \%$ & $-1.31 \%$ \\
\hline & & VR (1) 8,8, & & & & & \\
\hline
\end{tabular}


Table 62. Synchronous Generator Voltage Regulation Application (EL, LV) - LL

\begin{tabular}{|c|c|c|c|c|c|c|c|c|c|c|c|c|c|c|c|c|c|c|c|c|}
\hline \multirow{2}{*}{ Type } & \multirow[b]{2}{*}{ Power } & \multirow[b]{2}{*}{ Power } & \multirow[b]{2}{*}{ HVILV } & \multirow[b]{2}{*}{ Location } & \multirow[b]{2}{*}{ LTC Tap } & \multirow{2}{*}{\multicolumn{3}{|c|}{$\begin{array}{l}\text { Node } 01 \\
\end{array}$}} & \multicolumn{3}{|c|}{$\begin{array}{l}\text { Lowest } 3 \Phi \text { Voltage } \\
\end{array}$} & \multirow{2}{*}{$\begin{array}{c}\text { Lowest } \\
1 \Phi \\
\end{array}$} & \multirow{2}{*}{\begin{tabular}{|c} 
Highest \\
$12 / 11 \%$ \\
\end{tabular}} & \multirow{2}{*}{$\begin{array}{l}\text { Highest } \\
\text { V2/V1\% }\end{array}$} & \multirow{2}{*}{$\begin{array}{l}\% \mathrm{~kW} \\
\text { Loss }\end{array}$} & \multirow{2}{*}{\begin{tabular}{|c|} 
Total \\
kVar Loss
\end{tabular}} & \multirow{2}{*}{\multicolumn{3}{|c|}{$12 / 11 \%$}} & \multirow[b]{2}{*}{ Capacity } \\
\hline & & & & & & & & & VA & VB & $\mathrm{VC}$ & & & & & & & & $(<100)$ & \\
\hline SG & $50 \%$ & 0 & $\mathrm{LV}$ & $\mathrm{EL}$ & 16 & 212.2903 & 247.5062 & 240.9265 & 123.2606 & 123.1026 & 122.3766 & & 100.1499 & 0.5769 & $3.1 \%$ & \begin{tabular}{|l|l|}
443.41 \\
\end{tabular} & 5.0066 & 0.5875 & 85.37 & 63.1138 \\
\hline & & $\operatorname{VR}(1) 7,7$, & & & & & & & & & & & & & & & & & & \\
\hline SG & $75 \%$ & 0 & LV & EL & 16 & 203.6169 & 238.6617 & \begin{tabular}{|l|l|}
232.2108 \\
\end{tabular} & 123.3787 & 123.1704 & 122.4535 & $\begin{array}{ll}122.0762 \\
\end{array}$ & 100.1498 & 0.5716 & $3.0 \%$ & 409.53 & 5.1741 & 0.5871 & 85.3673 & 64.4319 \\
\hline & & $\operatorname{VR}(1) 7,7$, & & & & & & & & & & & & & & & & & & \\
\hline SG & $100 \%$ & 0 & LV & EL & 16 & 195.1622 & 229.9907 & 223.688 & 123.4919 & 123.2341 & 122.5252 & \begin{tabular}{l|l|l}
122.1478 \\
\end{tabular} & 100.1498 & 0.567 & $2.9 \%$ & 379.28 & 5.3492 & 0.5858 & 85.3647 & 65.7242 \\
\hline$S G$ & $106.7 \%$ & $\frac{V R(1) 7,7,}{0}$ & LV & EL & 16 & 192.934 & 227.6962 & 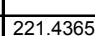 & 123.5215 & 123.2505 & 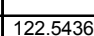 & \begin{tabular}{|c|}
2.1661 \\
\end{tabular} & 100.1497 & 0.5659 & $2.9 \%$ & 371.78 & 5.3975 & 0.5853 & 85.3639 & 66.0661 \\
\hline & & $\operatorname{VR}(1) 7,7$ & & & & & & & & & & & & 0.000 & & & & 0.0000 & & \\
\hline$S G$ & $100 \%$ & $100 \%$ & LV & EL & 15 & 181.0127 & 216.1996 & 209.5588 & 124.0533 & 123.7631 & 123.14 & \begin{tabular}{l|l|}
122.7609 \\
\end{tabular} & 100.1496 & 0.5089 & $2.7 \%$ & 327.99 & 5.8192 & 0.5394 & 85.3772 & 67.7795 \\
\hline & & $\operatorname{VR}(1) 7,7$, & & Fl & 16 & 2130625 & 2471357 & 2411868 & 1221223 & 1218917 & 1210974 & 1207239 & 100140 & & & & & & 852501 & \\
\hline SG & $100 \%$ & $-100 \%$ & LV & & & 213.0625 & 247.1357 & 241.1868 & 122.1223 & 121.8917 & \begin{tabular}{|l|l|}
121.0994 \\
\end{tabular} & 120.7239 & 100.1499 & 0.6305 & $3.2 \%$ & 461.79 & 4.8718 & 0.6474 & 85.3501 & 63.169 \\
\hline$S G$ & $25 \%$ & $\frac{1}{2(1)} 10,11$ & $\overline{L V V}$ & EL & 15 & 08.6222 & 243.9988 & 237.0845 & 123.7093 & 123.5693 & 122.9178 & 25393 & 100.1497 & 0.5263 & $30 \%$ & 42905 & 51957 & 0.5371 & 853851 & 636365 \\
\hline & & $\frac{1}{\operatorname{VR}(1) 6,6,}$ & & & & & & & & & & & & 0.0200 & $0.0 \%$ & 429.050 & & & 00.0051 & 63.6365 \\
\hline SG & $25 \%$ & $75 \%$ & LV & $\mathrm{EL}$ & 15 & 211.2622 & 246.6659 & 239.7802 & 123.3795 & 123.2477 & \begin{tabular}{|l|l|}
122.5779 \\
\end{tabular} & 122.2004 & 100.1498 & $\begin{array}{l}0.5403 \\
\end{array}$ & $3.0 \%$ & $\begin{array}{l}438.23 \\
\end{array}$ & 5.1119 & $\begin{array}{l}0.5508 \\
\end{array}$ & 85.382 & 63.2391 \\
\hline & & $\operatorname{VR}(1) 7,7$ & & & & & & & & & & & & & & & & & & \\
\hline SG & $25 \%$ & $50 \%$ & LV & $\mathrm{EL}$ & 15 & 213.8455 & 249.2062 & 242.3909 & \begin{tabular}{|l|l|}
123.0608 \\
23.0608
\end{tabular} & 122.937 & 122.2478 & 121.8712 & 100.1498 & 0.5541 & $3.1 \%$ & 448.04 & 5.0287 & 0.5633 & 85.3789 & 62.8605 \\
\hline & & $\operatorname{VR}(1) 7,7$ & & & & & & & & & & & & & & & & & & \\
\hline SG & $25 \%$ & $25 \%$ & $\underline{L V}$ & $E L$ & 15 & 216.7438 & $\begin{array}{l}252.02 \\
\end{array}$ & 245.2842 & 122.7333 & 122.6182 & \begin{tabular}{|l|l}
121.9086 \\
\end{tabular} & 121.5328 & 173.4712 & 0.5683 & $3.1 \%$ & 460.03 & 4.94 & 0.5761 & 98.6865 & 62.4411 \\
\hline & & $\operatorname{VRR}(1) 7,7$ & & & & & & & & & & & & & & & & & & \\
\hline$S G$ & $25 \%$ & $0 \%$ & $\mathrm{LV}$ & $\mathrm{EL}$ & 16 & 221.4532 & \begin{tabular}{|l|l|}
256.8277 \\
\end{tabular} & 250.101 & 123.1283 & 123.0217 & 122.2865 & \begin{tabular}{l|l|}
121.9097 \\
\end{tabular} & 100.1499 & 0.5832 & $3.2 \%$ & 482.19 & 4.8432 & 0.5886 & 85.3727 & 61.7246 \\
\hline & & $\operatorname{VR}(1) 8,8$, & & & & & & & & & & & & & & & & & & \\
\hline$S G$ & $25 \%$ & $-50 \%$ & LV & $E L$ & 16 & 228.481 & 263.5061 & 256.9753 & 122.4626 & 122.3755 & \begin{tabular}{|l|l|}
121.5962 \\
\end{tabular} & 121.2214 & 100.15 & 0.6122 & $3.3 \%$ & 516.53 & 4.6532 & 0.6066 & 85.3663 & 60.7293 \\
\hline & & $\operatorname{VR}(1) 8,8$, & & & & & & & & & & & & & & & & & & \\
\hline$S G$ & $25 \%$ & \begin{tabular}{|l|}
$-100 \%$ \\
\end{tabular} & LV & $\mathrm{EL}$ & 16 & 237.6856 & 272.3481 & 266.1733 & \begin{tabular}{|l|l}
121.7458 \\
\end{tabular} & 121.681 & 120.8464 & 120.4737 & 100.15 & 0.6467 & $3.4 \%$ & 565.3 & 4.453 & 0.6718 & 85.3584 & 59.4116 \\
\hline & & (1) 12,12 & & & & & & & & & & & & & & & & & & \\
\hline
\end{tabular}

\begin{tabular}{|c|c|c|c|c|c|c|c|}
\hline \multicolumn{8}{|c|}{ Improvement } \\
\hline $\begin{array}{l}\text { Machine } \\
\text { Type }\end{array}$ & $\begin{array}{l}\text { Real } \\
\text { Power }\end{array}$ & $\begin{array}{l}\text { Reactive } \\
\text { Power }\end{array}$ & HV/LV & Location & $\begin{array}{l}\text { Lowest } \\
\text { Voltage }\end{array}$ & Losses & $\begin{array}{l}\text { Node 1 } \\
\text { Capacity }\end{array}$ \\
\hline SG & $50 \%$ & & $\mathrm{LV}$ & $E L$ & $0.63 \%$ & $0.34 \%$ & $2.54 \%$ \\
\hline & & $\operatorname{VR}(1) 7,7$, & & & & & \\
\hline SG & $75 \%$ & 0 & LV & $E \mathrm{EL}$ & $0.69 \%$ & $0.43 \%$ & $3.86 \%$ \\
\hline & & $2(1) 7,7$, & & & & & \\
\hline SG & $100 \%$ & 0 & LV & $E L$ & $0.75 \%$ & $0.51 \%$ & $5.15 \%$ \\
\hline & & VR (1) 7,7, & & & & & \\
\hline SG & $106.7 \%$ & 0 & LV & $E \mathrm{EL}$ & $0.77 \%$ & $0.52 \%$ & $5.49 \%$ \\
\hline & & VR & & & & & \\
\hline SG & $100 \%$ & $100 \%$ & LV & $\overline{E L}$ & $1.26 \%$ & $0.66 \%$ & $7.20 \%$ \\
\hline & & $\operatorname{VR}(1) 7,7$, & & & & & \\
\hline SG & $100 \%$ & $-100 \%$ & LV & $\mathrm{EL}$ & $-0.42 \%$ & $0.23 \%$ & $2.59 \%$ \\
\hline & & (1) 10,11 & 12 & & & & \\
\hline SG & $25 \%$ & $100 \%$ & LV & $E L$ & $1.08 \%$ & $0.39 \%$ & $3.06 \%$ \\
\hline & & $\frac{1}{V R(1) 6,6,}$ & & & & & \\
\hline$S G$ & $25 \%$ & $75 \%$ & LV & $E \mathrm{EL}$ & $0.80 \%$ & $0.36 \%$ & $2.66 \%$ \\
\hline & & (1) 7,7 , & & & & & \\
\hline SG & $25 \%$ & $50 \%$ & LV & $E L$ & $0.53 \%$ & $0.32 \%$ & $2.29 \%$ \\
\hline & & $\operatorname{VR}(1) 7,7$, & & & & & \\
\hline SG & $25 \%$ & $25 \%$ & LV & EL & $0.25 \%$ & $0.28 \%$ & $1.87^{\circ}$ \\
\hline & & $\operatorname{VR}(1) 7,7$, & & & & & \\
\hline SG & $25 \%$ & $0 \%$ & LV & $E L$ & $0.56 \%$ & $0.24 \%$ & $1.15 \%$ \\
\hline & & $\operatorname{VR}(1) 8,8$, & & & & & \\
\hline SG & $25 \%$ & & LV & $E \mathrm{EL}$ & $-0.01 \%$ & $0.12 \%$ & $0.15 \%$ \\
\hline & & R (1) & & & & & \\
\hline SG & $25 \%$ & $-100 \%$ & LV & EL & $-0.63 \%$ & $-0.04 \%$ & $-1.16 \%$ \\
\hline & & 2(1) 12,12 & & & & & \\
\hline
\end{tabular}


Table 63. Synchronous Generator Voltage Regulation Application (ML, LV) - LL

\begin{tabular}{|c|c|c|c|c|c|c|c|c|c|c|c|c|c|c|c|c|c|c|c|c|}
\hline \multirow[b]{2}{*}{$\begin{array}{c}\text { Machine } \\
\text { Type }\end{array}$} & \multirow[b]{2}{*}{$\begin{array}{l}\text { Real } \\
\text { Power }\end{array}$} & \multirow[b]{2}{*}{\begin{tabular}{|c|} 
Reactive \\
Power
\end{tabular}} & \multirow[b]{2}{*}{ HV / LV } & \multirow[b]{2}{*}{ Location } & \multirow[b]{2}{*}{ LTC Tap } & \multicolumn{3}{|c|}{ Node 01} & \multicolumn{3}{|c|}{ Lowest $3 \Phi$ Voltage } & \multirow{2}{*}{$\begin{array}{c}\text { Lowest } \\
1 \Phi \\
\text { Voltage } \\
\end{array}$} & \multirow[b]{2}{*}{\begin{tabular}{|l} 
Highest \\
$12 / 11 \%$ \\
\end{tabular}} & \multirow[b]{2}{*}{$\begin{array}{l}\text { Highest } \\
\text { V2/N1 \% }\end{array}$} & \multirow[b]{2}{*}{$\begin{array}{c}\% \mathrm{~kW} \\
\text { Loss }\end{array}$} & \multirow[b]{2}{*}{$\begin{array}{c}\text { Total } \\
\text { kVar Loss }\end{array}$} & \multicolumn{3}{|c|}{$12 / 11 \%$} & \multirow[b]{2}{*}{$\begin{array}{c}\text { Node } 1 \\
\text { Capacity }\end{array}$} \\
\hline & & & & & & IA & IB & IC & VA & VB & vc & & & & & & Node 0 & Low & $\begin{array}{l}\text { High } \\
(<100)\end{array}$ & \\
\hline SG & $50 \%$ & 0 & $\mathrm{LV}$ & $\mathrm{ML}$ & 16 & 212.3396 & 247.5788 & 240.9566 & 123.2559 & 123.0939 & 122.3713 & 121.9943 & 100.1499 & 0.5762 & $3.1 \%$ & 447.96 & 5.0013 & 0.5209 & 85.37 & 63.103 \\
\hline & & (1) 7,7 , & & & & & & & & & & & & & & & & & & \\
\hline SG & $75 \%$ & 0 & LV & $\mathrm{ML}$ & 16 & 203.6459 & 238.736 & 232.2155 & 123.3749 & 123.1601 & 122.4489 & 122.0716 & 100.1498 & 0.5704 & $3.0 \%$ & 414.53 & 5.1672 & 0.5243 & 85.3673 & 64.4209 \\
\hline & & $\operatorname{VR}(1) 7,7$ & & & & & & & & & & & & & & & & & & \\
\hline SG & $100 \%$ & 0 & LV & $\mathrm{ML}$ & 16 & 195.1391 & 230.0414 & 223.638 & 123.491 & 123.224 & 122.5235 & 122.1461 & 100.1497 & 0.5653 & $2.9 \%$ & 383.51 & 5.3416 & 0.5274 & 85.3646 & 65.7166 \\
\hline$S G$ & $1067^{\circ}$ & $\operatorname{VR}(1) 7,7$ & 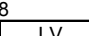 & $M$ & 16 & 1928913 & 2277359 & 2213666 & 1235217 & 1232408 & 122543 & 1221655 & 1001497 & 0564 & $29 \%$ & 37561 & 53899 & 0.5281 & 853639 & 660602 \\
\hline & & & & & & & 221.1000 & 221.0000 & 120.0211 & 120.2400 & 122.045 & 122.1000 & 100.1491 & 0.564 & $2.9 \%$ & $3 / 5.61$ & 5.3899 & 0.5281 & 0.00005 & 66.0602 \\
\hline SG & $100 \%$ & $100 \%$ & LV & $\mathrm{ML}$ & 15 & 180.3035 & 215.4447 & 208.831 & 124.0729 & 123.7724 & 123.1571 & 122.7779 & 100.1496 & 0.5063 & $2.8 \%$ & 330.64 & 5.8102 & 0.4986 & 85.3772 & 67.892 \\
\hline & & VR (1) 6, 6, & & & & & & & & & & & & & & & & & & \\
\hline SG & $100 \%$ & $-100 \%$ & LV & $\mathrm{ML}$ & 16 & 213.0786 & 247.3756 & 241.2184 & 122.1295 & 121.8875 & 121.1051 & 120.7316 & 100.1498 & 0.629 & $3.1 \%$ & 455.38 & 4.8838 & 0.5683 & 85.3502 & 63.1333 \\
\hline & 10010 & 2. & 12 & & & & & & & & & & & & & & & & & \\
\hline SG & $25 \%$ & $100 \%$ & LV & $\mathrm{ML}$ & 15 & 208.257 & 243.5636 & 236.709 & 123.7181 & 123.5753 & 122.926 & 122.5474 & 100.1497 & 0.5252 & $3.0 \%$ & 430.81 & 5.1856 & 0.4877 & 85.3852 & 63.7014 \\
\hline & & $\operatorname{VR}(1) 6,6$ & & & & & & & & & & & & & & & & & & \\
\hline SG & $25 \%$ & $75 \%$ & LV & $\mathrm{ML}$ & 15 & 211.0207 & 246.3693 & 239.5242 & 123.3835 & 123.2494 & 122.5814 & 122.2038 & 100.1498 & 0.5395 & $3.1 \%$ & 441.23 & 5.1026 & 0.496 & 85.3821 & 63.2833 \\
\hline & & $\operatorname{VR}(1) 7,7$, & & & & & & & & & & & & & & & & & & \\
\hline SG & $25 \%$ & $\begin{array}{l}\quad 50 \% \\
\end{array}$ & LV & $\mathrm{ML}$ & 15 & 213.7131 & 249.0361 & 242.2424 & \begin{tabular}{|l|l}
123.0612 \\
\end{tabular} & 122.9354 & \begin{tabular}{|l|l|}
122.2477 \\
\end{tabular} & $\begin{array}{l}121.871 \\
\end{array}$ & 100.1498 & 0.5534 & $3.1 \%$ & 451.66 & 5.0208 & 0.5033 & 85.379 & 62.8858 \\
\hline & & $\operatorname{VR}(1) 7,7$, & & & 16 & & & & & & & & & & & & & & & \\
\hline SG & $25 \%$ & $125 \%$ & LV & $\mathrm{ML}$ & 16 & 218.0283 & 253.4786 & 246.7043 & \begin{tabular}{|l|}
123.4602 \\
\end{tabular} & $\begin{array}{r}123.3425 \\
\end{array}$ & 122.6284 & 122.2507 & 100.1499 & 0.5684 & $3.1 \%$ & 470.68 & 4.9307 & 0.5103 & 85.3758 & 62.2237 \\
\hline & & $\mathrm{VR}(1) 7,7$ & & & & & & & & & & & & & & & & & & \\
\hline SG & $25 \%$ & $0 \%$ & LV & $\mathrm{ML}$ & 16 & 221.202 & 256.556 & 249.8464 & 123.1342 & 123.0254 & 122.2907 & 121.9139 & 100.1499 & 0.5826 & $3.2 \%$ & 0 & 4.8436 & 0.517 & 85.3727 & 61.7651 \\
\hline & & $\operatorname{VR}(1) 7,7$ & & & & & & & & & & & & & & & & & & \\
\hline$S G$ & $25 \%$ & $-50 \%$ & LV & ML & 16 & 228.6085 & 263.7029 & \begin{tabular}{|l|l|}
257.1074 \\
\end{tabular} & 122.4603 & \begin{tabular}{|l}
122.3707 \\
\end{tabular} & 121.5939 & 121.2191 & 100.15 & 0.612 & $3.3 \%$ & 515.86 & 4.6577 & 0.5312 & 85.3663 & 60.7 \\
\hline & & $\operatorname{VR}(1) 8,8$ & & & & & & & & & & & & & & & & & & \\
\hline$S G$ & $25 \%$ & $-100 \%$ & LV & $\mathrm{ML}$ & 16 & 237.7999 & 272.6093 & 266.318 & 121.7498 & 121.6811 & 120.8512 & 120.4784 & 100.15 & 0.6466 & $3.4 \%$ & 558.04 & 4.4672 & 0.5899 & 85.3584 & 59.3727 \\
\hline & & $2(1) 12,12$, & & & & & & & & & & & & & & & & & & \\
\hline
\end{tabular}

\begin{tabular}{|c|c|c|c|c|c|c|c|}
\hline \multicolumn{8}{|c|}{ Improvement } \\
\hline $\begin{array}{c}\text { Machine } \\
\text { Type }\end{array}$ & $\begin{array}{l}\text { Real } \\
\text { Power }\end{array}$ & $\begin{array}{c}\text { Reactive } \\
\text { Power }\end{array}$ & HV / LV & Location & $\begin{array}{l}\text { Lowest } \\
\text { Voltage }\end{array}$ & Losses & \begin{tabular}{|r|} 
Node 1 \\
Capacity
\end{tabular} \\
\hline SG & $50 \%$ & 0 & $\mathrm{LV}$ & ML & $0.63 \%$ & $0.32 \%$ & $2.53^{\circ}$ \\
\hline & & $\operatorname{VR}(1) 7,7$, & & & & & \\
\hline SG & $75 \%$ & 10 & LV & $\mathrm{ML}$ & $0.69 \%$ & $0.41 \%$ & $3.85 \%$ \\
\hline & & & & & & & \\
\hline SG & $100 \%$ & 0 & LV & ML & $0.75 \%$ & $0.49 \%$ & $5.14 \%$ \\
\hline & $1067 \%$ & $\operatorname{VR}(1) 7,7$ & V & & $0.77 \%$ & $051 \%$ & $549 \%$ \\
\hline SG & $100.17 \%$ & $\frac{1}{V} \frac{0}{V(1) 7.7}$ & LV & ML & $0.1 / \%$ & $0.51 \%$ & $5.49 \%$ \\
\hline$S G$ & $100 \%$ & $100 \%$ & LV & $\mathrm{ML}$ & $1.27 \%$ & $0.63 \%$ & $7.32 \%$ \\
\hline & & VR (1) 6, 6, & & & & & \\
\hline SG & $100 \%$ & $-100 \%$ & LV & $\mathrm{ML}$ & $-0.41 \%$ & $0.26 \%$ & $2.56 \%$ \\
\hline & & $R(1) 10,11$, & 12 & & & & \\
\hline SG & $25 \%$ & $100 \%$ & LV & $\mathrm{ML}$ & $1.08 \%$ & $0.38 \%$ & $3.13 \%$ \\
\hline & & $\frac{1}{\operatorname{VR}(1) 6,6,}$ & & & & & \\
\hline SG & $25 \%$ & $75 \%$ & LV & $\mathrm{ML}$ & $0.80 \%$ & $0.34 \%$ & $2.71 \%$ \\
\hline & & VR (1) 7,7 , & & & & & \\
\hline SG & $25 \%$ & $50 \%$ & LV & $\mathrm{ML}$ & $0.53 \%$ & $0.31 \%$ & $2.31 \%$ \\
\hline$S G$ & & $\operatorname{VR}(1), \%$ & 兴 & & $0,01 \%$ & $020 \%$ & \\
\hline 30 & $25 \%$ & $\frac{125 \%}{18(1) 77}$ & LV & ML & $0.84 \%$ & $0.28 \%$ & $1.65 \%$ \\
\hline SG & $25 \%$ & $0 \%$ & LV & $\begin{array}{l}\text { ML } \\
\end{array}$ & $0.56 \%$ & $0.23 \%$ & $1.19 \%$ \\
\hline & & $\mathrm{VR}(1) 7,7$ & & & & & \\
\hline SG & $25 \%$ & $-50 \%$ & $\overline{L V}$ & $\mathrm{ML}$ & $-0.01 \%$ & $0.12 \%$ & $0.13 \%$ \\
\hline & & $\operatorname{VR}(1) 8,8$, & & & & & \\
\hline SG & $25 \%$ & $-100 \%$ & LV & $\mathrm{ML}$ & $-0.62 \%$ & $0.00 \%$ & $-1.20 \%$ \\
\hline & & & & & & & \\
\hline
\end{tabular}




\section{Conclusions and Recommendations}

\subsection{Conclusions}

- Five methods to improve voltage regulation at the substation were explained and seven methods to improve voltage regulation on the distribution circuit were defined. It was shown that the voltage drop is six times less for three-phase circuit than single-phase circuit portions with the same load, yet most of the load is served by the single-phase portions of the circuit. Voltage and current equations were developed for the Type A and Type B step VRs and the bidirectional regulator, which is required for operation of DG on the circuit. The operation of the line drop compensator and how the settings are calculated when loads are tapped off the circuit before the regulation point were explained. In addition, equations were developed to determine the settings of the regulator when shunt capacitors are added to the circuit. Why grounded wye capacitors are used on four-wire wye-grounded systems and why delta-connected capacitors are used on ungrounded systems to alleviate series resonant conditions were explained.

- Causes for unbalanced voltage and current and how these conditions affect protective relaying were explained. The neutral relay is set to trip for ground faults, and the trip value may have to be increased to account for increased neutral current because of unbalance. This may cause a loss of sensitivity in clearing for faults.

- Significant unbalanced loading can occur even though voltages are balanced at the source. Reclosers and the substation breaker with ground fault-sensing circuits are affected by load imbalance. Unequal single-phase load connected line-to-line does not produce neutral current in the ground relay.

- Fuse preload because of unbalanced loading can cause fuses to become unselective with other protective devices such as reclosers and cause misoperation.

- Reducing unbalanced loading reduces the losses created by the neutral current in the neutral conductor.

- Unbalanced three-phase voltages have a significant effect on the heating of induction and synchronous generators. For example, a 5.5\% voltage unbalance can cause an approximate $25 \%$ increase in temperature rise. The phase currents with unbalanced voltages are greatly unbalanced, on the order of four to five times the voltage unbalance. If overload relay protection settings are raised because of unbalance, the generator may not be protected against overload and open phases.

- Heating of induction generators because of voltage unbalance is affected by phase rotation. Phase rotation affects which of the phases has the highest line currents. This means negative sequence current protection must be used to protect the induction generator to prevent failure because of voltage unbalance. The negative sequence losses are proportional to the square of the negative sequence voltage. The generator may have to reduce output below nameplate rating to avoid overheating with voltage unbalance. 
An equation was developed to calculate machine derating with unbalanced voltages with only the percent unbalanced voltage and ratio of the positive-to-negative sequence impedances of the induction generator. It was shown that a 5\% voltage unbalance causes a $3.2 \%-10.7 \%$ power output derate, depending on the positive-to-negative sequence impedance ratio.

- The 13-utility voltage survey showed a maximum percent unbalance of $5.94 \%$, with an average of $1.1 \%$. Eighty-five percent of all tests were $<2 \%$ voltage unbalance. The Milford Circuit showed the maximum percent unbalance to be $1.52 \%$ on the primary during HL conditions and $1.26 \%$ for LL. Typically, the maximum permissible current unbalance for synchronous generators is $10 \%$ of the rated stator current. A $15.44 \%$ negative sequence current is produced from a 3\% voltage unbalance for a single-cage induction generator, so care must be exercised to locate generating units on a distribution circuit for which the voltage unbalance is less than $3 \%$ and the unbalance current is less than $10 \%-20 \%$.

- Models were developed for the 10-MVA LTC delta-wye-grounded substation transformer, the 167-kVA bidirectional VRs, the three wye-grounded capacitors, the line impedances, and all (nine cases) the distribution circuit transformer connections.

- The line loss model was validated using three line configurations and balanced and unbalanced load conditions. The three line configurations were the balanced impedance triangular spacing configuration, or equilateral; the flat configuration nontransposed; and the flat configuration transposed. The purpose of the validation was to show that, with a balanced line impedance and balanced load, the kilowatt losses are the same in each phase and the total kilowatt losses are lowest. In addition, an example showed that, even though the kilowatt losses per phase are not correct because of the Kron reduction process from a four-by-four matrix to a three-by-three matrix, the total losses for the three phases are, in fact, correct.

- Models were developed for the 1,000-kW synchronous generator, the self-excited 400$\mathrm{kW}$ induction generator, and the 400-kW high-speed generator and inverter system.

- The line, equipment, and generation models were verified with the maximum phase current variance of $3.9 \%$ on July 17, 2006, and 5.7\% on July 29, 2006-except for Node I, where the percent unbalance current was very high. The maximum phase voltage variance was $1.5 \%$ on July 17 and $1.2 \%$ on July 29 . The maximum PF variance was $5.7 \%$ on July 17 and $3.4 \%$ on July 29 .

- The selected distribution circuit was a $13.2-\mathrm{kV}$, three-phase wye multi-grounded system that serves approximately $76.2 \%$ residential, $4 \%$ commercial, and $19.8 \%$ light industrial loads. The load on the summer peak day was 15.3 MVA, and the summer peak day minimum load was 5.91 MVA. Eight regulation tests were conducted for the HL load condition, and eight were conducted for the LL condition. The primary voltage to the substation was ranged from $95 \%$ to $105 \%$. The regulation methods consisted of the: 
○ LTC

- LTC and VR 1

O LTC, VR 1, and VR 2

- LTC and Cap 1

- LTC, Cap 1, and Cap 2

- LTC, Cap 1, Cap 2, and Cap 3

○ LTC, VR 1, VR 2, Cap 1, Cap 2, and Cap3.

The voltage spread, measured as the difference between the highest three-phase voltage and the lowest single-phase voltage, for the HL condition was $25.2 \mathrm{~V}$ with no regulation at the substation and on the distribution circuit. When all regulation was implemented, this voltage spread was reduced to only $10.4 \mathrm{~V}$, and there were no voltage criteria violations. For the LL condition, the voltage spread was $16.65 \mathrm{~V}$ for no regulation. With all regulation operating, the spread was only $2.91 \mathrm{~V}$.

- The load imbalance at the substation was $4 \%$ for HL and 5.45\% for LL. Adding regulation does not necessarily improve load imbalance; in fact, higher voltages generally cause higher loads and more imbalance.

- The VDC model best represented how the circuit load changes with changes in source voltage. The model consisted of $\% \Delta \mathrm{P} / \% \Delta \mathrm{V}=1.26$ and $\% \Delta \mathrm{Q} / \% \Delta \mathrm{V}=4.66 \%$. The CC model percent error was 3.9\%, the CP model percent error was $12.5 \%$, and the VDC model percent error was only $2 \%$.

- Three voltage control strategies were tested for the $400-\mathrm{kW}$ induction generator at LV and HV substation primary voltage for a total of six simulations. Three voltage control strategies were tested for the $400-\mathrm{kW}$ inverter-based generation at LV and HV substation primary voltage for a total of six simulations. And 13 voltage control strategies for $\mathrm{HV}$ and 13 for LV were tested for the synchronous generator located at the BL, ML, and EL of the circuit, for a total of 78 simulations. The maximum released capacity of $10.44 \%$ was achieved with the $1,000-\mathrm{kW}$ synchronous generator with $\mathrm{P}=100 \%$ and $\mathrm{Q}=100 \%$. The voltage improvement was $0.82 \%$, and the loss reduction was $0.56 \%$ out of a $5.4 \%$ base.

- The optimum location for the DG with the highest released capacity of $10.44 \%$ was at the source of the circuit because it directly offset the load current and load losses of the circuit. The optimum DR location to achieve the greatest loss reduction was at the EL of the circuit because adding generation here reduces on a prorata basis the load and the length of the circuit. There was little difference for improving the voltage regulation between locating the DR at the midpoint or end of the circuit. There was a slightly better improvement at the midpoint for circuits in which the conductor size of the entire three-phase backbone was the same. 
- The DG penetration study showed that a synchronous DG had a real power limit of $13,980 \mathrm{~kW}$ at the tag end of the circuit. The optimum location was at the midpoint, with the lowest single-phase voltage improvement of $1.7 \%$ and a real power loss savings of $2.04 \%$. The base case real power losses were $5.4 \%$. The DG penetration study found that the maximum real and reactive power output limit was $14,490 \mathrm{~kW}$ and 2,007 kVAr, which allows a larger DG to be installed than when only real power was injected. Again, the optimum location was at the midpoint of the circuit, but, in this case, the lowest single-phase voltage was improved $4.55 \%$ versus only $1.7 \%$ in the real power limit case. The real power loss savings were marginally better, with a $2.3 \%$ savings versus $2.04 \%$. This saving in losses represents a $57.4 \%$ reduction when the DG is located at the midpoint of the circuit.

\subsection{Recommendations}

- This project used validated circuit equipment and line models and a validated VDC source load model to test 78 synchronous generator voltage control strategies. It found the greatest improvement in released capacity reduced real losses and improvement in voltage regulation occurred when the synchronous generator produced the maximum real and reactive power. DG can be sized and located to produce these improvements, but the effects on system protection systems must be evaluated.

- It is recommended that a validated unbalanced three-phase power flow program be used to determine the percent unbalanced voltage and percent unbalanced current throughout the circuit. If the percent unbalanced voltage exceeds $3 \%$ and the percent unbalanced current exceeds $10 \%$ at the location where a synchronous generator is to be sited, it is highly possible it will never operate and could trip on voltage or current imbalance.

- It is highly recommended that a VDC source be used for the load model because it is more accurate than the $\mathrm{CP}$ and $\mathrm{CC}$ models.

- The optimum voltage regulation method that produced the least voltage spread used the substation transformer LTC, step regulators, capacitors, and DG. It is recommended that the DG be located at the midpoint of the circuit to produce the best overall improvements in voltage regulation, loss reduction, and released capacity.

- The circuit selected had voltage unbalance conditions that closely agreed with the average voltage unbalance measurements taken at 13 major utilities. Therefore, the results should be representative of what would be experienced on the average distribution circuit.

- Voltage regulation and system protection issues represent the most difficult problems for interconnecting DG with the distribution circuit. This report explains how voltage unbalance and current imbalance can affect system protection performance and the rating of the DG output. It is recommended that inverter-based generation be considered as the preferred DG type if the level of unbalanced voltage and current may prevent synchronous and induction generators from operating on the circuit. 
- Future research should fund the development of a real-time optimal control of DG to accomplish the optimum generator voltage control condition, including the control of the substation transformer LTC, the bidirectional step regulators, and the switched capacitors. The cost to perform this development work on the Milford Circuit DC 8103 would be substantially less than installing DG and metering equipment on a new circuit because all of this equipment is already installed and tested. Furthermore, the newly developed control strategies can be tested in the summer of 2008. The estimated cost for this project is less than $\$ 500,000$, and the development, testing, and analysis schedule is estimated to be approximately 1.5 years.

\subsection{Benefits to California}

Models were developed and validated with actual measured circuit and generator test data.

This project showed that adding DG to a distribution circuit can reduce generation capacity by $10 \%$ (released capacity), and these results can be achieved in 1 year-not 7-10 years-for traditional central-station generation. Also, improved voltage regulation results in fewer service voltage criteria violations and less distribution energy losses (i.e., approximately $10 \%$ ). 


\section{References}

Davis, M.W. (2003). Determine the Market Penetration Limits of Distributed Energy

Resources (by Distributed Generation Type) by the Year 2015. Department of

Energy/National Renewable Energy Laboratory report.

Davis, M.W.; Krupa, T.J.; Diedzic, M.J. “The Economics of Load Management on the Design and Operation of the Distribution System, Part II Load Characteristics." IEEE Transactions on Power Apparatus and Systems; Vol. PA8-102 No. 3, March 1983; pp. 654-674.

Electrical Distribution System Protection. 3rd edition. Pewaukee, WI: Cooper Power. Cooper Power Systems Bulletin 90020 February 2005. Available at www.cooperpower.com.

Gafford, B.N.; Duesterohoeft, N.C.; Mosher, C.C. III. "Heating of Induction Motors on Unbalanced Voltages." Prepared for the American Institute of Electrical Engineers Winter General Meeting, New York, Feb. 1-6, 1959; p. 284.

Kersting, W.H. "The Computation of Neutral and Dirt Currents and Power Losses." Prepared for the Power Systems Conference and Exposition 2004. IEEE PES, Vol. 10-13, October 2004; pp. 213-218.

G. E. Multilin SR 469 Motor Management Relay, 1998.

Mason, C.R. The Art and Science of Protective Relaying. General Electric online, http://www.geindustrial.com/industrialsystems/pm/notes/artsci/index.htm.

National Electrical Manufacturers Association Standard for Motors and Generators MG1, 1978.

Elmore, W.A. Protective Relaying Theory and Applications. 2nd edition. New York: Marcel Dekker Inc., 1994.

S\&C Electric Co. “Data Bulletin 350-195,” July 17, 2000; p. 1 of 4.

S\&C Electric Co. "Coordinaide: The S\&C Protection and Coordination Assistant." S\&C Electric Co. online, http://www.sandc.com/FSA/coordinaide.asp.

Tracey, G.F. "Maximum Allowable Continuous Output of Induction Motors Operating Under Unbalanced Voltages." April 1954. Discussion of J. E. Williams "Operation of Three-Phase Induction Motors on Unbalanced Voltages," Power Apparatus and Systems, April 1954, p. 132.

Wagner, C.F.; Evans, R.D. Symmetrical Components. New York: McGraw Hill Book Co. Inc., 1933; pp. 345-350.

Williams, J.E. "Operation of Three-Phase Induction Motors on Unbalanced Voltages." American Institute of Electrical Engineers Winter General Meeting; Jan. 18-22, 1954, New York. 


\section{Appendix A: Ground Detector and Ungrounded Systems}

For ungrounded delta systems, there is a normal leakage current of about 2 A to ground because of the distributed capacitance, as shown in Figure A-1. The VTs with a ratio of 40:1 are connected in a broken delta configuration on the secondary side. Under normal circumstances, the line-to-neutral voltages on the primary of the VT are $2,770 \mathrm{~V}$, and the voltage vectors on the secondary side have a magnitude of $69 \mathrm{~V}$ with an angle of $120^{\circ}$ between these vectors. These vectors add to zero, as in the phasor diagram of Figure A-2.

When a ground is applied to Phase Conductor A, as in Figure A-3, this phase goes to ground potential, while the two unfaulted phases have 4,800 V applied across each. This causes an increase in the ground current $\mathrm{I}_{\mathrm{G}}$ from $2 \mathrm{~A}$ to $6 \mathrm{~A}$ and an increase in voltage on the secondary of the broken delta from $0 \mathrm{~V}$ to $208 \mathrm{~V}$. This phasor diagram is shown in Figure A-4.

Grounding phase conductors of an ungrounded delta system produces a minimum current unbalance of $3.46 \mathrm{~A}$ in this case. Overcurrent relays are not normally installed to detect this small current unbalance. But there are instances in which as much as 20-30 A have been detected. This is still less than the typical overcurrent settings of $70 \mathrm{~A}$ for a recloser on the circuit and 1,000 A or higher for a breaker at the substation.

\section{A.1. Ungrounded Delta Ground Detection - Normal Condition, No Ground Applied to Phase Conductor}

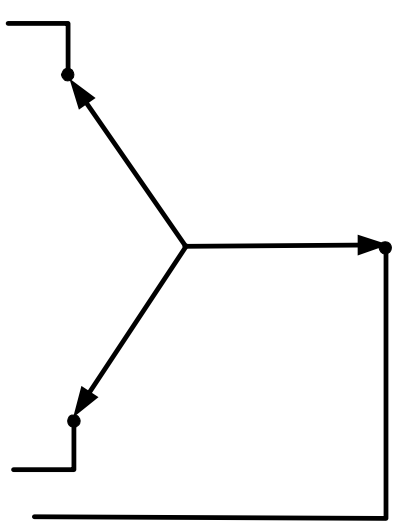

System Transformation Wye - Delta

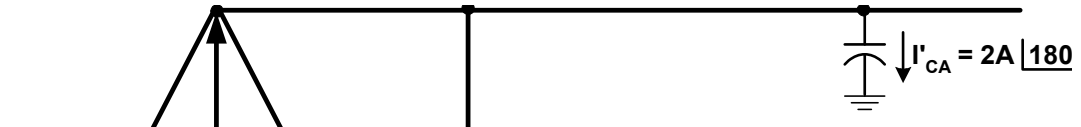

\section{$4.8 \mathrm{kV}$}

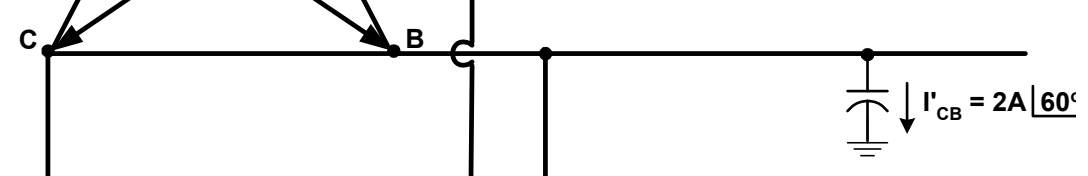

$$
\mathrm{VT}=40: 1=\frac{2771}{40}=69 \mathrm{~V}
$$
2771vi $40: 1$ 27?

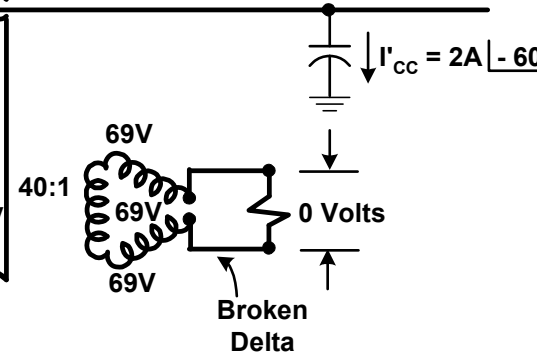

Figure A-1. Normal leakage currents to ground because of distributed capacitance 


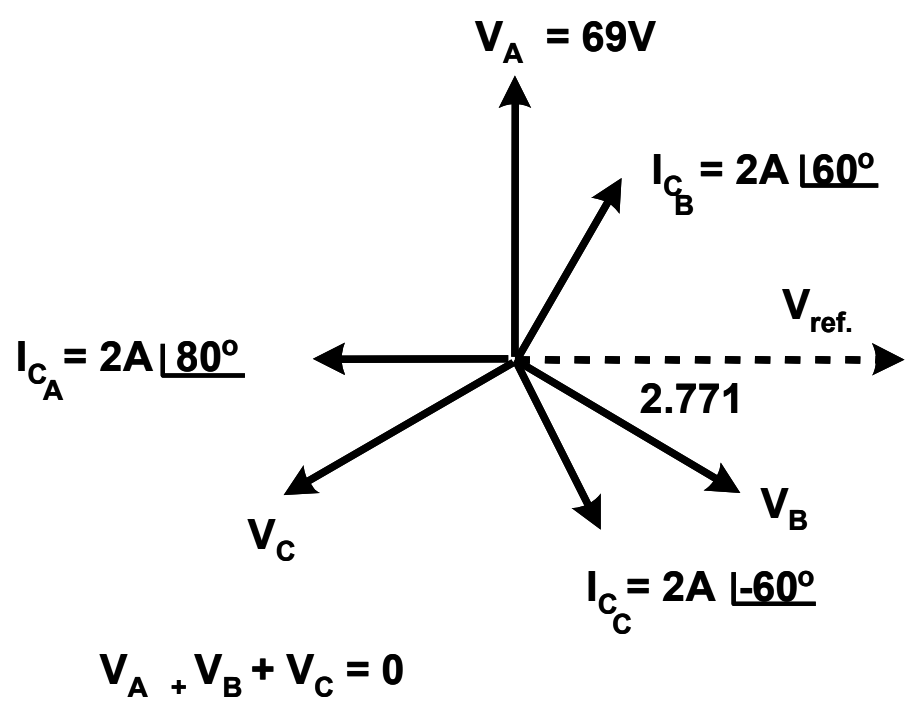

Figure A-2. Normal leakage currents to ground because of distributed capacitance - phasor diagram

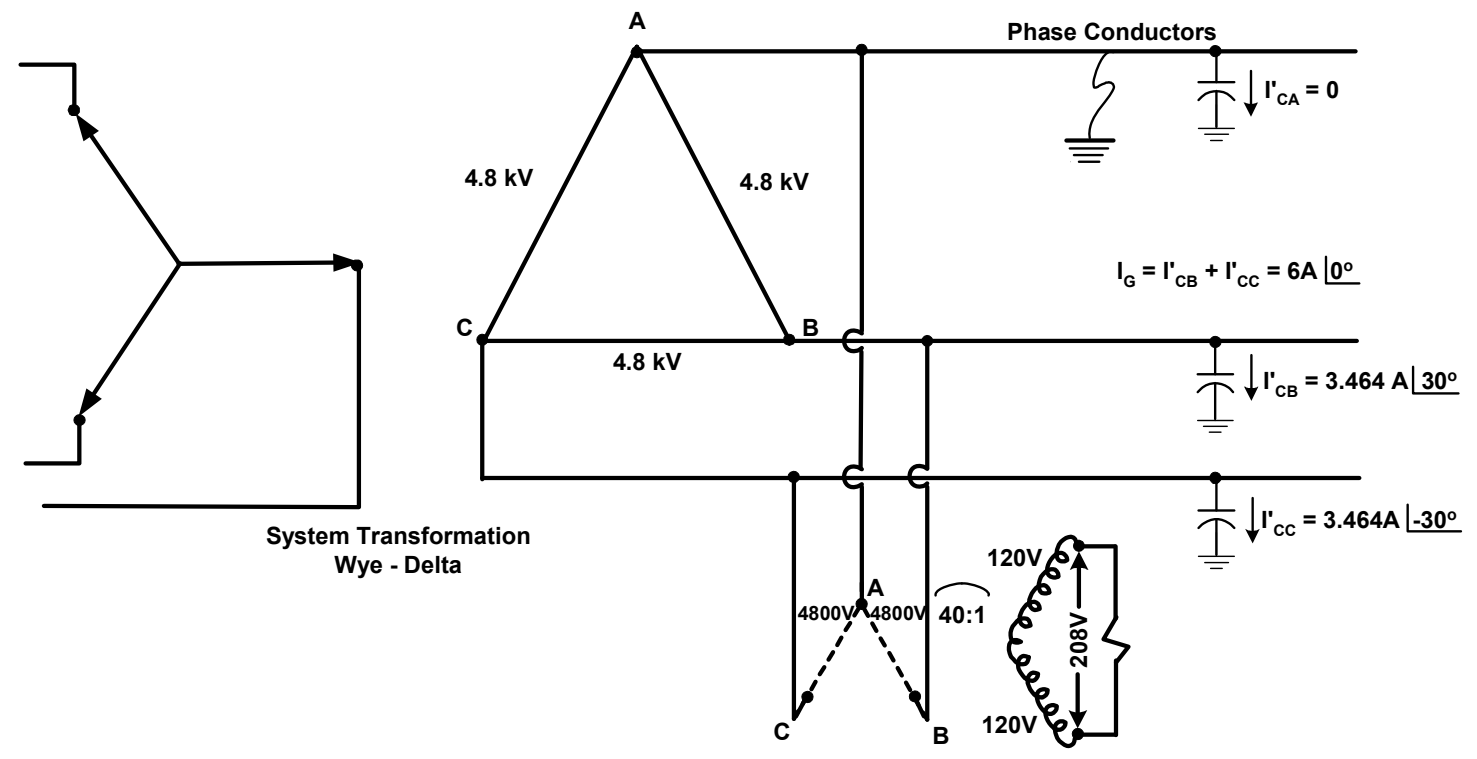

Figure A-3. Increased leakage current on the unfaulted phases and resultant ground current $I_{G}$ and resultant zero sequence voltage $3 V_{O}$ 


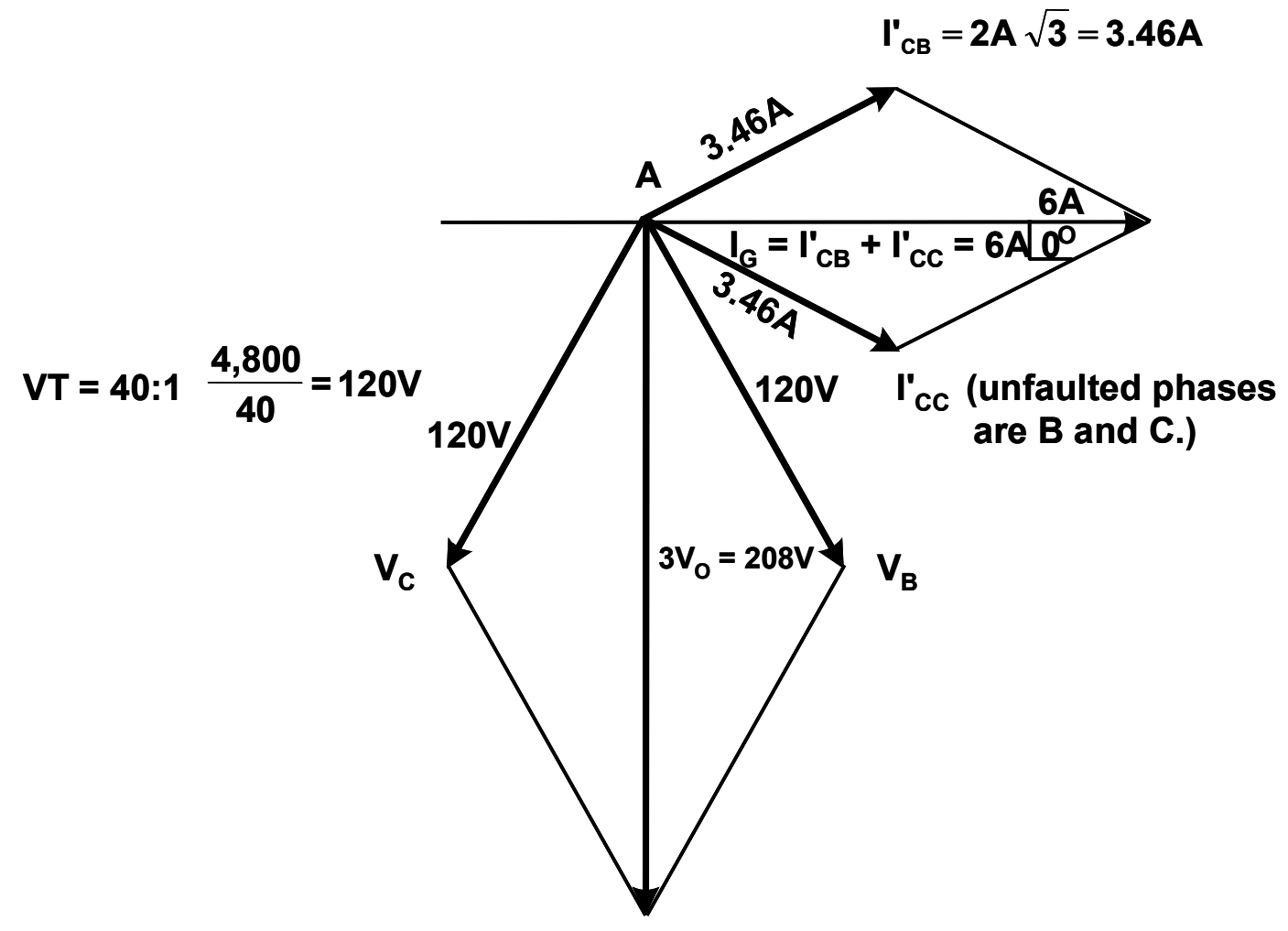

Figure A-4. Increased leakage current on the unfaulted phases and resultant ground current $I_{G}$ and resultant zero sequence voltage $3 V_{O}$ - phasor diagram

\section{A.2. Relay Settings}

Phase Settings at Substation

STATION: MILFD POSITION: T1-L

EQUIPMENT: TRF 1 TO DC 8103

SHEET\#: 1

SCHEME: A_REL

FUNCTION: OVERCURRENT XYZ-51

RELAY TYPE: CO-8 (4-12)

PT RATIO: $\quad 0: 1$

CT RATIO: $120: 1$

NORM:

CT NOTE: $\quad 600: 5 \mathrm{MR}$

T.O.:

$\mathrm{PU}=7.5 \mathrm{X} 120$

10 MVA TRF'S.

$\mathrm{L}=1.75$

40 CYCLES AT 37.5 AMPS

198 CYCLES AT 15 AMPS 
Neutral Settings at Substation

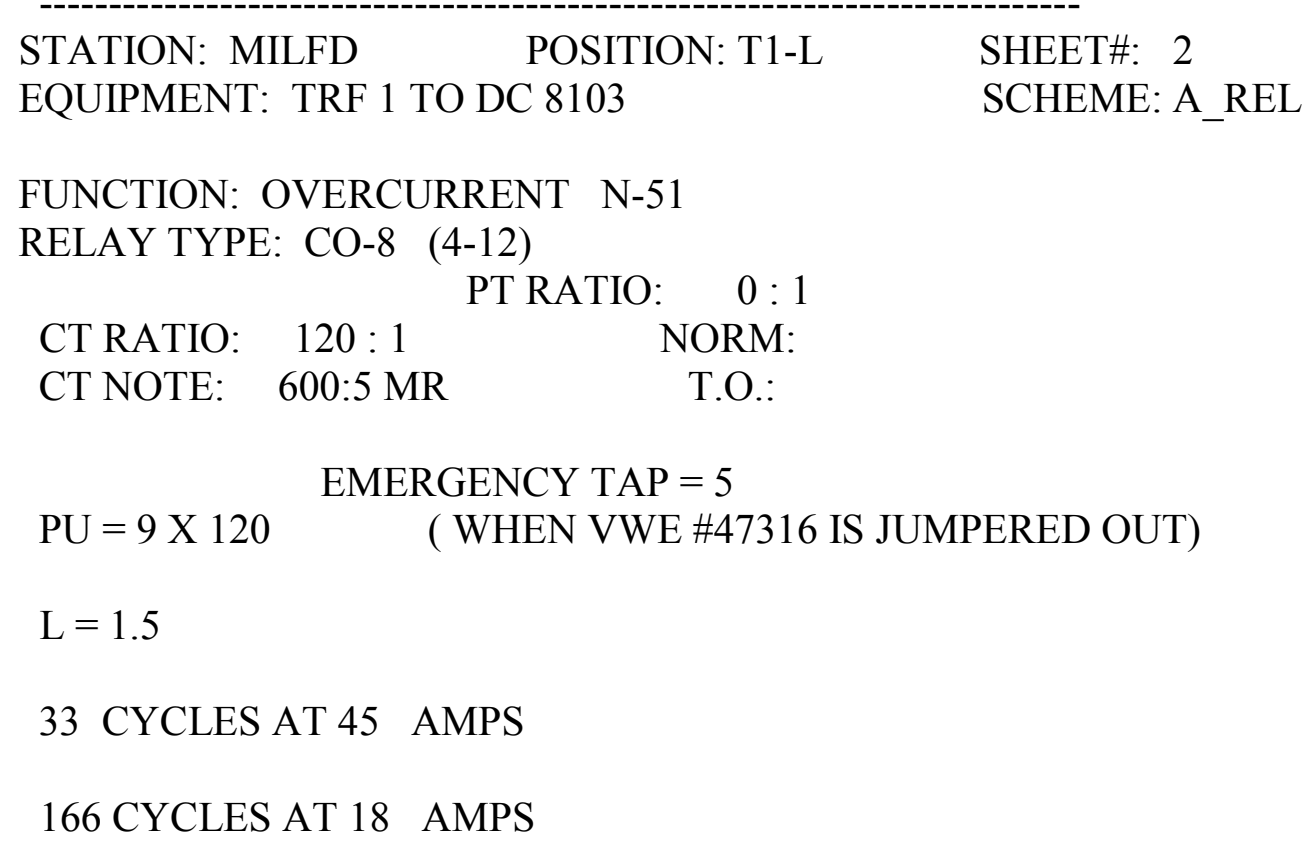

\section{Reclosing Settings at Substation}

\begin{tabular}{|c|c|c|c|}
\hline $\begin{array}{l}\text { STATION: MILFD } \\
\text { EQUIPMENT: TRF } 1\end{array}$ & $\begin{array}{r}\mathrm{PO} \\
\text { rO DC } 81\end{array}$ & $\begin{array}{l}\text { SITION: T1-L } \\
03\end{array}$ & $\begin{array}{l}\text { SHEET\#: } 3 \\
\text { SCHEME: A_REL }\end{array}$ \\
\hline FUNCTION: RECLO & ING 179 & & \\
\hline RELAY TYPE: PAR] & OF PR & & \\
\hline $\begin{array}{l}\text { CT RATIO: } \quad 0: 1 \\
\text { CT NOTE: }\end{array}$ & PT RA & $\begin{array}{l}\text { TIO: } 0: 1 \\
\text { NORM: } \\
\text { T.O.: }\end{array}$ & \\
\hline RECLOSE: 30 SEC & $30 \mathrm{SEC}$ & $30 \mathrm{SEC}$ & \\
\hline
\end{tabular}




\section{Phase Settings on Recloser}

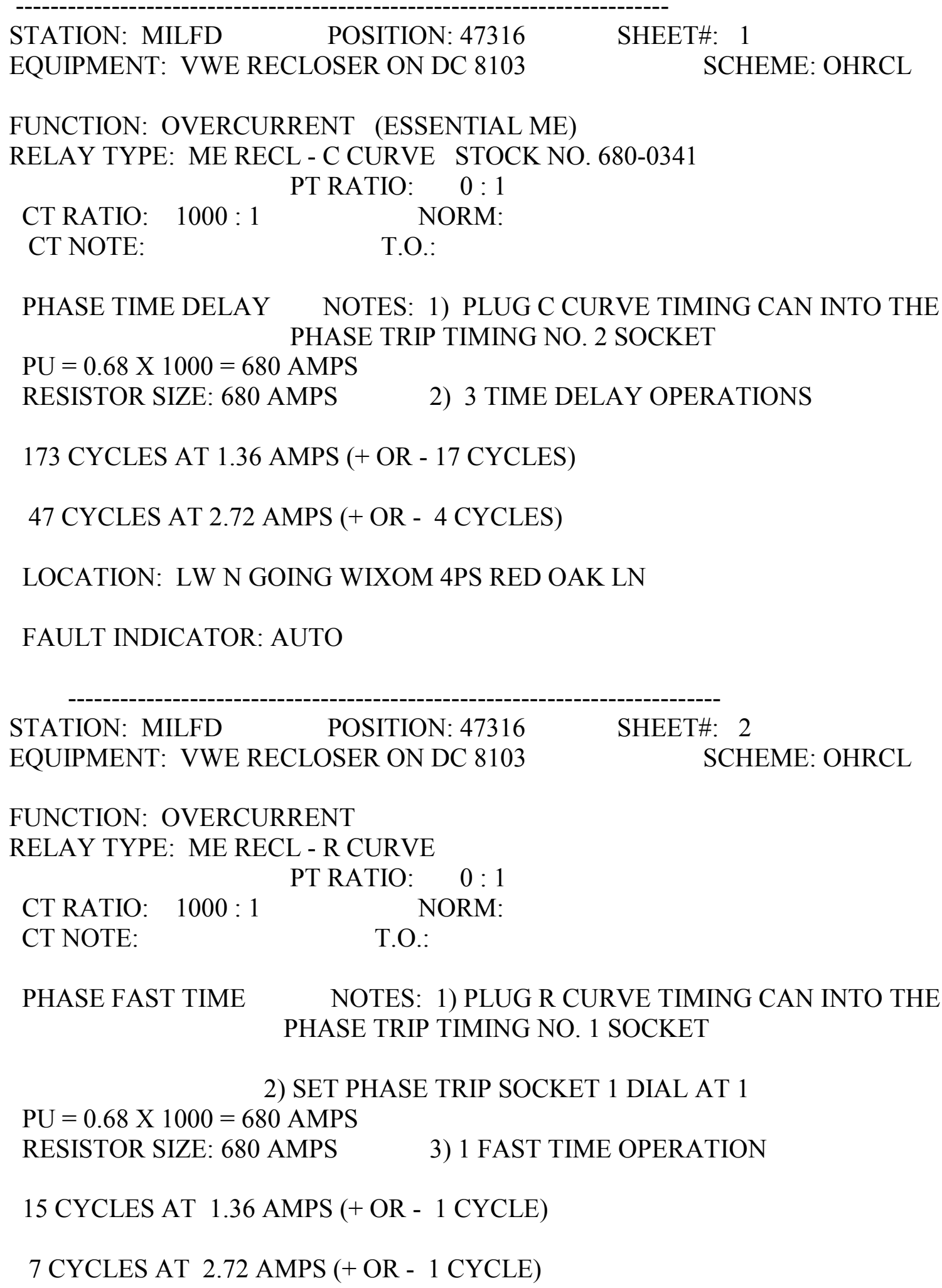




\section{Neutral Settings on Recloser}

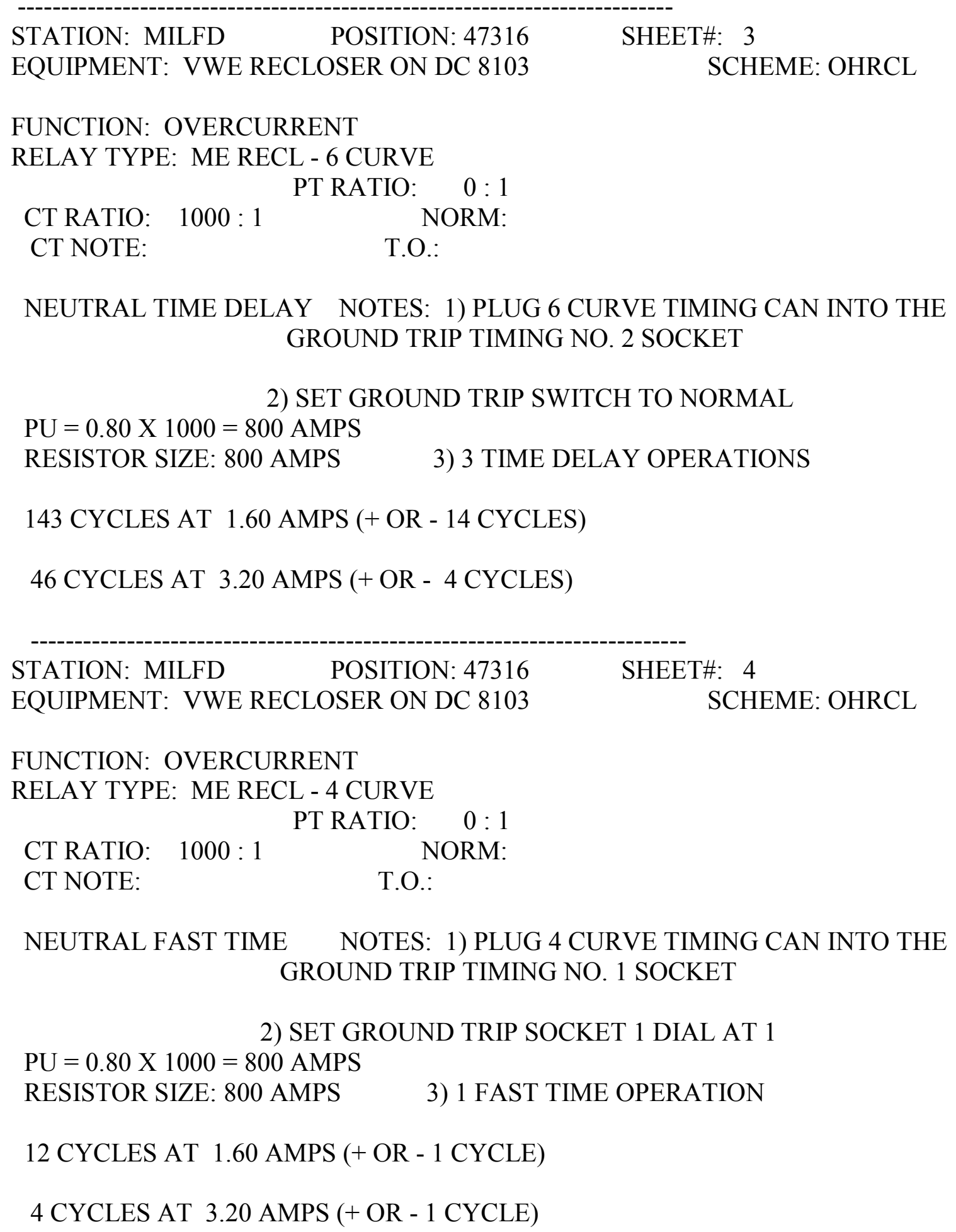




\section{Reclosing Setting on Recloser}

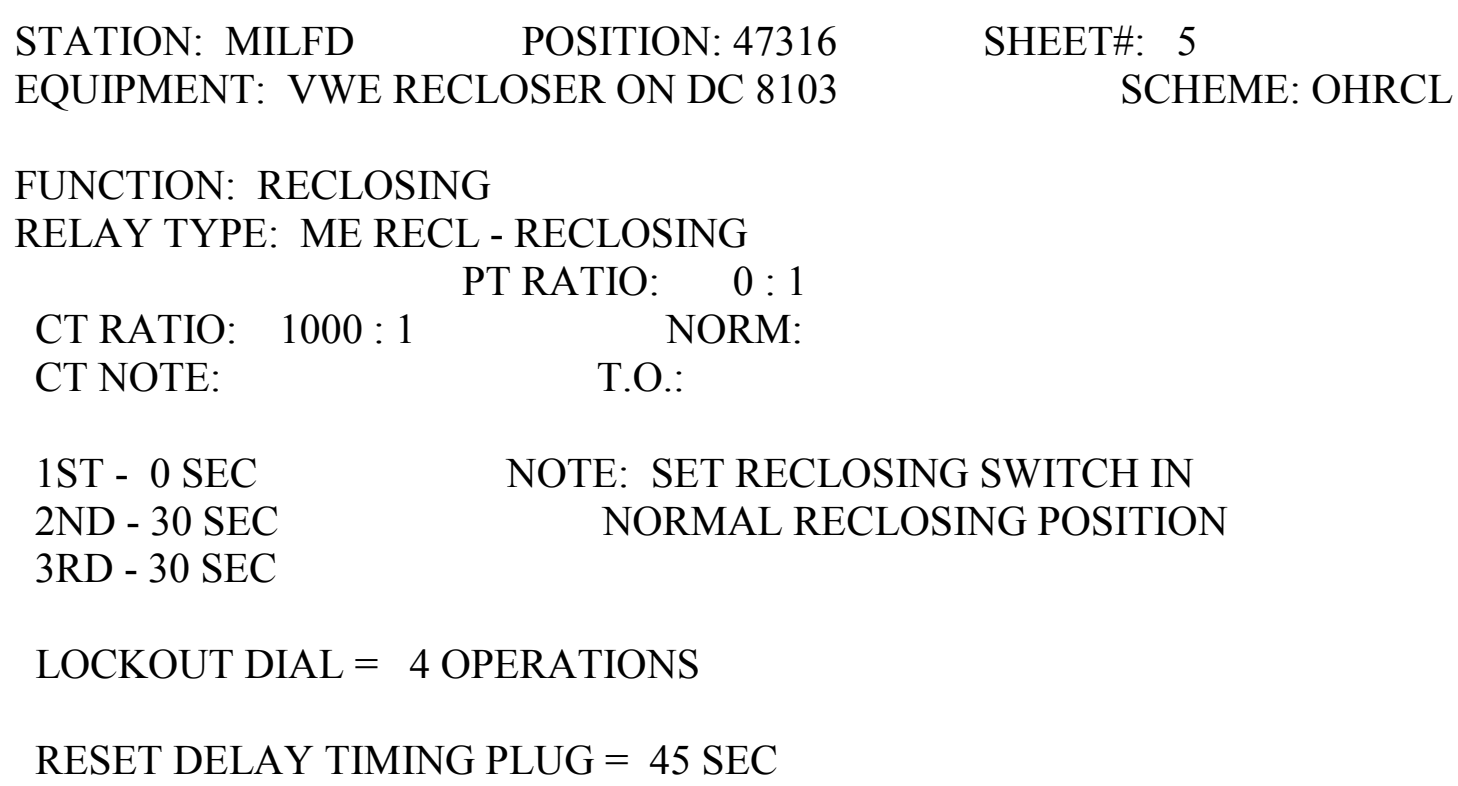

\section{Phase Neutral and Reclosing Setting on Form 5 Control}

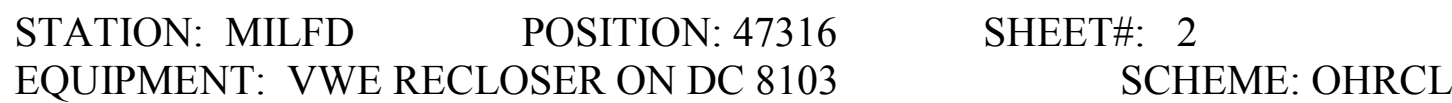

FUNCTION: OVERCURRRENT

RELAY TYPE: FORM5 (SHEET 2 OF 7)

PT RATIO: $0: 1$

CT RATIO: $0: 1 \quad$ NORM:
CT NOTE:

NORMAL ALT1 ALT2 ALT3 PHASE PROFILE|NORMAL ALT1 ALT2 ALT3 GROUND PROFILE OPERATIONS TO LOCKOUT:4 |OPERATIONS TO LOCKOUT:4 NORMAL PU $=0.68$ X $1000=680$ AMPS $\mid$ NORMAL PU $=0.60 \times 1000=600$ AMPS

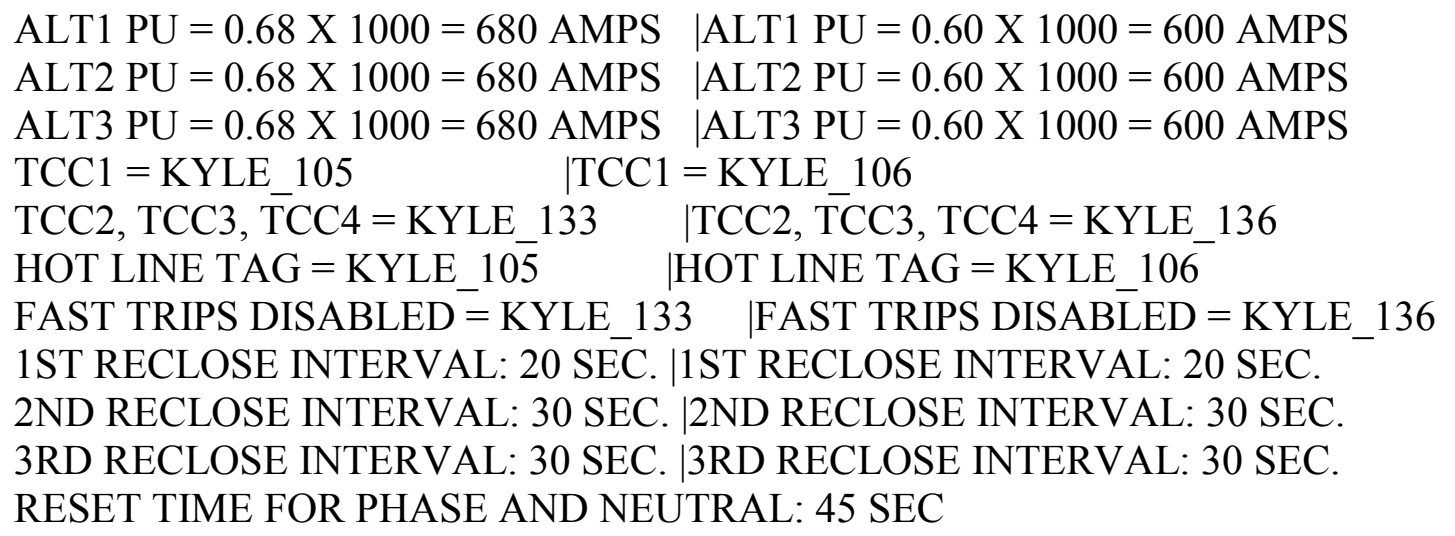




\section{Appendix B: Circuit Modeling}

\section{B.1 Circuit Modeling Verification}

The circuit was modeled using CC, CP, and VDC. Based on actual circuit-measured data taken from a circuit with a similar composition of load (Davis, Krups, and Diedzic 1983) as the Milford Circuit DC 8103, equations, B.1 and B.2 were developed for the summer period when the ambient temperature ranged from $80^{\circ} \mathrm{F}$ to $90^{\circ} \mathrm{F}$. The resultant change in real power $\mathrm{P}$ and reactive power $\mathrm{Q}$ for the range of source voltage $-2.8 \%(119.5 \mathrm{~V})$ to $0 \%(122.9 \mathrm{~V})$ to $+4.2 \%(128.0 \mathrm{~V})$ was

$$
\frac{\% \Delta \mathrm{P}}{\% \Delta \mathrm{V}}=1.26
$$

Equation B.1

and

$$
\frac{\% \Delta \mathrm{Q}}{\% \Delta \mathrm{V}}=4.66
$$

Equation B.2

To see which of the three model simulations best agrees with the measured circuit data, tests 7 and 8 were repeated for $95 \%$ primary voltage and $\mathrm{HL}$ conditions. By viewing the line current data of Table B-1 and comparing the differences between the actual and simulated values for each load model in Table B-2, it can be seen that the CC and VDC models best represent the load characteristics for the circuit.

To check the accuracy, the actual line currents at Node 0 were subtracted from the simulated values (shown as $\delta$ ). Then the absolute values of these phase current differences were summed and divided by three (the number of phases). For example, Test 7 for the $\mathrm{CC}$ model shows an absolute difference of 94 . To obtain a percent difference between the actual and simulated currents, the actual line current magnitudes were summed and divided by three.

This value $\$: \frac{\left|I_{3 \Phi}\right|}{3}$ forms the basis for comparing the deviation $\ddagger$ " $\frac{|\delta|}{3}$ with the basis for each model applied.

This resulted in $3.9 \%$ for the Test $7 \mathrm{CC}$ model. The CP model had a deviation of $12.5 \%$. The VDC model was slightly better (3.6\%) than the CC model. When this process was repeated for Test 8 at $95 \%$ primary voltage, the VDC model had a deviation of only $2 \%$, which is less than the measurement accuracy of $3 \%$.

The change in real power $\mathrm{P}$ and the change in reactive power $\mathrm{Q}$ as a function of change in source voltage V was defined (in (Davis, Krups, and Diedzic 1983) as

$$
\frac{\% \Delta \mathrm{P}}{\% \Delta \mathrm{V}}=1.26 \% \text { and } \frac{\% \Delta \mathrm{Q}}{\% \Delta \mathrm{V}}=4.66 \text {. }
$$


These percent changes in $\mathrm{P}$ and $\mathrm{Q}$ represent changes in flow at the source for changes in source voltage from $99.58 \%(119.5 \mathrm{~V})$ to $106.67 \%(128.0 \mathrm{~V})$.

To implement the load characteristics into the power flow algorithm, it becomes necessary to represent the percent changes in $\mathrm{P}$ and $\mathrm{Q}$ as percent change in current.

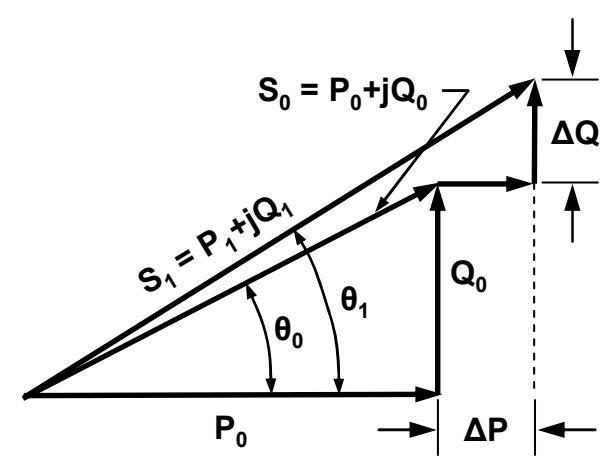

Figure B-1. Percent change in kilovolt-amperes or current from percent change in $P$ and $Q$

The three-phase kilovolt-ampere flow $\mathrm{S}$ is proportional to the phase circuit I. Therefore, the percent change in current (I) for a $1 \%$ change in voltage is equal to the percent change in kilovolt-amperes, or

$$
\% \Delta \mathrm{S}=\% \Delta \mathrm{I}=\frac{\mathrm{S}_{1}-\mathrm{S}_{0}}{\mathrm{~S}_{0}} \times 100 .
$$

For this study, at $\mathrm{HL}$ condition, the $\% \Delta \mathrm{I}=1.8 \%$ for a $1 \%$ change in voltage. The example below shows how the percent change in current of $1.9 \%$ is calculated for the initial values of $\mathrm{P}_{0}=2,000$ $\mathrm{kW}$ and $\mathrm{Q}_{0}=1,000 \mathrm{kVArs}$ for a $13.2-\mathrm{kV}$ system and a $1 \%$ change in voltage at the source.

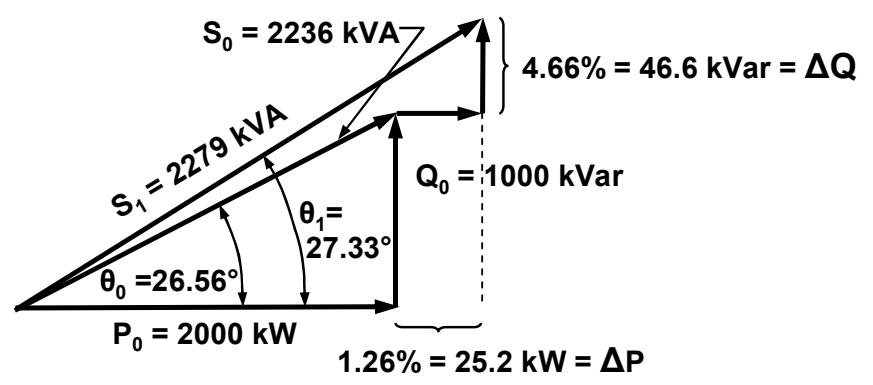

Figure B-2. Percent change in kilovolt-amperes or current 


$$
\begin{aligned}
& \% \Delta \mathrm{S}=\frac{\mathrm{S}_{1}-\mathrm{S}_{0}}{\mathrm{~S}_{0}} \times 100=\frac{2279-2236}{2236} \times 100=1.9 \% \\
& \mathrm{I}_{1}=\frac{2279 \mathrm{kVA}}{\sqrt{3} 13.2 \mathrm{kV}}=99.68 \mathrm{~A} \\
& \mathrm{I}_{0}=\frac{2236 \mathrm{kVA}}{\sqrt{3} 13.2 \mathrm{kV}}=97.80 \mathrm{~A} \\
& \% \Delta \mathrm{I}=\frac{\mathrm{I}_{1}-\mathrm{I}_{0}}{\mathrm{I}_{0}} \times 100=\frac{99.68-97.80}{97.80} \times 100=1.9 \%
\end{aligned}
$$

\section{B.2 Load Imbalance}

As previously noted, a 100\% load imbalance occurs at any node at which a line-to-line load or line-to-neutral load is connected to the wye-grounded systems. Proof of this statement is given below.

In Table B-4 (Test 8) at 95\% primary voltage and HL conditions with LTC, VR 1, VR 2, and capacitors 1,2, and 3 turned on, at Node $22 \mathrm{E}$, the phase current is 27.153 amperes, which indicates a line-to-neutral load. Now, from the zero sequence $\mathrm{I}_{0}$, positive sequence $\mathrm{I}_{1}$, and negative sequence $\mathrm{I}_{2}$ currents of $9.051 \mathrm{~A}$ from Table B-4, it can be shown that

$$
\begin{aligned}
\mathrm{I}_{\mathrm{A} 1} & =\left(\mathrm{I}_{\mathrm{A}}+\mathrm{I}_{\mathrm{B}} \mathbf{a}+\mathrm{I}_{\mathrm{C}} \mathbf{a}^{2}\right) / 3 \\
& =\left(27.153 \underline{0^{\circ}}+0+0\right) / 3 \\
& =9.051 \underline{0 \mathrm{O}} \\
\mathrm{I}_{\mathrm{A} 2} & =\left(\mathrm{I}_{\mathrm{A}}+\mathrm{I}_{\mathrm{B}} \mathbf{a}^{2}+\mathrm{I}_{\mathrm{C}} \mathbf{a}\right) / 3 \\
& =\left(27.153\left\lfloor 0^{\circ}+0+0\right) / 3\right. \\
& =9.051 \underline{0^{\circ}} \\
\mathrm{I}_{\mathrm{A} 0} & =\left(\mathrm{I}_{\mathrm{A}}+\mathrm{I}_{\mathrm{B}}+\mathrm{I}_{\mathrm{C}}\right) / 3 \\
& =\left(27.153\left\lfloor 0^{\circ}+0+0\right) / 3\right. \\
& =9.0510^{\circ}
\end{aligned}
$$

Equation B.3

Equation B.4

Furthermore,

$$
\begin{array}{ll}
\mathrm{I}_{\mathrm{B} 1}=\mathrm{I}_{\mathrm{A} 1} \mathbf{a}^{2}=9.051 \underline{1-120^{\circ}} & \text { Equation B.6 } \\
\mathrm{I}_{\mathrm{C} 1}=\mathrm{I}_{\mathrm{A} 1} \mathbf{a}=9.051 \underline{120^{\circ}} & \text { Equation B.7 } \\
\mathrm{I}_{\mathrm{B} 2}=\mathrm{I}_{\mathrm{A} 1} \mathbf{a}=9.051 \underline{120^{\circ}} & \text { Equation B.8 } \\
\mathrm{I}_{\mathrm{C} 2}=\mathrm{I}_{\mathrm{A} 1} \mathbf{a}^{2}=9.051\left\lfloor-120^{\circ}\right. & \text { Equation B.9 } \\
\mathrm{I}_{\mathrm{A} 0}=\mathrm{I}_{\mathrm{B} 0}=\mathrm{I}_{\mathrm{CO}}=9.0510^{\circ} & \text { Equation B. } 10
\end{array}
$$


and

$$
\begin{aligned}
\mathrm{I}_{\mathrm{A}} & =\mathrm{I}_{\mathrm{A} 1}+\mathrm{I}_{\mathrm{A} 2}+\mathrm{I}_{\mathrm{A} 0} \\
& =9.0510^{\circ}+9.051\left\lfloor 0^{\circ}+9.051\left\lfloor 0^{\circ}\right.\right. \\
& =27.1530^{\circ} .
\end{aligned}
$$

Equation B.11

The percent unbalanced current is then calculated from equations B.3 and B.4, or

$$
\frac{\mathrm{I}_{\mathrm{A} 2}}{\mathrm{I}_{\mathrm{A} 1}} \times 100=\frac{9.051}{9.051} \times 100=100 \% .
$$

Equation B.12

\section{B.3 System Zero Sequence Currents Become Negative Sequence Currents on the Generator Windings}

The zero sequence currents for each test are given in Table B-3. The values range from 45.43 A for Test 2 up to 50.24 A for Test 8 . Because most small synchronous machines are wyeconnected with an ungrounded neutral and the output terminals of these machines are normally connected to delta-wye transformers with the high-side wye grounded, there is no problem with these zero sequence current magnitudes if interconnected to the power system. For machines operating in an islanded mode with a delta-wye high-side transformer and a solidly grounded neutral, high zero sequence currents on the high side will appear as negative sequence currents on the machine windings. This is a very serious problem because it results in $100 \%$ negative sequence currents in the generator windings.

The zero sequence relays in the neutral at the substation are set to trip at current levels higher than the phase currents to ensure the neutral relay does not trip for single-phase switching operations on the circuit. But a time delay could be added to permit a lower neutral relay trip setting (to ignore single-phase switching conditions and maintain selectivity) so it becomes more sensitive to line-to-ground faults.

It will be shown that 100-A rated output current (on Phase A only) on the $13.2 \mathrm{kV}$ on the high side of the transformer of a generating unit results in $33.34 \mathrm{~A}$ of zero sequence current on the load side of the generator step-up transformer. This is a common value of zero sequence currents. This produces a negative sequence current of $916 \mathrm{~A}$ on the generator windings, or a $100 \%$ current imbalance or

$$
\frac{I_{2}}{I_{1}} \%=\frac{916 \mathrm{~A}}{916 \mathrm{~A}} \times 100=100 \% .
$$




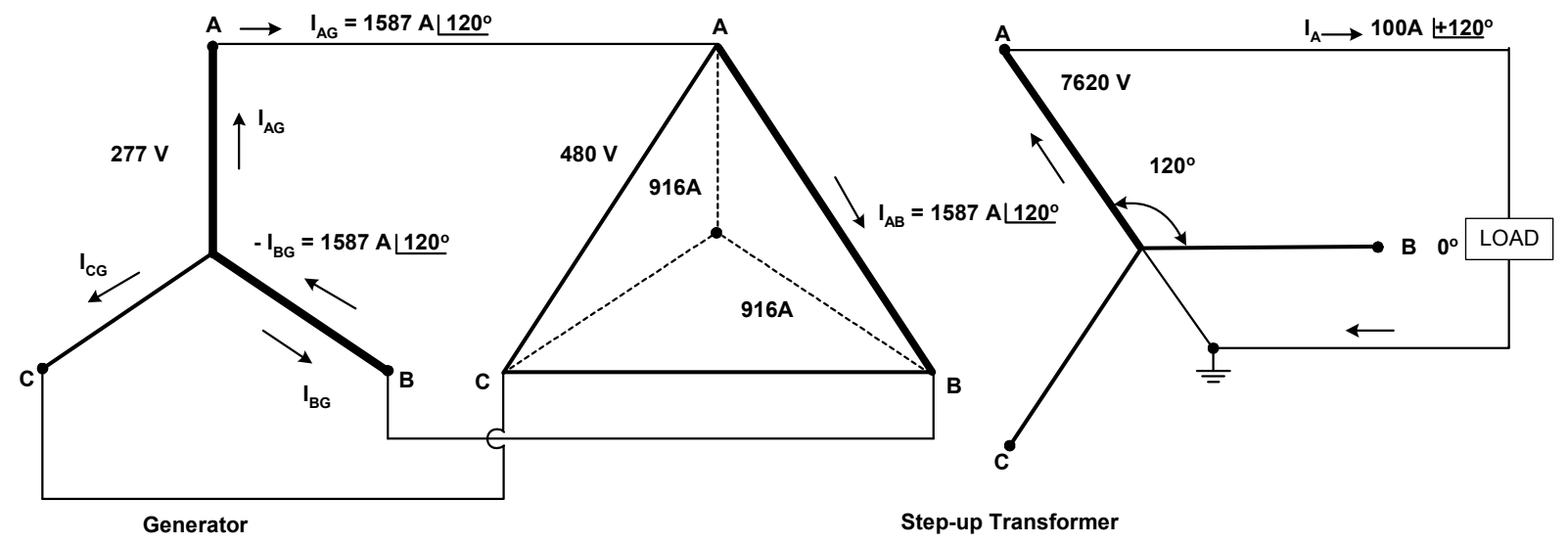

Figure B-3. Zero sequence current on load side becomes negative sequence current to the generator

Let, $\frac{\mathrm{V}_{1}}{\mathrm{~V}_{2}}=\frac{7620}{480}$ or $\frac{\mathrm{N}_{1}}{\mathrm{~N}_{2}}=15.875$, where $\mathrm{V}_{1}$ is the load-side voltage and $\mathrm{V}_{2}$ is the generator terminal voltage. The turns ratio is 15.875 .

By applying symmetrical components to the load side,

$$
\begin{aligned}
\mathrm{I}_{\mathrm{A}}= & \mathrm{I}_{\mathrm{A} 1}+\mathrm{I}_{\mathrm{A} 2}+\mathrm{I}_{\mathrm{A} 0}=100 \mathrm{~A} \perp 120^{\circ} \\
\mathrm{I}_{\mathrm{A} 1}= & \left(\mathrm{I}_{\mathrm{A}}+\mathrm{I}_{\mathrm{B}} \mathbf{a}+\mathrm{I}_{\mathrm{C}} \mathbf{a}^{2}\right) / 3 \\
& \left(100+120^{\circ}+0+0\right) / 3 \\
= & 33.34+\underline{120^{\circ}} \\
\mathrm{I}_{\mathrm{A} 2}= & \left(\mathrm{I}_{\mathrm{A}}+\mathrm{I}_{\mathrm{B}} \mathbf{a}^{2}+\mathrm{I}_{\mathrm{C}} \mathbf{a}\right) / 3 \\
= & 33.34+120^{\circ}+0+0
\end{aligned}
$$

Equation B.15

and

$$
\begin{aligned}
\mathrm{I}_{\mathrm{A} 0} & =\left(\mathrm{I}_{\mathrm{A}}+\mathrm{I}_{\mathrm{B}}+\mathrm{I}_{\mathrm{C}}\right) / 3 \\
& =33.34+\underline{120^{\circ}+0+0}
\end{aligned}
$$

$\therefore 3 \mathrm{I}_{\mathrm{A} 0}=100$ amperes.

Applying symmetrical components to the generator side,

$$
\begin{aligned}
& \mathrm{I}_{\mathrm{AG} 1}=\left(\mathrm{I}_{\mathrm{AG}}+\mathrm{I}_{\mathrm{BG}} \mathbf{a}+\mathrm{I}_{\mathrm{CG}} \mathbf{a}^{2}\right) / 3, \\
& \mathrm{I}_{\mathrm{AG} 2}=\left(\mathrm{I}_{\mathrm{AG}}+\mathrm{I}_{\mathrm{BG}} \mathbf{a}^{2}+\mathrm{I}_{\mathrm{CG}} \mathbf{a}\right) / 3,
\end{aligned}
$$

Equation B.18 
and

$$
\mathrm{I}_{\mathrm{AG} 0}=\left(\mathrm{I}_{\mathrm{AG}}+\mathrm{I}_{\mathrm{BG}}+\mathrm{I}_{\mathrm{CG}}\right) / 3 . \quad \text { Equation B.19 }
$$

Substituting the rated phase current from the generator into the three equations above results in:

$$
\begin{array}{ll}
\mathrm{I}_{\mathrm{AG} 1}=\left(1,587 \mathrm{~A}+\underline{120^{\circ}}+1,587 \mathrm{~A}\left\lfloor-60^{\circ} \mathbf{a}+0\right) / 3,\right. & \text { Equation B.20 } \\
\mathrm{I}_{\mathrm{AG} 2}=\left(1,587 \mathrm{~A}+\left\lfloor 120^{\circ}+1,587 \mathrm{~A}\left\lfloor-60^{\circ} \mathbf{a} 2+0\right) / 3,\right.\right. & \text { Equation B.21 }
\end{array}
$$

and

$$
\mathrm{I}_{\mathrm{AG} 0}=\left(1,587 \mathrm{~A}+\underline{L 120^{\circ}}+1,587 \mathrm{~A} L-60^{\circ}+0\right) / 3 . \quad \text { Equation B.22 }
$$

Note: $-\mathrm{I}_{\mathrm{BG}}$ in Figure B-3 shows the current flow path, but polarity on $-\mathrm{I}_{\mathrm{BG}}$ must be the opposite direction $+\mathrm{I}_{\mathrm{BG}}$ for correct symmetrical component calculation (i.e. $-\mathrm{I}_{\mathrm{BG}}=1,587\left\lfloor 120^{\circ}\right.$ $\mathrm{I}_{\mathrm{BG}}=1,587\left(-60^{\circ}\right)$.

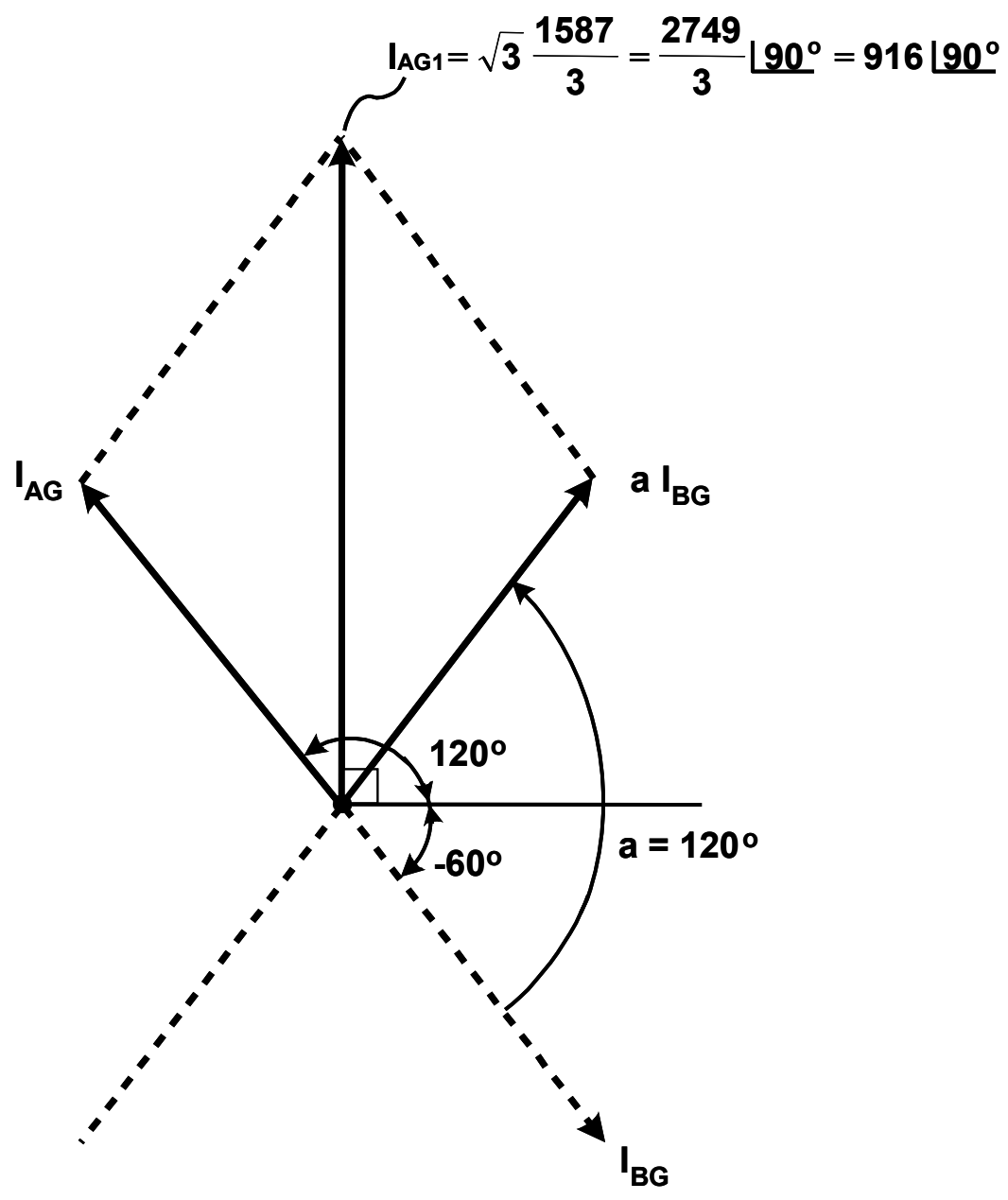

Figure B-4. Determining $\mathrm{I}_{\mathrm{AG} 1}$ 


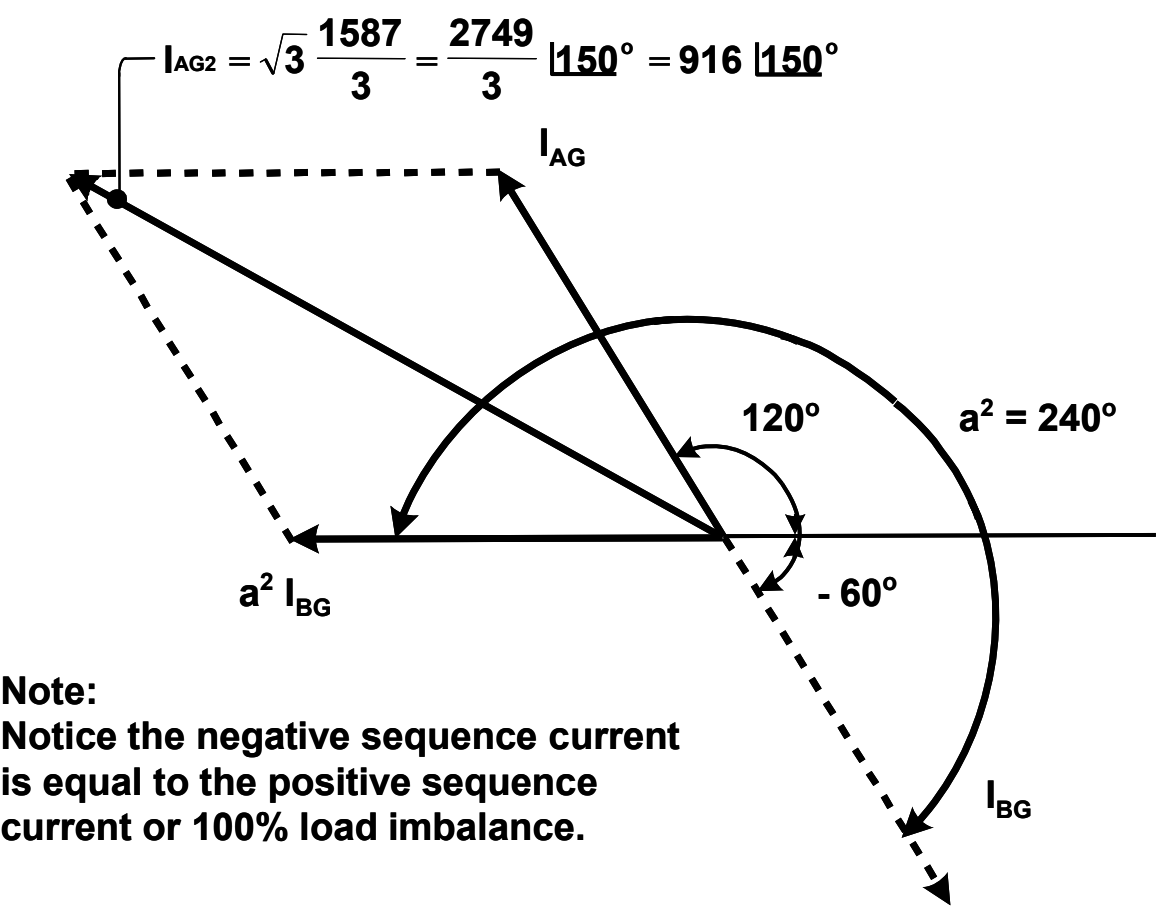

Figure B-5. Determining $I_{A G 2}$

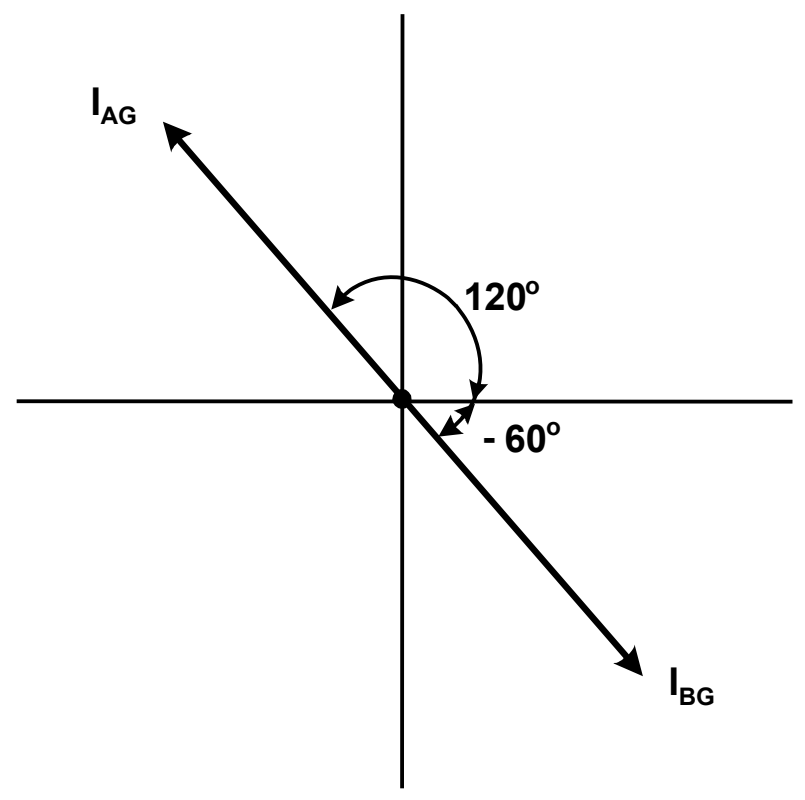

$\mathrm{I}_{\mathrm{AG} 0}=\mathbf{0}$ because there is no ground on the generator wye connection.

Figure B-6. Determining $I_{A G 0}$ 
The rated power for the generator is

$$
\begin{aligned}
P_{\text {rated generator }} & =\sqrt{3} \mathrm{~V}_{\mathrm{L}-\mathrm{L}} \mathrm{I}_{\mathrm{L}} \\
& =\sqrt{3}(13.2 \mathrm{kV})(100 \mathrm{~A})=2,286 \mathrm{kVA} \text { machine rating, }
\end{aligned}
$$

and the rated current is

$$
\mathrm{I}_{\text {rated generator }}=\frac{2286 \mathrm{kVA}}{\sqrt{3} 480 \mathrm{~V}}=2749 \mathrm{~A} \text { full-load rated current, or } \mathrm{I}_{\mathrm{AG} 1} \text {. }
$$

The percent unbalance loading based on machine rating is

$$
\frac{\mathrm{I}_{\mathrm{AG} 2}}{\mathrm{I}_{\mathrm{AG} 1}} \times 100=\frac{916 \mathrm{~A}}{2749 \mathrm{~A}} \times 100=33.3 \% \text {. }
$$

For a balanced load, $\mathrm{I}_{\mathrm{AG}}=\mathrm{I}_{\mathrm{BG}}=\mathrm{I}_{\mathrm{CG}}$, and

$$
\begin{aligned}
& \mathrm{I}_{\mathrm{AG} 1}=\left(\mathrm{I}_{\mathrm{AG}}+\mathrm{I}_{\mathrm{BG}} \mathbf{a}+\mathrm{I}_{\mathrm{CG}} \mathbf{a}^{2}\right) / 3, \\
& \mathrm{I}_{\mathrm{AG} 1}=\left(2,749+90^{\circ}+2,749{\underline{+90^{\circ}}}^{\circ}+2,749+90^{\circ}\right) / 3,
\end{aligned}
$$

and

$$
\mathrm{I}_{\mathrm{AG} 1}=2,749 \mathrm{~A} \lcm{90^{\circ}} .
$$

Even though the rated output on the high side of the transformer is $100 \mathrm{~A}$, only $33.34 \mathrm{~A}$ of zero sequence current (on the high side) causes a $100 \%$ unbalanced current $\frac{\mathrm{I}_{2}}{\mathrm{I}_{1}} \%$ on the generator, which has a rated generator currency of 2,747 A per phase. The negative sequence current of $916 \mathrm{~A}$ is $33 \%$ of the generator machine rating $(2,749 \mathrm{~A})$ current.

This negative sequence current of 916 A will cause the generator negative sequence relay to trip the unit because it is common to set this relay to $10 \%$ of the machine rating. Even with 33.4 A of single-phase load, which is one-third of the $100 \mathrm{~A}$ of rated phase current on the high side, the negative sequence relay will trip the unit. Notice that the generating unit could only run at $10 \%$ of full rated output (or 30 A divided by 300 A full-rated three-phase capacity) without tripping. 
Table B-1. Simulation Summary Data for Tests 7 and 8 at $95 \%$ Voltage and HL Conditions and a Comparison of Modeling Results

for CC, CP, and VDC

\begin{tabular}{|c|c|c|c|c|c|c|c|c|c|c|c|c|c|c|c|c|c|c|}
\hline \multirow[b]{2}{*}{ Test No. } & \multirow[b]{2}{*}{$\begin{array}{c}\text { Pri. Volt } \\
\%\end{array}$} & \multirow[b]{2}{*}{$\begin{array}{l}\text { Load } \\
\text { Type }\end{array}$} & \multirow{2}{*}{\begin{tabular}{|c} 
LTC Tap \\
/ Reg \\
Tap
\end{tabular}} & \multicolumn{3}{|c|}{ Node 00} & \multicolumn{3}{|c|}{ Lowest $3 \Phi$ Voltage } & \multirow[t]{2}{*}{$\begin{array}{c}\text { Lowest } \\
1 \Phi \\
\text { Voltage } \\
\end{array}$} & \multirow{2}{*}{$\begin{array}{l}\text { Highest } \\
\text { |2/11\% }\end{array}$} & \multirow{2}{*}{$\begin{array}{l}\text { Highest } \\
\text { V2/V1 \% }\end{array}$} & \multicolumn{3}{|c|}{ kW Losses/Ф \& Total } & \multicolumn{3}{|c|}{ kVAr Losses/ $\Phi$ \& Total } \\
\hline & & & & IA & IB & IC & VA & VB & VC & & & & A & B & C & A & B & C \\
\hline 7 & \begin{tabular}{l|l}
95 & \\
\end{tabular} & $\mathrm{CC}$ & 15 & 807.02 & 736.28 & 908.03 & 109.79 & 120.08 & 108.81 & 109.05 & 100.05 & 3.32 & 210.93 & 20.38 & 228.47 & 1119.86 & 820.68 & 1369.59 \\
\hline \multicolumn{4}{|c|}{ Cap (1) $900 \mathrm{kVAr}$} & & & & & & & & & & & 459.78 & & & 3310.13 & \\
\hline \multicolumn{19}{|c|}{ Cap (2) $900 \mathrm{kVAr}$} \\
\hline \multicolumn{19}{|c|}{ Cap (3) $1200 \mathrm{kVAr}$} \\
\hline 7 & \begin{tabular}{|l|l}
95 \\
\end{tabular} & $\mathrm{CP}$ & 16 & 871.31 & 720.56 & 1037.24 & 108.43 & 124.00 & 102.64 & 102.89 & 100.04 & 5.15 & 204.93 & -31.59 & 419.29 & 1392.44 & 729.90 & 1982.90 \\
\hline \multicolumn{4}{|c|}{ Cap (1) $900 \mathrm{kVAr}$} & & & & & & & & & & & 592.63 & & & 4105.24 & \\
\hline & & $0 \mathrm{kVAr}$ & & & & & & & & & & & & & & & & \\
\hline \multicolumn{19}{|c|}{ Cap (3) $1200 \mathrm{kVAr}$} \\
\hline 7 & \begin{tabular}{|c|}
95 \\
\end{tabular} & VDC & 14 & 756.47 & 723.87 & 844.61 & 110.88 & 118.45 & 110.72 & 110.79 & 100.05 & 2.61 & 190.80 & 40.20 & 170.49 & 953.41 & \begin{tabular}{|l|}
811.06 \\
\end{tabular} & 1162.98 \\
\hline \multicolumn{4}{|c|}{ Cap (1) $900 \mathrm{kVAr}$} & & & & & & & & & & & 401.49 & & & \begin{tabular}{|l|}
2927.45 \\
\end{tabular} & \\
\hline \multicolumn{19}{|c|}{ Cap (2) $900 \mathrm{kVAr}$} \\
\hline \multicolumn{19}{|c|}{ Cap (3) $1200 \mathrm{kVAr}$} \\
\hline 8 & \begin{tabular}{|l|l}
95 \\
9
\end{tabular} & $\mathrm{CC}$ & 15 & 828.54 & 744.03 & 928.42 & 114.70 & 122.11 & 111.24 & 110.46 & 100.05 & 3.08 & 221.99 & 16.61 & 244.04 & 1205.74 & 850.27 & 1447.40 \\
\hline \multicolumn{4}{|c|}{$\operatorname{VR}(1) 32,14,32$} & & & & & & & & & & & 482.64 & & & 3503.41 & \\
\hline \multicolumn{19}{|c|}{$\operatorname{VR}(2) 32,10,32$} \\
\hline \multicolumn{19}{|c|}{ Cap (1) $900 \mathrm{kVAr}$} \\
\hline \multicolumn{19}{|c|}{ Cap (2) $900 \mathrm{kVAr}$} \\
\hline \multirow{2}{*}{\multicolumn{19}{|c|}{ Cap (3) $1200 \mathrm{kVAr}$}} \\
\hline & & & & & & & & & & & & & & -18.75 & 403.81 & 1371.63 & 751.93 & 1988.70 \\
\hline & $\operatorname{VR}(1) 3$ & $2,8,32$ & & & & & & & & & & & & 585.36 & & & 4112.26 & \\
\hline & $\operatorname{VR}(2) 3$ & 4,32 & & & & & & & & & & & & & & & & \\
\hline & Cap (1) 9 & $0 \mathrm{kVAr}$ & & & & & & & & & & & & & & & & \\
\hline & Cap (2) 9 & $0 \mathrm{kVAr}$ & & & & & & & & & & & & & & & & \\
\hline & Cap (3) 12 & $00 \mathrm{kVA}$ & & & & & & & & & & & & & & & & \\
\hline 8 & \begin{tabular}{|l|l|}
95 \\
\end{tabular} & VDC & 15 & 801.31 & 756.68 & 888.74 & 115.48 & 121.15 & 113.13 & 112.50 & 100.05 & 2.52 & 215.99 & 38.73 & 193.39 & \begin{tabular}{|l|}
1092.14 \\
\end{tabular} & 894.21 & 1295.33 \\
\hline & $\operatorname{VR}(1) 32$ & 22,32 & & & & & & & & & & & & 448.11 & & & 3281.68 & \\
\hline & $\operatorname{VR}(2) 32$ & 15,32 & & & & & & & & & & & & & & & & \\
\hline & Cap (1) 9 & $0 \mathrm{kVAr}$ & & & & & & & & & & & & & & & & \\
\hline & Cap (2) 9 & $0 \mathrm{kVAr}$ & & & & & & & & & & & & & & & & \\
\hline & Cap (3) 12 & $00 \mathrm{kVA}$ & & & & & & & & & & & & & & & & \\
\hline
\end{tabular}


Table B-2. Comparison of Line Currents for Simulated and Actual Measurements for Each Load Model and Each Test 7 and 8

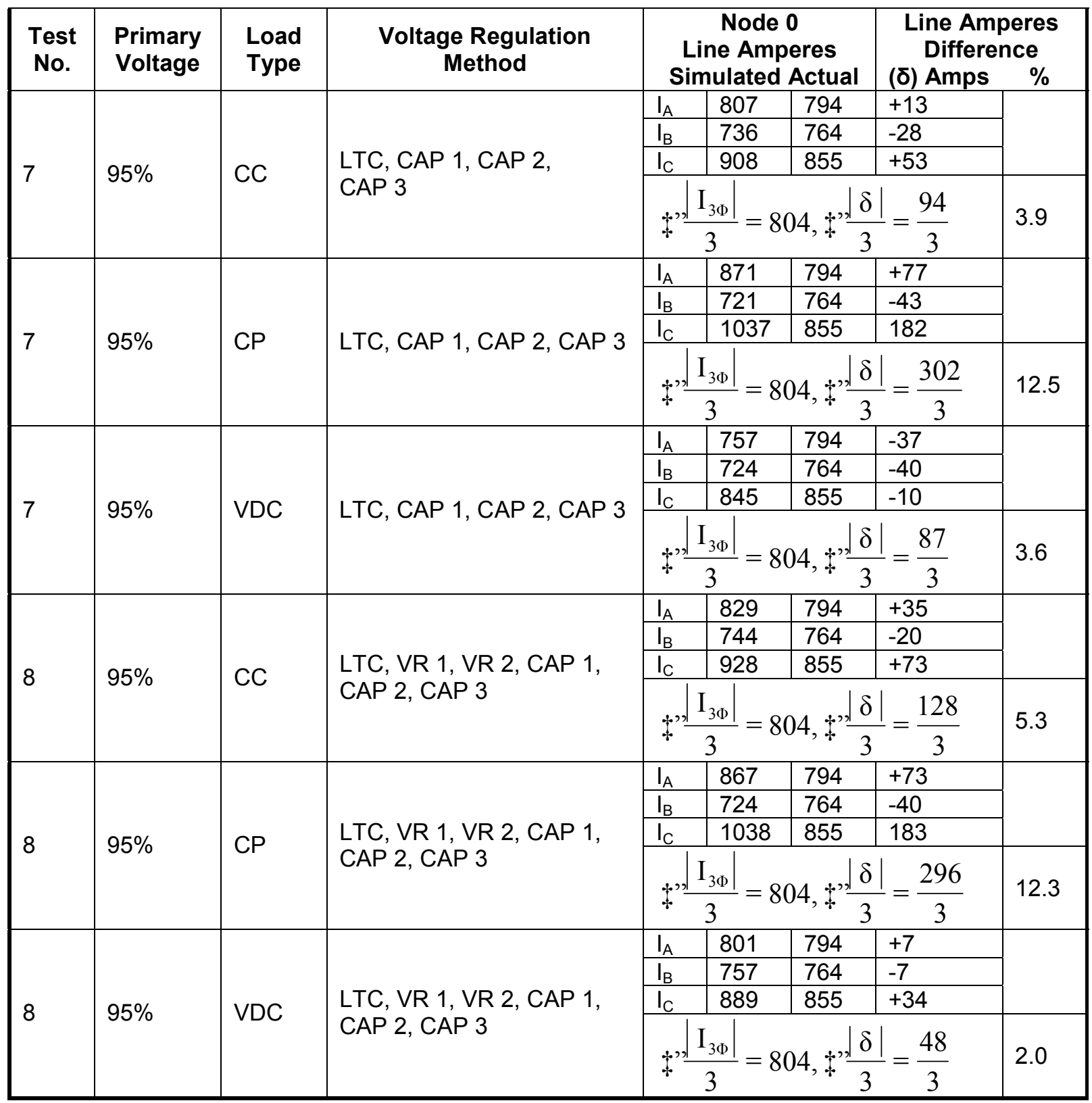


Table B-3. Sequence Currents for Each Test

\begin{tabular}{|c|c|c|c|c|c|c|}
\hline & & & & $\begin{array}{r}\text { Subs } \\
\mathrm{S}\end{array}$ & $\begin{array}{r}\text { tion Sec } \\
\text { uence } C \\
\text { Amp }\end{array}$ & $\begin{array}{l}\text { lary Bus } \\
\text { ents* }\end{array}$ \\
\hline $\begin{array}{l}\text { Test } \\
\text { No. }\end{array}$ & $\begin{array}{l}\text { Primary } \\
\text { Voltage }\end{array}$ & Load & Voltage Regulation & 10 & I1 & 12 \\
\hline $\begin{array}{l}1 \\
1\end{array}$ & $\begin{array}{l}95 \% \\
105 \%\end{array}$ & $\begin{array}{l}\mathrm{HL} \\
\mathrm{HL}\end{array}$ & $\begin{array}{l}\text { No LTC } \\
\text { No LTC }\end{array}$ & $\begin{array}{l}45.46 \\
45.44\end{array}$ & $\begin{array}{l}799.94 \\
780.11\end{array}$ & $\begin{array}{l}45.21 \\
45.08\end{array}$ \\
\hline $\begin{array}{l}2 \\
2\end{array}$ & $\begin{array}{l}95 \% \\
105 \%\end{array}$ & $\begin{array}{l}\mathrm{HL} \\
\mathrm{HL}\end{array}$ & $\begin{array}{l}\mathrm{LTC}+16 \\
\mathrm{LTC}+1\end{array}$ & $\begin{array}{l}45.43 \\
45.43\end{array}$ & $\begin{array}{l}780.12 \\
780.13\end{array}$ & $\begin{array}{l}45.08 \\
45.08\end{array}$ \\
\hline 3 & $\begin{array}{l}95 \% \\
105 \%\end{array}$ & $\begin{array}{l}\mathrm{HL} \\
\mathrm{HL}\end{array}$ & $\begin{array}{l}\text { LTC + 16 } \\
\text { VR 1, 32, 32, } 32 \\
\text { LTC + 3 } \\
\text { VR 1, 32, 20, } 32\end{array}$ & $\begin{array}{l}45.86 \\
47.37\end{array}$ & $\begin{array}{r}796.86 \\
794.95\end{array}$ & $\begin{array}{r}45.72 \\
47.34\end{array}$ \\
\hline 4 & $\begin{array}{l}95 \% \\
105 \%\end{array}$ & $\mathrm{HL}$ & $\begin{array}{l}\text { LTC + 16 } \\
\text { VR 1, 32, 32, } 32 \\
\text { VR 2, 32, 23, } 32 \\
\text { LTC + 3 } \\
\text { VR } 1,32,20,32 \\
\text { VR 2, 32, 11, } 32\end{array}$ & $\begin{array}{l}45.82 \\
47.61\end{array}$ & $\begin{array}{l}799.26 \\
796.96\end{array}$ & $\begin{array}{l}45.70 \\
47.69\end{array}$ \\
\hline 5 & $\begin{array}{l}95 \% \\
105 \%\end{array}$ & $\begin{array}{l}\mathrm{HL} \\
\mathrm{HL}\end{array}$ & $\begin{array}{l}\text { LTC }+16 \\
\text { CAP } 1 \\
\text { LTC }+1 \\
\text { CAP } 2\end{array}$ & $\begin{array}{l}45.34 \\
45.34\end{array}$ & $\begin{array}{l}760.27 \\
760.18\end{array}$ & $\begin{array}{l}45.87 \\
45.87\end{array}$ \\
\hline 6 & $\begin{array}{l}95 \% \\
105 \%\end{array}$ & $\begin{array}{l}\mathrm{HL} \\
\mathrm{HL}\end{array}$ & $\begin{array}{l}\text { LTC + 16 } \\
\text { CAP 1, CAP } 2 \\
\text { LTC + 1 } \\
\text { CAP 1, CAP } 2\end{array}$ & $\begin{array}{l}45.19 \\
45.18\end{array}$ & $\begin{array}{l}744.66 \\
744.49\end{array}$ & $\begin{array}{l}47.59 \\
47.59\end{array}$ \\
\hline 7 & $\begin{array}{l}95 \% \\
105 \%\end{array}$ & $\begin{array}{l}\mathrm{HL} \\
\mathrm{HL}\end{array}$ & $\begin{array}{l}\text { LTC + 15 } \\
\text { CAP 1, 2, } 3 \\
\text { LTC + 1 } \\
\text { CAP 1, 2, } 3\end{array}$ & $\begin{array}{l}45.69 \\
45.67\end{array}$ & $\begin{array}{l}724.07 \\
723.50\end{array}$ & $\begin{array}{l}49.55 \\
49.54\end{array}$ \\
\hline 8 & $95 \%$ & $\mathrm{HL}$ & $\begin{array}{l}\text { LTC + 15 } \\
\text { VR } 1,32,14,32 \\
\text { VR 2, 32, 10, } 32 \\
\text { CAP } 1,2,3 \\
\text { LTC + 1 } \\
\text { VR } 1,32,4,32 \\
\text { VR 2, 32, 7, } 32 \\
\text { CAP } 1,2,3\end{array}$ & 48.82 & 738.71 & 52.76 \\
\hline
\end{tabular}

${ }^{*}$ Note: The sequence currents are measured at the secondary of the transformer to indicate the magnitude of zero sequence current to be used to calculate the neutral relay settings. 
Table B-4. Simulation Data by Node for Test 8 at $95 \%$ HL with LTC, VR 1, VR 2, and Capacitors 1, 2, and 3 Regulation Methods Implemented (CC Model)

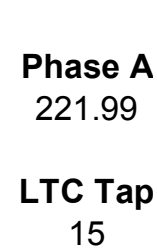

15
KW Losses:

Phase B

16.61

\section{Phase C \\ 244.04}

VR 1 A Tap 32

\section{Phase A 1205.74}

VR 1 B Tap

14
KVAR Losses:

Phase B

850.27

Phase C

1447.4
VR 1 C Tap

32

\section{Local Name \\ $0======$}

MILFD8103

$======$ Node $1======$

Node 1

======Node $2======$

$=====$ Node $3=====$

Node 3

Node $3 \mathrm{~N}$

622.6234

Node $3 \mathrm{E}$

602.6146

20.145

$======$ Node $4======$

Node 4

602.6207

Node $4 \mathrm{E}$

57.6258

Node $4 \mathrm{~N}$

$======$ Node $5=====$

Node 5

Node $5 \mathrm{E}$

Node $5 \mathrm{~N}$

$======$ Node $6======$

Node $6 \quad 38.6448$

$=====$ Node $7=====$

Node 7

Node $7 \mathrm{E}$

Node $7 \mathrm{~N}$

533.9893

370.1802

90.3277

81.0404

Node $7 \mathrm{~W}$

$======$ Node $8======$

Node 8

370.1825

======Node $9======$

Node 9

370.1834

$=====$ Node $10=====$

Node 10

==ニ===Node 11 ======

Node 11

Node $11 \mathrm{~N}$

Node $11 \mathrm{E}$

$=====$ Node 12======

Node 12

$=====$ Node $13=====$

Node 13

$======$ Node $14======$

Node 14

$=====$ Node $15======$

Node 15

\section{Amps B}

744.0258

659.7188

573.6905

562.2695

11.5252

553.9184

117.3591

437.5563

112.1439

112.1465

0.0186

39.825

425.5483

322.0644

48.7249

83.8447

312.0715

312.0719

0

154.4999

62.2984

94.8149

40.2759

53.442

60.0707

60.0719

\section{Amps C}

823.2208

685.2835

673.3692

11.923

673.3727

87.9715

589.768

77.187

68.3351

9.8124

38.713

579.9824

363.0935

107.2539

114.1217

363.0953

363.0959

$$
0
$$

183.0159

150.2312

43.9618

37.6143

49.2189

44.4666

44.4681
Cust Volts A Cust Volts B

114.0001

114.0001

$123.5558 \quad 124.6471$

118.8883

118.0738

118.8822

122.7722

122.4304

122.7727

$117.9749 \quad 122.3914$

$117.9713 \quad 122.3544$

$117.8738 \quad 122.3733$

$117.9394 \quad 121.8941$

$117.937 \quad 121.8709$

$117.9387 \quad 121.8952$

$117.8053 \quad 121.4029$

114.3333

114.3225

114.33

114.3293

114.0694

119.0288

123.7469

116.5839

123.0896

114.7319

114.7044

114.7126

122.7623

122.7646

122.7557

122.7774

114.5085

114.426

122.1848

114.3284

122.5585

124.3025 


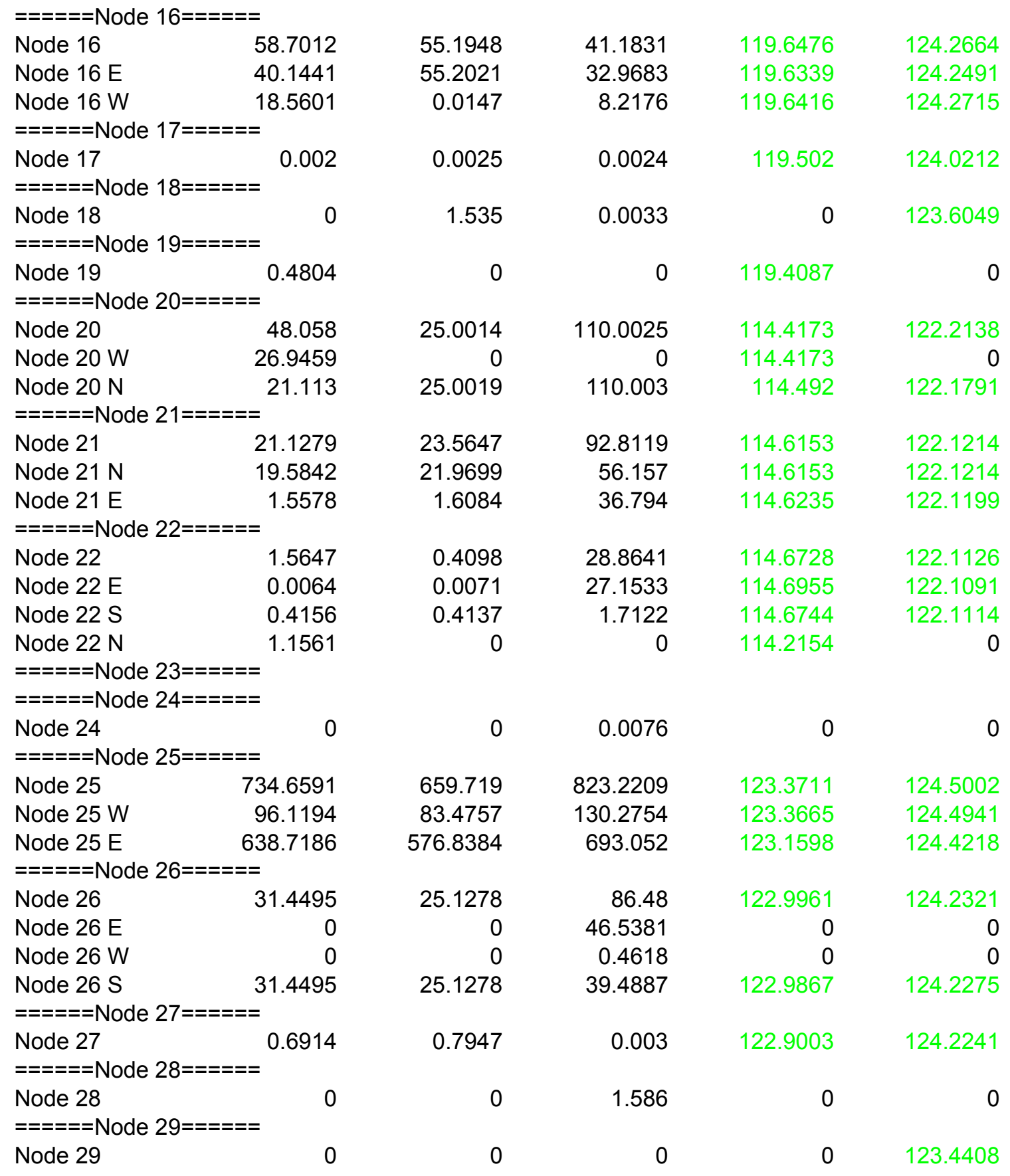

======Feeder Path Trace======

\begin{tabular}{|c|c|c|c|c|c|}
\hline \multicolumn{2}{|c|}{ Distance (1000r CustvoltsA } & CustVoltsB & CustVoltsC & \%CapacityA & \%CapacityB \\
\hline 0.1034 & 114.0001 & 114.0001 & 114.0001 & 23.6916 & 31.4756 \\
\hline 0.2378 & 123.6421 & 124.7267 & 123.8458 & 21.0044 & 29.0625 \\
\hline 0.3218 & 123.5558 & 124.6471 & 123.7504 & -9.4872 & 1.6813 \\
\hline 0.3218 & 123.5558 & 124.6471 & 123.7504 & 26.8996 & 34.3563 \\
\hline 0.5288 & 123.3711 & 124.5002 & 123.5386 & 26.8996 & 34.3563 \\
\hline 0.7648 & 123.1598 & 124.4218 & 123.3099 & 36.4459 & 42.6031 \\
\hline 1.3788 & 122.6107 & 124.2194 & 122.7203 & 36.4458 & 42.603 \\
\hline
\end{tabular}




\begin{tabular}{|c|c|c|c|c|c|}
\hline 2.583 & 121.5436 & 123.832 & 121.5709 & 36.4454 & 42.7524 \\
\hline 2.666 & 121.4705 & 123.8056 & 121.4921 & 36.4446 & 42.7622 \\
\hline 3.206 & 121.0131 & 123.62 & 120.9913 & 38.0481 & 42.7622 \\
\hline 3.429 & 120.825 & 123.5443 & 120.785 & 38.0477 & 42.8685 \\
\hline 3.716 & 120.5835 & 123.4475 & 120.5199 & 38.0476 & 42.9016 \\
\hline 5.754 & 118.8883 & 122.7722 & 118.6597 & 38.0474 & 42.9164 \\
\hline 6.798 & 118.0738 & 122.4304 & 117.7378 & 40.0383 & 44.0528 \\
\hline 6.9262 & 117.9749 & 122.3914 & 117.6237 & 40.0377 & 44.8837 \\
\hline 7.0603 & 117.8738 & 122.3733 & 117.5038 & 45.0297 & 56.4621 \\
\hline 7.2034 & 117.7663 & 122.3538 & 117.3759 & 45.1101 & 56.462 \\
\hline 7.3784 & 117.6352 & 122.33 & 117.2198 & 45.11 & 56.462 \\
\hline 7.7094 & 117.3887 & 122.2846 & 116.925 & 45.2256 & 56.4619 \\
\hline 7.9064 & 117.243 & 122.2573 & 116.75 & 45.3336 & 56.4618 \\
\hline 7.9064 & 117.243 & 122.2573 & 116.75 & 45.3335 & 56.4617 \\
\hline 8.2464 & 116.9892 & 122.2113 & 116.4532 & 45.3335 & 56.4617 \\
\hline 8.4174 & 116.8617 & 122.1883 & 116.3046 & 45.3333 & 56.4616 \\
\hline 8.8571 & 116.5373 & 122.1278 & 115.9235 & 45.5525 & 56.4616 \\
\hline 9.4411 & 116.1091 & 122.0486 & 115.419 & 45.5522 & 56.4911 \\
\hline 9.6082 & 115.9912 & 122.0231 & 115.2754 & 46.5649 & 56.4909 \\
\hline 10.0174 & 115.7046 & 121.9659 & 114.9215 & 46.5648 & 56.9883 \\
\hline 10.3374 & 115.4816 & 121.9213 & 114.6456 & 46.5885 & 56.9882 \\
\hline 10.5075 & 115.3628 & 121.8978 & 114.5002 & 46.5883 & 56.9881 \\
\hline 10.8129 & 115.1505 & 121.8583 & 114.2381 & 46.5882 & 57.2977 \\
\hline 10.9501 & 115.0555 & 121.8415 & 114.1201 & 46.588 & 57.5374 \\
\hline 11.0961 & 114.9547 & 121.824 & 113.9944 & 46.5879 & 57.6571 \\
\hline 11.6971 & 114.5436 & 121.7511 & 113.4784 & 46.7157 & 57.6571 \\
\hline 12.0081 & 114.3333 & 121.7128 & 113.2125 & 46.8667 & 57.6569 \\
\hline 12.0291 & 114.3225 & 121.709 & 113.2014 & 63.1661 & 67.9538 \\
\hline 12.0292 & 114.3225 & 121.709 & 113.2014 & 63.1661 & 67.9538 \\
\hline 12.2671 & 114.2006 & 121.6659 & 113.075 & 63.1661 & 67.9538 \\
\hline 12.3884 & 114.1384 & 121.6439 & 113.0107 & 63.166 & 67.9537 \\
\hline 12.5244 & 114.0694 & 121.6228 & 112.9367 & 63.1659 & 68.9481 \\
\hline 12.6619 & 118.9621 & 123.7245 & 117.7881 & 64.8529 & 69.5867 \\
\hline 12.7589 & 118.9155 & 123.7084 & 117.7392 & 65.0307 & 69.5867 \\
\hline 12.9495 & 118.824 & 123.6769 & 117.6433 & 65.0527 & 69.6087 \\
\hline 13.0549 & 118.7736 & 123.6594 & 117.5903 & 65.0905 & 69.6086 \\
\hline 13.3859 & 118.6107 & 123.605 & 117.4322 & 65.0904 & 69.6086 \\
\hline 13.6541 & 118.4865 & 123.5557 & 117.3054 & 66.2643 & 69.6085 \\
\hline 13.9121 & 118.3656 & 123.5085 & 117.1863 & 66.2641 & 69.6084 \\
\hline 14.1148 & 118.2716 & 123.4708 & 117.0929 & 66.4581 & 69.6083 \\
\hline 14.5219 & 118.0595 & 123.4005 & 116.9433 & 66.458 & 69.7846 \\
\hline 15.0699 & 117.7751 & 123.3105 & 116.74 & 66.4578 & 70.0693 \\
\hline 15.2599 & 117.6769 & 123.2793 & 116.6696 & 66.5157 & 70.0691 \\
\hline 15.4019 & 117.6053 & 123.2547 & 116.6173 & 67.0263 & 70.069 \\
\hline 15.5403 & 117.5361 & 123.2329 & 116.5651 & 67.0262 & 70.6868 \\
\hline 15.8453 & 117.3817 & 123.1853 & 116.4533 & 67.0261 & 70.6868 \\
\hline 16.1153 & 117.2443 & 123.1434 & 116.3557 & 67.0259 & 70.6867 \\
\hline 17.4453 & 116.5956 & 123.0812 & 115.7992 & 67.0258 & 74.9083 \\
\hline 17.6035 & 116.5257 & 123.0682 & 115.7365 & 69.1939 & 74.9076 \\
\hline 17.8465 & 116.4182 & 123.0483 & 115.641 & 69.1938 & 74.9076 \\
\hline 18.2915 & 116.2227 & 123.0111 & 115.4664 & 69.3132 & 74.9075 \\
\hline 18.4488 & 116.1534 & 122.9981 & 115.4051 & 69.313 & 74.9074 \\
\hline
\end{tabular}




\begin{tabular}{|c|c|c|c|c|c|}
\hline 18.6148 & 116.0804 & 122.9844 & 115.3405 & 69.3129 & 74.9074 \\
\hline 18.7818 & 116.0068 & 122.9707 & 115.2757 & 69.3128 & 74.9073 \\
\hline 19.1128 & 115.8612 & 122.9436 & 115.1473 & 69.3127 & 74.9073 \\
\hline 19.4183 & 115.7205 & 122.9196 & 115.0395 & 69.3125 & 74.9072 \\
\hline 20.0144 & 115.4673 & 122.8774 & 114.9176 & 74.42 & 78.4082 \\
\hline 20.3923 & 115.3085 & 122.8579 & 114.8363 & 74.4197 & 79.167 \\
\hline 20.7652 & 115.1688 & 122.8272 & 114.7579 & 76.2023 & 79.1669 \\
\hline 20.9525 & 115.0971 & 122.8121 & 114.7215 & 76.2021 & 79.1668 \\
\hline 21.4666 & 114.9004 & 122.7715 & 114.621 & 76.202 & 79.2307 \\
\hline 21.7311 & 114.7988 & 122.7509 & 114.5703 & 76.2017 & 79.2306 \\
\hline 21.9173 & 114.7319 & 122.7623 & 114.5207 & 76.2016 & 84.6269 \\
\hline 22.0473 & 114.7044 & 122.7646 & 114.4802 & 83.3553 & 93.8012 \\
\hline 22.1883 & 114.6741 & 122.7673 & 114.4374 & 83.3552 & 93.8011 \\
\hline 22.3543 & 114.6384 & 122.7703 & 114.3869 & 83.3552 & 93.801 \\
\hline 22.4763 & 114.6142 & 122.7716 & 114.3501 & 84.0002 & 93.8009 \\
\hline 22.8133 & 114.5463 & 122.7752 & 114.25 & 84.0002 & 93.8008 \\
\hline 22.9733 & 114.5484 & 122.7649 & 114.2008 & 91.0257 & 93.8006 \\
\hline 23.1957 & 114.5496 & 122.7507 & 114.135 & 91.0257 & 93.8005 \\
\hline 23.3361 & 114.5427 & 122.7479 & 114.0953 & 91.0256 & 93.8003 \\
\hline 23.3575 & 114.5417 & 122.7474 & 114.0893 & 91.0496 & 93.8244 \\
\hline 23.5515 & 114.5322 & 122.7435 & 114.0346 & 91.0496 & 93.8244 \\
\hline 23.7088 & 114.5248 & 122.74 & 113.9903 & 91.1444 & 93.8243 \\
\hline 24.0118 & 114.5108 & 122.7334 & 113.905 & 91.1443 & 93.8243 \\
\hline 24.1251 & 114.5055 & 122.7309 & 113.8731 & 91.1441 & 93.8241 \\
\hline 24.3851 & 114.4941 & 122.7247 & 113.8002 & 91.2696 & 93.8241 \\
\hline 24.6292 & 114.4822 & 122.719 & 113.734 & 91.2694 & 93.824 \\
\hline 24.8032 & 114.4753 & 122.7138 & 113.687 & 91.6332 & 93.8239 \\
\hline 25.0212 & 114.4659 & 122.7074 & 113.6295 & 91.6331 & 93.8238 \\
\hline 25.1302 & 114.4612 & 122.7044 & 113.6006 & 91.633 & 93.9099 \\
\hline 25.3432 & 114.4633 & 122.6909 & 113.5454 & 93.7495 & 93.9098 \\
\hline 26.3495 & 114.4243 & 122.6322 & 113.3672 & 93.7494 & 93.9098 \\
\hline 26.5235 & 114.4166 & 122.6221 & 113.3381 & 93.7487 & 93.9094 \\
\hline 26.9295 & 114.395 & 122.5991 & 113.2762 & 93.7486 & 93.9093 \\
\hline 27.0567 & 114.3887 & 122.5915 & 113.2569 & 93.8795 & 93.9091 \\
\hline 27.3222 & 114.3686 & 122.5765 & 113.2282 & 93.8794 & 93.9091 \\
\hline 27.3813 & 114.3621 & 122.5736 & 113.2252 & 93.8792 & 94.0228 \\
\hline 27.6828 & 114.3284 & 122.5585 & 113.2109 & 93.8792 & 94.0228 \\
\hline 27.8391 & 119.7117 & 124.2937 & 118.5691 & 94.5939 & 94.5492 \\
\hline 27.9641 & 119.698 & 124.2872 & 118.5641 & 94.1593 & 94.111 \\
\hline 28.1491 & 119.6779 & 124.2782 & 118.5565 & 94.1592 & 94.2556 \\
\hline 28.4312 & 119.6476 & 124.2664 & 118.5438 & 94.1591 & 94.508 \\
\hline 28.6137 & 119.6339 & 124.2491 & 118.5441 & 95.8614 & 94.3091 \\
\hline 28.983 & 119.607 & 124.2135 & 118.5448 & 95.9843 & 94.3089 \\
\hline 29.633 & 119.5614 & 124.1497 & 118.5465 & 96.1026 & 94.3087 \\
\hline 29.925 & 119.5436 & 124.1195 & 118.5476 & 96.4791 & 94.3082 \\
\hline 30.264 & 119.5336 & 124.0781 & 118.5499 & 97.7923 & 94.308 \\
\hline 30.904 & 119.5189 & 123.9977 & 118.5547 & 98.0387 & 94.3078 \\
\hline 31.183 & 119.5178 & 124.0023 & 118.5297 & 98.0383 & 99.9981 \\
\hline 31.4215 & 119.5173 & 124.0062 & 118.5092 & 98.1065 & 99.9985 \\
\hline 31.6365 & 119.5183 & 124.0089 & 118.4909 & 98.3796 & 99.9987 \\
\hline 31.7505 & 119.5191 & 124.0102 & 118.4812 & 98.4935 & 99.999 \\
\hline 31.8455 & 119.5177 & 124.0117 & 118.477 & 98.4935 & 99.9991 \\
\hline 32.0297 & 119.5114 & 124.0156 & 118.4756 & 98.4934 & 99.9992 \\
\hline
\end{tabular}




\begin{tabular}{cccccc} 
& kVA Flow SOC & & \multicolumn{3}{c}{ Released Capacity } \\
Phase A & Phase B & Phase C & Phase A & Phase B & Phase C \\
5998.63 & 5386.73 & 6721.75 & 6.55 & 8.84 & 6.36
\end{tabular}

VR 2 A Tap VR 2 B Tap VR 2 C Tap 32

10

32

\section{Cust Volts C Seq Amps 0 Seq Amps $1 \quad$ Seq Amps $2 \quad$ I2/l1 (\%)}

\begin{tabular}{|c|c|c|c|c|c|c|c|}
\hline 114.0001 & 55.0565 & 833.1159 & 59.4993 & 7.1418 & 0 & 7239.8662 & 0.1059 \\
\hline 123.7504 & 48.8179 & 738.7138 & 52.7574 & 7.1418 & 0.4098 & 7873.8943 & 42.2513 \\
\hline 118.6597 & 34.2333 & 626.6931 & 39.1943 & 6.2542 & 72.9172 & 7627.4228 & 114.1174 \\
\hline 117.7378 & 33.4637 & 612.2335 & 40.1371 & 6.5558 & 85.5513 & 7583.3448 & 127.8616 \\
\hline 118.6573 & 2.7552 & 14.5262 & 2.8859 & 19.8671 & 73.0231 & 7627.2546 & 114.1589 \\
\hline 117.6237 & 36.2142 & 609.4338 & 41.6729 & 6.838 & 87.2579 & 7577.9977 & 129.5801 \\
\hline 117.6249 & 18.2699 & 87.0192 & 19.2667 & 22.1408 & 85.6853 & 7577.1623 & 129.6009 \\
\hline 117.5038 & 50.857 & 526.0247 & 46.8835 & 8.9128 & 90.0076 & 7572.923 & 131.3654 \\
\hline 117.6675 & 18.7746 & 79.5263 & 19.8846 & 25.0038 & 65.6593 & 7567.6039 & 129.5358 \\
\hline 117.6747 & 20.0504 & 75.5901 & 21.1139 & 27.9321 & 64.5089 & 7567.2157 & 129.4562 \\
\hline 117.6499 & 2.8653 & 4.3419 & 2.9142 & 67.1179 & 66.1558 & 7567.2383 & 129.7394 \\
\hline 118.0132 & 0.1811 & 39.0583 & 0.6485 & 1.6602 & 35.0707 & 7561.6856 & 125.5384 \\
\hline 113.2125 & 50.5017 & 512.5785 & 47.365 & 9.2405 & 188.9092 & 7392.3764 & 196.3087 \\
\hline 113.2014 & 16.9106 & 351.6674 & 15.4637 & 4.3973 & 189.062 & 7391.8311 & 196.4311 \\
\hline 113.1866 & 28.4113 & 76.8408 & 25.1711 & 32.7574 & 190.4583 & 7392.1802 & 196.8204 \\
\hline 113.1848 & 10.0303 & 92.9083 & 11.8693 & 12.7752 & 189.3063 & 7391.44 & 196.3231 \\
\hline 112.9367 & 19.9915 & 348.328 & 18.7552 & 5.3844 & 192.8739 & 7379.0356 & 199.3641 \\
\hline 117.8575 & 19.9915 & 348.328 & 18.7552 & 5.3844 & 152.7909 & 7633.059 & 147.8634 \\
\hline 115.7925 & 0 & 0 & \multicolumn{2}{|c|}{$0 \mathrm{NA}$} & 177.6127 & 7523.7794 & 169.5996 \\
\hline 114.5207 & 27.8802 & 191.9652 & 23.6843 & 12.3378 & 201.5767 & 7450.8418 & 184.3941 \\
\hline 114.4802 & 33.0006 & 126.5865 & 32.1058 & 25.3628 & 203.2963 & 7449.4322 & 184.7586 \\
\hline 114.5306 & 18.1433 & 70.9456 & 15.5961 & 21.9831 & 201.1182 & 7450.5149 & 184.3036 \\
\hline 114.6636 & 1.0233 & 38.4793 & 0.956 & 2.4844 & 198.1103 & 7449.5857 & 185.0702 \\
\hline 112.5295 & 1.5608 & 50.8907 & 1.4428 & 2.8351 & 226.6332 & 7389.3299 & 209.4912 \\
\hline 113.2109 & 5.8404 & 55.3422 & 5.127 & 9.2642 & 231.6821 & 7409.7747 & 196.8352 \\
\hline 118.5772 & 5.8404 & 55.3422 & 5.127 & 9.2642 & 175.4698 & 7674.5193 & 132.419 \\
\hline
\end{tabular}

Seq Volts 0 Seq Volts 1 Seq Volts 2

(1)




\begin{tabular}{|c|c|c|c|c|c|c|c|}
\hline 118.5438 & 5.762 & 51.6853 & 4.9805 & 9.6362 & 174.758 & 7671.4176 & 132.3578 \\
\hline 118.5441 & 6.4845 & 42.7659 & 6.6492 & 15.5479 & 174.2237 & 7670.7728 & 132.2688 \\
\hline 118.5394 & 5.4573 & 8.9227 & 5.2855 & 59.2369 & 175.0594 & 7671.3023 & 132.4698 \\
\hline 118.4747 & 0 & 0.0023 & 0 & 0 & 169.8666 & 7661.7073 & 131.9398 \\
\hline 118.7155 & 0 & 0.5114 & 0.5109 NA & & 0 & 5128.758 & 2650.3923 \\
\hline 0 & 0.1601 & 0 & $0 \mathrm{NA}$ & & 2527.8226 & 0 & 0 \\
\hline 112.0482 & 25.6513 & 60.9544 & 25.2617 & 41.4437 & 242.4023 & 7379.2661 & 216.0309 \\
\hline & 8.982 & 0 & $0 \mathrm{NA}$ & & 2422.1572 & 0 & 0 \\
\hline 111.8079 & 28.4826 & 51.9765 & 29.6231 & 56.9932 & 248.7786 & 7374.8441 & 219.1163 \\
\hline 111.3929 & 23.03 & 45.7673 & 24.0853 & 52.6255 & 260.5602 & 7367.1183 & 224.4831 \\
\hline 111.3929 & 11.3733 & 32.4807 & 12.4718 & 38.3975 & 260.5602 & 7367.1183 & 224.4831 \\
\hline 111.3767 & 11.7106 & 13.3076 & 11.7767 & 88.4961 & 261.0967 & 7366.9025 & 224.7192 \\
\hline 111.2784 & 9.337 & 10.2756 & 9.2633 & 90.1485 & 264.4658 & 7365.61 & 226.1654 \\
\hline 111.2374 & 9.0519 & 9.0484 & 9.0526 & 100.0456 & 265.8927 & 7365.1032 & 226.776 \\
\hline 111.2734 & 0.4259 & 0.8465 & 0.4403 & 52.0152 & 264.6239 & 7365.5058 & 226.2304 \\
\hline 0 & 9.337 & 0 & $0 \mathrm{NA}$ & & 76.1436 & 0 & 0 \\
\hline 110.4629 & 0 & 0 & $0.0025 \mathrm{NA}$ & & 0 & 0 & 2338.3935 \\
\hline 123.5386 & 48.818 & 738.714 & 52.7573 & 7.1418 & 3.6133 & 7862.3871 & 43.5241 \\
\hline 123.5279 & 14.1559 & 103.2862 & 13.8139 & 13.3744 & 3.8854 & 7861.9354 & 43.618 \\
\hline 123.3099 & 37.0466 & 635.6931 & 38.9572 & 6.1283 & 6.7013 & 7851.4117 & 46.75 \\
\hline 122.3175 & 19.4346 & 47.6817 & 19.5383 & 40.9765 & 38.9384 & 7822.8104 & 60.8002 \\
\hline 122.2857 & 0 & 0 & $15.5124 \mathrm{NA}$ & & 0 & 0 & 2588.6705 \\
\hline 122.3169 & 0 & 0 & $0.1539 \mathrm{NA}$ & & 0 & 0 & 2589.3317 \\
\hline 122.3 & 4.0966 & 32.0209 & 4.2183 & 13.1736 & 39.3434 & 7822.1417 & 61.0528 \\
\hline 121.86 & 0.2209 & 0.4938 & 0.2777 & 56.238 & 55.2611 & 7810.8523 & 67.7209 \\
\hline 121.3932 & 0 & 0 & $0.5287 \mathrm{NA}$ & & 0 & 0 & 2569.776 \\
\hline 0 & 0 & 0 & $0 \mathrm{NA}$ & & 0 & 2613.1221 & 0 \\
\hline
\end{tabular}




\begin{tabular}{|c|c|c|c|c|c|c|}
\hline V2/V1 (\%) & pf $A$ & pf $B$ & pf C & kW Flow A & kW Flow B & kW Flow C \\
\hline 0.0015 & 0.8584 & 0.8849 & 0.8406 & 5149.1606 & 4766.8566 & 5650.2696 \\
\hline 0.5366 & 0.8879 & 0.9128 & 0.8763 & 5122.0445 & 4770.1754 & 5674.0669 \\
\hline 1.4961 & 0.9047 & 0.9274 & 0.8933 & 4313.6989 & 4171.0642 & 4685.7275 \\
\hline 1.6861 & 0.9133 & 0.933 & 0.9015 & 4155.377 & 4090.1585 & 4574.6012 \\
\hline 1.4967 & 0.8616 & 0.8777 & 0.8942 & 131.0445 & 78.8687 & 80.3424 \\
\hline 1.71 & 0.9167 & 0.936 & 0.9056 & 4142.5374 & 4031.3237 & 4559.5452 \\
\hline 1.7104 & -0.9968 & 0.9706 & 0.99 & 430.3626 & 885.3978 & 650.5856 \\
\hline 1.7347 & 0.8965 & 0.9249 & 0.8868 & 3710.6449 & 3145.7893 & 3907.0391 \\
\hline 1.7117 & -0.9868 & 0.975 & 0.9965 & 382.8852 & 846.4462 & 574.7759 \\
\hline 1.7108 & -0.9791 & 0.975 & 0.9997 & 362.179 & 846.4345 & 510.4917 \\
\hline 1.7145 & 0.858 & -0.081 & 0.8767 & 20.7062 & 0.0117 & 64.2842 \\
\hline 1.6602 & -1 & -1 & -1 & 0 & 0 & 0 \\
\hline 2.6556 & 0.9148 & 0.9339 & 0.9025 & 3553.3654 & 3073.0376 & 3772.1753 \\
\hline 2.6574 & 0.8864 & 0.8959 & 0.8899 & 2382.5191 & 2230.3426 & 2323.1687 \\
\hline 2.6625 & 0.9993 & -0.7408 & 0.9812 & 655.4244 & 279.0285 & 756.6251 \\
\hline 2.6561 & 0.8719 & 0.8705 & 0.8383 & 513.0646 & 564.1957 & 687.8519 \\
\hline 2.7018 & 0.8872 & 0.8979 & 0.8906 & 2380.7169 & 2164.8146 & 2320.9673 \\
\hline 1.9371 & 0.8872 & 0.8979 & 0.8906 & 2380.1093 & 2165.0119 & 2320.1343 \\
\hline 2.2542 & 0.014 & -0.1051 & 0.101 & 0 & 0 & 0 \\
\hline 2.4748 & 0.92 & 0.9521 & 0.932 & 1604.2112 & 1146.7663 & 1241.0627 \\
\hline 2.4802 & 0.8593 & 0.8597 & 0.8538 & 1047.4059 & 417.5658 & 932.8863 \\
\hline 2.4737 & 0.9945 & 0.9868 & -0.9626 & 556.3686 & 729.4639 & 307.7802 \\
\hline 2.4843 & -1 & -1 & -1 & 0 & 0 & 0 \\
\hline 2.835 & -1 & -1 & -1 & 0 & 0 & 0 \\
\hline 2.6564 & 0.8728 & 0.8599 & 0.8593 & 389.9266 & 402.0834 & 274.7443 \\
\hline 1.7254 & 0.8728 & 0.8599 & 0.8593 & 389.8517 & 402.1026 & 274.7275 \\
\hline
\end{tabular}




\begin{tabular}{|c|c|c|c|c|c|c|c|}
\hline & 1.7253 & 0.8735 & 0.8611 & 0.8598 & 389.7086 & 375.1131 & 266.6114 \\
\hline & 1.7243 & 0.8755 & 0.861 & 0.8619 & 267.0694 & 375.1165 & 213.9266 \\
\hline & 1.7268 & 0.8692 & -0.098 & 0.8514 & 122.5788 & 0.0114 & 52.6708 \\
\hline & 1.7221 & 0.0786 & 0.0777 & -0.1562 & -0.0012 & -0.0016 & 0.0028 \\
\hline NA & & 0 & 0.8569 & -0.2548 & 0 & 10.3257 & 0.0064 \\
\hline NA & & 0.9293 & 0 & 0 & 3.3855 & 0 & 0 \\
\hline & 2.9275 & 0.8611 & 0.8725 & 0.8317 & 300.7089 & 169.3145 & 651.1819 \\
\hline NA & & 0.8602 & 0 & 0 & 168.4344 & 0 & 0 \\
\hline & 2.9711 & 0.8624 & 0.8725 & 0.8318 & 132.3024 & 169.3082 & 651.1001 \\
\hline & 3.0471 & 0.8627 & 0.8721 & 0.8292 & 132.6442 & 159.397 & 544.9446 \\
\hline & 3.0471 & 0.8581 & 0.8677 & 0.8043 & 122.3268 & 147.8421 & 319.5401 \\
\hline & 3.0504 & 0.9149 & 0.9225 & 0.8652 & 10.3743 & 11.5081 & 225.2152 \\
\hline & 3.0706 & 0.9123 & 0.8937 & 0.8678 & 10.3955 & 2.8404 & 177.045 \\
\hline & 3.0791 & 0.1803 & -0.1022 & 0.8683 & -0.0084 & 0.0056 & 166.6303 \\
\hline & 3.0715 & 0.8774 & 0.8826 & 0.8593 & 2.6557 & 2.8318 & 10.3979 \\
\hline NA & & 0.9209 & 0 & 0 & 7.7537 & 0 & 0 \\
\hline NA & & 0 & 0 & 0 & 0 & 0 & 0 \\
\hline & 0.5536 & 0.8877 & 0.9127 & 0.8761 & 5117.5113 & 4766.5191 & 5668.3741 \\
\hline & 0.5548 & 0.8613 & 0.8621 & 0.8588 & 648.6462 & 568.9836 & 877.767 \\
\hline & 0.5954 & 0.8926 & 0.9196 & 0.8804 & 4466.9384 & 4194.1674 & 4787.3572 \\
\hline & 0.7772 & 0.8651 & 0.8701 & 0.8628 & 212.5206 & 172.4914 & 579.6498 \\
\hline NA & & 0 & 0 & 0.8562 & 0 & 0 & 309.537 \\
\hline NA & & 0 & 0 & 0.8614 & 0 & 0 & 3.0899 \\
\hline & 0.7805 & 0.8651 & 0.8701 & 0.8705 & 212.5206 & 172.4914 & 267.0229 \\
\hline & 0.867 & 0.9235 & 0.8599 & -0.0702 & 4.9835 & 5.3911 & 0.0016 \\
\hline NA & & 0 & 0 & 0.8578 & 0 & 0 & 10.4887 \\
\hline & 0 & 0 & 0 & 0 & 0 & 0 & 0 \\
\hline
\end{tabular}


kVAR Flow A kVAR Flow B kVAR Flow C

\begin{tabular}{|c|c|c|}
\hline 3077.2811 & 2508.765 & 3640.9319 \\
\hline 2653.9563 & 2134.0307 & 3119.0733 \\
\hline 2031.446 & 1682.7564 & 2357.103 \\
\hline 1853.4526 & 1578.1536 & 2196.1263 \\
\hline 77.2185 & 43.07 & 40.2268 \\
\hline 1805.4186 & 1515.9154 & 2135.9344 \\
\hline-34.6628 & 219.5798 & 92.699 \\
\hline 1834.1241 & 1292.804 & 2035.8337 \\
\hline-62.8923 & 192.8663 & 48.3931 \\
\hline-75.2874 & 193.01 & 13.1168 \\
\hline 12.3951 & -0.1436 & 35.2763 \\
\hline-289.1261 & -307.0551 & -290.1482 \\
\hline 1569.381 & 1176.185 & 1800.4953 \\
\hline 1244.4147 & 1105.9595 & 1190.9072 \\
\hline 24.4915 & -252.9738 & 148.9699 \\
\hline 288.1546 & 318.9365 & 447.3585 \\
\hline 1237.9948 & 1061.1047 & 1185.0185 \\
\hline 1235.644 & 1059.7503 & 1182.8978 \\
\hline-0.0028 & -0.0027 & -0.0025 \\
\hline 683.4916 & 368.2371 & 482.7818 \\
\hline 623.3742 & 248.0914 & 568.8417 \\
\hline 58.5656 & 119.7008 & -86.6172 \\
\hline-273.1699 & -314.0477 & -273.9112 \\
\hline-363.7023 & -414.6977 & -351.747 \\
\hline 218.0912 & 238.7374 & 163.5691 \\
\hline 217.9555 & 238.5926 & 163.5184 \\
\hline
\end{tabular}




\begin{tabular}{|c|c|c|}
\hline 217.2283 & 221.5176 & 158.3322 \\
\hline 147.3893 & 221.5439 & 125.8585 \\
\hline 69.7442 & -0.1153 & 32.4542 \\
\hline-0.0154 & -0.02 & -0.0175 \\
\hline 0 & 6.2119 & -0.0243 \\
\hline 1.3455 & 0 & 0 \\
\hline 177.5406 & 94.8042 & 434.753 \\
\hline 99.8401 & 0 & 0 \\
\hline 77.671 & 94.8264 & 434.5321 \\
\hline 77.7599 & 89.4539 & 367.2787 \\
\hline 73.1979 & 84.7146 & 236.0576 \\
\hline 4.5774 & 4.814 & 130.505 \\
\hline 4.665 & 1.4261 & 101.3997 \\
\hline-0.0457 & -0.0546 & 95.1758 \\
\hline 1.4522 & 1.5085 & 6.1891 \\
\hline 3.2815 & 0 & 0 \\
\hline 0 & 0 & -0.0531 \\
\hline 2653.9597 & 2134.0345 & 3119.0764 \\
\hline 382.6578 & 334.5056 & 523.6688 \\
\hline 2256.2813 & 1792.011 & 2578.3209 \\
\hline 123.2254 & 97.73 & 339.5784 \\
\hline 0 & 0 & 186.7663 \\
\hline 0 & 0 & 1.8222 \\
\hline 123.2254 & 97.73 & 150.99 \\
\hline 2.07 & 3.2 & -0.0231 \\
\hline 0 & 0 & 6.2854 \\
\hline 0 & 0 & 0 \\
\hline
\end{tabular}




\section{B.4. Heavy- and Light-Load Base Case Data and Measured Data at Node 01}

Table B-5. Summary of HL Base Case

(LTC control only, tap $=14)$

[\#0 MXAT services included]

\begin{tabular}{|c|c|c|c|c|c|c|}
\hline Location & \multicolumn{3}{|c|}{ Simulated Data } & \multicolumn{3}{|c|}{ Measured Data July 17, 2006} \\
\hline Node 01 & $\begin{array}{c}\mathrm{I}_{\mathrm{A}} \\
578.13\end{array}$ & $\begin{array}{c}I_{B} \\
654.17\end{array}$ & $\begin{array}{c}I_{C} \\
636.62\end{array}$ & $\begin{array}{c}\mathrm{I}_{\mathrm{A}} \\
565\end{array}$ & $\begin{array}{c}I_{B} \\
651\end{array}$ & $\begin{array}{c}I_{C} \\
637\end{array}$ \\
\hline Node 01 & $\begin{array}{c}V_{A} \\
125.90\end{array}$ & $\begin{array}{c}V_{B} \\
125.55\end{array}$ & $\begin{array}{c}V_{C} \\
124.98\end{array}$ & $\begin{array}{c}V_{A} \\
126.0\end{array}$ & $\begin{array}{c}V_{B} \\
126.0\end{array}$ & $\begin{array}{c}V_{C} \\
126.0\end{array}$ \\
\hline $\begin{array}{l}\text { Node } 01 \\
\text { Node } 01 \\
\end{array}$ & $\begin{array}{c}\mathrm{PF}_{\mathrm{A}} \\
0.843\end{array}$ & $\begin{array}{c}\mathrm{PF}_{\mathrm{B}} \\
0.843\end{array}$ & $\begin{array}{c}\mathrm{PF}_{\mathrm{C}} \\
0.841\end{array}$ & & & \\
\hline & \multicolumn{3}{|c|}{ kVA Flow } & Total & & \\
\hline \multirow[t]{2}{*}{ Node 01} & $\begin{array}{l}\text { Phase A } \\
4,622.62\end{array}$ & $\begin{array}{l}\text { Phase B } \\
5,216.17\end{array}$ & $\begin{array}{l}\text { Phase C } \\
5,053.13\end{array}$ & $14,891.92$ & & \\
\hline & \multicolumn{3}{|c|}{ kW Flow } & & & \\
\hline \multirow[t]{2}{*}{ Node 01} & $\begin{array}{l}\text { Phase A } \\
3,897.94\end{array}$ & $\begin{array}{l}\text { Phase B } \\
4,399.49 \\
\end{array}$ & $\begin{array}{l}\text { Phase C } \\
4,249.92 \\
\end{array}$ & $12,547.35$ & & \\
\hline & \multicolumn{3}{|c|}{ kVAr Flow } & & & \\
\hline \multirow[t]{2}{*}{ Node 01} & $\begin{array}{l}\text { Phase A } \\
2,484.88\end{array}$ & $\begin{array}{l}\text { Phase B } \\
2,802.29\end{array}$ & $\begin{array}{l}\text { Phase C } \\
2,735.55\end{array}$ & $8,020.72$ & & \\
\hline & \multicolumn{3}{|c|}{ kVAr Losses } & Total \% & & \\
\hline \multirow[t]{3}{*}{ Node 01} & $\begin{array}{c}\text { Phase A } \\
877.31\end{array}$ & $\begin{array}{c}\text { Phase B } \\
1,132.1\end{array}$ & $\begin{array}{l}\text { Phase C } \\
1,086.42\end{array}$ & $\begin{array}{l}3095.83 \\
38.59 \%\end{array}$ & & \\
\hline & \multicolumn{3}{|c|}{ kW Losses } & Total $\%$ & & \\
\hline & $\begin{array}{c}\text { Phase A } \\
246.56\end{array}$ & $\begin{array}{c}\text { Phase B } \\
202.22\end{array}$ & $\begin{array}{c}\text { Phase C } \\
221.79\end{array}$ & $\begin{array}{c}670.57 \\
5.34 \%\end{array}$ & & \\
\hline Node 01 & $\begin{array}{c}\mathrm{I}_{0} \\
23.01 \\
\mathrm{~V}_{0} \\
0.1936\end{array}$ & $\begin{array}{c}\mathrm{I}_{1} \\
622.96 \\
\mathrm{~V}_{1} \\
7968.87\end{array}$ & $\begin{array}{c}\mathrm{I}_{2} \\
23.01 \\
\mathrm{~V}_{2} \\
33.92\end{array}$ & $\begin{array}{c}\mathrm{I}_{2} / \mathrm{I}_{1} \% \\
3.69 \\
\mathrm{~V}_{2} / \mathrm{V}_{1} \% \\
0.4257\end{array}$ & & \\
\hline
\end{tabular}

Note: All capacitors and line regulators were turned off. The substation primary voltage was set at $100 \%(120 \mathrm{~V})$. The base case used for the synchronous generator, inverter-based generator, and induction generator simulations had all three capacitors on one regulator turned on and all generation turned off. The losses in this case were $5.4 \%$. 


$\begin{array}{ccccc}\text { Phase A } & \text { Phase B } & \text { Phase C } & \text { Phase A } & \text { Phase B } \\ 246.56 & 202.22 & 221.79 & 877.31 & 1132.1 \\ & & & & \\ \text { LTC Tap } & & \text { VR 1 A Tap } & \text { VR 1 B Tap } & \text { VR 1 C Tap } \\ \text { 14 } & & \text { NA } & \text { NA } & \text { NA }\end{array}$

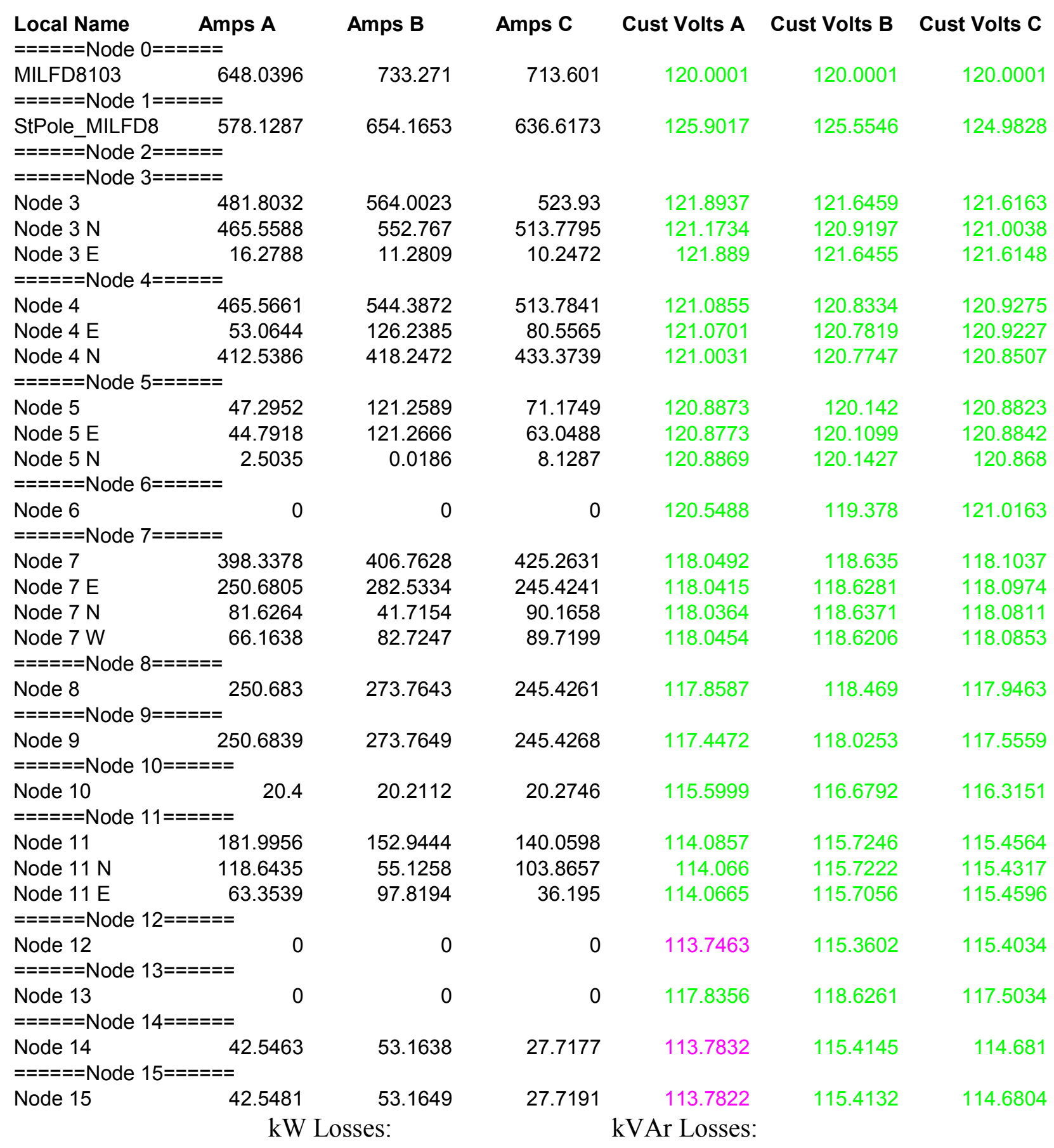




\begin{tabular}{|c|c|c|c|c|c|c|}
\hline $\begin{array}{l}\text { Node } 17 \\
======\text { Node } 18=====\end{array}$ & $==0.0019$ & 0.0024 & 0.0022 & 113.6089 & 115.1328 & 114.6841 \\
\hline $\begin{array}{l}\text { Node } 18 \\
======\text { Node } 19======\end{array}$ & $=$ & 1.3445 & 0.0032 & 0 & 114.7666 & 114.8794 \\
\hline $\begin{array}{l}\text { Node } 19 \\
======\text { Node } 20=====\end{array}$ & $==0.3952$ & 0 & 0 & 113.2432 & 0 & 0 \\
\hline Node 20 & 37.9451 & 26.6195 & 77.7292 & 117.8058 & 118.6012 & 117.2153 \\
\hline Node $20 \mathrm{~W}$ & 19.3002 & 0 & 0 & 117.8058 & 0 & 0 \\
\hline $\begin{array}{l}\text { Node } 20 \mathrm{~N} \\
======\text { Node } 21=====\end{array}$ & $==$ & 26.62 & 77.7296 & 117.8475 & 118.553 & 117.0723 \\
\hline Node 21 & 18.6604 & 25.3668 & 65.2933 & 117.9146 & 118.4698 & 116.8272 \\
\hline Node $21 \mathrm{~N}$ & 17.3276 & 23.6725 & 37.8338 & 117.9146 & 118.4698 & 116.8272 \\
\hline $\begin{array}{l}\text { Node } 21 \mathrm{E} \\
======\text { Node } 22=====\end{array}$ & $==^{1.3462}$ & 1.7079 & 27.4619 & 117.9204 & 118.4682 & 116.8158 \\
\hline Node 22 & 1.3529 & 0.411 & 21.7288 & 117.9554 & 118.4604 & 116.7453 \\
\hline Node $22 \mathrm{E}$ & 0.0066 & 0.007 & 20.4099 & 117.9718 & 118.457 & 116.7157 \\
\hline Node $22 \mathrm{~S}$ & 0.3388 & 0.4147 & 1.3202 & 117.9565 & 118.4591 & 116.7418 \\
\hline $\begin{array}{l}\text { Node } 22 \mathrm{~N} \\
======\text { Node } 23====== \\
=====\text { Node } 24=====\end{array}$ & $==^{1.0205}$ & 0 & 0 & 117.118 & 0 & 0 \\
\hline $\begin{array}{l}\text { Node } 24 \\
======\text { Node } 25=====\end{array}$ & $=\quad 0$ & 0 & 0 & 0 & 0 & 116.1101 \\
\hline Node 25 & 578.129 & 654.1655 & 636.6175 & 125.7425 & 125.3385 & 124.8545 \\
\hline Node $25 \mathrm{~W}$ & 82.7048 & 87.1324 & 106.8792 & 125.7381 & 125.3313 & 124.848 \\
\hline $\begin{array}{l}\text { Node } 25 \mathrm{E} \\
======\text { Node } 26=====\end{array}$ & $\begin{array}{l}495.5384 \\
==\end{array}$ & 567.2441 & 530.0965 & 125.5643 & 125.1707 & 124.7026 \\
\hline Node 26 & 26.4142 & 25.8686 & 69.1744 & 125.3649 & 124.9546 & 124.0365 \\
\hline Node $26 \mathrm{E}$ & 0 & 0 & 36.6633 & 0 & 0 & 123.9562 \\
\hline Node 26 W & 0 & 0 & 0.3888 & 0 & 0 & 124.0349 \\
\hline $\begin{array}{l}\text { Node } 26 \mathrm{~S} \\
======\text { Node } 27=====\end{array}$ & $\begin{aligned} & 26.4142 \\
= & \end{aligned}$ & 25.8686 & 32.1314 & 125.3562 & 124.9477 & 124.0252 \\
\hline $\begin{array}{l}\text { Node } 27 \\
======\text { Node } 28======\end{array}$ & $=0.6813$ & 0.7658 & 0.003 & 125.2701 & 124.9092 & 123.7098 \\
\hline $\begin{array}{l}\text { Node } 28 \\
======\text { Node } 29=====\end{array}$ & $=$ & 0 & 1.293 & 0 & 0 & 122.477 \\
\hline Node 29 & 0 & 0 & 0 & 0 & 114.6208 & 0 \\
\hline
\end{tabular}

\begin{tabular}{|c|c|c|c|c|c|c|}
\hline \multicolumn{7}{|c|}{$======$ Feeder Path Trace====== } \\
\hline Distance (1000r & voltsA & CustVoltsB & CustVoltsC & \%CapacityA & \%CapacityB & \%CapacityC \\
\hline 0.1034 & 116.4234 & 116.2687 & 116.0501 & 40.3159 & 32.4661 & 34.2777 \\
\hline 0.2168 & 125.9662 & 125.6275 & 125.0536 & 37.8356 & 29.6596 & 31.5465 \\
\hline 0.3008 & 125.9017 & 125.5546 & 124.9828 & 13.8407 & 2.5089 & 5.1241 \\
\hline 0.3008 & 125.9017 & 125.5546 & 124.9828 & 42.4748 & 34.9089 & 36.655 \\
\hline 0.5078 & 125.7425 & 125.3385 & 124.8545 & 42.4747 & 34.9089 & 36.655 \\
\hline 0.7438 & 125.5643 & 125.1707 & 124.7026 & 50.6927 & 43.5578 & 47.2541 \\
\hline 1.3578 & 125.0996 & 124.7357 & 124.3115 & 50.6925 & 43.5577 & 47.4227 \\
\hline 2.562 & 124.191 & 123.8923 & 123.5481 & 50.6921 & 43.7146 & 47.4224 \\
\hline
\end{tabular}




\begin{tabular}{|c|c|c|c|c|c|c|}
\hline 2.645 & 124.1284 & 123.8344 & 123.4958 & 50.6912 & 43.7247 & 47.4219 \\
\hline 3.185 & 123.7363 & 123.4468 & 123.1639 & 52.0601 & 43.7247 & 47.8681 \\
\hline 3.408 & 123.5746 & 123.2877 & 123.0271 & 52.0598 & 43.8324 & 47.8679 \\
\hline 3.695 & 123.3667 & 123.0835 & 122.8512 & 52.0596 & 43.8643 & 47.8678 \\
\hline 5.733 & 121.8937 & 121.6459 & 121.6163 & 52.0594 & 43.8804 & 47.8677 \\
\hline 6.777 & 121.1734 & 120.9197 & 121.0038 & 53.6757 & 44.9983 & 48.8777 \\
\hline 6.9052 & 121.0855 & 120.8334 & 120.9275 & 53.675 & 45.8321 & 48.8772 \\
\hline 7.0393 & 121.0031 & 120.7747 & 120.8507 & 58.9514 & 58.3834 & 56.8782 \\
\hline 7.1824 & 120.9154 & 120.712 & 120.7689 & 59.0161 & 58.3833 & 56.8782 \\
\hline 7.3574 & 120.8081 & 120.6353 & 120.6689 & 59.016 & 58.3832 & 56.8781 \\
\hline 7.6884 & 120.6062 & 120.49 & 120.4802 & 59.1066 & 58.3831 & 56.878 \\
\hline 7.8854 & 120.4865 & 120.4034 & 120.3681 & 59.1883 & 58.383 & 56.8779 \\
\hline 7.8854 & 120.4865 & 120.4034 & 120.3681 & 59.1882 & 58.3829 & 56.9812 \\
\hline 8.2254 & 120.2777 & 120.255 & 120.1788 & 59.1882 & 58.3829 & 57.413 \\
\hline 8.3964 & 120.1726 & 120.1804 & 120.0841 & 59.1879 & 58.3827 & 57.4847 \\
\hline 8.8361 & 119.9043 & 119.9879 & 119.8411 & 59.3508 & 58.3826 & 57.4846 \\
\hline 9.4201 & 119.5486 & 119.7333 & 119.5193 & 59.3505 & 58.4097 & 57.4844 \\
\hline 9.5872 & 119.4502 & 119.6584 & 119.4278 & 60.131 & 58.4094 & 57.4842 \\
\hline 9.9964 & 119.2101 & 119.48 & 119.2011 & 60.1309 & 58.9101 & 57.4841 \\
\hline 10.3164 & 119.0227 & 119.3406 & 119.0243 & 60.152 & 58.9099 & 57.4839 \\
\hline 10.4865 & 118.9227 & 119.2667 & 118.9312 & 60.1518 & 58.9098 & 57.6858 \\
\hline 10.7919 & 118.7436 & 119.1363 & 118.7632 & 60.1517 & 59.188 & 57.6858 \\
\hline 10.9291 & 118.6633 & 119.0785 & 118.6874 & 60.1514 & 59.3977 & 57.6856 \\
\hline 11.0751 & 118.578 & 119.0175 & 118.6066 & 60.1514 & 59.5265 & 57.6856 \\
\hline 11.6761 & 118.2288 & 118.7656 & 118.2748 & 60.2484 & 59.5264 & 57.6855 \\
\hline 11.9871 & 118.0492 & 118.635 & 118.1037 & 60.3644 & 59.5261 & 57.6853 \\
\hline 12.0081 & 118.0415 & 118.6281 & 118.0974 & 75.0567 & 71.8872 & 75.5797 \\
\hline 12.0082 & 118.0415 & 118.6281 & 118.0974 & 75.0567 & 71.8872 & 75.5797 \\
\hline 12.2461 & 117.9535 & 118.5502 & 118.0255 & 75.0567 & 71.8872 & 75.5797 \\
\hline 12.3674 & 117.9086 & 118.5105 & 117.9889 & 75.0565 & 71.8871 & 75.5796 \\
\hline 12.5034 & 117.8587 & 118.469 & 117.9463 & 75.0564 & 72.7598 & 75.5795 \\
\hline 12.6409 & 117.3969 & 117.9836 & 117.5131 & 75.0563 & 72.7597 & 75.5794 \\
\hline 12.7379 & 117.3617 & 117.954 & 117.4829 & 75.1868 & 72.7597 & 75.5794 \\
\hline 12.9285 & 117.2927 & 117.8959 & 117.4238 & 75.2038 & 72.7806 & 75.5962 \\
\hline 13.0339 & 117.2546 & 117.8638 & 117.3911 & 75.2311 & 72.7805 & 75.5961 \\
\hline 13.3649 & 117.1317 & 117.7635 & 117.2942 & 75.231 & 72.7804 & 76.3121 \\
\hline 13.6331 & 117.0379 & 117.6782 & 117.2165 & 76.1338 & 72.7803 & 76.3119 \\
\hline 13.8911 & 116.9465 & 117.5965 & 117.144 & 76.1336 & 72.7802 & 76.6399 \\
\hline 14.0938 & 116.8754 & 117.5319 & 117.0871 & 76.2787 & 72.7801 & 76.6398 \\
\hline 14.5009 & 116.7155 & 117.4069 & 117.0003 & 76.2786 & 72.9292 & 79.629 \\
\hline 15.0489 & 116.5008 & 117.2425 & 116.8818 & 76.2783 & 73.1781 & 79.629 \\
\hline 15.2389 & 116.4266 & 117.1856 & 116.8408 & 76.3222 & 73.1779 & 79.6287 \\
\hline 15.3809 & 116.3724 & 117.1421 & 116.8104 & 76.7063 & 73.1778 & 79.6286 \\
\hline 15.5193 & 116.32 & 117.1016 & 116.7796 & 76.7062 & 73.7591 & 79.6285 \\
\hline 15.8243 & 116.2031 & 117.0129 & 116.714 & 76.7061 & 73.7591 & 79.9307 \\
\hline 16.0943 & 116.0991 & 116.9345 & 116.657 & 76.7059 & 73.759 & 80.0757 \\
\hline 17.4243 & 115.6043 & 116.6686 & 116.3121 & 76.7057 & 77.462 & 80.0756 \\
\hline 17.5825 & 115.5473 & 116.6314 & 116.2701 & 76.29 & 75.5126 & 78.5313 \\
\hline 17.8255 & 115.4594 & 116.5744 & 116.2061 & 76.2899 & 75.5126 & 78.604 \\
\hline 18.2705 & 115.2994 & 116.4695 & 116.0892 & 76.391 & 75.5125 & 78.6039 \\
\hline 18.4278 & 115.2427 & 116.4325 & 116.0483 & 76.3907 & 75.5123 & 78.7047 \\
\hline 18.5938 & 115.1828 & 116.3936 & 116.0051 & 76.3906 & 75.5123 & 78.7047 \\
\hline
\end{tabular}




\begin{tabular}{|c|c|c|c|c|c|c|}
\hline 18.7608 & 115.1225 & 116.3544 & 115.9618 & 76.3905 & 75.5122 & 78.7362 \\
\hline 19.0918 & 115.0031 & 116.277 & 115.876 & 76.3904 & 75.5121 & 78.7361 \\
\hline 19.3973 & 114.8882 & 116.2064 & 115.8045 & 76.3902 & 75.512 & 79.7979 \\
\hline 19.9934 & 114.6846 & 116.0857 & 115.7242 & 80.5779 & 79.2287 & 85.5329 \\
\hline 20.3713 & 114.5566 & 116.0157 & 115.6697 & 80.5775 & 79.9467 & 85.5325 \\
\hline 20.7442 & 114.4426 & 115.9384 & 115.6173 & 81.8918 & 79.9466 & 85.5323 \\
\hline 20.9315 & 114.3841 & 115.8998 & 115.5929 & 81.8916 & 79.9464 & 85.9615 \\
\hline 21.4456 & 114.2239 & 115.7948 & 115.5258 & 81.8915 & 80.0014 & 85.9614 \\
\hline 21.7101 & 114.1411 & 115.741 & 115.4919 & 81.8912 & 80.0012 & 86.0638 \\
\hline 21.8963 & 114.0857 & 115.7246 & 115.4564 & 81.891 & 84.7817 & 86.0637 \\
\hline 22.0263 & 114.066 & 115.7222 & 115.4317 & 88.1947 & 94.5148 & 89.6651 \\
\hline 22.1673 & 114.0442 & 115.7197 & 115.4055 & 88.1946 & 94.5148 & 89.8794 \\
\hline 22.3333 & 114.0185 & 115.7167 & 115.3747 & 88.1946 & 94.5147 & 89.8793 \\
\hline 22.4553 & 114.001 & 115.7138 & 115.3522 & 88.6467 & 94.5146 & 89.8792 \\
\hline 22.7923 & 113.952 & 115.706 & 115.2914 & 88.6466 & 94.5145 & 90.0361 \\
\hline 22.9523 & 113.953 & 115.694 & 115.2609 & 93.6863 & 94.5143 & 90.0359 \\
\hline 23.1747 & 113.9531 & 115.6775 & 115.2202 & 93.6862 & 94.5142 & 90.3605 \\
\hline 23.3151 & 113.9476 & 115.6713 & 115.1959 & 93.6861 & 94.5141 & 90.6615 \\
\hline 23.3365 & 113.9468 & 115.6704 & 115.1922 & 93.7046 & 94.5372 & 90.6802 \\
\hline 23.5305 & 113.9392 & 115.6619 & 115.1587 & 93.7046 & 94.5372 & 90.6802 \\
\hline 23.6878 & 113.9334 & 115.6548 & 115.1315 & 93.7713 & 94.5371 & 90.6801 \\
\hline 23.9908 & 113.9222 & 115.6412 & 115.0792 & 93.7712 & 94.5371 & 90.68 \\
\hline 24.1041 & 113.918 & 115.6361 & 115.0597 & 93.771 & 94.537 & 90.6953 \\
\hline 24.3641 & 113.9089 & 115.624 & 115.015 & 93.8589 & 94.5369 & 90.6953 \\
\hline 24.6082 & 113.8994 & 115.6128 & 114.9746 & 93.8588 & 94.5368 & 90.9637 \\
\hline 24.7822 & 113.8938 & 115.604 & 114.946 & 94.1175 & 94.5368 & 90.9636 \\
\hline 25.0002 & 113.8862 & 115.5932 & 114.9111 & 94.1173 & 94.5367 & 91.1438 \\
\hline 25.1092 & 113.8824 & 115.5879 & 114.8935 & 94.1172 & 94.6128 & 91.1437 \\
\hline 25.3222 & 113.8832 & 115.5721 & 114.8601 & 95.6735 & 94.6127 & 91.1436 \\
\hline 26.3285 & 113.8533 & 115.5023 & 114.7593 & 95.6734 & 94.6127 & 93.4976 \\
\hline 26.5025 & 113.8474 & 115.4904 & 114.7431 & 95.6728 & 94.6123 & 93.7918 \\
\hline 26.9085 & 113.8313 & 115.4628 & 114.7094 & 95.6727 & 94.6122 & 94.2053 \\
\hline 27.0357 & 113.8266 & 115.454 & 114.6989 & 95.7668 & 94.6121 & 94.2051 \\
\hline 27.3012 & 113.8119 & 115.4363 & 114.685 & 95.7667 & 94.612 & 95.4719 \\
\hline 27.3603 & 113.8073 & 115.4327 & 114.6843 & 95.7666 & 94.7101 & 97.1667 \\
\hline 27.6618 & 113.7832 & 115.4145 & 114.681 & 95.7665 & 94.7101 & 97.242 \\
\hline 27.8754 & 113.768 & 115.3986 & 114.6763 & 96.0813 & 95.1035 & 97.4471 \\
\hline 28.0004 & 113.7579 & 115.391 & 114.6752 & 95.7663 & 94.7099 & 97.3313 \\
\hline 28.1854 & 113.743 & 115.3805 & 114.6733 & 95.7662 & 94.8365 & 97.3313 \\
\hline 28.4675 & 113.7204 & 115.3658 & 114.6696 & 95.7661 & 95.0503 & 97.3312 \\
\hline 28.65 & 113.7102 & 115.3493 & 114.674 & 96.9771 & 94.871 & 97.8451 \\
\hline 29.0193 & 113.6904 & 115.3154 & 114.683 & 97.0786 & 94.8709 & 97.845 \\
\hline 29.6693 & 113.657 & 115.2549 & 114.6991 & 97.1762 & 94.8707 & 97.8449 \\
\hline 29.9613 & 113.6438 & 115.2265 & 114.7065 & 97.4539 & 94.8703 & 97.8447 \\
\hline 30.3003 & 113.6364 & 115.1891 & 114.7161 & 98.4128 & 94.8701 & 97.8446 \\
\hline 30.9403 & 113.6248 & 115.1169 & 114.7343 & 98.575 & 94.8699 & 97.8444 \\
\hline 31.2193 & 113.6227 & 115.12 & 114.7189 & 98.5747 & 99.9982 & 97.8437 \\
\hline 31.4578 & 113.6212 & 115.1226 & 114.7062 & 98.6242 & 99.9986 & 97.9187 \\
\hline 31.6728 & 113.621 & 115.1243 & 114.6949 & 98.8229 & 99.9988 & 97.9186 \\
\hline 31.7868 & 113.621 & 115.1251 & 114.6889 & 98.9169 & 99.9991 & 97.9185 \\
\hline 31.8818 & 113.6201 & 115.126 & 114.6859 & 98.9169 & 99.9992 & 98.786 \\
\hline 32.066 & 113.6155 & 115.1288 & 114.6849 & 98.9168 & 99.9993 & 99.8769 \\
\hline
\end{tabular}




\section{Phase B}

5216.17
Phase C

5053.13

\section{kVA Flow SOC}

Phase A

NA
Phase B

NA
Released Capacity

Phase C

NA

\section{Seq Amps 0}

Seq Amps 1

25.7965

698.2907

Seq Amps 2

12/11 (\%)

Seq Volts 0

Seq Volts 1

Seq Volts 2

23.0135

622.9587

25.7941

3.6939

23.0114

3.6939

0.1936

7620.9118

0.1115

22.4991

23.9031

1.7735

523.2323

510.6884

12.597

21.8127

21.6972

7.5855

507.8991

86.6161

421.3687

24.9978

26.5738

4.7776

5.2035

15.7225

24.1295

20.9846

5.4263

4.7508

24.2271

1.2878

21.9777

79.9071

23.1378

2.3733

76.367

21.6109

22.9815

2.4374

27.045

30.0935

68.828

$0 \mathrm{NA}$

9.2072

10.8543

15.1231

5.932

7.9299

7.9299

0.2618

11.8567

19.7917

17.419

410.102

259.536

71.1666

79.493

7.2384

12.4159

14.7461

8.307

256.6139

9.5945

256.6139

9.5945

20.2921

0.2301

158.322

92.5382

65.7855

13.0377

18.5969

18.2442

20.0965

27.7329

1.765
4.7839
20.7205
10.45

3.7389

3.7389

1.1341

0 NA

0 NA

6.7516

6.7516

41.1329

8.0044

19.4599

8.0044

19.4599

7.3816

6.15
8.1408

41.1329

39.6964

3.9063

7729.7254

7686.0193

7729.5842

57.4956

49.9106

7680.7011

7679.1639

7676.0831

63.5193

61.4762

7660.636

7659.7703

7660.3411

7639.7369

122.8907

7509.8745

61.7245

7509.8745

7509.4287

7509.1726

7509.0973

60.9885

61.7607

7498.9618

84.9248

$64.834 \quad 7472.586$

83.9778

$83.6019 \quad 7378.426$

94.7981

103.7461

7307.7226

101.2307

$102.826 \quad 7306.745$

7306.9653

101.1012

101.1667

118.8639

7291.4843

101.5956

47.9228

7492.5132

90.5568

$87.6444 \quad 7278.4331$

107.7743

87.6602

7278.3672

107.7566

89.473

7275.8145

107.4898

58.7658

90.1313

7275.3363

7275.737

107.3031

107.4865 


\begin{tabular}{|c|c|c|c|c|c|c|}
\hline 0 & 0.0022 & 0 & 0 & 96.7325 & 7268.7484 & 105.6876 \\
\hline 0 & 0.4479 & 0.4474 & 99.8923 & 0 & 4861.3551 & 2416.1432 \\
\hline 0.1317 & 0 & $0 \mathrm{NA}$ & & 2397.3029 & 0 & 0 \\
\hline 15.4031 & 47.4282 & 15.5983 & 32.8881 & 45.8041 & 7485.2458 & 94.603 \\
\hline 6.4334 & 0 & $0 \mathrm{NA}$ & & 2493.8892 & 0 & 0 \\
\hline 18.3321 & 40.9954 & 18.6891 & 45.5884 & 48.7395 & 7482.0428 & 97.0537 \\
\hline 14.3794 & 36.4373 & 14.735 & 40.4393 & 55.2777 & 7476.4375 & 101.2469 \\
\hline 5.8922 & 26.2761 & 6.2311 & 23.714 & 55.2777 & 7476.4375 & 101.2469 \\
\hline 8.6301 & 10.1643 & 8.6697 & 85.2956 & 55.6248 & 7476.2813 & 101.4468 \\
\hline 6.9913 & 7.8281 & 6.9199 & 88.3989 & 57.7939 & 7475.3449 & 102.6636 \\
\hline 6.8041 & 6.8009 & 6.8047 & 100.056 & 58.8266 & 7474.9831 & 103.1873 \\
\hline 0.3134 & 0.691 & 0.3175 & 45.9455 & 57.9135 & 7475.2644 & 102.7101 \\
\hline 6.9913 & 0 & $0 \mathrm{NA}$ & & 78.0787 & 0 & 0 \\
\hline 0 & 0 & $0 \mathrm{NA}$ & & 0 & 0 & 2457.9386 \\
\hline 23.0135 & 622.9589 & 23.0114 & 3.6939 & 2.9102 & 7958.2171 & 32.5265 \\
\hline 7.3151 & 92.2339 & 7.587 & 8.2258 & 3.0394 & 7957.833 & 32.4707 \\
\hline 19.3679 & 530.9452 & 22.1074 & 4.1638 & 4.6354 & 7947.6736 & 32.8143 \\
\hline 14.1796 & 40.4839 & 14.5118 & 35.8459 & 19.7449 & 7924.7918 & 34.2899 \\
\hline 0 & 0 & $12.2208 \mathrm{NA}$ & & 0 & 0 & 2624.0328 \\
\hline 0 & 0 & $0.1296 \mathrm{NA}$ & & 0 & 0 & 2625.6999 \\
\hline 1.8314 & 28.1349 & 2.1963 & 7.8065 & 19.8955 & 7924.2239 & 34.3331 \\
\hline 0.2178 & 0.4812 & 0.267 & 55.4879 & 29.4606 & 7914.9081 & 35.5305 \\
\hline 0 & 0 & $0.431 \mathrm{NA}$ & & 0 & 0 & 2592.7205 \\
\hline 0 & 0 & $0 \mathrm{NA}$ & & 0 & 2426.4122 & 0 \\
\hline
\end{tabular}




\begin{tabular}{|c|c|c|c|c|c|c|}
\hline V2/V1 (\%) & pf A & pf B & pf C & kW Flow A & kW Flow B & kW Flow C \\
\hline 0.0015 & 0.7952 & 0.7913 & 0.7921 & 3927.3825 & 4422.1394 & 4307.9145 \\
\hline 0.4257 & 0.8432 & 0.8434 & 0.841 & 3897.9469 & 4399.4995 & 4249.9173 \\
\hline 0.6692 & 0.8446 & 0.849 & 0.8452 & 3188.3499 & 3743.2235 & 3454.8722 \\
\hline 0.7481 & 0.8476 & 0.8549 & 0.8514 & 3054.845 & 3650.9841 & 3378.6615 \\
\hline 0.669 & 0.8733 & 0.8936 & 0.9131 & 110.047 & 77.876 & 72.2701 \\
\hline 0.758 & 0.8495 & 0.8578 & 0.8553 & 3043.6655 & 3586.0975 & 3376.8882 \\
\hline 0.7622 & 0.8675 & 0.8754 & 0.883 & 354.002 & 848.0526 & 546.2885 \\
\hline 0.7659 & 0.8474 & 0.8528 & 0.8504 & 2688.3306 & 2737.1724 & 2830.3302 \\
\hline 0.815 & 0.8673 & 0.8779 & 0.8829 & 314.935 & 812.2552 & 482.4273 \\
\hline 0.8172 & 0.8675 & 0.8778 & 0.8814 & 298.3348 & 812.2442 & 426.6433 \\
\hline 0.8177 & 0.8637 & -0.0773 & 0.8939 & 16.6002 & 0.011 & 55.784 \\
\hline 0.8471 & -1 & -1 & -1 & 0 & 0 & 0 \\
\hline 1.11 & 0.8568 & 0.8628 & 0.8628 & 2562.5809 & 2647.2655 & 2755.9769 \\
\hline 1.1109 & 0.8478 & 0.8526 & 0.8594 & 1593.3367 & 1815.0299 & 1582.0433 \\
\hline 1.1131 & 0.8666 & 0.8835 & 0.8775 & 530.33 & 277.6918 & 593.4315 \\
\hline 1.1116 & 0.8809 & 0.8884 & 0.861 & 436.9675 & 553.7387 & 579.382 \\
\hline 1.1325 & 0.8482 & 0.8529 & 0.86 & 1592.1555 & 1757.4092 & 1581.5949 \\
\hline 1.1238 & 0.8482 & 0.8529 & 0.86 & 1591.7548 & 1757.3321 & 1581.4082 \\
\hline 1.2848 & 0.9637 & 0.9637 & 0.9637 & -144.3264 & -144.3264 & -144.3265 \\
\hline 1.3853 & 0.8659 & 0.8754 & 0.8739 & 1142.3289 & 984.1866 & 897.71 \\
\hline 1.3837 & 0.8671 & 0.8766 & 0.8741 & 745.3779 & 355.1598 & 665.6767 \\
\hline 1.3845 & 0.8641 & 0.8751 & 0.8737 & 396.658 & 629.1174 & 231.8776 \\
\hline 1.3933 & -1 & -1 & -1 & 0 & 0 & 0 \\
\hline 1.2086 & -1 & -1 & -1 & 0 & 0 & 0 \\
\hline 1.4807 & 0.8804 & 0.8773 & 0.8798 & 270.7401 & 341.9209 & 177.6084 \\
\hline 1.4805 & 0.8804 & 0.8773 & 0.8798 & 270.6931 & 341.9206 & 177.6115 \\
\hline
\end{tabular}




\begin{tabular}{|c|c|c|c|c|c|c|c|}
\hline & 1.4774 & 0.8805 & 0.878 & 0.88 & 270.6305 & 320.0234 & 171.8888 \\
\hline & 1.4749 & 0.883 & 0.8779 & 0.8817 & 186.9862 & 320.0152 & 134.214 \\
\hline & 1.4773 & 0.8749 & -0.0824 & 0.8738 & 83.604 & 0.0084 & 37.6769 \\
\hline & 1.454 & 0.0586 & 0.097 & -0.1554 & 0 & -0.0017 & 0.0025 \\
\hline & 49.701 & 0 & 0.8733 & -0.2558 & 0 & 8.558 & 0.0059 \\
\hline NA & & 0.9297 & 0 & 0 & 2.6429 & 0 & 0 \\
\hline & 1.2639 & 0.8665 & 0.8856 & 0.8791 & 245.9844 & 177.5682 & 508.7365 \\
\hline NA & & 0.866 & 0 & 0 & 125.055 & 0 & 0 \\
\hline & 1.2972 & 0.867 & 0.8856 & 0.8791 & 120.9412 & 177.5618 & 508.7018 \\
\hline & 1.3542 & 0.8669 & 0.8854 & 0.8807 & 121.1206 & 169.0124 & 426.8469 \\
\hline & 1.3542 & 0.8622 & 0.8815 & 0.879 & 111.8729 & 156.9953 & 246.7566 \\
\hline & 1.3569 & 0.9201 & 0.932 & 0.8835 & 9.2763 & 11.9755 & 180.0152 \\
\hline & 1.3734 & 0.9171 & 0.9059 & 0.8858 & 9.2938 & 2.8014 & 142.7331 \\
\hline & 1.3804 & 0.1516 & -0.0919 & 0.8862 & -0.0075 & 0.0049 & 134.0994 \\
\hline & 1.374 & 0.885 & 0.8955 & 0.881 & 2.246 & 2.794 & 8.6234 \\
\hline NA & & 0.9235 & 0 & 0 & 7.0599 & 0 & 0 \\
\hline NA & & 0 & 0 & 0 & 0 & 0 & 0 \\
\hline & 0.4087 & 0.8432 & 0.8434 & 0.841 & 3897.9475 & 4399.4991 & 4249.9171 \\
\hline & 0.408 & 0.8688 & 0.8769 & 0.8799 & 573.8255 & 608.1993 & 745.6672 \\
\hline & 0.4129 & 0.8395 & 0.8387 & 0.8338 & 3322.0806 & 3786.9672 & 3504.6396 \\
\hline & 0.4327 & 0.8735 & 0.886 & 0.8836 & 183.6974 & 181.8916 & 481.4931 \\
\hline NA & & 0 & 0 & 0.8765 & 0 & 0 & 253.1318 \\
\hline NA & & 0 & 0 & 0.8747 & 0 & 0 & 2.679 \\
\hline & 0.4333 & 0.8735 & 0.886 & 0.8916 & 183.6974 & 181.8916 & 225.6823 \\
\hline & 0.4489 & 0.9254 & 0.875 & -0.0565 & 5.016 & 5.3157 & 0.0013 \\
\hline NA & & 0 & 0 & 0.8751 & 0 & 0 & 8.8011 \\
\hline & 0 & 0 & 0 & 0 & 0 & 0 & 0 \\
\hline
\end{tabular}


kVAR Flow A kVAR Flow B kVAR Flow C

\begin{tabular}{|c|c|c|}
\hline 2994.4404 & 3416.6517 & 3319.2982 \\
\hline 2484.8771 & 2802.2932 & 2733.5539 \\
\hline 2020.8649 & 2329.1825 & 2184.851 \\
\hline 1912.3068 & 2215.1469 & 2081.2881 \\
\hline 61.4043 & 39.1233 & 32.2663 \\
\hline 1890.091 & 2148.7621 & 2045.9234 \\
\hline 202.9733 & 468.2726 & 290.3765 \\
\hline 1684.3503 & 1676.1461 & 1751.1922 \\
\hline 180.7191 & 443.0079 & 256.5759 \\
\hline 171.0321 & 443.1493 & 228.6038 \\
\hline 9.6871 & -0.1414 & 27.9721 \\
\hline 0 & 0 & 0 \\
\hline 1542.3544 & 1550.7972 & 1615.0629 \\
\hline 996.676 & 1112.2177 & 941.1572 \\
\hline 305.3682 & 147.2087 & 324.3699 \\
\hline 234.7624 & 286.0857 & 342.3179 \\
\hline 994.3771 & 1075.6567 & 938.4383 \\
\hline 993.5189 & 1074.3983 & 937.4523 \\
\hline-40.0011 & -40.0009 & -40.0006 \\
\hline 659.9751 & 543.3731 & 499.4587 \\
\hline 428.2069 & 194.9498 & 369.9944 \\
\hline 231.0125 & 347.9404 & 129.1095 \\
\hline 0 & 0 & 0 \\
\hline 0 & 0 & 0 \\
\hline 145.8217 & 187.0529 & 95.9727 \\
\hline 145.772 & 186.9251 & 95.955 \\
\hline
\end{tabular}




\begin{tabular}{|c|c|c|}
\hline 145.7273 & 174.5042 & 92.7921 \\
\hline 99.4138 & 174.5241 & 71.8258 \\
\hline 46.2855 & -0.1015 & 20.9698 \\
\hline-0.014 & -0.0174 & -0.016 \\
\hline 0 & 4.7749 & -0.0224 \\
\hline 1.047 & 0 & 0 \\
\hline 141.7294 & 93.1255 & 275.851 \\
\hline 72.193 & 0 & 0 \\
\hline 69.5248 & 93.1382 & 275.7495 \\
\hline 69.6617 & 88.7318 & 229.5944 \\
\hline 65.7403 & 84.1135 & 133.8224 \\
\hline 3.9478 & 4.6589 & 95.4486 \\
\hline 4.0411 & 1.3092 & 74.7599 \\
\hline-0.0486 & -0.0527 & 70.1198 \\
\hline 1.1817 & 1.3887 & 4.6312 \\
\hline 2.9325 & 0 & 0 \\
\hline 0 & 0 & 0 \\
\hline 2484.8807 & 2802.297 & 2733.557 \\
\hline 326.9982 & 333.3856 & 402.7434 \\
\hline 2150.222 & 2459.0224 & 2320.6186 \\
\hline 102.3847 & 95.1695 & 255.1357 \\
\hline 0 & 0 & 139.0539 \\
\hline 0 & 0 & 1.4846 \\
\hline 102.3847 & 95.1695 & 114.5972 \\
\hline 2.055 & 2.9415 & -0.0236 \\
\hline 0 & 0 & 4.8678 \\
\hline 0 & 0 & 0 \\
\hline
\end{tabular}


Table B-6. Summary of LL Base Case

(LTC control only, tap =7)

[\#0 MXAT services included]

\begin{tabular}{|c|c|c|c|c|c|c|}
\hline Location & \multicolumn{3}{|c|}{ Simulated Data } & \multicolumn{3}{|c|}{ Measured Data July 17, 2006} \\
\hline Node 01 & $\begin{array}{c}\mathrm{I}_{\mathrm{A}} \\
227.46\end{array}$ & $\begin{array}{c}\mathrm{I}_{\mathrm{B}} \\
262.61\end{array}$ & $\begin{array}{c}\mathrm{I}_{\mathrm{C}} \\
255.85\end{array}$ & $\begin{array}{c}I_{A} \\
225\end{array}$ & $\begin{array}{c}I_{B} \\
260\end{array}$ & $\begin{array}{c}I_{C} \\
254\end{array}$ \\
\hline Node 01 & $\begin{array}{c}V_{A} \\
125.76\end{array}$ & $\begin{array}{c}V_{B} \\
125.60\end{array}$ & $\begin{array}{c}V_{C} \\
125.31\end{array}$ & $\begin{array}{c}V_{A} \\
126.0\end{array}$ & $\begin{array}{c}V_{B} \\
126.0\end{array}$ & $\begin{array}{c}V_{C} \\
126.0\end{array}$ \\
\hline Node 01 & $\begin{array}{c}\mathrm{PF}_{\mathrm{A}} \\
0.867\end{array}$ & $\begin{array}{c}\mathrm{PF}_{\mathrm{B}} \\
0.862\end{array}$ & $\begin{array}{c}P_{C} \\
0.857\end{array}$ & & & \\
\hline & \multicolumn{3}{|c|}{ kVA Flow } & Total & & \\
\hline Node 01 & $\begin{array}{l}\text { Phase A } \\
1,816.66\end{array}$ & $\begin{array}{l}\text { Phase B } \\
2,094.65\end{array}$ & $\begin{array}{l}\text { Phase C } \\
2,036.10\end{array}$ & $5,947.41$ & & \\
\hline & \multicolumn{3}{|c|}{ kW Flow } & & & \\
\hline Node 01 & $\begin{array}{l}\text { Phase A } \\
1,575.70\end{array}$ & $\begin{array}{l}\text { Phase B } \\
1,806.23\end{array}$ & $\begin{array}{l}\text { Phase C } \\
1,743.88\end{array}$ & $5,125.81$ & & \\
\hline & \multicolumn{3}{|c|}{ kVAr Flow } & & & \\
\hline \multirow[t]{2}{*}{ Node 01} & $\begin{array}{c}\text { Phase A } \\
904.12\end{array}$ & $\begin{array}{l}\text { Phase B } \\
1,060.70\end{array}$ & $\begin{array}{l}\text { Phase C } \\
1,050.99\end{array}$ & $3,015.81$ & & \\
\hline & \multicolumn{3}{|c|}{ kW Losses } & Total \% & & \\
\hline \multirow[t]{3}{*}{ Node 01} & $\begin{array}{c}\text { Phase A } \\
59.58\end{array}$ & $\begin{array}{c}\text { Phase B } \\
48.67\end{array}$ & $\begin{array}{c}\text { Phase C } \\
58.98\end{array}$ & $\begin{array}{l}167.23 \\
3.26 \%\end{array}$ & & \\
\hline & \multicolumn{3}{|c|}{ kVAr Losses } & Total \% & & \\
\hline & $\begin{array}{c}\text { Phase A } \\
135.74\end{array}$ & $\begin{array}{c}\text { Phase B } \\
182.47\end{array}$ & $\begin{array}{c}\text { Phase C } \\
178.03\end{array}$ & $\begin{array}{l}496.24 \\
16.45 \%\end{array}$ & & \\
\hline $\begin{array}{l}\text { Node } 01 \\
\text { Node } 01\end{array}$ & $\begin{array}{c}\mathrm{I}_{0} \\
9.97 \\
\mathrm{~V}_{0} \\
0.084 \\
\end{array}$ & $\begin{array}{c}\mathrm{l}_{1} \\
248.62 \\
\mathrm{~V}_{1} \\
7973.66\end{array}$ & $\begin{array}{c}\mathrm{I}_{2} \\
11.71 \\
\mathrm{~V}_{2} \\
16.65\end{array}$ & $\begin{array}{c}\mathrm{I}_{2} / \mathrm{I}_{1} \% \\
4.71 \\
\mathrm{~V}_{2} / \mathrm{V} 1 \% \\
0.2088 \\
\end{array}$ & & \\
\hline
\end{tabular}

Note: All capacitors and line regulators were turned off, and the substation primary voltage was set at $100 \%(120 \mathrm{~V})$. 
kW Losses:

Phase A

59.58

Phase B
48.67

LTC Tap

7

48.67
Local Name Am

MILFD8103 244.0341

$=====$ Node $1======$

StPole_MILFD8 227.4606

======Node $2======$

$=====$ Node $3=====$

Node 3

Node $3 \mathrm{~N}$

195.3918

Node $3 \mathrm{E}$ 189.6574

======Node $4======$

\section{Node 4}

Node $4 \mathrm{E}$

5.7509

Node $4 \mathrm{~N}$

189.6645

17.4388

172.2271

$======$ Node $5=====$

\section{Node 5}

Node $5 \mathrm{E}$

15.6842

Node $5 \mathrm{~N}$

14.9015

0.7828

======Node $6======$

Node 6

===-==Node 7 ===-==

Node 7

Node $7 \mathrm{E}$

Node $7 \mathrm{~N}$

167.4328

Node $7 \mathrm{~W}$

105.0159

32.9179

29.5179

$======$ Node $8======$

Node $8 \quad 105.0185$

=====-Node $9=====$

Node 9

$======$ Node $10======$

Node 10

======Node $11======$

Node 11

Node $11 \mathrm{~N}$

65.79

Node $11 \mathrm{E}$

43.6761

22.1163

$=====$ Node $12======$

Node 12

======Node 13======

Node 13

$=====$ Node $14======$
Node 14

$======$ Node $15======$

636_B

17.6897

kVAr Losses:

$\begin{array}{ccccc}\text { Phase C } & \text { Phase A } & \text { Phase B } & \text { Phase C } & \text { Phase A } \\ 58.98 & 135.74 & 182.47 & 178.03 & 1816.66\end{array}$

VR 1 A Tap VR 1 B Tap VR 1 C Tap

NA NA NA

Amps B

281.7392

262.605

233.8487

229.6123

4.256

226.3778

45.8427

180.5432

44.2623

44.27

0.019

0

176.0287

118.6366

20.8533

36.562

115.4683

115.4688

0

55.7814

19.9716

35.8106

48.2718

35.7606

12.5122

0

0

19.3124

19.3144

Amps C

274.4897

255.8479

218.4801
213.8667
4.6576

213.8713

29.0388

184.8588

25.1048

22.0038

3.1026

180.8732

102.1838

41.1011

37.6357

102.1858

102.1865

0

9.9032

9.6032
121.6699

121.0926

121.085

121.0856

120.9692

122.5989

120.9486

120.9384

\section{Cust Volts B}

120.0001

125.5965

124.0145

123.7173

124.0142

123.6818

123.6625

123.6565

123.4232

123.4112

123.4233

123.1477

122.732

122.7292

122.7314

122.7259

122.665

122.4842

121.9238

121.5547

121.5542

121.5474

121.414

122.6963

121.4649

121.4575
Cust Volts C

120.0001

125.3097

123.9459

123.6939

123.945

123.6624

123.6608

123.6305

123.6495

123.6505

123.6441

123.7001

122.4825

122.48

122.4726

122.4746

122.4185

122.2595

121.7519

121.4505

121.4419

121.4519

121.4392

122.2159 


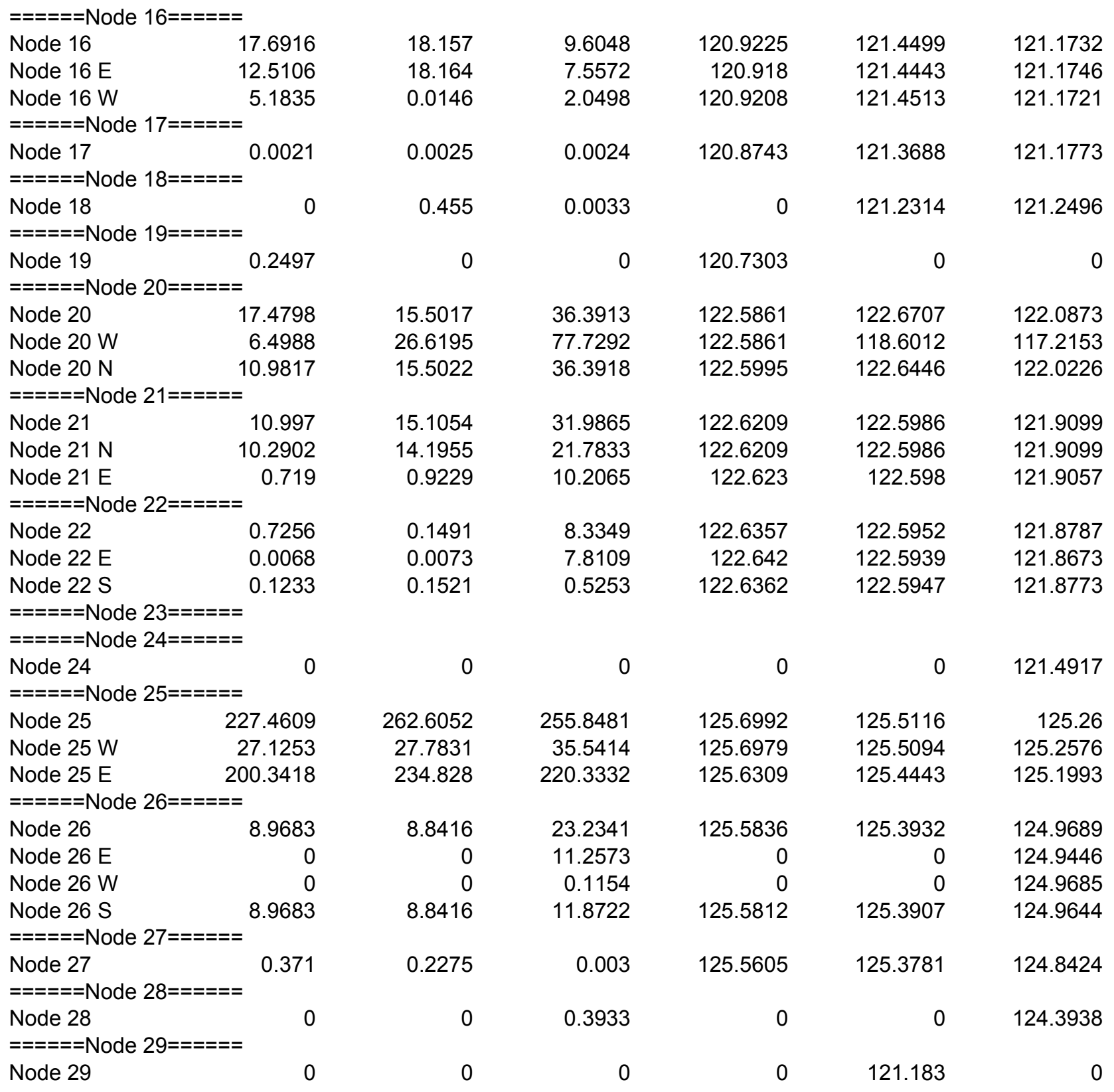

$======$ Feeder Path Trace======

Distance (1000r CustvoltsA CustVoltsB CustVoltsC \%CapacityA \%CapacityB \%CapacityC

$\begin{array}{rrrrrrr}0.1034 & 118.7851 & 118.7178 & 118.6043 & 77.5246 & 74.052 & 74.7196 \\ 0.2168 & 125.7839 & 125.6264 & 125.3387 & 75.5419 & 71.7629 & 72.4895 \\ 0.3008 & 125.7579 & 125.5965 & 125.3097 & 66.1013 & 60.8636 & 61.8707 \\ 0.3008 & 125.7579 & 125.5965 & 125.3097 & 77.3671 & 73.8702 & 74.5425 \\ 0.5078 & 125.6992 & 125.5116 & 125.26 & 77.3671 & 73.8701 & 74.5425 \\ 0.7438 & 125.6309 & 125.4443 & 125.1993 & 80.0655 & 76.634 & 78.0763 \\ 1.3578 & 125.453 & 125.2693 & 125.0424 & 80.0653 & 76.6339 & 78.1271 \\ 2.562 & 125.1045 & 124.9284 & 124.7351 & 80.0649 & 76.6811 & 78.1268\end{array}$




\begin{tabular}{|c|c|c|c|c|c|c|}
\hline 2.645 & 125.0805 & 124.905 & 124.714 & 80.0641 & 76.6837 & 78.1263 \\
\hline 3.185 & 124.9297 & 124.7482 & 124.5792 & 80.5588 & 76.6837 & 78.2612 \\
\hline 3.408 & 124.8674 & 124.6837 & 124.5236 & 80.5584 & 76.7162 & 78.2609 \\
\hline 3.695 & 124.7873 & 124.6008 & 124.4519 & 80.5582 & 76.7257 & 78.2608 \\
\hline 5.733 & 124.2194 & 124.0145 & 123.9459 & 80.558 & 76.7315 & 78.2607 \\
\hline 6.777 & 123.9386 & 123.7173 & 123.6939 & 81.1286 & 77.153 & 78.7197 \\
\hline 6.9052 & 123.9043 & 123.6818 & 123.6624 & 81.1279 & 77.4748 & 78.7193 \\
\hline 7.0393 & 123.8714 & 123.6565 & 123.6305 & 82.863 & 82.0355 & 81.6061 \\
\hline 7.1824 & 123.8364 & 123.6295 & 123.5964 & 82.8828 & 82.0354 & 81.606 \\
\hline 7.3574 & 123.7935 & 123.5965 & 123.5548 & 82.8827 & 82.0354 & 81.606 \\
\hline 7.6884 & 123.7128 & 123.5339 & 123.4762 & 82.9105 & 82.0353 & 81.6059 \\
\hline 7.8854 & 123.6649 & 123.4965 & 123.4294 & 82.9353 & 82.0351 & 81.6057 \\
\hline 7.8854 & 123.6649 & 123.4965 & 123.4294 & 82.9352 & 82.035 & 81.6379 \\
\hline 8.2254 & 123.5809 & 123.4326 & 123.3508 & 82.9352 & 82.035 & 81.8885 \\
\hline 8.3964 & 123.5386 & 123.4004 & 123.3114 & 82.935 & 82.0349 & 81.9108 \\
\hline 8.8361 & 123.4305 & 123.3175 & 123.2102 & 82.9856 & 82.0348 & 81.9107 \\
\hline 9.4201 & 123.287 & 123.2075 & 123.076 & 82.9853 & 82.0431 & 81.9105 \\
\hline 9.5872 & 123.2471 & 123.1753 & 123.0377 & 83.2611 & 82.0428 & 81.9103 \\
\hline 9.9964 & 123.1497 & 123.0984 & 122.9429 & 83.261 & 82.2412 & 81.9102 \\
\hline 10.3164 & 123.0736 & 123.0382 & 122.8689 & 83.2733 & 82.241 & 81.91 \\
\hline 10.4865 & 123.033 & 123.0063 & 122.8299 & 83.2731 & 82.2409 & 82.0033 \\
\hline 10.7919 & 122.9602 & 122.9497 & 122.7596 & 83.2729 & 82.3374 & 82.0032 \\
\hline 10.9291 & 122.9275 & 122.9246 & 122.7279 & 83.2727 & 82.4083 & 82.0031 \\
\hline 11.0751 & 122.8929 & 122.8981 & 122.6939 & 83.2726 & 82.4851 & 82.003 \\
\hline 11.6761 & 122.7506 & 122.7887 & 122.5546 & 83.3033 & 82.485 & 82.0029 \\
\hline 11.9871 & 122.6773 & 122.732 & 122.4825 & 83.34 & 82.4847 & 82.0027 \\
\hline 12.0081 & 122.6741 & 122.7292 & 122.48 & 89.5507 & 88.1954 & 89.8325 \\
\hline 12.0082 & 122.6741 & 122.7292 & 122.48 & 89.5506 & 88.1954 & 89.8325 \\
\hline 12.2461 & 122.6381 & 122.6978 & 122.4507 & 89.5506 & 88.1954 & 89.8325 \\
\hline 12.3674 & 122.6197 & 122.6818 & 122.4358 & 89.5505 & 88.1953 & 89.8323 \\
\hline 12.5034 & 122.5993 & 122.665 & 122.4185 & 89.5504 & 88.5106 & 89.8323 \\
\hline 12.6409 & 122.416 & 122.4672 & 122.2421 & 89.5503 & 88.5106 & 89.8322 \\
\hline 12.7379 & 122.4016 & 122.4551 & 122.2298 & 89.5935 & 88.5105 & 89.8321 \\
\hline 12.9285 & 122.3732 & 122.4315 & 122.2057 & 89.5989 & 88.5171 & 89.8375 \\
\hline 13.0339 & 122.3575 & 122.4184 & 122.1924 & 89.6074 & 88.5171 & 89.8374 \\
\hline 13.3649 & 122.3068 & 122.3775 & 122.153 & 89.6074 & 88.517 & 90.1356 \\
\hline 13.6331 & 122.268 & 122.3427 & 122.1214 & 89.958 & 88.5169 & 90.1354 \\
\hline 13.8911 & 122.2303 & 122.3094 & 122.0917 & 89.9579 & 88.5168 & 90.2428 \\
\hline 14.0938 & 122.2008 & 122.283 & 122.0684 & 90.0051 & 88.5167 & 90.2427 \\
\hline 14.5009 & 122.1348 & 122.2319 & 122.0327 & 90.0049 & 88.5661 & 91.4448 \\
\hline 15.0489 & 122.0461 & 122.1643 & 121.9841 & 90.0047 & 88.6493 & 91.4446 \\
\hline 15.2389 & 122.0154 & 122.1409 & 121.9673 & 90.0186 & 88.6491 & 91.4443 \\
\hline 15.3809 & 121.9929 & 122.123 & 121.9548 & 90.16 & 88.649 & 91.4442 \\
\hline 15.5193 & 121.9712 & 122.1063 & 121.9422 & 90.1599 & 88.8767 & 91.4442 \\
\hline 15.8243 & 121.9227 & 122.0697 & 121.9153 & 90.1598 & 88.8766 & 91.5733 \\
\hline 16.0943 & 121.8795 & 122.0374 & 121.8918 & 90.1596 & 88.8765 & 91.6211 \\
\hline 17.4243 & 121.6732 & 121.9215 & 121.7536 & 90.1594 & 90.1983 & 91.621 \\
\hline 17.5825 & 121.6506 & 121.9062 & 121.738 & 90.7546 & 90.1974 & 91.7572 \\
\hline 17.8255 & 121.6158 & 121.8827 & 121.7141 & 90.7545 & 90.1973 & 91.7819 \\
\hline 18.2705 & 121.5526 & 121.8392 & 121.6706 & 90.8164 & 90.1972 & 91.7818 \\
\hline 18.4278 & 121.5302 & 121.8238 & 121.6555 & 90.8161 & 90.1971 & 91.8435 \\
\hline 18.5938 & 121.5065 & 121.8076 & 121.6395 & 90.816 & 90.197 & 91.8434 \\
\hline
\end{tabular}




\begin{tabular}{|c|c|c|c|c|c|c|}
\hline 18.7608 & 121.4826 & 121.7914 & 121.6234 & 90.8159 & 90.1969 & 91.8536 \\
\hline 19.0918 & 121.4353 & 121.7591 & 121.5916 & 90.8158 & 90.1969 & 91.8535 \\
\hline 19.3973 & 121.3899 & 121.7297 & 121.5652 & 90.8156 & 90.1968 & 92.2613 \\
\hline 19.9934 & 121.3147 & 121.6853 & 121.5392 & 93.0173 & 92.4638 & 95.0126 \\
\hline 20.3713 & 121.2676 & 121.6599 & 121.5211 & 93.0169 & 92.7741 & 95.0121 \\
\hline 20.7442 & 121.2252 & 121.6321 & 121.5037 & 93.4546 & 92.7739 & 95.0119 \\
\hline 20.9315 & 121.2035 & 121.6182 & 121.4957 & 93.4544 & 92.7738 & 95.1624 \\
\hline 21.4456 & 121.144 & 121.5803 & 121.4735 & 93.4542 & 92.7918 & 95.1623 \\
\hline 21.7101 & 121.1132 & 121.5608 & 121.4623 & 93.4539 & 92.7916 & 95.197 \\
\hline 21.8963 & 121.0926 & 121.5547 & 121.4505 & 93.4537 & 94.4496 & 95.1968 \\
\hline 22.0263 & 121.085 & 121.5542 & 121.4419 & 95.6541 & 98.0128 & 96.4417 \\
\hline 22.1673 & 121.0766 & 121.5536 & 121.4329 & 95.6541 & 98.0127 & 96.5147 \\
\hline 22.3333 & 121.0667 & 121.5529 & 121.4222 & 95.654 & 98.0126 & 96.5146 \\
\hline 22.4553 & 121.0599 & 121.5522 & 121.4144 & 95.8112 & 98.0125 & 96.5145 \\
\hline 22.7923 & 121.041 & 121.5502 & 121.3934 & 95.8111 & 98.0124 & 96.5674 \\
\hline 22.9523 & 121.0402 & 121.5465 & 121.3827 & 97.5132 & 98.0122 & 96.5672 \\
\hline 23.1747 & 121.0387 & 121.5413 & 121.3683 & 97.5131 & 98.0121 & 96.6775 \\
\hline 23.3151 & 121.0358 & 121.5397 & 121.3597 & 97.513 & 98.012 & 96.7796 \\
\hline 23.3365 & 121.0353 & 121.5394 & 121.3584 & 97.5204 & 98.0212 & 96.787 \\
\hline 23.5305 & 121.0313 & 121.5372 & 121.3467 & 97.5204 & 98.0211 & 96.787 \\
\hline 23.6878 & 121.0282 & 121.5353 & 121.3371 & 97.5426 & 98.0211 & 96.7869 \\
\hline 23.9908 & 121.0222 & 121.5317 & 121.3187 & 97.5425 & 98.021 & 96.7868 \\
\hline 24.1041 & 121.0199 & 121.5303 & 121.3118 & 97.5423 & 98.0209 & 96.7918 \\
\hline 24.3641 & 121.0149 & 121.5271 & 121.2961 & 97.5718 & 98.0209 & 96.7917 \\
\hline 24.6082 & 121.0099 & 121.5241 & 121.2818 & 97.5716 & 98.0208 & 96.882 \\
\hline 24.7822 & 121.0067 & 121.5217 & 121.2717 & 97.6614 & 98.0207 & 96.882 \\
\hline 25.0002 & 121.0025 & 121.5187 & 121.2594 & 97.6613 & 98.0206 & 96.9438 \\
\hline 25.1092 & 121.0004 & 121.5173 & 121.2532 & 97.6611 & 98.0462 & 96.9437 \\
\hline 25.3222 & 120.9992 & 121.5125 & 121.2414 & 98.21 & 98.0461 & 96.9437 \\
\hline 26.3285 & 120.9818 & 121.4915 & 121.2059 & 98.2098 & 98.0461 & 97.7627 \\
\hline 26.5025 & 120.9786 & 121.4879 & 121.2001 & 98.2092 & 98.0457 & 97.8604 \\
\hline 26.9085 & 120.9702 & 121.4796 & 121.1882 & 98.2091 & 98.0456 & 97.9984 \\
\hline 27.0357 & 120.9677 & 121.4769 & 121.1844 & 98.2404 & 98.0455 & 97.9982 \\
\hline 27.3012 & 120.9608 & 121.4715 & 121.1794 & 98.2403 & 98.0454 & 98.42 \\
\hline 27.3603 & 120.9588 & 121.4705 & 121.1791 & 98.2401 & 98.0784 & 98.9894 \\
\hline 27.6618 & 120.9486 & 121.4649 & 121.1777 & 98.2401 & 98.0784 & 99.0146 \\
\hline 27.858 & 120.9427 & 121.4598 & 121.176 & 98.3709 & 98.2212 & 99.0878 \\
\hline 27.983 & 120.9384 & 121.4575 & 121.1755 & 98.2398 & 98.0782 & 99.0445 \\
\hline 28.168 & 120.9321 & 121.4543 & 121.1747 & 98.2398 & 98.1203 & 99.044 \\
\hline 28.4501 & 120.9225 & 121.4499 & 121.1732 & 98.2396 & 98.1933 & 99.0443 \\
\hline 28.6326 & 120.918 & 121.4443 & 121.1746 & 98.7102 & 98.1274 & 99.220 \\
\hline 29.0019 & 120.9094 & 121.4327 & 121.1777 & 98.7743 & 98.1273 & 99.220 \\
\hline 29.6519 & 120.8952 & 121.4116 & 121.1833 & 98.8357 & 98.1271 & 99.2207 \\
\hline 29.9439 & 120.8896 & 121.4016 & 121.186 & 98.9576 & 98.1266 & 99.2205 \\
\hline 30.2829 & 120.8864 & 121.388 & 121.1894 & 99.373 & 98.1264 & 99.2203 \\
\hline 30.9229 & 120.8813 & 121.3619 & 121.1959 & 99.4283 & 98.1262 & 99.2202 \\
\hline 31.2019 & 120.8802 & 121.3633 & 121.1902 & 99.4279 & 99.9981 & 99.2194 \\
\hline 31.4404 & 120.8794 & 121.3645 & 121.1855 & 99.4477 & 99.9985 & 99.2464 \\
\hline 31.6554 & 120.8791 & 121.3653 & 121.1813 & 99.5151 & 99.9988 & 99.2464 \\
\hline 31.7694 & 120.8791 & 121.3657 & 121.1791 & 99.5744 & 99.999 & 99.2463 \\
\hline 31.8644 & 120.8787 & 121.3661 & 121.178 & 99.5744 & 99.9991 & 99.5357 \\
\hline 32.0486 & 120.8769 & 121.3672 & 121.1776 & 99.5743 & 99.9992 & 99.9585 \\
\hline
\end{tabular}


kVA Flow SOC

Phase B 2094.65
Phase C

2036.10
Phase A
NA

Phase B

NA
Released Capacity

Phase C

NA

\begin{tabular}{|c|c|c|c|c|c|c|}
\hline Seq Amps 0 & Seq Amps 1 & Seq Amps 2 & I2/I1 (\%) & Seq Volts 0 & Seq Volts 1 & Seq Volts 2 \\
\hline 10.6997 & 266.7397 & 12.5661 & 4.711 & 0 & 7620.9118 & 0.1115 \\
\hline 9.973 & 248.6242 & 11.7127 & 4.711 & 0.084 & 7973.6646 & 16.649 \\
\hline 10.0093 & 215.8935 & 12.4044 & 5.7456 & 21.2443 & 7878.6813 & 24.3383 \\
\hline 10.3777 & 211.0309 & 12.9244 & 6.1244 & 25.6344 & 7861.0895 & 26.6788 \\
\hline 0.3748 & 4.886 & 0.5281 & 10.8088 & 21.2226 & 7878.627 & 24.3351 \\
\hline 9.6275 & 209.9567 & 12.0089 & 5.7197 & 26.1204 & 7858.9441 & 26.9705 \\
\hline 8.3286 & 30.7726 & 8.1616 & 26.5225 & 26.8884 & 7858.3973 & 27.1083 \\
\hline 3.754 & 179.1924 & 4.3125 & 2.4066 & 26.1587 & 7857.0354 & 27.1938 \\
\hline 8.4174 & 28.3499 & 8.3981 & 29.6229 & 36.6073 & 7851.8011 & 28.8003 \\
\hline 8.8219 & 27.0579 & 8.8711 & 32.7856 & 37.1205 & 7851.4935 & 28.871 \\
\hline 0.9194 & 1.2925 & 0.9472 & 73.2813 & 36.506 & 7851.6931 & 28.8793 \\
\hline 0 & 0 & 01 & & 48.6106 & 7844.5683 & 29.8772 \\
\hline 3.9833 & 174.7588 & 4.5671 & 2.6134 & 28.0842 & 7787.8378 & 36.715 \\
\hline 4.5087 & 108.6062 & 5.673 & 5.2234 & 28.1123 & 7787.6568 & 36.7426 \\
\hline 5.9934 & 31.6231 & 5.769 & 18.243 & 27.8664 & 7787.5197 & 36.811 \\
\hline 1.8431 & 34.5322 & 3.5023 & 10.142 & 28.1004 & 7787.4935 & 36.7669 \\
\hline 3.4602 & 107.5518 & 4.6405 & 4.3146 & 28.733 & 7783.4062 & 37.3958 \\
\hline 3.4602 & 107.5518 & 4.6405 & 4.3146 & 29.1752 & 7772.7694 & 37.063 \\
\hline 0 & 0 & 01 & & 35.9934 & 7733.8786 & 41.7965 \\
\hline 5.0211 & 56.61 & 5.1669 & 9.1272 & 43.847 & 7707.4137 & 44.0908 \\
\hline 7.2945 & 33.1324 & 6.6414 & 20.0449 & 43.514 & 7707.0621 & 44.0182 \\
\hline 6.6666 & 23.4789 & 6.8538 & 29.1915 & 44.2314 & 7707.1408 & 44.0789 \\
\hline 0 & 0 & 01 & & 49.8449 & 7701.5484 & 44.3862 \\
\hline 0 & 0 & 01 & & 24.7761 & 7779.7819 & 39.7308 \\
\hline 2.6065 & 15.6289 & 3.198 & 20.4621 & 38.0717 & 7696.7125 & 45.7633 \\
\hline 2.7067 & 15.5305 & 3.2965 & 21.2261 & 38.2758 & 7696.2939 & 45.6723 \\
\hline
\end{tabular}




\begin{tabular}{|c|c|c|c|c|c|c|}
\hline 2.5008 & 15.1462 & 3.0505 & 20.1403 & 38.6129 & 7695.7453 & 45.627 \\
\hline 2.8332 & 12.7392 & 3.2962 & 25.8747 & 38.8364 & 7695.5626 & 45.5523 \\
\hline 1.55 & 2.4084 & 1.4676 & 60.9367 & 38.5379 & 7695.7163 & 45.6194 \\
\hline 0 & 0.0023 & 0 & 0 & 41.1339 & 7693.0855 & 44.9112 \\
\hline 0 & 0.1514 & 0.1509 & 99.6866 & 0 & 5133.0788 & 2558.1215 \\
\hline 0.0832 & 0 & $0 \mathrm{NA}$ & & 2555.801 & 0 & 0 \\
\hline 6.6367 & 23.1235 & 6.6805 & 28.8905 & 25.1359 & 7776.2405 & 41.4469 \\
\hline 2.1663 & 47.4282 & 15.5983 & 32.8881 & 2595.0857 & 7485.2458 & 94.603 \\
\hline 7.7805 & 20.9578 & 7.8723 & 37.5624 & 26.7462 & 7774.5972 & 42.5068 \\
\hline 6.3754 & 19.3623 & 6.4694 & 33.4126 & 29.9628 & 7771.6767 & 44.346 \\
\hline 3.3308 & 15.4226 & 3.4172 & 22.157 & 29.9628 & 7771.6767 & 44.346 \\
\hline 3.1224 & 3.9442 & 3.1421 & 79.6646 & 30.0955 & 7771.6168 & 44.4178 \\
\hline 2.659 & 3.0675 & 2.619 & 85.3787 & 30.9206 & 7771.2542 & 44.8628 \\
\hline 2.6044 & 2.6012 & 2.6051 & 100.1499 & 31.3241 & 7771.1159 & 45.0595 \\
\hline 0.1292 & 0.2665 & 0.1305 & 48.9528 & 30.9698 & 7771.2238 & 44.8812 \\
\hline 0 & 0 & $0 \mathrm{NA}$ & & 0 & 0 & 2571.8618 \\
\hline 9.973 & 248.6244 & 11.7127 & 4.711 & 1.214 & 7969.5736 & 16.1522 \\
\hline 2.8208 & 30.149 & 2.5843 & 8.5717 & 1.2553 & 7969.4473 & 16.1338 \\
\hline 8.7835 & 218.4865 & 11.2937 & 5.169 & 1.9729 & 7965.4167 & 16.3163 \\
\hline 4.7697 & 13.6807 & 4.7844 & 34.9721 & 7.611 & 7958.4629 & 16.5635 \\
\hline 0 & 0 & $3.7523 \mathrm{NA}$ & & 0 & 0 & 2644.9557 \\
\hline 0 & 0 & $0.0385 \mathrm{NA}$ & & 0 & 0 & 2645.4616 \\
\hline 0.9805 & 9.8936 & 1.0013 & 10.1203 & 7.7004 & 7958.2634 & 16.5774 \\
\hline 0.0977 & 0.1987 & 0.1188 & 59.808 & 11.8744 & 7954.9772 & 16.8805 \\
\hline 0 & 0 & $0.1311 \mathrm{NA}$ & & 0 & 0 & 2633.2957 \\
\hline 0 & 0 & $0 \mathrm{NA}$ & & 0 & 2565.3263 & 0 \\
\hline
\end{tabular}


pf FLOW

\begin{tabular}{|c|c|c|c|c|c|c|}
\hline V2/V1 (\%) & pf $A$ & pf $B$ & pf C & kW Flow A & kW Flow B & kW Flow C \\
\hline 0.0015 & 0.8498 & 0.8427 & 0.838 & 1580.4571 & 1809.311 & 1752.9747 \\
\hline 0.2088 & 0.8674 & 0.8623 & 0.8565 & 1575.6953 & 1806.2348 & 1743.876 \\
\hline 0.3089 & 0.8682 & 0.8656 & 0.8586 & 1344.3375 & 1601.8058 & 1482.6379 \\
\hline 0.3394 & 0.8692 & 0.8676 & 0.8603 & 1300.5374 & 1568.9705 & 1448.3102 \\
\hline 0.3089 & 0.8833 & 0.8992 & 0.917 & 40.0761 & 30.1402 & 33.6184 \\
\hline 0.3432 & 0.87 & 0.8686 & 0.8619 & 1298.7358 & 1544.9531 & 1447.9904 \\
\hline 0.345 & 0.8734 & 0.8766 & 0.8812 & 119.8594 & 315.6539 & 200.9773 \\
\hline 0.3461 & 0.8697 & 0.8667 & 0.8589 & 1178.6613 & 1229.138 & 1246.9655 \\
\hline 0.3668 & 0.8736 & 0.8777 & 0.8789 & 107.7664 & 304.5262 & 173.2681 \\
\hline 0.3677 & 0.8739 & 0.8775 & 0.8769 & 102.4174 & 304.5142 & 151.515 \\
\hline 0.3678 & 0.8689 & -0.0799 & 0.8928 & 5.349 & 0.0119 & 21.7531 \\
\hline 0.3809 & -1 & -1 & -1 & 0 & 0 & 0 \\
\hline 0.4714 & 0.8735 & 0.8707 & 0.8631 & 1140.0921 & 1195.1526 & 1215.1032 \\
\hline 0.4718 & 0.8703 & 0.8658 & 0.8684 & 712.0695 & 800.647 & 690.2474 \\
\hline 0.4727 & 0.8711 & 0.8755 & 0.8713 & 223.4181 & 142.3008 & 278.5733 \\
\hline 0.4721 & 0.8882 & 0.8844 & 0.8406 & 204.2579 & 252.041 & 246.0894 \\
\hline 0.4805 & 0.8704 & 0.8664 & 0.8686 & 711.8635 & 779.4645 & 690.1703 \\
\hline 0.4768 & 0.8704 & 0.8664 & 0.8686 & 711.792 & 779.4513 & 690.1382 \\
\hline 0.5404 & -0.0038 & -0.1003 & 0.1056 & 0 & 0 & 0 \\
\hline 0.5721 & 0.871 & 0.8715 & 0.8649 & 440.7576 & 375.3048 & 322.0443 \\
\hline 0.5711 & 0.8731 & 0.8727 & 0.8648 & 293.2731 & 134.5544 & 238.5249 \\
\hline 0.5719 & 0.8669 & 0.8709 & 0.8652 & 147.4439 & 240.7619 & 83.502 \\
\hline 0.5763 & -1 & -1 & -1 & 0 & 0 & 0 \\
\hline 0.5107 & -1 & -1 & -1 & 0 & 0 & 0 \\
\hline 0.5946 & 0.8897 & 0.8722 & 0.8735 & 120.8866 & 129.9473 & 66.5753 \\
\hline 0.5934 & 0.8896 & 0.8722 & 0.8738 & 120.8783 & 129.9443 & 64.5752 \\
\hline
\end{tabular}




\begin{tabular}{|c|c|c|c|c|c|c|}
\hline 0.5929 & 0.8896 & 0.8729 & 0.8737 & 120.8727 & 122.2559 & 64.5766 \\
\hline 0.5919 & 0.8923 & 0.8725 & 0.8766 & 85.7247 & 122.2442 & 50.9787 \\
\hline 0.5928 & 0.8828 & -0.0877 & 0.8621 & 35.1431 & 0.0099 & 13.599 \\
\hline 0.5838 & 0.0618 & 0.0941 & -0.1546 & 0 & -0.0018 & 0.0028 \\
\hline 49.836 & 0 & 0.8623 & -0.2553 & 0 & 3.0209 & 0.0066 \\
\hline NA & 0.9291 & 0 & 0 & 1.7787 & 0 & 0 \\
\hline 0.533 & 0.8675 & 0.8753 & 0.8709 & 118.0516 & 105.7122 & 245.7587 \\
\hline 1.2639 & 0.8668 & 0.8856 & 0.8791 & 43.8564 & 177.5682 & 508.7365 \\
\hline 0.5467 & 0.8679 & 0.8753 & 0.8709 & 74.1985 & 105.7093 & 245.7518 \\
\hline 0.5706 & 0.8672 & 0.8748 & 0.8714 & 74.2613 & 102.8992 & 215.8611 \\
\hline 0.5706 & 0.8617 & 0.8699 & 0.8684 & 69.0483 & 96.1532 & 146.4653 \\
\hline 0.5715 & 0.933 & 0.9368 & 0.878 & 5.2243 & 6.7311 & 69.3783 \\
\hline 0.5773 & 0.9273 & 0.9447 & 0.8814 & 5.2405 & 1.0966 & 56.8652 \\
\hline 0.5798 & 0.1525 & -0.0924 & 0.8814 & -0.0081 & 0.0052 & 53.2874 \\
\hline 0.5775 & 0.9217 & 0.9196 & 0.8792 & 0.8852 & 1.0887 & 3.575 \\
\hline NA & 0 & 0 & 0 & 0 & 0 & 0 \\
\hline 0.2027 & 0.8674 & 0.8623 & 0.8565 & 1575.6959 & 1806.2345 & 1743.8758 \\
\hline 0.2024 & 0.8765 & 0.871 & 0.8745 & 189.8084 & 192.8982 & 247.2446 \\
\hline 0.2048 & 0.8664 & 0.8615 & 0.8539 & 1385.5885 & 1612.6247 & 1496.6866 \\
\hline 0.2081 & 0.8819 & 0.8846 & 0.876 & 63.0834 & 62.2831 & 161.5408 \\
\hline NA & 0 & 0 & 0.8606 & 0 & 0 & 76.8906 \\
\hline NA & 0 & 0 & 0.8599 & 0 & 0 & 0.7877 \\
\hline 0.2083 & 0.8819 & 0.8846 & 0.89 & 63.0834 & 62.2831 & 83.8625 \\
\hline 0.2122 & 0.9261 & 0.8671 & -0.0568 & 2.7398 & 1.5706 & 0.0014 \\
\hline NA & 0 & 0 & 0.8601 & 0 & 0 & 2.6726 \\
\hline 0 & 0 & 0 & 0 & 0 & 0 & 0 \\
\hline
\end{tabular}


kVAR Flow A kVAR Flow B kVAR Flow C

\begin{tabular}{|c|c|c|}
\hline 980.2917 & 1156.1179 & 1141.532 \\
\hline 904.118 & 1060.7034 & 1050.9932 \\
\hline 768.4928 & 926.5778 & 885.2514 \\
\hline 739.75 & 899.2868 & 858.1904 \\
\hline 21.2659 & 14.6679 & 14.6282 \\
\hline 736.1879 & 881.3615 & 852.071 \\
\hline 66.8173 & 173.2785 & 107.7932 \\
\hline 668.9267 & 707.346 & 743.5245 \\
\hline 60.0243 & 166.2396 & 94.0404 \\
\hline 56.9766 & 166.3883 & 83.067 \\
\hline 3.0477 & -0.1486 & 10.9734 \\
\hline 0 & 0 & 0 \\
\hline 635.5206 & 675.2037 & 710.8971 \\
\hline 402.9679 & 462.6705 & 394.1484 \\
\hline 125.9318 & 78.5502 & 156.8848 \\
\hline 105.6756 & 133.0068 & 158.5757 \\
\hline 402.5919 & 449.2327 & 393.7029 \\
\hline 402.4412 & 449.008 & 393.533 \\
\hline-0.003 & -0.0027 & -0.0027 \\
\hline 248.6105 & 211.181 & 186.9282 \\
\hline 163.7406 & 75.2687 & 138.5139 \\
\hline 84.785 & 135.8595 & 48.387 \\
\hline 0 & 0 & 0 \\
\hline 0 & 0 & 0 \\
\hline 62.0248 & 72.8681 & 37.0982 \\
\hline 62.046 & 72.8788 & 35.9416 \\
\hline
\end{tabular}




\begin{tabular}{|c|c|c|}
\hline 62.063 & 68.3221 & 35.9626 \\
\hline 43.3819 & 68.4443 & 27.9884 \\
\hline 18.6962 & -0.1121 & 7.9934 \\
\hline-0.0158 & -0.0193 & -0.0179 \\
\hline 0 & 1.774 & -0.0249 \\
\hline 0.7079 & 0 & 0 \\
\hline 67.6977 & 58.3967 & 138.6665 \\
\hline 25.2285 & 93.1255 & 275.851 \\
\hline 42.4745 & 58.4064 & 138.6507 \\
\hline 42.6478 & 56.9816 & 121.4951 \\
\hline 40.6686 & 54.5054 & 83.6167 \\
\hline 2.0144 & 2.5146 & 37.8295 \\
\hline 2.1153 & 0.3805 & 30.4788 \\
\hline-0.0525 & -0.0563 & 28.5605 \\
\hline 0.3725 & 0.4652 & 1.937 \\
\hline 0 & 0 & 0 \\
\hline 904.1216 & 1060.7072 & 1050.9963 \\
\hline 104.2233 & 108.7893 & 137.1452 \\
\hline 798.7348 & 950.3567 & 912.2053 \\
\hline 33.7157 & 32.8395 & 88.9252 \\
\hline 0 & 0 & 45.5001 \\
\hline 0 & 0 & 0.4675 \\
\hline 33.7157 & 32.8395 & 42.9576 \\
\hline 1.1161 & 0.9025 & -0.024 \\
\hline 0 & 0 & 1.5853 \\
\hline 0 & 0 & 0 \\
\hline
\end{tabular}




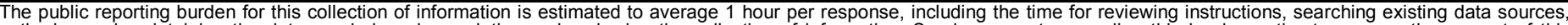

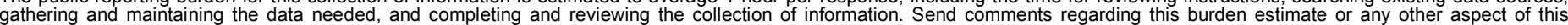

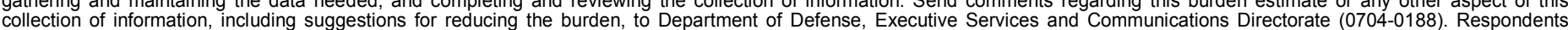

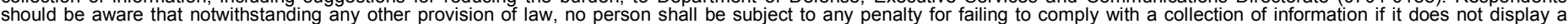

should be aware that notwithstanding

PLEASE DO NOT RETURN YOUR FORM TO THE ABOVE ORGANIZATION.

\begin{tabular}{l|l|l|l} 
1. REPORT DATE $(D D-M M-Y Y Y Y)$ & 2. REPORT TYPE & 3. DATES COVERED (FrOm - TO)
\end{tabular}

July 2007

Subcontract report

4. TITLE AND SUBTITLE
Modeling and Testing of Unbalanced Loading and Voltage

Regulation: Final Report

5a. CONTRACT NUMBER

DE-AC36-99-G010337

5b. GRANT NUMBER

5c. PROGRAM ELEMENT NUMBER

6. AUTHOR(S)

M.W. Davis, R. Broadwater, and J. Hambrick

5d. PROJECT NUMBER

NREL/SR-581-41805

5e. TASK NUMBER

WW88.2003

5f. WORK UNIT NUMBER
7. PERFORMING ORGANIZATION NAME(S) AND ADDRESS(ES)

National Renewable Energy Laboratory

1617 Cole Blvd.

Golden, CO 80401-3393
8. PERFORMING ORGANIZATION REPORT NUMBER

NREL/SR-581-41805

9. SPONSORING/MONITORING AGENCY NAME(S) AND ADDRESS(ES)

10. SPONSOR/MONITOR'S ACRONYM(S)

NREL

11. SPONSORING/MONITORING AGENCY REPORT NUMBER

12. DISTRIBUTION AVAILABILITY STATEMENT

National Technical Information Service

U.S. Department of Commerce

5285 Port Royal Road

Springfield, VA 22161

13. SUPPLEMENTARY NOTES

14. ABSTRACT (Maximum 200 Words)

This paper is a final report on a project to (1) explain how voltage regulation reduces voltage spread; (2) define the effects of unbalanced loading and voltage on system protection and distributed generation output ratings; (3) develop models for an actual distribution circuit, its voltage regulation equipment, and distributed generation; (4) validate the models by comparing power flow simulations and voltage profiles with actual measured circuit data; (5) determine the optimum generator operating conditions to provide the greatest improvement in released capacity, reduced energy losses, and voltage regulation, and (6) determine the maximum distributed generation penetration limits with synchronous generator real and reactive power injections.

15. SUBJECT TERMS

distributed generation; DG; distributed resources; DR; interconnection; voltage regulation; modeling; penetration limit; National Renewable Energy Laboratory; NREL; DTE Energy

\begin{tabular}{|c|c|c|c|c|c|}
\hline 16. SECURITY & CLASSIFICATI & N OF: & 17. LIMITATION & 18. NUMBER & 19a. NAME OF RESPONSIBLE PERSON \\
\hline $\begin{array}{l}\text { a. REPORT } \\
\text { Unclassified }\end{array}$ & $\begin{array}{l}\text { b. ABSTRACT } \\
\text { Unclassified }\end{array}$ & $\begin{array}{l}\text { c. THIS PAGE } \\
\text { Unclassified }\end{array}$ & $\begin{array}{c}\text { OF ABSTRACT } \\
\text { UL }\end{array}$ & & 19b. TELEPHONE NUMBER (Include area code) \\
\hline
\end{tabular}

